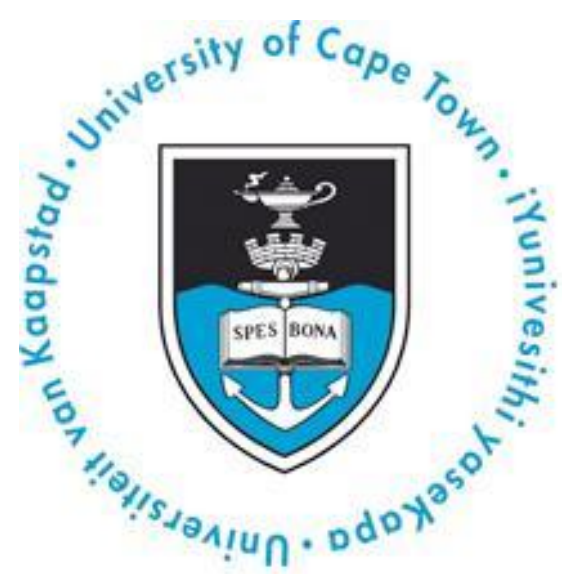

\title{
MULTI-OBJECTIVE OPTIMISATION UNDER DEEP UNCERTAINTY
}

\section{Babooshka Shavazipour}

\author{
Thesis Presented for the Degree of \\ DOCTOR OF PHILOSOPHY \\ Department of Statistical Sciences \\ UNIVERSITY OF CAPE TOWN
}

January 28, 2018

Supervised by: Prof. Theodor J Stewart 
The copyright of this thesis vests in the author. No quotation from it or information derived from it is to be published without full acknowledgement of the source. The thesis is to be used for private study or noncommercial research purposes only.

Published by the University of Cape Town (UCT) in terms of the non-exclusive license granted to UCT by the author. 
To my parents, Nasrin and Hamid

who provided me with a better opportunity to study than was available to themselves

and

To my beloved wife, Sarah

for all of her love and support 


\begin{abstract}
Most of the decisions in real-life problems need to be made in the absence of complete knowledge about the consequences of the decision. Furthermore, in some of these problems, the probability and/or the number of different outcomes are also unknown (named deep uncertainty). Therefore, all the probability-based approaches (such as stochastic programming) are unable to address these problems. On the other hand, involving various stakeholders with different (possibly conflicting) criteria in the problems brings additional complexity.

The main aim and primary motivation for writing this thesis have been to deal with deep uncertainty in Multi-Criteria Decision-Making (MCDM) problems, especially with long-term decision-making processes such as strategic planning problems.

To achieve these aims, we first introduced a two-stage scenario-based structure for dealing with deep uncertainty in Multi-Objective Optimisation (MOO)/MCDM problems. The proposed method extends the concept of two-stage stochastic programming with recourse to address the capability of dealing with deep uncertainty through the use of scenario planning rather than statistical expectation. In this research, scenarios are used as a dimension of preference (a component of what we term the meta-criteria) to avoid problems relating to the assessment and use of probabilities under deep uncertainty. Such scenario-based thinking involved a multi-objective representation of performance under different future conditions as an alternative to expectation, which fitted naturally into the broader multi-objective problem context. To aggregate these objectives of the problem, the Generalised Goal Programming (GGP) approach is used. Due to the capability of this approach to handle large numbers of objective functions/criteria, the GGP is significantly useful in the proposed framework. Identifying the goals for each criterion is the only action that the Decision Maker (DM) needs to take without needing to investigate the trade-offs between different criteria.

Moreover, the proposed two-stage framework has been expanded to a three-stage structure and a moving horizon concept to handle the existing deep uncertainty in more complex problems, such as strategic planning. As strategic planning problems will deal with more than two stages and real processes are continuous, it follows that more scenarios will continuously be unfolded that may or may not be periodic. "Stages", in this study, are artificial constructs to structure thinking of an indefinite future. A suitable length of the planning window and stages in the proposed methodology are also investigated.

Philosophically, the proposed two-stage structure always plans and looks one step ahead while the three-stage structure considers the conditions and consequences of two upcoming steps in advance, which fits well with our primary objective. Ignoring longterm consequences of decisions as well as likely conditions could not be a robust strategic approach. Therefore, generally, by utilising the three-stage structure, we may expect a more robust decision than with a two-stage representation.

Modelling time preferences in multi-stage problems have also been introduced to solve the fundamental problem of comparability of the two proposed methodologies because of the different time horizon, as the two-stage model is ignorant of the third stage. This concept has been applied by a differential weighting in models. Importance weights, then, are primarily used to make the two- and three-stage models more directly comparable, and only secondarily as a measure of risk preference. Differential weighting can help us
\end{abstract}


apply further preferences in the model and lead it to generate more preferred solutions.

Expanding the proposed structure to the problems with more than three stages which usually have too many meta-scenarios may lead us to a computationally expensive model that cannot easily be solved, if it all. Moreover, extension to a planning horizon that too long will not result in an exact plan, as nothing in nature is predictable to this level of detail, and we are always surprised by new events. Therefore, beyond the expensive computation in a multi-stage structure for more than three stages, defining plausible scenarios for far stages is not logical and even impossible. Therefore, the moving horizon models in a T-stage planning window has been introduced.

To be able to run and evaluate the proposed two- and three-stage moving horizon frameworks in longer planning horizons, we need to identify all plausible meta-scenarios. However, with the assumption of deep uncertainty, this identification is almost impossible. On the other hand, even with a finite set of plausible meta-scenarios, comparing and computing the results in all plausible meta-scenarios are hardly possible, because the size of the model grows exponentially by raising the length of the planning horizon. Furthermore, analysis of the solutions requires hundreds or thousands of multi-objective comparisons that are not easily conceivable, if it all. This issues motivated us to perform a Simulation-Optimisation study to simulate the reasonable number of meta-scenarios and enable evaluation, comparison and analysis of the proposed methods for the problems with a T-stage planning horizon. In this Simulation-Optimisation study, we started by setting the current scenario, the scenario that we were facing it at the beginning of the period. Then, the optimisation model was run to get the first-stage decisions which can implement immediately. Thereafter, the next scenario was randomly generated by using Monte Carlo simulation methods. In deep uncertainty, we do not have enough knowledge about the likelihood of plausible scenarios nor the probability space; therefore, to simulate the deep uncertainty we shall not use anything of scenario likelihoods in the decision models. The two- and three-stage Simulation-Optimisation algorithms were also proposed.

A comparison of these algorithms showed that the solutions to the two-stage moving horizon model are feasible to the other pattern (three-stage). Also, the optimal solution to the three-stage moving horizon model is not dominated by any solutions of the other model. So, with no doubt, it must find better, or at least the same, goal achievement compared to the two-stage moving horizon model. Accordingly, the three-stage moving horizon model evaluates and compares the optimal solution of the corresponding twostage moving horizon model to the other feasible solutions, then, if it selects anything else it must either be better in goal achievement or be robust in some future scenarios or a combination of both. However, the cost of these supremacies must be considered (as it may lead us to a computationally expensive problem), and the efficiency of applying this structure needs to be approved.

Obviously, using the three-stage structure in comparison with the two-stage approach brings more complexity and calculations to the models. It is also shown that the solutions to the three-stage model would be preferred to the solutions provided by the two-stage model under most circumstances. However, by the "efficiency" of the threestage framework in our context, we want to know that whether utilising this approach and its solutions is worth the expense of the additional complexity and computation.

The experiments in this study showed that the three-stage model has advantages 
under most circumstances(meta-scenarios), but that the gains are quite modest. This issue is frequently observed when comparing these methods in problems with a shortterm (say less than five stages) planning window. Nevertheless, analysis of the length of the planning horizon and its effects on the solutions to the proposed frameworks indicate that utilising the three-stage models is more efficient for longer periods because the differences between the solutions of the two proposed structures increase by any iteration of the algorithms in moving horizon models. Moreover, during the long-term calculations, we noticed that the two-stage algorithm failed to find the optimal solutions for some iterations while the three-stage algorithm found the optimal value in all cases.

Thus, it seems that for the planning horizons with more than ten stages, the efficiency of the three-stage model be may worth the expenses of the complexity and computation. Nevertheless, if the DM prefers to not use the three-stage structure because of the complexity and/or calculations, the two-stage moving horizon model can provide us with some reasonable solutions, although they might not be as good as the solutions generated by a three-stage framework.

Finally, to examine the power of the proposed methodology in real cases, the proposed two-stage structure was applied in the sugarcane industry to analyse the whole infrastructure of the sugar and bioethanol Supply Chain (SC) in such a way that all economics (Max profit), environmental ( $\mathrm{Min} \mathrm{CO}_{2}$ ), and social benefits (Max job-creations) were optimised under six key uncertainties, namely sugarcane yield, ethanol and refined sugar demands and prices, and the exchange rate. Moreover, one of the critical design questions - that is, to design the optimal number and technologies as well as the best place(s) for setting up the ethanol plant(s) - was also addressed in this study. The general model for the strategic planning of sugar-bioethanol supply chains (SC) under deep uncertainty was formulated and also examined in a case study based on the South African Sugar Industry. This problem is formulated as a Scenario-Based MixedInteger Two-Stage Multi-Objective Optimisation problem and solved by utilising the Generalised Goal Programming Approach.

To sum up, the proposed methodology is, to the best of our knowledge, a novel approach that can successfully handle the deep uncertainty in MCDM/MOO problems with both short- and long-term planning horizons. It is generic enough to use in all MCDM problems under deep uncertainty. However, in this thesis, the proposed structure only applied in Linear Problems (LP). Non-linear problems would be an important direction for future research. Different solution methods may also need to be examined to solve the non-linear problems. Moreover, many other real-world optimisation and decision-making applications can be considered to examine the proposed method in the future. 



\section{Acknowledgements}

Firstly, I would like to express my sincere gratitude to my supervisor Prof. Theodor J Stewart for the continuous support during my Ph.D study and related research. I am exceptionally grateful for his patience, motivation, and immense knowledge. His guidance helped me throughout research and writing phases of this thesis. Without his guidance and persistent help this dissertation would not have been possible. I could not have imagined having a better supervisor and mentor for my Ph.D study.

I would also like to thank the Department of Statistical Sciences and Faculty of Science for funding this work via the SCIENCE FACULTY PhD FELLOWSHIP 20142016. Without this fellowship, I would not have been able to take part in this graduate program.

I would like to thank Dr Jonas Stray, who either worked with me as a colleague during the preparation of the final chapter (Sugarcane Application) or who cheered me up as a friend.

I would also like to thank Dr Karis Moxley for the grammatical editing of my thesis. Without her flawless suggestions, this thesis would simply not be readable.

I could not find suitable words to express my deepest appreciation for my parents, family and friends for their continuous love and support.

Finally, I would like to dedicate this thesis to my beloved wife, Sarah, who was always there to support me. She sacrificed her time and stood by my side through thick and thin, and was my rock through smooth and rough times. Sarah helped me throughout the endless hours and days that I was working on this project and I am deeply grateful for her.

25th January 2018

Babooshka Shavazipour 



\section{Contents}

1 Introduction 1

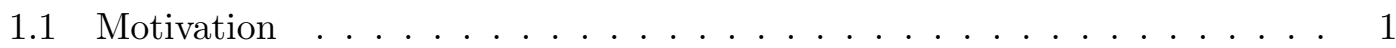

1.2 Research objectives . . . . . . . . . . . . . . . 2

1.3 Overview of the dissertation . . . . . . . . . . . . . . 2

2 Uncertainty in Multi-Objective Optimisation Problems 5

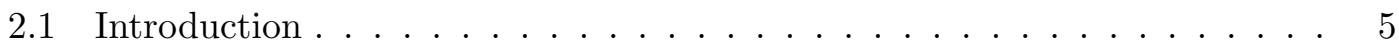

2.2 Uncertainty . . . . . . . . . . . . . . . . . . . 6

2.2 .1 General definitions . . . . . . . . . . . . . . . . . . 6

2.2.2 Classification of uncertainty from optimisation viewpoint . . . . 7

2.2 .3 Dealing with uncertainty . . . . . . . . . . . . . . 12

2.3 Multi-Objective Optimisation . . . . . . . . . . . . . . . 15

2.4 Goal Programming under uncertainty . . . . . . . . . . . . . 16

2.4 .1 General formulation . . . . . . . . . . . . . . . . 16

2.4.2 Stochastic Goal Programming (SGP) . . . . . . . . . . . . 18

2.4 .3 Scenario-based Goal programming . . . . . . . . . . . . . . 25

2.4.4 Fuzzy Goal Programming (FGP) . . . . . . . . . . . . . . 27

2.4.5 Robust Goal Programming (RGP) . . . . . . . . . . . . 30

2.4.6 Dynamic Goal Programming . . . . . . . . . . . . . . . . 31

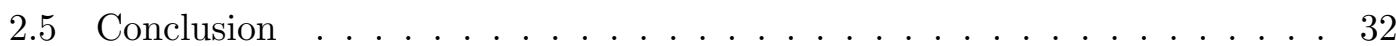

3 Two-stage Scenario-Based Multi-Criteria Decision-Making/ Multiple $\begin{array}{lr}\text { Objective Optimisation Structure } & \mathbf{3 7}\end{array}$

3.1 Introduction . . . . . . . . . . . . . . . . . . . 37

3.2 Proposed two-stage approach . . . . . . . . . . . . . . . 39

3.2 .1 Mathematical Formulation . . . . . . . . . . . . . . . . 41

3.3 Solving by Generalised Goal Programming (GGP) . . . . . . . . . . . . . 43

3.4 Simple farming example . . . . . . . . . . . . . . . . . . . 46

3.4.1 Discussion of contributions to planning and understanding . . . . . 51

3.4.2 Analysis of the variations in importance weights . . . . . . . . 52

3.4 .3 Analysis of the variations in goal levels . . . . . . . . . . . . 53

3.5 Example illustrating scenario effects . . . . . . . . . . . . . 55

3.5.1 Comparison with single-scenario models . . . . . . . . . . . . 57

3.5.2 Comparison with Robust Goal Programming . . . . . . . . . . . . 60

3.6 Conclusion . . . . . . . . . . . . . . . . . . 61

3.7 Appendix: Model formulations for case studies . . . . . . . . . . . . 63 
3.7.1 The two-stage multi-objective optimisation model of example 3.4 . 63

3.7.2 The equivalent GP formulated model of example 3.4 (after normalising) . . . . . . . . . . . . . . . 6 64

3.7.3 The GP model for $3.5 .1 \ldots \ldots . \ldots . \ldots 65$

3.7.4 Single-scenario models for example $3.5 .2 \ldots \ldots$. . . . . . . . 66

4 Three-stage Scenario-Based Multi-Criteria Decision Making Structure 69

4.1 Introduction . . . . . . . . . . . . . . . . . . . . 69

4.2 Proposed three-stage structure . . . . . . . . . . . . . . 71

4.2.1 Extension of the concept of meta-criterion in the three-stage struc-

ture ............................ 74

4.2 .2 Mathematical Formulation . . . . . . . . . . . . . . . 74

4.3 Two-stage vs. Three-stage structure . . . . . . . . . . . . . 78

4.3.1 Mathematical distinctions . . . . . . . . . . . . . . . . . 79

4.3.2 Philosophical distinctions . . . . . . . . . . . . . 80

4.4 Two-stage moving horizon model . . . . . . . . . . . . . . . 80

4.5 Illustrative example (Sequential Investment). . . . . . . . . . . . . . . 84

4.5.1 Numerical comparison of two-stage model approximation and full

three-stage solution . . . . . . . . . . . . 88

4.5.2 Sensitivity analysis . . . . . . . . . . . . . . . . . . 92

4.6 Two-stage robustness . . . . . . . . . . . . . . . . . . . . . . . . . . 95

4.7 Conclusion . . . . . . . . . . . . . . . . . . 97

4.8 Appendix . . . . . . . . . . . . . . . . . . . . . . 100

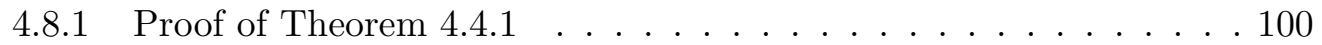

4.8.2 Two-stage model for example 4.5.1 . . . . . . . . . . . . . 102

4.8.3 Two-stage GP model for example 4.5.1 . . . . . . . . . . . . . . 102

4.8 .4 Two-stage model . . . . . . . . . . . . . . . . . . . 103

4.8.5 Three-stage GP model for example 4.5.1 . . . . . . . . . . . 103

4.8 .6 Notations for example $4.5 .1 \ldots$. . . . . . . . . . . . 103

5 A Simulation-Optimisation Study on Moving Horizon Structure under $\begin{array}{lr}\text { Deep Uncertainty } & 105\end{array}$

5.1 Introduction . . . . . . . . . . . . . . . . . . . . . . . . 105

5.1.1 Chapter Motivations Summary . . . . . . . . . . . . 106

5.2 Moving horizon models in a T-stage planning window . . . . . . . . . . 108

5.2 .1 Rolling two-stage structure . . . . . . . . . . . . . . . . 109

5.2 .2 Rolling three-stage structure . . . . . . . . . . . . . . . 111

5.2.3 Comparing the rolling two-stage pattern with the three-stage framework in a moving horizon structure . . . . . . . . . . . 113

5.3 Modelling of Time Preferences in Multi-Objective Multi-stage Models . . 115

5.3 .1 Differential Weighting . . . . . . . . . . . . . 116

5.3 .2 Discounted Weights . . . . . . . . . . . . . . . 117

5.4 Simulation-Optimisation study . . . . . . . . . . . . . . 117

5.4.1 Simulating the two-stage moving horizon structure in a T-stage planning horizon . . . . . . . . . . . . . . . . 118

5.4.2 Simulating three-stage moving horizon structure in a T-stage planning horizon . . . . . . . . . . . . . . . . . 119 
5.4.3 How many simulations need to be run? . . . . . . . . . . . . . . . 119

5.5 Illustrative example . . . . . . . . . . . . . . . . . . . . 120

5.5.1 Discussion on trade-offs and Pareto Front . . . . . . . . . . . . 121

5.6 Effects of the Length of Planning Horizon . . . . . . . . . . . . . . . . 132

5.7 Conclusion . . . . . . . . . . . . . . . . . . . . 135

6 Multi-Objective Optimisation of the Sugar and Bioethanol Supply Chain under Deep Uncertainty 139

6.1 Introduction . . . . . . . . . . . . . . . . . . . . . 139

6.2 Problem description . . . . . . . . . . . . . . . . . . . . . 141

6.2.1 Existing Uncertainties . . . . . . . . . . . . . . . . . . . . . 143

6.3 Model structure and formulation . . . . . . . . . . . . . . . . . . 145

6.3 .1 Two-stage structure . . . . . . . . . . . . . . . . . . . . . 145

6.3 .2 Objective functions . . . . . . . . . . . . . . . . . . 146

6.3 .3 Constraints . . . . . . . . . . . . . . . . . . . . 151

6.3.4 Solving by Generalised Goal Programming (GGP) . . . . . . . . 153

6.4 Case study (SA sugar-ethanol supply chain) . . . . . . . . . . . . . . . 154

6.4 .1 Modelling assumptions . . . . . . . . . . . . . . 155

6.4 .2 Uncertainties and scenarios . . . . . . . . . . . . . . 156

6.4 .3 Solution procedure and goals . . . . . . . . . . . . 158

6.5 Case study results and discussion . . . . . . . . . . . . . . . 158

6.5.1 First-stage/initial decisions . . . . . . . . . . . . . . . . 159

6.5.2 Second-stage/recourse decisions . . . . . . . . . . . . . . . 160

6.5.3 Objective functions and achievements . . . . . . . . . . . 161

6.5.4 Sensitivity analysis on various goals . . . . . . . . . . . . 163

6.6 Conclusions . . . . . . . . . . . . . . . . . 166

6.7 Notation . . . . . . . . . . . . . . . . . . . . . . . . 169

6.8 Appendix . . . . . . . . . . . . . . . . . . . . 174

7 Conclusions and Future Directions $\quad 177$

7.1 Summary and Conclusions . . . . . . . . . . . . . . . . 177

7.2 Future Directions . . . . . . . . . . . . . . . . . . . . 181

Appendix A: Case study data 183

$\begin{array}{ll}\text { Appendix B: Case study results (Figures) } & 187\end{array}$

$\begin{array}{ll}\text { Appendix C: Case study results (Tables) } & 191\end{array}$ 


\section{List of Figures}

2.1 Different sources of the nature of uncertainty $\ldots \ldots \ldots$. . . . . 8

2.2 Different Degrees of Uncertainty . . . . . . . . . . . . . . . . . . . . . 10

2.3 The general shape of the satisfaction functions $\ldots \ldots \ldots 21$

3.1 Two-stage decision-making process with $p$ scenarios . . . . . . . . . . . . 39

3.2 The schematic of two-stage decision-making process $\ldots \ldots$. . . . . . 40

4.1 Three-stage decision-making process with $p(1) \times p(2)$ scenarios $\ldots \ldots . .72$

4.2 the schematic of three-stage decision making process $\ldots \ldots$. . . . . 73

4.3 Rolling two-stage structure compared to three-stage structure . . . . . . . 81

4.4 Scenarios of the two-stage structure in comparison with meta-scenarios of the three-stage structure . . . . . . . . . . . . . 86

4.5 Scenarios of the $2 \times$ two-stage structure $\ldots \ldots \ldots \ldots$. . . . . . 89

4.6 Results of the $2 \times$ two-stage structure in comparison with the three-stage structure . . . . . . . . . . . . . . . . . . . . 91

4.7 Results of the $2 \times$ two-stage structure in comparison with the three-stage structure. . . . . . . . . . . . . . . . . . . . 92

5.1 Rolling $T-1$ consecutive two-stage and T-2 following three-stage in a T-stage horizon . . . . . . . . . . . . . . . . . . 108

5.2 Optimisation and Simulation process . . . . . . . . . . . . . 118

5.3 Mean of cash flow and withdrawal at the end of the stages in 700 simulation runs $(T=6)$, comparison of rolling two-stage and the three-stage structures. . . . . . . . 122

5.4 Simulated Pareto frontiers of rolling two-stage and the three-stage structures in the worst case meta-scenario (3-2-1-1-1-1) (1000 simulation runs with random weights between 1 and 30 in a discounting manner for 5 -stage planning horizon $(T=6))$. . . . . 124

5.5 Simulated Pareto frontiers of rolling two-stage and the three-stage structures in some specific meta-scenarios (1000 simulation runs with random weights between 1 and 30 in a discounting manner for 5 -stage planning horizon $(T=6))$. . . . . . . . . 126

5.6 Simulated Pareto frontiers of rolling two-stage and the three-stage structures in some specific meta-scenarios (1000 simulation runs with random weights between 1 and 30 in a discounting manner for 5 -stage planning horizon $(T=6))$. . . . . . . . . 127

5.7 Combined picture of cash flow and total withdrawal at the end of the fifth stage in 1000 simulation runs $(T=6)$, showing a comparison between the rolling two- and the three-stage structures with and without applying time preferences. . . . . . . . . . . 129 
5.8 Combined picture of cash flow and total withdrawal at the end of the different stages with discounted weights in 300 simulation runs $(T=11,16,21)$, to compare the rolling two-stage and the three-stage structures. . . . . . . . . . . . . . . 134

6.1 Sugar and Bioethanol SC . . . . . . . . . . . . . . . . . . . . 142

6.2 Sugar and ethanol production technologies used in mills and refineries . . 143

6.3 Set of production technologies in an ethanol plant . . . . . . . . . . . 144

6.4 SA sugar Mills and the central refinery locations . . . . . . . . . . . 155

6.5 Ethanol plants locations . . . . . . . . . . . . . . . . . . 159

6.6 A combined picture of objectives in different scenarios(from two various angles $\ldots \ldots \ldots \ldots \ldots \ldots 16 \ldots \ldots \ldots \ldots$

6.7 Economics objective in different scenarios $\left(Z_{1 k}\right) \ldots \ldots$. . . . . . . 162

6.8 Environmental objective in different scenarios $\left(Z_{2 k}\right) \ldots \ldots . \ldots 162$

6.9 Social benefits objective in different scenarios $\left(Z_{3 k}\right) \ldots \ldots$. . . . . . 163

1 Ethanol importation in different meta-scenarios . . . . . . . . . . . . 187

2 By-product Sale in different meta-scenarios . . . . . . . . . . . . . . 187

3 Molasses Sale in different meta-scenarios . . . . . . . . . . . . . . . . 188

4 Molasses send back to ethanol plants (Recourse decision) in different metascenarios . . . . . . . . . . . . . . . . . . . . 188

5 Raw sugar send back to ethanol plants (Recourse decision) in different meta-scenarios . . . . . . . . . . . . . . . . . 188

$6 \quad$ White sugar send back to ethanol plants (Recourse decision) in different meta-scenarios . . . . . . . . . . . . . . . . . . . 189

$7 \quad$ White sugar Exportation in different meta-scenarios . . . . . . . . . . 189

8 Raw sugar Exportation in different meta-scenarios . . . . . . . . . . . . . 189

9 Total Molasses Production Recourse in different meta-scenarios . . . . . . 190

10 Total Raw sugar Production Recourse in different meta-scenarios . . . . . 190 


\section{List of Tables}

2.1 Different classifications of the location of uncertainty. . . . . . . . . . . 9

2.2 Different classifications of the depth of uncertainty. . . . . . . . . . . . 11

2.3 GP review on different methodologies . . . . . . . . . . . . . 35

2.4 GP review on nature and degrees of uncertainty . . . . . . . . . . . 36

3.1 Farming data set . . . . . . . . . . . . . . . . . . . . 47

3.2 Goals and weights . . . . . . . . . . . . . . . . . . 49

3.3 The result of the proposed two-stage MOLP for farming example . . . . . 50

3.4 Goal achievements variations in different importance weights . . . . . . . 54

3.5 Goal achievements variations in different goal levels . . . . . . . . . . . . 55

3.6 The amount of the coefficients of the model in the case of five different

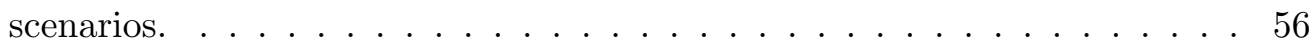

3.7 The result of the proposed two-stage MOLP for example 3.5.1 . . . . . 56

3.8 Comparing different solutions of various models . . . . . . . . . . 57

3.9 Comparing the feasibility of each solution in different scenario realisations 57

3.10 Achievements which could be reached by applying the solution of scenario 1 model as an initial solution together with the possible recourse action in example $3.5 .1 \ldots \ldots \ldots$. . . . . . . . . . . . . . . 59

3.11 Achievements which could be reached by applying the solution of scenario 2 model as an initial solution together with the possible recourse action in example $3.5 .1 \ldots \ldots \ldots$. . . . . . . . . . . . . . . . 59

3.12 Achievements which could be reached by applying the solution of scenario 3 model as an initial solution together with the possible recourse action

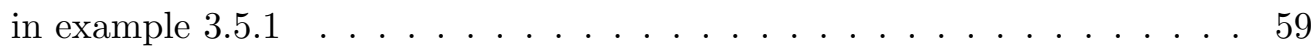

3.13 Achievements which could be reached by applying the solution of scenario 4 model as an initial solution together with the possible recourse action in example $3.5 .1 \ldots \ldots \ldots$. . . . . . . . . . . . . . . 59

3.14 Achievements which could be reached by applying the solution of scenario 5 model as an initial solution together with the possible recourse action in example $3.5 .1 \ldots \ldots \ldots \ldots$. . . . . . . . . . . . . . . 59

3.15 A summary of differences between the objective function $(\varphi)$ of the proposed two-stage model and the others in various scenarios . . . . . . . . 60

3.16 The proposed Two-Stage approach in comparison with the Robust GP . . 61

4.1 Possible transition between different scenarios . . . . . . . . . . . . . . . 84

4.2 Percentage growths for each investment under each scenario . . . . . . . . 84 
4.3 Percentage of loss of funds (penalty cost) for transferring between each pair of investment and withdrawal in state $S_{1} \ldots \ldots$. . . . . 85

4.4 Percentage of loss of funds (penalty cost) for transferring between each pair of investment and withdrawal in state $S_{2} \ldots \ldots \ldots$. . . . . 85

4.5 Percentage of loss of funds (penalty cost) for transferring between each pair of investment and withdrawal in state $S_{3} \ldots \ldots \ldots$. . . . . 85

4.6 Percentage of loss of funds (penalty cost) for transferring between each pair of investment and withdrawal in state $S_{4} \ldots \ldots \ldots$. . . . . 85

4.7 Percentage of loss of funds (penalty cost) for transferring between each pair of investment and withdrawal in state $S_{5} \ldots \ldots \ldots$. . . . 85

4.8 Desirable levels of total remained funds(million Rands) after consumption in each state . . . . . . . . . . . . . . . . . 86

4.9 Result of three-stage, two-stage, and $2 \times$ two-stage model . . . . . . . . . 90

4.10 Results comparison between three-stage and $2 \times$ two-stage models . . . . . 91

4.11 Percentage growths for each investment under each scenario . . . . . . . . 93

4.12 Results comparison between three-stage and $2 \times$ two-stage models . . . . . 93

4.13 Results comparison between three-stage and $2 \times$ two-stage models . . . . . 94

4.14 Percentage growths for each investment under each scenario . . . . . . . . 96

4.15 Percentage of loss of funds (penalty cost) for transferring between each pair of investment and withdrawal . . . . . . . . . . . . 996

4.16 Results comparison between three-stage and $2 \times$ two-stage models . . . . . 96

4.17 Results comparison between three-stage and $2 \times$ two-stage models . . . . . 97

4.18 Example 4.5 .1 variables notation . . . . . . . . . . . . . . . . . . 104

5.1 E3 Comparison results of 700 simulation runs in a five-stage horizon . . . . . . . . . 121

5.2 Analysis of various weights . . . . . . . . . . . . . 131

5.3 Meta-criteria of the second stage in a two or three-stage model. . . . . . . 132

5.4 Comparison results of 300 simulations in long-term horizons $(5,10,15,20,25$, and 30

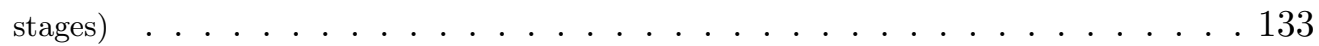

6.1 Some recent related works proposing models for optimising biofuel SCs under uncertainty . . . . . . . . . . . . . . . . . . 140

6.2 Demand . . . . . . . . . . . . . . . . . . . . . 157

6.3 Prices (estimated from last 30 years prices) . . . . . . . . . . . 157

6.4 Sugarcane yields and exchange rate in different scenarios (estimated from

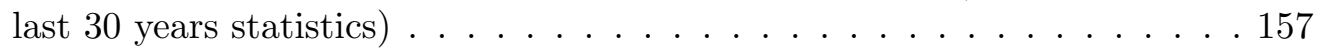

6.5 Sugarcane yields and exchange rate in different scenarios . . . . . . . . 158

6.6 Land use . . . . . . . . . . . . . . . . . . . . . . . . 160

6.7 Sensitivity analysis on various goals . . . . . . . . . . . . 165

6.8 variables notation . . . . . . . . . . . . . . . . . . . . . . . 169

6.9 Estimated available land(Thousand hectares) . . . . . . . . . . . . . 174

6.10 Distances . . . . . . . . . . . . . . . . . . . . . . 175

6.11 Production Capacity (Thousand Tonnes) (guessed) . . . . . . . . . . . 175

1 SA sugar-ethanol SC data . . . . . . . . . . . . . . . . . 183

2 Mills Operations . . . . . . . . . . . . . . . . . . 191 
3 Recourse decisions part $1 \ldots \ldots$. . . . . . . . . . . . 191

4 Recourse decisions part $2 \ldots \ldots$. . . . . . . . . . . . 197 


\section{Chapter 1}

\section{Introduction}

\subsection{Motivation}

This thesis concerns the disability of the existing multi-criteria decision-making (MCDM) methodologies in dealing with higher degrees of uncertainty which are frequently observed in many real-life processes, such as long-term strategic planning problems. In most of these problems, the decisions must be made before one can begin to explore the consequences of the decision or gain sufficient awareness of all other options. Furthermore, this kind of uncertainty cannot be reduced (such as by gathering more information) nor can it be predicted (by predictive models); it is also not statistical in nature. Therefore, there is no statistical (probability-based) approach nor predictive modelling that can help to deal with this kind of uncertainty.

In real-world MCDM or Multi-Objective Optimisation (MOO) problems, decision- or policy-makers are faced with several (possibly conflicting) criteria/objectives. Satisfying these conflicting criteria/objectives is not simply possible and brings more complexity to the model.

As mentioned above, by "higher" degrees/levels of uncertainty (or deeper uncertainty) in this thesis we mean that we are looking for that kind of uncertainty beyond regular measurable statistical uncertainties or well understood stochastic events. In fact, we are concerned with the existing uncertainty in many real-life problems in which there is no sufficient knowledge about the outcomes (or other parameters) and their probabilities/distributions. The classification of different degrees of uncertainty and their definitions have been explained in the next chapter (Chapter 2). The degrees of uncertainty have a crucial role in modelling the multi-criteria decision-making and optimisation problems. Therefore, the classification of different degrees of uncertainty can help us to choose the most suitable strategy and improve the decision-making process in real-world problems.

Most of the early works and applications in MCDM/MOO have ignored the higher degrees of uncertainty because of its complexity. Many of these studies have been limited to lower levels of uncertainty by assuming some probability distributions (mostly the Normal distribution). To the best of our knowledge, none of the existing methodologies in MCDM/MOO literature are able to deal with higher degrees of uncertainty. Therefore, the primary aim of this thesis is to address this important gap in MCDM and provide a way forward for these complex problems. 


\section{$1.2 \quad$ Research objectives}

According to the above discussion, our primary purpose for writing this thesis was to demonstrate a way of dealing with higher degrees of uncertainty in MCDM/MOO problems. To achieve this aim, we concentrated on motivating a multi-objective model-based structure to integrate the Scenario Planning and Goal Programming (GP). We also tried to investigate the use of the meta-criterion (combination of criteria-scenarios) concept, introduced by Stewart et al. [2013], in goal programming for dynamic decision-making under higher degrees of uncertainty. The secondary objectives are listed below:

1. To motivate and express the two-stage MOO/GP in the simplest form including the following concepts/features:

- Extension to stochastic programming with recourse

- For higher degrees of uncertainty using scenarios not expectations, with a multi-objective representation

2. To extend the proposed two-stage structure to three- and (possibly) multi-stage problems because strategic planning will be more than two stages.

3. To simulate a study of a moving horizon approach to examine the effect of horizon length.

4. To investigate/discuss whether there is any merit in going beyond three stages for the proposed multi-stage structure.

5. To present practical examples to examine the proposed methodology and evaluate the results.

\subsection{Overview of the dissertation}

This dissertation consists of seven chapters which, after a brief introduction in this chapter (i.e. Chapter 1), the rest will be structured as follows:

Chapter 2 contains a literature review which is split into (a) Uncertainty and (b) Goal Programming (GP) under Uncertainty. In the first part, initially, definitions and dimensions of uncertainty are reviewed. Thereafter, a new classification of uncertainty is introduced. This classification of degrees of uncertainty which includes Mild, Moderate, and Deep uncertainty, help us find the best methodology to deal with different degrees of uncertainty in optimisation problems. In the second part, after a review and comparison of the GP methods with uncertain parameters in various applications, some crucial shortcomings of these models are highlighted, and possible future developments and directions are identified.

Chapter 3 introduces a generic structure to deal with the higher degrees of uncertainty (moderate and deep) in multi-criteria decision-making problems. The main idea was to extend the concept of two-stage stochastic programming with recourse to address the capability of dealing with deep uncertainty through the use of scenario planning rather than statistical expectation. Such scenario-based thinking involved a 
multi-objective representation of performance under different future conditions as an alternative to expectation, which fitted naturally into the broader multi-objective problem context. This two-stage scenario-based multi-objective optimisation structure can pave the way for us to handle deep uncertainty in complex long-term strategic planning.

Chapter 4 extends the proposed two-stage structure to longer period problems (i.e. three-stage). This expansion helped us to execute our structure in the problems with more than two stages, as most of the real-world applications, such as strategic planning, have more than two stages of the planning horizon. Therefore, the three-stage scenariobased multi-criteria decision-making structure as an extension of the proposed two-stage structure is proposed and compared to the two-stage moving horizon structure.

Chapter $\mathbf{5}$ is allocated to further extension of the proposed methodology by introducing the two- and three-stage moving horizon models in problems with a T-stage $(T>3)$ planning window. Although looking further ahead can help us improve the robustness, it may have some costs such as expensive computation and complexity that leads to the generation of some unfavourable solutions. The length of the planning horizon is also analysed in this chapter, and the effects of the planning horizon on the solutions to the proposed frameworks are investigated.

Chapter 6 applied the proposed concepts and methodology to address the strategic planning problem of sugar-bioethanol supply chains (SC) under deep uncertainty. The whole infrastructure of the sugar-bioethanol supply chain is analysed in the way that all economics, environmental and social aspects could be optimised. Moreover, the design problem of finding the best place(s) to build the ethanol plant(s) amongst available areas is also investigated. A scenario-based mixed-integer two-stage multi-objective optimisation problem was solved by utilising the generalised goal programming approach (reference point method). Three objectives (Max profit, Min $\mathrm{CO}_{2}$-emissions, and Max job-creations) are considered in this problem under six uncertain parameters (sugarcane yield, ethanol and refined sugar demands and prices, and the exchange rate). Finally, the case study of the South African sugarcane industry is utilised to study and examine the proposed methodology.

Chapter $\mathbf{7}$ provides a final summary of as well as the conclusions of the thesis. Some possible directions for future studies are also presented. 



\section{Chapter 2}

\section{Uncertainty in Multi-Objective Optimisation Problems}

\subsection{Introduction}

In the past few decades, decision sciences which function under conditions of uncertainty, for example forecasting future markets in significant competitive and unstable environments, has turned into a highly controversial issue amongst managers and policy makers. There are many important questions in this field, such as: What is the exact meaning of the uncertainty? How should we classify ranges of uncertainties? Can we develop deterministic models to solve the problems which have different levels of uncertainty? How do we deal with high levels of uncertainty, when even probability spaces are not definable? It is important to note that uncertainty takes on different meanings in various fields, which means there are multiple definitions for this term.

Furthermore, multiple objectives with conflicting criteria create another area of complexity which decision makers (DM)need to deal with. Goal Programming (GP) is a famous and powerful methodology for multiple objective decision making, especially during the design and screening stages of strategy analysis. When decision makers face conflicting goals in a context of substantial uncertainties, the complexity becomes much more extreme. Although a few GP-based approaches, such as stochastic goal programming, fuzzy goal programming and dynamic goal programming, have been proposed for addressing such complex problems, many of these are limited to the less deep areas of uncertainty.

This chapter includes a literature review which is divided into two sections. The first section reviews definitions and dimensions of uncertainty and provides a proper classification of uncertainty, which could be used in optimisation problems. In the second part, the Goal Programming methods with uncertain parameters are reviewed and compared in various real-world applications. Some crucial issues and shortcomings of these models are highlighted, and possible future developments are suggested. 


\subsection{Uncertainty}

\subsubsection{General definitions}

On many occasions, we have to make a decision and choose the one alternative while we do not have enough information or knowledge about the consequences of our decision or we may not even know about all other options. Many other unpredictable variables and/or events could affect the outcomes of the decisions (such as policy-makers' and stakeholders' risk-taking, habits, relations, behaviours, religious beliefs, previous decisions, power, and money). This uncertainty also will be more complex when it is related to the future. Let us use a metaphor to explore this concept. Consider, for example, a complete and detailed map as the ideal situation in which we know everything with absolute certainty.If such a complete detailed map were reached, consequences of all alternatives could be seen and compared. Therefore, selecting the best alternative would be done simply in this situation. However, sometimes, the decisions need to be made when the complete, detailed map is unavailable. Lack of any details in the map could be viewed as uncertainty.

It is important to note that, the absence of the complete, detailed map is not merely the lack of knowledge and information. Uncertainty can exist in a situation where lots of information are available [van Asselt and Rotmans, 2002]. However, new knowledge can either increase or decrease uncertainty. In many instances, the emergence of new information and knowledge on complex processes can reveal uncertainties that were previously unknown or were understated. In this case, more knowledge clarifies that the processes are more complex or that our cognition is more limited than previously thought [van der Sluijs, 1997].

There are also different definitions of uncertainty in the literature; some of them are as follows:

- "Any deviation from the unachievable ideal of completely deterministic knowledge of the relevant system" [Walker et al., 2003].

- "At a most fundamental level, uncertainty relates to a state of the human mind, i.e. lack of complete knowledge about something" [Stewart, 2005].

- "Incomplete information about a particular subject" [Ascough II et al., 2008].

- "Lack of confidence in knowledge related to a specific question" [Sigel et al., 2010].

- "In general, uncertainty can be defined as limited knowledge about the future, the past, or current events" [Walker et al., 2013a].

As seen above, all of these definitions highlight the lack of knowledge and information, or confidence in them, or about something (such as some events, processes, subject, etc.). This lack of knowledge represents the missing details from the complete, detailed metaphorical map that was previously used to clarify the meaning of uncertainty.

The aim of writing this chapter is to present the state-of-the-art of the Goal Programming (GP) methodologies in which uncertainty can manifest itself in objectives and/or constraint parameters. The classification of different types of uncertainty, which may important in modelling multi-objective optimisation problems, is also presented. Moreover, 
some gaps in the literature which could be used as potential directions for future work in this field are highlighted. Furthermore, different dimensions of uncertainty are reviewed and a novel classification of different degrees of uncertainty in Multi-Criteria DecisionMaking (MCDM) problems is proposed. The importance of handling uncertainty and methods of uncertainty treatment are discussed in section 2.2.2. This is followed by a detailed review of the Goal Programming under Uncertainty in Section 2.4. In the last Section(2.5), presents some classified brief reviews and conclusions which highlight the existing gap in the literature and identify opportunities for our research.

\subsubsection{Classification of uncertainty from optimisation viewpoint}

The next question worth considering refers to the dimensions of uncertainty. Where could uncertainty manifest itself? What is the entire spectrum of different degrees of knowledge? What is the source of uncertainty? When could it manifest itself? Walker et al. [2003] introduced three dimensions of uncertainty related to model-based decision support exercises: nature, location and level.

The nature of uncertainty can be split into (a) epistemic uncertainty (mainly because of the lack of wisdom/information and may be diminished by more information and research) and (b) aleatory or variability uncertainty (mainly because of innate instability/variability).

Furthermore, Van Asselt [2000] distinguished different sources of variability as the inherent randomness of nature, value diversity, human behaviour, societal randomness, and technological surprises. There are also two different groups of epistemic uncertainty (lack of knowledge)-unreliability and structural or systematic uncertainty. Unreliability can include inexactness, lack of observations or measurements [Funtowicz and Ravetz, 1990]. Conflicting evidence, ignorance, to indeterminacy are also referred to as structural or systematic uncertainty [Henrion and Fischhoff, 1986, Morgan et al., 1992]. Different sources of uncertainty have been portrayed in Figure 2.1. Recognising the nature of uncertainty may help to determine a useful methodology which can treat the uncertainty. For instant, lack of wisdom may be decreased by additional research and knowledge while uncertainty reduction may not be possible in the case of variability. More information about the nature of uncertainty and its two features can be found in Walker et al. [2003], van Asselt and Rotmans [2002].

The location of uncertainty mostly refers to the various sources of uncertainty in the model or modelling-related phenomena, such as parameters, outcomes, and inputs. Generally, as Stewart [2005] classified, the location of uncertainty could be divided into two major categories-internal and external uncertainty. Internal uncertainty relates to DM judgement and his/her preferences while external uncertainty relates to lack of knowledge about outcomes of the model. There are many classifications of the location of uncertainty in the literature [French, 1995, Walker et al., 2003, Hawkins and Sutton, 2009, Marchau et al., 2010, Kwakkel and Walker, 2010, Walker et al., 2013b]. For example, French [1995] recognized three major locations where uncertainty may manifest itself: during (a) modelling (includes what might happen, ambiguity, and related decisions), (b) exploration of the models (lack of knowledge, the evolution of future beliefs and performances, judgement, and the accuracy of calculations), and (c) interpretation (appropriateness of a descriptive and normative model and the depth of analysis). Recently, Walker et al. [2013b] recognized four different locations of uncer- 
tainty (a) context, (b) system model, (c) system outcomes, and (d) weights on outcomes. Table 2.1 shows a short review of these taxonomies.

More important than where the uncertainty can appear, the depth or level of uncertainty plays a key role in handling uncertainty. Knight [1921] were the first researchers to make the distinction that the calculable and controllable part of the unknown is referred as risk, while the incalculable and uncontrollable part of the unknown refers to uncertainty. Generally, statistical methods and/or probabilities have been utilised and developed by many authors to handle the controllable and calculable parts ([Quade, 1989] and [Morgan et al., 1992]). For example, Quade [1989] called the calculable part stochastic uncertainty and named the second part real uncertainty which covers the future state of the world and human factors for which we know absolutely nothing about probability distribution and little more about possible outcomes. Recently, this part of uncertainty (i.e. real uncertainty) has been called deep or severe uncertainty by Lempert et al. [2003] and Ben-Haim [2006], respectively.

The term deep uncertainty has been used recently by several authors in various

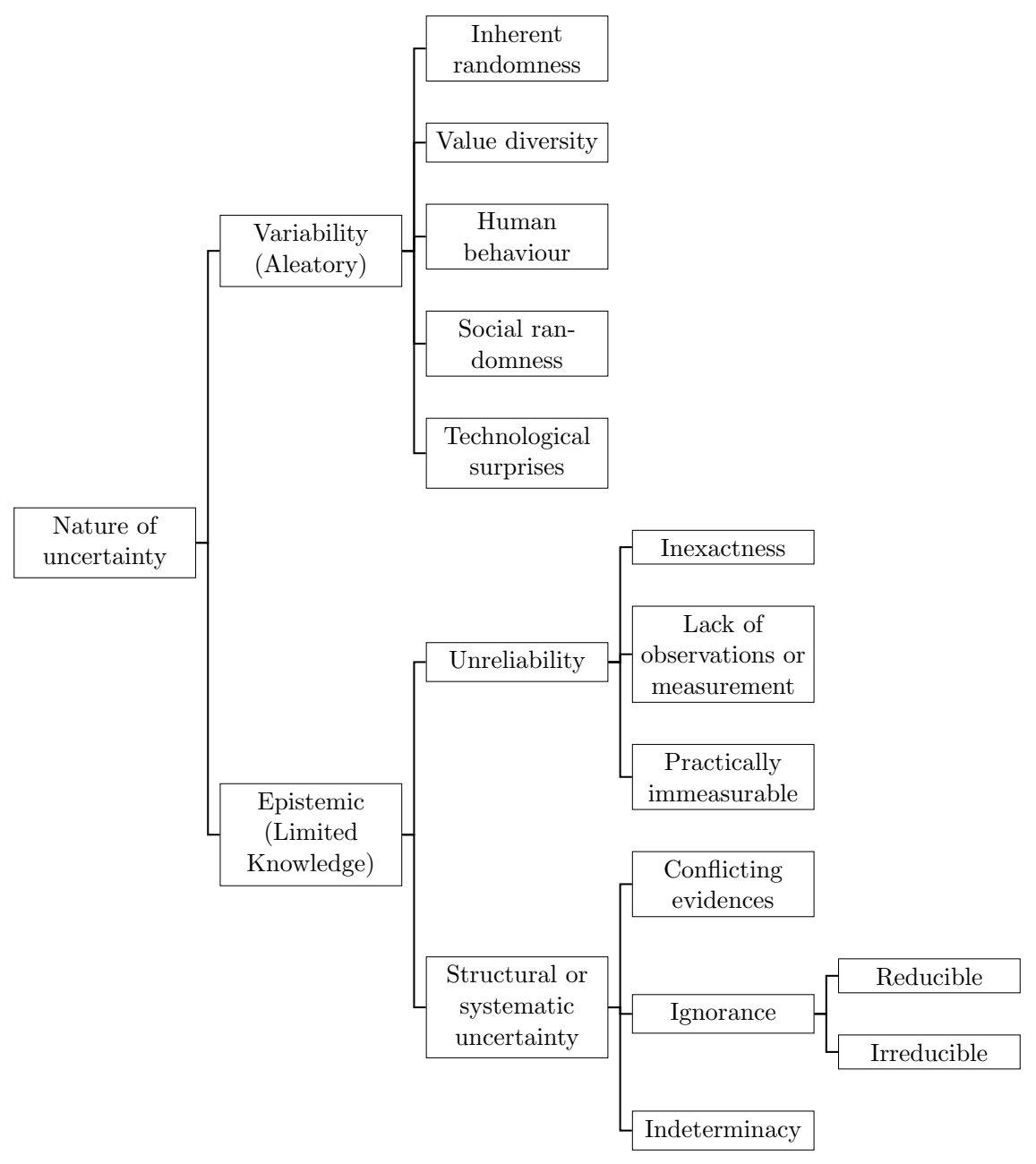

Figure 2.1: Different sources of the nature of uncertainty 
contexts, especially in climate change studies. However, only a few authors have been concerned about its definition and classification. Amongst them Lempert et al. [2003] have defined deep uncertainty as "the condition in which analysts do not know or the parties to a decision cannot agree upon the following subjects:

- The appropriate models to describe interactions among a system's variables,

- The probability distributions to represent uncertainty about key parameters in the models,

- How to value the desirability of alternative outcomes".

In the realm of deep uncertainty, the terms "do not know" and "do not agree upon" are utilised by Walker et al. [2013a] regarding individual and group decision making, respectively.

Moreover, the term deep uncertainty has been used previously by some other authors. For example, Bankes [2002] wrote: "There are phenomena that are prosaically

\begin{tabular}{|c|c|c|}
\hline Author(s) & & ifferent categories \\
\hline \multirow{10}{*}{ French [1995] } & \multirow{3}{*}{$\begin{array}{l}\text { Uncertainties expressed during } \\
\text { modeling }\end{array}$} & What might happen or what can be done \\
\hline & & Meaning/ambiguity \\
\hline & & Related decisions \\
\hline & \multirow{4}{*}{$\begin{array}{l}\text { Uncertainties expressed during } \\
\text { exploration of the models }\end{array}$} & Physical randomness or lack of knowledge \\
\hline & & The evolution of future beliefs and performances \\
\hline & & Judgments \\
\hline & & The accuracy of calculations \\
\hline & \multirow{3}{*}{$\begin{array}{l}\text { Uncertainty expressed during } \\
\text { interpretation }\end{array}$} & Appropriateness of a descriptive model \\
\hline & & Appropriateness of a normative model \\
\hline & & the depth of analysis \\
\hline \multirow{4}{*}{ Walker et al. [2003] } & \multicolumn{2}{|l|}{ Context } \\
\hline & Model & $\begin{array}{l}\text { Structure } \\
\text { Technology }\end{array}$ \\
\hline & Input & $\begin{array}{l}\text { Controllable } \\
\text { Uncontrollable }\end{array}$ \\
\hline & \multicolumn{2}{|l|}{$\begin{array}{l}\text { parameter } \\
\text { Model outcome }\end{array}$} \\
\hline Stewart [2005] & \multicolumn{2}{|l|}{$\begin{array}{l}\text { Internal } \\
\text { External }\end{array}$} \\
\hline Hawkins and Sutton [2009] & \multicolumn{2}{|l|}{$\begin{array}{l}\text { Internal variability of the system } \\
\text { Model Uncertainty } \\
\text { Scenario Uncertainty }\end{array}$} \\
\hline Marchau et al. [2010] & \multicolumn{2}{|c|}{$\begin{array}{l}\text { The value system(s) to be used to rank alternative policies } \\
\text { The system models } \\
\text { How the future will develop }\end{array}$} \\
\hline Kwakkel and Walker [2010] & \multicolumn{2}{|l|}{$\begin{array}{l}\text { External Forces } \\
\text { Relations within a system } \\
\text { Outcomes of interest } \\
\text { Weights }\end{array}$} \\
\hline Walker et al. [2013b] & \multicolumn{2}{|l|}{$\begin{array}{l}\text { Context } \\
\text { System model } \\
\text { System Outcome } \\
\text { Weights } \\
\text { Weights on outcome }\end{array}$} \\
\hline
\end{tabular}

Table 2.1: Different classifications of the location of uncertainty. 
described as uncertain that are not well modelled by the tools of probability and statistics. Although controversial, this pragmatic reality has been recognised for some time. The term I use to describe such phenomena is deep uncertainty".

Knowing the depth of uncertainty paves the way for us to choose the right methodology to treat the existing uncertainty. This concept of the depth of uncertainty is frequently used by many authors in other literature under the label of alternative representations of uncertainty (e.g. [Ben-Haim, 2004, Helton and Oberkampf, 2004, Helton et al., 2004]). Nevertheless, uncertainty experts unanimously support the idea that there are two extreme levels of uncertainty: complete certainty (or deterministic knowledge) and total ignorance, with some intermediate levels (e.g. [Courtney, 2001, Walker et al., 2003, 2013a, Kwakkel and Walker, 2010, Van der Pas et al., 2010]). However, to the best of our knowledge, there is not any suitable definition with which we can classify and model the optimisation problems under uncertainty. To reach this goal, we classified the depth of uncertainty based on a suitable definition which could be used in optimisation problems under uncertainty.

We use the term degree of uncertainty as depth or level of uncertainty and define uncertainty of degree ' 0 ' as absolute certainty or deterministic knowledge, and uncertainty of infinity degree as total ignorance. We also propose three major levels of uncertainty: the mild uncertainty with an uncertainty of first-degree, the moderate uncertainty with an uncertainty of second-degree, and deep uncertainty which can be labelled as the third-degree of uncertainty. Figure 2.2 shows how they stand on the spectrum while the definitions of these three intermediate levels have been described as follows:

- Mild uncertainty (First-degree): Outcomes can be enumerated and probabilities (or probability distribution) are specified.

- Moderate uncertainty (Second-degree): Outcomes can be enumerated but probabilities (or probability distribution) are difficult to specify generally.

- Deep uncertainty (Third-degree): Outcomes cannot be completely enumerated, so that, probabilities are not definable.

Table 2.2 describes our proposed classification of different degrees of uncertainty in comparison with some other classifications that are found in the literature.

$\begin{array}{ccccc}\begin{array}{c}\text { Absolute } \\ \text { Certainty }\end{array} & \text { Mild } & \text { Moderate } & \text { Deep } & \begin{array}{c}\text { Total } \\ \text { Ignorance }\end{array} \\ 0 & 1 & 2 & 3 & \infty\end{array}$

Figure 2.2: Different Degrees of Uncertainty 


\begin{tabular}{|c|c|c|c|c|c|c|c|}
\hline Proposed Classification & \multirow{4}{*}{ 焉 } & \multicolumn{2}{|c|}{ Mild uncertainty (First-degree) } & \multicolumn{2}{|c|}{ Moderate uncertainty (Second-degree) } & \multirow{2}{*}{$\begin{array}{c}\text { Deep uncertainty (Third-degree) } \\
\text { Level } 5 \\
\text { (Unknown } \\
\text { future) }\end{array}$} & \\
\hline Walker et al. [2013a] & & $\begin{array}{c}\text { Level 1 } \\
\text { (A clear } \\
\text { enough future) }\end{array}$ & $\begin{array}{c}\text { Level } 2 \\
\text { (Alternate futures } \\
\text { with probabilities) }\end{array}$ & $\begin{array}{c}\text { Level } 3 \\
\text { (Alternate futures } \\
\text { with ranking) } \\
\end{array}$ & $\begin{array}{c}\text { Level } 4 \\
\text { (Multiplicity } \\
\text { of futures) }\end{array}$ & & \\
\hline Walker et al. [2003] & & \multirow{2}{*}{\multicolumn{2}{|c|}{ statistical uncertainty }} & scenario uncertainty & \multicolumn{2}{|c|}{ Recognized ignorance } & \\
\hline & & & & & Reducible & Irreducible & \\
\hline Kwakkel et al. [2010] & 8 & \multicolumn{2}{|c|}{ Shallow Uncertainty (Level 1) } & Medium Uncertainty (Level 2) & Deep Uncertainty (Level 3) & Recognized Ignorance (Level 4) & \\
\hline Courtney [2001] & \multirow[t]{3}{*}{ 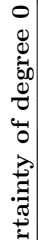 } & $\begin{array}{l}\text { Level } 1 \text { (so low that the } \\
\text { traditional methods that } \\
\text { employ point forecasts can } \\
\text { be used with great success) }\end{array}$ & $\begin{array}{l}\text { Level } 2 \text { (manager can identify } \\
\text { a set of distinct possible } \\
\text { outcomes, one of } \\
\text { which will occur) }\end{array}$ & \multicolumn{2}{|c|}{$\begin{array}{c}\text { Level } 3 \\
\text { (manager can bound } \\
\text { the range of } \\
\text { possible outcomes) } \\
\end{array}$} & $\begin{array}{c}\text { Level } 4 \\
\text { (analysis cannot } \\
\text { even bound the range } \\
\text { of possibilities) }\end{array}$ & \\
\hline Morgan et al. [1992] & & \multicolumn{2}{|c|}{ Uncertainties can be treated through probabilities } & \multicolumn{3}{|c|}{ Uncertainties cannot be treated probabilistically } & \\
\hline Quade [1989] & & \multicolumn{4}{|c|}{ Stochastic uncertainty } & Real uncertainty & \\
\hline Knight [1921] & है & \multicolumn{4}{|c|}{ Risk } & Uncertainty & \\
\hline
\end{tabular}

Table 2.2: Different classifications of the depth of uncertainty. 
As a result of the second-degree of uncertainty, uncertainty can be further divided into ranked and unranked uncertainty. In the case of ranked moderate uncertainty, outcomes rank in terms of their likelihood, but how much less likely or more likely cannot be specified. In this situation, outcomes can be enumerated and, for any two outcomes, the first is either 'ranked higher than'(more likely), 'ranked lower than'(less likely) or 'ranked equal to'(equally likely) the second while the quantity of this cannot be calculated. Under conditions of unranked moderate uncertainty, outcomes can be enumerated but their ranking cannot be calculated or cannot be agreed upon.

When different degrees of uncertainty are simultaneously observed in a problem/model, the highest degree of existing uncertainty determines the degree of uncertainty in that problem/model. For example, consider a problem which has outcomes that can be enumerated and the probability distribution of some parameters of a model are attainable while the probabilities are not seizable for some other parameters. Therefore, this example has the second degree of uncertainty.

The proposed classification will be utilised as the base of our comparisons between previous works and finding opportunities and gaps in the literature that highlight some advantageous directions for our studies in optimisation problems. However, before that let us pursue our review on uncertainty and different approaches which have been used to treat different degrees of uncertainty. The next section attempts to answer some questions such as: Why must uncertainty be treated? And how can we treat it?

\subsubsection{Dealing with uncertainty}

\subsubsection{Why must uncertainty be treated?}

Although we struggle with uncertainty on a daily basis, many authors for many years have spent a great deal of time and energy on ignoring uncertainty and still some others try to avoid the third-degree of uncertainty (deep uncertainty). However, the reality is that researchers will have to face this challenge eventually. Some of the traditional applied scientific works in the engineering, social, and natural sciences have been built on the supposition that the existing uncertainties are caused by a lack of information, which has led to an emphasis on uncertainty reduction through ever-increasing information seeking and processing [McDaniel and Driebe, 2005], or caused by random variation, which has concentrated efforts on stochastic processes and statistical analysis. Nevertheless, some of the vital strategic planning problems currently faced by decision makers are characterised by deep uncertainty(e.g. uncertainties about the future) that may not be reduced by gathering more information, nor are they statistical in nature. Accordingly, many decision makers in different fields of study are facing uncertain changing conditions with the third degree uncertainty. Under such circumstances, a decision is not only one that is able to seize objectives related to society, economy, and environment; it also requires sustainable planning that robust and adaptable, meaning that the decision must perform satisfactorily under a broad variety of futures (robust) and it can be adapted to changing unpredictable future conditions [Haasnoot et al., 2011]. Therefore, a major challenge in dealing with uncertainty is the requirement to accept, understand, and manage uncertainty, mainly due to the fact that:

1. Not all uncertainties about the future or human behaviour can be eliminated; 
2. Ignoring uncertainty could mean that we limit our ability to make corrective action in the future and result in positions that could have been avoided;

3. Ignoring uncertainty can throw away the opportunity of studying real-world problems, and/or lead to get some unsustainable approaches.

Some authors argue that third-degree uncertainty does not exist while decades of practical experience prove otherwise. It is important to consider the conditions of deep uncertainty in real problems. This is because the causes of deep uncertainty are pragmatic limitations in our capability to utilise the representative formalisms of statistical decision theory as well as limitations in our ability to display our knowledge about complex adaptive systems and their relates policy issues. Several reasons can be found to show the fact that, in a real problem, probability and statistics are not sufficient to represent our entire knowledge and, therefore, some supplementary tools should be used in addition to probabilistic methods[Bankes, 2002].

\subsubsection{How can we deal with uncertainty?}

Typically, there are two general approaches to deal with uncertainty ([Marchau et al., 2010]):

1. The predict and act approach, which assumes that we can predict the future well enough to create a static policy generating reasonable outcomes in most plausible future worlds.

2. The monitor and adapt or dynamic approach, which assumes that the future cannot be predicted. Dynamic adaptive policies are implemented.

The first approach is currently utilised in some policy-making. This approach limits handling of uncertainty and can lead to policy failures[Marchau et al., 2010]. McInerney et al. [2012] liken this to "dancing on the tip of a needle". Although the presence of deep uncertainty in real problems, as well as the inability to treat them by utilising the probabilities or getting more knowledge, is not deniable, some strategic planners and policy makers tend to expand their plans through predictive models. Furthermore, time passes quickly and everything in the world changes continuously. Accordingly, there is a need to apply these changes to long-term plans and adapt them continuously. However, such adaptations are seldom planned in advance[Walker et al., 2013b].

From another point of view, Walker et al. [2013b], identified four, not mutually exclusive, methods to treat the higher degrees of uncertainty that have been used by some DMs to make a sustainable plan. These approaches have been summarised as the following:

1. Resistance: Plan for the worst plausible scenario in the future.

2. Resilience: Concentrate on recovering the system quickly without considering possible future events.

3. Static robustness: Decrease vulnerability in the widest possible range of different futures. 
4. Dynamic robustness (or flexibility): Plan for continuous change and adaptation regarding upcoming situations.

The first approach, resistance planning, may well cost too much as it plans for the worst case scenario that rarely happens. On the other hand, because of surprises, or painful events, which are called "Black Swans" by Taleb [2007] and seem to be occurring more often[Walker et al., 2013b], there is no guarantee that this approach will work well in all cases. Taleb [2007] defines the Black Swan event as an unexpected scenario that extremely impacts on the plan. This event can be explained only after scenario realisation. Negative system performances (or short-term pains) are acceptable in the resilience approach; however, they concentrate on recovery.

Instead of producing the forecasts, the last two approaches identify the best predictive models that will be solved for an optimal plan, although this action weakly depend on assumptions. Alternatives with robust actions seem more reasonable in the case of higher degrees of uncertainty. In a robust plan, the outcomes are supposed to be satisfied by some evaluation criteria in terms of a broad range of plausible futures[Rosenhead and Mingers, 2001]. It is in contrast to the optimality definition in which the best results should be provided by the plan; nevertheless, there is no guarantee of doing so outside of the restricted circumstances. Lempert and Collins [2007] argue that analytic methods which include robust decisions are more appropriate than the others under conditions of deep uncertainty and provide DMs with an extensive array of options.

In the case of the second degree of uncertainty, to identify static robust policies, we should focus on exploring how different assumptions about the future values of the uncertain parameters would affect the decisions actually being faced [Walker et al., 2013a]. Scenario planning and Exploratory Modelling and Analysis (EMA) can be useful to identify static robust policies. Scenario planning assumes that, although the probabilities are not specific, a range of alternatives can be specified well enough to identify a static policy [Van der Heijden, 1996]. EMA considers what one would need to believe was true to discard one possible policy in favour of another[Walker et al., 2013a].

Handling the third degree of uncertainty (deep uncertainty) and making policies in this situation, generally, need to utilise dynamic adaptive policies which are able to adapt to changing conditions over time and/or in the case of facing surprises or Black Swans. A dynamic adaptive policy is developed over time when new information becomes available or surprises/Black Swans manifest themselves. Eriksson and Weber [2008] proposed an adaptive foresight approach in which they suggested that some strategic decisions should be deferred until more information is available. We should also invest in real options which would facilitate the implementation of such decisions if taken some future time. Various approaches can be found in the literature and these attempt to treat the higher degrees of uncertainty. For instance, Walker et al. [2001] proposed an approach for developing adaptive policies, called Dynamic Adaptive Policy-making (DAP). Robust Decision Making (RDM) is a similar approach introducing by Lempert et al. [2003, 2006] which leads a vulnerability and response option analysis. This approach utilises the EMA to compare and determine robust strategies.

To sum up, it seems that mild uncertainty (the first degree of uncertainty), which is commonly encountered by decision makers in short-term planning, is mostly treated by statistical approaches and probability theory while long-term programming, which largely involves deeper uncertainties (second and third degree of uncertainty), cannot 
deal with regular probability models and regular statistic approaches. We need to use some approaches which could be more robust, adaptive and dynamic.

Furthermore, most problems in nature have multiple objectives with conflicting criteria which create another area of complexity which DMs need to deal with. The next section briefly introduces multi-objective optimisation which is followed by a more detailed review of Goal Programming methods with uncertain parameters.

\subsection{Multi-Objective Optimisation}

From the simplest daily decisions, such as finding the shortest path to work or buying the cheapest products whilst ensuring quality, to the most complex space projects, decision makers (DMs) always seek to make better decisions or optimise their decisions. In other words, in optimisation problems, we are looking for the best, or near best, solution(s) for minimising/maximising some particular objectives subject to satisfying certain conditions and limitations of the problem. Optimisation is an interesting topic in almost every field of study; for example, most business decisions, almost all engineering design and much of data analysis involves optimisation.

In some problems, only one objective/criterion is considered which usually produces a single optimal solution. This optimal solution provides us with the best performance based on the given criteria/objective while simultaneously satisfies all the constraints/limitations of the problem. However, most problems by nature have several, possibly conflicting, objectives to be satisfied at the same time which leads us to multiobjective optimisation (MOO) problems. The existing conflicts between the various criteria/objectives in these problems usually prevent us from obtaining a single optimal solution which works well in all objectives. Therefore, instead of a single optimal solution, the multi-objective problems have several, usually infinitely many, alternatives, called Pareto optimal (or non-dominated) solutions, with various trade-offs among objectives. The existence of these multiple alternatives means that there is a need to choose one solution among the others. This choice is made by decision maker or an informed expert, referred to as the $\operatorname{DM}(\mathrm{s})$, and is based on their preferences. Therefore, multi-objective optimisation problems include two important parts; (a) identifying the Pareto optimal solutions (optimisation part), and (b) determining the most preferred solution (decision-making part).

Multi-Criteria Decision-Making (MCDM) is the field of research which studies decision problems, and covers both discrete and continuous problems with multiple conflicting criteria/objectives. Discrete problems contain an infinite, usually predefined, set of solutions while the set of feasible solutions to continuous problems (known as multicriteria/multi-objective optimisation) is not completely pre-identified and only limited by some constraints. Accordingly, the DM becomes involved in solution process to find the best-preferred solution by applying his/her preferences into the optimisation model. This involvement can be applied before (known as "priori" methods) or after (called "posteriori" methods) the solution process. In some other methods, the DM iteratively modifies his/her preferences during the solution process. In these so-called "interactive" methods, the DM can learn about the relations between the objectives/criteria through an analysis of the Pareto solutions and their trade-offs, as well as from a range of achievable solutions in different criteria and balanced solutions. More information 
about different approaches in MCDM can be found in [Belton and Stewart, 2002, Deb and Miettinen, 2008, Hwang and Masud, 2012, Miettinen, 2012].

Over the years, multi-criteria optimisation problems have been solved by a wide range of various solution approaches mainly based on a problem's nature and the domain of application, such as Multi-Attribute Utility Theory (MAUT), Goal Programming (GP), Analytical Hierarchy Process(AHP)([Saaty, 1980]), Technique for Order of Preference by Similarity to Ideal Solution (TOPSIS), and Data Envelopment Analysis (DEA). Amongst them, the GP approach is one of the most well-known and flexible techniques in multicriteria optimisation problems. It applies the DM preferences through a set of goals or aspiration levels for criteria in the GP model that tries to minimise the deviations between the objectives and these goals. Applying the DM's preferences prior to solving the model puts the standard GP approach in the priori group, although several interactive GP models are also proposed in the literature, such as Goal Programming model of the Reference Point Method([Ogryczak, 1994]). It is also possible to combine GP with another technique, such as discrete-event or Monte-Carlo simulation, for symbiotic advantage ([Oddoye et al., 2009] and [Kosmidou and Zopounidis, 2004]). A broad variety of GP applications can be found in different fields of study from engineering and management to social sciences. A state-of-the-art review of the GP and its applications can be found in [Tamiz et al., 1998] and [Colapinto et al., 2015], respectively.

Although the classical GP approaches are widely applied in deterministic problems, several methods can be found in the literature that successfully expand GP to problems with uncertain parameters (e.g. Stochastic(SGP) and Fuzzy goal programming (FGP)).

Overall, as mentioned above, Goal Programming (GP) is a popular and powerful methodology for multiple objective decision-making, especially during the design and screening stages of strategy analysis. When decision makers face conflicting objectives in a context of substantial uncertainty, the complexity becomes much more extreme. Although a few GP-based approaches, such as stochastic goal programming, fuzzy goal programming and dynamic goal programming, have been proposed for addressing such complex problems, many of them are limited to the less deep areas of uncertainty. The following section will review different developments of GP under uncertainty and will highlight the advantages and/or shortcomings of these methods.

\subsection{Goal Programming under uncertainty}

\subsubsection{General formulation}

In the literature dedicated to multi-objective optimisation, especially when the goals are conflicting, the Goal Programming (GP) approach, developed by Charnes et al. [1955], is one of the most popular and oldest Multi-Criteria Decision Analysis (MCDA), also known as Multi-Criteria Decision Making (MCDM), technique. The GP model is a distance function; it can be a vector or a weighted sum dependent on the goal programming variant used, where the deviations $\left(\delta_{i}\right)$ between the achievement and aspiration levels are to be minimised. The standard mathematical formulation of the GP model introduced first time by Charnes and Cooper (see [Charnes and Cooper, 1952, Charnes et al., 1955, Charnes and Cooper, 1959]) is as follows (in a linear programming context): 


$$
\begin{array}{lll}
\text { Min } \Delta=\sum_{i=1}^{m}\left(\delta_{i}^{+}+\delta_{i}^{-}\right) & \\
\text {s.t. } & \sum_{j=1}^{n} c_{i j} x_{j}-\left(\delta_{i}^{+}-\delta_{i}^{-}\right)=g_{i}, & i=1, \cdots, m ; \\
& \sum_{j=1}^{n} a_{r j} x_{j} \leq b_{r}, & r=1, \cdots, R ; \\
& \delta_{i}^{+}, \delta_{i}^{-}, x_{j} \geq 0, & \forall i, j .
\end{array}
$$

where $\delta_{i}=\delta_{j}^{+}-\delta_{i}^{-}$describes the positive and the negative deviations ${ }^{1}$ with respect to the aspiration level (goals) $g_{i}$, respectively. $z_{i}(x)=\sum_{j=1}^{n} c_{i j} x_{j}$ represents the $i^{\text {th }}$ objective function and $\sum_{j=1}^{n} a_{r j} x_{j} \leq b_{r}, r=1, \cdots, R$; are the initial problem constraints which indicate the feasible region of our problem.

The decision maker (DM) can apply more preferences by setting some so-called importance weights on different goals. If $w_{i}^{+}$and $w_{i}^{-}(i=1, \cdots, m)$ are the DM's additional preferences, the Weighted Goal Programming (WGP) model can be formulated as follows:

$$
\begin{array}{lll}
\operatorname{Min} & \sum_{i=1}^{m}\left(w_{i}^{+} \delta_{i}^{+}+w_{i}^{-} \delta_{i}^{-}\right) & \\
\text {s.t. } & \sum_{j=1}^{n} c_{i j} x_{j}-\left(\delta_{i}^{+}-\delta_{i}^{-}\right)=g_{i}, & i=1, \cdots, m ; \\
& \sum_{j=1}^{n} a_{r j} x_{j} \leq b_{r}, & r=1, \cdots, R ; \\
& \delta_{i}^{+}, \delta_{i}^{-}, x_{j} \geq 0, & \forall i, j .
\end{array}
$$

Since then, GP has been frequently developed and applied by many authors in a wide variety of real-world applications such as financial management, human resources, marketing, agriculture, transport, quality control, allocation problems, and production. (e.g. [Ijiri, 1965, Lee and Clayton, 1972, Lee, 1973, Ignizio, 1976, Lee et al., 1981, Goicoechea et al., 1982, White, 1982, Lee and Olson, 1985, Sawaragi et al., 1985, Romero, 1991, Martel and Aouni, 1998, Lee and Olson, 1999, Aouni et al., 2005, El-Wahed and Lee, 2006, Kharrat et al., 2007, Aouni et al., 2014]). More information about the history of GP and its applications can be found in [Jones and Tamiz, 2002, Caballero et al., 2009, Jones and Tamiz, 2010, Aouni et al., 2014, Colapinto et al., 2015].

The literature on goal programming under uncertainty is less popular than certain GP but several approaches could be found in the literature which developed GP models in an uncertain environment. To contrast the GP model with uncertain parameters, it is sufficient to consider model 2.1 (or 2.2) with some uncertain parameters in objective and/or constraints. These approaches have been reviewed in the next sections.

\footnotetext{
${ }^{1}$ In most cases, a one-sided deviation may be important in which the positive deviations in Maximization and the negative deviations in Minimization problems would be an advantage. However, for general formulation here, the two-side deviation is considered and both positive and negative deviations are undesirable.
} 


\subsubsection{Stochastic Goal Programming (SGP)}

[Caballero et al., 2001] defined Stochastic programming problems as follows:

"If in a problem some parameters take unknown values at the time of making a decision, and these parameters are random variables, then the resulting problem is called a stochastic programming problem".

Therefore, the Stochastic Goal Programming (SGP) approach developed GP models in the presence of random parameters with a known or unknown probability distribution. In many situations, suppose that the probability distribution is known or can be approximated via sampling, tests, experiences and expertises, etc. Note that these methods may fail in determining accurate values for the probability distribution [Abdelaziz and Masri, 2005]. However, existing uncertainty in these problems is classified as the first degree of uncertainty (mild uncertainty). In many other situations, the probability distribution is unknown and information about possible outcomes is limited. If plausible futures (alternatives) can be enumerated and the probability space is finite, these problems have a second-degree uncertainty (moderate uncertainty). Otherwise, if counting alternatives is difficult or impossible and the probability space is infinite, the problems are classified as a third-degree uncertainty (deep uncertainty).

Optimisation of conflicting random objectives leads us to solve Multi-Objective Stochastic Programs (MOSP) in which objectives and/or constraints contain random parameters. The general formulation of the MOSP can be formulated as follows:

$$
\begin{aligned}
& \operatorname{Max} \tilde{F}(x)=\left(\tilde{f}_{1}(x), \tilde{f}_{2}(x), \ldots, \tilde{f_{m}}(x)\right) \\
& \text { s.t. } \tilde{B}_{r}(x) \leq \tilde{b_{r}}, \quad r=1, \cdots, R \text {; } \\
& x \in E \text {. }
\end{aligned}
$$

where, $\tilde{F}(x)=\left(\tilde{f}_{1}(x), \tilde{f}_{2}(x), \ldots, \tilde{f_{m}}(x)\right)$ is a vector of $m$ random objectives, $\tilde{B}_{r}(x)$ and $\tilde{b_{r}}$ $(r=1, \cdots, R$;) describe the random constraints (defined on a probability space $(\Omega, \Xi, p)$ ), and the convex set $E$ is deterministic. The linear form of 2.3, in which both constraints and objectives have a linear form, can be written as follows:

$$
\begin{aligned}
& \operatorname{Max} \sum_{j=1}^{n} \tilde{c_{i j}} x_{j} \quad i=1, \cdots, m ; \\
& \text { s.t. } \sum_{j=1}^{n} \tilde{a_{r j}} x_{j} \leq \tilde{b_{r}}, \quad r=1, \cdots, R \text {; } \\
& x_{j} \geq 0, \quad j=1, \cdots, n .
\end{aligned}
$$

where $\tilde{\mathbf{b}}=\left[b_{1}, b_{2}, \ldots, b_{R}\right]_{R \times 1}^{T}$ is a random vector and $\tilde{\mathbf{C}}=\left[c_{i j}\right]_{m \times n}$ and $\tilde{\mathbf{A}}=\left[a_{r j}\right]_{R \times m}$ are the random matrices defined on a probability space $(\Omega, \Xi, p)$. The notation " " represents the uncertain/random parameters and does not consider any specification in the corresponding states of nature in their distributions. 
This problem (model 2.4) is equivalent to minimising the distances between achievements $\left(\sum_{j=1}^{n} c_{i j} x_{j}\right)$ and the aspiration levels (goals) $\left(g_{i}\right)$. These deviations are shown by $\delta_{i}$. Therefore, the equivalent linear SGP model can be formulated as follows:

$$
\begin{array}{lll}
\operatorname{Min} & \sum_{i=1}^{m}\left(\tilde{\delta}_{i}^{+}+\tilde{\delta}_{i}^{-}\right) & \\
\text {s.t. } & \sum_{j=1}^{n} \tilde{c_{i j}} x_{j}+\tilde{\delta}_{i}^{-}-\tilde{\delta}_{i}^{+}=\tilde{g_{i}}, & i=1, \cdots, m ; \\
& \sum_{j=1}^{n} \tilde{a_{r j}} x_{j} \leq \tilde{b_{r}}, & r=1, \cdots, R ; \\
\tilde{\delta}_{i}{ }^{+}, \tilde{\delta}_{i}{ }^{-}, x_{j} \geq 0, & \forall i, j .
\end{array}
$$

where $\tilde{\delta}_{i}=\left(\tilde{\delta}_{i}^{+}-\tilde{\delta}_{i}^{-}\right)$. If $\tilde{\delta}_{i}^{+}>0$, then $\tilde{\delta}_{i}^{-}=0$, and conversely.

The basic idea used in stochastic optimisation is to convert the stochastic model to an equivalent deterministic model. The resulting model is then solved by standard linear or non-linear programming methods. In 1968, Contini [1968] made the first formulation of the Stochastic Goal Programming (SGP) model which includes the assumption of a stochastic relation between the goals $\left(\mathbf{g}=\left[g_{i}\right]_{m \times 1}, i=1, \cdots, m\right)$ and decision variables, $\tilde{g}_{i}=\sum_{j=1}^{n} c_{i j} x_{j}+\tilde{\delta}_{i}, i=1, \cdots, m$; where $\tilde{\delta}$ is a random variable vector with a normal distribution with mean vector ' $\mathbf{0}$ ' and known non-singular variance-covariance matrix $\boldsymbol{\Sigma}$. So, $\tilde{\mathbf{g}}$ is a normally distributed vector with known means $\left(\sum_{j=1}^{n} c_{i j} x_{j}\right)$ and variancecovariance matrix. He also assumed that both matrices $\mathbf{C}$ and $\mathbf{A}$, as well as vector $\mathbf{b}$, are fixed and known. Then Contini sets $G$ as a well defined region in $E^{m}$ such that $\mathbf{g} \in G$ and maximised the probability that this random vector of goals $(\tilde{\mathbf{g}}=\mathbf{C x}+\tilde{\delta})$ will lie inside the $G$ (i.e. the probability that the consequence of the decision belongs to a region containing the uncertain goals is maximised [Abdelaziz, 2012]). The following quadratic programming problem is proposed by [Contini, 1968] as an equivalent program to the $\operatorname{SGP}(2.5)$ :

$$
\begin{aligned}
& \operatorname{Min}[\mathbf{K}+\mathbf{x} \cdot \mathbf{L} \cdot \dot{\mathbf{x}}+2 \dot{\rho} \cdot \mathbf{x}] \\
& \text { s.t. } \mathbf{A} \cdot \mathbf{x} \leq \mathbf{b} \\
& \quad \mathbf{x} \geq \mathbf{0}
\end{aligned}
$$

where $\mathbf{L}_{n \times n}=\mathbf{C} \cdot \boldsymbol{\Sigma}^{-1} \cdot \mathbf{C}$ is positive-definite if $m \geq n$, and is positive-semidefinite in otherwise. $\mathbf{K}=\mathbf{g} \cdot \boldsymbol{\Sigma}^{-1} \cdot \mathbf{g}$ is a constant which is independent of decision variable $\mathbf{x}$, $\rho_{n \times 1}=-\mathbf{C} . \boldsymbol{\Sigma}^{-1} \cdot \mathbf{g}=-\mathbf{g} . \boldsymbol{\Sigma}^{-1} . \mathbf{C}$, and the variance-covariance matrix $\boldsymbol{\Sigma}_{m \times m} . \mathbf{C}_{m \times n}$, $\mathbf{A}_{r \times n}, \mathbf{x}_{n \times 1}, \mathbf{g}_{m \times 1}$ and $\mathbf{b}_{r \times 1}$ are the matrix form of $c_{i j}, a_{r j}, x_{j}, g_{i}$ and $b_{r}$, respectively.

In this model, the random distance from an arbitrary neighbourhood of the optimal solution is minimised. Since [Contini, 1968] showed that the SGP is equivalent to a 
quadratic programming problem in standard form, model 2.6 can be solved by any of the existing algorithms. Finally, the effectiveness of this approach is evaluated by the concept of confidence region.

Then, after 1968, SGP models were developed and solved by many authors over time (e.g.[Stancu-Minasian, 1984], [Keown, 1978, Keown and III, 1980, Lee and Olson, 1985], [Martinez and Aguado, 1998], [Ballestero, 2001, Sahoo and Biswal, 2005] and [Aouni et al., 2012]). Ballestero [2001], for instance, utilised an Absolute Risk Aversion (ARA) approach based on standard expected utility theory to propose a mean-variance model of stochastic weighted GP under uncertainty, as follows:

$$
\begin{aligned}
& \operatorname{Min} \sum_{i=1}^{m} w_{i} \cdot R_{A i} \cdot \sigma_{i}^{2} \\
& \text { s.t. } E\left(\sum_{i=1}^{m} \tilde{c_{i j}} x_{j}\right) \geq \gamma_{i} \quad i=1, \cdots, m \text {; } \\
& \sum_{j=1}^{n} a_{r j} x_{j} \leq b_{r}, \quad r=1, \cdots, R ; \\
& w_{i}, x_{j} \geq 0, \quad i=1, \cdots, m ; j=1, \cdots, n .
\end{aligned}
$$

where $w_{i}$ is the importance weight for the $i^{t h}$ deviation variable, $\delta_{i}^{-}=(1 / 2) R_{A i} \cdot \sigma_{i}^{2}(i=$ $1, \ldots, m)$ are the only undesirable variables mainly due to the assumption that every goal behaves such that more is better, and the worse is better goal can be converted to more is better goal. $\gamma_{i}$ is the $i^{\text {th }}$ goals or aspiration level for the normalised expected utility of the $\tilde{g}_{i}$. $\tilde{g}_{i}=\sum_{j=1}^{n} \tilde{c_{i j}} x_{j}, i=1, \ldots, m ; x_{j}$ is the decision variable and $E\left(\tilde{g}_{i}\right)=$ $E\left(\sum_{i=1}^{m} \tilde{c_{i j}} x_{j}\right)$ is the mean value of $\tilde{g}_{i} . \quad R_{A i}=(-1)\left[\ddot{U}_{i} / \dot{U}_{i}\right]$ is Arrow's absolute risk aversion coefficient for random changes in the goal level at point $E\left(\tilde{g}_{i}\right) . U_{i}$ is the decision maker's utility corresponding to each random value of $\tilde{g}_{i}$ which satisfies the standard properties of increasing utility and decreasing marginal utility. $\dot{U}_{i}$ and $\ddot{U}_{i}$ are the first and second derivative at point $E\left(\tilde{g}_{i}\right) . \sigma_{i}^{2}$ is the variance of the $i^{t h}$ goal. This model only can be applied in problems with the first degree of uncertainty or mild uncertainty.

Later, [Ballestero, 2005] extended this approach by considering the combination of both random and non-random goals and utilising the SGP with a lexicographic achievement function. This author also applied his proposed approach to solve various practical problems. More developments of this mean-variance approach were carried out by [Ballestero and Garcia-Bernabeu, 2012] to portfolio selection with multiple time horizons.

[Aouni et al., 2005, 2013] used the satisfaction functions, which defined on the interval $[0,1]$, number ' 1 ' shows the total DM's satisfaction and decreasing monotonically until ' 0 ' that describes their unsatisfaction before veto threshold, to explicitly integrate preferences into GP in an uncertain environment. Consider model 2.5 while $\tilde{g}_{i} \in \mathrm{N}\left(\mu_{i}, \sigma^{2}{ }_{i}\right)$, $\mu_{i}$ and $\sigma^{2}{ }_{i}$ are known (mild uncertainty). By adding the satisfaction functions $\left(F_{i}\left(\delta_{i}\right)\right)$ their SGP model can be formulated as follows: 


$$
\begin{aligned}
& \operatorname{Min} \sum_{i=1}^{m}\left(w_{i}{ }^{+} F_{i}{ }^{+}\left(\tilde{\delta}_{i}^{+}\right)+w_{i}{ }^{-} F_{i}{ }^{-}\left(\tilde{\delta}_{i}^{-}\right)\right) \\
& \text {s.t. } \sum_{j=1}^{n} c_{i j} x_{j}+\tilde{\delta}_{i}^{-}-\tilde{\delta}_{i}^{+}=\mu_{i}, \quad i=1, \cdots, m \text {; } \\
& \sum_{j=1}^{n} a_{r j} x_{j} \leq b_{r}, \quad r=1, \cdots, R ; \\
& 0 \leq \tilde{\delta}_{i}^{+} \leq \alpha_{i v}{ }^{+}, \quad i=1, \cdots, m ; \\
& 0 \leq \tilde{\delta}_{i}{ }^{-} \leq \alpha_{i v}{ }^{-}, \quad i=1, \cdots, m ; \\
& x_{j} \geq 0, \quad j=1, \cdots, n .
\end{aligned}
$$

where $F_{i}{ }^{+}\left(\tilde{\delta}_{i}{ }^{+}\right)$and ${F_{i}}^{-}\left(\tilde{\delta}_{i}{ }^{-}\right)$are satisfaction functions which have thresholds. The $w_{i}{ }^{+}$ and $w_{i}{ }^{-}$represent the intrinsic components of the objective relative importance and $\alpha_{i v}{ }^{+}$and $\alpha_{i v}{ }^{-}$are the veto thresholds determining by the DM. The general shape of the satisfaction function $F_{i}\left(\delta_{i}\right)$ associated with the deviation $\delta_{i}$ is shown in figure 2.3.

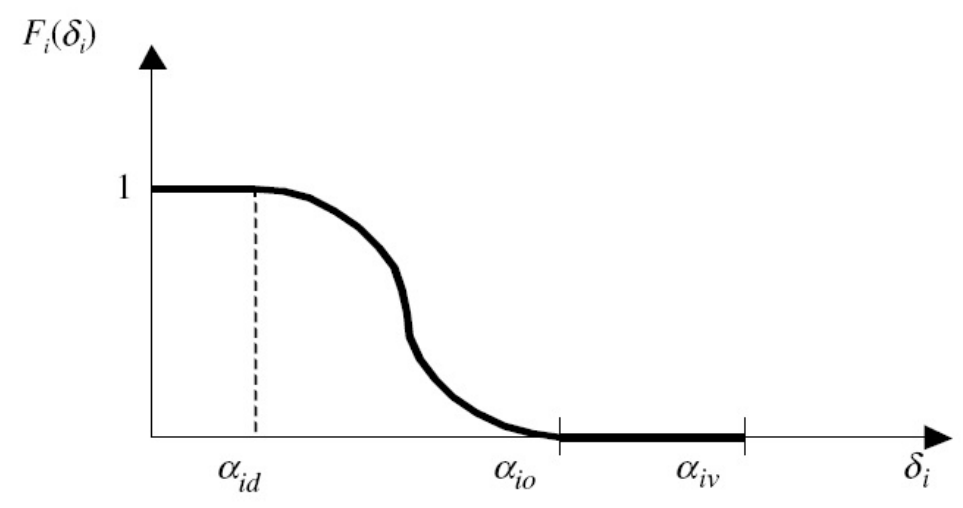

Figure 2.3: The general shape of the satisfaction functions: $F_{i}\left(\delta_{i}\right)$ is the satisfaction function associated with the deviation $\left.\delta_{i}\right) ; \alpha_{i d}$ describes the indifference threshold; $\alpha_{i o}$ expresses the dissatisfaction threshold and $\alpha_{i v}$ represents the veto threshold. (Source: [Aouni et al., 2005])

The literature contains various SPG models which deal with uncertainty in different applications, such as farm management [Ballestero, 2001], water management [AlZahrani and Ahmad, 2004, Bravo and Gonzalez, 2009], portfolio selection [Abdelaziz et al., 2007, Ben Abdelaziz et al., 2009, Aouni et al., 2005, Aouni, 2010a], risk management [Maggis and La Torre, 2012], textile industry [Ballestero, 2005], and resource allocation [Martel and Price, 1981, Abdelaziz and Mejri, 2001, Jayaraman et al., 2016]. Two popular solution methods which are frequently used to solve the SGP problems 
are: (a) Chance Constrained Programming $(C C P)$ developed by Charnes and Cooper ([Charnes and Cooper, 1952, 1959, 1963]) and, (b) stochastic programming with recourse (e.g. [Dantzig and Medansky, 1961, Dantzig, 1963, Van Slyke and Wets, 1969, Wets, 1983, Ermoliev, 1988, Kall and Wallace, 1994, Dupačová, 1995, Dantzig, 1998, Martinez and Aguado, 1998, Shapiro and Homem-de Mello, 1998]).

\subsubsection{Chance Constrained Programming (CCP) and Chance Constrained Goal Programming (CCGP)}

The Chance Constrained Programming (CCP) technique, developed by Charnes and Cooper ([Charnes and Cooper, 1952, 1959, 1963]), can be used to solve problems involving chance/probabilistic constraints. Charnes and Cooper [1963] proposed a deterministic equivalent model of 2.4, while matrices $\mathbf{C}$ and $\mathbf{A}$ are deterministic and the only random variables are in the resources vector $\tilde{\mathbf{b}}$.

The chance constrained approach is frequently used to solve stochastic problems when the feasible solution satisfies the uncertain constraints. This approach supposes that the probability of the constraints is known. The CCP approach converts the abovementioned form of model 2.4 into the following equivalent deterministic model ([Prkopa, 1995]):

$$
\begin{aligned}
& \operatorname{Max} \mathrm{E}\left(\sum_{j=1}^{n} \tilde{c_{i j}} x_{j}\right) \quad i=1, \cdots, m \text {; } \\
& \text { s.t. } \mathrm{P}\left(\sum_{j=1}^{n} \tilde{a_{r j}} x_{j} \leq \tilde{b_{r}}\right) \geq 1-\eta_{r}, \quad r=1, \cdots, R \text {; } \\
& x_{j} \geq 0, \quad j=1, \cdots, n .
\end{aligned}
$$

where $\mathrm{E}\left(\sum_{j=1}^{n} \tilde{c_{i j}} x_{j}\right)$ is the vector of expected value of the objectives function with regards to the random state of nature. $\eta_{r}\left(\eta_{r} \in[0,1], r=1, \cdots, R ;\right)$ are threshold values of constraints that are determined by the DM.

Integration of SGP and CCP was first proposed by [Keown and Martin, 1977] for working capital management. They utilised the lexicographic form of stochastic goal programming which could be formulated as follows:

$$
\begin{array}{lll}
\text { Lex } \operatorname{Min} L=\left(\sum_{i \in I_{p}}\left(\tilde{\delta}_{i}^{+}+\tilde{\delta}_{i}^{-}\right)\right) & p=1, \ldots m ; \\
\text { s.t. } & \mathrm{P}\left(\sum_{j=1}^{n} \tilde{c_{i j}} x_{j} \leq \tilde{g}_{i}\right) \geq \eta_{i}, & i=1, \cdots, m ; \\
& \sum_{j=1}^{n} a_{r j} x_{j} \leq b_{r}, & r=1, \cdots, R ; \\
& \tilde{\delta}_{i}^{+}, \tilde{\delta}_{i}^{-}, x_{j} \geq 0, & j=1, \cdots, n ; i=1, \cdots, m ; .
\end{array}
$$


where $\delta_{i}=\delta_{j}^{+}-\delta_{i}^{-}$describes the positive and the negative deviations with respect to the aspiration level (goals) $g_{i}$, respectively.

Most applications of the probabilistic models assume a normal distribution for model coefficients [Sahoo and Biswal, 2005]. However, other distributions have been considered for model coefficients [Goicoechea et al., 1982, Infanger, 1994]. The Chance Constrained Goal Programming (CCGP) model was developed and applied in different fields of study such as finance [Keown, 1978, Muhlemann et al., 1978, Keown and III, 1980, P. K. De and Sahu, 1982, Brockett et al., 1992], optimal utilisation of resources and the reservoir operation problem [Helm et al., 1984, Changchit and Terrell, 1993, Nembou and Murtagh, 1996, Abdelaziz and Mejri, 2001], and scheduling problem [Easton and Rossin, 1996].

In conclusion, although it seems that the CCGP can be utilised as an efficient technique to deal with uncertainty, the treatment of some complex problems by CCP becomes cumbersome mainly due to some disadvantages of the CCP approach, such as the difficulty in computing the probability in CCP models. The CCP utilises the most probable scenario to reduce the set of random constraints and this has been enumerated as a main advantage of the CCP approach, while this assumption limits the capability of this approach to treat mild uncertainty. Much worse, the probabilistic constraints of CCP models are generally non-convex. Furthermore, Martinez and Aguado [1998] shows that, in general, CCGP or other GP models with probability constraints may not be compatible with Utility Theory and Bayesian Decision Theory. Furthermore, because of the presence of probabilities in CCGP models, they only would be applied under mild uncertainty situations.

\subsubsection{Stochastic Goal Programming (SGP) with recourse}

Generally, to deal with uncertainty about the future the Here-and-Now decision problems are faced and the decision must be taken before some parameters are known. It seems logical that corrective action, named recourse, should be taken once the unknown parameters are known, meaning that some penalties must be paid for any shortfalls (deviation from the goals). The stochastic programming with recourse, first considered by Beale [1955], Dantzig [1955], Dantzig and Medansky [1961], Dantzig [1963] under the name of two-stage linear programs under uncertainty, can be summarised in three sections Linderoth [2003]:

1. Basic decision is made. (The decision maker (DM) makes a basic decision (referred to Here-and-know decision)).

2. Nature decision is made (Black Swan or Surprise events may be revealed, it seems that uncertainty can be moderate or deep).

3. Corrective decision is made. (The DM makes a corrective decision-referred to as a Wait-and-See decision- that attempts to renovate the ruins by nature in (2) by paying some penalties) (Recourse).

The basic formulation of a two-stage stochastic linear programming problem can be found as follows [Shapiro et al., 2009]: 


$$
\begin{aligned}
& \operatorname{Min}_{\mathbf{x} \in \Re^{n}} \mathbf{c}^{T} \cdot \mathbf{x}+\mathrm{E}[Q(\mathbf{x}, \theta)] \\
& \text { s.t. } \mathbf{A} \cdot \mathbf{x} \leq \mathbf{b}, \\
& \quad \mathbf{x} \geq \mathbf{0} .
\end{aligned}
$$

where $Q(\mathbf{x}, \theta)$ is the optimal value of the second-stage problem (some named the recourse function) and $\mathrm{E}(\mathrm{x})$ is the expected value of $x$ :

$$
\begin{aligned}
& \operatorname{Min}_{\mathbf{y} \in \Re^{m}} \quad \mathbf{q}^{T} \cdot \mathbf{y} \\
& \text { s.t. } \mathbf{T} \cdot \mathbf{x}+\mathbf{W} \cdot \mathbf{y}=\mathbf{h}, \\
& \quad \mathbf{y} \geq \mathbf{0} .
\end{aligned}
$$

where $\theta=(\mathbf{q}, \mathbf{h}, \mathbf{T}, \mathbf{W})$ are data of the second-stage problem, some or all elements of vector $\theta$ are random and the expectation operator at the first-stage problem (2.11) is taken with respect to the probability distribution of $\theta$. The recourse decision is presented by vector $\mathbf{y}$ while $\mathbf{W}$ shows the matrix of recourse variables. This two-stage problem is said to have fixed recourse if the matrix $\mathbf{W}$ is not random (fixed). More information about the stochastic programming, its stability and solving approaches can be found in [Van Slyke and Wets, 1969, Olsen, 1976a,b, Kall and Stoyan, 1982, Dupacov, 1984, 1986, Kall and Wallace, 1994, Shapiro and Homem-de Mello, 1998, Shapiro et al., 2009]. Moreover, several applications of Stochastic Programming with recourse can be found in the literature such as petroleum refinery planning [Khor et al., 2008], developing regional economic-ecological sustainability [Li et al., 2011], a collection of test problem [Shapiro and Homem-de Mello, 1998], transportation planning [Barbarosoglu and Arda, 2004], and agriculture [K. Darby-Dowman and Parsons, 2000].

Several alternative formulations for Stochastic Goal Programming are discussed by Martinez and Aguado [1998]. These authors showed that only one of their models, which is a particular case of the Stochastic Linear Programs with Recourse (consider programs 2.11 and 2.12 when $\mathbf{c}=\mathbf{0}$ and $\mathbf{W}=\mathbf{I}$ ), is compatible with the Utility theory and Bayesian Decision Theory under certain conditions. This feature implies that DM's preferences agree with the axioms of the rationality of Utility Theory ${ }^{2}$. Moreover, all Bayesian decision-making techniques (such as measuring techniques of the value of new information) could be utilised in this context ([Martinez and Aguado, 1998]). Their model can be formulated as follows:

\footnotetext{
${ }^{2}$ The utility function can be defined over the possible consequences of the DM's decisions if his/her preferences are consistent with certain axioms of rational behaviour. Therefore, one feasible decision will be preferred to another if, and only if, the expected utility of the possible consequences is larger for the first decision than it is for the second([Martinez and Aguado, 1998] and [DeGroot, 2005]).
} 


$$
\begin{aligned}
& \operatorname{Min}_{\mathbf{x} \in \Re^{n}} \quad \mathrm{E}_{\theta}[Q(\mathbf{x}, \theta)] \\
& \text { s.t. } \quad \mathbf{A} \cdot \mathbf{x}=\mathbf{b} \\
& \quad \mathbf{x} \geq \mathbf{0} .
\end{aligned}
$$

where $Q(\mathbf{x}, \theta)=\left\{\operatorname{Min}_{\mathbf{y} \in \Re^{m}} \mathbf{q}^{T} \cdot \mathbf{y} ; \mathbf{T} \cdot \mathbf{x}+\mathbf{y}=\mathbf{h}, \mathbf{y} \geq \mathbf{0}\right\}$.

Masmoudi and Abdelaziz [2012] utilised a recourse goal programming approach for a portfolio selection problem. They optimised the risk and the return by modelling a biobjective stochastic problem and transferred this stochastic problem to the deterministic equivalent problem using the goal programming and recourse approach. They applied a similar approach to a hospital bed planning problem in Ben Abdelaziz and Masmoudi [2012] in which they utilised a mixture of the recourse approach, GP, and CCP to transfer the multi-objective problem to the deterministic equivalent one. Masri et al. [2016] applied a recourse stochastic goal programming approach to a vehicle routeing problem in which the demands and travel durations are uncertain.

Overall, although it seems that integrating the goal programming and stochastic programming with recourse, i.e. Stochastic Goal Programming with recourse, gives the DM an opportunity to react in an intelligent/optimal way. This could also be useful to treat the second or third degree of uncertainty if we can find an alternative for expectation which is limited to mild uncertainty. However, to the best of our knowledge, there is not any scientific work in the literature in which the recourse goal programming approach is utilised to treat the second and/or third degrees of uncertainty. Only a few authors have tried to use this context while all of them limited their problems to the first degree of uncertainty. It seems that the concept of two-stage stochastic programming or, more general, multi-stage stochastic programming in which decisions should be made sequentially at certain periods of time based on available information at each time period, together with recourse actions could be a useful direction for future work. It could also pave a way for us to find a suitable dynamic long-term programming approach to handle the second and third-degree uncertainty.

\subsubsection{Scenario-based Goal programming}

There are several approaches in the literature that somehow combine the term "scenario" and goal programming. Most of them utilised the GP to compare different alternatives and select a preferable one amongst different criteria. For example, Kim et al. [1999] utilised the combination of the goal programming and the Analytical Hierarchy Process (AHP) to choose the best nuclear fuel scenario in Korea. In addition, Diaz-Balteiro and Romero [2004] introduced a general procedure based on discrete goal programming to determine the system with a higher level of achievement.

Scenario-Based Goal Programming (SBGP) methodology for discrete decision-making problems was well described and structured by Durbach and Stewart [2003] and used to an assess alternatives and select a preferable one over the uncertainty of plausible scenarios. These authors provided a simple SBGP model based on the decomposition of the decision problem into some scenario-specific goal programming problems, one for each 
scenario. This scenario-specific problems were used to rank the alternatives. Suppose that there are $J$ criteria and $K$ scenarios and goals can be set for each criterion-scenario combination. The goal programming formulation to evaluate $I$ discrete alternatives can be found as follows:

Min $\Delta_{i k}=\left[\sum_{j=1}^{J}\left(w_{j k} \delta_{i j k}\right)^{p}\right]^{1 / p}, \quad i=1, \ldots, I ; k=1, \ldots, K$

where $w_{j k}$ is the cumulative weight of deviations $\delta_{i j k}$ from goal $g_{j k}$ for each criterion $j$ and scenario $k . p$ denotes the choice of norm. Durbach and Stewart [2003] examined two famous norms, $(p=1)$ and $(p=\infty)$, and discussed different types of decision-making strategies. Tchebycheff aggregation $\left(L_{\infty}\right)$ ought to be used when the DM considers robust behaviour over criteria/scenarios more desirable. The other norm (i.e. $L_{1}$ ) is suitable for compensatory behaviour. The Super-GP formulation has also been proposed by these authors to aggregation purpose as follows:

$$
\operatorname{Min} \Delta_{i}=\left[\sum_{k=1}^{K}\left(\left[\sum_{j=1}^{J}\left(w_{j k} \delta_{i j k}\right)^{p}\right]^{1 / p}\right)^{q}\right]^{1 / q}, \quad i=1, \ldots, I \text {. }
$$

where $p$ and $q$ are selected metrics for the individual and super problems, respectively. There is not any scenario probability that can be found in these formulations. However, these formulations can be easily modified when the DM prefers to make some judgements regarding the probability of each scenario. Although their model does not generate the solutions, their methodology has great potential to deal with the deeper uncertainties.

On the other hand, some of the studies which have been carried out in an uncertain world include multi-objective or multi-stage stochastic programming which are somehow integrated with scenarios; some are named scenario-based models ([Watkins et al., 2000, Ahmed et al., 2003, Alonso-Ayuso et al., 2003, Abdelaziz and Masri, 2005, Ji et al., 2005, Azaron et al., 2008, Ballestero et al., 2009, Aouni, 2010b,a, Li et al., 2011, Stoyan and Kwon, 2011, Aouni et al., 2013]). For example, Aouni [2010a], Aouni et al. [2010] proposed a different SGP model with a random solution vector $\mathbf{x}$. They assumed the finite probability space $(\Omega, \Im, P)$, where $\Omega=\left\{\omega_{1}, \cdots, \omega_{K}\right\}$ is the sample space of all possible events or scenarios with known associated probabilities $p\left(\omega_{k}\right)=p_{k}$, $\Im$ is a $\sigma$ algebra on $\Omega$ and $P$ is a probability measure (which refers to mild uncertainty). If $z_{i j k}=z_{i}\left(x, \omega_{k}\right)=\sum_{j=1}^{n} c_{i j}\left(\omega_{k}\right) x_{j}\left(\omega_{k}\right), i=1, \cdots, m$; are the $i^{\text {th }}$ objective function with the corresponding goal $g_{i}$ which depends on scenario $\omega_{k}$, the Scenario-Based Stochastic Goal Programming model (for a particular $k$ ) can be formulated as follows: 


$$
\begin{array}{lll}
\text { Min } \sum_{i=1}^{m}\left(\delta_{i}^{+}\left(\omega_{k}\right)+\delta_{i}^{-}\left(\omega_{k}\right)\right) & \\
\text { s.t. } & \sum_{j=1}^{n} c_{i j}\left(\omega_{k}\right) x_{j}\left(\omega_{k}\right)+\delta_{i}^{-}\left(\omega_{k}\right)-\delta_{i}^{+}\left(\omega_{k}\right)=g_{i}\left(\omega_{k}\right), & i=1, \cdots, m ; \\
& \sum_{j=1}^{n} a_{r j}\left(\omega_{k}\right) x_{j}\left(\omega_{k}\right) \leq b_{r}\left(\omega_{k}\right), & r=1, \cdots, R ; \\
& \delta_{i}^{+}\left(\omega_{k}\right), \delta_{i}^{-}\left(\omega_{k}\right), x_{j}\left(\omega_{k}\right) \geq 0, & \forall i, j .
\end{array}
$$

Although Aouni [2010a], Aouni et al. [2010] suggested that this model must run for all $K$ scenarios to find $K$ solutions and the optimal solution will correspond to the one which shows the highest probability, these authors did not prove whether or not this optimal solution would be a feasible solution in other scenarios (one can easily find a counterexample to show in-feasibility in this problem). This is an important gap in their work which needs more discussion and debate. However, Aouni et al. [2013] added the satisfaction functions to this model and applied a Scenario-Based SGP with satisfaction functions for venture capital investment decision-making.

\subsubsection{Fuzzy Goal Programming (FGP)}

At the beginning of the $21^{\text {st }}$ century, Fuzzy sets and theory, introduced by Zadeh [1965] and Bellman and Zadeh [1970], had turned into a popular approach to handle imprecise data amongst a vast majority of authors. It is not surprising that many authors have been found to use and apply the fuzzy logic in their works. Zimmermann [1978] introduced fuzzy mathematical programming for dealing with multi-objective problems. A few years later, the Fuzzy Goal Programming (FGP) was introduced by Narasimhan [1980] to specify imprecise aspiration levels of the goals in a fuzzy environment. Fuzzy goal programming was developed by [Narasimhan, 1981, Hannan, 1981a,b,c, 1982, Ignizio, 1982a,b, Tiwari et al., 1986, 1987]. Since then, as Aouni et al. [2009] confirmed, there is no fundamental scientific contribution related to the FGP and most of the published papers try to either ameliorate the efficiency of Hannan's FGP formulation ([Hannan, 1981a,b,c]) by developing some different algorithms or utilise the FGP approach in different applications.

Let us consider a GP problem in which objectives with imprecise aspiration levels can be treated as fuzzy goals. The FGP formulation of Hannan [1981a] can be formulated as follows: 
$\operatorname{Max} \lambda$

$$
\begin{array}{lll}
\text { s.t. } & \frac{\sum_{j=1}^{n} c_{i j} x_{j}}{\Delta_{i}}+\delta_{i}^{-}-\delta_{i}^{+}=\frac{g_{i}}{\Delta_{i}}, & i=1, \cdots, m ; \\
\lambda+\delta_{i}^{-}+\delta_{i}^{+} \leq 1, & i=1, \cdots, m ; \\
\sum_{j=1}^{n} a_{r j} x_{j} \leq b_{r}, & r=1, \cdots, R ; \\
\lambda, \delta_{i}^{+}, \delta_{i}^{-}, x_{j} \geq 0, & \forall i, j .
\end{array}
$$

By simple replacements in the objective function and the second pack of constraints, the additive FGP model, as an alternative to the above Max-Min model (2.17), can be reformulated as follows:

$$
\begin{array}{lll}
\operatorname{Min} & \sum_{i=1}^{m}\left(\delta_{i}^{+}+\delta_{i}^{-}\right) & \\
\text {s.t. } & \frac{\sum_{j=1}^{n} c_{i j} x_{j}}{\Delta_{i}}+\delta_{i}^{-}-\delta_{i}^{+}=\frac{g_{i}}{\Delta_{i}}, & i=1, \cdots, m ; \\
& \sum_{j=1}^{n} a_{r j} x_{j} \leq b_{r}, & r=1, \cdots, R ; \\
\delta_{i}^{+}, \delta_{i}^{-}, x_{j} \geq 0, & \forall i, j .
\end{array}
$$

where $\Delta_{i}$, which is specified by the DM, is the constant of deviation of the aspiration levels $g_{i}$.

The FGP formulations utilise the concept of membership functions to deal with the fuzziness of the goals. The concept of membership functions, based on the fuzzy set theory, has been introduced and used by Zimmermann [1976, 1978, 1983, 1996] and Freeling [1980] for modelling the fuzziness related to decision-making context parameters. Triangular membership functions of the solution set $\left(0 \leq \mu_{i}(x) \leq 1, i=1, \cdots, m\right.$; ) are defined to fuzziness in models 2.17 and 2.18 as:

$$
\mu_{i}= \begin{cases}0 & \text { if } \sum_{j=1}^{n} c_{i j} x_{j} \leq\left(g_{i}-\Delta_{i}\right) \\ \frac{\sum_{j=1}^{n} c_{i j} x_{j}-\left(g_{i}-\Delta_{i}\right)}{\Delta_{i}} & \text { if }\left(g_{i}-\Delta_{i}\right) \leq \sum_{j=1}^{n} c_{i j} x_{j} \leq g_{i} \\ \frac{g_{i}+\Delta_{i}-\sum_{j=1}^{n} c_{i j} x_{j}}{\Delta_{i}} & \text { if } g_{i} \leq \sum_{j=1}^{n} c_{i j} x_{j} \leq\left(g_{i}+\Delta_{i}\right) \\ 0 & \text { if } \sum_{j=1}^{n} c_{i j} x_{j} \geq\left(g_{i}+\Delta_{i}\right)\end{cases}
$$


The FGP formulations of Dhingra et al. [1992] and Zimmermann [1978] have developed an approximation procedure for the non-linear membership functions. Moreover, the FGP models have frequently been developed by many authors (e.g. [Rubin and Narasimhan, 1984, Yang et al., 1991, Rao et al., 1992, Gen et al., 1993, Wang and Fu, 1997, Liu and Iwamura, 1998, Kim and Whang, 1998, Mohammed, 2000, Ramik, 2000, Parra et al., 2001, Chen and Tsai, 2001, El-Wahed and Abo-Sinna, 2001, Rasmy et al., 2002, Pal and Moitra, 2003, Pal et al., 2003, Lin, 2004, Xu, 2004, Saad, 2005, Wang et al., 2005, Akz and Petrovic, 2007, Chang, 2007, Hu et al., 2007, Pramanik and Roy, 2007, Yaghoobi and Tamiz, 2007, Yaghoobi et al., 2008, Arora and Gupta, 2009, Baky, 2009, Jana and Sharma, 2010, Kara et al., 2009, Khalili-Damghani et al., 2013, Mouslim et al., 2014, Chen, 1994]).

Aouni et al. [2009] classified the different FGP formulations into four categories: (i) Lexicographic FGP, (ii) Weighted FGP, (iii) Fuzzy MINMAX Goal Programming and (iv) Interactive FGP. More information of different types of FGP can be found in [Aouni et al., 2009, Mohamed, 1997, Li, 2012].

The FGP also has a wide variety of applications in the literature such as supply chain management [Kumar et al., 2004, Selim and Ozkarahan, 2008, Selim et al., 2008, Torabi1 and Hassini, 2009, Tsai and Hung, 2009], waste management systems [Chang and Wang, 1997], water quality management [Lee and Wen, 1997], project networking [Arkan and Gngr, 2001], agricultural planning [Sinha et al., 1988, Biswas and Pal, 2005, Sharma and Jana, 2009], forestry [Pickens and Hof, 1991], energy planning [Jinturkar and Deshmukh, 2011], engineering design in Quality Function Deployment (QFD) processes [Chen and Weng, 2006], portfolio selection [Watada, 1997, Parra et al., 2001, Wang and Zhu, 2002, Bilbao et al., 2007, Mansour et al., 2007, Azmi and Tamiz, 2010, Bilbao-Terol et al., 2012] and management [Sharma et al., 2009], investment management [Gupta and Bhattacharjee, 2010, Gupta and Bhattacharya, 2010], transportation problems [Chalam, 1994, El-Wahed and Lee, 2006, Pramanik and Roy, 2008, Giri et al., 2014], machine tool selection [Rai et al., 2002, Chan et al., 2005, Mishra et al., 2006], production planning [Chan and Swarnkar, 2006, Javadi et al., 2008, Belmokaddem et al., 2009, Jamalnia and Soukhakian, 2009, Kara et al., 2009, Özcan and Toklu, 2009, Mkidiche et al., 2013], nurse scheduling [Tamiz and Yaghoobi, 2010], demand coverage [Giannikos, 2010] etc.

Abdelaziz and Masri [2005] introduced stochastic programming with an imprecise (fuzzy linear partial information) probability distribution. They supposed that the probability distribution of random data exists but its exact value is not reachable. Two cases for partial information are considered by these authors: (1) statistical tools fail to determine the exact values of probabilities (for example, the probability value lies between two fixed numbers), (2) the probability distribution was approximated (for example, the probability value is a fixed number). Then their problem transfers to a stochastic linear program where the probability distribution is known to belong to the set $\tilde{\phi}_{j}=\left\{p_{j} \in \Re ; \sum_{j=1}^{n} a_{i j} p_{j}<b_{j} ; \sum_{j=1}^{n} p_{j}=1, p_{j} \geq 0, j=1, \ldots, n\right.$. $\}$. They proposed a two-step solution strategy in which the first step is allocated to transferring the fuzzy problem to an equivalent deterministic one (they used the $\alpha$-cut technique). The randomness of the parameters is then solved by a stochastic programming approach in the second step.

Although it seems that their approach may be able to handle moderate uncertainty, it still supposes that there is a finite probability space includes some vague probability 
values. This is closer to the definition of mild uncertainty.

\subsubsection{Robust Goal Programming (RGP)}

Robust optimisation (RO) is one of the more recent approaches to treat uncertainty in optimisation problems. However, its origin goes back to the use of worst-case analysis to deal with uncertainty in the foundation of the modern decision theory in the 1950s and has been developed and applied in several fields of study over the years. Recently, with the advent of super-fast computers and after some works of Bental and Nemirovski in the late 1990s and early 2000s (ex. [Ben-Tal and Nemirovski, 1997, 1998, 1999, 2000b]), a new wave of the tendency in Robust Optimisation (RO) has been observed amongst a vast majority of authors in many fields of study. Robust optimization (RO), generally, includes several methods to help DMs in dealing with two different types of uncertainty: (a) the uncertainty of feasibility (i.e. the feasibility of a solution is affected by uncertainty) or (b) the uncertainty of optimality (i.e. the optimality of a solution is affected by uncertainty). In RO, mainly based on the worst case ${ }^{3}$, the DM attempts to capture a solution that will be feasible (robust feasible solution) or optimal (robust optimal solution) for any realisation of the uncertainty in a given set. Generally, this means that a robust feasible/optimal solution is a solution which retains its feasibility/optimality even if some of the decision parameters are changed. Gabrel et al. [2014] and Bertsimas et al. [2011] provide information about RO and its value. The literature contains examples of various RO models which treat uncertainty (e.g. [Soyster, 1973, Ben-Tal and Nemirovski, 2000a, Bertsimas and Sim, 2004]). Amongst them, Bertsimas and Sim [2004] proposed a linear robust optimisation programming approach in which the DM can control the conservatism of solution. Kuchta [2004] was first adopted the Bertisimas and Sim's RO method to introduce a robust optimal solution of a goal programming problem with negative variations in the left-hand sides of the goals. Later, Ghahtarani and Najafi [2013] developed this approach to find a robust feasible solution in portfolio selection. The Kuchta's model can be formulated as follows:

$$
\begin{array}{lll}
\text { Min } & \sum_{i=1}^{m}\left(w_{i}^{+} \delta_{i}^{+}+w_{i}^{-} \delta_{i}^{-}\right) & \\
\text {s.t. } & \sum_{j=1}^{n} \underline{\mathrm{c}}_{i j} x_{j}+\max _{\left(S_{i} \subset\{1, \ldots, n\},\left|S_{i}\right| \leq t_{i}\right)} \sum_{j \in S_{j}} \theta_{i j} x_{j}+\delta_{i}^{-}-\delta_{i}^{+}=g_{i}, & i=1, \cdots, m ; \\
& \sum_{j=1}^{n} a_{r j} x_{j} \leq b_{r}, & r=1, \cdots, R ; \\
& \delta_{i}^{+}, \delta_{i}^{-}, x_{j} \geq 0, & \forall i, j .
\end{array}
$$

where coefficients $c_{i j} \in\left[\underline{\mathrm{c}}_{i j}, \bar{c}_{i j}\right],(i=1, \ldots, n ; j=1, \ldots, m)$ represent the uncertainty which impresses the attainment of goals in a negative way. $c_{i j}$ may well take on a normal (most possible) value (i.e. $\underline{\mathrm{c}}_{i j}$ ) for all $i$ and $j$, but it is possible that they take on any value from the interval $\left[\underline{\mathrm{c}}_{i j}, \bar{c}_{i j}\right] .\left|S_{i}\right|$ denotes the power of set $S_{i}, \theta_{i j}=\bar{c}_{i j}-\underline{\mathrm{c}}_{i j},(i=$ $1, \ldots, n ; j=1, \ldots, m)$ and $t_{i},(i=1, \ldots, m)$ is an integer number $(\leq n)$ which is given by

\footnotetext{
${ }^{3}$ The most unfavourable realisation of uncertainty is used to evaluate a solution.
} 
the DM to indicate how many coefficients can be changed at the most in constraint $i$. The normal optimal solution (optimistic solution) is obtained when $t_{i}=0,(i=1, \ldots, m)$ and it means that nothing will go wrong, while the pessimistic solution will be reached if $t_{i}=n,(i=1, \ldots, m)$. In other words, $t_{i}$ shows the DM's degree of pessimism with respect to each goal.

If, in each $g_{i},(i=1, \ldots, m)$, the coefficients $c_{i j}$ are taken from the least favourable values, the optimal values of the objective function are the worst optimal value of total deviation. The optimal value of the total deviation is extremely sensitive to changes in values of $t_{i}$.

The worst possible optimal (pessimistic optimal) value of the total deviation from the goals according to the worst scenario can be seized by this pessimistic approach. By changing one parameter per goal, different solutions for various degrees of the DM's pessimism or uncertainty can be reached. However, this may not necessarily be what the DMs want. In general, it seems that the solutions of robust goal programming could be an unsatisfactory solution which is too risk averse (conservative) and/or dominated by one bad scenario.

\subsubsection{Dynamic Goal Programming}

Generally, multi-objective dynamic programming treats multi-period decision processes. Two main approaches to multi-objective dynamic problems can be found in the literature, namely vector dynamic and scalarization. Brown and Strauch [1965] introduced the Vector Dynamic approach (VD) as a version of the fundamental functional equation of dynamic programming for a group of problems in which the return space is only partially ordered. The purpose of solving VD programming problems is to find a set of efficient solutions and Pareto-optimal vectors in the criterion space [Klotzler, 1978]. Scalarization methods transformed the multi-objective dynamic problem into an equivalent single objective dynamic problem based on the DM's preference. Levary [1984] first utilised the goal programming approach as a scalarization method to solve a multi-objective dynamic program.

Charnes et al. [1989] proposed a dynamic goal programming model as a framework for planning joint investment in agriculture to achieve self-sufficiency in food production in the Middle East and the fair allocation of the returns. Their linear goal programming formulation assumed the penalty cost, which is taken as the import price, was constant for each period and the sensitivity analysis was performed to assess the model solution in each period. Game theory (synthesise a game in chance-constrained characteristic function from the optimal solution to their goal programming model) was utilised as well.

A few dynamic GP models were described by Trzaskalik [1997] to discuss different aspects of the goal programming approach in multiple objective dynamic programming. These approaches have been listed as follows:

1. Dynamic Goal Programming Model. This method considers values of multiperiod criteria and multi-period goals and looks for the nearest solution while utilising weight coefficients to set different importances of deviations from multiperiod goals. 
2. Dynamic Hierarchical Goal Programming Model. Weight coefficients are not used in this method. Instead, a hierarchy is set for deviations from the DM's goals.

3. Dynamic Period Goal Programming Model. Multi-period criteria's values are set by DM and deviations from given target period are measured by using weight coefficients. The nearest solution is looked for again.

4. Dynamic Hierarchical Period Goal Programming Model. In this approach, the hierarchy of deviations from period target values is set by the DM.

To sum up, dynamic GP approach has potential to cope with higher degrees of uncertainty, but it needs to more development, robustness and some real-world applications. Also, as discussed in Section 2.2.3, dynamic robustness is the most suggestible approach to handling the higher degrees of uncertainty in long-term strategic planning. Therefore, we have integrated the concepts of robustness and dynamic multi-stage programming to propose a dynamic robust multi-objective optimisation structure which is able to treat the higher degrees of uncertainty in complex MCDM/MOO problems (such as long-term strategic planning) in this thesis.

\subsection{Conclusion}

This review has described the definition of uncertainty and has shown that it can be studied from different dimensions such as nature, location and degrees of uncertainty. It is contended by many authors that uncertainty takes on different meanings in various fields, which means that it needs different classifications. Therefore, the classification of different degrees of uncertainty, which could be useful in the structuring of multi-criteria decision making (MCDM) and optimisation problems, has been introduced and compared with some other classifications in the literature. Throughout this study, the term deep uncertainty refers to the third degree of uncertainty. This term has a different meaning in comparison with previous definitions of deep uncertainty. For example, as seen in Table 2.2, deep uncertainty (level 3) of Kwakkel et al. [2010] is classified as a second degree of uncertainty in our definition. The importance of accepting the uncertainty in real-world problems and different suggestions to handle higher degrees of uncertainty have also been reviewed.

As discussed in section 2.2.3, dynamic robustness is the most suggestible approach to handle the higher degrees of uncertainty in long-term planning. However, to the best of our knowledge, there is a paucity of studies which have utilised this approach (dynamics robustness) to handle uncertainty in multi-objective optimisation (see [Ahmed et al., 2003, Ji et al., 2005, Dupacov et al., 2000, Watkins et al., 2000]). Nonetheless, it is frequently applied as a powerful tool to treat the higher degrees of uncertainty in several fields of study. Utilising this approach in MCDM and optimisation problems under higher degrees of uncertainty could be an interesting direction for research. Nevertheless, a high level of complexity has prevented authors from working on these kind of problems. Modelling these kinds of problems need to be well structured and carefully developed.

In the second part of this review, goal programming as one of the most popular and well established multi-objective optimisation approaches, as well as its developments in 
dealing with uncertainty, has been reviewed with details. Stochastic Goal Programming, Fuzzy Goal Programming, Scenario-Based Goal Programming, Robust Goal Programming and Dynamic Goal Programming are reviewed. The advantages and disadvantages of each approach are highlighted and some possible directions for future research are noted as well. We believe that the limitation of GP to modelling uncertainty relating to future states of the world can be rectified by scenario planning. Integrating the scenario planning and goal programming, if well structured and defined, could be helpful in this complex multi-criteria decision-making problems. Furthermore, amongst different kinds of Stochastic Goal Programming (SGP), the SGP with recourse seems able to match with the concept of dynamic robustness and deep uncertainty if we can find a way to avoid using the probabilities and expectations in Stochastic Programming.

Therefore, it seems that integrating these different concepts (i.e. dynamic robustness, SGP with recourse, and scenario planning) can be an effective approach to handle deep uncertainty in MCDM/MOO. thus, as one of our initial purposes in this thesis, we tried to find a way to integrate these different concepts in one suitable framework that could help us deal with deep uncertainty in MCDM/MOO problems.

At the end of this review, a quick overview of the literature has been provided in Tables 2.3 and 2.4 which indicates different goal programming approaches under uncertainty. Table 2.3 reviewed some recent applications of GP under uncertainty. Different degrees of uncertainty that are able to be dealt with, the methodology which was used to treat the uncertainty, and the number of objectives that were considered by the authors in these applications have been described in this table. As seen in Table 2.3, none of them are designed to treat deep uncertainty.

A 3-dimensional matrix including the nature and degrees of uncertainty as well as the number of objectives and different applications, have been portrayed in Table 2.4. As described in this table, similar to the previous table, none of the previous scientific works can deal with deep uncertainty in multi-objective optimisation (MOO) problems neither in internal nor in external uncertainty. This is an important gap in the literature and is the basis of our research (i.e. dealing with deep uncertainty in MCDM/MOO). Table 2.4 also shows that only a few authors have attempted to treat the second degree of uncertainty in this area. Therefore, further development of some real-world applications in multi-objective optimisation problems is necessary.

Hence, as mentioned in this review, many real-world applications involve multiobjective optimisation under conditions of deep uncertainty. However, most of the previous works in this field of study ignore deep uncertainty because of its complexity, and are limited to a low level of uncertainty with known probability distributions (namely mild uncertainty). To the best of our knowledge, there is limited or no literature or scientific work concerning deep uncertainty in MCDM/MOO and how these problems can be addressed.

Ignorance of deep uncertainty in decision-making problems can lead us to make an improper decision and waste a vast majority of our limited and valuable resources. In the current fast-growing and highly competitive world, all companies and policy-makers pay a lot of money for a only little improvement in their performances. Therefore, considering the deep uncertainty can help them to tremendously improve their sustainability and performances. This thesis tried to provide a way forward as well as further understanding of the complex problems associated with deep uncertainty in MCDM/MOO. 
To attain this purpose, as mentioned in Chapter 1, this thesis first defined and developed some necessary concepts and structures, then introduced a generic model to deal with deep uncertainty in MCDM/MOO problems. Dynamic robustness, SGP with recourse, and scenario planning are contributed in motivating our proposed methodology. Further discussion, examples, and expansions are also provided in this thesis, as well as a real case study to examine the reliability and validity of the proposed methodology in practice. 


\begin{tabular}{|c|c|c|c|c|c|c|c|c|}
\hline \multirow{2}{*}{ Methodology } & \multirow{2}{*}{ Authors } & \multicolumn{3}{|c|}{ Degree of uncertainty } & \multicolumn{3}{|c|}{ Number of objectives } & \multirow{2}{*}{ Application } \\
\hline & & 1st & 2nd & 3rd & 1 & 2 or 3 & $>3$ & \\
\hline \multirow{12}{*}{ Resilience } & Aouni et al. (2005) & $\star$ & & & & $\star$ & & Finance (Portfolio selection) (Tunisian stock exchange) \\
\hline & Aouni et al. (2013) & $\star$ & & & & & $\star$ & Finance (Italian venture capital fund) \\
\hline & Abdelaziz et al. (2007) & $\star$ & & & & $\star$ & & Finance (portfolio management) \\
\hline & Abdelaziz and Masri (2005) & $\star$ & & & $\star$ & & & - \\
\hline & AL-ZAHRANI and AHMAD (2004) & $\star$ & & & & & $\star \star$ & Manage domestic water supply \\
\hline & Aouni and Torre (2010) & $\star$ & & & & & $\star$ & - \\
\hline & Ballestero (2001) & $\star$ & & & & $\star$ & & Farm management \\
\hline & Ballestero (2005) & $\star$ & & & & $\star$ & & Textile industry \\
\hline & Bravo and Gonzalez (2009) & $\star$ & & & & $\star$ & & Water use planning \\
\hline & Bravo et al. (2010) & $\star$ & & & & $\star$ & & Finance \\
\hline & Li et al. (2011) & $\star$ & & & $\star$ & & & Planning of resources management \\
\hline & Martinez (1998) & $\star$ & & & & & $\star$ & - \\
\hline \multirow{10}{*}{ Static robustness } & Muoz and Ruiz (2009) & $\star$ & & & & $\star$ & & - \\
\hline & Sahoo and Biswal (2005) & $\star$ & & & & $\star$ & & - \\
\hline & Steuer et al. (2005) & $\star$ & & & & & $\star$ & - \\
\hline & Stoyan and Kwon (2011) & $\star$ & & & & & $\star$ & Finance \\
\hline & ALONSO-AYUSO ET AL. (2003) & $\star$ & & & & $\star$ & & A sample of Strategic Supply Chain Planning \\
\hline & Kuchta 2004) & $\star$ & & & & & $\star$ & - \\
\hline & Ghahtarani and Najafi (2013) & $\star$ & & & & & $\star$ & Portfolio selection \\
\hline & Malcolm and Zenios (1994) & $\star$ & & & & $\star$ & & Power system planning \\
\hline & Khor et al. (2008) & $\star$ & & & * & & & Midterm refinery planning problem \\
\hline & Azaron et al. (2008) & $\star$ & & & & $\star$ & & A sample of wine company is willing to design its Supply Chain. \\
\hline \multirow{4}{*}{ Dynamic robustness } & SHABBIR AHMED et al. (2003) & $\star$ & & & $\star$ & & & - \\
\hline & Xiao-Dong Ji et al. (2005) & $\star$ & & & & $\star \star$ & & Finance (portfolio management) \\
\hline & DUPACOV ET AL. (2000) & $\star$ & & & & & $\star$ & - \\
\hline & Watkins Jr et al. (2000) & & $\star$ & & & $\star$ & & Management of the Highland Lakes \\
\hline
\end{tabular}

Table 2.3: Some applications of GP under uncertainty, degrees of uncertainties and the methodologies that used by their authors to treat these uncertainties over the last years. 


\begin{tabular}{|c|c|c|c|c|c|c|c|c|}
\hline \multirow{2}{*}{ Nature/Sources of Uncertainty } & \multirow{2}{*}{ Authors } & \multicolumn{3}{|c|}{ Degree of uncertainty } & \multicolumn{3}{|c|}{ Number of objectives } & \multirow{2}{*}{ Application } \\
\hline & & 1st (Mild) & 2nd (Moderate) & 3rd (Deep) & 1 & 2 or 3 & $>3$ & \\
\hline \multirow{28}{*}{ Variability (Objective/External) } & Xiao-Dong Ji et al. (2005) & $\star$ & & & & $\star$ & & Finance (portfolio management) \\
\hline & Stoyan and Kwon (2011) & $\star$ & & & & & $\star$ & Finance \\
\hline & Abdelaziz et al. (2007) & $\star$ & & & & $\star$ & & Finance (portfolio management) \\
\hline & Abdelaziz and Masri (2005) & $\star$ & & & $\star$ & & & - \\
\hline & Ballestero (2001) & $\star$ & & & & $\star$ & & Farm management \\
\hline & Ballestero (2005) & $\star$ & & & & $\star$ & & Textile industry \\
\hline & Sahoo and Biswal (2005) & $\star$ & & & & $\star$ & & - \\
\hline & Muoz and Ruiz (2009) & $\star$ & & & & $\star$ & & - \\
\hline & Bravo and Gonzalez (2009) & $\star$ & & & & $\star$ & & Water use planning \\
\hline & Aouni et al. (2005) & $\star$ & & & & $\star$ & & Finance (Portfolio selection) \\
\hline & AL-ZAHRANI and AHMAD (2004) & $\star$ & & & & & $\star$ & Manage domestic water supply \\
\hline & Azaron et al. (2008) & $\star$ & & & & $\star$ & & A sample of wine company is willing to design its Supply Chain. \\
\hline & Aouni et al. (2013) & $\star$ & & & & & ᄎ & Finance (Italian venture capital fund) \\
\hline & Aouni and Torre (2010) & $\star$ & & & & & $\star$ & - \\
\hline & Bravo et al. (2010) & $\star$ & & & & $\star$ & & Finance \\
\hline & Steuer et al. (2005) & $\star$ & & & & & * & - \\
\hline & SHABBIR AHMED et al. (2003) & $\star$ & & & $\star$ & & & - \\
\hline & ALONSO-AYUSO ET AL. (2003) & $\star$ & & & & $\star$ & & A sample of Strategic Supply Chain Planning \\
\hline & Khor et al. (2008) & $\star$ & & & * & & & mMidterm refinery planning problem \\
\hline & Watkins Jr et al. (2000) & & $\star$ & & & $\star$ & & Management of the Highland Lakes \\
\hline & Li et al. (2011) & $\star$ & & & $\star \star$ & & & Planning of resources management \\
\hline & Abdelaziz (2012) & $\star$ & & & & & * & Six different samples \\
\hline & DUPACOV ET AL. (2000) & $\star$ & & & & & * & - \\
\hline & Abbas and Bellahcene (2006) & $\star$ & & & & & $\star$ & - \\
\hline & Malcolm and Zenios (1994) & $\star$ & & & & $\star$ & & Power system planning \\
\hline & Martinez (1998) & $\star$ & & & & & $\star$ & - \\
\hline & Kuchta 2004) & $\star$ & & & & & $\star$ & - \\
\hline & Ghahtarani and Najafi (2013) & $\star$ & & & & & * & Portfolio selection \\
\hline \multirow{13}{*}{ Limited knowledge (Subjective/Internal) } & Charnes et al. (1989) & $\star$ & & & & & ᄎ & Planning joint investments \\
\hline & Bordley and Kirkwood (2004) & $\star$ & & & & & $\star$ & New Product Development and decision making \\
\hline & Durbach and Stewart (2003) & $\star$ & $\star$ & & & & $\star$ & - \\
\hline & Hannan (1981) & $\star$ & & & & $\star$ & $\star$ & - \\
\hline & Sharma and Jana (2009) & $\star$ & & & & $\star$ & & Rice Crop Planning \\
\hline & Arora and Gupta (2009) & $\star$ & & & & $\star$ & & \\
\hline & Jinturkar and Deshmukh (2011) & $\star$ & & & & & * & Rural cooking and heating energy planning \\
\hline & Jamalnia and Soukhakian (2009) & $\star$ & & & & & * & Aggregate production planning \\
\hline & Saad (2005) & $\star$ & & & & $\star$ & $\star$ & \\
\hline & Chang (2007) & $\star$ & & & & $\star$ & & Establishing E-learning web servers \\
\hline & Tamiz and Yaghoobi (2010) & $\star$ & & & & $\star$ & & Nurse Scheduling \\
\hline & Silva and Marins (2014) & $\star$ & & & & & $\star$ & Brazilian Sugar mill \\
\hline & Shahrezaie et al. (2014) & $\star$ & & & & & $\star$ & Sample of man power scheduling \\
\hline
\end{tabular}

Table 2.4: 3-dimensional matrix includes nature and degrees of uncertainty as well as number of objectives and different applications of GP under uncertainty over the last years. 


\section{Chapter 3}

\section{Two-stage Scenario-Based Multi-Criteria Decision-Making/ Multiple Objective Optimisation Structure}

\subsection{Introduction}

The primary motivation for writing this thesis was that we were concerned that many optimisation models do not treat the higher degrees of uncertainty and risk observing many real-world problems, especially, with long-term decision-making processes such as strategic planning problems. We were exclusively interested in the multi-objective optimisation problems under deep uncertainty as most real-life problems, by nature, have several (possibly conflicting) objectives to be satisfied. In order to address these problems, a number of decisions need to be made, and one alternative/solution must be chosen before one can begin to explore the consequences of the decision or gain sufficient awareness of all other options.

In other words, some of the fundamental strategic planning problems currently faced by policy makers are characterised by uncertainties about the future, classified as deep uncertainty, which is unpredictable. These uncertainties are almost impossible to reduce by gathering more information and are not statistical in nature. Therefore, in dealing with such a problem, a decision is not only one that can seize different conflicting objectives; it also relies sustainable planning that should be robust and adaptable. This means that the decision should perform satisfactorily under a broad variety of futures (it is robust) and it can be adapted to changing variable future conditions [Haasnoot et al., 2011].

Classical approaches, such as stochastic goal programming, are probability based and such mean/variance models require a correctly-defined complete sample space, strong assumptions (e.g. normality), or both. They have circumvented the variability by utilising a mean/approximated/fixed value of the parameters, thus losing the valuable information about the variability in the final optimised solution. However, amongst them, stochastic programming with recourse, which is a two-stage approach, can make sense but the 
calculating expectation in this model is still difficult and prevents us from dealing with deep uncertainty.

Moreover, under conditions of the deep uncertainty, qualitative components in complex problems are incompletely understood and potential outcomes not enumerable. In such situations, it becomes useful to utilise the concept of scenarios to representations of coherent futures as a framework for thought and critical conversation [Durbach and Stewart, 2012]. The term scenario is used in this study as "logical descriptions of possible futures in which the outcomes of decisions will emerge". In Multi-Criteria Decision Making(MCDM), the performance of decision regarding each criterion under conditions of each scenario is a valid dimension of preference, for which the term meta-criteria was introduced by Stewart et al. [2013]. This term has been used to evaluate and compare different decisions concerning all criteria-scenario combinations.

Therefore, in this thesis, we extend the meta-criteria concept, and utilise scenarios as a dimension of preference (a component of the meta-criteria) to avoid problems relating to the assessment and use of probabilities under deep uncertainty. These concepts promoted our motivation to adopt a scenario-based multi-objective optimisation approach under deep uncertainty.

In this regard, robust decision-making seeks good performance under each scenario (especially the worst case scenario) where performance is typically based on multiple criteria. It seems that robust goal programming may solve the problem. Nevertheless, sometimes the robust solution may be an unsatisfactory solution which is too risk-averse (conservative), dominated by a bad scenario, or both. Extending the concept of robustness, in a way that a less risk-averse solution can be reached, enhances an extraordinary motivation to our study. Also, as discussed in Chapter 2, dynamic robustness is the most suggestible approach to handling the higher degrees of uncertainty in long-term strategic planning. However, to the best of our knowledge, this approach has never been used to treat the deep uncertainty in multi-objective optimisation. It can be an exciting direction and extra motivation for use in this thesis.

In this chapter, we aimed to introduce a two-stage scenario-based structure to dealing with the higher degrees of uncertainty (moderate and deep) in multi-criteria decisionmaking problems. The main idea was to extend the concept of two-stage stochastic programming with recourse to address the capability of dealing with deep uncertainty through the use of scenario planning rather than statistical expectation. Such scenariobased thinking involved a multi-objective representation of performance under different future conditions as an alternative to expectation, which fitted naturally into the broader multi-objective problem context. This dynamic robust multi-objective optimisation structure which could pave the way for us to handle moderate and deep uncertainty in complex long-term strategic planning was introduced and formulated in Section 3.2. Section 3.3 applied the Generalised Goal Programming (Wierzbicki reference point model) to solve the linear form of the proposed two-stage model. In the Following Section (3.4), a simple farming example is presented and followed by discussion on results, contribute to planning and understanding. Section 3.5 utilised another example to further illustrate the proposed two-stage approach. This section also provided a comparison between the proposed framework and popular existing methods, such as single scenario optimisation and robust optimisation. It further highlighted some superiorities of the proposed framework. Finally, this chapter will be summarised and concluded in Section 3.6. 


\subsection{Proposed two-stage approach}

The simplest recourse model is two-stage (i.e. a set of recourse variables to be applied after that scenario is fully revealed only). Consider a two-stage decision-making process as shown in figure 3.1. Suppose that the decision maker (DM) makes and implements an initial decision $\left(x^{0} \in \mathbf{X}^{0}, \mathbf{X}^{0}\right.$ is a feasible set of the initial decisions) at stage ' 0 ' which is a scenario free decision. Then we will wait and see what happens in the future. Suppose $p$ scenarios $\left(s_{k} \in\left\{s_{1}, \ldots, s_{p}\right\}\right)$ are defined to describe the plausible future space. The subsequent decision will be taken by DM, as a recourse, if scenario $k$ occurs at stage ' 1 ' $\left(x_{k}^{1} \in \mathbf{X}^{1}\left(\mathbf{x}^{0}, k\right), k=1, \ldots, p ;\right)$. Therefore, $p$ contingent decisions will be identified, one for each scenario. The union of the initial decision and the relevant contingent decision will constitute the full decision of the problem. In the proposed structure, we suggest $p$ different decisions - one for each plausible scenario. However, only one contingent decision will be implemented after the realisation of the scenario.

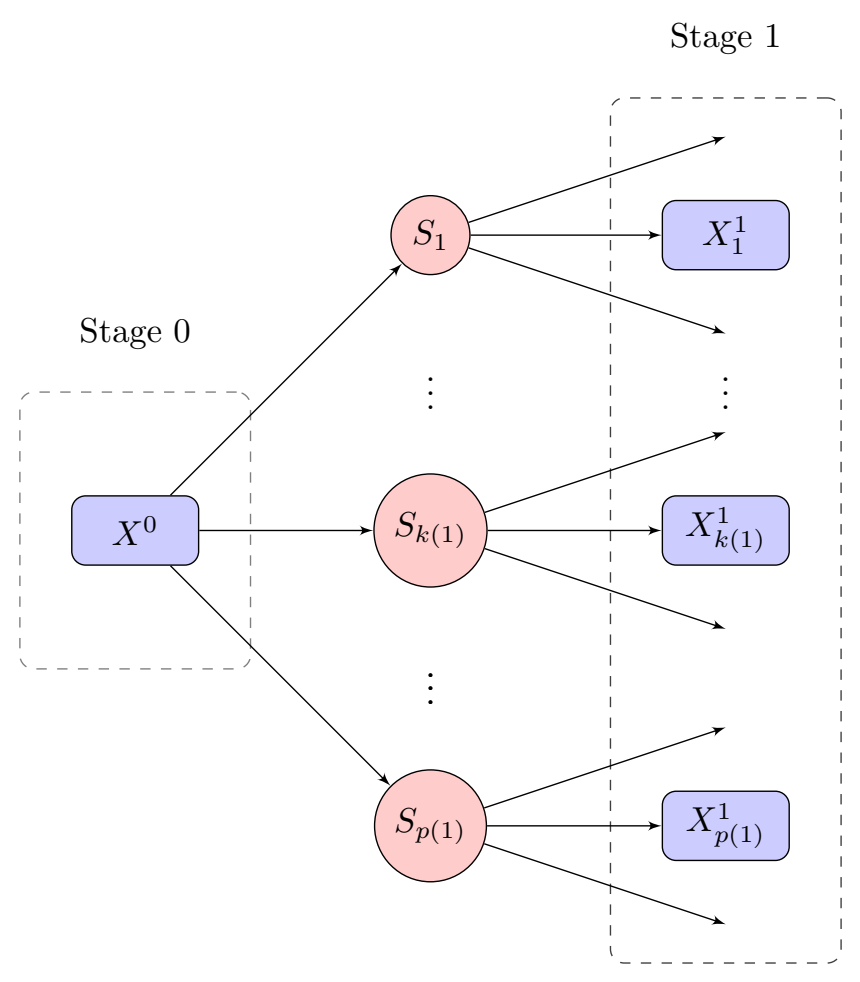

Figure 3.1: Two-stage decision-making process with $p$ scenarios

In this structure, we try to optimise the aggregation of the initial decision $\left(x^{0}\right)$ and dependent (recourse) decisions $\left(x_{k}^{1}, k=1, \ldots, p\right)$ which should be defined for each and every plausible scenario. In fact, we are looking for a robust initial decision which does not need to be feasible for every single scenario, but the aggregation of the initial decision $\left(x^{0}\right)$ and the subsequent scenario-dependent decision $\left(x_{k}^{1}, k=1, \ldots, p\right)$ must be feasible for every scenario $k(k=1, \ldots, p)$. In other words, DM makes an initial scenario-free decision. Then, after scenario revelation, he/she has a chance to adapt his/her decision, if it is necessary, by a subsequent scenario-dependent decision (recourse) while may well pay 
some penalties for any deviation from initial goals. Figure 3.2 provides the schematic of the proposed two-stage approach. In this structure, we do not utilise models to produce forecasts but try instead to identify the reasonable initial decision (which is compensable, whatever happens in the future) and a suitable contingent scenario-dependent decision for every single plausible scenario, implemented after scenario revelation. This process can be summarised as follows:

Two-stage decision-making process

Stage 0: An initial decision is made before any scenario revelation (Scenario free decision).

Stage 1: A contingent decision to be taken if the scenario $k$ is revealed (Scenario dependent decision).

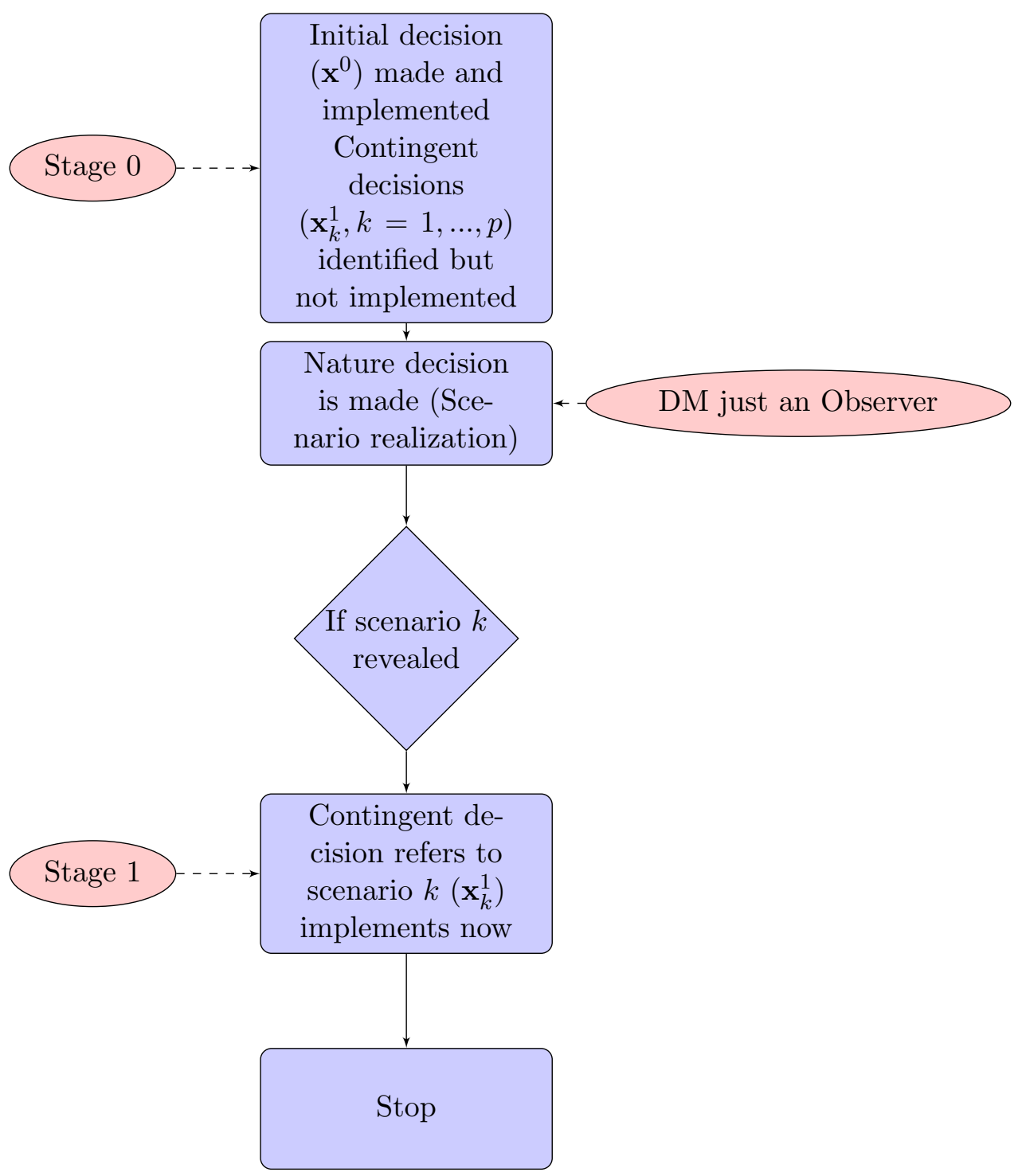

Figure 3.2: The schematic of two-stage decision-making process 


\subsubsection{Mathematical Formulation}

As mentioned earlier, in the realm of multi-criteria decision making, decisions in a scenario-based model needs to be evaluated in terms of all criteria under conditions of each scenario as a dimension of preferences. Stewart et al. [2013] introduced the term "meta-criteria" and used this to assess and compare different alternatives regarding a two-dimensional $(m \times p)$ array of performance measures (all criteria-scenario combinations). Moreover, to avoid challenges of considering probabilities under deep uncertainty, we utilise scenarios as a dimension of preference (a component of the meta-criteria) and, to some extent, extend the meta-criteria concept to find a robust decision and adopt a scenario-based multi-criteria structure under deep uncertainty that can provide us with a robust decision.

Moreover, the decisions/solutions need to be evaluated and compared (on the basis of performance) in terms of each criterion under conditions of an uncertain (or a random) scenario. Therefore, the main purpose of this section is to introduce a model to find the best possible decision/solution by which the performance measures regarding each criterion $i(i=1, \ldots, m)$ under conditions of an uncertain (or a random) scenario $k$ $(k=1, \ldots, p)$ must be optimised. These performance measures can be modelled as objective functions/meta-criteria which represent different dimension of preferences. In other words, each objective function/meta-criterion represents preferences in terms of a criterion under conditions of a scenario. In fact, each meta-criterion (performance measure under a particular scenario) is treated as an objective or dimension of preference.

In the proposed two-stage structure, we break the decision into two parts and defer one part of the decision long enough to realise the uncertain parameter(s). In other words, in the two-stage structure, we are looking one step ahead, postpone part of the decision and leave room for possible adaptation later (after scenario realisation). We identified suitable adaptive plans for every plausible scenario in advance. A robust decision in our philosophy (let us call it a dynamic-robust decision) is a split decision containing two subgroups of decisions: the initial decision(s) followed by recourse deci$\operatorname{sion}(\mathrm{s})$, in which the full decision is robust across scenarios. Unlike the regular robust solution, the initial decision does not need to be optimal or even feasible under conditions of all scenarios. Instead, it must be good enough, with beneficial foresight, to lead us to the optimal aggregations of this initial decision(s) and the following recourse decisions.

Furthermore, the full decision does not necessarily include both initial and contingent decisions for every scenario, and it may need no recourse action under conditions of some scenarios(as you will see later in an example). In such a situation the problem reached its optimality by applying the initial decision(s), and no more optimisation is possible under conditions of that particular scenario(s).

As mentioned in the previous section, we need to formulate an optimisation model that evaluates the overall performances for all $m$ criteria in the conditions of all $p$ scenarios. Each overall performance must include the performances of the initial decision $\left(x^{0}\right)$ and one recourse decision $\left(x_{k}^{1}, k=1, \ldots, p\right)$ related to scenario $k$. The initial decision is common amongst all scenarios while recourse decisions particularly identify for a relevant scenario.

In other words, by applying the concept of meta-criteria, we are seeking an aggregation of the above decisions that provides us with the best performance measure in all $m \times p$ meta-criteria. Therefore, a multi-criteria two-stage model includes optimisa- 
tion of the $m \times p$ meta-criteria/objectives under conditions of some problem constraints. Hence, generally, the two-stage multi-objective optimisation problem under uncertainty, in general form, can be formulated as follows:

$$
\begin{array}{ll}
\operatorname{Opt}_{\left(\mathbf{x}^{0}, \mathbf{x}_{k}^{1}\right)} \mathbf{F}=f_{i k}^{1}\left(\mathbf{x}^{0}, \mathbf{x}_{k}^{1}\right) ; & k=1, \ldots, p ; i=1, \ldots, m ; \\
\text { s.t. } & u_{r}^{0}\left(\mathbf{x}^{0}\right) \leq 0, \\
& r=1, \ldots, R_{0} ; \\
& \begin{aligned}
1 \\
u_{r}^{1}\left(\mathbf{x}^{0}, \mathbf{x}_{k}^{1}\right) \leq 0,
\end{aligned} \\
& k=1, \ldots, p ; \\
& r=R_{0}+1, \ldots, R_{0}+\ldots+R_{k-1}+R_{k} ;
\end{array}
$$

where $\mathbf{x}^{0}=\left(x_{1}^{0}, \ldots, x_{n}^{0}\right) \in \mathbf{X}^{0}$ is an $n$-dimensional initial scenario-free decision variable vector which was made in stage ' 0 ' before scenario $k$ happens and $\mathbf{X}_{0}$ is an initial decision space.

$\mathbf{x}_{k}^{1}=\left(x_{1 k}^{1}, \ldots, x_{n k}^{1}\right) \in \mathbf{X}^{1}\left(\mathbf{x}^{0}, k\right),(k=1, \ldots, p)$; is an $n$-dimensional contingent scenariodependent decision vector which is taken in stage ' 1 ' if scenario $k$ is revealed and $\mathbf{X}^{1}\left(\mathbf{x}^{0}, k\right)$ is a contingent decision space when scenario $k$ is unfolded.

$f_{i k}^{1}\left(\mathbf{x}^{0}, \mathbf{x}_{k}^{1}\right),(k=1, \ldots, p ; i=1, \ldots, m)$, is $i^{t h}$ meta-criterion/objective includes scenariofree performances, if scenario $k$ is revealed. In fact, they indicate preferences regarding criterion $i$ under conditions of scenario $k$.

$u_{r}^{0}\left(\mathbf{x}^{0}\right)$ is the set of inequality constraints in stage ' 0 '.

$u_{r}^{1}\left(\mathbf{x}^{0}, \mathbf{x}_{k}^{1}\right)$ is the set of inequality constraints in stage ' 1 '.

The problem consists in optimising $m \times p$ objectives under $\left(R_{0}+R_{1}+\ldots+R_{p}\right)$ constraints. Both objectives and constraints can be linear or non-linear. However, this thesis focuses on the linear problems.

Moreover, to make the model more general, some initial objectives could be considered for stage ' 0 ' (i.e. before any uncertainty $\left(f_{i_{0}}^{0}\left(\mathbf{x}^{0}\right),\left(i_{0}=1, \ldots, m_{0}\right)\right)$, In this case, the model contains optimisation of $\mathbf{F}=\left[f_{i_{0}}^{0}\left(\mathbf{x}^{0}\right) ; f_{i k}^{1}\left(\mathbf{x}^{0}, \mathbf{x}_{k}^{1}\right)\right]$. Then, the total number of objectives equals to $m_{0}+(m \times p)$ (if $m_{0}=m$, then the total is equal to $m \times(p+1)$ ).

It is also possible that a different number of objectives is considered in each scenario which could lead us to model the higher levels of uncertainty. In this situation, the problem consists in Optimising $\left(m_{0}+\left(m_{1}+\ldots+m_{p}\right)\right)$ objectives.

\subsubsection{Two-stage Multi-Objective Linear Programming (MOLP)}

Without loss of generality, It can be assumed that the objectives are to be minimised (maximisation of $\mathbf{f}(\mathbf{x})$ is equivalent to minimising $-\mathbf{f}(\mathbf{x})$ ). If the objectives and constraints are linear and each decision evaluated on $m$ criteria denoted by $C=\left\{C_{1}, \ldots, C_{m}\right\}$, problem 3.1 is a Multi-Objective Linear Programming (MOLP) with $m \times p$ objectives which can be formulated as follows: 


$$
\begin{array}{ll}
\text { Min } \quad Z_{i k}=\sum_{j=1}^{n} c_{i j}^{0} x_{j}^{0}+\left(\sum_{j=1}^{n} c_{i j k}^{0} x_{j}^{0}+\sum_{j=1}^{n} c_{i j k}^{1} x_{j k}^{1}\right) ; & i=1, \ldots, m ; k=1, \ldots, p ; \\
\text { s.t. } \quad \sum_{j=1}^{n} a_{r j}^{0} x_{j}^{0} \leq b_{r}^{0}, & r=1, \ldots, R_{0} ; \\
& \sum_{j=1}^{n} a_{r j k}^{0} x_{j}^{0}+\sum_{j=1}^{n} a_{r j k}^{1} x_{j k}^{1} \leq b_{r k}^{1}, \\
& k=1, \ldots, p ; \\
& r=R_{0}+1, \ldots, R_{0}+\ldots+R_{k} ; \\
x_{j}^{0}, x_{j k}^{1} \geq 0 . & j=1, \ldots, n ; k=1, \ldots, p .
\end{array}
$$

where $Z_{i k}$ describe the $i^{\text {th }}$ linear meta-criterion/objective function representing preferences in terms of criterion $i$ under conditions pertaining to scenario $k$, and includes some initial goals (scenario-free) before any uncertainties $\left(\sum_{j=1}^{n} c_{i j}^{0} x_{j}^{0}\right)$, consequences of the initial decision in the second stage if scenario $k$ revealed and it's outcomes $\left(\sum_{j=1}^{n} c_{i j k}^{0} x_{j}^{0}+\sum_{j=1}^{n} c_{i j k}^{1} x_{j k}^{1}\right)$. The sets of linear inequalities for the first and the second stage are respectively described in the constraints as well as the non-negativity constraints.

\subsection{Solving by Generalised Goal Programming (GGP)}

The Goal Programming (GP) approach, introduced by Charnes et al. [1955] and Charnes et al. [1961], is one of the most popular and oldest Multi-Criteria Decision Analysis (MCDA) techniques. The most appealing idea about GP is that, firstly, the goals are set by $\mathrm{DM}$ in objective space then try to come close to it through minimising the deviation or distance measure (this deviation represents by a norm) between this specific goal vector (or aspiration levels) and the decision outcome (obtainable objective vector).

However, without some extra assumptions, there is no norm minimization can generate efficient/vector-optimal solutions. Then, the above-mentioned idea(coming close to a goal) is mathematically contradictory to the concept of efficiency (vector optimality) [Wierzbicki, 1998]. Therefore, this idea can lead to dominated solutions in the standard form of goal programming approach. This represents the main shortcoming of the typical goal programming approach [Wierzbicki, 1998, Ogryczak, 1994]. Hence, in regular goal programming, a distance function is utilised to evaluate the performances. If the distance function is zero, then there is no more optimisation possible. That is mainly due to the fact that we cannot get a negative value for a distance function and so the optimisation process is stopped [Wierzbicki, 1998].

Amongst different GP models, it has been shown by Ogryczak [1994] that the solutions of the Generalised Goal Programming (GGP) (also called Reference Point Method $(\mathrm{RPM}))$ are always Pareto efficient. Although methods exist to correct standard GP for non-efficiency (see [Jones and Tamiz, 2010] and [Romero, 1991]), the reference point approach is a direct and simple approach to achieve this end. The Reference Point Method (RPM) uses the so-called quasi-satisficing decisions introduced by Wierzbicki [1982]. In this approach, optimisation is carried out (even after reaching the desirable goals) and 
the goals/reference points are interpreted consistently, based on the vector optimisation (Pareto efficiency) concept. Therefore, the solutions/decisions can be improved beyond the primary targets, and aspiration levels/reference points are obtainable with some surplus (i.e. the achievements are beyond our ideal expectations, surplus achievements compared to the ideal). Therefore, unlike the standard goal programming, GGP continues searching for better points even if the predetermined goals/reference points have already been obtained. In fact, the RPM uses the underlying optimisation philosophy instead of the underlying satisfying philosophy which is utilised in typical GP.

The efficient/Pareto solutions in RPM are generated by minimising some so-called scalarization achievement functions (which are a kind of utility or value functions) instead of minimising the distance measures (norm) which is a strategy used in regular GP methods. In other words, the RPM utilises the reference levels (instead of aspiration levels in GP) to control and lead the parameters. This change in philosophy provides the ability for the model to accept negative weights regarding the negative deviations in the models. Therefore, the RPM continues searching for better points (even if the predetermined reference levels have already been obtained) and, therefore, generates the efficient/optimal solution.

These scalarization functions utilise an arbitrary small positive scalar $\epsilon$ to keep searching for better solutions (even after obtaining the reference levels). This scalar $(\epsilon)$ guarantee efficiency/optimality in the case of multiple optimal solutions which is a normal outcome in MCDM problems. Different scalarization functions have been introduced in the literature. One of the simplest forms of scalarization achievement function is formulated as follows [Ogryczak, 1994]:

$$
\operatorname{Max}_{1 \leq i \leq m}\left\{\omega_{i}\left(f_{i}(x)-g_{i}\right)\right\}+\epsilon \sum_{i=1}^{m}\left(\omega_{i}\left(f_{i}(x)-g_{i}\right)\right),
$$

where $\omega_{i}>0(i=1, \ldots, m)$ are scaling factors, $g_{i}$ explain reference levels, and $\epsilon$ is an arbitrary small positive number.

Minimising this scalarization achievement function (3.3) over the feasible set of a decision problem can produce an efficient solution. By defining $\delta_{i}(i=1, \ldots, m)$ for representing the goal deviations, we will have $f_{i}(x)-g_{i}=\delta_{i}$. Therefore, the corresponding GGP model can be formulated as follows:

$$
\begin{aligned}
& \text { Min } \psi=\varphi+\epsilon \sum_{i=1}^{m}\left(\omega_{i} \delta_{i}\right) \\
& \text { s.t. } \omega_{i} \delta_{i} \leq \varphi \quad i=1, \ldots, m, \\
& f_{i}(x)-\delta_{i}=g_{i} \quad i=1, \ldots, m, \\
& \mathbf{x} \in \mathbf{X}
\end{aligned}
$$

where $\varphi=\operatorname{Max}_{1 \leq i \leq m}\left\{\omega_{i} \delta_{i}\right\}, \mathbf{x}$ is the vector of decision variables, and $\mathbf{X}$ is the set of feasible solutions of the problem. 
The RPM is an interactive technique and the DM preferences/expectations will be reflected by the reference levels which are determined by the DM (similar to the typical GP). The optimal/efficient solution could be compared with previous/other solutions by the DM, and could be modified (via some changes in the reference levels) if necessary. Furthermore, the GGP is significantly useful in the proposed framework as it can easily handle large numbers of objective functions/criteria. Identifying the goals for each criterion is the only action that the DM needs to carry out in the GP approach. This, as pointed out by Belton and Stewart [2002], "facilitates the formal incorporation of the treatment of uncertainty through use of scenarios". The two-stage structure needs to evaluate and compare alternatives/solutions taking into account all meta-criteria (all criteria-scenario combinations) that, especially in a real-world problem, may give rise to dealing with very large numbers of meta-criteria. Therefore, the DMs need only to set their desirable goals for each meta-criterion without trade-off assessments or further calculations. This can markedly reduce computation which would make the process of setting goals easier and help us gain robust solutions which perform satisfactorily on all meta-criteria. We may need to note that, although utilising the $L_{1}$ based model is more common in GP, it requires consideration of trade-offs, whereas the $L_{\infty}$ based model focusses more on worst-case performance which is more robust in our context of including deep not always measurable uncertainties. Furthermore, the use of GGP could also be effective in strategic planning problems that might have some unknown consequences and in which identifying the suitable set of goals is much more challenging. In such a scenario, GGP could assist at the elementary level of screening to produce a short-list of possible solutions [Belton and Stewart, 2002].

In the two-stage structure proposed in the current study, reference points represent aspiration levels/goals for each meta-criterion by which the DMs' preferences apply in the evaluation process and lead the model to construct an efficient solution which is the "closest or better" to the preferred reference points. Moreover, each meta-criterion associates with a measurable attribute value(goal/reference point) which represents a natural interplay between meta-criteria and the reference point mode of value elicitation.

Accordingly, all the above reasons motivate us to utilise the generalised goal programming (GGP) to solve the multi-objective models. In such models, we would seek to minimise all $m \times p$ objective functions (or performance measures $\left(Z_{i k}\right)$ ). If we define $m \times p$ goals $g_{i k}$ for all meta-criteria corresponding to deviation variables $\delta_{i k}$ and, then consider the generalised Goal Programming approach, the associated constraints can be formulated as follows:

$$
Z_{i k}-\delta_{i k}=\sum_{j=1}^{n} c_{i j}^{0} x_{j}^{0}+\left(\sum_{j=1}^{n} c_{i j k}^{0} x_{j}^{0}+\sum_{j=1}^{n} c_{i j k}^{1} x_{j k}^{1}\right)-\delta_{i k}=g_{i k}, \quad i=1, . ., m, k=1, \ldots, p
$$

So, the equivalent GGP model can be formulated as follows: 


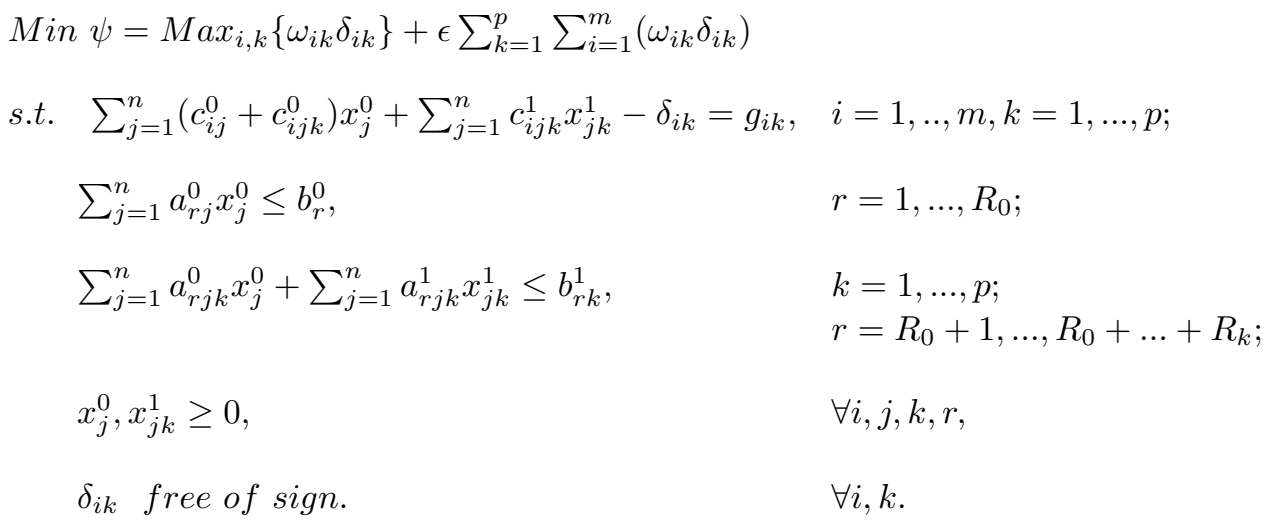

where $\omega_{i k} \geq 0,(i=1, . ., m, k=1, \ldots, p)$; are the importance weighting of deviations which set by DM. $\epsilon$ is an arbitrarily small positive number.

Moreover, by setting $\phi=\operatorname{Max}_{i, k}\left\{\omega_{i k} \delta_{i k}\right\}$, the linear form can be formulated as follows:

$$
\begin{aligned}
& \text { Min } \psi=\phi+\epsilon \sum_{k=1}^{p} \sum_{i=1}^{m}\left(\omega_{i k} \delta_{i k}\right) \\
& \text { s.t. } \omega_{i k} \delta_{i k}-\phi \leq 0, \quad i=1, . ., m, k=1, \ldots, p \text {; } \\
& \sum_{j=1}^{n}\left(c_{i j}^{0}+c_{i j k}^{0}\right) x_{j}^{0}+\sum_{j=1}^{n} c_{i j k}^{1} x_{j k}^{1}-\delta_{i k}=g_{i k}, \quad i=1, . ., m, k=1, \ldots, p ; \\
& \sum_{j=1}^{n} a_{r j}^{0} x_{j}^{0} \leq b_{r}^{0}, \quad r=1, \ldots, R_{0} ; \\
& \sum_{j=1}^{n} a_{r j k}^{0} x_{j}^{0}+\sum_{j=1}^{n} a_{r j k}^{1} x_{j k}^{1} \leq b_{r k}^{1}, \quad k=1, \ldots, p ; \\
& r=R_{0}+1, \ldots, R_{0}+\ldots+R_{k} ; \\
& x_{j}^{0}, x_{j k}^{1} \geq 0, \quad \forall i, j, k, r, \\
& \phi, \delta_{i k} \text { free of sign. } \forall i, k .
\end{aligned}
$$

\subsection{Simple farming example}

In this section, we show that how the proposed two-stage model works under deep uncertainty using a simple farming example ${ }^{1}$. We will explain how this structure can be modelled, implemented, and solved by Goal Programming. Further discussion and sensitivity analysis of the variations in goals and importance weights will be presented at the final part of this section.

Example 3.4.1. Assume that a farmer can organically grow wheat, corn or soy-beans on the entirety of his 1000 hectare plot. From this land, he needs to produce 400 tonnes of wheat and 440 tonnes of corn to feed his cattle. If the farmer fails to produce enough feed, the shortfall must be bought from a wholesaler at the cost of $\mathbf{R} 2000 /$ tonne of wheat and $\mathbf{R} 1600 /$ tonne of corn. However, if he produces an excess of feed, this can be sold for $\mathbf{R} 1850 /$ tonne of wheat and R $1450 /$ tonne of corn.

${ }^{1}$ The idea has been taken from Linderoth [2003] 


\begin{tabular}{|l|c|c|c|c|c|c|c|c|c|}
\hline & \multicolumn{3}{|c|}{ Wheat } & \multicolumn{3}{c|}{ Corn } & \multicolumn{3}{c|}{ Soy-beans } \\
\cline { 2 - 11 } & $s_{1}$ & $s_{2}$ & $s_{3}$ & $s_{1}$ & $s_{2}$ & $s_{3}$ & $s_{1}$ & $s_{2}$ & $s_{3}$ \\
\hline Yield (Tonnes/hectare) & 4 & 3.6 & 3.3 & 9 & 6 & 5 & 2.2 & 2 & 1.5 \\
\hline Planting Cost (R/hectare) & 2400 & 2500 & 2750 & 4300 & 4500 & 4750 & 2900 & 3400 & 3900 \\
\hline Selling Price (R/tonnes) & 1600 & 1850 & 2100 & 1250 & 1450 & 1650 & 3050 & 3300 & 3500 \\
\hline Environmental benefit coefficient (percent) & 14 & 13 & 12 & 12 & 13 & 14 & 22 & 24 & 25 \\
\hline Purchase Price (R/tonnes) & 1800 & 2000 & 2300 & 1400 & 1600 & 1800 & N/A & N/A & N/A \\
\hline Minimum Requirement (tonnes) & 400 & 400 & 400 & 440 & 440 & 440 & N/A & N/A & N/A \\
\hline
\end{tabular}

Table 3.1: Farming data set

The farmer can also grow soy-beans which sell for R3300/tonne. He knows well enough that his yields are not always precise. As the yields are highly dependant on weather, he decides to consider three different scenarios for weather conditions. Assume three scenarios: $\operatorname{good}\left(s_{1}\right)$, average $\left(s_{2}\right)$ and bad $\left(s_{3}\right)$ weather. All data have been described in table 3.1.

Furthermore, the farmer is an advocate for organic farming practices which promote certain environmental benefits such as sustainable soil and climate, as well as negligible impact on ecosystems. As a result of this, the farmer benefits from reduced taxes which are calculated by an environmental benefit coefficient that is specific each product and affected by a range of different factors. Related environmental benefit coefficients for each product under various scenarios are described in Table 3.1. The farmer also needs to consider planting costs which for wheat, corn and soy-beans are $R 2400, R 4300$, and $R 2900$, respectively. He has to pay these minimum costs at the beginning of the year. In addition these costs, extra charges may need to paid depending on scenario revelation-that is, if scenario 2 or 3 happen. To further clarify, this means that the farmer will have to pay extra if (and only if) one of the scenarios 2 or 3 unfold. These extra costs would be payable after scenario realisation. It must be noted that there is a recourse/adaptation option at the second stage of the decision-making process. This means that, just after observing weather conditions (and in the second stage of decision-making), the farmer would have a chance to decide how much of each crop to purchase or sell. It may need to be noted that in this stage(second stage) the farmer has enough knowledge about the current weather conditions (the scenario that unfolded). This information can help him to estimate his yields at the end of the period and adapt his initial decision(s).

To formulate the model, let us set the following variables:

\begin{tabular}{|ll|}
\hline \multicolumn{1}{|c|}{ Setting variables } \\
\hline$x_{i}^{0}, i=1,2,3:$ & Hectares of Wheat, Corn,and Soy-beans Planted (initial decision), respectively. \\
$x_{i k}^{1}, i=1,2,3:$ & Tonnes of Wheat, Corn, and Soy-beans sold if scenario $k(k=1,2,3)$ unfolded, respectively. \\
$x_{4 k}^{1} ; x_{5 k}^{1}:$ & Tonnes of Wheat and Corn purchased if scenario $k(k=1,2,3)$ revealed, respectively. \\
\hline
\end{tabular}

Suppose that there are three criteria $\left(C_{i}, i=1,2,3 ;\right)$ which define as follows:

\section{Criteria/Objectives}

$\mathbf{C}_{\mathbf{1}}$ : $\quad$ Minimising the sum of current investment costs.

$\mathbf{C}_{2}$ : Maximising the liquidity at the end of the year which earn from selling crops.

$\mathbf{C}_{\mathbf{3}}$ : Maximising the environmental benefits.

Then, the objectives have been formulated in equations 3.8-3.10. 


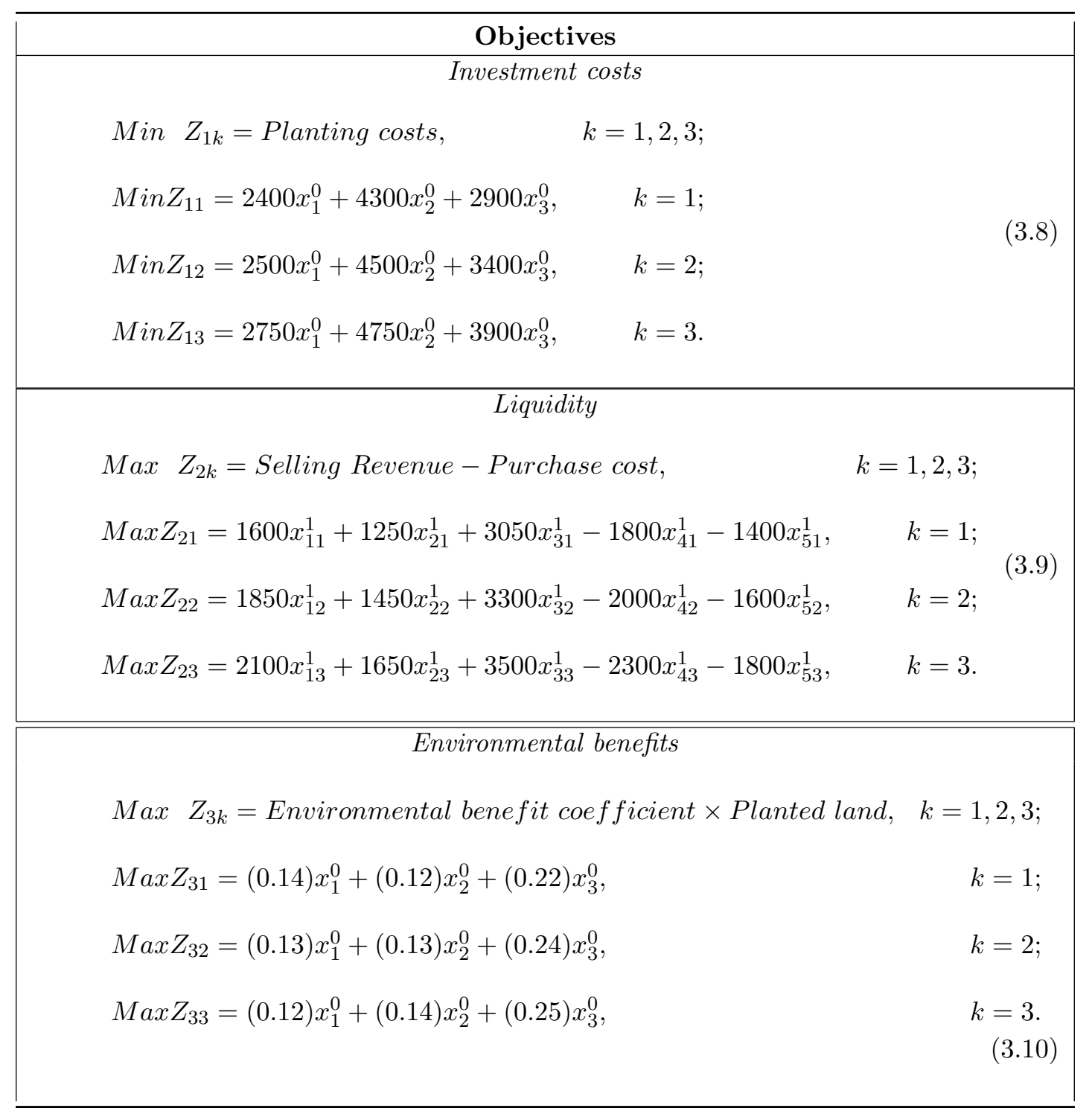

It is important to note at this stage that the model is not unit-invariant. Therefore, if various units exist for different objectives, to getting the best result, it is better to normalise the data before running the model. For instance, dividing by the maximum or utilising some weights can make the model unit-invariant (a review of some normalisation methods can be found in [Tamiz et al., 1998]).

Also, the problem's constraints would be written as follows (equations 3.11-3.14):

\begin{tabular}{|c|c|}
\hline \multicolumn{3}{|c|}{ Problem Constraints } \\
\hline \hline \multicolumn{3}{|c|}{ Initial constraint (scenario free constraint) } \\
\hline$x_{1}^{0}+x_{2}^{0}+x_{3}^{0}=1000 \quad$ (Land limitation) \\
\hline
\end{tabular}




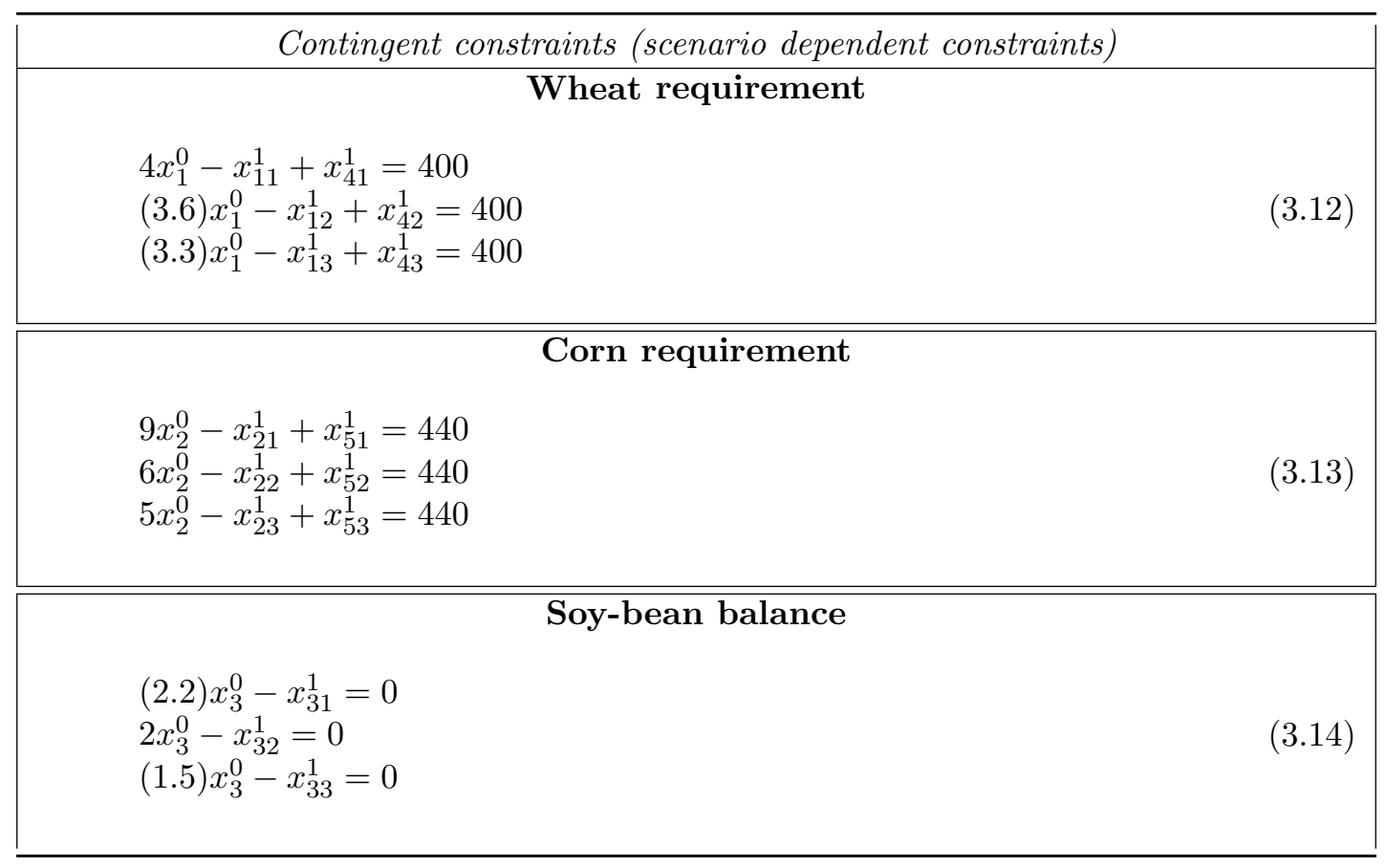

Moreover, by appending non-negative constraints the two-stage multi-objective optimisation model of this problem can be formulated (see model 3.16 in chapter Appendix 3.7.1).

To solve this problem using the generalised goal programming (GGP) approach, the farmer is asked to set his goals for each objective, as well as some weights for deviations from these goals. This information has been portrayed in Table 3.2, and the GGP model of this problem is formulated as model 3.17 in chapter Appendix 3.7.2.

After normalising units, the final linear model (model 3.17 in chapter Appendix 3.7.2) was coded in Matlab and the result, as well as the optimal values of objectives that can be reached from the results/solutions, are presented in Table 3.3. Furthermore, the initial decision $\mathbf{X}^{0}$, second decision (recourse) $\mathbf{X}_{k}^{1}$ and the deviations from the initial goals $\Delta$ for $k$ different scenarios $(k=1,2,3)$ together with the current costs, total earning (liquidity) and environmental benefits for these three plausible scenarios have been presented in this table. Table 3.3 also compare the results when the importance weights are changed and this data will be used for further discussions in the next sections.

The aspiration levels and importance weights identified by $\mathrm{DM}$ (here the farmer is the DM) can add respectively the first and second order of his/her preferences into the model and generate the solutions as close as possible to what the $\mathrm{DM}$ (farmer) likes the most. For example, as seen in Table 3.2, more weights have been allocated to the last two criteria in all scenarios that surely will lead us to the solutions with more goal achievements for these objectives. Analysis of these

\begin{tabular}{|c|c|c|c|c|c|c|}
\hline Scenario/(Goals,weights) & $g_{1 k}$ & $g_{2 k}$ & $g_{3 k}$ & $\omega_{1 k}$ & $\omega_{2 k}$ & $\omega_{3 k}$ \\
\hline$k=1$ & R 1400000 & R 10000000 & 160 & 1 & 4 & 7 \\
\hline$k=2$ & R 1550000 & R 8000000 & 165 & 1 & 3.5 & 7 \\
\hline$k=3$ & R 1750000 & R 6500000 & 170 & 1.5 & 5 & 8 \\
\hline
\end{tabular}

Table 3.2: Goals and weights 


\begin{tabular}{|c|c|c|c|c|c|c|c|c|}
\hline \multicolumn{3}{|c|}{ Importance weights } & \multirow{2}{*}{ Scenario } & \multirow{2}{*}{$\begin{array}{l}X^{0}=\left(x_{1}^{0}, x_{2}^{0}, x_{3}^{0}\right) \\
X_{k}^{1}=\left(x_{1 k}^{1}, x_{2 k}^{1}, x_{3 k}^{1}, x_{4 k}^{1}, x_{5 k}^{1}\right)\end{array}$} & \multirow{2}{*}{$\begin{array}{c}\text { Current costs } \\
\left(Z_{1 k}\right) \\
\end{array}$} & \multirow{2}{*}{$\begin{array}{c}\text { Liquidity } \\
\left(Z_{2 k}\right)\end{array}$} & \multirow{2}{*}{$\begin{array}{c}\text { Environmental } \\
\text { benefits }\left(Z_{3 k}\right)\end{array}$} & \multirow{2}{*}{$\Delta=\left(\delta_{1 k}, \delta_{2 k}, \delta_{3 k}\right)$} \\
\hline$\omega_{i 1}$ & $\omega_{i 2}$ & $\omega_{i 3}$ & & & & & & \\
\hline \multirow{4}{*}{$\begin{array}{l}\omega_{11}=1 \\
\omega_{21}=4 \\
\omega_{31}=7\end{array}$} & \multirow{4}{*}{$\begin{array}{l}\omega_{12}=1 \\
\omega_{22}=3.5 \\
\omega_{32}=7\end{array}$} & \multirow{4}{*}{$\begin{array}{l}\omega_{13}=1.5 \\
\omega_{23}=5 \\
\omega_{33}=8\end{array}$} & scenario free & $\bar{X}^{0}=(148.2,779.4,72.4)$ & - & - & - & 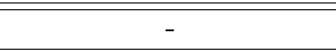 \\
\hline & & & $k=1$ & $X_{1}^{1}=(192.8,6574.8,159.2,0,0)$ & 3917093 & 9012636 & 130.2 & $(529.91,207.87,119.19)$ \\
\hline & & & $k=2$ & $X_{2}^{1}=(133.5,4236.5,144.8,0,0)$ & 4123988 & 6867679 & 138 & $(541.89,238.38,108.15)$ \\
\hline & & & $k=3$ & $X_{3}^{1}=(89,3457.5,108.2,0,0)$ & 4392083 & 6271172 & 145 & $(556.23,48.17,100)$ \\
\hline \multirow{4}{*}{$\begin{array}{l}\omega_{11}=1 \\
\omega_{21}=1 \\
\omega_{31}=1\end{array}$} & \multirow{4}{*}{$\begin{array}{l}\omega_{12}=1 \\
\omega_{22}=1 \\
\omega_{32}=1\end{array}$} & \multirow{4}{*}{$\begin{array}{l}\omega_{13}=1 \\
\omega_{23}=1 \\
\omega_{33}=1\end{array}$} & scenario free & $X^{0}=(446.7,553.3,0)$ & - & - & - & 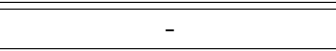 \\
\hline & & & $k=1$ & $X_{1}^{1}=(1386.9,4539.6,0,0,0)$ & 3452241 & 7893431 & 128.9 & $(431.84,443.49,124.26)$ \\
\hline & & & $k=2$ & $X_{2}^{1}=(1208.2,2879.7,0,0,0)$ & 3606569 & 6409805 & 130 & $(432.96,334.78,140)$ \\
\hline & & & $k=3$ & $X_{3}^{1}=(1074.2,2326.5,0,0,0)$ & 3856693 & 6093716 & 131 & $(443.49,85.53,155.74)$ \\
\hline \multirow{4}{*}{$\begin{array}{l}\omega_{11}=20 \\
\omega_{21}=4 \\
\omega_{31}=7\end{array}$} & \multirow{4}{*}{$\begin{array}{l}\omega_{12}=20 \\
\omega_{22}=3.5 \\
\omega_{32}=7\end{array}$} & \multirow{4}{*}{$\begin{array}{l}\omega_{13}=20 \\
\omega_{23}=5 \\
\omega_{33}=8\end{array}$} & scenario free & $X^{0}=(1000,0,0)$ & - & - & - & - \\
\hline & & & $k=1$ & $X_{1}^{1}=(3600,0,0,0,440)$ & 2400000 & 5135605 & 140 & $(210.53,1024,80)$ \\
\hline & & & $k=2$ & $X_{2}^{1}=(3200,0,0,0,440)$ & 2500000 & 5215845 & 130 & $(200,586.14,140)$ \\
\hline & & & $k=3$ & $X_{3}^{1}=(2900,0,0,0,440)$ & 2750000 & 5297611 & 120 & $(210.53,253.14,200)$ \\
\hline \multirow{4}{*}{$\begin{array}{l}\omega_{11}=1 \\
\omega_{21}=9 \\
\omega_{31}=7\end{array}$} & \multirow{4}{*}{$\begin{array}{l}\omega_{12}=1 \\
\omega_{22}=9 \\
\omega_{32}=7\end{array}$} & \multirow{4}{*}{$\begin{array}{l}\omega_{13}=1.5 \\
\omega_{23}=9 \\
\omega_{33}=8\end{array}$} & scenario free & $\overline{X^{0}=(0,1000,0)}$ & - & - & - & - \\
\hline & & & $k=1$ & $X_{1}^{1}=(0,8560,0,400,0)$ & 4300000 & 9980000 & 120 & $(610.52,42.11,160)$ \\
\hline & & & $k=2$ & $X_{2}^{1}=(0,5560,0,400,0)$ & 4500000 & 7262000 & 130 & $(621,155.37,140)$ \\
\hline & & & $k=3$ & $X_{3}^{1}=(0,4560,0,400,0)$ & 4750000 & 6604000 & 140 & $(631.58,-21.9,120)$ \\
\hline \multirow{4}{*}{$\begin{array}{l}\omega_{11}=1 \\
\omega_{21}=4 \\
\omega_{31}=7000\end{array}$} & \multirow{4}{*}{$\begin{array}{l}\omega_{12}=1 \\
\omega_{22}=3.5 \\
\omega_{32}=7000\end{array}$} & \multirow{4}{*}{$\begin{array}{l}\omega_{13}=1.5 \\
\omega_{23}=5 \\
\omega_{33}=8000\end{array}$} & scenario fre & $X^{0}=(0,600.5,399.5)$ & 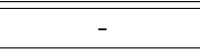 & - & 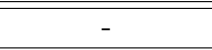 & 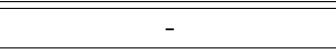 \\
\hline & & & $k=1$ & $X_{1}^{1}=(0,4965,878.8,400,0)$ & 3740772 & 8166503 & 159.95 & $(492.8,386,0.22)$ \\
\hline & & & $k=2$ & $X_{2}^{1}=(0,3169,798.9,400,0)$ & 4060606 & 6422317 & 173.94 & $(528.55,332.14,-35.75)$ \\
\hline & & & $k=3$ & $X_{3}^{1}=(0,2563,599.2,400,0)$ & 4410469 & 5405604 & 183.94 & $(560,230.4,-55.75)$ \\
\hline
\end{tabular}

Table 3.3: The result of the proposed two-stage MOLP for farming example 
factors can clearly show this fact, and this will be performed in the next sections.

\subsubsection{Discussion of contributions to planning and understanding}

There are three criteria/objectives that need to be considered in this example. Also, three scenarios are identified. Therefore, the decisions must be evaluated and compared in all criterionscenario combinations, called meta-criteria. Here, these combinations are represented by $Z_{i k}, i=$ $1,2,3 ; k=1,2,3$; and can be found in equations 3.8-3.10, followed by scenario-free (fundamental limitations) and scenario-dependent constraints (uncertain upcoming restrictions) that completely constitute the proposed two-stage scenario-based multi-objective optimisation model for this example. To solve this multi-objective model with Generalised Goal Programming, the DM defined his/her goals and importance weights, and the final results have been described in Table 3.3 .

The DM (farmer here) has to make his initial decision (that is how to allocate different parts of the land to planting various types of crops) at the beginning of the period/year before he has any information about the future events (uncertain weather in this particular example). These uncertain events will have an effect on the outcomes of the initial decision. For example, effects could be in terms of yield, expenses, selling prices, and environmental benefits in the current example. Moreover, the DM (farmer) only can make the recourse decisions (sales and purchase decisions) after scenario realisations (seeing his yield). Clearly, it is not possible to find a perfect decision that keeps its optimality in all scenarios, even in this simple example.

The first criterion is minimising the planting costs, if we take a glance over the expenses of planting different crops in Table 3.1. It is evident that growing wheat is the least expensive activity in all scenarios, and the differences between the plausible scenarios are not significant(the maximum difference is less than $15 \%$ per hectare i.e. between $R 2400$ and $R 2750$ ). However, the selling price, as an effective factor for the second criteria, will be increased from the first scenario(good weather) to the last one(bad weather). Then, following a simple calculation, which is dependent on the different yields in each scenario, it will be apparent that the farmer can earn greater profits following bad weather than following good weather. The profit for planting a hectare of wheat in different scenarios $s_{1}, s_{2}$, and $s_{3}$ will be R4000, R4160, and R4180), respectively. Furthermore, the profit values for planting corn in a hectare of land under these scenarios are $\mathbf{R} 6950, \mathbf{R} 4200$, and $\mathbf{R} 3500$, respectively while those of growing soy-beans will be $\mathbf{R} 3810, \mathbf{R} 3200$, and R1350, respectively. Although by planting wheat the farmer may meet the minimum costs and the average profit in all plausible scenarios, one might not be able to forego the attractive profit that would be made by growing corn if scenario $s_{1}$ unfolds-that is, if there is bad weather. Therefore, it seems that growing soy-bean does not make sense from an economical standpoint because this crop would yield the lowest profit under all conditions, whilst also being the more expensive crop compared to wheat. The only favourable factor that might encourage the farmer to grow soy-bean is its environmental benefits (considered as the third criterion). That is, the values for soy-bean are almost double the environmental benefits of the other crops. This concept is clearly visible in Table 3.3. As defined in 3.4.1, $x_{3}^{0}$ represents hectares of land that are allocated to planting soy-bean. Comparing suggested values for this variable in various cases at Table 3.3 indicates that only the first and the last cases suggest planting soy-bean in some parts of the land. In both cases, the farmer's(DM's) tended to apply further weights on the third criterion (i.e. environmental benefits) by increasing related importance weights. Therefore, it is sensible that the model allocated parts of the land to planting soy-bean when assigning greater importance to environmental benefits than the other criteria.

However, the proposed two-stage framework looks for the best initial decision that, together with the subsequent recourse decisions, provide us with the best possible performances under all circumstances. It is worth remembering at this stage that the initial decision is common among all scenarios while recourse decisions offer specific contingent decisions for the relevant scenario. In other words, we are simultaneously optimising the aggregation of an initial decision (i.e. allocate land for various crops), which is common for all scenarios, and the contingent decisions (i.e. the 
sell and/or purchase decisions) in all plausible scenarios, viz. one initial decision (or one group of decisions) aggregates with $\mathrm{k}$ different (groups of) recourse/contingent decisions. Therefore, in total, we will face $\mathrm{k}$ joint decisions for $k$ plausible scenarios (one particular joint decision for each scenario). In practice, at the first, the initial decision is implemented before scenario realisation. After getting information about the scenario, the relevant recourse decision will be implemented to complete the two-stage decision-making process. This framework helps us make a reasonable initial decision that can constitute the best possible aggregation with the recourse decisions of all plausible scenarios while considering the DM's preferences. In fact, in the proposed two-stage structure, the $\mathrm{DMs}($ farmer $)$ make(s) and implement(s) the best possible initial decision(s) today while looking one step ahead, evaluating the consequences of this decision, and preparing well for all plausible futures/scenarios. Finally, once new information has been received, and a specific scenario has unfolded, they can simply implement the appropriate recourse decision(s) that was previously planned.

As it could be expected, satisfying the animal feeds requirements by growing wheat and corn on some parts of the land is economically optimal. This is because costs associated with the production of these commodities is less than if they purchased from the market. As it could be expected, satisfying the animal feeds requirements by growing wheat and corn on some parts of the land is economically optimal. This is because costs associated with the production of these commodities is less than if they purchased from the market. However, sometimes, other criteria may be more important than economics, or other attractive products can provide the farmer(DM) with further profits than what he needs to pay for his animal feeds.

These results also described in the Table 3.3. As shown in this table, the model tends to provide solutions in which all requirements to wheat and corn can be satisfied from farming rather than through the purchase of these commodities from the other growers (follow the purchasing portion of wheat and corn presented respectively by $x_{4 k}^{1}$ and $x_{5 k}^{1}$ in solutions). However, there is an exception to this. In the last three cases, the model is led to take more advantages of some preferred meta-criteria by setting large importance weights to them.

It will be shown (later in Sections 3.5.1 and 3.5.2) that the proposed two-stage model can provide us with an optimal solution when no common feasible solution can be found by other models, such as the single-scenario and robust models. In fact, the aggregation of the solutions/decisions which is provided by the proposed two-stage model guarantees the feasibility and overall optimality if the feasible region under conditions of any scenario is not empty (i.e. for each scenario, at least one point can be found satisfying all restrictions).

\subsubsection{Analysis of the variations in importance weights}

By changing the importance weight, different solutions can be generated and trade-offs between the objectives can be observed. We may need to note that, in this study, we do not focus on the system of goal and weight choice. As we believe, because of existing too many goals/weights, elicitation of the DM preferences is hardly possible in practice and needs a separate study which lies in our future directions of research. Therefore, here, we only use some different weights(and goals) to show some specific situations or cover a wide range of possible consequences. If the same weights are assumed for all meta-criteria (namely, all weights set at 1) then the goal achievements in the second and the third objectives would be decreased. As expected, these reductions are caused by setting fewer importance weights (compared to the previous weighting) for the above-mentioned criteria. This weighting also provides better achievement in the first three meta-criteria (i.e. current costs in three plausible scenarios). Therefore, it can be clearly seen that the DM's preferences, which act as importance weights, affect the solutions in such a way that the model will generate solutions that satisfy his/her preferences the most.

As the planting costs of wheat are the lowest compared to the two other crops, it is expected that the lowest overall costs would be reached when all the land is allocated to growing wheat. In this case, which could be gained by increasing the importance weights of the first objective (Min costs) to ' 20 ' $\left(\omega_{11}=\omega_{12}=\omega_{13}=20\right)$, the lower bonds (ideal values) for the first criteria 
in each scenario would be equal to $2.4,2.5$, and 2.75 million Rands, respectively. These solutions will completely satisfy the DM in this criteria. However, achievements in the second and third objective functions will decrease sharply by these variations that made them unbalanced solutions, particularly for the last scenario in which the lowest amount of environmental benefits (pessimistic amount) would be reached at 120 units. Also, one of the lowest values will be gained by the second objective at 5.55 million Rands. Therefore, the ideal values in the first three meta-criteria conflict with the others which highlights the trade-offs between the goals.

Putting more weights on the second criteria, for instance set $\omega_{21}=\omega_{22}=\omega_{23}=9$, while keeping the others at their initial values, as defined in Table 3.2, would lead us to obtain the pessimistic values of the first three meta-criteria. Simultaneously, this would provide the ideal amounts for the second criterion in all scenarios by dedicating the entire land to corn. These weights, on the one hand, show the worst plausible (pessimistic) values for the costs for all three plausible scenarios (4.3, 4.5, and 4.75 million Rands, respectively). Conversely, utilising these weights leads the model to generate the best possible(ideal) amounts for liquidity under circumstances of all scenarios - 9.98, 7.262, and 6.604 million Rands, respectively. The third criterion also presents the pessimistic values under conditions of the first two scenarios (120 and 130 units, respectively) and almost the worst amount for the last scenario(140 units). Thus, in this case, the second objective can reach its maximum values for all scenarios while the other two objectives get their worst amount under conditions of all plausible scenarios.

Furthermore, increasing the third criterion's importance weights would obtain the ideal amounts for the environmental objective for different scenarios at 159.945, 173.939, and 183.939 (against the goals of 160,165, and 170). Therefore, the last two meta-criteria not only meet the DM's initial preferences but also provide some over-achievements for them. The achievement of the third objective under conditions of the first scenario also almost be met by only $0.055\left(\delta_{31}\right)$ difference. However, the goal achievements for other meta-criteria are distant from their initial aspiration levels because of the immense conflict between the objectives(see Table 3.4 for detail results and comparison).

More importantly, the solutions change much more quickly when the weights of the second criteria change, and the slope of variations in the last group (third criterion) is not as sharp as the others. The improvement in the last goal achievements slowly continues as the weights increase and these finally converge to 160,174 , and 184 in scenario $s_{1}, s_{2}$, and $s_{3}$, respectively. Table 3.4 represents achievement changes while the weights are raising in different objectives. As seen in this table, increasing the importance weights of the first criterion is effective until these weights have grown 20 times and, after that, no changes are possible by raising them. The most changes in the second objective function $Z_{2 k}$ occur when its relevant importance weights were doubled(see the third row in the second part of Table 3.4. Conversely, as shown in the last part of the table, enhancement in weights related to the last(third) criterion still affected the results (even after they were raised one thousand times), although their effects would not extensive after 100 times increase. Therefore, importance weights for the first objective and, specifically, for the second criterion are more sensitive and a slight variation in the weights can give rise to extensive changes in solutions. Nevertheless, this is different in the third criterion's importance weights in which the solution is less sensitive to the variations in this objective. This fact also can be seen by comparing the range of changes in achievements represented in Table 3.4.

\subsubsection{Analysis of the variations in goal levels}

It is also useful to study sensitivity to changes in the aspiration levels identified. Determining the aspiration levels is the first order of applying the DM's preferences to the model in goal programming approaches, while identifying the importance weights is the second order. In fact, in generalised GP, the DM preferences are largely modelled by choice of goals. The weights are primarily used to achieve a comparative scaling on each objective and the use of the term "importance weights" in GP is arguable. In other words, in GP, the importance of a criterion (or meta-criterion in our definitions) is mostly dependent on the goals (that determined by the 
DM) and, the weight parameters play a different role. Nevertheless, as an effect, a re-scaling of the objective values is presented by the weights to certify suitable levels of the trade-off between objective functions in the presence of the goals.

Here, goal variations have been investigated. Accordingly, any variations in $g_{11}$ and $g_{12}$ have almost no effect on the solutions; while changes in $g_{13}$ have a direct impact on the solution. In particular, increasing or decreasing $g_{13}$ raises or reduces all the achievements except $g_{23}$ which would decrease or increase slightly. An individual reduction or increase in any $g_{2 k}$ has no effect on the solution while a joint decrease or increase (e.g. in two of them) affects the solutions.Finally, the most sensitive goals of the third group belong to the first scenario $\left(g_{31}\right)$, while the other two scenarios are affected the solutions only when raising. More variations have been examined and the results are indicated in Table 3.5.

Changing the values of the goals on the first criterion, while keeping the initial values of the other criteria, changes the objective values with a direct relationship. For instance, as shown in the sixth row of Table 3.5 , increasing $g_{11}$ to $3.4, g_{22}$ to 3.55 , and $g_{23}$ to 3.75 , slightly boost all objective functions, while any reduction in these goals declines the objective values(see row three and four in Table 3.5). However, the goal of the other functions prevents more variations in this case and we could not reach the pessimistic nor the optimistic values. In other words, the goals that were set on the other meta-criteria could not be reached by the current maximum deviation. This, then, prevents the model from allocating any more reduction to the first objective values in the final solution. In this example, growing wheat has the minimum costs, however, if the model allocated more land than what is assign in the second row (324 hectares, Table 3.5) it would not end with an optimal solution as it will decrease the revenue sharply.

Planting corn provides us with the highest tonnage of yield and, therefore, would provide the highest revenue compared to the other two crops. Therefore, it is expected that the maximum revenue and then maximum liquidity would be reached when all the land is dedicated to growing

\begin{tabular}{|lll|lll|lll|lll|}
\hline \multicolumn{2}{|c|}{ Importance weights } & \multicolumn{2}{|c|}{$Z_{1 k}$ (million Rands) } & \multicolumn{2}{|c|}{$Z_{2 k}$ (million Rands) } & \multicolumn{3}{c|}{$Z_{3 k}$ (Units) } \\
\hline \hline$\omega_{11}$ & $\omega_{12}$ & $\omega_{13}$ & $s_{1}$ & $s_{2}$ & $s_{3}$ & $s_{1}$ & $s_{2}$ & $s_{3}$ & $s_{1}$ & $s_{2}$ & $s_{3}$ \\
\hline 1 & 1 & 1 & 3.451 & 3.607 & 3.857 & 7.89 & 6.410 & 6.094 & 128.9 & 130 & 131 \\
\hline 1 & 1 & 1.5 & 3.917 & 4.124 & 4.392 & 9.013 & 6.868 & 6.271 & 130 & 138 & 145 \\
\hline $1 \times 2$ & $1 \times 2$ & $1.5 \times 2$ & 3.609 & 3.772 & 4.022 & 8.296 & 6.58 & 6.204 & 127.28 & 130 & 132.72 \\
\hline $1 \times 5$ & $1 \times 5$ & $1.5 \times 5$ & 3.044 & 3.178 & 3.4278 & 6.854 & 5.973 & 5.811 & 133.22 & 130 & 126.78 \\
\hline $1 \times 10$ & $1 \times 10$ & $1.5 \times 10$ & 2.560 & 2.668 & 2.918 & 5.618 & 5.454 & 5.471 & 138.32 & 130 & 121.68 \\
\hline $1 \times 20$ & $1 \times 20$ & $1.5 \times 20$ & 2.4 & 2.5 & 2.75 & 5.137 & 5.185 & 5.296 & 140 & 130 & 120 \\
\hline $1 \times 100$ & $1 \times 100$ & $1.5 \times 100$ & 2.4 & 2.5 & 2.75 & 5.139 & 5.214 & 5.298 & 140 & 130 & 120 \\
\hline \hline$\omega_{21}$ & $\omega_{22}$ & $\omega_{23}$ & $s_{1}$ & $s_{2}$ & $s_{3}$ & $s_{1}$ & $s_{2}$ & $s_{3}$ & $s_{1}$ & $s_{2}$ & $s_{3}$ \\
\hline 1 & 1 & 1 & 3.451 & 3.607 & 3.857 & 7.89 & 6.410 & 6.094 & 128.9 & 130 & 131 \\
\hline 4 & 3.5 & 5 & 3.917 & 4.124 & 4.392 & 9.013 & 6.868 & 6.271 & 130 & 138 & 145 \\
\hline $4 \times 2$ & $3.5 \times 2$ & $5 \times 2$ & 4.292 & 4.494 & 4.745 & 9.955 & 7.25 & 6.587 & 120.55 & 130.6 & 140.6 \\
\hline $4 \times 2.5$ & $3.5 \times 2.5$ & $5 \times 2.5$ & 4.3 & 4.5 & 4.75 & 9.98 & 7.262 & 6.604 & 120 & 130 & 140 \\
\hline $4 \times 5$ & $3.5 \times 5$ & $5 \times 5$ & 4.3 & 4.5 & 4.75 & 9.98 & 7.262 & 6.604 & 120 & 130 & 140 \\
\hline \hline$\omega_{31}$ & $\omega_{32}$ & $\omega_{33}$ & $s_{1}$ & $s_{2}$ & $s_{3}$ & $s_{1}$ & $s_{2}$ & $s_{3}$ & $s_{1}$ & $s_{2}$ & $s_{3}$ \\
\hline 1 & 1 & 1 & 3.451 & 3.607 & 3.857 & 7.89 & 6.410 & 6.094 & 128.9 & 130 & 131 \\
\hline 7 & 7 & 8 & 3.917 & 4.124 & 4.392 & 9.013 & 6.868 & 6.271 & 130 & 138 & 145 \\
\hline $7 \times 2$ & $7 \times 2$ & $8 \times 2$ & 3.97 & 4.241 & 4.549 & 8.909 & 6.76 & 5.896 & 143.6 & 155.95 & 165.95 \\
\hline $7 \times 5$ & $7 \times 5$ & $8 \times 5$ & 3.861 & 4.155 & 4.484 & 8.558 & 6.604 & 5.664 & 151.3 & 164.46 & 174.46 \\
\hline $7 \times 10$ & $7 \times 10$ & $8 \times 10$ & 3.808 & 4.113 & 4.451 & 8.385 & 6.524 & 5.55 & 155.14 & 168.65 & 178.65 \\
\hline $7 \times 20$ & $7 \times 20$ & $8 \times 20$ & 3.776 & 4.088 & 4.432 & 8.281 & 6.475 & 5.481 & 157.4 & 171.1 & 181.1 \\
\hline $7 \times 100$ & $7 \times 100$ & $8 \times 100$ & 3.748 & 4.066 & 4.415 & 8.189 & 6.433 & 5.420 & 159.45 & 173.4 & 183.4 \\
\hline $7 \times 1000$ & $7 \times 1000$ & $8 \times 1000$ & 3.74 & 4.061 & 4.411 & 8.16 & 6.422 & 5.406 & 159.95 & 173.94 & 183.94 \\
\hline
\end{tabular}

Table 3.4: Goal achievements variations in different importance weights 
corn. In this case, which could be gained by doubling the goals in the second criterion (Max liquidity) and keeping the other criteria at their initial values, the pessimistic values (anti-ideal) of the first three meta-criteria will be obtained while simultaneously providing the ideal amounts of objective for the second criterion in all scenarios. Therefore, on the one hand, the worst plausible (pessimistic) values for the first objective function (costs) in all three plausible scenarios will be obtained (4.3, 4.5, and 4.75 million Rands, respectively). On the other hand, the best possible amounts for liquidity (second criterion) under circumstances of all scenarios could be generated, that is $9.98,7.262$, and 6.604 million Rands, respectively. These amounts highlight the existing conflict between different criteria and the trade-offs between the goals.

The third criterion also presents the pessimistic values under conditions of the first two scenarios (120 and 130 units, respectively) and near the worst amount for the last scenario(140 units). Thus, in this case, the second objective can reach its maximum values for all scenarios while the other two objectives get their worst amount under conditions of all plausible scenarios. Contrary results would be reached when these goals, in the second group, have been reduced. In this case, objective values in both costs and liquidity will be decrease to near their worst case, while objectives related to the third criterion rise dramatically (see rows 12 and 13 in Table 3.5).

Finally, conflicts between the third criteria and the other two have been recognised by considering the last part of the Table in which in the values of the last criterion in all scenarios increase when their goals increase, and vice versa. The best plausible values for the third objective are reachable when the land is completely dedicated to growing soy-beans which provide the highest rate of the environmental benefits. This rise in environmental benefits is accompanied by a reduction in other objective values (costs and liquidities) that will experience one of their worst cases. Also, the opposite results could be observed by decreasing the goals related to the third criterion (see Table 3.5 for detail results and comparison).

\begin{tabular}{|c|c|c|c|c|c|c|c|c|c|c|c|c|}
\hline \multicolumn{3}{|c|}{ Goals } & \multirow{2}{*}{$\begin{array}{c}X^{0}=\left(x_{1}^{0}, x_{2}^{0}, x_{3}^{0}\right) \\
\text { (Wheat, Corn }, \text { Soyb })\end{array}$} & \multicolumn{3}{|c|}{$Z_{1 k}$ (million Rands) } & \multicolumn{3}{|c|}{$Z_{2 k}$ (million Rands) } & \multicolumn{3}{|c|}{$Z_{3 k}$ (Units) } \\
\hline$g_{11}$ & $g_{12}$ & $g_{13}$ & & $s_{1}$ & $s_{2}$ & $s_{3}$ & $s_{1}$ & $s_{2}$ & $s_{3}$ & $s_{1}$ & $s_{2}$ & $s_{3}$ \\
\hline 0.04 & 0.055 & 0.075 & $(324,676,0)$ & 3.68 & 3.85 & 4.10 & 8.49 & 6.66 & 6.26 & 126.5 & 130 & 133.5 \\
\hline 0.4 & 0.55 & 0.75 & $(268,720,12)$ & 3.77 & 3.95 & 4.20 & 8.70 & 6.75 & 6.29 & 126.5 & 131.3 & 136 \\
\hline 1.4 & 1.55 & $1.75\left(^{*}\right)$ & $(148,780,72)$ & 3.917 & 4.124 & 4.392 & 9.013 & 6.868 & 6.271 & 130 & 138 & 145 \\
\hline 3.4 & 3.55 & 3.75 & $(0,867,133)$ & 4.11 & 4.35 & 4.64 & 9.38 & 6.98 & 6.21 & 133.3 & 144.6 & 154.6 \\
\hline 6.4 & 6.55 & 6.75 & $(0,867,133)$ & 4.11 & 4.35 & 4.64 & 9.38 & 6.98 & 6.21 & 133.3 & 144.6 & 154.6 \\
\hline$g_{21}$ & $g_{22}$ & $g_{23}$ & $(,)$, & $s_{1}$ & $s_{2}$ & $s_{3}$ & $s_{1}$ & $s_{2}$ & $s_{3}$ & $s_{1}$ & $s_{2}$ & $s_{3}$ \\
\hline $10 \times 0.02$ & $8 \times 0.02$ & $6.5 \times 0.02$ & $(716,0,284)$ & 2.54 & 2.76 & 3.08 & 5.23 & 5.20 & 4.82 & 162.7 & 161.2 & 156.9 \\
\hline $10 \times 0.2$ & $8 \times 0.2$ & $6.5 \times 0.2$ & $(716,0,284)$ & 2.54 & 2.76 & 3.08 & 5.23 & 5.20 & 4.82 & 162.7 & 161.2 & 156.9 \\
\hline 10 & 8 & $6.5\left(^{*}\right)$ & $(148,780,72)$ & 3.917 & 4.124 & 4.392 & 9.013 & 6.868 & 6.271 & 130 & 138 & 145 \\
\hline $10 \times 2$ & $8 \times 2$ & $6.5 \times 2$ & $(0,1000,0)$ & 4.3 & 4.5 & 4.75 & 9.98 & 7.24 & 6.6 & 120 & 130 & 140 \\
\hline $10 \times 300$ & $8 \times 300$ & $6.5 \times 300$ & $(0,1000,0)$ & 4.3 & 4.5 & 4.75 & 9.98 & 7.24 & 6.6 & 120 & 130 & 140 \\
\hline$g_{31}$ & $g_{32}$ & $g_{33}$ & $(,)$, & $s_{1}$ & $s_{2}$ & $s_{3}$ & $s_{1}$ & $s_{2}$ & $s_{3}$ & $s_{1}$ & $s_{2}$ & $s_{3}$ \\
\hline $160 \times 0.02$ & $165 \times 0.02$ & $170 \times 0.02$ & $(210,790,0)$ & 3.9 & 4.08 & 4.33 & 9.04 & 6.89 & 6.41 & 124.2 & 130 & 135.8 \\
\hline $160 \times 0.2$ & $165 \times 0.2$ & $170 \times 0.2$ & $(210,790,0)$ & 3.9 & 4.08 & 4.33 & 9.04 & 6.89 & 6.41 & 124.2 & 130 & 135.8 \\
\hline 160 & 165 & $170\left(^{*}\right)$ & $(148,780,72)$ & 3.451 & 3.607 & 3.857 & 7.89 & 6.410 & 6.094 & 128.9 & 130 & 131 \\
\hline $160 \times 2$ & $165 \times 2$ & $170 \times 2$ & $(0,130,870)$ & 3.08 & 3.54 & 4.01 & 6.03 & 5.44 & 4.0 & 206.9 & 225.6 & 236.6 \\
\hline $160 \times 5$ & $165 \times 5$ & $170 \times 5$ & $(0,0,1000)$ & 2.9 & 3.4 & 3.9 & 5.35 & 5.08 & 3.54 & 220 & 240 & 250 \\
\hline $160 \times 50$ & $165 \times 50$ & $170 \times 50$ & $(0,0,1000)$ & 2.9 & 3.4 & 3.9 & 5.37 & 5.09 & 3.54 & 220 & 240 & 250 \\
\hline
\end{tabular}

Table 3.5: Goal achievements variations in different goal levels

\subsection{Example illustrating scenario effects}

The aim of this example is to provide comparison with some popular existing scenario-based goal programming methods, specifically the single-scenario models and the robust GP. The purpose is to highlight the superiorities of the proposed two-stage structure in comparison with these approaches. Especially, this superiority is crucial when the intersection of the constraints of all scenarios is empty (i.e. the integrated problem has no feasible solution). Furthermore, it will 
be shown that, even if (after the revelation of scenarios) we have a chance to adapt the decision in corresponding single-scenario optimisation models, their solutions could not be as good as the solutions to the proposed two-stage approach which considers the required adaptations in advance (before scenario realisation).

Example 3.5.1. Let us consider the following model:

$$
\begin{aligned}
& \operatorname{Min} \mathbf{F}=\left[f_{1 k}, f_{2 k}\right]=\sum_{k=1}^{5} \sum_{j=1}^{2}\left(c_{i j k}^{0} x_{j}^{0}+c_{i j k}^{1} x_{k}^{1}\right) ; \quad i=1,2 ; k=1, \ldots, 5 ; \\
& \text { s.t. } \sum_{j=1}^{2} a_{r j k}^{0} x_{j}^{0}+a_{r k}^{1} x_{k}^{1} \leq b_{r k}^{1}, \quad r=1,2,3 ; k=1, \ldots, 5 ; \\
& x_{j}^{0}, x_{k}^{1} \geq 0 . \quad j=1,2 ; k=1, \ldots, 5 .
\end{aligned}
$$

where the coefficients of the model in the case of five different scenarios have been shown in Table 3.6 .

To solve a problem using the goal programming approach, we need to set some goals $\left(g_{i k}\right)$ for each objective which could be established by DM or we may use the optimum solution of the specific problem when there is not any uncertainty in the model. For example, if it is known that scenario ' 1 ' will unfold we could solve two simple single objective models, related to two objectives, and subject to specific constraints of scenario ' 1 '. These goals, which are obtained by solving two single objective problems subjected to specific constraints of scenario $k,(k=1, . ., 5)$, have also been demonstrated in Table 3.6.

If $c_{i j k}^{1}=1$, and $a_{r k}^{1}=-1$, for all $i, j, r$, and $k$, then the proposed two-stage model for example 3.5.1 can be formulated (see model 3.18 in Chapter Appendix 3.7.3). By solving this linear model the following solution, which shown in Table 3.7, can be found.

As shown in Table 3.7, the proposed 2-stage model suggests that $X^{0}=\left(x_{1}^{0}, x_{2}^{0}\right)=(2.62,1.32)$ (the solutions have been rounded off to two decimal places) as an initial decision. The contingent (recourse) decisions $\left(x_{k}^{1}, k=1, . ., 5\right)$, which will be implemented after scenario realisation, have been presented in column three of Table 3.7. Number ' 0 ' for scenarios ' 1 ' and ' 2 ' describe that there is no need for any more contingent decisions, and that the DM needs to do nothing if one

\begin{tabular}{|c|c|c|c|c|c|c|c|c|c|c|c|c|c|c|c|}
\hline Scenario/coefficients & $c_{11 k}^{0}$ & $c_{12 k}^{0}$ & $g_{1 k}$ & $c_{22 k}^{0}$ & $c_{21 k}^{0}$ & $g_{2 k}$ & $a_{11 k}^{0}$ & $a_{12 k}^{0}$ & $b_{1 k}^{1}$ & $a_{21 k}^{0}$ & $a_{22 k}^{0}$ & $b_{2 k}^{1}$ & $a_{31 k}^{0}$ & $a_{32 k}^{0}$ & $b_{3 k}^{1}$ \\
\hline $\mathrm{K}=1$ & -5 & 4 & -15 & 1 & 2 & 0 & 1 & 1 & 4 & 1 & 0 & 3 & 0 & 1 & 3 \\
\hline $\mathrm{K}=2$ & -3 & 1.5 & -9 & 2 & 3 & 2 & -1 & -1 & -1 & 1 & 0 & 3 & 0 & 1 & 3 \\
\hline $\mathrm{K}=3$ & 0.5 & 9 & 28 & 2 & 1 & 7 & 1 & 1 & 5 & -1 & 0 & -2 & 0 & -1 & -3 \\
\hline $\mathrm{K}=4$ & 0.7 & 7 & 16.1 & 2.2 & 1.3 & 9.2 & -1 & -1 & -2 & -1 & 0 & -3 & 0 & -1 & -2 \\
\hline $\mathrm{K}=5$ & -7 & 4 & -12 & 1.5 & 2.5 & 13 & -1 & -1 & -6 & 1 & 0 & 4 & 0 & -1 & -4 \\
\hline
\end{tabular}

Table 3.6: The amount of the coefficients of the model in the case of five different scenarios.

\begin{tabular}{|c|c|c|c|c|c|c|}
\hline Scenario/solutions & $X^{0}=\left(x_{1}^{0}, x_{2}^{0}\right)$ & $x_{k}^{1}$ & $F=\left(f_{1 k}, f_{2 k}\right)$ & $G=\left(g_{1 k}, g_{2 k}\right)$ & $\Delta=\left(\delta_{1 k}, \delta_{2 k}\right)$ & $\varphi$ \\
\hline$k=1$ & $(2.62,1.32)$ & 0 & $(-7.8,5.25)$ & $(-15,0)$ & $(7.2,5.25)$ & 7.2 \\
\hline$k=2$ & $(2.62,1.32)$ & 0 & $(-5.87,9.2)$ & $(-9,2)$ & $(3.13,7.2)$ & 7.2 \\
\hline$k=3$ & $(2.62,1.32)$ & 7.64 & $(20.8,14.2)$ & $(28,7)$ & $(-7.2,7.2)$ & 7.2 \\
\hline$k=4$ & $(2.62,1.32)$ & 3.94 & $(15,11.4)$ & $(16.1,9.2)$ & $(-1.1,2.2)$ & 2.2 \\
\hline$k=5$ & $(2.62,1.32)$ & 4.55 & $(-8.5,11.78)$ & $(-12,13)$ & $(3.5,-1.22)$ & 3.5 \\
\hline
\end{tabular}

Table 3.7: The result of the proposed two-stage MOLP for example 3.5.1 
of these two scenarios is revealed. There would be a corrective decision for any other scenario realisation. The value of objective functions (achievements) $\left(F=\left(f_{1 k}, f_{2 k}\right)\right)$ and their goals have been presented in columns 4 and 5, respectively. The column 6 of Table 3.7 shows the deviations $\left(\delta_{i k}\right)$ from the initial goals. Positive and negative signs represent the positive and negative deviations from the goals ${ }^{2}$. Finally, the maximum deviations from the goals $(\varphi)$ are demonstrated in the last column of the Table. For example, consider the last row where scenario ' 5 ' occurred. If the suggested initial decision $\left(X^{0}=\left(x_{1}^{0}, x_{2}^{0}\right)=(2.62,1.32)\right)$ was applied, now and after the revelation of scenario ' 5 ', the contingent decision $\left(x_{5}^{1}=4.55\right)$ needs to be implemented as a recourse in which the values of ' $-8.5^{\text {' }}$ and ' $11.78^{\prime}$ ' could be reached for the first and the second objectives, respectively. The goals were ' -12 ' and ' 13 ' so, in comparison with our achievements ('-8.5' and ' 11.78 '), these show ' 3.5 ' units positive deviation from the first goal and ' 1.22 ' units negative deviation from $g_{25}$ that represent over achievement in this meta-criteria. Obviously, the maximum deviation in this scenario is ' $\varphi=3.5$ '. More discussion on results of example 3.5.1 and comparison with other approaches can be found in the next subsection.

\subsubsection{Comparison with single-scenario models}

In this section, example 3.5.1 is revisited and solved by some single-scenario models. In these models, we assume that the future scenario is known and the problem is solved for this specific scenario. Therefore, the problem needs to be solved under the conditions of every plausible scenario.

Example 3.5.2. Consider example 3.5.1 for which five single-scenario models need to be run (see models 3.19-3.23 in Chapter Appendix 3.7.4.1-3.7.4.5). By solving these five specific models, five different solutions can be reached which have been portrayed in Table 3.8 together with the proposed two-stage solution.

At first, the feasibility of these solutions in every single scenario has been checked, and the result has been presented in Table 3.9. Check marks indicate that the solution is feasible for that specific scenario realisation, and the cross marks represent the infeasibility of the related solutions under conditions of that scenario.

It can be clearly seen that, except for the solution of the proposed two-stage model, none of the solutions of the five single-scenario models is feasible in all scenarios. In fact, the intersection

\footnotetext{
${ }^{2}$ Sometimes, a one-side deviation may be considered in which the positive deviations in Maximisation problem, and the negative deviations in Minimisation problem would be an advantage. However, for this specific example, the two-sided deviation is considered and both positive and negative deviations are undesirable.
}

\begin{tabular}{|c|c|c|c|c|c|c|}
\hline Scenario/goals & $S_{1}$ & $S_{2}$ & $S_{3}$ & $S_{4}$ & $S_{5}$ & proposed 2-stage model \\
\hline$X^{0}=\left(x_{1}^{0}, x_{2}^{0}\right)$ & $(2.5,0)$ & $(2.2,0)$ & $(2,3)$ & $(3,2)$ & $(3.65,4)$ & $(2.62,1.32)+x_{k}^{1}$ \\
\hline
\end{tabular}

Table 3.8: Comparing different solutions of various models

\begin{tabular}{|c|c|c|c|c|c|c|}
\hline Scenario/models & $S_{1}$ & $S_{2}$ & $S_{3}$ & $S_{4}$ & $S_{5}$ & proposed 2-stage model \\
\hline$s_{1}$ & $\checkmark$ & $\checkmark$ & $\times$ & $\times$ & $\times$ & $\checkmark$ \\
\hline$s_{2}$ & $\checkmark$ & $\checkmark$ & $\checkmark$ & $\checkmark$ & $\times$ & $\checkmark$ \\
\hline$s_{3}$ & $\times$ & $\times$ & $\checkmark$ & $\times$ & $\times$ & $\checkmark$ \\
\hline$s_{4}$ & $\times$ & $\times$ & $\times$ & $\checkmark$ & $\checkmark$ & $\checkmark$ \\
\hline$s_{5}$ & $\times$ & $\times$ & $\times$ & $\times$ & $\checkmark$ & $\checkmark$ \\
\hline
\end{tabular}

Table 3.9: Comparing the feasibility of each solution in different scenario realisations 
of the constraints of these five scenarios is empty, and it is impossible to find a feasible solution that satisfies all constraints. However, by utilising the proposed two-stage approach the initial solution together with a recourse action could solve this problem. These results present the power of our proposed approach in comparison with existing single scenario-based goal programming methods, especially when the intersection of the constraints of all scenarios is empty, and the integrated problem has no feasible solution. The question that may raise here is: what if it is possible to correct the solutions of these five single scenario models after scenario realisation? It should be quite clear that the solution of the proposed two-stage approach must perform equally or better than the other approaches because it is an optimal solution. However, to further illustration this, these solutions have been applied to a two-stage structure (Tables 3.10-3.14).

In this case, suppose that there is a possibility of adapting the first decision after scenario revelation ${ }^{3}$. This means that one of the solutions of these five single scenario optimisation models is applied and then, after scenario revelation, we will try to adapt the decision to the extent possible, if there is a need for such a correction. Therefore, we consider a two-stage process in which the initial decision referred to the solution of the single-scenario optimisation models, and the contingent solutions for every single scenario revelation are what we are looking for. Tables 3.10-3.14 in page 59 describe and compare the contingent solutions, objective functions, goals, deviations and maximum deviation from the goals for every single scenario realisation.

In all these tables (Tables 3.10-3.14), the initial and contingent decisions have been presented in the second and third columns, respectively. Columns 4-6 have respectively described the achievements (values of the objective functions), goals and the deviations from the goals. Finally, the last column shows the maximum deviations from the goals that would be obtained if scenario $k(k=1,2,3,4,5 ;)$ is revealed. By taking a glance over these five tables and comparing them to Table 3.7, which indicates the result of our proposed two-stage model, it is clear that the proposed two-stage approach has the minimum of maximum deviations from goals (Min - Max $\left.\delta_{(2 \text { stage })}=7.2\right)$ in comparison with single-scenario models $\left(\right.$ Min - Max $\delta_{(\text {single-scenarios })}=$ $12.375,12.15,18,10$ and 18.03 , respectively). So, it vividly demonstrates the optimality of the proposed model as it is expected, mainly due to the fact it is looking ahead, and must, therefore, find better goal achievement than the single-scenario models. For example, let us compare the proposed solution to the solution which was obtained from the single-scenario model related to the forth scenario, which has the least difference compared to the proposed model. This solution is the best solution, if and only if, scenario ' 4 ' manifested in which all goals would be reached completely. Otherwise, except for scenario ' 5 ', the proposed two-stage model provides us with better performance than this solution.

A summary of differences between the objective function $(\varphi)$ of the proposed two-stage model and the others in various scenarios has been recorded in Table 3.15(see page 60). Negative numbers describe the advantages of the objective function $(\varphi)$ of the proposed model in comparison with the single-scenario models. As shown in the last row of this Table, the aggregation of these differences over all scenarios is a negative number in all columns which highlights the optimality of the solutions of the proposed two-stage model.

As shown in the above example, the proposed two-stage model can provide us with an optimal solution when no common feasible solution can be found by the single-scenario models and the intersection of the combined constraints is empty. In fact, by applying the proposed two-stage structure, we are always able to find a feasible solution if, at least, one feasible solution can be found for every single-scenario model. We need to note that this feasible solution is not necessarily feasible in all single-scenario models. In other words, the necessary and sufficient condition for the feasibility of the full solution(union of initial and recourse solutions) in the proposed two-stage model is the feasibility of the particular single-scenario models(i.e. each single-scenario model is feasible and can provide at least one feasible solution that satisfies its

\footnotetext{
${ }^{3}$ More often, in real situations, there is not enough time to correct the decisions if there is not any preparation.
} 


\begin{tabular}{|c|c|c|c|c|c|c|}
\hline Scenario/solutions & $X^{0}=\left(x_{1}^{0}, x_{2}^{0}\right)$ & $x_{k}^{1}$ & $F=\left(f_{1 k}, f_{2 k}\right)$ & $G=\left(g_{1 k}, g_{2 k}\right)$ & $\Delta=\left(\delta_{1 k}, \delta_{2 k}\right)$ & $\varphi$ \\
\hline$k=1$ & $(2.5,0)$ & 0 & $(-15,3)$ & $(-15,0)$ & $(2.5,2.5)$ & 2.5 \\
\hline$k=2$ & $(2.5,0)$ & 0 & $(-9,6)$ & $(-9,2)$ & $(1.5,3)$ & 3 \\
\hline$k=3$ & $(2.5,0)$ & 14.375 & $(15.25,19.75)$ & $(28,7)$ & $(-12.375,12.375)$ & 12.375 \\
\hline$k=4$ & $(2.5,0)$ & 10.743 & $(12.08,16.68)$ & $(16.1,9.2)$ & $(-3.61,7.48)$ & 7.48 \\
\hline$k=5$ & $(2.5,0)$ & 8.031 & $(-12.4,13.1)$ & $(-12,13)$ & $(2.53,-1.22)$ & 2.53 \\
\hline
\end{tabular}

Table 3.10: Achievements which could be reached by applying the solution of scenario 1 model as an initial solution together with the possible recourse action in example 3.5.1

\begin{tabular}{|c|c|c|c|c|c|c|}
\hline Scenario/solutions & $X^{0}=\left(x_{1}^{0}, x_{2}^{0}\right)$ & $x_{k}^{1}$ & $F=\left(f_{1 k}, f_{2 k}\right)$ & $G=\left(g_{1 k}, g_{2 k}\right)$ & $\Delta=\left(\delta_{1 k}, \delta_{2 k}\right)$ & $\varphi$ \\
\hline$k=1$ & $(2.2,0)$ & 0 & $(-15,3)$ & $(-15,0)$ & $(4,2.2)$ & 4 \\
\hline$k=2$ & $(2.2,0)$ & 0 & $(-9,6)$ & $(-9,2)$ & $(2.4,2.4)$ & 2.4 \\
\hline$k=3$ & $(2.2,0)$ & 14.75 & $(15.25,19.75)$ & $(28,7)$ & $(-12.15,12.15)$ & 12.15 \\
\hline$k=4$ & $(2.2,0)$ & 10.93 & $(12.08,16.68)$ & $(16.1,9.2)$ & $(-3.63,6.57)$ & 6.57 \\
\hline$k=5$ & $(2.2,0)$ & 8.03 & $(-12.4,13.1)$ & $(-12,13)$ & $(4.9,-1.4)$ & 4.9 \\
\hline
\end{tabular}

Table 3.11: Achievements which could be reached by applying the solution of scenario 2 model as an initial solution together with the possible recourse action in example 3.5.1

\begin{tabular}{|c|c|c|c|c|c|c|}
\hline Scenario/solutions & $X^{0}=\left(x_{1}^{0}, x_{2}^{0}\right)$ & $x_{k}^{1}$ & $F=\left(f_{1 k}, f_{2 k}\right)$ & $G=\left(g_{1 k}, g_{2 k}\right)$ & $\Delta=\left(\delta_{1 k}, \delta_{2 k}\right)$ & $\varphi$ \\
\hline$k=1$ & $(2,3)$ & 1 & $(3,9)$ & $(-15,0)$ & $(18,9)$ & 18 \\
\hline$k=2$ & $(2,3)$ & 0 & $(-1.5,13)$ & $(-9,2)$ & $(7.5,11)$ & 11 \\
\hline$k=3$ & $(2,3)$ & 0 & $(28,7)$ & $(28,7)$ & $(0,0)$ & 0 \\
\hline$k=4$ & $(2,3)$ & 1 & $(23.4,9.3)$ & $(16.1,9.2)$ & $(7.3,0.1)$ & 7.3 \\
\hline$k=5$ & $(2,3)$ & 2.04 & $(0.04,12.54)$ & $(-12,13)$ & $(12.04,-0.46)$ & 12.04 \\
\hline
\end{tabular}

Table 3.12: Achievements which could be reached by applying the solution of scenario 3 model as an initial solution together with the possible recourse action in example 3.5.1

\begin{tabular}{|c|c|c|c|c|c|c|}
\hline Scenario/solutions & $X^{0}=\left(x_{1}^{0}, x_{2}^{0}\right)$ & $x_{k}^{1}$ & $F=\left(f_{1 k}, f_{2 k}\right)$ & $G=\left(g_{1 k}, g_{2 k}\right)$ & $\Delta=\left(\delta_{1 k}, \delta_{2 k}\right)$ & $\varphi$ \\
\hline$k=1$ & $(3,2)$ & 1 & $(-6,8)$ & $(-15,0)$ & $(9,8)$ & 9 \\
\hline$k=2$ & $(3,2)$ & 0 & $(-6,12)$ & $(-9,2)$ & $(3,10)$ & 10 \\
\hline$k=3$ & $(3,2)$ & 6.36 & $(25.86,14.36)$ & $(28,7)$ & $(-2.14,7.36)$ & 7.36 \\
\hline$k=4$ & $(3,2)$ & 0 & $(16.1,9.2)$ & $(16.1,9.2)$ & $(0,0)$ & 0 \\
\hline$k=5$ & $(3,2)$ & 2.84 & $(-10.16,12.44)$ & $(-12,13)$ & $(1.84,-0.66)$ & 1.84 \\
\hline
\end{tabular}

Table 3.13: Achievements which could be reached by applying the solution of scenario 4 model as an initial solution together with the possible recourse action in example 3.5.1

\begin{tabular}{|c|c|c|c|c|c|c|}
\hline Scenario/solutions & $X^{0}=\left(x_{1}^{0}, x_{2}^{0}\right)$ & $x_{k}^{1}$ & $F=\left(f_{1 k}, f_{2 k}\right)$ & $G=\left(g_{1 k}, g_{2 k}\right)$ & $\Delta=\left(\delta_{1 k}, \delta_{2 k}\right)$ & $\varphi$ \\
\hline$k=1$ & $(3.65,4)$ & 3.65 & $(8,12)$ & $(-15,0)$ & $(16.4,15.3)$ & 16.4 \\
\hline$k=2$ & $(3.65,4)$ & 1 & $(1,17)$ & $(-9,2)$ & $(5.05,18.03)$ & 18.03 \\
\hline$k=3$ & $(3.65,4)$ & 2.65 & $(38,9)$ & $(28,7)$ & $(12.475,6.96)$ & 12.475 \\
\hline$k=4$ & $(3.65,4)$ & 0 & $(30.4,10.6)$ & $(16.1,9.2)$ & $(14.46,4.03)$ & 14.46 \\
\hline$k=5$ & $(3.65,4)$ & 0 & $(2,13)$ & $(-12,13)$ & $(2.45,2.475)$ & 2.475 \\
\hline
\end{tabular}

Table 3.14: Achievements which could be reached by applying the solution of scenario 5 model as an initial solution together with the possible recourse action in example 3.5.1 
constraints). Then, if that decision could not meet the limitations of the other models, the scenario-related recourse arrangements, with appropriate coefficients, help to compensate for the differences.

Obviously, if the feasible region under conditions of any scenario is not empty(i.e. for each scenario, at least one point can be found satisfying all restrictions), we are able to construct some feasible solutions for the proposed two-stage model by setting appropriate coefficients for the recourse decisions. This feature indicates the natural superiority of generating the dynamicrobust solutions in the proposed two-stage structure that highly motivated our study in complex MCDA problems. This superiority will be further highlighted in the next section, where it is compared to the regular robust goal programming approaches.

\begin{tabular}{|c|c|c|c|c|c|}
\hline \multirow{2}{*}{ Scenario } & \multicolumn{5}{|c|}{ Differences of $\varphi^{\prime}$. } \\
\cline { 2 - 6 } & 2 stage $-s_{1}$ & 2 stage $-s_{2}$ & 2 stage $-s_{3}$ & 2 stage $-s_{4}$ & 2 stage $-s_{5}$ \\
\hline$k=1$ & 4.7 & 3.2 & -10.8 & -1.8 & -9.2 \\
\hline$k=2$ & 4.2 & 4.8 & -3.8 & -2.8 & -10.83 \\
\hline$k=3$ & -5.175 & -4.95 & 7.2 & -0.16 & -5.275 \\
\hline$k=4$ & -5.28 & -4.38 & -5.1 & 2.2 & -12.26 \\
\hline$k=5$ & 0.97 & -1.9 & -8.54 & 1.66 & 1.025 \\
\hline Total & -0.585 & -3.22 & -21.04 & -0.9 & -36.54 \\
\hline
\end{tabular}

Table 3.15: A summary of differences between the objective function $(\varphi)$ of the proposed two-stage model and the others in various scenarios

\subsubsection{Comparison with Robust Goal Programming}

As seen in the previous section, the intersection of the constraints of plausible scenarios is empty, in example 3.5.2, and it is impossible to find a feasible solution nor a robust one, which satisfies all constraints. Therefore, none of the single-scenario or robust approaches can solve the generated example but the proposed two-stage framework can. In fact, in the proposed two-stage framework, there is no need to find a common feasible initial solution that works for all scenarios, including the worst scenario. Rather, it is sufficient to be good enough as a starting point that enables the construction of the best possible combined decisions, together with scenario-related recourse decisions, in every single plausible scenario. This feature expands the robust decisions philosophy in our context and methodology by introducing and applying the concept of dynamicrobust decisions by which the limitation of the regular robust strategy (i.e. a risk-averse solution because of a bad scenario) is also reduced, its advantages are developed, and an extra motivation is appended to our study.

Therefore, for further illustration, let us make a comparison between the proposed two-stage structure and the robust goal programming approach, which was introduced by [Kuchta, 2004]. Accordingly, we changed some coefficients in this example to find a non-empty intersection in which we can find a robust solution. Now we can compare this robust decision with the decision suggested by the proposed Two-stage structure.

Example 3.5.3. To reach this goal, it is sufficient to change $b_{11}^{1}$ from ' 4 ' to ' 6 ' and $b_{13}^{1}$ from ' 5 ' to ' 6 ' in example 3.5.1. Then, $X^{0}=\left(x_{1}^{0}, x_{2}^{0}\right)=(3,3)$ would be a robust solution, and the solution of the proposed two-stage model remained Pareto optimum with no change $((2.61,1.32))$. Results and comparison between the two, have been shown in Table 3.16. It can be clearly seen that except for the realisation of scenario ' 3 ' in which the robust solution will provide a better performance, implementing the proposed two-stage solution has a minimum deviations $(\operatorname{Min} \varphi)$ 
from the goals if scenario ' 1 ', '2' or ' 4 ' unfolded. In the case of the last scenario, both approaches have almost the same results with slight advantages for the robust solution in comparison with the proposed two-stage solution. Overall, it is evident that the proposed two-stage solution can provide us with better performance for this example.

\begin{tabular}{|c|c|c|c|c|c|c|c|}
\hline \multirow{2}{*}{ Scenario/solutions } & \multicolumn{3}{|c|}{ Robust Solution } & \multirow{2}{*}{ Goals } & \multicolumn{3}{c|}{ Proposed Two-Stage Solution } \\
\cline { 2 - 3 } \cline { 6 - 7 } & $F=\left(f_{1 k}, f_{2 k}\right)$ & $\Delta=\left(\delta_{1 k}, \delta_{2 k}\right)$ & $\varphi$ & $G=\left(g_{1 k}, g_{2 k}\right)$ & $F=\left(f_{1 k}, f_{2 k}\right)$ & $\Delta=\left(\delta_{1 k}, \delta_{2 k}\right)$ & $\varphi$ \\
\hline$k=1$ & $(-3,9)$ & $(12,9)$ & 12 & $(-15,0)$ & $(-7.8,5.25)$ & $(7.2,5.25)$ & 7.2 \\
\hline$k=2$ & $(-4.5,15)$ & $(4.5,13)$ & 13 & $(-9,2)$ & $(-5.87,9.2)$ & $(3.13,7.2)$ & 7.2 \\
\hline$k=3$ & $(28.5,9)$ & $(0.5,2)$ & 2 & $(28,7)$ & $(20.8,14.2)$ & $(-7.2,7.2)$ & 7.2 \\
\hline$k=4$ & $(23.1,10.5)$ & $(7,1.3)$ & 7 & $(16.1,9.2)$ & $(15,11.4)$ & $(-1.1,2.2)$ & 2.2 \\
\hline$k=5$ & $(-9,12)$ & $(3,1)$ & 3 & $(-12,13)$ & $(-8.5,11.78)$ & $(3.5,-1.22)$ & 3.5 \\
\hline
\end{tabular}

Table 3.16: The proposed Two-Stage approach in comparison with the Robust GP

Note that the solution of the robust model is dominated by the solution of the proposed twostage model mainly due to the fact it is searching in the same feasible region as the robust model, plus it leaves room for the possibility of adaptation. If there is no more improvement possible by any contingent decisions in any scenario, the solutions of both approaches are expected to be equal. However, remember that the two-stage structure can provide a meaningful solution even if no "robust" solution exists.

\subsection{Conclusion}

The focus and primary motivation throughout this chapter have been on dealing with deep uncertainty and risk observing in many multi-criteria decision-making problems, especially, with long-term decision-making processes such as strategic planning problems in which the decision needs to be made (and one alternative must be chosen) before getting enough knowledge about consequences of the decision or even sufficient awareness of all other options.

Therefore, this chapter introduced a novel robust-dynamic optimisation approach, named the scenario-based two-stage framework, to deal with multi-objective optimisation problems under deep uncertainty. The main idea was extended by the two-stage stochastic programming with recourse to address the capability of dealing with deep uncertainty through the use of scenario planning rather than statistical expectation. Scenarios are utilised as a dimension of preference (a component of the meta-criteria) to avoid problems of evaluating probabilities under deep uncertainty. Moreover, to some extent, the meta-criteria concept is extended to find a robust decision. Such scenario-based thinking involves a multi-objective representation of performance under different future conditions as an alternative to expectation, which fits naturally into the broader multi-objective problem context. This dynamic-robust multi-objective optimisation structure can help us handle moderate and deep uncertainty in complex MCDA problems. A dynamic-robust decision in our philosophy is a split decision and contains two subgroups of decisions, the initial decision(s) followed by recourse decision(s), in which the full decision is robust across scenarios.

In the proposed two-stage structure, we do not utilise models to produce forecasts but try instead identify the reasonable initial decision (which is compensable, whatever happens in the future) and an appropriate scenario-dependent recourse decision for every single plausible scenario, implemented after scenario revelation. In fact, we break the decision into two parts and defer one part of the decision long enough to realise the uncertain parameter(s). In other words, in the two-stage structure, we are looking one step ahead postpone part of the decision and leave room for possible adaptation later (after scenario realisation), while we identified suitable adaptive plans for every plausible scenario in advance.

It is shown that the proposed two-stage structure not only helps us cope with higher degrees of uncertainty but also can contribute to solving the scenario-based problems without a feasible 
nor robust solution. This approach does not look for a solution that could be feasible in every single scenario. Such a solution, if it exists, may well cost too much as it needs to satisfy all constraints of all plausible scenarios, while only one scenario will happen. Sometimes, as seen in example 3.5.1, the intersection of the constraints of all scenarios is empty, and no feasible solution can be found for the problem. In general, a few scenarios, which may never unfold, may promote a conservative solution that often does not satisfy DM when another possible scenario manifested. Moreover, these scenarios cannot be ignored or eliminated due to the fact they exist and may happen. Furthermore, waiting for scenario realisation without suitable preparations may neglect the opportunity for possible adaptations.

To deal with these issues, the proposed approach tries to make a reasonable decision at the first stage, as an initial decision that is made in the absence of any knowledge about scenarios, followed by a subsequent decision stage after gaining information (scenario revelation). In other words, our first aim is to avoid choosing an irrecoverable decision at the first stage. Secondly, we want to look for a convenient contingent decision for each and every plausible scenario at the next stage. The aggregation of the decisions in both stages provide us with the overall optimality to the two-stage problem. Therefore, by using the proposed method, any specific cost would be postponed to the second stage and after scenario realisation. Thus, there is no need to pay some costs for some scenarios that would never be revealed.

Furthermore, by applying the proposed two-stage structure, we are always able to find a feasible solution if, at least, one feasible solution can be found for every single-scenario model. We need to note that this feasible solution is not necessarily feasible in all single-scenario models. The necessary and sufficient condition for the feasibility of the full solution(union of initial and recourse solutions) in the proposed two-stage model is the feasibility of the particular singlescenario models(i.e. each single-scenario model is feasible and can provide at least one feasible solution that satisfies its constraints). If that decision could not meet the limitations of the other models, then the scenario-related recourse arrangements, with appropriate coefficients, help to compensate the differences.

Robust Optimisation (RO) approaches are helpful to improve system robustness by optimising the problem under worst case scenarios. Nevertheless, the solutions of RO approaches are often considered to be very conservative (risk averse), and the total charge tends to be significantly high. However, the proposed two-stage approach avoids over conservatism by providing a chance for modification after the revelation of scenario. Furthermore, because of the complexity of multi-objective optimisation problems with higher degrees of uncertainty, identifying the worst case is hardly possible (if ever possible).

However, the proposed two-stage structure does not look for an initial decision that satisfies the conditions of all scenarios. Instead, it looks for a suitable initial decision as a starting point that provides us with the best possible overall performances if followed by a convenient recourse decision for all plausible scenarios. In fact, the proposed methodology introduces the concept of dynamic-robust decisions by extending the robust decision philosophy. These kinds of decisions, reduce the shortcoming of the regular robust strategies such as generating a risk-averse solution because of a bad scenario. Also, the advantages of the robust solutions are developed. All the above-mentioned features motivated us to pursue our study of the proposed two-stage structure. 


\subsection{Appendix: Model formulations for case studies}

\subsubsection{The two-stage multi-objective optimisation model of example 3.4}

$$
\begin{aligned}
& \operatorname{Min} Z_{11}=2400 x_{1}^{0}+4300 x_{2}^{0}+2900 x_{3}^{0} \\
& \operatorname{Min} Z_{12}=2500 x_{1}^{0}+4500 x_{2}^{0}+3400 x_{3}^{0} \\
& \operatorname{Min} Z_{13}=2750 x_{1}^{0}+4750 x_{2}^{0}+3900 x_{3}^{0} \\
& \operatorname{Max}_{21}=1600 x_{11}^{1}+1250 x_{21}^{1}+3050 x_{31}^{1}-1800 x_{41}^{1}-1400 x_{51}^{1} \\
& M a x Z_{22}=1850 x_{12}^{1}+1450 x_{22}^{1}+3300 x_{32}^{1}-2000 x_{42}^{1}-1600 x_{52}^{1} \\
& \operatorname{Max}_{23}=2100 x_{13}^{1}+1650 x_{23}^{1}+3500 x_{33}^{1}-2300 x_{43}^{1}-1800 x_{53}^{1} \\
& \operatorname{Max} Z_{31}=(0.14) x_{1}^{0}+(0.12) x_{2}^{0}+(0.22) x_{3}^{0} \\
& \operatorname{Max} Z_{32}=(0.13) x_{1}^{0}+(0.13) x_{2}^{0}+(0.24) x_{3}^{0} \\
& \operatorname{Max} Z_{33}=(0.12) x_{1}^{0}+(0.14) x_{2}^{0}+(0.25) x_{3}^{0} \\
& \text { s.t } x_{1}^{0}+x_{2}^{0}+x_{3}^{0}=1000 \\
& 4 x_{1}^{0}-x_{11}^{1}+x_{41}^{1}=400 \\
& \text { (3.6) } x_{1}^{0}-x_{12}^{1}+x_{42}^{1}=400 \\
& (3.3) x_{1}^{0}-x_{13}^{1}+x_{43}^{1}=400 \\
& 9 x_{2}^{0}-x_{21}^{1}+x_{51}^{1}=440 \\
& 6 x_{2}^{0}-x_{22}^{1}+x_{52}^{1}=440 \\
& 5 x_{2}^{0}-x_{23}^{1}+x_{53}^{1}=440 \\
& (2.2) x_{3}^{0}-x_{31}^{1}=0 \\
& 2 x_{3}^{0}-x_{32}^{1}=0 \\
& (1.5) x_{3}^{0}-x_{33}^{1}=0 \\
& x_{1}^{0}, x_{2}^{0}, x_{3}^{0}, x_{1 k}^{1}, x_{2 k}^{1}, x_{3 k}^{1}, x_{4 k}^{1}, x_{5 k}^{1} \geq 0
\end{aligned}
$$

$$
k=1,2,3 \text {. }
$$




\subsubsection{The equivalent GP formulated model of example 3.4 (after nor- malising)}

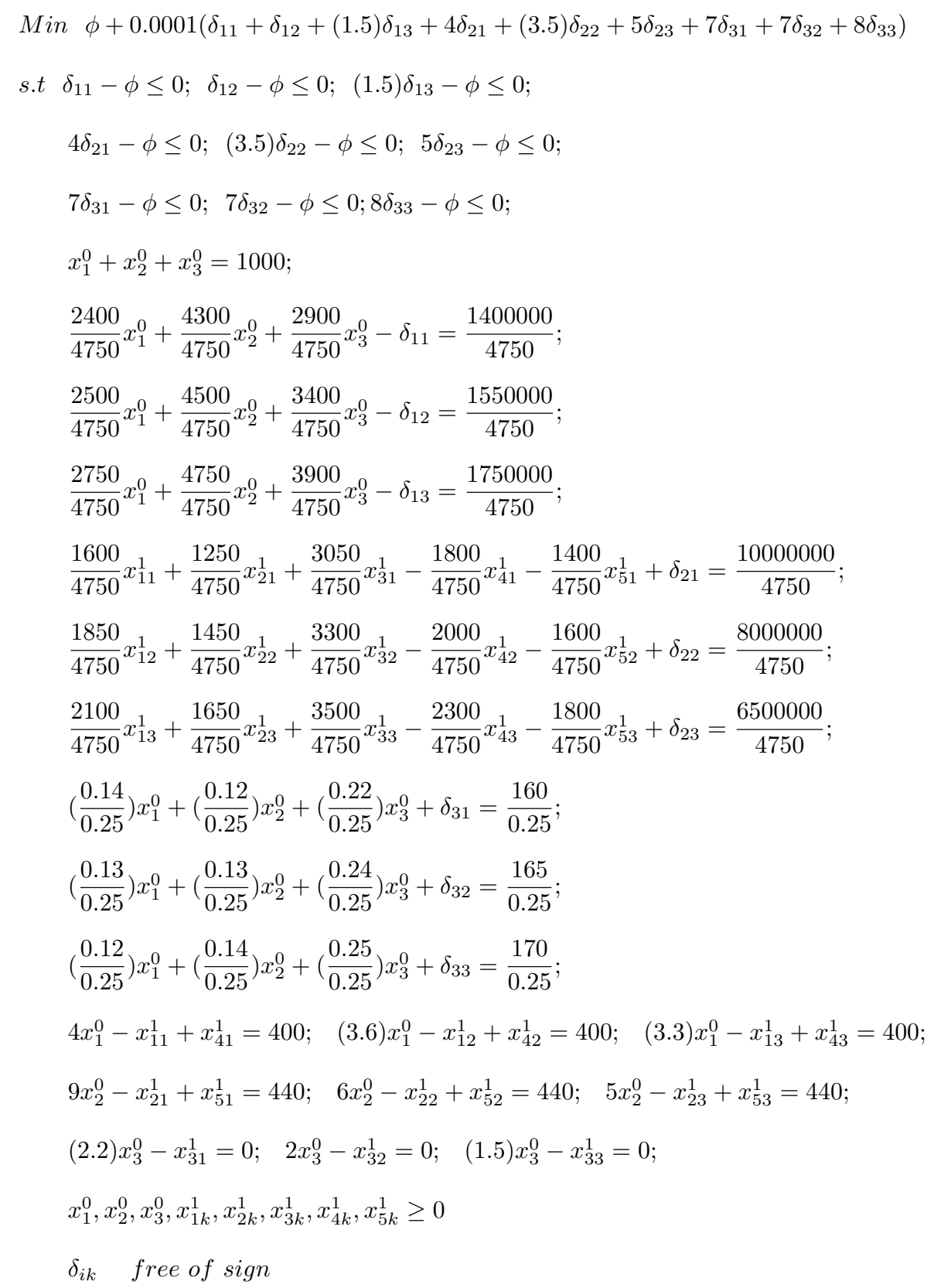

Note that the coefficients in some constraints divided by some numbers(4750 or 0.25 ) to normalising different units. 


\subsubsection{The GP model for 3.5.1}

$$
\begin{aligned}
& \operatorname{Min} \psi=\varphi+\varepsilon \sum_{k=1}^{5}\left[\sum_{i=1}^{2}\left(\delta_{i k}^{+}+\delta_{i k}^{-}\right)\right] \\
& \text {s.t. }-5 x_{1}^{0}+4 x_{2}^{0}+x_{1}^{1}-\delta_{11}^{+}+\delta_{11}^{-}=-15 \\
& x_{1}^{0}+2 x_{2}^{0}+x_{1}^{1}-\delta_{21}^{+}+\delta_{21}^{-}=0 \\
& -3 x_{1}^{0}+(1.5) x_{2}^{0}+x_{2}^{1}-\delta_{12}^{+}+\delta_{12}^{-}=-9 \\
& 2 x_{1}^{0}+3 x_{2}^{0}+x_{2}^{1}-\delta_{22}^{+}+\delta_{22}^{-}=2 \\
& (0.5) x_{1}^{0}+9 x_{2}^{0}+x_{3}^{1}-\delta_{13}^{+}+\delta_{13}^{-}=28 \\
& 2 x_{1}^{0}+x_{2}^{0}+x_{3}^{1}-\delta_{23}^{+}+\delta_{23}^{-}=7 \\
& (0.7) x_{1}^{0}+7 x_{2}^{0}+x_{4}^{1}-\delta_{14}^{+}+\delta_{14}^{-}=16.1 \\
& (2.2) x_{1}^{0}+(1.3) x_{2}^{0}+x_{4}^{1}-\delta_{24}^{+}+\delta_{24}^{-}=9.2 \\
& -7 x_{1}^{0}+4 x_{2}^{0}+x_{5}^{1}-\delta_{15}^{+}+\delta_{15}^{-}=-12 \\
& (1.5) x_{1}^{0}+(2.5) x_{2}^{0}+x_{5}^{1}-\delta_{25}^{+}+\delta_{25}^{-}=13 \\
& x_{1}^{0}+x_{2}^{0}-x_{1}^{1} \leq 4 \\
& x_{1}^{0}-x_{1}^{1} \leq 3 \\
& x_{2}^{0}-x_{1}^{1} \leq 3 \\
& -x_{1}^{0}-x_{2}^{0}-x_{2}^{1} \leq-1 \\
& x_{1}^{0}-x_{2}^{1} \leq 3 \\
& x_{2}^{0}-x_{2}^{1} \leq 3 \\
& x_{1}^{0}+x_{2}^{0}-x_{3}^{1} \leq 5 \\
& -x_{1}^{0}-x_{3}^{1} \leq-2 \\
& -x_{2}^{0}-x_{3}^{1} \leq-3 \\
& -x_{1}^{0}-x_{2}^{0}-x_{4}^{1} \leq-2 \\
& -x_{1}^{0}-x_{4}^{1} \leq-3 \\
& -x_{2}^{0}-x_{4}^{1} \leq-2 \\
& -x_{1}^{0}-x_{2}^{0}-x_{5}^{1} \leq-6 \\
& x_{1}^{0}-x_{5}^{1} \leq 4 \\
& -x_{2}^{0}-x_{5}^{1} \leq-4 \\
& \left(\delta_{i k}^{+}+\delta_{i k}^{-}\right)-\varphi \leq 0 \\
& i=1,2, k=1, . ., 5 \text {; } \\
& \delta_{i k}^{+}, \delta_{i k}^{-}, x_{j}^{0}, x_{k}^{1} \geq 0, \\
& \forall i, j, k \text {. }
\end{aligned}
$$




\subsubsection{Single-scenario models for example 3.5.2}

\subsubsection{Scenario 1 model for example 3.5.2}

$$
\begin{aligned}
& \operatorname{Min} \psi=\varphi+\varepsilon \sum_{i=1}^{2}\left(\delta_{i 1}^{+}+\delta_{i 1}^{-}\right) \\
& \text {s.t. }-5 x_{1}^{0}+4 x_{2}^{0}-\delta_{11}^{+}+\delta_{11}^{-}=-15 \\
& x_{1}^{0}+2 x_{2}^{0}-\delta_{21}^{+}+\delta_{21}^{-}=0 \\
& x_{1}^{0}+x_{2}^{0} \leq 4 \\
& x_{1}^{0} \leq 3 \\
& x_{2}^{0} \leq 3 \\
& \delta_{i 1}-\varphi \leq 0 \quad i=1,2 \text {; } \\
& \delta_{i 1}^{+}, \delta_{i 1}^{-}, x_{j}^{0} \geq 0, \quad i=j=2 .
\end{aligned}
$$

\subsubsection{Scenario 2 model for example 3.5.2}

$$
\begin{aligned}
& \operatorname{Min} \psi=\varphi+\varepsilon \sum_{i=1}^{2}\left(\delta_{i 2}^{+}+\delta_{i 2}^{-}\right) \\
& \text {s.t. }-3 x_{1}^{0}+(1.5) x_{2}^{0}-\delta_{12}^{+}+\delta_{12}^{-}=-9 \\
& 2 x_{1}^{0}+3 x_{2}^{0}-\delta_{22}^{+}+\delta_{22}^{-}=2 \\
& -x_{1}^{0}-x_{2}^{0} \leq-1 \\
& x_{1}^{0} \leq 3 \\
& x_{2}^{0} \leq 3 \\
& \delta_{i 2}-\varphi \leq 0 \quad i=1,2 \\
& \delta_{i 2}^{+}, \delta_{i 2}^{-}, x_{j}^{0} \geq 0, \quad i=j=2 .
\end{aligned}
$$




\subsubsection{Scenario 3 model for example 3.5 .2}

$$
\begin{aligned}
& \operatorname{Min} \psi=\varphi+\varepsilon \sum_{i=1}^{2}\left(\delta_{i 3}^{+}+\delta_{i 3}^{-}\right) \\
& \text {s.t. }(0.5) x_{1}^{0}+9 x_{2}^{0}-\delta_{13}^{+}+\delta_{13}^{-}=28 \\
& 2 x_{1}^{0}+x_{2}^{0}-\delta_{23}^{+}+\delta_{23}^{-}=7 \\
& x_{1}^{0}+x_{2}^{0} \leq 5 \\
& -x_{1}^{0} \leq-2 \\
& -x_{2}^{0} \leq-3 \\
& \delta_{i 3}-\varphi \leq 0 \quad i=1,2 \\
& \delta_{i 3}^{+}, \delta_{i 3}^{-}, x_{j}^{0} \geq 0, \quad i=j=2 .
\end{aligned}
$$

\subsubsection{Scenario 4 model for example 3.5 .2}

$$
\begin{aligned}
& M i n \psi=\varphi+\varepsilon \sum_{i=1}^{2}\left(\delta_{i 4}^{+}+\delta_{i 4}^{-}\right) \\
& \text {s.t. }(0.7) x_{1}^{0}+7 x_{2}^{0}-\delta_{14}^{+}+\delta_{14}^{-}=16.1 \\
& (2.2) x_{1}^{0}+(1.3) x_{2}^{0}-\delta_{24}^{+}+\delta_{24}^{-}=9.2 \\
& -x_{1}^{0}-x_{2}^{0} \leq-2 \\
& -x_{1}^{0} \leq-3 \\
& -x_{2}^{0} \leq-2 \\
& \delta_{i 4}-\varphi \leq 0 \\
& i=1,2 \text {; } \\
& \delta_{i 4}^{+}, \delta_{i 4}^{-}, x_{j}^{0} \geq 0, \\
& i=j=2 .
\end{aligned}
$$




\subsubsection{Scenario 5 model for example 3.5.2}

$$
\begin{aligned}
& \operatorname{Min} \psi=\varphi+\varepsilon \sum_{i=1}^{2}\left(\delta_{i 5}^{+}+\delta_{i 5}^{-}\right) \\
& \text {s.t. }-7 x_{1}^{0}+4 x_{2}^{0}-\delta_{15}^{+}+\delta_{15}^{-}=-12 \\
& (1.5) x_{1}^{0}+(2.5) x_{2}^{0}-\delta_{25}^{+}+\delta_{25}^{-}=13 \\
& -x_{1}^{0}-x_{2}^{0} \leq-6 \\
& x_{1}^{0} \leq 4 \\
& -x_{2}^{0} \leq-4 \\
& \delta_{i 5}-\varphi \leq 0 \\
& i=1,2 \text {; } \\
& \delta_{i 5}^{+}, \delta_{i 5}^{-}, x_{j}^{0} \geq 0, \quad i=j=2 .
\end{aligned}
$$




\section{Chapter 4}

\section{Three-stage Scenario-Based Multi-Criteria Decision Making Structure}

\subsection{Introduction}

As discussed in the previous chapter, our main aim for this study was to deal with deep uncertainty in multi-criteria decision analytic models, especially in long-term strategic planning problems. Moreover, integrating performances under different scenarios with the extended concept of the meta-criteria is also used to motivate for a scenario-based two-stage structure for multi-criteria decision making under deep uncertainty. Utilising scenarios, as a dimension of preference (a component of the meta-criteria) to avoid problems of evaluating probabilities under deep uncertainty was an additional motivation to propose this structure. Scenarios, in this thesis, are utilised to structure and organise the present uncertainty by defining different plausible scenarios for uncertain statements of the problem. Using scenarios as a tool for representing uncertainty in a problem can allow for the DM to think, discuss, and plan for that uncertainty.

In the proposed two-stage structure, the decision-making process has been divided into two parts/stages: In the first part, knowledge about some states of the problem is lacking and this is the initial decision. The second part occurs after unfolding the uncertain states, as an adaptation/recourse decision. Therefore, a more reasonable initial decision, which is common among all plausible scenarios, together with a set of contingent decisions relating to different scenarios, one for every single plausible scenario, is made. However, only the initial decision will be implemented at the first stage while the implementation of the recourse decision is postponed to the second stage, after scenario realisation. Then, the relevant recourse decision, which depends on the unfolded scenario, would be implemented.

As mentioned previously, the proposed two-stage framework can help us reach some parts of our primary purposes. In the two-stage structure, we are looking one step ahead, and postponing part of the decision, which allows for possible adaptation after scenario realisation, if it is ever possible or optimum. The successful experience in applying this scenario-based two-stage structure in dealing with deep uncertainty, by which we get rid of the probabilities' conditions and calculation, motivates us to extend the proposed two-stage structure to longer period problems. Furthermore, in the proposed two-stage structure, we are also capable of evaluating the consequences of the initial decision after the achievement of every plausible scenario. Nevertheless, the effects of the first decision after a longer period, as well as the outcomes of the recourse decisions, need to be investigated in a longer structure.

On the other hand, to deal with deep uncertainty in strategic planning problems, as one of 
our first themes, more than two stages would be needed. Moreover, real processes are continuous in which scenarios are not independent events by stages and continuously unfold, generally with some depending. Note that "stages" here, are artificial to structure thinking of an indefinite future.

In the proposed two-stage framework, as seen in the last section of the previous chapter, there is no need to find a common feasible initial solution that works for all scenarios, including the worst scenario. Rather, it is sufficient for the solution to be "good enough" as a start point that would enable us to construct the best possible combined decisions, together with scenario-related recourse decisions, in every single plausible scenario. Applying this dynamic-robust philosophy to long-term strategic planning problems and finding a less risk-averse robust solution boost our motivations for this chapter.

All the above-mentioned reasons, motivated us to take a step forward, in this infinite future, and extend this methodology to the problems with a longer horizon and provide the capability of dealing with more uncertainty. We were also interested in examining the suitable length of the planning window and stages that could be investigated in this structure. Accordingly, the original purpose of this chapter, and the next one, is to answer the following question: How many stages is it worth looking ahead if the DM is proposed to think of discrete stages $(t=1,2, \ldots)$.

To develop a robust strategy, the following options might be used:

- Purely myopic: Forget future contingencies.

- One step moving horizon: Always plan as if it is a two-stage horizon.

- Multi-step moving horizon: Always plan as if it is a T-stage horizon.

The first option is not logical, and has been rejected by several policy-makers. Furthermore, it is entirely out of the scope of this dissertation. A comparison of the other two options could begin the process of achieving our primary objective which was mentioned above. To avoid too many complexities and to gain better insights into the context without loss of generality, we focus on the three-stage structure and examined it in detail (it can be simply extended to more than three stages by adding more stages if necessary). Therefore, two alternatives that may extend the two-stage structure to a three-stage horizon can be described as follows:

1. Applying another two-stage structure at the end of the first period, i.e. the second twostage structure starting at the end of the first two-stage structure $2 \times$ two-stage. In other words, we can roll the two-stage structure continuously.

2. Extend to a three-stage structure in which the consequences of the first stage recourse decisions, as well as the outcomes of the initial decision after two consecutive periods, are evaluated (looking two steps ahead).

The next section (4.2) introduces in detail a three-stage scenario-based multi-criteria decisionmaking structure as an extension of the proposed two-stage structure. Some essential concepts, such as stages, planning horizon, scenarios, and meta-criteria, have been reviewed, extended, or defined and the mathematical formulations are fully expanded while presenting the solution procedure. In section 4.3, the proposed three-stage structure and its solutions are compared, mathematically and philosophically, while some advantages, disadvantages and the suitable conditions in which these structures should be utilised will be highlighted and discussed. The two-stage moving horizon model concepts, mathematical formulations, some properties, the cost of robustness and comparison with the three-stage structure are all fully debated in section 4.4, which is followed by a sensitivity analysis of a numerical example. The next section (4.5) provides an illustrative example by which the solutions of the full three-stage model is compared with the solutions of the two-stage models. Finally, before the conclusion section in 4.7, the possibility of any improvement in the robustness of the proposed two-stage moving horizon structure is highlighted, and some initial ideas that may help us reach this purpose are provided, as a direction of future study, in section 4.6. 


\subsection{Proposed three-stage structure}

In the three-stage structure, the decision process is divided into three parts/stages during the time horizon which is arbitrarily chosen depending on the problem ${ }^{1}$. These parts/stages can help us discuss, think about, and formulate the model. The stages do not show some specific points in the time horizon and could be adapted to different conditions, as scenarios unfold continuously and the real problems are continuous as well. We use stages to distinguish separate periods of the decision-making process and scenario realisation, i.e. the time that one/some uncertain state(s) of the problem is/are unfolded and the consequences of our previous decision(s) manifest(s) themselves by then.

In other words, each stage demonstrates the time when the uncertain states that could affect the decision-making process are unfolded. At this point, the DM has just obtained enough information about some previously unknown/unsure states and the outcomes of the prior deci$\operatorname{sion}(\mathrm{s})$. Then, he/she can either do something to recover the prior decisions or make/implement another decision(s) while still having no idea about some other states for the next period. In the three-stage structure, two periods of unfolding scenarios are considered during the chosen/given planning horizon which, together with the initial decision, will draw the three-stage structure of decision-making.

Consider a three-stage decision-making process as shown in Figure 4.1. Suppose that an initial decision $\left(\mathbf{x}^{0} \in \mathbf{X}^{0}\right)$ has been made and implemented, firstly at stage ' 0 ' which is the scenario free decision. Then, we need to wait and see what will happen in the future. Suppose $p_{1}$ scenarios $\left(s_{k(1)} \in\left\{s_{1}, \ldots, s_{p(1)}\right\}\right)$ are defined to represent the plausible future space that can be revealed in stage ' 1 '. As soon as knowledge about the uncertain states in the first period (say scenario $k(1)$ unfold) has been obtained, the first contingent decision $\left(\mathbf{x}_{k(1)}^{1} \in \mathbf{X}^{1}\left(\mathbf{x}^{0}, k(1)\right), k(1)=1, \ldots, p(1) ;\right)$ can be implemented. This may include the recourse/adaptation decision to recover the initial decision, in the condition of relevant scenarios, and/or another decision that may count as a new initial decision for the next period. Afterwards, we need to wait again and observe the nature decision (what will happen), about the unknown states (say scenario $k(2)$ revealed in this stage subject to $\left.s_{k^{\prime}(2)}^{\prime} \in\left\{s_{1}^{\prime}, \ldots, s_{p(2)}^{\prime}\right\}\right)$, in the second period of scenario realisation(stage ' 2 '). Finally, the last decision, which is dependent upon new information that has just been received as well as what was revealed in prior stage, can be implemented $\left(\mathbf{x}_{k(1), k(2)}^{2} \in \mathbf{X}^{2}\left(\mathbf{x}^{0}, \mathbf{x}_{k(1)}^{1}, k(2)\right), k(1)=\right.$ $1, \ldots, p(1), k(2)=1, \ldots, p(2) ;)$.

Definition 4.2.1. Meta-decision: In the three-stage structure, we are looking to evaluate and compare some aggregation of the initial decision and two more consecutive decisions concerning all performance measures. From now on, we utilise the term "meta-decision" to indicate that aggregation. In other words, a meta-decision represents a chain of some consecutive decisions (related to different stages of the decision-making process) regarding each meta-criterion. For example, in three-stage structure a meta-decision will be indicated by $\mathbf{X}=\left(\mathbf{x}^{0}, \mathbf{x}_{k(1)}^{1}, \mathbf{x}_{k(1) k(2)}^{2}\right)$.

Moreover, each scenario includes the realisation of scenario $k(1)$ in stage ' 1 ' and the revelation of scenario $k(2)$ in stage ' 2 '. Therefore, for enumerating all scenarios, we need to count all combinations of scenario realisation over both stages (i.e. $p(1) \times p(2)$ ). However, it could be natural that some scenarios in two consecutive periods of scenario realisation may be dependent on the previous scenario, i.e. related to the scenario which was revealed in the last stage, one specific group of plausible scenarios will be considered. Therefore, the total number of plausible combinations could be less than $p(1) \times p(2)$, where $p(1)$ and $p(2)$ are the maximum numbers of plausible scenarios in stage ' 1 ' and ' 2 ', respectively.

Definition 4.2.2. Meta-scenario: The term "meta-scenario" is utilised to describe a plausible combination of scenarios in two scenario realisation stages (it may be showed by paths in a

\footnotetext{
${ }^{1}$ Note that real processes are continuous, and by the time horizon, we try to structure and model a specific part of an infinite future.
} 


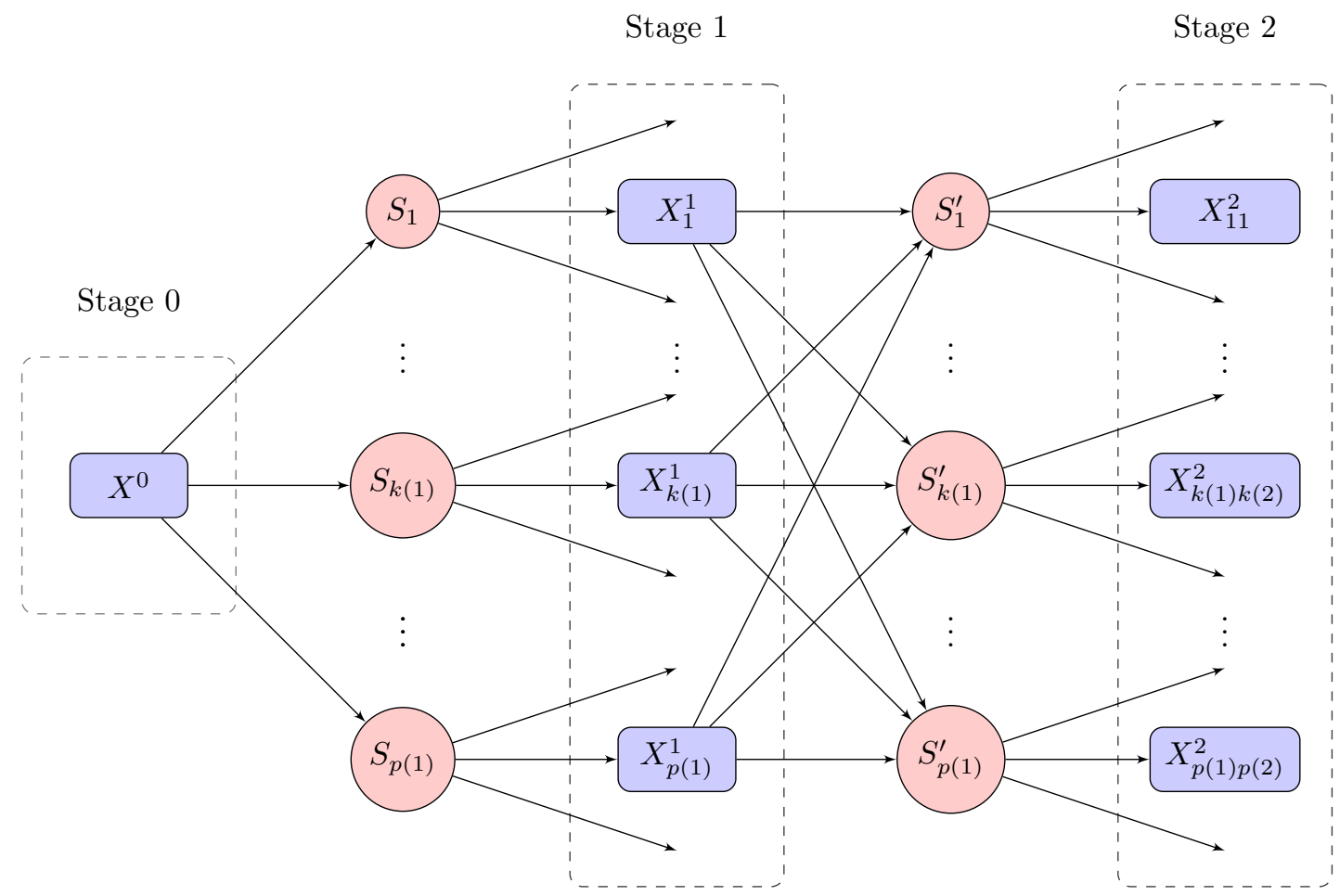

Figure 4.1: Three-stage decision-making process with $p(1) \times p(2)$ scenarios

scenario tree). Then, the number of meta-scenarios, in three-stage structure would be $p \leq$ $p(1) \times p(2)$.

Here, we try to optimise meta-decisions(the aggregation of the initial decision $\left(\mathbf{x}^{0}\right)$ and two dependent (recourse) decisions (i.e. $\mathbf{x}_{k(1)}^{1}, k(1)=1, \ldots, p(1)$ after the revelation of scenario $k(1)$ in stage ' 1 ' and $\mathbf{x}_{k(1), k(2)}^{2}, k(1)=1, \ldots, p(1), k(2)=1, \ldots, p(2)$ after $k(2)$ realisation) which would be defined for each and every plausible meta-scenario (combination of scenarios of these two stages). In fact, we are looking for a dynamic-robust meta-decision in which an initial decision does not need to be feasible for every single meta-scenario, but any meta-decision must be feasible in a relevant meta-scenario. In other words, the DM makes an initial decision then, after scenario revelation in each stage, he/she has a chance to adapt his/her decision, if it is necessary, by a subsequent scenario-dependent decision (recourse). In this structure, we do not utilise models to produce forecasts but try instead identify a reasonable initial decision (which is compensable, whatever happens in the future) and two contingent scenario-dependent decisions for every single meta-scenario that may occur, which is implemented after scenario revelation in the relevant stage (see the schematic of the proposed three-stage approach in Figure 4.2). This process can be summarised as follows:

1. Stage 0: An initial decision is made before any scenario revelation (scenario-free decision).

2. Stage 1: The first contingent/recourse decision is made, which is dependent on scenario $k(1)$ which was revealed in stage ' 1 '(scenario-dependent decision).

3. Stage 2: The second contingent/recourse decision is made, which is dependent on scenario $k(1)$ which unfolded at stage ' 1 ' and the one $(k(2))$ which manifested in stage '2' (scenario-dependent decision). 


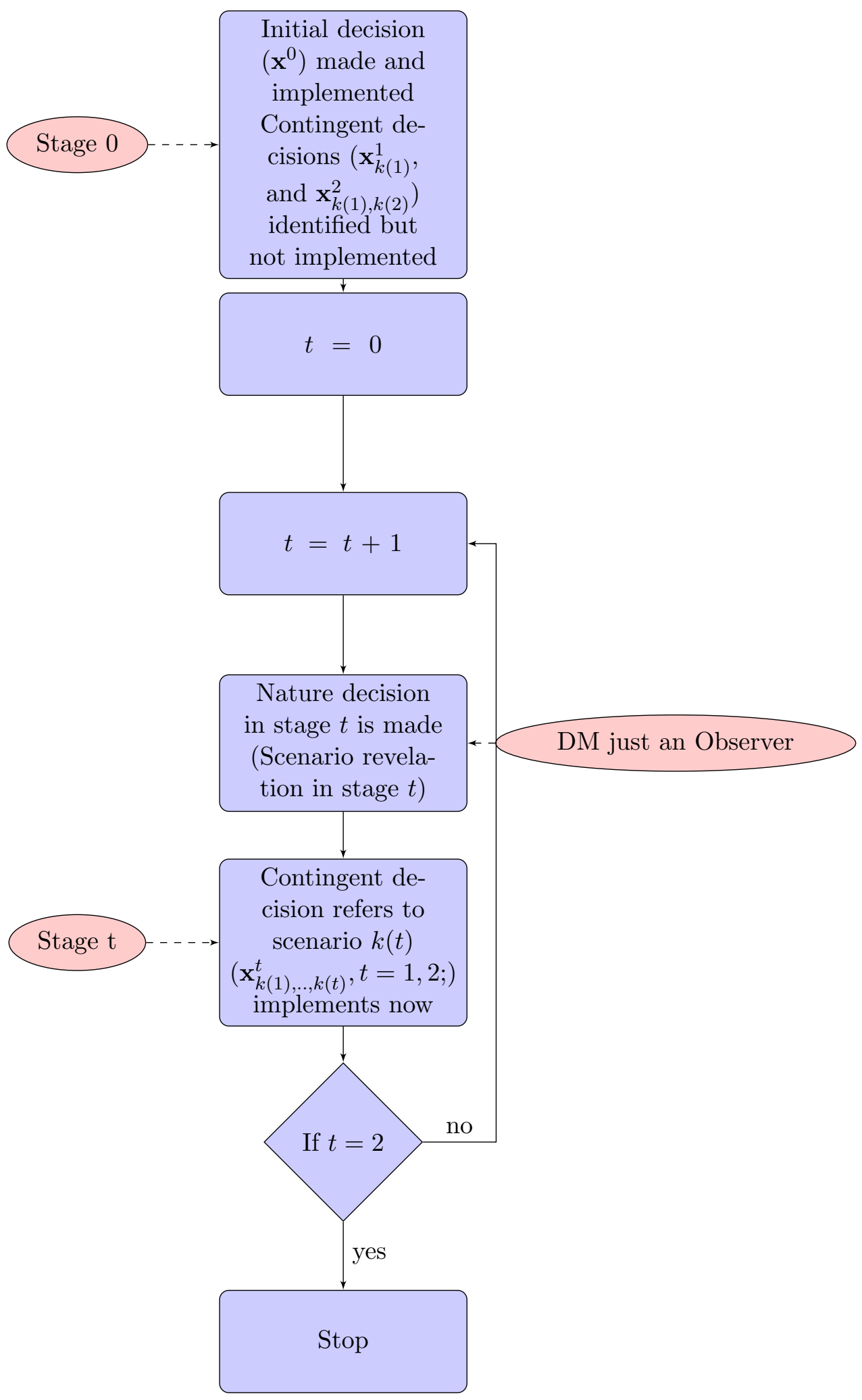

Figure 4.2: the schematic of three-stage decision making process 


\subsubsection{Extension of the concept of meta-criterion in the three-stage structure}

Before formulating the model, the concept of meta-criterion in a three-stage process should be discussed. As mentioned earlier, Stewart et al. [2013] introduced the term "meta-criterion" (criterion-scenario combination) as a dimension of preference in a scenario-based MCDA structure. That is, the performance of each decision regarding each criterion under conditions of each scenario is evaluated and compared. In our three-stage framework, each meta-decision is related to a meta-scenario for which an evaluation needs to be done to find out how well a meta-decision performs concerning each criterion under the conditions of each meta-scenario. It means that each meta-criterion, in the proposed three-stage structure, represents preferences concerning a criterion under conditions relating to a meta-scenario. Therefore, meta-criterion here is defined as the combination of each criterion and meta-scenario in which the number of all meta-criteria is equal to $m \times p$ ( $m$ and $p$ describe the total number of criteria and meta-scenarios, respectively).

Also, meta-scenarios, as a component of the meta-criteria, represent a valid dimension of preference and are utilised to avoid problems of assessing and using probabilities under deep uncertainty. Furthermore, this motivates for the use of a scenario-based three-stage (multi-stage) multi-criteria decision-making framework.

\subsubsection{Mathematical Formulation}

In this section, we use the extended concept of the meta-criteria to formulate a mathematical model that is able to find the optimum set of meta-decisions and expand the concept of robustness and dynamic-robust decision/solution in a three-stage structure.

In the proposed three-stage structure, we break the meta-decision into three parts and defer two parts of the decision long enough to allow for the unfolding of the uncertain parameter(s) (one part after each stage of scenario realisation). A dynamic-robust decision in this threestage structure is a divided decision which contains three subgroups of decisions: the initial decision(s) followed by two other contingent recourse decision(s), in which the meta-decisions are robust across meta-scenarios. Similar to the two-stage decisions, the initial and the first recourse decisions do not need to be optimal or even feasible under conditions of all metascenarios. Instead, they must be good enough, with beneficial foresight, to lead us to the optimal meta-decisions (aggregations of the initial decision(s) and the two future recourse decisions).

According to definitions 4.2 .1 and 4.2 .2 , in a multi-criteria three-stage structure, there are $m \times p$ meta-criteria ${ }^{2}$ that must be optimised, while some uncertainties will unfold after the decision-making step. Therefore, the model includes $m \times p$ objectives in which the performance of the meta-decisions are compared and evaluated subject to the satisfaction of some conditions that could be stage-oriented. Each meta-decision contains an initial decision $\left(\mathrm{x}^{0}\right)$, which is common amongst all $p$ meta-decisions, a first contingent decision $\left(\mathbf{x}_{k(1)}^{1}\right)$, which is scenario-dependent and shared among all meta-decisions referring to $k(1)$, and the second contingent decision $\left(\mathbf{x}_{k(1) k(2)}^{2}\right)$ which is unique for any particular meta-scenario. Hence, there is a meta-decision for each and every meta-scenario that gives us a less risk-averse Pareto optimal dynamic-robust decision, but which is not necessarily optimal for all meta-scenarios, mainly because of the trade-offs between the different meta-scenarios (remember that meta-scenarios are used as a dimension of preferences).

Furthermore, some separate objectives $\left(m_{1}\right)$ may be considered for the first stage. Then, the number of objectives, in comparison with the two-stage model, will increase to $\left(m_{1} \times p(1)\right)+$ $(m \times p)$. Thus, the proposed three-stage multi-objective optimisation problem under uncertainty, which treats each meta-criteria in stage ' 1 ' and stage ' 2 ', can be formulated as follows:

\footnotetext{
${ }^{2}$ Without loss of generality, to simplify models and readability, in all models suppose that $p=$ $p(1) \times p(2)$.
} 


$$
\begin{array}{ll}
O p t_{\left(\mathbf{x}^{\mathbf{0}}, \mathbf{x}_{\mathbf{k}(\mathbf{1})}^{1}, \mathbf{x}_{\mathbf{k}(1) \mathbf{k}(\mathbf{2})}^{2}\right)} \mathbf{F}=\left[f_{i_{1} k(1)}^{1}\left(\mathbf{x}^{0}, \mathbf{x}_{k(1)}^{1}\right) ; f_{i k(1) k(2)}^{2}\left(\mathbf{x}^{0}, \mathbf{x}_{k(1)}^{1}, \mathbf{x}_{k(1) k(2)}^{2}\right)\right] ; & i_{1}=1, \ldots, m_{1} ; i=1, \ldots, m ; \\
& k(1)=1, \ldots, p(1) ; k(2)=1, \ldots, p(2) ; \\
\text { s.t. } \quad u_{r}^{0}\left(\mathbf{x}^{\mathbf{0}}\right) \leq 0, & r=1, \ldots, R_{0} ; \\
u_{r}^{1}\left(\mathbf{x}^{0}, \mathbf{x}_{k(1)}^{1}\right) \leq 0, & k(1)=1, \ldots, p(1) ; \\
& r=R_{0}+1, \ldots, R_{0}+R_{k(1)} ; \\
u_{r}^{2}\left(\mathbf{x}^{0}, \mathbf{x}_{k(1)}^{1}, \mathbf{x}_{k(1) k(2)}^{2}\right) \leq 0, & k(1)=1, \ldots, p(1) ; k(2)=1, \ldots, p(2) ; \\
& r=R_{0}+1, \ldots, R_{0}+R_{k(1) . k(2)} ;
\end{array}
$$

where $\mathbf{x}^{0}=\left(x_{1}^{0}, \ldots, x_{n}^{0}\right) \in \mathbf{X}^{0}$ is an $n$-dimensional initial decision variable vector which is common among all meta-decisions and implemented in stage ' 0 ' before scenario $k(1)$ is revealed and $\mathbf{X}_{0}$ is an initial decision space.

$\mathbf{x}_{k(1)}^{1}=\left(x_{1 k(1)}^{1}, \ldots, x_{n k(1)}^{1}\right) \in \mathbf{X}^{1}\left(\mathbf{x}^{0}, k(1)\right),(k(1)=1, \ldots, p(1))$; is an $n$-dimensional contingent decision vector which is taken and implemented in stage ' 1 ' if scenario $k(1)$ unfolded and $\mathbf{X}^{1}\left(\mathbf{x}^{0}, k(1)\right)$ is a contingent decision space when scenario $k(1)$ manifests.

$\mathbf{x}_{k(1) k(2)}^{2}=\left(x_{1 k(1) k(2)}^{2}, \ldots, x_{n k(1) k(2)}^{2}\right) \in \mathbf{X}^{2}\left(\mathbf{x}^{0}, \mathbf{x}_{k(1)}^{1}, k(2)\right),(k(1)=1, \ldots, p(1) ; k(2)=1, \ldots, p(2)) ;$ is an $n$-dimensional contingent decision vector which is taken in stage ' 2 ' if meta-scenario $k(1) k(2)$ is revealed. Therefore, $\mathbf{X}^{2}\left(\mathbf{x}^{0}, \mathbf{x}_{k(1)}^{1}, k(2)\right)$ is a contingent decision space when meta-scenario $k(1) k(2)$ is manifested. This, and the two previous decisions, would form the meta-decision $\mathbf{X}=\left(\mathbf{x}^{0}, \mathbf{x}_{k(1)}^{1}, \mathbf{x}_{k(1) k(2)}^{2}\right)$.

$f_{i_{1} k(1)}^{1}\left(\mathbf{x}^{0}, \mathbf{x}_{k(1)}^{1}\right),\left(k(1)=1, \ldots, p(1) ; i_{1}=1, \ldots, m_{1}\right)$, is $i_{1}^{\text {th }}$ objective of stage ' 1 ', if scenario $k(1)$ unfolded. These objectives represent the exclusive preferences of the first stage regarding criterion $i_{1}$ under conditions of scenario $k(1)$.

$f_{i k(1) k(2)}^{2}\left(\mathbf{x}^{0}, \mathbf{x}_{k(1)}^{1}, \mathbf{x}_{k(1) k(2)}^{2}\right),(k(1)=1, \ldots, p(1) ; k(2)=1, \ldots, p(2) ; i=1, \ldots, m)$, is $i^{\text {th }}$ meta-criterion/objective function, if meta-scenario $k(1) k(2)$ is revealed. These meta-criteria demonstrate the $i^{\text {th }}$ linear objective function indicating preferences concerning criterion $i$ under conditions pertaining to meta-scenario $k(1) k(2)$.

$u_{r}^{0}\left(\mathbf{x}^{0}\right)$ is the set of inequality constraints in stage ' 0 '.

$u_{r}^{1}\left(\mathbf{x}^{0}, \mathbf{x}_{k(1)}^{1}\right)$ is the set of inequality constraints in stage ' 1 '.

$u_{r}^{2}\left(\mathbf{x}^{0}, \mathbf{x}_{k(1)}^{1}, \mathbf{x}_{k(1) k(2)}^{2}\right)$ is the set of inequality constraints in stage ' 2 '.

The problem consists in optimising $\left[\left(m_{1} \times p(1)\right)+(m \times p(1) \times p(2))\right]$ objectives under $\left(R_{0}+\right.$ $\left.R_{p(1)}+R_{p(1) \times p(2)}\right)$ constraints. Both objectives and constraints can be linear or non-linear; but, in this thesis, we are focusing on the linear problems.

Moreover, to make the model more general, some initial objectives could be considered for stage ' 0 ' (i.e. before the advent of any uncertain parameter $\left(f_{i_{0}}^{0}\left(\mathbf{x}^{0}\right),\left(i_{0}=1, \ldots, m_{0}\right)\right)$, In this case, the model contains optimisation of $\mathbf{F}=\left[f_{i_{0}}^{0}\left(\mathbf{x}^{0}\right) ; f_{i_{1} k(1)}^{1}\left(\mathbf{x}^{0}, \mathbf{x}_{k(1)}^{1}\right) ; f_{i k(1) k(2)}^{2}\left(\mathbf{x}^{\mathbf{0}}, \mathbf{x}_{\mathbf{k}(\mathbf{1})}^{\mathbf{1}}, \mathbf{x}_{\mathbf{k}(\mathbf{1}) \mathbf{k}(\mathbf{2})}^{\mathbf{2}}\right)\right]$. Then, the total number of objectives would be equal to $m_{0}+\left(m_{1} \times p(1)\right)+(m \times p(1) \times p(2))$.

It is also possible that a different number of objectives could be considered in each scenario which could lead us to model the higher levels of uncertainty. In this situation, the problem consists in optimising $\left(m_{0}+\left(m_{1}+\ldots+m_{p(1)}\right)+\left(m_{11}+m_{12}+\ldots+m_{p(1) p(2)}\right)\right)$ objectives. 


\subsubsection{Three-stage Multi-Objective Linear Programming (3ST MOLP)}

Without loss of generality, it can be assumed that the objectives are to be minimised. If the objectives and constraints are linear and each decision is evaluated on $m_{1}$ criteria in the first period and $m$ criteria in the second one, then the problem (4.1) is a Multi-Objective Linear Programming (MOLP) which can be formulated as follows:

$$
\begin{aligned}
& \operatorname{Min} Z_{i_{1} k(1)}=\sum_{j=1}^{n} c_{i_{1} j}^{0} x_{j}^{0}+\left(\sum_{j=1}^{n} c_{i_{1} j k(1)}^{0} x_{j}^{0}+\sum_{j=1}^{n} c_{i_{1} j k(1)}^{1} x_{j k(1)}^{1}\right), \quad i_{1}=1, \ldots, m_{1} \\
& k(1)=1, \ldots, p(1) \\
& \text { Min } Z_{i k(1) k(2)}=\sum_{j=1}^{n} c_{i j}^{0} x_{j}^{0}+\left(\sum_{j=1}^{n} c_{i j k(1)}^{0} x_{j}^{0}+\sum_{j=1}^{n} c_{i j k(1)}^{1} x_{j k(1)}^{1}\right)+\quad i=1, \ldots, m \text {; }
\end{aligned}
$$

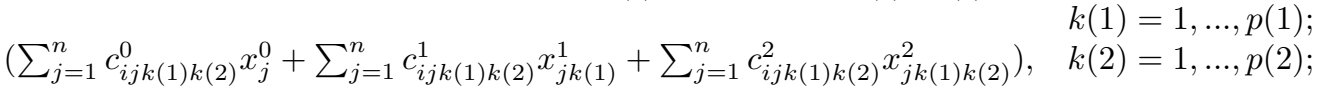

$$
\begin{aligned}
& \text { s.t. } \\
& \sum_{j=1}^{n} a_{r j}^{0} x_{j}^{0} \leq b_{r}^{0}, \\
& \sum_{j=1}^{n} a_{r j k(1)}^{0} x_{j}^{0}+\sum_{j=1}^{n} a_{r j k(1)}^{1} x_{j k(1)}^{1} \leq b_{r k(1)}^{1}, \\
& \sum_{j=1}^{n} a_{r j k(1) k(2)}^{0} x_{j}^{0}+\sum_{j=1}^{n} a_{r j k(1) k(2)}^{1} x_{j k(1)}^{1} \\
& +\sum_{j=1}^{n} a_{r j k(1) k(2)}^{2} x_{j k(1) k(2)}^{2} \leq b_{r k(1) k(2)}^{2}, \\
& x_{j}^{0}, x_{j k(1)}^{1}, x_{j k(1) k(2)}^{2} \geq 0 . \\
& r=1, \ldots, R_{0} \\
& k(1)=1, \ldots, p(1) ; \\
& r=R_{0}+1, \ldots \text {, } \\
& R_{0}+\ldots+R_{k(1)} \\
& k(1)=1, \ldots, p(1) \\
& k(2)=1, \ldots, p(2) \text {; } \\
& r=R_{0}+\ldots+R_{p(1)}+1 \text {, } \\
& \ldots, R_{0}+\ldots+R_{k(1) . k(2)} \text {; } \\
& j=1, \ldots, n \text {; } \\
& k(1)=1, \ldots, p(1) \\
& k(2)=1, \ldots, p(2) \text {. }
\end{aligned}
$$

where $Z_{i_{1}(1)}$ represents the exclusive preferences of the first stage regarding criterion $i_{1}$ under conditions of scenario $k(1) . Z_{i k(1) k(2)}$ demonstrates the $i^{\text {th }}$ linear meta-criterion/objective function indicating preferences concerning criterion $i$ under conditions pertaining to meta-scenario $k(1) k(2)$. The latter group $\left(Z_{i k(1) k(2)}\right)$ contains some initial goals (scenario-free) which will be implemented before the advent of any uncertain parameter $\left(\sum_{j=1}^{n} c_{i j}^{0} x_{j}^{0}\right)$. These meta-criteria also include the consequences of the initial decision and its outcomes (i.e. $\sum_{j=1}^{n} c_{i j k(1)}^{0} x_{j}^{0}+$ $\left.\sum_{j=1}^{n} c_{i j k(1)}^{1} x_{j k(1)}^{1}\right)$ in the second stage if scenario $k(1)$ is revealed. Furthermore, the consequences of the initial decision and the first contingent decision in the third stage under conditions of meta-scenario $k(1) k(2)$, together with the consequences of the last contingent decision will be constructed the last part of these objective functions/meta-criteria. These concepts are presented by $\sum_{j=1}^{n} c_{i j k(1) k(2)}^{0} x_{j}^{0}+\sum_{j=1}^{n} c_{i j k(1) k(2)}^{1} x_{j k(1)}^{1}+\sum_{j=1}^{n} c_{i j k(1) k(2)}^{2} x_{j k(1) k(2)}^{2}$ in the above formulation.

\subsubsection{Solving three-stage multi-objective programming by generalized goal programming (Wierzbicki reference point model)}

As mentioned in the previous chapter, we are using the Generalised Goal Programming (GGP), based on Wierzbicki's works on reference point method ([Wierzbicki, 1982] and [Wierzbicki, 1998]), in which our aim is to minimise the $\mathbf{m}_{\mathbf{1}} \times \mathbf{p}(\mathbf{1})$ performance measures of the first stage 
$\left(Z_{i_{1} k(1)}\right)$ plus $\left(\mathbf{m} \times \mathbf{p}_{\mathbf{1}} \times \mathbf{p}_{\mathbf{2}}\right)$ performance measures $\left(Z_{i k(1) k(2)}\right)$. If we define $m \times p_{1} \times p_{2}$ goals/aspiration levels $\left(g_{i k(1) k(2)}\right)$ for all meta-criteria (corresponding to deviation variables $\left.\delta_{i k(1) k(2)}\right)$, and $m_{1} \times p(1)$ goals $\left(g_{i_{1} k(1)}\right)$ for the deviations from the first-stage objectives, by considering the Generalised Goal Programming approach, associated constraints can be formulated as follows:

$$
\begin{gathered}
Z_{i_{1} k(1)}-\delta_{i_{1} k(1)}=\sum_{j=1}^{n} c_{i_{1} j}^{0} x_{j}^{0}+\left(\sum_{j=1}^{n} c_{i_{1} j k(1)}^{0} x_{j}^{0}+\sum_{j=1}^{n} c_{i_{1} j k(1)}^{1} x_{j k(1)}^{1}\right)-\delta_{i_{1} k(1)}=g_{i_{1} k(1)}, \\
i_{1}=1, . ., m_{1}, k(1)=1, \ldots, p(1)
\end{gathered}
$$

$$
\begin{gathered}
Z_{i k(1) k(2)}-\delta_{i k(1) k(2)}=\sum_{j=1}^{n} c_{i j}^{0} x_{j}^{0}+\left(\sum_{j=1}^{n} c_{i j k(1)}^{0} x_{j}^{0}+\sum_{j=1}^{n} c_{i j k(1)}^{1} x_{j k(1)}^{1}\right)+ \\
\left(\sum_{j=1}^{n} c_{i j k(1) k(2)}^{0} x_{j}^{0}+\sum_{j=1}^{n} c_{i j k(1) k(2)}^{1} x_{j k(1)}^{1}+\sum_{j=1}^{n} c_{i j k(1) k(2)}^{2} x_{j k(1) k(2)}^{2}\right)-\delta_{i k(1) k(2)}=g_{i k(1) k(2)}, \\
i=1, . ., m, k(1)=1, \ldots, p(1) ; k(2)=1, \ldots, p(2)
\end{gathered}
$$

Also, the equivalent generalised goal programming model can be formulated as follows:

$$
\begin{aligned}
& \operatorname{Min} \psi=\operatorname{Max}\left\{\delta_{1}, \delta_{2}\right\}+\epsilon\left[\sum_{k(1)=1}^{p(1)} \sum_{i_{1}=1}^{m_{1}}\left(\omega_{i_{1} k(1)} \delta_{i_{1} k(1)}\right)\right. \\
& \left.+\sum_{k(2)=1}^{p(2)} \sum_{k(1)=1}^{p(1)} \sum_{i=1}^{m}\left(\omega_{i k(1) k(2)} \delta_{i k(1) k(2)}\right)\right] \\
& \text { s.t. } \\
& Z_{i_{1} k(1)}-\delta_{i_{1} k(1)}=g_{i_{1} k(1)}, \\
& Z_{i k(1) k(2)}-\delta_{i k(1) k(2)}=g_{i k(1) k(2)}, \\
& \sum_{j=1}^{n} a_{r j}^{0} x_{j}^{0} \leq b_{r}^{0} \\
& \sum_{j=1}^{n} a_{r j k(1)}^{0} x_{j}^{0}+\sum_{j=1}^{n} a_{r j k(1)}^{1} x_{j k(1)}^{1} \leq b_{r k(1)}^{1}, \\
& \sum_{j=1}^{n} a_{r j k(1) k(2)}^{0} x_{j}^{0}+\sum_{j=1}^{n} a_{r j k(1) k(2)}^{1} x_{j k(1)}^{1} \\
& +\sum_{j=1}^{n} a_{r j k(1) k(2)}^{2} x_{j k(1) k(2)}^{2} \leq b_{r k(1) k(2)}^{2}, \\
& x_{j}^{0}, x_{j k(1)}^{1}, x_{j k(1) k(2)}^{2} \geq 0, \\
& \delta_{i_{1} k(1)}, \delta_{i k(1) k(2)} \text { free of sign. } \\
& i_{1}=1, . ., m_{1} \text {, } \\
& k(1)=1, \ldots, p(1) \text {; } \\
& i=1, . ., m \text {, } \\
& k(1)=1, \ldots, p(1) \text {; } \\
& k(2)=1, \ldots, p(2) \text {; } \\
& r=1, \ldots, R_{0} \\
& k(1)=1, \ldots, p(1) ; \\
& r=R_{0}+1, \ldots, R_{0}+\ldots+R_{k(1)} \text {; } \\
& k(1)=1, \ldots, p(1) \text {; } \\
& k(2)=1, \ldots, p(2) \text {; } \\
& r=R_{0}+R_{k(1)}+1, \ldots, R_{0} \\
& +\ldots+R_{k(1)}+R_{k(1) k(2)} \text {; } \\
& j=1, \ldots, n \text {; } \\
& k(1)=1, \ldots, p(1) \text {; } \\
& \forall i, i_{1}, k(1), k(2) \text {. }
\end{aligned}
$$


where $\quad \delta_{1}=\operatorname{Max}_{i_{1}, k(1)}\left\{\omega_{i_{1} k(1)} \delta_{i_{1} k(1)}\right\}, \quad \delta_{2}=\operatorname{Max}_{i, k(1), k(2)}\left\{\omega_{i k(1) k(2)} \delta_{i k(1) k(2)}\right\}$, $\omega_{i_{1} k(1)} \geq 0,\left(i_{1}=1, . ., m_{1} ; k(1)=1, \ldots, p(1)\right.$, and $\omega_{i k(1) k(2)} \geq 0,(i=1, . ., m ; k(1)=1, \ldots, p(1) ; k(2)=$ $1, \ldots, p(2))$; are the importance weighting of deviations set by the DM. $\epsilon$ is an arbitrarily small positive number.

Moreover, by setting $\phi=\operatorname{Max}\left\{\delta_{1}, \delta_{2}\right\}$, the linear form can be formulated as follows:

$$
\begin{array}{ll}
\text { Min } \psi=\phi+\epsilon\left[\sum_{k(1)=1}^{p(1)} \sum_{i_{1}=1}^{m_{1}}\left(\omega_{i_{1} k(1)} \delta_{i_{1} k(1)}\right)+\right. & \left.\sum_{k(2)=1}^{p(2)} \sum_{k(1)=1}^{p(1)} \sum_{i=1}^{m}\left(\omega_{i k(1) k(2)} \delta_{i k(1) k(2)}\right)\right] \\
\text { s.t. } & \\
\omega_{i_{1} k(1)} \delta_{i_{1} k(1)}-\phi \leq 0, & i_{1}=1, \ldots, m_{1}, k(1)=1, \ldots, p(1) ; \\
\omega_{i k(1) k(2)} \delta_{i k(1) k(2)}-\phi \leq 0, & i=1, . ., m, k(1)=1, \ldots, p(1) ; \\
& k(2)=1, \ldots, p(2) ; \\
Z_{i_{1} k(1)}-\delta_{i_{1} k(1)}=g_{i_{1} k(1)}, & i_{1}=1, . ., m_{1}, k(1)=1, \ldots, p(1) ; \\
Z_{i k(1) k(2)}-\delta_{i k(1) k(2)}=g_{i k(1) k(2)}, & i=1, \ldots, m, k(1)=1, \ldots, p(1) ; \\
& k(2)=1, \ldots, p(2) ; \\
\sum_{j=1}^{n} a_{r j}^{0} x_{j}^{0} \leq b_{r}^{0}, & r=1, \ldots, R_{0} ; \\
\sum_{j=1}^{n} a_{r j k(1)}^{0} x_{j}^{0}+\sum_{j=1}^{n} a_{r j k(1)}^{1} x_{j k(1)}^{1} \leq b_{r k(1)}^{1}, & k(1)=1, \ldots, p(1) ; \\
& r=R_{0}+1, \ldots, R_{0}+\ldots+R_{k(1)} ; \\
\sum_{j=1}^{n} a_{r j k(1) k(2)}^{0} x_{j}^{0}+\sum_{j=1}^{n} a_{r j k(1) k(2)}^{1} x_{j k(1)}^{1}+ & \\
\sum_{j=1}^{n} a_{r j k(1) k(2)}^{2} x_{j k(1) k(2)}^{2} \leq b_{r k(1) k(2)}^{2}, & k(1)=1, \ldots, p(1) ; k(2)=1, \ldots, p(2) ; \\
& r=R_{0}+R_{k(1)}+1, \ldots, R_{0}+\ldots+R_{k(1)}+R_{k(1) k(2)} ; \\
x_{j}^{0}, x_{j k(1)}^{1}, x_{j k(1) k(2)}^{2} \geq 0, & j=1, \ldots, n ; k(1)=1, \ldots, p(1) ; \\
\phi, \delta_{i_{1} k(1)}, \delta_{i k(1) k(2)} \text { free of sign. } & k(2)=1, \ldots, p(2) ; \\
& \forall i, i_{1}, k(1), k(2) .
\end{array}
$$

\subsection{Two-stage $v s$. Three-stage structure}

In the previous chapter, a scenario-based two-stage structure has been introduced to deal with deep uncertainty in an MCDM problem. The successful experience in applying scenarios to treat the deep uncertainty in the proposed two-stage framework and the dynamic-robust decisions that were reached from this approach motivate us to extend the proposed methodology to a longer horizon structure, three-stage, in this chapter. Here, we shall compare these two structures to highlight their similarity and differences, and find out whether there is any merit in going beyond two stages.

In this comparison, there is a fundamental problem with comparability of the two methods because of the different time horizons, as the two-stage model is ignorant of the third stage. This difference is an issue of future flows of objective function performance. Therefore, we need to compare these methods in the same period; then, one may think of two ways of comparison. These two ways are as follows: 
- Comparison between the first stage (initial) solutions

- Iterate the two-stage model starting at the end of the first stage to compare the whole period of the three stages.

Thus, as mentioned above, to be able to compare the results of these two methods, the same time horizon must be considered. Therefore, in this section, the full solution of the threestage model is compared, conceptually and mathematically, with the first stage decisions from a two-stage analysis concentrating on the robustness of the solutions.

On the other hand, comparing the result of the three-stage model with the results of two sequential two-stage models, in a three stage horizon, would be an interesting comparison that led us to introduce "moving horizon models" later in this chapter (see section 4.4) and also will be the basis for Chapter 5. As will be discussed later, the initial decision of the two-stage model will be implemented first. Then, after scenario realisation, another two-stage model will be run, which can cover the decisions of the second and third stage.

\subsubsection{Mathematical distinctions}

We may need to note that, according to the way that we defined, structured, and formulated the two proposed frameworks, the two-stage structure is a part of the three-stage structure, and the three-stage structure extends the two-stage structure for one more stage/period. Therefore, it should be clear that the first part of the optimal solution of the three-stage model must be feasible for the two-stage model.

Theorem 4.3.1. Each feasible solution of the three-stage model (4.2) corresponds to a feasible solution to the two-stage model(3.2).

Proof. Mathematical proof can be simply concluded as the three-stage model includes all the constraints of the two-stage model together with some other restrictions. So, the feasible region of the two-stage model involves the image of the feasible region of the three-stage model in this dimension. Therefore, the relevant part of any feasible solution, and also the related part of the optimal solution, of the three-stage model can satisfy all constraints of the two-stage model and then corresponds to a feasible solution in the two-stage model (Suppose that $\mathbf{X}=\left(\mathbf{x}^{0}, \mathbf{x}^{1}, \mathbf{x}^{2}\right)$ is a feasible solution for the three-stage model, then $\mathbf{X}^{\prime}=\left(\mathbf{x}^{0}, \mathbf{x}^{1}\right)$ will be feasible in the two-stage model).

Corollary 4.3.1.1. The converse is not always true; that is, the feasibility of a solution in the two-stage model does not guarantee the feasibility of the corresponding solution in the three-stage model.

Proof. Obviously, the two-stage solution may not satisfy the additional constraints of the threestage model.

This theorem and corollary indicate the fact that although the initial solutions of the twostage model are the optimal initial solutions of the problem in the first period, the consequences of the plausible scenarios in the following phase can play a fundamental role in the optimality, or the feasibility, of the meta-decision in the combined problem. In other words, although the solution of the two-stage model provides us with the optimality under the uncertain conditions of the first period, it may not be even feasible and recoverable under additional uncertainties and circumstances of the new phase that would be appended in the three-stage structure. Moreover, the three-stage structure, by a more futuristic vision, may suggest a suboptimal initial decision that could make feasible robust meta-decisions for each and every plausible meta-scenarios. This property automatically expands, one more time ${ }^{3}$, the concept of the dynamic-robust solutions for

\footnotetext{
${ }^{3}$ It is worth being reminded here that the proposed two-stage structure is naturally robust-oriented since it looks one step ahead, chooses suboptimal initial decisions, and leaves room for recourse decisions. In this way, it is able to find a dynamic-robust decision under conditions of each plausible scenario.
} 
the three-stage framework that naturally existed in the proposed two-stage structure. Thus, the three-stage model provides us with a more robust initial solution than the two-stage structure. Of course, it may not be optimal in the first stage, but the full dynamic-robust meta-decisions will be both feasible and optimal in the three-stage model.

\subsubsection{Philosophical distinctions}

Philosophically, the proposed two-stage structure always plans and looks one step ahead while the three-stage structure considers the conditions and consequences of two upcoming steps in advance. In the two-stage structure, it does not matter what may happen after the following stage and the model concentrates only on the conditions of the next stage, initial decision's consequences in the next stage and the contingent decisions that must be made at the following stage depend on the unfolding scenario.

On the other hand, the three-stage structure looks further ahead than the two-stage structure and considers, in addition to the above, the consequences of the initial and the first contingent decisions in the last stage as well as the second contingent decisions, depending on the unfolding meta-scenario.

To sum up, ignoring the consequences and likely conditions of long-term decisions cannot be a robust strategic strategy. Therefore, generally, by utilising the three-stage structure, we may expect a more robust/provident decision. This robustness, of course, is dependent upon the complexity of the problem and the possibility of considering more stages. However, more uncertain statements bring more complexity to the problem and boost the computation that may make the efficiency of using the three-stage structure unclear. It is worth explaining here that by the "efficiency" of the three-stage framework in our context, we want to know whether utilising this approach and its solutions can justify the expense of the additional complexity and computation.

In fact, the ability to model more uncertainty, make more robust decisions and consider the longer term consequences of the decisions can be counted as the advantages of the three-stage approach, if it is parsimonious. Since the number of meta-scenarios increases exponentially by considering more stages, using an approach with more stages could result in a model that is too complex, computationally expensive and difficult to solve simply. The two questions we face here are: (1) "Is the dynamic-robust solution using the three-stage model significant enough to warrant the added computation?" (2) "Are there variations to the two-stage model which may work better as the first step analysis and enhance its robustness?"

In section 4.5, these concepts will be reviewed, tested and illustrated using a simple, but not trivial, example. Before defining the example, the next section is dedicated to explaining the two-stage moving horizon model in detail. Mathematical formulations will be provided, and some properties and comparison with the three-stage structure will be illustrated with an example by a sensitivity analysis in 4.5.2. Overall, these comparisons and analyses may help to find the answers to the above questions.

\subsection{Two-stage moving horizon model}

Consider a decision-making problem with three-stage planning horizon that includes three stages of decision-making and two steps of scenario realisation, as shown on the top of Figure 4.3. Suppose that $\mathbf{x}^{0}, \mathbf{x}^{1}$, and $\mathbf{x}^{2}$ present the decision vectors related to each stage, respectively. $S\left(k_{1}\right)$ and $S\left(k_{2}\right)$ indicate the scenario spaces regarding the first and the second steps of scenario revelation, respectively. In a one-step moving horizon model we always plan as if it is a two-stage horizon. This means that, at first, the proposed two-stage model (model 3.2) is used to get the first stage decisions $\left(\mathrm{x}^{0}\right)$, that could be immediately implemented; then we need to wait and see which scenario from $S\left(k_{1}\right)$ will unfold. After that, another two-stage model (model 4.7) starting at the second stage is applied to reach the contingent decisions $\left(\mathbf{x}^{1}\right.$ and $\left.\mathbf{x}^{2}\right)$. That is, the second 
two-stage structure starts at the end of the first two-stage structure $(2 \times$ two - stage $)$. In other words, we roll the two-stage structure continuously.

Note that the recourse decisions $\left(\mathbf{x}^{1}\right)$ provided by the first two-stage model are not implemented, and they may be used as an approximation that will substitute with the initial decisions gained from the new two-stage model applying after scenario realisation in the first period. In other words, the first recourse decisions that have no idea about the next period are not considered. Instead, after iteration of the two-stage model (while the current stage (i.e. stage ' 0 ') moves one step ahead), the recourse decisions change their role and act as the initial decisions of the second two-stage model. This can help us to adapt the decision with new plausible scenarios in the next stage.

We also need to mention that, for sensitivity analysis of all plausible meta-scenarios and to compare the solutions of these two following two-stage models with the three-stage model's solutions, the second two-stage model needs to be run for all plausible scenarios of $S\left(k_{1}\right)$ (one for each plausible scenario in $S\left(k_{1}\right)$ ) to gain all meta-decisions. Figure 4.3 demonstrates how to apply the two-stage moving horizon models and their solutions in comparison with the three-stage model.

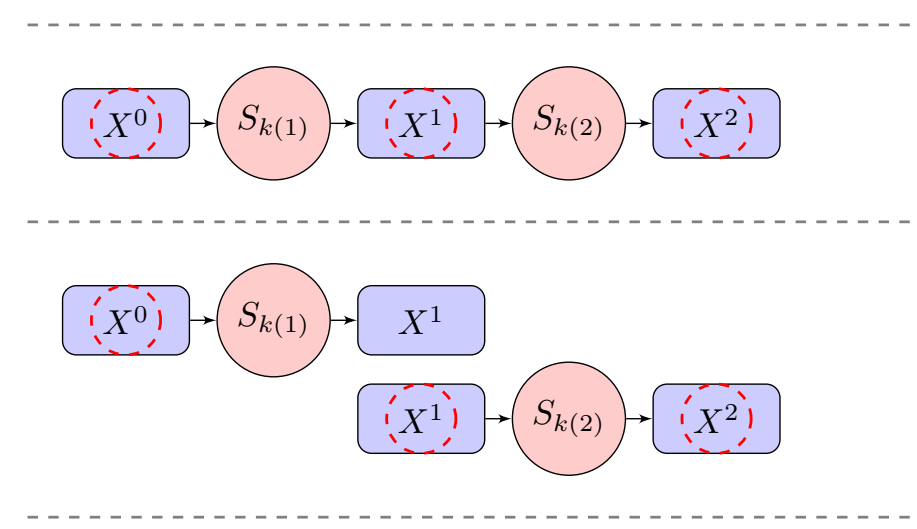

Figure 4.3: Rolling two-stage structure compared to three-stage structure

The formulation of the first two-stage model is similar to the original two-stage model formulation in the last chapter(model 3.2 ${ }^{4}$. The second two-stage models, corresponding to scenario $k(1)$, can be formulated by eliminating the previous stage's variables from the objective functions and their related constraints from the three-stage model (4.2). Furthermore, in some constraints, there might be a need for moving of the previous stage's variables, which are determined by the first two-stage models, from the left side of constraints to the right side with the identified values. Therefore, if scenario $k(1)$ manifested itself at the first step of scenario realisation, the second two-stage model related to this scenario can be formulated as follows:

${ }^{4}$ Note that $k$ in model 3.2 corresponds to $k_{1}$ in model 4.7 and the other patterns in this chapter. 


$$
\begin{array}{ll}
\text { Min } \quad Z_{i k(1)}=\sum_{j=1}^{n} c_{i j k(1)}^{1} x_{j k(1)}^{1}+\sum_{j=1}^{n} c_{i j k(1) k(2)}^{2} x_{j k(1) k(2)}^{2} ; & i=1, \ldots, m ; k(2)=1, \ldots, p(2) ; \\
\text { s.t. } \quad \sum_{j=1}^{n} a_{r j k(1)}^{1} x_{j k(1)}^{1} \leq \beta_{r k(1)}^{1}, & r=1, \ldots, R_{k(1)} ; \\
\sum_{j=1}^{n} a_{r j k(1) k(2)}^{1} x_{j k(1)}^{1}+\sum_{j=1}^{n} a_{r j k(1) k(2)}^{2} x_{j k(1) k(2)}^{2} \leq \beta_{r k(1) k(2)}^{2}, & k(2)=1, \ldots, p(2) ; \\
& r=R_{k(1)}+1, \ldots, R_{k(1)}+\ldots+R_{k(1) . k(2)} ; \\
x_{j k(1)}^{1}, x_{j k(1) k(2)}^{2} \geq 0 . & j=1, \ldots, n ; k(1)=1, \ldots, p(1), \\
& k(2)=1, \ldots, p(2) .
\end{array}
$$

where $\beta_{r k(1)}^{1}=b_{r k(1)}^{1}-\sum_{j=1}^{n} a_{r j k(1)}^{0} x_{j}^{0 *}, \beta_{r k(1) k(2)}^{2}=b_{r k(1) k(2)}^{2}-\sum_{j=1}^{n} a_{r j k(1) k(2)}^{0} x_{j}^{0 *}$ and $x_{j}^{0 *},(j=$ $1, \ldots, n)$; is the optimal solution given from the first two-stage model(model 3.2).

Definition 4.4.1. (Feasible solutions in a two-stage moving horizon model). Vector $\mathbf{X}=\left(\underline{\mathbf{x}}^{0}, \overline{\mathbf{x}}_{k(1)}^{1}, \overline{\mathbf{x}}_{k(1) k(2)}^{2}\right)$ is feasible for a two-stage moving horizon model if it simultaneously satisfies the constrains in both two-stage models(3.2 and 4.7), and also $\forall k=k(1) \in S^{1}$, and $\forall k(2) \in S^{2}, \exists \underline{\mathbf{X}}=\left(\underline{\mathbf{x}}^{0}, \underline{\mathbf{x}}_{k(1)}^{1}\right)$, and $\overline{\mathbf{X}}=\left(\overline{\mathbf{x}}_{k(1)}^{1}, \overline{\mathbf{x}}_{k(1) k(2)}^{2}\right)$, s.t. $\underline{\mathbf{X}}$ and $\overline{\mathbf{X}}$ are feasible for the first(4.17) and the second(4.13) two-stage models, respectively.

Theorem 4.4.1. Any feasible solution (and hence the optimal one) of the two-stage moving horizon model (models 3.2 and 4.7) in a three-stage planning window is a feasible solution for the corresponding three-stage model (model 4.2) and vice versa (i.e. the two-stage moving horizon model is feasible iff the three-stage model is feasible).

Proof. The proof of this theorem can be found in chapter Appendix (see section 4.8.1).

Theorem 4.4.1 shows that any feasible solution to the two-stage moving horizon model is a feasible solution to the three-stage model and vice versa. However, in the first two-stage model, we are not able to consider the consequences of the initial decisions and the conditions of the scenarios in the third stage. Therefore, it is possible that, although there is a feasible solution for both three-stage and two-stage moving horizon models, the optimal initial decisions $\left(\mathbf{x}^{0}\right)$ provided by the first two-stage model may give rise to a situation in which, for some meta-scenarios in the next two-stage model, we cannot find any feasible combination of this initial decision and the two contingent decisions. i.e. although any feasible solution for the $2 \times$ two-stage structure is also feasible for the three-stage structure, there may in fact be no feasible solution found at the second two-stage optimisation. However, this issue will not occur if the three-stage model is applied. This highlights the robustness of the decisions in the proposed three-stage structure and confirms that the initial solutions provided by the proposed three-stage structure are more robust than initial solutions of the two-stage model. For further illustration, let us mathematically describe this specific situation in a simple example.

Example 4.4.1. Suppose that the following constraints indicate the feasible region for the first two-stage optimisation model of a three-stage planning horizon.

$$
\begin{aligned}
& x^{0} \leq 1, \\
& x^{0}-x^{1} \leq 1, \\
& x^{0}, x^{1} \geq 0,
\end{aligned}
$$


Moreover, the limitations of the second two-stage optimisation model for the appropriate two-stage moving horizon model under conditions of a scenario have been explained in 4.9.

$$
\begin{aligned}
& x^{1} \leq 1-x^{0 *}, \\
& x^{1}+x^{2} \leq \frac{1}{2}-x^{0 *}, \\
& x^{1}, x^{2} \geq 0
\end{aligned}
$$

where $x^{0 *}$ is an optimal solution to the first two-stage optimisation model (model 4.8).

Let consider $\overline{\mathbf{X}}=\left(\overline{\mathbf{x}}^{0}, \overline{\mathbf{x}}^{1}\right)=(1,0)$, as a feasible solution for 4.8. If we substitute $x^{0 *}=1$ into 4.9 , then no feasible solution can be found.

$$
\begin{aligned}
& x^{1} \leq 1-1=0 \rightarrow x^{1} \leq 0\left(^{*}\right) \text { and } x^{1}, x^{2} \geq 0 \quad(* *) \\
& \left(^{*}\right) \text { and }(* *) \text { imply that } x^{1}=0, \Rightarrow x^{1}+x^{2}=0+x^{2} \leq \frac{1}{2}-1=-\frac{1}{2} \longrightarrow x^{2} \leq-\frac{1}{2} \text { "Contradiction!". }
\end{aligned}
$$

The feasible region for the corresponding three-stage model can be described by equations in 4.10 which includes simultaneous satisfaction of all the constraints in the first and second two-stage optimisation models (models 4.8 and 4.9).

$$
\begin{aligned}
& x^{0} \leq 1, \\
& x^{0}-x^{1} \leq 1, \\
& x^{0}+x^{1}+x^{2} \leq \frac{1}{2}, \\
& x^{0}, x^{1}, x^{2} \geq 0
\end{aligned}
$$

In contrast to the $2 \times$ two-stage models, too many feasible solutions can be found for the corresponding three-stage model in the same scenario, such as $x^{0}=x^{1}=x^{2}=\frac{1}{n}, \forall n>6$. For example, by setting " $n=\frac{1}{8}$ ", we have

$$
\begin{aligned}
& \frac{1}{8} \leq 1, \\
& \frac{1}{8}-\frac{1}{8}=0 \leq 1, \\
& \frac{1}{8}+\frac{1}{8}+\frac{1}{8}=\frac{3}{8} \leq \frac{1}{2}, \\
& \frac{1}{8} \geq 0 .
\end{aligned}
$$

As discussed in this section, any feasible solution, and thus the optimal one, to the two-stage moving horizon model is a feasible solution to the three-stage model. Also, since the three-stage model is looking further ahead than the two-stage model in each stage, and the constraints in the three-stage model are simultaneously satisfied, then the optimal solution of the three-stage model is no worse than the optimal solution of the two-stage moving horizon model. Therefore, the three-stage models must find a better, or at least the same, goal achievement compared to the two-stage model.

Overall it is shown that the decisions provided by the three-stage model are more robust than those gained from the two-stage moving horizon model, as the three-stage structure considers the consequences of the decisions of the initial stages and the conditions of different meta-scenarios with a longer future vision. Furthermore, by applying the three-stage model, we can expect better goal achievements in comparison with the two consecutive two-stage models. Although these advantages may be enough for some decision makers to utilise the three or even the extended multi-stage structure of the proposed framework, we are still concerned about the challenges of 
computationally expensive situations that may arise in some real-life problems. We are still looking for an answer to our initial question about the cost of these superiorities (the efficiency of the three-stage structure). To investigate this possible issue, in section 4.5.2, a sensitivity analysis has been carried out in example 4.5.1.

\subsection{Illustrative example (Sequential Investment).}

Example 4.5.1. Here, a simple, but not trivial, hypothetical example is presented that will be used for some comparisons in this and following chapters. We believe that this simple example could be a suitable example for illustration and comparison of the different aspects of the proposed two- and three-stage structures.

Suppose that there are five investment options $I_{1}, \ldots, I_{5}$ (for example, Gold, Equities, Bonds, US Dollar, and Property)which differ in terms both of risk and of growth of funds. Furthermore, suppose that an amount of R1 000000 is available for each investment.

Assume that there are five states $S_{1}, \ldots, S_{5}$ (high inflation, stagflation, baseline inflation, deflation, and low inflation) in any one stage which represent the set of plausible scenarios ${ }^{5}$ which depend on the scenario in previous stage, with the following transition possibilities:

\begin{tabular}{cccccc}
\hline Previous stage & \multicolumn{5}{c}{ State at next stage } \\
\cline { 2 - 6 } State & $S_{1}$ & $S_{2}$ & $S_{3}$ & $S_{4}$ & $S_{5}$ \\
\hline$S_{1}$ & $\checkmark$ & $\checkmark$ & & & \\
$S_{2}$ & $\checkmark$ & $\checkmark$ & $\checkmark$ & & \\
$S_{3}$ & & $\checkmark$ & $\checkmark$ & $\checkmark$ & \\
$S_{4}$ & & & $\checkmark$ & $\checkmark$ & $\checkmark$ \\
$S_{5}$ & & & & $\checkmark$ & $\checkmark$ \\
\hline
\end{tabular}

Table 4.1: Possible transition between different scenarios

The anticipated growth in funds over each state have been portrayed in Table 4.2. For each investment option, there is an opportunity to withdraw part of the investment as cash in each stage which could be spent for current costs or this money could be transferred to another investment option for re-investment, but some percentages of the total fund will be lost upon transfer between investments, and this loss could be state-related as shown in tables 4.34.7. Otherwise, the money remains until the next investment maturity at the next stage. No switching of funds between investment options is ever possible between stages.

\begin{tabular}{cccccc}
\hline & \multicolumn{5}{c}{ Growth under scenario } \\
\cline { 2 - 6 } Investment & $S_{1}$ & $S_{2}$ & $S_{3}$ & $S_{4}$ & $S_{5}$ \\
\hline$I_{1}$ & $-20 \%$ & $+4 \%$ & $+16 \%$ & $+20 \%$ & $+50 \%$ \\
\hline$I_{2}$ & $-2 \%$ & $+8 \%$ & $+11.5 \%$ & $+20 \%$ & $+30 \%$ \\
\hline$I_{3}$ & $+8 \%$ & $+8.5 \%$ & $+9 \%$ & $+9.5 \%$ & $+10 \%$ \\
\hline$I_{4}$ & $+4 \%$ & $+7 \%$ & $+12 \%$ & $+16 \%$ & $+20 \%$ \\
\hline$I_{5}$ & $-15 \%$ & $+6 \%$ & $+15 \%$ & $+20 \%$ & $+35 \%$ \\
\hline
\end{tabular}

Table 4.2: Percentage growths for each investment under each scenario

${ }^{5}$ Note that to simulate deep uncertainty we shall not specify scenario's probabilities 


\begin{tabular}{|cccccc|c|}
\hline \multirow{2}{*}{$\begin{array}{c}\text { Transfer } \\
\text { from }\end{array}$} & \multicolumn{5}{c|}{ to } & \\
\cline { 2 - 6 } & $I_{1}$ & $I_{2}$ & $I_{3}$ & $I_{4}$ & $I_{5}$ & Withdrawal \\
\hline$I_{1}$ & 0 & $-2.5 \%$ & $-3 \%$ & $-3 \%$ & $-2 \%$ & $-3 \%$ \\
\hline$I_{2}$ & $-0.05 \%$ & 0 & $-1 \%$ & $-0.1 \%$ & $-0.1 \%$ & $-0.3 \%$ \\
\hline$I_{3}$ & $-0.01 \%$ & $-0.1 \%$ & 0 & $-0.01 \%$ & $-0.01 \%$ & $-0.1 \%$ \\
\hline$I_{4}$ & $-0.01 \%$ & $-0.01 \%$ & $-0.8 \%$ & 0 & $-0.01 \%$ & $-0.2 \%$ \\
\hline$I_{5}$ & $-0.1 \%$ & $-2.5 \%$ & $-3 \%$ & $-3 \%$ & 0 & $-2.5 \%$ \\
\hline
\end{tabular}

Table 4.3: Percentage of loss of funds (penalty cost) for transferring between each pair of investment and withdrawal in state $S_{1}$

\begin{tabular}{|cccccc|c|}
\hline \multirow{2}{*}{$\begin{array}{c}\text { Transfer } \\
\text { from }\end{array}$} & \multicolumn{5}{c|}{ to } & \\
\cline { 2 - 6 } & $I_{1}$ & $I_{2}$ & $I_{3}$ & $I_{4}$ & $I_{5}$ & Withdrawal \\
\hline$I_{1}$ & 0 & $-1 \%$ & $-1.2 \%$ & $-1.0 \%$ & $-0.7 \%$ & $-2 \%$ \\
\hline$I_{2}$ & $-0.5 \%$ & 0 & $-1.0 \%$ & $-0.5 \%$ & $-0.3 \%$ & $-0.4 \%$ \\
\hline$I_{3}$ & $-0.7 \%$ & $-0.2 \%$ & 0 & $-0.01 \%$ & $-0.2 \%$ & $-0.3 \%$ \\
\hline$I_{4}$ & $-0.5 \%$ & $-1 \%$ & $-1.5 \%$ & 0 & $-0.1 \%$ & $-0.4 \%$ \\
\hline$I_{5}$ & $-0.2 \%$ & $-1 \%$ & $-1.5 \%$ & $-0.1 \%$ & 0 & $-1.5 \%$ \\
\hline
\end{tabular}

Table 4.4: Percentage of loss of funds (penalty cost) for transferring between each pair of investment and withdrawal in state $S_{2}$

\begin{tabular}{|cccccc|c|}
\hline \multirow{2}{*}{$\begin{array}{c}\text { Transfer } \\
\text { from }\end{array}$} & \multicolumn{5}{c|}{ to } & \\
\cline { 2 - 6 } & $I_{1}$ & $I_{2}$ & $I_{3}$ & $I_{4}$ & $I_{5}$ & Withdrawal \\
\hline$I_{1}$ & 0 & $-0.4 \%$ & $-0.5 \%$ & $-0.3 \%$ & $-1.0 \%$ & $-1.0 \%$ \\
\hline$I_{2}$ & $-1.1 \%$ & 0 & $-0.2 \%$ & $-0.01 \%$ & $-1.1 \%$ & $-1.2 \%$ \\
\hline$I_{3}$ & $-1.2 \%$ & $-1 \%$ & 0 & $-0.3 \%$ & $-1.0 \%$ & $-2 \%$ \\
\hline$I_{4}$ & $-1.1 \%$ & $-1.5 \%$ & $-0.7 \%$ & 0 & $-1.0 \%$ & $-2 \%$ \\
\hline$I_{5}$ & $-0.8 \%$ & $-0.3 \%$ & $-0.3 \%$ & $-0.2 \%$ & 0 & $-0.8 \%$ \\
\hline
\end{tabular}

Table 4.5: Percentage of loss of funds (penalty cost) for transferring between each pair of investment and withdrawal in state $S_{3}$

\begin{tabular}{|cccccc|c|}
\hline \multirow{2}{*}{$\begin{array}{c}\text { Transfer } \\
\text { from }\end{array}$} & \multicolumn{5}{c|}{ to } & \\
\cline { 2 - 6 } & $I_{1}$ & $I_{2}$ & $I_{3}$ & $I_{4}$ & $I_{5}$ & Withdrawal \\
\hline$I_{1}$ & 0 & $-0.01 \%$ & $-0.01 \%$ & $-0.01 \%$ & $-0.5 \%$ & $-0.1 \%$ \\
\hline$I_{2}$ & $-2 \%$ & 0 & $-0.1 \%$ & $-0.1 \%$ & $-2 \%$ & $-1.5 \%$ \\
\hline$I_{3}$ & $-3 \%$ & $-2.5 \%$ & 0 & $-0.7 \%$ & $-3 \%$ & $-2.5 \%$ \\
\hline$I_{4}$ & $-3 \%$ & $-2 \%$ & $-0.1 \%$ & 0 & $-3 \%$ & $-2.5 \%$ \\
\hline$I_{5}$ & $-5 \%$ & $-0.01 \%$ & $-0.01 \%$ & $-0.01 \%$ & 0 & $-0.1 \%$ \\
\hline
\end{tabular}

Table 4.6: Percentage of loss of funds (penalty cost) for transferring between each pair of investment and withdrawal in state $S_{4}$

\begin{tabular}{|cccccc|c|}
\hline \multirow{2}{*}{$\begin{array}{c}\text { Transfer } \\
\text { from }\end{array}$} & \multicolumn{5}{c|}{ to } & \\
\cline { 2 - 6 } & $I_{1}$ & $I_{2}$ & $I_{3}$ & $I_{4}$ & $I_{5}$ & Withdrawal \\
\hline$I_{1}$ & 0 & $-0.01 \%$ & $-0.01 \%$ & $-0.01 \%$ & $-1.5 \%$ & $-0.2 \%$ \\
\hline$I_{2}$ & $-1.5 \%$ & 0 & $-0.05 \%$ & $-0.1 \%$ & $-2.5 \%$ & $-1.5 \%$ \\
\hline$I_{3}$ & $-3 \%$ & $-2.5 \%$ & 0 & $-1 \%$ & $-3 \%$ & $-2.5 \%$ \\
\hline$I_{4}$ & $-2.5 \%$ & $-2 \%$ & $-0.1 \%$ & 0 & $-3 \%$ & $-2.5 \%$ \\
\hline$I_{5}$ & $-0.01 \%$ & $-0.01 \%$ & $-0.01 \%$ & $-0.01 \%$ & 0 & $-0.1 \%$ \\
\hline
\end{tabular}

Table 4.7: Percentage of loss of funds (penalty cost) for transferring between each pair of investment and withdrawal in state $S_{5}$ 
Moreover, two criteria $\left(C_{n}, n=1,2 ;\right)$ have been considered as follows:

1. $\mathbf{C}_{\mathbf{1}}$ : Maximising the desirable level of total funds available after withdrawals.

2. $\mathbf{C}_{2}$ : Maximising the consumption at each stage (i.e. at the end of the stage) between an absolute minimum and a desirable maximum, with goals that may be state-dependent.

Also, the goals depend on different states, and stages are represented in Table 4.8.

\begin{tabular}{|c|c|c|c|c|c|c|}
\hline \multirow[b]{2}{*}{ Goals } & & \multicolumn{5}{|c|}{ Plausible states (scenarios) } \\
\hline & & $S_{1}$ & $S_{2}$ & $S_{3}$ & $S_{4}$ & $S_{5}$ \\
\hline \multirow{2}{*}{ Stage '0’ } & $g_{1}^{0}$ & 5 & 5.25 & 5.5 & 6 & 7 \\
\hline & $g_{2}^{0}$ & 0.5 & 0.5 & 0.75 & 1 & 1.5 \\
\hline \multirow{2}{*}{ Stage ' 1 ' } & $g_{1}^{1}$ & 6 & 6.5 & 7 & 7.5 & 9 \\
\hline & $g_{2}^{1}$ & 0.5 & 0.5 & 0.75 & 1 & 1.5 \\
\hline \multirow{2}{*}{ Stage '2' } & $g_{1}^{2}$ & 7 & 7.5 & 8 & 9 & 11 \\
\hline & $g_{2}^{2}$ & 0.5 & 0.5 & 0.75 & 1 & 1.5 \\
\hline
\end{tabular}

Table 4.8: Desirable levels of total remained funds(million Rands) after consumption in each state

Suppose that, currently, we are facing the economic situation of baseline inflation (i.e. state $\left.S_{3}\right)$. Then, in the two-stage structure scenarios are collected from $K_{1}=\left\{S_{2}, S_{3}, S_{4}\right\}$. However, in the three-stage structure, meta-scenarios can be set as follows: $k(1) \in K_{1}=\left\{S_{2}, S_{3}, S_{4}\right\}$ and $k(2) \in K_{2}$ but $K_{2}$ would be dependent upon $K_{1}$. If $K_{1}=S_{2}$ then $K_{2}=\left\{S_{1}, S_{2}, S_{3}\right\}$ and for $K_{1}=S_{3}$ and $K_{1}=S_{4}, K_{2}$ would be respectively equal to $\left\{S_{2}, S_{3}, S_{4}\right\}$ and $\left\{S_{3}, S_{4}, S_{5}\right\}$. Figure 4.4 describes and compares scenarios of the two-stage structure and meta-scenarios of the three-stage structure as well as their stages and solutions.

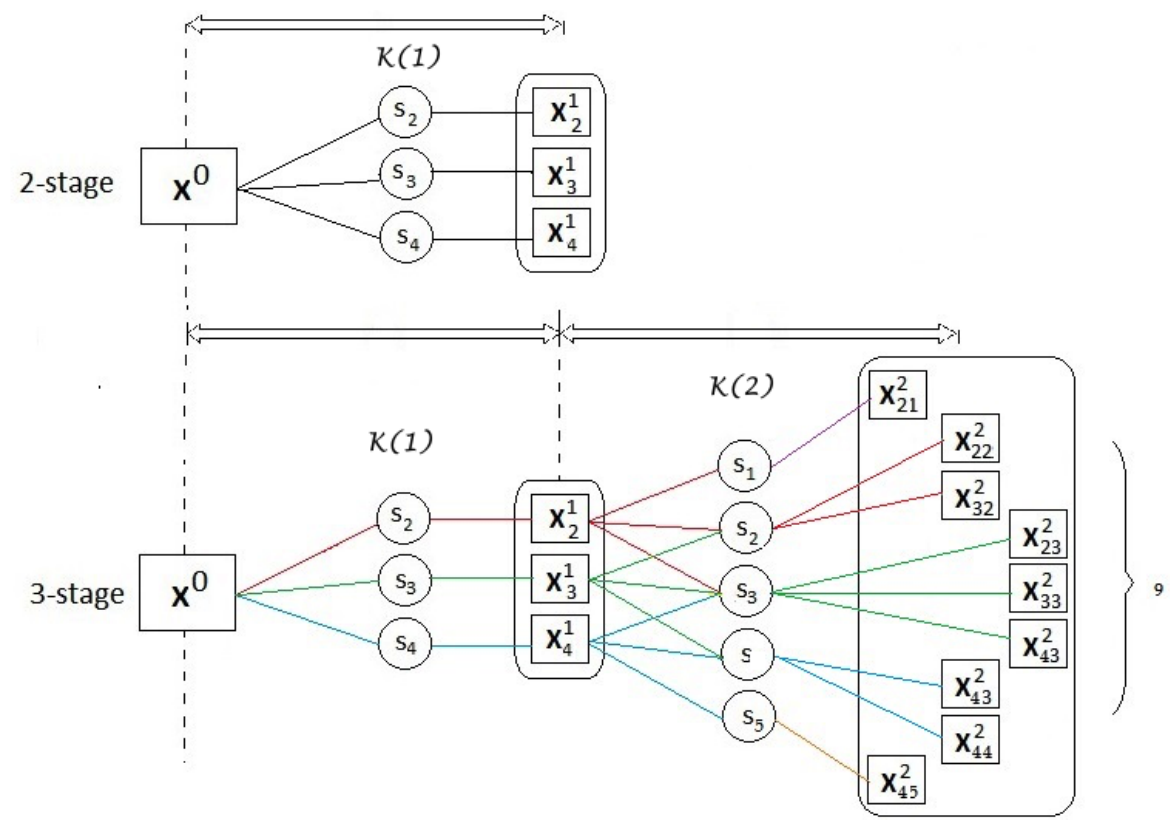

Figure 4.4: Scenarios of the two-stage structure in comparison with meta-scenarios of the three-stage structure 
Accordingly, there are three scenarios in a two-stage structure that could establish six metacriteria in combination with two existing criteria. In a three-stage structure, each of these three scenarios would be followed by a different group of three plausible scenarios that will construct nine meta-scenarios in which the performance measures must be evaluated and compared regarding the two criteria. In other words, alternative decisions must be evaluated in terms of all eighteen (six, in two-stage structure) meta-criteria which will give us nine(three) meta-decisions. Although finding the best group of nine (three) meta-decisions that simultaneously satisfy all eighteen(six) meta-criteria is, generally, impossible ${ }^{6}$, a Pareto-optimum decision would be reached by solving the three-stage(two-stage) model. Therefore, the three-stage multi-objective optimisation model for example 4.5.1 can be formulated as follows:

$$
\begin{array}{lll}
\operatorname{Max} Z_{1 k(1) k(2)}=\sum_{j=1}^{5} \sum_{i=1}^{5}\left(x_{i j}^{0}+x_{i j k(1)}^{1}\right)+\sum_{i=1}^{5} x_{i 7 k(1) k(2)}^{2} & \forall k(1), k(2) \\
\text { Max } Z_{2 k(1) k(2)}=\sum_{i=1}^{5}\left(x_{i 6}^{0}+x_{i 6 k(1)}^{1}+x_{i 6 k(1) k(2)}^{2}\right) & \forall k(1), k(2) \\
\text { s.t. } & &
\end{array}
$$

(Funds balance constraints)

$$
\begin{array}{ll}
\sum_{j=1}^{6}\left(1+p_{i j}\right) x_{i j}^{0}=b_{i}^{0}, & i=1, \ldots, 5 ; \\
\sum_{j=1}^{6}\left(1+p_{i j}\right) x_{i j k(1)}^{1}=\sum_{j=1}^{5}\left(1+c_{i k(1)}\right) x_{j i}^{0} & \forall i, k(1) ; \\
\left(1+p_{i 6}\right) x_{i 6 k(1) k(2)}^{2}+x_{i 7 k(1) k(2)}^{2}=\sum_{i=1}^{5}\left(1+c_{j k(1) k(2)}\right) x_{j i k(1)}^{1} & \forall i, k(1), k(2) ;
\end{array}
$$

\section{(Minimum withdrawal constraints)}

$$
\begin{array}{ll}
\sum_{i=1}^{5} x_{i 6}^{0} \geq b_{6}^{0}, & \\
\sum_{i=1}^{5} x_{i 6 k(1)}^{1} \geq b_{6 k(1)}^{1}, & \forall k(1) ; \\
\sum_{i=1}^{5} x_{i 6 k(1) k(2)}^{0} \geq b_{6 k(1) k(2)}^{2}, & \forall k(1), k(2) ; \\
(N \text { on }- \text { negativity constraints }) & \\
x_{i j}^{0}, x_{i j k(1)}^{1}, x_{i 6 k(1) k(2)}^{2}, x_{i 7 k(1) k(2)}^{2} \geq 0 . & i=1, \ldots, 5 ; j=1, \ldots, 6 ; \\
& \forall k(1), k(2) .
\end{array}
$$

Note that, in this example, no separate goals are assumed for the initial and middle stages, and only the overall targets have been considered. This means that only the overall/final results are important for the DM and he/she must accept some suboptimal values for the initial and

\footnotetext{
${ }^{6}$ This is mainly due to the conditions of multi-criteria decision-making problems in which any metadecision has better efficiency/performance on some meta-criteria while reflecting a lesser efficiency on the others that represent the trade-offs between performances on each meta-criteria.
} 
middle stages of the process if it can help to improve the final achievements. In other words, this three-stage model naturally puts more weight on the latter stages. More discussions concerning the differences and effects of setting specific goals for different stages will be presented later.

By eliminating the third stage and its relevant states and variables from the three-stage model, the two-stage multi-objective optimisation model for the given example can be formulated (see model 4.15 in Chapter Appendix 4.8.2). Parameters and decision variables that are utilised to formulate the two- and three-stage models are listed in the Chapter Appendix 4.18 as well as the relevant GP model for both (see 4.16 and 4.18 in Chapter Appendix 4.8.3 and 4.8.5).

It can be clearly seen that, by comparing the two- and three-stage models, any variation of states in the third stage will have no effect on the two-stage initial decision mainly because there is not any third stage's variable in the two-stage model. For example, the two-stage model has no idea about state $S_{1}$ and $S_{5}$ and the initial decision on this model, in some cases, may not work well enough in the future if one of those states unfolded. Such a variation may have some influences in the initial decision of the three-stage model. As propounded in 4.3.1, the future decisions might be affected by current decisions in a continues problem. Therefore, considering different plausible futures and consequences of current decisions on each future that could be provided by the three-stage structure seems helpful in such a problem. This also generates a more robust initial solution than the two-stage structure. Of course, it may not be optimal in the first stage, but the full dynamic-robust meta-decisions will be both feasible and optimal to the three-stage model. Therefore, with no doubt, it must find better, or at least the same, goal achievement compared to the two-stage model. The next section is allocated to more numerical comparison to illustrate these concepts.

\subsubsection{Numerical comparison of two-stage model approximation and full three-stage solution}

As mentioned in section 4.3, to be able to compare the results of these two methods, the same time horizon must be considered. The results of the two-stage model would surely be on the Pareto-frontier at this stage, which leaves us no choice of any comparison. However, comparing the result of the three-stage model with the results of the two following two-stage models, in a three stage horizon, would be an interesting comparison. To do this, firstly, the initial decision of the two-stage model is implemented. Then, after scenario realisation, another two-stage model will be run, which can cover the decisions of the second and third stage. To evaluate and compare the solutions of these two following two-stage models with three-stage model's solutions, we need to run the second two-stage model three times, one for each plausible scenario, to produce nine meta-decisions for nine meta-scenarios.

In other words, we will suppose that one scenario revealed in the first scenario realisation section; say, for example, $S_{k(1)}$ unfolded. Then, the consequences of the initial decision under conditions of state $S_{k(1)}$ are simulated and the second two-stage model with the new amount of money in each investment will be considered. Therefore, the model will provide us with a particular investment decision for each plausible scenario in this stage (stage ' 1 '). Thus, there are three different investment suggestions depending on three plausible scenarios $\left(S_{k(1)} ; k(1)=\left\{S_{2}, S_{3}, S_{4}\right\}\right)$ in this stage where the relevant decision will be implemented after scenario realisation. Thereafter, a new two-stage model needs to be run for each of them. The initial decisions of these new two-stage models will consider as the first contingent decision $\left(X_{2}^{1}, X_{3}^{1}\right.$, and $\left.X_{4}^{1}\right)$, while the contingent decisions of these two-stage models will represent the second contingent decisions in the three-stage structure $\left(X_{21}^{2}, \ldots, X_{45}^{2}\right.$ in figure 4.5$)$.

Accordingly, as shown in figure 4.5 in example 4.5.1, if $S_{2}$ is revealed then solution $X_{2}^{1}$ will implemented and then the set of plausible scenarios in the next period starting at this stage will be $\left\{S_{1}, S_{2}, S_{3}\right\} . \quad\left\{S_{2}, S_{3}, S_{4}\right\}$ determines the set of plausible scenarios in the next stage if $S_{3}$ unfolds and $X_{3}^{1}$ is executed. Also, in the case of $S_{4}$ realisation, $X_{4}^{1}$ will be performed and the plausible scenarios can be presented by $\left\{S_{3}, S_{4}, S_{5}\right\}$. Thus, by solving these three two-stage 


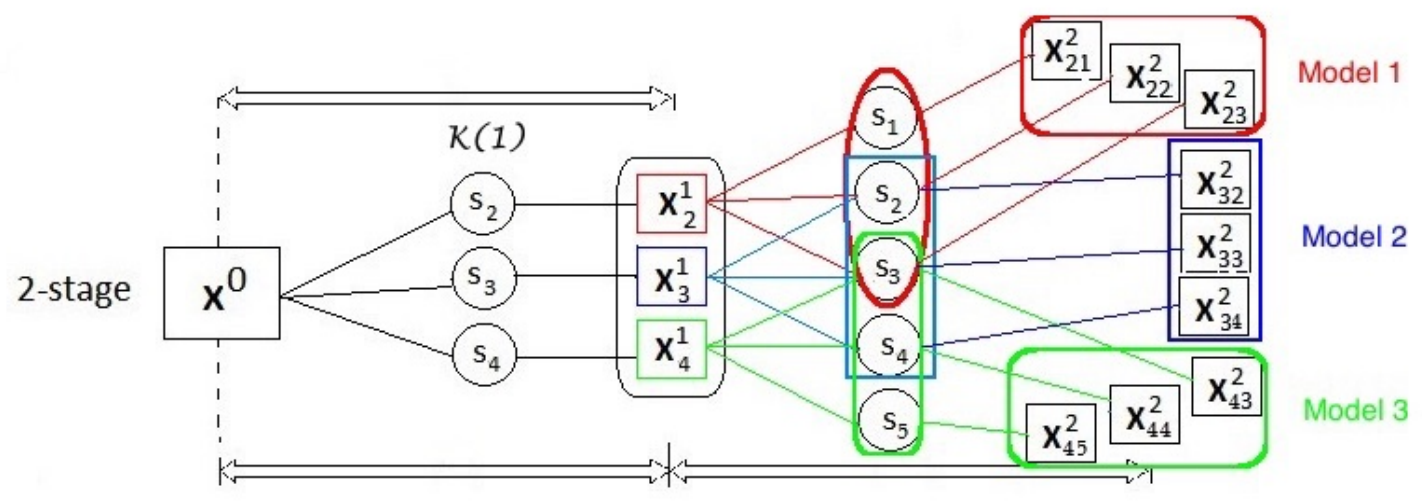

Figure 4.5: Scenarios of the $2 \times$ two-stage structure

models comparable solutions would be gained which are described in the third part of the Table 4.9 .

Hence, example 4.5.1 has been solved by the proposed three-stage model (model 4.18) and the results are portrayed in the first part of Table 4.9, while the results of the two-stage model (model 4.16) are described in the second part. The third part of Table 4.9 shows the suggested decisions of example 4.5.1 from the two-stage moving horizon approach, as well as the final results.

As shown in Table 4.9, the second column indicates the suggested amount of investment for each investment option at the beginning of the horizon relating to the initial decision $\left(X^{0}\right)$. The first recourse action, that will be implemented at the end of the second stage, has been described in the third column. As seen in this table, the two-stage model cannot determine any amount of reinvestment in this stage as it will end at this stage and does not continue in the third stage, while the other models provide three different solutions, one for each plausible $\operatorname{scenario}\left(X_{k(1)}^{1}, k(1) \in\left\{S_{2}, S_{3}, S_{4}\right\}\right)$. Available capital at the end of the related time horizon in each structure has been portrayed in the fourth column. Clearly, the length of the two-stage framework is two stages in which three plausible scenarios may be realised while the others have a three-stage planning horizon and then contain $3 \times 3=9$ meta-scenarios. Finally, the last column includes the other recourse actions (i.e. withdrawal) at each stage, together with the total withdrawal at the end of the relevant window. The withdrawal in the first, second, and the third stage are demonstrated by $X_{6}^{0}, X_{6}^{1}$, and $X_{6}^{2}$, respectively. Again, it is evident that no value has been provided for the third stage in the two-stage structure.

Figure 4.6 compares the objective functions of these two methods. As seen in this figure, the three-stage model provides better achievements for the second objective (total withdrawals) while the two-stage model contains better achievements for the first objective (available funds). However, because the three-stage model is looking further ahead than the two-stage model, the three-stage model is expected to find better goal achievement than the two-stage model ${ }^{7}$. It is difficult to identify this superiority (better achievements of the three-stage model) in this example. In the meantime, we need to note that, in this example, assigning more budget to consumption (withdrawal), by itself, gives rise to obtaining less interest in the next stages. Therefore, the less available fund for investment in the three-stage solutions could be the cause of the fewer capital for this model that were allocated further withdrawals, in comparison with the two-stage model.

\footnotetext{
${ }^{7}$ It will show that, in Theorem 4.4.1, the optimal solution to the two-stage moving horizon model is feasible for the three-stage model so that if the three-stage model selects anything else, it must be better in goal achievement.
} 


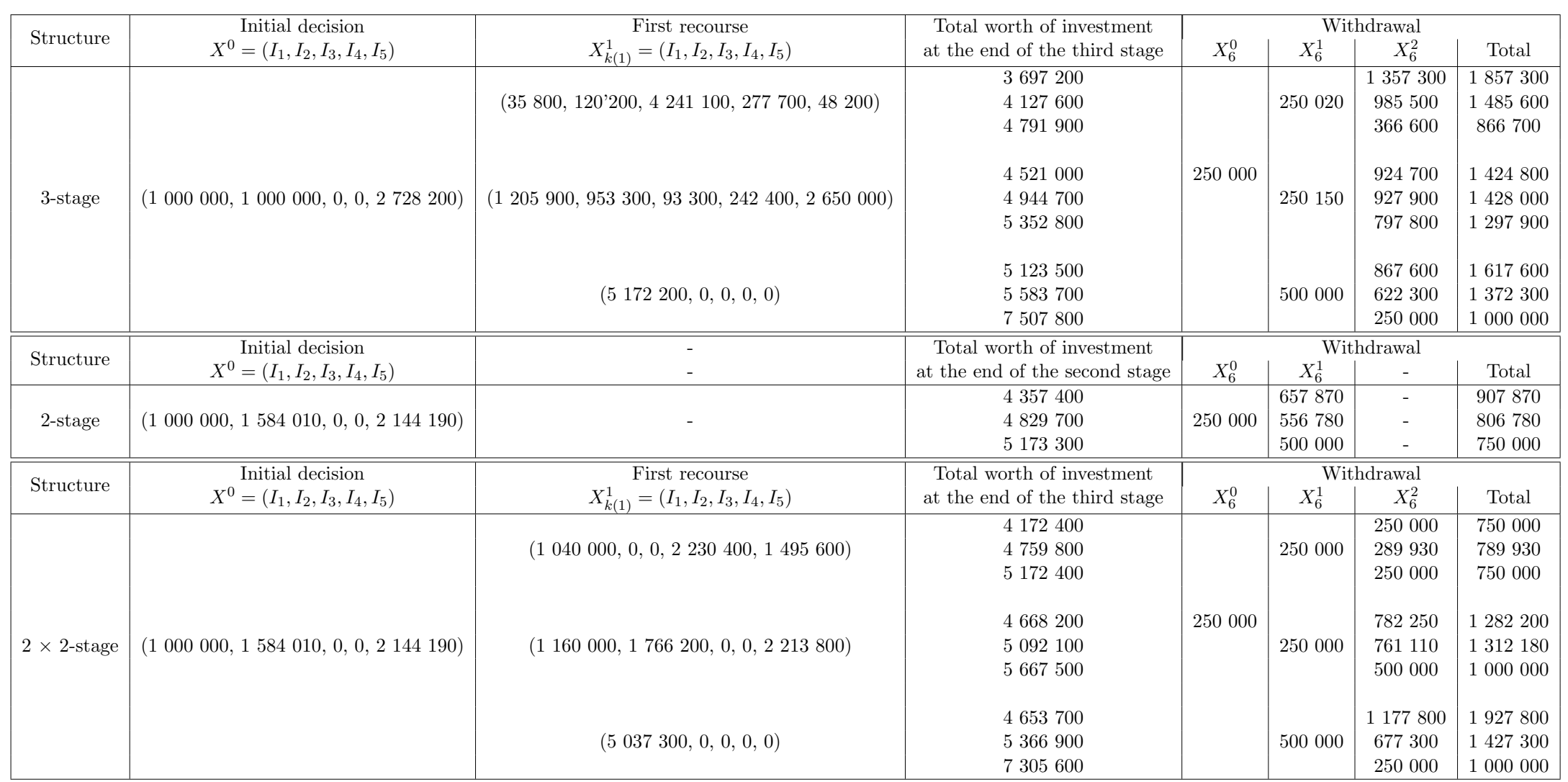

Table 4.9: Result of three-stage, two-stage, and $2 \times$ two-stage model 

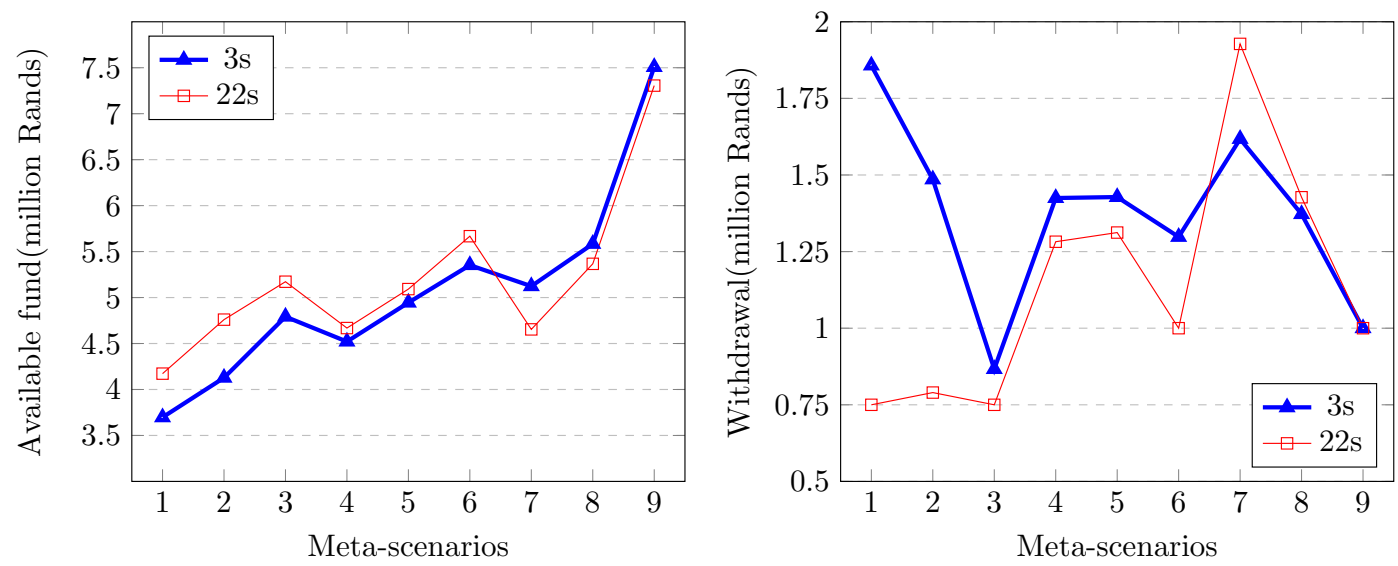

Figure 4.6: Results of the $2 \times$ two-stage structure in comparison with the three-stage structure

Due to the multi-objective orientation of the problem, higher performance in one objective/criterion and a lower performance in the other means that comparing separate objectives may not be useful, and a different measure should be utilised to evaluate and compare the results. Here, we are using the total profit over the given horizon as a measure to compare the results of these two approaches. Although this criterion is not added to the model directly, as an objective or a constraint, it will always remain an important hidden goal of all policy-makers.

Table 4.10 and graphs in Figure 4.7 evaluate the benefits that could obtained by utilising each approach after two periods of investment. The profit on investments is computed by subtracting the summation of all withdrawals and remaining money in investments at the end of the horizon from our initial capital (i.e. 5 million Rands).

Overall, as shown in Table 4.10, the total differences between the two approaches describe better achievements for the three-stage approach with $R 953370$ difference to the achievements compared to the two-stage approach. Except for the third, fourth, and the sixth meta-scenario which are shown in the rows in Table 4.10) the profits that may be reached by using the suggested meta-decisions of the three-stage model in most of the meta-scenarios are more desirable if the total profit is the measure. The differences between the profit of these two methods are very close in the fourth and sixth meta-scenarios, unlike in the third meta-scenario in which the two two-stage approach is the winner of the comparison by $R 263860$ more than the profit of the three-

\begin{tabular}{|c|c|c|c|c|c|c|c|c|}
\hline \multirow{2}{*}{\multicolumn{2}{|c|}{$\begin{array}{c}\text { Structure } \\
\text { Meta-scenario }\end{array}$}} & \multicolumn{3}{|c|}{3 -stage } & \multicolumn{3}{|c|}{$2 \times 2$-stage } & \multirow{2}{*}{$\begin{array}{c}\text { Difference } \\
(3 \mathrm{~S}-2 \mathrm{~S})\end{array}$} \\
\hline & & Remained fund & Withdrawal & Profit & Remained fund & Withdrawal & Profit & \\
\hline \multirow{3}{*}{$k(1)=S_{2}$} & $k(2)=S_{1}$ & 3697200 & 1857300 & 554600 & 4172400 & 750000 & -77640 & 632240 \\
\hline & $k(2)=S_{2}$ & 4127600 & 1485600 & 613200 & 4759800 & 789930 & 549710 & 63490 \\
\hline & $k(2)=S_{3}$ & 4791900 & 866700 & 658500 & 5172400 & 750000 & 922360 & -263860 \\
\hline \multirow{3}{*}{$k(1)=S_{3}$} & $k(2)=S_{2}$ & 4521000 & 1424800 & 945800 & 4668200 & 1282200 & 950500 & -4700 \\
\hline & $k(2)=S_{3}$ & 4944700 & 1428000 & 1372700 & 5092100 & 1312180 & 1353200 & 19500 \\
\hline & $k(2)=S_{4}$ & 5352800 & 1297900 & 1650700 & 5667500 & 1000000 & 1667500 & -16800 \\
\hline \multirow{3}{*}{$k(1)=S_{4}$} & $k(2)=S_{3}$ & 5123500 & 1617600 & 1741100 & 4653700 & 1927800 & 1581600 & 159500 \\
\hline & $k(2)=S_{4}$ & 5583700 & 1372300 & 1956000 & 5366900 & 1427300 & 1794200 & 161800 \\
\hline & $k(2)=S_{5}$ & 7507800 & 1000000 & 3507800 & 7305600 & 1000000 & 3305600 & 202200 \\
\hline Total & & & & & & & & 953370 \\
\hline
\end{tabular}

Table 4.10: Results comparison between three-stage and $2 \times$ two-stage models 


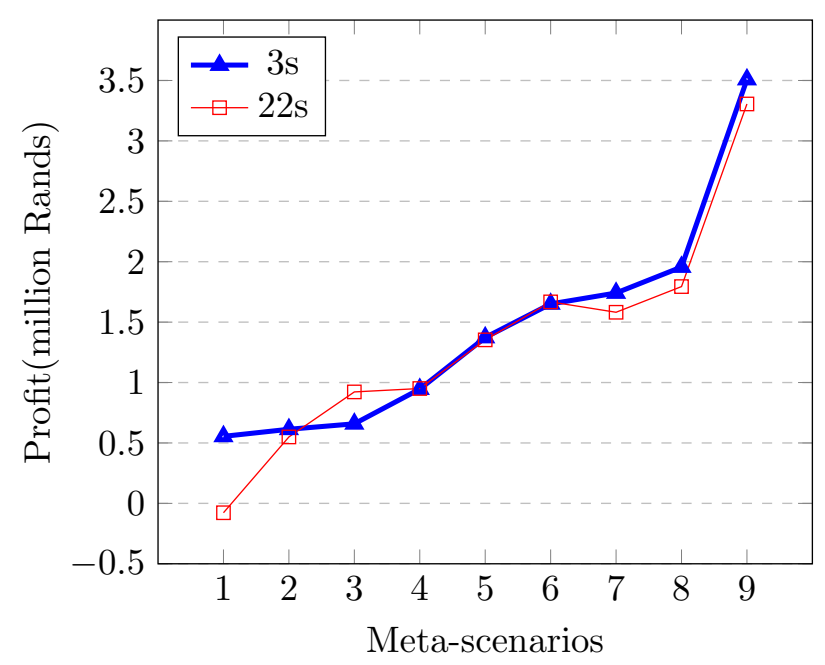

Figure 4.7: Results of the $2 \times$ two-stage structure in comparison with the three-stage structure.

stage model. The differences between the two methods in the fourth and sixth meta-scenarios are $R 4700$ and $R 195000$, respectively.

Furthermore, the superior feature of the three-stage model in comparison with the two twostage approach, in this example, has appeared in the first meta-scenario where utilising two following two-stage models end with the negative profit in this meta-scenario which may not satisfy the DM. This result clearly shows the cost of ignoring the consequences of the initial decision on the two following two-stage approach that may terminate to an irrecoverable decision, or even infeasible solution, in some meta-scenarios. This issue also highlights the robustness of the three-stage approach, compared to the two consecutive two-stage model.

Therefore, the above example confirms our expectations and demonstrates that the threestage approach might be preferable compared to the two consecutive two-stage models. Surely, looking at more stages must improve the solution. However, does the robustness of the threestage structure have no cost or is it always parsimonious? Is the better performance utilising a three-stage model good enough to warrant the added calculations? Remember, adding more stages would exponentially increase the size of the model. This would be a major limitation to appending more stages to the model and, in practice, we may not be able to run a model with more than two or three stages. Then, proving the efficiency of using the three-stage model rather than the two successive two-stage models is necessary.

In the next section, by sensitivity analysis and changing some data in the example 4.5.1, we will show that, despite all the excellences mentioned above, sometimes, utilising the three-stage structure with more calculations may not be preferable compared to using the two successive two-stage models. Therefore, the efficiency of the solutions to the three-stage model needs to be considered.

\subsubsection{Sensitivity analysis}

Example 4.5.2. Consider example 4.5.1 with different investment's growth as described in Table 4.11 .

Table 4.12 compares the results of applying the three-stage model and the two-stage moving horizon pattern. As shown in the last column of Table 4.12, except the first meta-scenario in which the profit of the three-stage model has a higher value than the profit reaching from the two successive two-stage models by the difference of $R 279$ 600, the superiority of the three-stage 


\begin{tabular}{cccccc}
\hline & \multicolumn{5}{c}{ Growth under scenario } \\
\cline { 2 - 6 } Investment & $S_{1}$ & $S_{2}$ & $S_{3}$ & $S_{4}$ & $S_{5}$ \\
\hline$I_{1}$ & $-5 \%$ & $+5 \%$ & $+16 \%$ & $+20 \%$ & $+30 \%$ \\
\hline$I_{2}$ & $+4 \%$ & $+8 \%$ & $+11.5 \%$ & $+15 \%$ & $+20 \%$ \\
\hline$I_{3}$ & $+8 \%$ & $+8.5 \%$ & $+8.7 \%$ & $+9 \%$ & $+10 \%$ \\
\hline$I_{4}$ & $+7 \%$ & $+7 \%$ & $+8 \%$ & $+10 \%$ & $+11 \%$ \\
\hline$I_{5}$ & $-3 \%$ & $+7 \%$ & $+15 \%$ & $+22 \%$ & $+35 \%$ \\
\hline
\end{tabular}

Table 4.11: Percentage growths for each investment under each scenario

\begin{tabular}{|c|c|c|c|c|c|c|c|c|}
\hline \multirow{2}{*}{\multicolumn{2}{|c|}{$\begin{array}{c}\text { Structure } \\
\text { Meta-scenario }\end{array}$}} & \multicolumn{3}{|c|}{3 -stage } & \multicolumn{3}{|c|}{$2 \times 2$-stage } & \multirow{2}{*}{$\begin{array}{c}\text { Difference } \\
(3 \mathrm{~S}-2 \mathrm{~S})\end{array}$} \\
\hline & & Remained fund & Withdrawal & Profit & Remained fund & Withdrawal & Profit & \\
\hline \multirow{3}{*}{$k(1)=S_{2}$} & $k(2)=S_{1}$ & 2881400 & 2442300 & 323600 & 4287700 & 756310 & 44000 & 279600 \\
\hline & $k(2)=S_{2}$ & 3261700 & 2298400 & 560100 & 4774700 & 750000 & 524700 & 35400 \\
\hline & $k(2)=S_{3}$ & 3674400 & 2102300 & 776800 & 5274700 & 750000 & 1024700 & -247900 \\
\hline \multirow{3}{*}{$k(1)=S_{3}$} & $k(2)=S_{2}$ & 3436900 & 2557100 & 994000 & 4607300 & 1407600 & 1014900 & -20900 \\
\hline & $k(2)=S_{3}$ & 3841300 & 2401000 & 1242200 & 5099700 & 1355400 & 1455100 & -212900 \\
\hline & $k(2)=S_{4}$ & 4676200 & 1755000 & 1431200 & 5806000 & 1000000 & 1806000 & -374800 \\
\hline \multirow{3}{*}{$k(1)=S_{4}$} & $k(2)=S_{3}$ & 4294800 & 2473100 & 1767900 & 4436100 & 2324000 & 1760100 & 7800 \\
\hline & $k(2)=S_{4}$ & 5314900 & 1832800 & 2147700 & 5061600 & 2076400 & 2137900 & 9800 \\
\hline & $k(2)=S_{5}$ & 6830400 & 1000000 & 2830400 & 6819800 & 1000000 & 2819800 & 10600 \\
\hline Total & & & & & & & & -513300 \\
\hline
\end{tabular}

Table 4.12: Results comparison between three-stage and $2 \times$ two-stage models

approach is insignificant for the rest of the meta-scenarios. The differences between the profits of the two methods for the second meta-scenario is just $R 35400$, and it is less than $R 11000$ for the last three. However, this difference in three of four meta-scenarios is less than $R-200000$. These negative values present further profits that are provided by the two following two-stage models compared to the three-stage structure. Furthermore, in these nine meta-scenarios, these differences $(3 S-2 S)$ have a sum of -513300 which may show the superiority of the two-stage moving horizon approach.

We may, nonetheless, need to be reminded that, as discussed in this chapter, without any doubt, the solution of the three-stage model must find better goal achievement (Min-Max deviation from the goals on GP) than the two-stage moving horizon model. This is because, as proved in Theorem 4.4.1, the optimal solution to the two-stage moving horizon model is a feasible solution of the three-stage model so that if the three-stage model selects anything else, it must be better in goal achievement(less Max deviation). The cause of the above result may back to the trade-offs between the Pareto optimal solutions in the three-stage model. A comparison of the objectives in Table 4.12 indicate that the solutions in the three-stage model suggested the higher amount of withdrawal (and thus, fewer funds will remain at the end of each stage) than what was provided by the other method, which clearly gives rise to higher benefits for the latter model. As briefly mentioned in section 4.5.1, only the overall goals have been assumed for the three-stage model in the above example that inherently lead to putting more weight on the last stages. So, we cannot say that the solutions of the $2 \times$ two-stage model are better than the solutions of the three-stage model. Instead, we shall say that the first one is preferred by the DM if the benefit is the base of the comparison. However, this issue in the three-stage model might be solved by adding some extra goals for achievements in the initial and/or middle stages. In fact, by appending objectives, equal weights will be considered in both stages. Then, to study this idea, 
let us append some other specific goals for maximisation of the level of the available fund at the end of the first stage and after consumption, plus maximising the consumption(withdrawal) at the end of stage ' 1 '. These objectives that represent the particular preferences of the first stage concerning criteria $C_{1}$ and $C_{2}$ under consequences of the scenario $k(1)$ can be mathematically formulated as follows:

$$
\begin{array}{lll}
\operatorname{Max} & Z_{1 k(1)}=\sum_{j=1}^{5} \sum_{i=1}^{5}\left(x_{i j}^{0}+x_{i j k(1)}^{1}\right) & \forall k(1), \\
M a x & Z_{2 k(1)}=\sum_{i=1}^{5}\left(x_{i 6}^{0}+x_{i 6 k(1)}^{1}\right) & \forall k(1),
\end{array}
$$

Thus, by defining some relevant variables for deviations from the goals of these objectives, $\delta_{n k(1)}^{\prime},(n=1,2 ; k(1)=1,2,3)$, and solving the related GP model, the results presented in Table 4.13 can be reached.

As described in Table 4.13, by appending some separate goals on achievements in stage ' 1 ', the DM is able to apply more preferences for this middle stage that gives rise to generate different solutions. In these solutions, more amounts are allocated to the first group of objectives (remained fund) that, by nature, boost the benefits. The positive number for summation of differences between the solutions of this new three-stage model and the two-stage moving horizon pattern in the last column of Table 4.13 confirm this increase in profits if the solutions of the new three-stage model are utilised. However, despite the first meta-scenario, the differences are not remarkable and do not seem to warrant the additional computation of the three-stage model in comparison with the $2 \times$ two-stage structure. It is worth remembering that both of these solutions are Pareto optimal and the differences explain the trade-offs between the goals. Therefore, if the benefit is the basis of the DM's judgement, as supposed in this example, adding some more goals for the initial stages may help us reach our purposes. Note that we also assumed equal importance weights for all meta-criteria in this example. Nonetheless, the DM can easily apply various weights to exert his/her secondarily preferences, as the primary preferences have already been applied by goal selection, and a more preferred Pareto optimum solution has been generated.

To sum up, on the one hand, ignoring the consequences of current decisions in a far future, which occurs by utilising the two-stage moving horizon structure, could lead to an unsatisfactory situation that costs too much, and may even lead to an irrecoverable situation under conditions of some meta-scenarios. On the other hand, although the three-stage structure may prepare more robust solutions than the $2 \times$ two-stage, the differences between the results of these two

\begin{tabular}{|c|c|c|c|c|c|c|c|c|}
\hline \multirow{2}{*}{\multicolumn{2}{|c|}{$\begin{array}{c}\text { Structure } \\
\text { Meta-scenario }\end{array}$}} & \multicolumn{3}{|c|}{ modified 3-stage } & \multicolumn{3}{|c|}{$2 \times 2$-stage } & \multirow{2}{*}{$\begin{array}{c}\text { Difference } \\
(3 \mathrm{~S}-2 \mathrm{~S})\end{array}$} \\
\hline & & Remained fund & Withdrawal & Profit & Remained fund & Withdrawal & Profit & \\
\hline \multirow{3}{*}{$k(1)=S_{2}$} & $k(2)=S_{1}$ & 3844100 & 1642600 & 493800 & 4287700 & 756310 & 44000 & 395800 \\
\hline & $k(2)=S_{2}$ & 3958800 & 1645300 & 611600 & 4774700 & 750000 & 524700 & 86900 \\
\hline & $k(2)=S_{3}$ & 4134500 & 1569500 & 719100 & 5274700 & 750000 & 1024700 & -305600 \\
\hline \multirow{3}{*}{$k(1)=S_{3}$} & $k(2)=S_{2}$ & 4519400 & 1477400 & 1008200 & 4607300 & 1407600 & 1014900 & -6700 \\
\hline & $k(2)=S_{3}$ & 4866400 & 1523500 & 1401000 & 5099700 & 1355400 & 1455100 & -54100 \\
\hline & $k(2)=S_{4}$ & 5086200 & 1638100 & 1730200 & 5806000 & 1000000 & 1806000 & -75800 \\
\hline \multirow{3}{*}{$k(1)=S_{4}$} & $k(2)=S_{3}$ & 5173000 & 1601900 & 1781700 & 4436100 & 2324000 & 1760100 & 21600 \\
\hline & $k(2)=S_{4}$ & 5374200 & 1773600 & 2148800 & 5061600 & 2076400 & 2137900 & 10900 \\
\hline & $k(2)=S_{5}$ & 6830400 & 1000000 & 2830600 & 6819800 & 1000000 & 2819800 & 10800 \\
\hline Total & & & & & & & & +83800 \\
\hline
\end{tabular}
approaches would vary in different problems, and the efficiency of the three-stage approach should

Table 4.13: Results comparison between three-stage and $2 \times$ two-stage models 
be considered in any problem. Nevertheless, the future vision of the three-stage structure may sometimes lead to unsatisfactory solutions. In this circumstances, putting separate aspiration levels on initial stages and the use of various weights may help the DM add more preferences into the model and detect a more preferred solution from the Pareto frontier. Nonetheless, as seen in the previous example, the cost of robustness in the modified solutions may still not be worth enough for additional calculation caused, in some cases, by handling more uncertainty in the three-stage structure.

However, the DM is the one who must choose the preferred approach by considering the cost of robustness, depending on his/her needs and priorities. Therefore, the cost of robustness in a three-stage structure and the differences of results that can be reached by utilising this structure, in comparison to the two-stage moving horizon approach, should be considered in advance. Then, the DM can make his/her preferred decision of the model that he/she wants to apply to solve the problem.

The last section of this chapter is allocated to the examination of some simple, but useful, ideas that may help us boost the robustness of the initial decisions provided by the two-stage moving horizon model. This improvement would assist in the case when applying the three-stage model is not parsimonious.

\subsection{Two-stage robustness}

As discussed in the previous sections, although the decisions provided by the two-stage moving horizon model would be a less conservative and, in the same time, a robust decision for a single two-stage horizon, the robustness of these decisions in longer horizons, as seen in the example 4.5.1, could not be guaranteed. In this situation, a three-stage (or more) structure would return a more robust initial decision, although it may not be a Pareto-optimum solution for the first stage. This is because, like any other robust solution, this sub-optimal initial solution together with the other two recourse decisions could construct a Pareto-optimum solution for the three-stage structure.

In this section, we seek to find a way to reach a more robust initial decision from a twostage structure without utilising the three-stage approach. Our motivation for this could be the situations in which use of the three-stage model is not parsimonious, and/or when the results of the three-stage approach do not satisfy the DM (such as what happened in example 4.5.2). Moreover, using a three-stage structure would be too complex to be modelled or computationally too expensive to be solved, because of many meta-scenarios (and hence too many meta-criteria), or the higher degrees of uncertainty that even scenario space cannot completely determine before the next stage. It is also possible that, in some problems, recognising the plausible scenarios of the future stages are strictly dependent on some unexpectable outcomes of the following stage(s) that are completely unknown at the time of initial decision-making. In these circumstances, the outcomes of the problem would be out of imagination, and DMs are not able to look more than one (a few) step(s) ahead. This condition would be led us to treat that kind of deep uncertainty in which enumerating the whole set of the problem's outcomes is impossible.

Accordingly, the aim of this section is to find a way to get a more robust solution from the two-stage moving horizon model in some situations, such as what we saw in the previous example (negative profit). It will be shown that some variations in the two-stage parameters (for instance, various weights on the second stage in the first two-stage model) can help us reach this purpose. However, it must be noted that the way of setting the weights may be ad hoc (i.e. different problems may need specific weight selection), but the idea would still be useful. Therefore, let us make some sensitivity analysis in a new example to examine this idea.

Example 4.6.1. Consider the example 4.5.1 with different investment growths that are described in Table 4.14. For simplicity purposes, only one penalty cost table applies in all states (as shown in Table 4.15). Table 4.16 compares the results of solving this problem by the two approaches. 


\begin{tabular}{cccccc}
\hline & \multicolumn{5}{c}{ Growth under scenario } \\
\cline { 2 - 6 } Investment & $S_{1}$ & $S_{2}$ & $S_{3}$ & $S_{4}$ & $S_{5}$ \\
\hline$I_{1}$ & $-20 \%$ & $-10 \%$ & $+10 \%$ & $+20 \%$ & $+50 \%$ \\
\hline$I_{2}$ & $-5 \%$ & $+12 \%$ & $+17 \%$ & $+16 \%$ & $+10 \%$ \\
\hline$I_{3}$ & $+12 \%$ & $+10.5 \%$ & $+12 \%$ & $+15 \%$ & $+30 \%$ \\
\hline$I_{4}$ & $-30 \%$ & $+10 \%$ & $+20 \%$ & $+14 \%$ & $+20 \%$ \\
\hline$I_{5}$ & $+15 \%$ & $+10 \%$ & $+17 \%$ & $+13 \%$ & $+45 \%$ \\
\hline
\end{tabular}

Table 4.14: Percentage growths for each investment under each scenario

\begin{tabular}{|cccccc|c|}
\hline \multirow{2}{*}{$\begin{array}{c}\text { Transfer } \\
\text { from }\end{array}$} & \multicolumn{5}{c|}{ to } & \\
\cline { 2 - 6 } & $I_{1}$ & $I_{2}$ & $I_{3}$ & $I_{4}$ & $I_{5}$ & Withdrawal \\
\hline$I_{1}$ & 0 & $-2 \%$ & $-15.1 \%$ & $-1.9 \%$ & $-25 \%$ & $-1.5 \%$ \\
\hline$I_{2}$ & $-5 \%$ & 0 & $-20.1 \%$ & $-5.4 \%$ & $-20 \%$ & $-5 \%$ \\
\hline$I_{3}$ & $-0.01 \%$ & $-0.51 \%$ & 0 & $-0.41 \%$ & $-1.01 \%$ & $-0.01 \%$ \\
\hline$I_{4}$ & $-0.6 \%$ & $-1.1 \%$ & $-0.61 \%$ & 0 & $-1.6 \%$ & $-0.6 \%$ \\
\hline$I_{5}$ & $-1 \%$ & $-1.5 \%$ & $-1.01 \%$ & $-1.4 \%$ & 0 & $-1 \%$ \\
\hline
\end{tabular}

Table 4.15: Percentage of loss of funds (penalty cost) for transferring between each pair of investment and withdrawal

\begin{tabular}{|c|c|c|c|c|c|c|c|c|}
\hline \multirow{2}{*}{\multicolumn{2}{|c|}{$\begin{array}{c}\text { Structure } \\
\text { Meta-scenario }\end{array}$}} & \multicolumn{3}{|c|}{3 -stage } & \multicolumn{3}{|c|}{$2 \times 2$-stage } & \multirow{2}{*}{$\begin{array}{c}\text { Difference } \\
(3 \mathrm{~S}-2 \mathrm{~S})\end{array}$} \\
\hline & & Remained fund & Withdrawal & Profit & Remained fund & Withdrawal & Profit & \\
\hline \multirow{3}{*}{$k(1)=S_{2}$} & $k(2)=S_{1}$ & 4826100 & 750000 & 576100 & 4105200 & 750000 & -144750 & 720850 \\
\hline & $k(2)=S_{2}$ & 4826100 & 750000 & 576100 & 4105200 & 750000 & -144750 & 720850 \\
\hline & $k(2)=S_{3}$ & 4921400 & 952000 & 873400 & 4248600 & 978520 & 227090 & 646310 \\
\hline \multirow{3}{*}{$k(1)=S_{3}$} & $k(2)=S_{2}$ & 4816300 & 929800 & 746200 & 4921800 & 750000 & 671800 & 74400 \\
\hline & $k(2)=S_{3}$ & 5294400 & 750000 & 1044400 & 5109900 & 1149000 & 1258900 & -214500 \\
\hline & $k(2)=S_{4}$ & 4994400 & 1000000 & 994400 & 5461800 & 1000000 & 1461800 & -467400 \\
\hline \multirow{3}{*}{$k(1)=S_{4}$} & $k(2)=S_{3}$ & 5137300 & 1029500 & 1166900 & 5115300 & 1273800 & 1389300 & -222400 \\
\hline & $k(2)=S_{4}$ & 5406400 & 1250000 & 1656400 & 5526300 & 1250000 & 1776300 & -119900 \\
\hline & $k(2)=S_{5}$ & 5973200 & 2148200 & 3121400 & 5645800 & 2204400 & 2850500 & 270900 \\
\hline Total & & & & & & & & 1409110 \\
\hline
\end{tabular}

Table 4.16: Results comparison between three-stage and $2 \times$ two-stage models

Here, we are using different importance weights for the first two-stage, and comparing the results in Table 4.17. The current set of importance weights is $(1,1,1,1,1,1)$. Table 4.17 compares benefits that could be reached by utilising the three-stage model and two following two-stage models, while different sets of importance weights are applied in the first two-stage model.

As seen in Table 4.17, by increasing the importance weight of scenario $S_{2}$ or $S_{3}$ at the first two-stage model and implementing its initial solution that followed by the second two-stage model (the importance weights of scenarios in the second stage had no change and remained at their first values $(1,1,1,1,1,1))$, then the differences between the solutions of the three-stage model (which also has no changes in its importance weight) and these two following models have decreased significantly (see columns 7-9 in Table 4.17). With these importance weights, the 
negative values of the profits (which are presented in the first two meta-scenarios in the eighth column of Table4.16) have turned into the positive values. Furthermore, the total differences of these profits and the profits of the three-stage model change into $R-18340$ and $R-1456200$, respectively. These amounts, especially the last one, can probably assure the DM that utilising the two-stage moving horizon models with these importance weights is preferable to the threestage structure.

Nevertheless, reverse results are reached by applying the third importance weights. As described in the fourth column of Table 4.17, less desirable values would be gained in this problem, especially in the first three meta-scenarios. Therefore, increasing the priority of some objectives in the first two-stage may help us obtain a more desirable initial solution and improve the robustness of the two-stage moving horizon approach. However, this enhancement might produce the worse solution, as explained earlier.

To sum up, as mentioned earlier, various combinations of weights may be needed in different problems and we may not be able to apply a constant set of weights for all problems. Nonetheless, the idea of utilising different weights/parameters can still useful and applicable in most processes. Furthermore, in the next chapter, we will expand this idea to explain some interesting subjects such as time preferences and differential weighing in multi-stage moving horizon models that may help us obtain more desirable decisions.

\begin{tabular}{|c|c|c|c|c|c|c|c|c|}
\hline \multirow{2}{*}{\multicolumn{2}{|c|}{$\begin{array}{c}\text { Structure } \\
\text { Meta-scenario }\end{array}$}} & \multicolumn{3}{|c|}{$2 \times 2$-S with different importance weights } & $3 S$ & \multicolumn{3}{|c|}{ Difference(3S-2S) } \\
\hline & & $(2,1,1,1,1,1)$ & $(1,2,1,1,1,1)$ & $(1,1,2,1,1,1)$ & $(1,1,1,1,1,1)$ & 1 & 2 & 3 \\
\hline \multirow{3}{*}{$k(1)=S_{2}$} & $k(2)=S_{1}$ & 529520 & 822500 & -551900 & 576100 & 46580 & -246400 & 1128000 \\
\hline & $k(2)=S_{2}$ & 529520 & 822500 & -551900 & 576100 & 46580 & -246400 & 1128000 \\
\hline & $k(2)=S_{3}$ & 805600 & 1020400 & -279030 & 873400 & 67800 & -147000 & 1152430 \\
\hline \multirow{3}{*}{$k(1)=S_{3}$} & $k(2)=S_{2}$ & 958500 & 955400 & 585700 & 746200 & -212300 & -209200 & 160500 \\
\hline & $k(2)=S_{3}$ & 1540000 & 1573600 & 1160300 & 1044400 & -495600 & -529200 & -115900 \\
\hline & $k(2)=S_{4}$ & 1714500 & 1745400 & 1375700 & 994400 & -720100 & -751000 & -381300 \\
\hline \multirow{3}{*}{$k(1)=S_{4}$} & $k(2)=S_{3}$ & 1357500 & 1173300 & 1460200 & 1166900 & -190600 & -6400 & -293300 \\
\hline & $k(2)=S_{4}$ & 1513800 & 1540700 & 1856100 & 1656400 & 142600 & 115700 & -199700 \\
\hline & $k(2)=S_{5}$ & 1824700 & 2557700 & 2947400 & 3121400 & 1296700 & 563700 & 174000 \\
\hline \multicolumn{2}{|l|}{ Total } & & & & & -18340 & -1456200 & 1737530 \\
\hline
\end{tabular}

Table 4.17: Results comparison between three-stage and $2 \times$ two-stage models

\subsection{Conclusion}

In the previous chapter, we introduced a scenario-based two-stage structure for multi-criteria decision making under deep uncertainty. The proposed two-stage framework reaps benefits from the concept of meta-criteria to integrate performances under different scenarios. Scenarios are utilised as a dimension of preference to avoid problems of evaluating probabilities under deep uncertainty. Using scenarios as a tool for representing uncertainty in a problem can pave the way for the DM to think, discuss, and plan for that uncertainty. The successful experience in applying this scenario-based two-stage structure in dealing with deep uncertainty, by which we get rid of the probabilities conditions and calculations, motivates us to extend the proposed twostage structure to longer period problems. Furthermore, in the proposed two-stage structure, we are also capable of evaluating the consequences of the initial decision after the achievement of every plausible scenario. Nevertheless, measuring the effects of the first decision after a longer period, as well as the outcomes of the recourse decisions, need to be investigated in a longer structure. On the other hand, to deal with the deep uncertainty in strategic planning problems, as one of our first themes, more than two stages would be needed. In the two-stage structure, we 
are looking one step ahead, and postponing part of the decision, which leaves room for possible adaptation after scenario realisation, if it is ever possible or optimum. However, real processes always face an indefinite future in which scenarios are not independent events by stages and continuously unfold. We have used some artificial stages to be able to discuss, think about, and formulate the model in these problems. Therefore, the central question that is raised in this work is: If we proposed to think of discrete stages, how many steps is it worth looking ahead? To begin to answer this question, this chapter has introduced the three-stage scenario-based multi-criteria decision-making structure as an extension of the proposed two-stage structure and compared it with the two-stage moving horizon structure.

Philosophically, the proposed two-stage structure always plans and looks one step ahead while the three-stage structure considers the conditions and consequences of two upcoming steps in advance, which fits well with our primary objective. Ignoring long-term decisions consequences and likely conditions could not be a robust strategic strategy. Therefore, generally, by utilising the three-stage structure, we may expect a more robust/provident decision. It is shown that, although the solution of the two-stage model provides us with the optimality under the uncertain conditions of the first period, it may not be even feasible and recoverable under additional uncertainties and circumstances of the new phase that would be appended in the three-stage structure. However, the three-stage structure, by a more futuristic vision, may suggest a suboptimal initial decision that could make feasible robust meta-decisions for each and every plausible meta-scenario. This property automatically expands the concept of the dynamic-robust solutions for the three-stage framework.

In comparing the two-stage versus the three-stage structure, the fundamental problem is the comparability of the two methods because of the different time horizons, as the two-stage model is ignorant of the third stage. Therefore, we need to compare these methods in the same period. For this, the two-stage moving horizon approach was introduced in which another two-stage model, starting at the end of the first stage, was iterated to cover the entire period of three stages. In comparing the two-stage moving horizon model with the three-stage pattern, it was shown that any feasible solution, and thus the optimal one, to the two-stage moving horizon model, is a feasible solution to the three-stage structure. Also, since the three-stage model is looking further ahead than the two-stage model in each stage, and the constraints in the threestage model are simultaneously satisfied, then the optimal solution of the three-stage model is no worse than the optimal solution of the two-stage moving horizon model. So, with no doubt, the three-stage model must find a better, or at least the same, goal achievement compared to the two-stage model.

Obviously, the ability to model more uncertainty, produce more robust decisions and consider the longer term consequences of the decisions are the main advantages of the three-stage approach. However, more uncertain statements bring more complexity to the problem, since the number of meta-scenarios is raised exponentially by considering more stages. Furthermore, using an approach with more stages could lead to a model that is too complex and which is computationally expensive and may not be solved simply. Therefore, we needed to indicate whether the better performance using a three-stage model is significant enough to warrant the added computation. A sensitivity analysis in an example showed that, despite all the excellences mentioned above, the three-stage structure may not always be preferable compared to the two successive two-stage models. Dynamic-robust decisions of the proposed three-stage model, in some cases, may end up with an unsatisfactory solution, as a cost of robustness. Also, the three-stage structure naturally puts more weights on the latter stages that may lead to some undesirable solutions. In these circumstances, putting separate aspiration levels on initial stages and the use of various weights may help the DM add more preferences into the model and obtain a more preferred solution from the Pareto frontier. Nonetheless, the modified solutions may still not be worth enough to warrant additional calculation, in some cases, caused by handling more uncertainty in the three-stage structure. Therefore, the efficiency of the solutions of the three-stage model needs to be considered. More discussions will be presented for this issue in 
the next chapter.

Finally, in the last section of the current chapter, we described that some variations in the priority of certain objectives in the first two-stage can help us obtain a more desirable initial solution and improve the robustness of the two-stage moving horizon approach. However, these alternations might be ad hoc (i.e. various combinations of weights may be needed in different problems). Nonetheless, the idea of utilising different weights/parameters can still useful and applicable in most processes.

To sum up, the scenario-based three-stage structure provides us with dynamic-robust decisions in dealing with deep uncertainty in MCDM problems with a three-stage planning horizon. These decisions are more robust than the solutions of the two-stage moving horizon models and, certainly, must find better goal achievement/Min-Max deviation by looking further ahead than the $2 \times$ two-stage models. Nevertheless, the differences between the results of these two approaches would vary in different problems, and the efficiency of the three-stage approach should be considered in any problem. It is also possible to boost the robustness of the initial decisions provided by the two-stage moving horizon model in cases when the application of the three-stage model is not efficient and/or the solutions of the rolling two-stage models are not robust enough to cover all plausible meta-scenarios or satisfy the DM's goals.

Therefore, looking further ahead can help us improve the robustness but it may have some costs such as expensive computation or unfavourable solutions. Accordingly, the DM is the one who must choose the preferred approach by considering the cost of robustness, depending on his/her needs and priorities. Thus, the cost of robustness in a three-stage structure and the differences of results that can be reached by utilising this structure, in comparison to the twostage moving horizon approach, should be considered in advance. Thereafter, the DM can make his/her preferred decision in terms of which model he/she wants to apply to solve the problem.

The successful experience of dealing with deep uncertainty in the two- and three-stage models motivated us to extend these structures to problems with a longer planning horizon. However, the high level of complexity and expensive computation may prevent us from applying four- or fivestage models. Even if we are able to apply the proposed methodology to problems with more than three stages, the resultant solutions might not be efficient. Nevertheless, according to the positive results from rolling the two-stage model in a three-stage horizon in this chapter, utilising the moving horizon models seems more reasonable in long-term decision-making problems. Therefore, the next chapter will introduce, examine, and compare the two- and three-stage moving horizon models in a T-stage planning window. Moreover, as we saw in the last parts of this chapter, some variations in the first stage parameters, in both approaches, may enhance the robustness in the moving horizon models or generate a more preferred Pareto-optimal solution in the three-stage model. This idea will be expanded in the next chapter to express some interesting subjects such as time preferences and differential weighing in multi-stage moving horizon models that may help us obtain more desirable decisions. More comparison of these two methods in longer horizons will be made, by a simulation study, and some other issues, such as the effects of horizon length in moving horizon models, will also be investigated. 


\subsection{Appendix}

\subsubsection{Proof of Theorem 4.4.1}

Theorem 4.4.1. Any feasible solution (and hence the optimal one) of the two-stage moving horizon model (models 3.2 and 4.7) in a three-stage planning window is a feasible solution for the corresponding three-stage model (model 4.2) and vice versa (i.e. the two-stage moving horizon model is feasible iff the three-stage model is feasible).

Proof. Let us, for simplicity, consider the vector form of the models. Model 4.17 utilise for the first two-stage model and getting the initial solution $\left(\mathbf{x}^{0}\right)$. The vector form of the model 4.7 as the second two-stage model providing us with the recourse solutions can be rewritten as follows:

$$
\begin{array}{lll}
\text { Min } & \mathbf{Z}_{k(1)}=\mathbf{c}_{k(1)}^{0} \mathbf{x}_{k(1)}^{1}+\mathbf{c}_{k(1) k(2)}^{2} \mathbf{x}_{k(1) k(2)}^{2} ; & k(2)=1, \ldots, p(2) \\
\text { s.t. } & & \\
& \mathbf{A}_{k(1)}^{1} \mathbf{x}_{k(1)}^{1} \leq \mathbf{b}_{k(1)}^{1}-\mathbf{A}_{k(1)}^{0} \mathbf{x}^{0 *}, & \\
& \mathbf{A}_{k(1) k(2)}^{1} \mathbf{x}_{k(1)}^{1}+\mathbf{A}_{k(1) k(2)}^{2} \mathbf{x}_{k(1) k(2)}^{2} \leq \mathbf{b}_{k(1) k(2)}^{2}-\mathbf{A}_{k(1) k(2)}^{0} \mathbf{x}^{0 *}, & k(2)=1, \ldots, p(2), \\
& \mathbf{x}_{k(1)}^{1}, \mathbf{x}_{k(1) k(2)}^{2} \geq \mathbf{0}, & k(2)=1, \ldots, p(2) .
\end{array}
$$

Also, the vector form of the three-stage model (4.2) is set down as the following:

$$
\begin{array}{cr}
\text { Min } \quad \mathbf{Z}_{k(1)}=\mathbf{c}^{\prime 0} \mathbf{x}^{0}+\mathbf{c}_{k(1)}^{\prime 0} \mathbf{x}^{0}+\mathbf{c}_{k(1)}^{\prime 1} \mathbf{x}_{k(1)}^{1} & k(1)=1, \ldots, p(1) ; \\
\text { Min } \quad \mathbf{Z}_{k(1) k(2)}=\mathbf{c}^{0} \mathbf{x}^{0}+\mathbf{c}_{k(1)}^{0} \mathbf{x}^{0}+\mathbf{c}_{k(1)}^{1} \mathbf{x}_{k(1)}^{1}+\mathbf{c}_{k(1) k(2)}^{0} \mathbf{x}^{0} & k(1)=1, \ldots, p(1) ; \\
+\mathbf{c}_{k(1) k(2)}^{1} \mathbf{x}_{k(1)}^{1}+\mathbf{c}_{k(1) k(2)}^{2} \mathbf{x}_{k(1) k(2)}^{2} ; & k(2)=1, \ldots, p(2) ; \\
\text { s.t. } \quad \mathbf{A}^{0} \mathbf{x}^{0} \leq \mathbf{b}^{0},(*) & \\
\quad \mathbf{A}_{k(1)}^{0} \mathbf{x}^{0}+\mathbf{A}_{k(1)}^{1} \mathbf{x}_{k(1)}^{1} \leq \mathbf{b}_{k(1)}^{1},(* *) & k(1)=1, \ldots, p(1) ; \\
\quad \mathbf{A}_{k(1) k(2)}^{0} \mathbf{x}^{0}+\mathbf{A}_{k(1) k(2)}^{1} \mathbf{x}_{k(1)}^{1}+\mathbf{A}_{k(1) k(2)}^{2} \mathbf{x}_{k(1) k(2)}^{2} \leq \mathbf{b}_{k(1) k(2)}^{2},(* * *) & k(1)=1, \ldots, p(1) ; \\
\mathbf{x}^{0}, \mathbf{x}_{k(1)}^{1}, \mathbf{x}_{k(1) k(2)}^{2} \geq 0, & k(2)=1, \ldots, p(2) ; \\
& k(1)=1, \ldots, p(1) ; \\
& k(2)=1, \ldots, p(2) .
\end{array}
$$

$\Rightarrow)$ Now, due to the assumption, suppose that the two-stage moving horizon model is feasible, then $\forall k=k(1) \in S^{1}$, and $\forall k(2) \in S^{2}, \exists \underline{X}=\left(\underline{\mathbf{x}}^{0}, \underline{\mathbf{x}}_{k(1)}^{1}\right)$, and $\exists \overline{\mathbf{X}}=\left(\overline{\mathbf{x}}_{k(1)}^{1}, \overline{\mathbf{x}}_{k(1) k(2)}^{2}\right)$, where $\underline{X}$, and $\overline{\mathbf{X}}$, are, respectively, the vectors of feasible solutions for the first(4.17) and the second(4.13) two-stage models. Therefore, $\mathbf{X}=\left(\underline{\mathbf{x}}^{0}, \overline{\mathbf{x}}_{k(1)}^{1}, \overline{\mathbf{x}}_{k(1) k(2)}^{2}\right)$ is a vector of feasible solutions for the two-stage moving horizon model that satisfies its constraints, then, we have

(1) $\underline{\mathbf{x}}^{0}, \overline{\mathbf{x}}_{k(1)}^{1}, \overline{\mathbf{x}}_{k(1)}^{2} \geq \mathbf{0}, \quad \forall k(1), k(2)$

(2) $\quad \mathbf{A}^{0} \underline{\mathbf{x}}^{0} \leq \mathbf{b}^{0}, \Rightarrow(*)$ is satisfied. 
(3) $\forall k(1), \mathbf{A}_{k(1)}^{1} \overline{\mathbf{x}}_{k(1)}^{1} \leq \mathbf{b}_{k(1)}^{1}-\mathbf{A}_{k(1)}^{0} \underline{\mathbf{x}}^{0} ;$

$\Rightarrow \mathbf{A}_{k(1)}^{0} \underline{\mathbf{x}}^{0}+\mathbf{A}_{k(1)}^{1} \overline{\mathbf{x}}_{k(1)}^{1} \leq \mathbf{b}_{k(1)}^{1} \Rightarrow(* *)$, is satisfied.

(4) $\forall k(1), k(2), \mathbf{A}_{k(1) k(2)}^{1} \overline{\mathbf{x}}_{k(1)}^{1}+\mathbf{A}_{k(1) k(2)}^{2} \overline{\mathbf{x}}_{k(1) k(2)}^{2} \leq \mathbf{b}_{k(1) k(2)}^{2}-\mathbf{A}_{k(1) k(2)}^{0} \underline{\mathbf{x}}^{0}$;

$\Rightarrow \mathbf{A}_{k(1) k(2)}^{0} \underline{\mathbf{x}}^{0}+\mathbf{A}_{k(1) k(2)}^{1} \overline{\mathbf{x}}_{k(1)}^{1}+\mathbf{A}_{k(1) k(2)}^{2} \overline{\mathbf{x}}_{k(1) k(2)}^{2} \leq \mathbf{b}_{k(1) k(2)}^{2} \Rightarrow(* * *)$, is satisfied. Thus,

$\mathbf{X}$ is a feasible solution for the three-stage model.

$\Leftarrow)$ Suppose that the 3 -stage model is feasible, then,

$\forall k=k(1) \in S^{1}$, and $\forall k(2) \in S^{2}, \exists X^{\prime}=\left(\widehat{\mathbf{x}}^{0}, \widehat{\mathbf{x}}_{k(1)}^{1}, \widehat{\mathbf{x}}_{k(1) k(2)}^{2}\right)$, subject to:

(i) $\widehat{\mathbf{x}}^{0}, \widehat{\mathbf{x}}_{k(1)}^{1}, \widehat{\mathbf{x}}_{k(1) k(2)}^{2} \geq \mathbf{0}, \quad \forall k(1), k(2) ;$

(ii) $\quad \mathbf{A}^{0} \widehat{\mathbf{x}}^{0} \leq \mathbf{b}^{0}$;

(iii) $\forall k(1), \mathbf{A}_{k(1)}^{0} \widehat{\mathbf{x}}^{0}+\mathbf{A}_{k(1)}^{1} \widehat{\mathbf{x}}_{k(1)}^{1} \leq \mathbf{b}_{k(1)}^{1} ;$

(iv) $\forall k(1), k(2), \mathbf{A}_{k(1) k(2)}^{0} \widehat{\mathbf{x}}^{0}+\mathbf{A}_{k(1) k(2)}^{1} \widehat{\mathbf{x}}_{k(1)}^{1}+\mathbf{A}_{k(1) k(2)}^{2} \widehat{\mathbf{x}}_{k(1) k(2)}^{2} \leq \mathbf{b}_{k(1) k(2)}^{2}$;

(i), (ii), and (iii), satisfy the constraints of the first two-stage model in 4.17, while $(i),(i i i)$, and $(i v)$, convince the limitations of the model 4.13. Therefore, the two-stage moving horizon model is feasible, and the proof is complete. 


\subsubsection{Two-stage model for example 4.5.1}

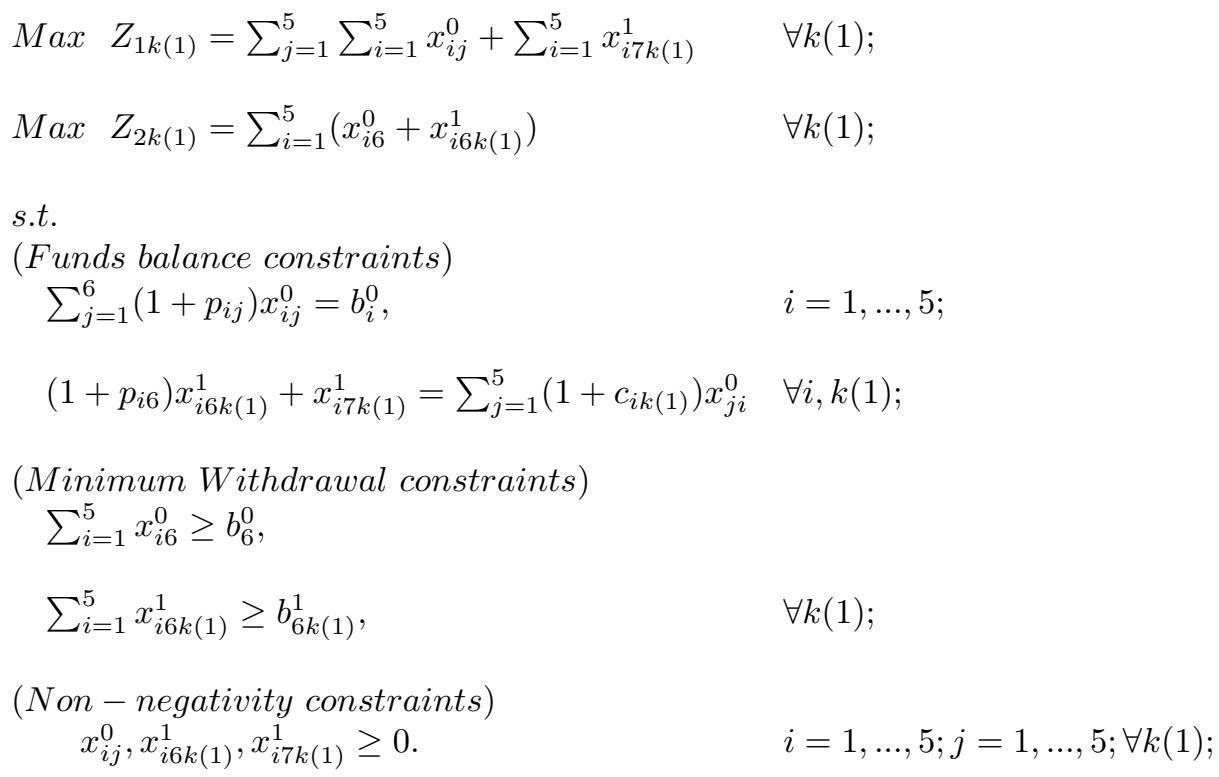

\subsubsection{Two-stage GP model for example 4.5.1}

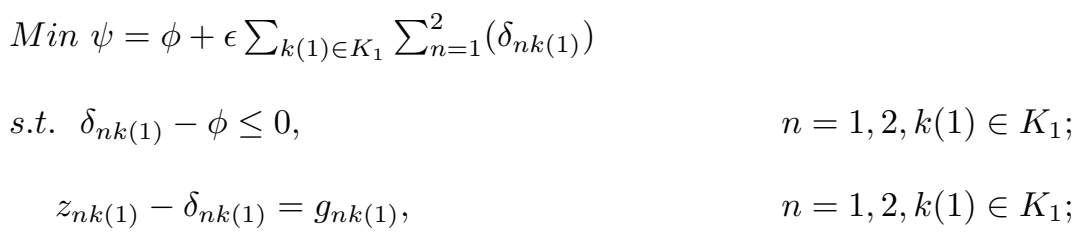

(Funds balance constraints)

$$
\begin{array}{ll}
\sum_{j=1}^{6}\left(1+p_{i j}\right) x_{i j}^{0}=b_{i}^{0}, & i=1, \ldots, 5 ; \\
\left(1+p_{i 6}\right) x_{i 6 k(1)}^{1}+x_{i 7 k(1)}^{1}=\sum_{j=1}^{5}\left(1+c_{i k(1)}\right) x_{j i}^{0} & \forall i, k(1) ;
\end{array}
$$

(Minimum Withdrawal constraints)

$\sum_{i=1}^{5} x_{i 6}^{0} \geq b_{6}^{0}$,

$$
\sum_{i=1}^{5} x_{i 6 k(1)}^{1} \geq b_{6 k(1)}^{1}, \quad \forall k(1)
$$

(Non - negativity constraints)

$$
\begin{array}{ll}
x_{i j}^{0}, x_{i 6 k(1)}^{1}, x_{i 7 k(1)}^{1} \geq 0 . & i=1, \ldots, 5 ; j=1, \ldots, 5 ; \forall k(1) ; \\
\phi, \delta_{n k(1)} \text { free of sign. } & \forall n, k(1) .
\end{array}
$$




\subsubsection{Two-stage model}

$\operatorname{Min} \mathbf{Z}_{k}=\mathbf{c}^{0} \mathbf{x}^{0}+\left(\mathbf{c}_{k}^{0} \mathbf{x}^{0}+\mathbf{c}_{k}^{1} \mathbf{x}_{k}^{1}\right) ; \quad k=1, \ldots, p ;$

s.t. $\quad \mathbf{A}^{0} \mathbf{x}^{0} \leq \mathbf{b}^{0}$,

$$
\begin{array}{ll}
\mathbf{A}_{k}^{0} \mathbf{x}^{0}+\mathbf{A}_{k}^{1} \mathbf{x}_{k}^{1} \leq \mathbf{b}_{k}^{1}, & k=1, \ldots, p \\
\mathbf{x}^{0}, \mathbf{x}_{k}^{1} \geq \mathbf{0} . & k=1, \ldots, p .
\end{array}
$$

\subsubsection{Three-stage GP model for example 4.5.1}

$$
\begin{array}{ll}
\text { Min } \psi=\phi+\epsilon \sum_{k(2) \in K_{2}} \sum_{k(1) \in K_{1}} \sum_{n=1}^{2}\left(\delta_{n k(1) k(2)}\right) & \\
\text { s.t. } \delta_{n k(1) k(2)}-\phi \leq 0, & n=1,2, \forall k(1), k(2) ; \\
Z_{n k(1) k(2)}-\delta_{n k(1) k(2)}=g_{n k(1) k(2)}, & n=1,2, \forall k(1), k(2) ; \\
(\text { Funds balance constraints }) & \\
\sum_{j=1}^{6}\left(1+p_{i j}\right) x_{i j}^{0}=b_{i}^{0}, & i=1, \ldots, 5 ; \\
\sum_{j=1}^{6}\left(1+p_{i j}\right) x_{i j k(1)}^{1}=\sum_{j=1}^{5}\left(1+c_{i k(1)}\right) x_{j i}^{0} & i=1,2,3,4,5 ; \\
\left(1+p_{i 6}\right) x_{i 6 k(1) k(2)}^{2}+x_{i 7 k(1) k(2)}^{2}=\sum_{i=1}^{5}\left(1+c_{j k(1) k(2)}\right) x_{j i k(1)}^{1} & \forall k(1) ; \\
(\text { Minimum Withdrawal constraints }) & \forall i, k(1), k(2) ; \\
\sum_{i=1}^{5} x_{i 6}^{0} \geq b_{6}^{0}, & \\
\sum_{i=1}^{5} x_{i 6 k(1)}^{1} \geq b_{6 k(1)}^{1}, & \forall k(1) ; \\
\sum_{i=1}^{5} x_{i 6 k(1) k(2)}^{0} \geq b_{6 k(1) k(2)}^{2}, & \forall k(1), k(2) ; \\
(N o n-n e g a t i v i t y \text { constraints }) & \\
\quad x_{i j}^{0}, x_{i j k(1)}^{1}, x_{i 6 k(1) k(2)}^{2}, x_{i 7 k(1) k(2)}^{2} \geq 0 . & \\
\quad \phi, \delta_{n k(1) k(2)} \text { free of sign. } & \forall k(1), k(2) . \\
& \forall n, k(1), k(2) .
\end{array}
$$

\subsubsection{Notations for example 4.5.1}




\begin{tabular}{|c|c|}
\hline $\begin{array}{l}\text { Notation } \\
I ; I=1,2,3,4,5\end{array}$ & Set of investment options. \\
\hline \multicolumn{2}{|c|}{ Decision variables (stage ' 0 ') } \\
\hline$x_{i j}^{0} \in \Re:$ & $\begin{array}{l}\text { Amount of fund transferring from investment option } i \in I \text { to } \\
\text { investment option } j \in I \text { in stage ' } 0 \text { '. }\end{array}$ \\
\hline$x_{i 6}^{0} \in \Re:$ & $\begin{array}{l}\text { Amount of fund withdrawing from investment option } i \in I \text { to } \\
\text { consumption expenditure in stage ' } 0 \text { '. }\end{array}$ \\
\hline Decision variables (stage ' 1 ') & \\
\hline $\begin{array}{l}x_{i 6 k(1)}^{1} \in \Re: \\
x_{i 7 k(1)}^{1} \in \Re: \\
\text { 3-stage model }\end{array}$ & $\begin{array}{l}\text { Amount of fund withdrawing from investment option } i \in I \\
\text { if scenario } S_{k(1)} \text { revealed }\left(S_{k(1)} \in S_{2}, S_{3}, S_{4}\right) \text {. } \\
\text { Amount of fund available in investment option } i \in I \\
\text { if scenario } S_{k(1)} \text { revealed }\left(S_{k(1)} \in S_{2}, S_{3}, S_{4}\right) \text {. }\end{array}$ \\
\hline$x_{i j k(1)}^{1} \in \Re:$ & $\begin{array}{l}\text { Amount of fund transferring from investment option } i \in I \text { to } \\
\text { investment option } j \in I \text { in stage ' } 1 \text { ', } \\
\text { if state } S_{k(1)} \text { revealed }\left(S_{k(1)} \in S_{2}, S_{3}, S_{4}\right) \text {. }\end{array}$ \\
\hline \multicolumn{2}{|c|}{ Decision variables (stage '2') } \\
\hline$x_{i 6 k(1) k(2)}^{2} \in \Re:$ & $\begin{array}{l}\text { Amount of fund withdrawing from investment option } i \in I \\
\text { if meta-scenario } S_{k(1) k(2)} \text { revealed }\left(S_{k(1) k(2)} \in S_{1}, S_{2}, S_{3}, S_{4}, S_{5}\right) \text {. }\end{array}$ \\
\hline$x_{i 7 k(1) k(2)}^{2} \in \Re:$ & $\begin{array}{l}\text { Amount of fund available in investment option } i \in I \\
\text { if meta-scenario } S_{k(1) k(2)} \text { revealed }\left(S_{k(1) k(2)} \in S_{1}, S_{2}, S_{3}, S_{4}, S_{5}\right) \text {. }\end{array}$ \\
\hline \multicolumn{2}{|l|}{ Parameters } \\
\hline$c_{j k(1)}:$ & $\begin{array}{l}\text { The percentage of the growth of investment option } j \in I \text {, } \\
\text { if state } S_{k(1)} \text { revealed }\left(S_{k(1)} \in S_{2}, S_{3}, S_{4}\right) \text {. }\end{array}$ \\
\hline$c_{j k(1) k(2)}$ & $\begin{array}{l}\text { The percentage of the growth of investment option } j \in I \\
\text { if meta-scenario } S_{k(1) k(2)} \text { revealed }\left(S_{k(1) k(2)} \in S_{1}, S_{2}, S_{3}, S_{4}, S_{5}\right) \text {. }\end{array}$ \\
\hline$p_{i j}:$ & $\begin{array}{l}\text { Percentage of loss of funds (penalty cost) for transferring } \\
\text { between each pair of investment }(i, j) \text {. }\end{array}$ \\
\hline$p_{i 6}:$ & $\begin{array}{l}\text { Percentage of loss of funds (penalty cost) for withdrawal money } \\
\text { from investment } i \in I \text {. }\end{array}$ \\
\hline$b_{i}^{0}:$ & Available funds in investment options $i \in I$. \\
\hline$b_{6}^{0}:$ & Minimum required fund to withdrawal at stage ' 0 '. \\
\hline$b_{6 k(1)}^{1}:$ & $\begin{array}{l}\text { Minimum required fund to withdrawal at stage ' } 1 \text { '. } \\
\text { if state } S_{k(1)} \text { revealed }\left(S_{k(1)} \in S_{2}, S_{3}, S_{4}\right) \text {. }\end{array}$ \\
\hline$b_{6 k(1) k(2)}^{1}:$ & $\begin{array}{l}\text { Minimum required fund to withdrawal at stage ' } 1 \text { '. } \\
\text { if meta-scenario } S_{k(1) k(2)} \text { revealed }\left(S_{k(1) k(2)} \in S_{1}, S_{2}, S_{3}, S_{4}, S_{5}\right) \text {. }\end{array}$ \\
\hline$g_{n k(1)} \in \Re,:$ & Goal $n$ in scenario $k(1)$ in 2 -stage model. \\
\hline$\delta_{n k(1)} \in \Re,:$ & Deviations from the goal $n$ in scenario $k(1)$ in 2 -stage model. \\
\hline $\begin{array}{l}g_{n k(1) k(2)} \in \Re,: \\
\delta_{n k(1) k(2)} \in \Re,:\end{array}$ & $\begin{array}{l}\text { Goal } n \text { in meta-scenario } k(1) k(2) \text { in } 3 \text {-stage model. } \\
\text { Deviations from the goal } n \text { in meta-scenario } k(1) k(2) \text { in } 3 \text {-stage model. }\end{array}$ \\
\hline
\end{tabular}

Table 4.18: Variables notation 


\section{Chapter 5}

\section{A Simulation-Optimisation Study on Moving Horizon Structure under Deep Uncertainty}

\subsection{Introduction}

Indefinite real world problems need an indeterminate planning horizon and infinite horizon models. However, handling this level of uncertainty is almost impossible with the current technologies and knowledge. Therefore, we have to limit the endless real horizon into a restricted perspective, then rolling this bounded horizon may help us portray that infinite horizon problem as we move ahead into the future. Integrating the proposed structures, moving horizon models, and deep uncertainty seem to be perfectly matched with this concept. Meta-scenarios and stages fit well in this case as well as the concept of deep uncertainty that naturally exists in the long-term horizons. Also, the successful results from rolling the two-stage model in a three-stage horizon in the previous chapter boost our motivation to extend these concepts to the long-term planning horizon problems. Moreover, identifying plausible meta-scenarios and their conditions for twenty, ten, or even five stages, is not simply possible and some surprises may arise over the extended period and affect the future. The application of moving horizon models gives us an opportunity to make modifications during the planning horizon if new information brings some unexpected surprises that were not considered in advance. This adaptability will be useful and meaningful for long-term strategic decision-making problems in our fast growing world. For example, imagine one is running a company in which he/she uses a technology to produce some products. In the event of the emergence of a new technology that suddenly takes control of the market, this company would need to develop the current technology immediately, redesign the planning and determine new scenarios and their conditions so all these modifications can perform simply in the proposed structure. This feature (versatility of the proposed approach) increases our motivation to extend the proposed structures to these kinds of problems. However, the important question concerns the most valuable length of the decision-making process (two or three stages) for a long-term moving horizon structure in practice (i.e. how many stages should we look ahead at each stage?).

As discussed in the previous chapter, we used some artificial stages to delineate the planning horizon into different periods that were separated by the time of various scenario realisations in which we will get new knowledge by unfolding an unknown scenario. In the proposed twostage structure we always plan for the next stage, while the three-stage structure looks two steps ahead. The previous chapter also indicated the fact that, although the solution of the two-stage model provides us with the optimality under the uncertain conditions of the first period, it 
may not be even feasible and recoverable under additional uncertainties and circumstances of the new phase that would be appended in the three-stage structure. However, the three-stage structure, by a more futuristic vision, may suggest a suboptimal initial decision that could make feasible robust meta-decisions for each and every plausible meta-scenario. This property extends the dynamic-robust solution concept for the three-stage framework that naturally existed in the proposed two-stage structure. Thus, the three-stage model provides us with a more robust initial decision than the two-stage approach. Of course, this robust decision may not be optimal in the first stage, but the full dynamic-robust meta-decisions will be both feasible and optimal to the three-stage model. Furthermore, it was shown that the optimal solution to the two-stage moving horizon model is a feasible solution to the three-stage model. Therefore, applying the solutions of the three-stage model must give rise to better, or at least the same, goal achievement (Min-Max deviation from the goals on GP) than the other decisions.

All the above advantages of the three-stage structure confirm the superiority of utilising this approach. However, sensitivity analysis and more comparison between these patterns in a threestage horizon in the previous chapter described that, despite some advantages of the three-stage structure, in some cases, it may not be preferable compared to the two successive two-stage models. Moreover, it was shown in the previous chapter that there is a fundamental problem of comparability between the two methods because of the different time horizons. This is because the solution to the three-stage structure, by nature, has a robust orientation that manifests itself by choosing a sub-optimal initial solution and postpones some parts of the decision until after the scenario realisation steps in the distant future. This property has no place in the twostage moving horizon models that are ignorant of the third stage conditions. This difference is an issue of future flows of objective function performance. Therefore, we need to compare these methods in the same period for which the two-stage moving horizon model has been introduced. The three-stage model naturally puts more weights on the posterior targets as the model considers all consequences of the initial decisions on the latter stages. Also, this model tries to satisfy all following limitations under all scenarios as well as the conditions of the next stage, especially when no specific condition has been considered for the first and/or intermediate stages. However, as demonstrated briefly in Chapter 3, appending some additional objectives that act as the particular preferences of the first stage concerning all meta-criteria, and the use of various weights for different stages, may help the DM obtain a more preferred solution from the Pareto frontier and makes the two and three-stage approaches more directly comparable. These issues motivated us to introduce modelling of time preferences in multi-stage problems in the current chapter.

On the other hand, adding more stages would exponentially increase the size of the model, and this would be a major limitation. In practice, for real world applications with multiple uncertain parameters, even modelling a problem with three stages would be too complex and solving that model, if ever possible, is computationally expensive. Therefore, considering more than four or five stages in real problems may not be practical.

\subsubsection{Chapter Motivations Summary}

According to the above discussion, the main motivations for extending our proposed structure to the moving horizon models in the current chapter can be summarised as follows:

1 The main motivation was dealing with deep uncertainty in multi-criteria decision analytic models/multi-objective optimisation in long-term strategic planning problems. Introducing the two-stage model and extending it to three-stage (multi-stage) successfully led us to handle deep uncertainty in MCDM problems with a short-term (two and three stages) planning horizon. However, we still need to conclude how many stages we should consider in advance in an infinite horizon.

2 Dynamic-robust solutions are adaptable (i.e. they look some steps ahead, postpone part of the decision and leave room for possible adaptation later after scenario realisation) and 
seem remarkably attractive and meaningful in this concept and may satisfy the DM.

3 The proposed scenario-based framework works naturally with the moving horizons models, because there is no need to find a common feasible initial solution that works for all scenarios. It is sufficient for the solution to be good enough as a starting point and have a feasible union with the recourse decisions in every plausible scenario.

4 Drawing a horizon that is too long would not be an exact plan, as nothing in nature is predictable, and we are always surprised by new events. Therefore, beyond the expensive computation in a multi-stage structure for more than three stages, defining plausible scenarios for far stages is not logical nor even possible. However, identifying plausible scenarios for the next two stages (near future) is much easier and acceptable. These are also entirely matched with the dynamic nature of the proposed frameworks which motivated us to extend the concept to a moving horizon structure.

5 The difference between the solutions to the two and three-stage structure is an issue of future flows of objective function performances, and time plays an important role in these kinds of problems. Applying time preferences in multi-stage problems would be an interesting idea to explore.

Therefore, in this chapter, we will extend and compare the two proposed models to investigate the length of horizon needed in a moving horizon structure for an unlimited horizon. We also consider the following questions:

- How many scenarios can be handled by the proposed structures?

- In examining the effect of horizon length in moving horizon models, can we draw conclusions as to the optimum length of the planning horizon to use in practice?

Then, suppose that $\mathbf{T}(T \in \mathbb{N}, T \geq 3)$ is the number of stages that construct the whole period of study or planning horizon. Two algorithms, based on the proposed two-stage and three-stage models will be used to form the moving horizon models and their results can be compared. The question, still, is: "How many stages, one or two, should we look ahead in a moving horizon structure?".

To understand this, we need to analyse a vast majority of decisions generated by various models under different conditions that, even for five or six stages, requires hundreds and thousands of multi-objective comparisons that are not easily conceivable, if ever possible. This issue, that will be explained later (section 5.2.3), motivated us to conduct a Simulation-Optimisation study to be able to evaluate, compare and analyse these methods for problems with a T-stage planning horizon.

In this simulation study, we will attempt to answer the following questions:

- How do we generate random scenarios for the simulation?

- How do we compare the results of the simulations?

- How many simulations need to be run?

- How large should the length of horizon be?

Structuring of the moving horizon models in a T-stage planning window, as well as their mathematical formulations, have been fully described and compared in Section 5.2. Modelling of time preferences in multi-objective multi-stage models has been introduced in Section 5.3. Section 5.4 is dedicated to introducing a simulation optimisation approach and two algorithms that can help us carry out our study and compare the proposed moving horizon structures in a T-stage planning horizon. In Section 5.5, these concepts and structures will be illustrated in detail through a simple but non-trivial problem (sequential investments) in which the proposed structures and algorithms are applied and the results are compared and interpreted. In Section 5.6 , the length of the planning horizon and its effects on the solutions to the proposed frameworks will be examined and this will be followed by the conclusion in 5.7. 


\subsection{Moving horizon models in a T-stage planning window}

In a T-stage decision-making structure, the decision process is divided into $\mathrm{T}$ stages, from $\mathbf{X}^{0}$ to $\mathbf{X}^{(T-1)}$, during the time horizon. Horizons are arbitrarily chosen depending on the problem as real processes are continuous, and by the time horizon, we try to structure and model a specific part of an infinite future. Stages and time horizons can help us talk, think about, and formulate the model. Real problems are continuous, and scenarios are unfolding continuously. Also, the stages are utilised to distinguish separate periods of the decision-making process and scenario realisation, viz. the time that some uncertain states of the problem are unfolded and the consequences of our previous decisions manifest themselves. In other words, each stage points out to an artificial time when the uncertain states are expected to be recognised. In fact, by this time, the DM will have received enough information about some previously unknown states and the outcomes of the prior decision(s). Then, the recourse action can be implemented to recover from the prior decisions, while the next states are still unknown.

Consider a decision-making problem with a T-stage planning horizon including $\mathrm{T}$ stages of decision-making and T-1 steps of scenario realisation, as shown at the top of Figure 5.1. Suppose that $\mathbf{x}^{0}, \mathbf{x}^{1}, \ldots$, and $\mathbf{x}^{T-1}$ present the decision vectors related to each stage, respectively. $S\left(k_{1}\right)$, $S\left(k_{2}\right), \ldots$, and $S\left(k_{T-1}\right)$ indicate the scenario spaces regarding the first, second, and $(T-1)^{t h}$ steps of scenario revelation, respectively. In a one-step moving horizon model we always plan as if it is a two-stage horizon, while the two-step moving horizon model acts as a three-stage model. Note that in both structures, at each stage only the first initial decision is implemented after running the model (red dashed circles, in Figure 5.1, demonstrate the decisions that will be implemented), except the last series in which we reach the end of the planning horizon and all decisions will be executed.

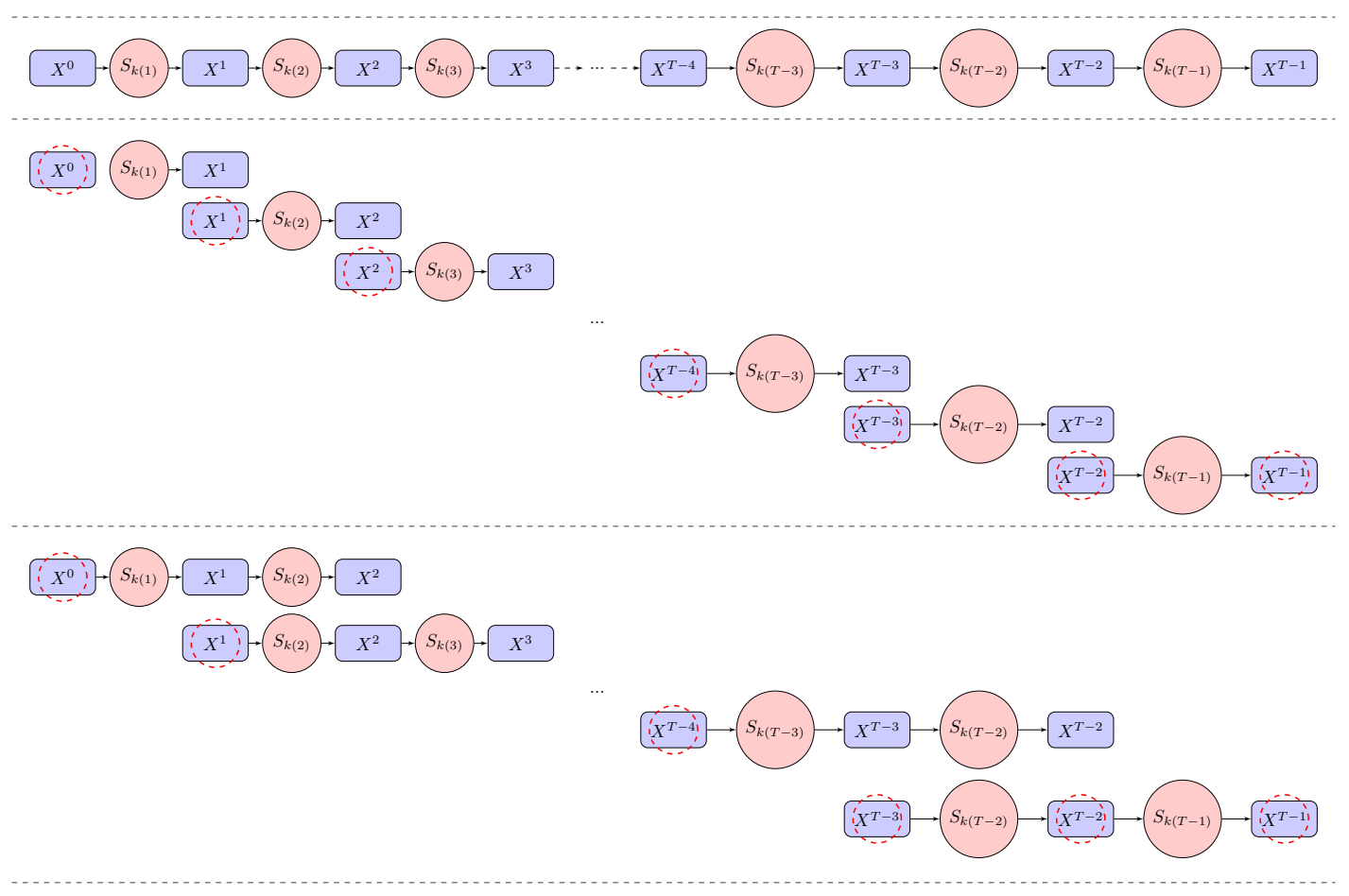

Figure 5.1: Rolling T-1 consecutive two-stage and T-2 following three-stage in a T-stage horizon 
Definition 5.2.1. Meta-decision: In a T-stage structure, a "meta-decision", shown by $\mathbf{X}=$ $\left(\mathbf{x}^{0}, \mathbf{x}_{k(1)}^{1}, \ldots, \mathbf{x}_{k(1) k(2) \ldots k(T-1)}^{T-1}\right)$, including a chain of some consecutive decisions which are made in different stages of the decision-making process regarding each meta-criterion. In other words, meta-decisions contain the initial decision and $(T-1)$ more consecutive decisions concerning all performance measures.

Definition 5.2.2. Meta-scenario: The term "meta-scenario" in a T-stage planning window describes a plausible combination of scenarios in all $(T-1)$ scenario realisation stages (it may be shown by paths in a scenario tree).

Each scenario includes the realisation of scenario $k(1)$ in stage ' 1 ', the revelation of scenario $k(2)$ in stage ' 2 ', ..., scenario $k(T-1)$ unfolding in the last stage $((T-1))$. Depending on the number of plausible scenarios in each stage, the total number of possible combinations would be equal or less than $p(1) \times p(2) \times \ldots \times p(T-1)$, where $p(1), p(2), \ldots$, and $p(T-1)$ are the maximum numbers of plausible scenarios in each relevant stage.

Furthermore, similar to the three-stage framework, in a T-stage structure, each meta-decision is related to a meta-scenario for which an evaluation needs to be done to find out how well a metadecision performs regarding each criterion under conditions of each meta-scenario. This means that each meta-criterion, in a T-stage structure, represents preferences in terms of a criterion under conditions relating to a meta-scenario. Therefore, "meta-criterion" here is defined as the combination of each criterion and meta-scenario in which the number of all meta-criteria is equal to $m \times p$ ( $m$ and $p$ describe, respectively, the total number of criteria and meta-scenarios). Furthermore, in a scenario-based moving horizon multi-criteria decision-making framework under deep uncertainty, meta-scenarios (as a component of the meta-criteria) express a true dimension of preferences and are utilised to avoid problems of assessing and using probabilities under deep uncertainty.

\subsubsection{Rolling two-stage structure}

A two-stage moving horizon model in a three-stage planning window has been fully described in the previous chapter. Therefore, for a T-stage horizon, a two-stage model can be simply iterated until we reach the end of the planning horizon. This means that, at first, the proposed two-stage model (model 3.2) is used to obtain the first stage decisions $\left(\mathbf{x}^{0}\right)$, that could be immediately implemented. Then, we need to wait and see which scenario from $S\left(k_{1}\right)$ will unfold. After recognising the uncertain states in the first stage, another two-stage model (model 4.7) starting at the second stage is applied to reach the decisions $\left(\mathbf{x}^{1}\right)$ that must be executed in the second stage. This process is iterated continuously until the two-stage model starting at the (T-2)-th stage runs and generates the last two decisions $\left(\mathbf{x}^{T-2}\right.$ and $\left.\mathbf{x}^{T-1}\right)$, i.e. $(T-1) \times$ two - stage.

It is worth remembering at this point that the recourse decisions provided by the intermediate two-stage models are not implemented, and they may be used as an approximation that will substitute with the initial decisions gained from the next two-stage model applied after the first scenario realisation of the current model. In other words, after applying the next two-stage model (while the current stage is moved one step ahead), the recourse decisions of the first model are substituted with the initial decisions of the second two-stage model. The last run would be slightly different because, in this stage, we may implement both initial and recourse decisions of the last model.This movement can help us to adapt the decision continuously with the new plausible scenarios in the next stages.

\subsubsection{Mathematical formulations}

The formulation of the first two-stage model is similar to the original two-stage model(model $3.2)^{1}$ ). The second two-stage model, corresponding to scenario $k(1)$, is also formulated in 4.7.

\footnotetext{
${ }^{1}$ Note that $k$ in model 3.2 corresponds to $k_{1}$ in model 4.7 .
} 
The following two-stage models can also be formulated, similar to the second one, by eliminating the previous stages' variables from the objective functions and their related constraints, as well as by moving of the identified variables (by all previous two-stage models) from the left to the right side of some constraints. Generally, the $L^{t h}(L=2, \ldots,(T-1))$ two-stage model, corresponding to scenario $k(L)$, can be formulated as follows:

$$
\begin{aligned}
& \text { Min } Z_{i k(L)}=\sum_{j=1}^{n} \widehat{c}_{i j k(1) k(2) . . k(L-1)}^{L-1} x_{j k(1) k(2) . . k(L-1)}^{L-1} \\
& +\sum_{j=1}^{n} c_{i j k(1) k(2) . . k(L)}^{L} x_{j k(1) k(2) . . k(L)}^{L}, \\
& i=1, \ldots, m \text {; } \\
& \text { s.t. } \\
& \sum_{j=1}^{n} a_{r j k(1) . . k(L-1)}^{L-1} x_{j k(1) . . k(L-1)}^{L-1} \leq \beta_{r k(1) . . k(L-1)}^{L-1}, \\
& r=1, \ldots, R_{1} \\
& \sum_{j=1}^{n} a_{r j k(1) . . k(L)}^{L-1} x_{j k(1) . . k(L-1)}^{L-1}+\sum_{j=1}^{n} a_{r j k(1) . . k(L)}^{L} x_{j k(1) . . k(L)}^{L} \leq \beta_{r k(1) . . k(L)}^{L}, \quad r=1, \ldots, R_{l} \\
& k(L)=1, \ldots, p(L) \text {; } \\
& x_{j}^{0}, x_{j k(1)}^{1}, \ldots, x_{j k(1) . . k(l)}^{l} \geq 0 . \\
& j=1, \ldots, n \text {; } \\
& k(l)=1, \ldots, p(l) \text {; } \\
& l=2, \ldots, L \text {. }
\end{aligned}
$$

where,

$$
\begin{aligned}
\beta_{r k(1) . . k(L-1)}^{L-1}= & b_{r k(1) . . k(L-1)}^{L-1}-\sum_{j=1}^{n} a_{r j k(1) . . k(L-1)}^{0} x_{j}^{0 *}-\sum_{j=1}^{n} a_{r j k(1) . . k(L-1)}^{1} x_{j k(1)}^{1 *}-\ldots \\
& -\sum_{j=1}^{n} a_{r j k(1) . . k(L-1)}^{L-2} x_{j k(1) . . k(L-2)}^{L-2 *}
\end{aligned}
$$

and

$$
\begin{gathered}
\beta_{r k(1) . . k(L)}^{L}=b_{r k(1) . . k(L)}^{L}-\sum_{j=1}^{n} a_{r j k(1) . . k(L)}^{0} x_{j}^{0 *}-\sum_{j=1}^{n} a_{r j k(1) . . k(L)}^{1} x_{j k(1)}^{1 *}-\ldots \\
\quad-\sum_{j=1}^{n} a_{r j k(1) . . k(L)}^{L-2} x_{j k(1) . . k(L-2)}^{L-2 *} .
\end{gathered}
$$

shown the constraints relating to the $(L-1)^{t h}$ and the $L^{t h}$ stages, respectively.

$\sum_{j=1}^{n} \widehat{c}_{i j k(1) . . k(L-1)}^{L-1}=\sum_{j=1}^{n} c_{i j k(1) . . k(L-2)}^{L-1}+\sum_{j=1}^{n} c_{i j k(1) . . k(L-1)}^{L-1}$ explains initial goals and consequences of the previous decisions if meta-scenario $k(1) k(2) . . k(L-1)$ is revealed.

$Z_{i k(L)}$ describes the $i^{\text {th }}$ linear meta-criterion/objective function representing preferences regarding criterion $i$ under conditions pertaining to scenario $k(L)$, and includes some initial goals and consequences of the previous decisions if meta-scenario $k(1) k(2) . . k(L-1)$ is revealed, before any uncertainties of the $L^{t h}$ stage $\left(\sum_{j=1}^{n} \widehat{c}_{i j k(1) . . k(L-1)}^{L-1} x_{j k(1) . . k(L-1)}^{L-1}\right)$, and the recourse decisions for the realisation of $k(L)\left(\sum_{j=1}^{n} c_{i j k(1) . . k(L)}^{L} x_{j k(1) . . k(L)}^{L}\right)$.

By determining $m \times p(L-1)$ aspiration levels for the $(L-1)^{t h}$ stage and solving the equivalent Generalised GP model, as performed in the previous chapters, the solutions to the $L^{\text {th }}$ two-stage $\operatorname{model}\left(\mathbf{X}^{L-1}\right.$ and $\left.\mathbf{X}^{L}\right)$ can be reached. Therefore, rolling the $T-1$ two-stage model in a T-stage horizon provides us with a set of decisions for the two-stage moving horizon model. 
Definition 5.2.3. (Feasible solutions to the two-stage moving horizon model).

Vector $\mathbf{X}=\left(\mathbf{x}^{0\langle 1\rangle}, \mathbf{x}_{k(1)}^{1\langle 2\rangle}, \mathbf{x}_{k(1) k(2)}^{2\langle 3\rangle}, \cdots, \mathbf{x}_{k(1) k(2) \cdots k(T-3)}^{T-3\langle\mathfrak{T}-2\rangle}, \mathbf{x}_{k(1) k(2) \cdots k(T-2)}^{T-2\langle\mathfrak{T}-1\rangle}, \mathbf{x}_{k(1) k(2) \cdots k(T-1)}^{T-1\langle\mathfrak{T}-1\rangle}\right)$

is feasible for a two-stage moving horizon model in the T-stage planning window if it

simultaneously satisfies the constrains in all $(T-1)$ consecutive two-stage models(5.1), also,

$\forall k=k(1) \in S^{1}, \forall k(2) \in S^{2}, \cdots, \forall k(T-1) \in S^{T-1}$

$\exists \mathbf{X}^{\langle 1\rangle}=\left(\mathbf{x}^{0\langle 1\rangle}, \mathbf{x}_{k(1)}^{1\langle 1\rangle}\right), \mathbf{X}^{\langle 2\rangle}=\left(\mathbf{x}_{k(1)}^{1\langle 2\rangle}, \mathbf{x}_{k(1) k(2)}^{2\langle\langle\rangle}\right), \cdots, \mathbf{X}^{\langle\mathfrak{T}-1\rangle}=\left(\mathbf{x}_{k(1) k(2) \cdots k(T-2)}^{T-2\langle\mathfrak{T}-1\rangle}, \mathbf{x}_{k(1) k(2) \cdots k(T-1)}^{T-1\langle\mathfrak{T}-1\rangle}\right) ;$

s.t.

$\mathbf{X}^{\langle 1\rangle}, \mathbf{X}^{\langle 2\rangle}, \cdots$, and $\mathbf{X}^{\langle\mathfrak{T}-1\rangle}$ are, respectively, feasible for the first, second, $\ldots,(T-1)$-th two-stage models.

\subsubsection{Rolling three-stage structure}

Consider, again, the decision-making problem with a T-stage planning horizon described in Figure 5.1. As shown at the bottom of this figure, in a three-stage moving horizon model in a T-stage planning window, firstly, the proposed three-stage model (model 4.2) is used to obtain the first stage decisions $\left(\mathbf{x}^{0}\right)$, which could be immediately executed. Then, we need to wait and see which scenario from $S\left(k_{1}\right)$ will unfold. After that, another three-stage model starting at the second stage is applied to reach the second group of decisions $\left(\mathbf{x}^{1}\right)$ that must be implemented at the second stage. This process is iterated continuously until the three-stage model, starting at the (T-3)-th stage, runs and produces the last three decisions $\left(\mathbf{x}^{T-3}, \mathbf{x}^{T-2}\right.$ and $\left.\mathbf{x}^{T-1}\right)$, i.e. $(T-2) \times$ three-stage models rolling continuously over the planning horizon. Therefore, except for the last run, we always implement the initial decisions in each iteration while looking two steps ahead and evaluate the consequences of these early decisions on different plausible scenarios of the two next stages. However, the recourse decisions of the models in intermediate iterations are not executed. They play their role as an approximation that will substitute with the initial decisions obtained from the next following three-stage models applied after the first scenario realisation of the current model. This movement can help us to adapt the decision continuously with the new plausible scenarios in the next stages.

\subsubsection{Mathematical formulation}

The formulation of the first three-stage model is equivalent to the original three-stage model (model 4.2). The following three-stage models are also formulated similarly to the two-stage moving horizon models. In these models, variables of the previous stages are eliminated from the objective functions and their related constraints. Also, the values for some variables are solved by the previous three-stage models. Therefore, these values now need to be substituted into the model. These variables are then transferred to the right side of the constraints as constant numbers. Generally, the $L^{\text {th }}(L=2, \ldots,(T-2))$ three-stage model, which corresponds to scenario $k(L)$, can be formulated as follows: 
Min $Z_{i_{1} k(L-1)}=\sum_{j=1}^{n} c_{i_{1} j k(1) . . k(L-2)}^{L-2} x_{j k(1) . . k(L-2)}^{L-2}$

$$
+\sum_{j=1}^{n} c_{i_{1} j k(1) . . k(L-1)}^{L-1} x_{j k(1) . . k(L-1)}^{L-1}
$$

Min $Z_{i k(1) . . k(L)}=\sum_{j=1}^{n} \widehat{c}_{i j k(1) . . k(L)}^{L-2} x_{j k(1) . . k(L-2)}^{L-2}$

$$
\begin{aligned}
& +\sum_{j=1}^{n} \widehat{c}_{i j k(1) . . k(L)}^{L-1} x_{j k(1) . . k(L-1)}^{L-1} \\
& +\sum_{j=1}^{n} c_{i j k(1) . . k(L)}^{L} x_{j k(1) . . k(L)}^{L}
\end{aligned}
$$

s.t.

$$
\begin{aligned}
& \sum_{j=1}^{n} a_{r j k(1) . . k(L-2)}^{L-2} x_{j k(1) . . k(L-2)}^{L-2} \leq \beta_{r k(1) . . k(L-2)}^{L-2}, \\
& \sum_{j=1}^{n} a_{r j k(1) . . k(L-1)}^{L-2} x_{j k(1) . . k(L-2)}^{L-2}+\sum_{j=1}^{n} a_{r j k(1) . . k(L-1)}^{L-1} x_{j k(1) . . k(L-1)}^{L-1} \\
& \leq \beta_{r k(1) . . k(L-1)}^{L-1}, \\
& \sum_{j=1}^{n} a_{r j k(1) . . k(L)}^{L-2} x_{j k(1) . . k(L-2)}^{L-2}+\sum_{j=1}^{n} a_{r j k(1) . . k(L)}^{L-1} x_{j k(1) . . k(L-1)}^{L-1} \\
& +\sum_{j=1}^{n} a_{r j k(1) k(2) . . k(L)}^{L} x_{j k(1) . . k(L)}^{L} \\
& \leq \beta_{r k(1) . . k(L)}^{L}, \\
& x_{j k(1) . . k(L-2)}^{L-2}, x_{j k(1) . . k(L-1)}^{L-1}, x_{j k(1) . . k(L)}^{L} \geq 0 .
\end{aligned}
$$

$$
\begin{aligned}
& i_{1}=1, \ldots, m_{1} ; \\
& k(l)=1, \ldots, p(l) ; \\
& l=1, \ldots,(T-1) ; \\
& i=1, \ldots, m ; \\
& k(l)=1, \ldots, p(l) ; \\
& l=1, \ldots,(T-1) ;
\end{aligned}
$$

$$
\begin{aligned}
& r=1, \ldots, R_{0} ; \\
& k(l)=1, \ldots, p(l) ; \\
& l=1, \ldots,(T-1) ; \\
& r=R_{0}+1, \ldots, R_{0}+\ldots+R_{k(1)} ; \\
& \quad k(l)=1, \ldots, p(l) ; \\
& l=1, \ldots,(T-1) \\
& r=R_{0}+\ldots+R_{p(L-1)}+1, \ldots, \\
& \quad R_{0}+\ldots+R_{k(L-1) . k(L-2)} ; \\
& \\
& j=1, \ldots, n ; \\
& k(l)=1, \ldots, p(l) ; \\
& l=1, \ldots,(T-1) .
\end{aligned}
$$

where,

$$
\begin{aligned}
& \beta_{r k(1) . . k(L-2)}^{L-2}=b_{r k(1) . . k(L-2)}^{L-2}-\sum_{j=1}^{n} a_{r j k(1) . . k(L-2)}^{0} x_{j}^{0 *}-\sum_{j=1}^{n} a_{r j k(1) . . k(L-2)}^{1} x_{j k(1)}^{1 *}-\ldots \\
& -\sum_{j=1}^{n} a_{r j k(1) . . k(L-2)}^{L-3} x_{j k(1) . . k(L-3)}^{L-3 *}, \\
& \beta_{r k(1) . . k(L-1)}^{L-1}=b_{r k(1) . . k(L-1)}^{L-1}-\sum_{j=1}^{n} a_{r j k(1) . . k(L-1)}^{0} x_{j}^{0 *}-\sum_{j=1}^{n} a_{r j k(1) . . k(L-1)}^{1} x_{j k(1)}^{1 *}-\ldots \\
& -\sum_{j=1}^{n} a_{r j k(1) . . k(L-1)}^{L-3} x_{j k(1) . . k(L-3)}^{L-3 *}, \text { and } \\
& \beta_{r k(1) . . k(L)}^{L}=b_{r k(1) . . k(L)}^{L}-\sum_{j=1}^{n} a_{r j k(1) . . k(L)}^{0} x_{j}^{0 *}-\sum_{j=1}^{n} a_{r j k(1) . . k(L)}^{1} x_{j k(1)}^{1 *}-\ldots \\
& -\sum_{j=1}^{n} a_{r j k(1) . . k(L)}^{L-3} x_{j k(1) . . k(L-3)}^{L-3 *} .
\end{aligned}
$$

describe the constraints relating to the $(L-2)^{t h},(L-1)^{t h}$, and the $L^{t h}$ stages, respectively.

$Z_{i_{1} k(L-1)}$ represent the exclusive preferences of the $(L-1)^{t h}$ stage regarding criterion $i_{1}$ under conditions of scenario $k(L-1)$.

$\sum_{j=1}^{n} \widehat{c}_{i j k(1) . . k(L)}^{L-2}=\sum_{j=1}^{n} c_{i j k(1) . . k(L-2)}^{L-2}+\sum_{j=1}^{n} c_{i j k(1) . . k(L-1)}^{L-2}+\sum_{j=1}^{n} c_{i j k(1) . . k(L)}^{L-2}$ expresses initial goals, consequences of the prior decisions under conditions of meta-scenario $k(1) k(2) . . k(L-1)$ in stage ' $(L-1)$ ', and meta-scenario $k(1) k(2) . . k(L)$ in stage ' $L$ '. 
$\sum_{j=1}^{n} \widehat{c}_{i j k(1) . . k(L)}^{L-1}=\sum_{j=1}^{n} c_{i j k(1) . . k(L-1)}^{L-1}+\sum_{j=1}^{n} c_{i j k(1) . . k(L-2)}^{L-2}$ presents outcomes in stage ' $(L-1)$ ', and consequences of the recourse decisions at this stage, under conditions of meta-scenario $k(1) k(2) . . k(L-1)$.

$Z_{i k(1) . . k(L)}$ demonstrate the $i^{t h}$ linear meta-criterion/objective function indicating preferences concerning criterion $i$ under conditions pertaining to meta-scenario $k(1) k(2) . . k(L)$.

If $m \times p(L-1) \times p(L-2)$ aspiration levels are identified (by the DM) for the $L^{\text {th }}$ stage, by solving the equivalent Generalised GP model the solutions to the $L^{\text {th }}$ three-stage model $\left(\mathbf{X}^{L-2}, \mathbf{X}^{L-1}\right.$, and $\left.\mathbf{X}^{L}\right)$ will be obtained. Therefore, a set of decisions for the three-stage moving horizon model can be reached by rolling the $T-2$ three-stage model in a T-stage planning horizon.

Definition 5.2.4. (Feasible solutions to the three-stage moving horizon model). Vector $\mathbf{X}=\left(\mathbf{x}^{0\langle\mathbf{1}\rangle}, \mathbf{x}_{k(1)}^{1\langle 2\rangle}, \mathbf{x}_{k(1) k(2)}^{2\langle 3\rangle}, \cdots, \mathbf{x}_{k(1) k(2) \cdots k(T-3)}^{T-3\langle\mathfrak{T}-2\rangle}, \mathbf{x}_{k(1) k(2) \cdots k(T-2)}^{T-2\langle\mathfrak{T}-2\rangle}, \mathbf{x}_{k(1) k(2) \cdots k(T-1)}^{T-1\langle\mathfrak{T}-2\rangle}\right)$

is feasible for a three-stage moving horizon model in the T-stage planning window if it simultaneously satisfies the constrains in all $(T-2)$ consecutive three-stage models(5.2). Also,

$\forall k(1) \in S^{1}, \forall k(2) \in S^{2}, \cdots, \forall k(T-1) \in S^{T-1}$

$\exists \mathbf{X}^{\langle 1\rangle}=\left(\mathbf{x}^{O\langle 1\rangle}, \mathbf{x}_{k(1)}^{1\langle\mathbf{1}\rangle}, \mathbf{x}_{k(1) k(2)}^{2\langle 1\rangle}\right), \mathbf{X}^{\langle 2\rangle}=\left(\mathbf{x}_{k(1)}^{1\langle 2\rangle}, \mathbf{x}_{k(1) k(2)}^{2\langle 2\rangle}, \mathbf{x}_{k(1) k(2) k(3)}^{3\langle 2\rangle}\right), \cdots$,

$\mathbf{X}^{\langle\mathfrak{T}-2\rangle}=\left(\mathbf{x}_{k(1) k(2) \cdots k(T-3)}^{T-3\langle\mathfrak{T}-2\rangle}, \mathbf{x}_{k(1) k(2) \cdots k(T-2)}^{T-2\langle\mathfrak{T}-2\rangle}, \mathbf{x}_{k(1) k(2) \cdots k(T-1)}^{T-1\langle\mathfrak{T}-2\rangle}\right)$

s.t.

$\mathbf{X}^{\langle 1\rangle}, \mathbf{X}^{\langle 2\rangle}, \cdots$, and $\mathbf{X}^{\langle\mathfrak{T}-2\rangle}$ are, respectively, feasible for the first, second, ..., $(T-2)$-th threestage models.

\subsubsection{Comparing the rolling two-stage pattern with the three-stage framework in a moving horizon structure}

he previous sections introduced and formulated the rolling two- and the three-stage moving horizon in a longer planning window. These models can help us reach our primary aim of dealing with deep uncertainty in long-term strategic planning problems. However, the important question, which we will attempt to answer in the current chapter, concerns the most valuable length of the decision-making process(two or three stages) for a long-term moving horizon structure in practice (i.e. how many stages should be look ahead at each stage?). In this section, and throughout the rest of the chapter, these structures and their concepts, solutions, and formulations are evaluated and compared to investigate and highlight their advantages and disadvantages.

Lemma 5.2.1. Any feasible solution (and hence the optimal one) of any pair of two successive two-stage models (model 5.1) is a feasible solution for the corresponding three-stage model (model 5.2 ) and vice versa (i.e. the two-stage moving horizon model is feasible iff the three-stage model is feasible).

Proof. The proof of this theorem will be simply concluded by replacing any three successive stages (e.g. replace stages 0,1 , and 2 by $(L-2),(L-1)$, and $L$ ) and the related patterns in proof of Theorem 4.4.1 in 4.8.1. 
Theorem 5.2.2. Any feasible (and hence the optimal) solution to the two-stage moving horizon model in a T-stage $(T \in \mathbb{N}, T \geq 3)$ horizon is a feasible solution for the corresponding three-stage moving horizon model.

Proof. The proof will be simply concluded by using the principle of mathematical induction. The theorem 4.4.1 states that the above statement is true for $\mathrm{T}=3$. Then, we can assume that the statement is true for (T-1)-stage horizon. Therefore, by utilising the induction assumption, and applying the Lemma 5.2.1 for the two last stages (i.e. for (T-1)-th and T-th stages), clearly, the statement is true for the T-stage horizon.

Corollary 5.2.2.1. The optimal solution to the three-stage moving horizon model in a problem with T-stage planning window is not dominated by any solution of the corresponding two-stage moving horizon model.

Proof. The proof is directly concluded from Theorem 5.2.2.

The three-stage model is looking further ahead than the two-stage model in each stage, and the constraints in the three-stage model, including all the limitations of the corresponding twostage model, are simultaneously satisfied. Furthermore, the above corollary confirms that the optimal solution to the three-stage moving horizon model is not dominated by any solutions of the other model. So, with no doubt, it must find a better, or at least the same, goal achievement compared to the two-stage moving horizon model. Also, it was indicated in the previous chapter that the three-stage structure provides more robust solutions than the two-stage pattern, because the three-stage structure considers the consequences of the decisions of the initial stages and the conditions of different meta-scenarios with a longer future vision. Accordingly, the three-stage moving horizon model evaluates and compares the optimal solution of the corresponding twostage moving horizon model to the other feasible solutions. Then, if it selects anything else, it must either be better in goal achievement or be robust in some future scenarios, or have a combination of both these features.

To sum up, the superiority of the three-stage moving horizon model has been proven. However, as discussed in the previous chapter, the cost of these supremacies must be considered (as it may lead us to a computationally expensive problem), and the efficiency of applying this structure still needs to be proved.

It must be mentioned at this point that, for the analysis of all plausible meta-scenarios and comparison of the solutions to these two moving horizon models in a T-stage planning window, too many models would need to be run. In fact, after the performance of the first stage in the first model which generates the initial decisions, we would need to run $p_{t}$ different models for each scenario at each subsequent stage, where $p_{t}$ is the total number of plausible scenarios in that stage (stage ' $t$ ', $t=0, . .,(T-1)$ ). Therefore, the total number of two- and three-stage models that needs to be run in a T-stage problem (present by $p$ ) can be calculated as follows:

$$
\begin{aligned}
& p^{\langle 2-\text { stage }\rangle}=1+p_{1}+p_{1} \times p_{2}+p_{1} \times p_{2} \times p_{3}+\cdots+p_{1} \times p_{2} \times \ldots \times p_{T-1} . \\
& p^{\langle 3-\text { stage }\rangle}=1+p_{1}+p_{1} \times p_{2}+p_{1} \times p_{2} \times p_{3}+\cdots+p_{1} \times p_{2} \times \ldots \times p_{T-2} .
\end{aligned}
$$

If we assume that $p_{t}$ for all scenarios in all stages are the same and equal to $\rho$, then the total number of models that need to be run to cover all plausible scenarios in the whole period can be computed by the following geometric series:

$$
\begin{aligned}
& p^{\langle 2-\text { stage }\rangle}=1+\rho+\rho^{2}+\cdots+\rho^{T-1}=\sum_{k=0}^{T-1} \rho^{k}=\frac{1-\rho^{T}}{1-\rho} \\
& p^{\langle 3-\text { stage }\rangle}=1+\rho+\rho^{2}+\cdots+\rho^{T-2}=\sum_{k=0}^{T-2} \rho^{k}=\frac{1-\rho^{T-1}}{1-\rho}
\end{aligned}
$$


Accordingly, for instance, if $p_{t}=\rho=3$ for all scenarios in all stages in a problem with six-stage planning window $(T=6)$, the total number of required two-stage models is

$p_{\{6\}}^{\langle 2-\text { stage }\rangle}=\sum_{k=0}^{5} 3^{k}=\frac{1-3^{6}}{1-3}=364\left(p_{\{6\}}^{\langle 3-\text { stage }\rangle}=121\right)$.

This number for the planning window of seven and ten stages are, respectively, equals to

$p_{\{7\}}^{\langle 2-\text { stage }\rangle}=\frac{1-3^{7}}{2}=1093$ and $p_{\{10\}}^{\langle 2-\text { stage }\rangle}=\frac{1-3^{10}}{2}=29524$ two-stage models (for three-stage these numbers are $p_{\{7\}}^{\langle 3-\text { stage }\rangle}=\frac{1-3^{6}}{2}=364$ and $p_{\{10\}}^{\langle 3-\text { stage }\rangle}=\frac{1-3^{9}}{2}=9841$, respectively).

Therefore, carrying an analysis of the solutions of these numbers of models, even for five or six stages, requires hundreds and thousands of multi-objective comparisons that are not easily conceivable, if ever possible. This issue motivated us to perform a simulation study to evaluate, compare and analyse these methods for problems with a T-stage planning horizon. Section 5.4 is dedicated to introducing a simulation optimisation approach that can help us compare the twoand three-stage moving horizon models structures in a T-stage planning window. However, before that, we need to discuss the comparability between the proposed two- and three-stage moving horizon models with different planning windows. Thus, let us first talk about this problem and present a solution. This issue will be solved by identifying some proper weights in the equivalence Goal Programming models that lead us to introduce the "time preferences" in multi-objective multi-stage problems in the next section.

\subsection{Modelling of Time Preferences in Multi-Objective Multi- stage Models}

As mentioned in the previous chapter, there is a fundamental problem of comparability between the two methods because of the different time horizons. In turn, these different time horizons emerge because the two-stage model is ignorant of the third stage. This difference is an issue of future flows of objective function performance. Therefore, we need to compare these methods in the same period. To reach this purpose the two-stage moving horizon models have been introduced. Accordingly, as an extension of the two-stage moving horizon models, the two- and three-stage moving horizon models in a T-stage planning window have been proposed in this chapter. The main purpose of this chapter is to compare these two methods and provide some visual signs of the superiority of them. Thus, a meaningful comparison is necessary.

Moreover, it was shown in the previous chapter that the three-stage model naturally puts more weights on the posterior targets because it considers all consequences of the initial decisions on the latter stages. Also, the three-stage model tries to satisfy all following limitations under all scenarios as well as the conditions of the next stage, especially when no specific condition has been considered for the first and/or intermediate stages. On the other hand, the solutions to the three-stage structure, by nature, have a robust orientation that manifests themselves by choosing a sub-optimal initial solution and postponing some parts of the decision until after scenario realisation steps in the distant future. This property has no place in the two-stage moving horizon models that is ignorant of the third stage conditions. However, as demonstrated briefly in Chapter 3, some variations in importance weights, and of the equivalent Goal Programming models, can help us make the two and three-stage approaches more directly comparable. These issues motivated us to investigate the "modelling of time preferences in multi-stage problems" in this section.

Note that the terms "time preferences" or "time discounting" have a different meaning and definition in economics and psychology. Here, the term "time preferences" is utilised to classify the relative importance of the stages and the activities that related to them. 
As frequently mentioned before, the Generalised Goal Programming approach has been utilised in this study to solve the multi-objective problems. Determining the aspiration levels is the first order of the DM's preferences in GP approaches, while identifying the importance weights is the second order. In fact, in generalised GP, the DM's preferences are largely modelled by choice of goals. The weights are primarily to achieve a comparative scaling on each objective. In other words, as the importance of a criterion (or meta-criterion in our definitions) is displayed greatly by the goals which are identified by the $\mathrm{DM}$, while the weight parameters (in GP) play a different role as the target values. Nevertheless, as an effect, a re-scaling of the objective values is presented by the weights to certify suitable levels of the trade-off between objective functions in the presence of the goals.

We suggest the use of importance weights to make the two and three-stage solutions comparable. This will be done by assigning various weights for different activities in some specific stages(time) and these weights will represent the priority of the stages (some specific time during the planning horizon) for the DM in multi-stage decision making. To reach this purpose, two ways of weighting as "differential weighting" and "discounting weights" will be introduced in the following sections.

\subsubsection{Differential Weighting}

As mentioned above, the three-stage model has a natural tendency to put more weights on future events and tries to satisfy them as much as possible. However, as discussed in the previous chapter, adding some particular constraints for the first and middle stages can help to balance the solutions and obtain more reasonable decisions for the three-stage structure. Furthermore, in a complex multi-criteria problem with an infinite planning horizon under deep uncertainty, while the future parameters are changing and updating continuously, putting more weights on the unknown future and concentrating on satisfying all the expectations, does not seem to be a robust strategy. Therefore, it would be more meaningful if more weights are applied on the first stage of the three-stage structure than the second and the third stages. This weighting could lead the model to generate balanced solutions whilst also applying the DM's time preferences in the presence of the preferred goals.

On the other hand, the two-stage framework does not consider the third stage's conditions at all and this would lead us to some dis-satisfactory or even infeasible solutions in the next stages. This shortcoming, as discussed in Chapter 3, can improve when some weights are applied to this model which would help to produce more robust initial decisions than the equal weighting. These weights would also make much more sense if more weights are applied on the outcomes of the model in the second stage. This weighting can present the fact that the outcomes of the second stage in a two-stage moving horizon structure are a substitute for all the future returns rather than a single stage, although it may, in some cases, need separate definitions of the objectives for each stage rather than the overall goals.

Hence, we utilise the weights primarily to make these models (the two- and three-stage models) more directly comparable. Remember that we are using the GP to solving the models in our study by which the DM's preferences have been largely applied to the model via determining the goals/aspiration levels as the first-order preferences, and the weights are primarily to achieve a comparative scaling on each objective. To reach this aim, according to the above arguments, we suggest two ways of weighting as follows:

- Up-weight the second stage's outcomes in a two-stage model to reflect the fact that the second stage is, in a sense, a surrogate for all future returns rather than just a single stage.

- Apply some sort of discounting to future objective function values in the proposed threestage model.

Differential weighting on various stages (i.e. putting more weights on the first stage while applying fewer weights on the following stages) of each run in a moving horizon model seems 
meaningful and can help us obtain balanced solutions. By applying fewer weights on the late stages, we are able to apply the second-order of preferences (remember that the first-order preferences in the GP-based models were applied by identifying the goals) which lead the model to focus on the first stages while still considering the future to avoid infeasible solutions and provide robustness. Moreover, putting some importance weights into a two-stage model might help us produce more robust initial decisions than the equal weighting. These various weights, applied in a manner of up-weighting the outcomes of the second stage in the two-stage structure, can also help the two-stage framework to provide more sound solutions, and both approaches can benefit from this differential weighting. These ideas will be examined and illustrated more by applying this differential weighting on simulations in the form of some examples in Section 5.5 .

\subsubsection{Discounted Weights}

As an extension of the differential weighting scheme, in problems with longer planning horizons it would be more interesting and also meaningful to apply the differential weighting in a discounting manner, viz. put the heaviest weight on the first stage, then decrease the weights for the second stage and so on. For example, for a problem with a five-stage planning horizon, we can put on the weights of 5, 4, 3, 2, 1 from the first stage to the fifth. Descending weighting in long-term problems seems more meaningful because, in most real-life problems, our predictions and evaluations for a far, unknown future regularly fail. We always need to update and adapt the decisions as we are moving ahead. Therefore, the most attention should be dedicated to the near future. However, to keep the robustness and to avoid (or at least reduce) some difficulties that may be predictable, we should not forget the distant future. Thus, decreasing the weights, as we are considering, in the next stages would be logical and meaningful. Furthermore, this concept also completely matches with the dynamic-robust orientation of the proposed structures in which we generate a robust initial solution in each stage and always adapt the information and decisions, if necessary, as we move forward in the planning horizon. These ideas will be examined and illustrated more by using simulations later in this chapter (see sections 5.5.1.2 and 5.5.1.3).

The next section is allocated to introducing a Simulation-Optimisation approach in MultiCriteria Decision-Making problems with a T-stage planning horizon under deep uncertainty.

\subsection{Simulation-Optimisation study}

To be able to run and evaluate the proposed two- and three-stage moving horizon frameworks in longer horizons, we need to identify all plausible meta-scenarios. However, with the assumption of deep uncertainty, this identification is almost impossible. On the other hand, even with a finite set of plausible meta-scenarios, comparing and computing the results in all plausible metascenarios are hardly possible because of the fact that the size of the model grows exponentially by increasing the length of the planning horizon. Furthermore, as seen in Section 5.2.3, analysis of the solutions requires hundreds or thousands of multi-objective comparisons that are not easily conceivable, if ever possible. These issues motivated us to perform a simulation study to simulate the reasonable number of meta-scenarios and to evaluate, compare and analyse the proposed methods for the problems with T-stage planning horizon.

In this simulation study, we will start by setting the current scenario (i.e. the scenario that we are currently facing); then, the optimisation model will be run to obtain the first stage decisions which can be implemented immediately. Afterwards, the next scenario will be randomly generated by using the Monte Carlo simulation method. As mentioned earlier, in deep uncertainty, we do not have enough knowledge about the likelihood of plausible scenarios nor the probability space; therefore, to be able to simulate deep uncertainty we shall not use anything of scenario likelihoods. Accordingly, in each stage, we consider the set of plausible scenarios of that stage $(S=\{s(1), s(2), \ldots, s(p)\})$, then randomly choose one scenario from the list (say, 


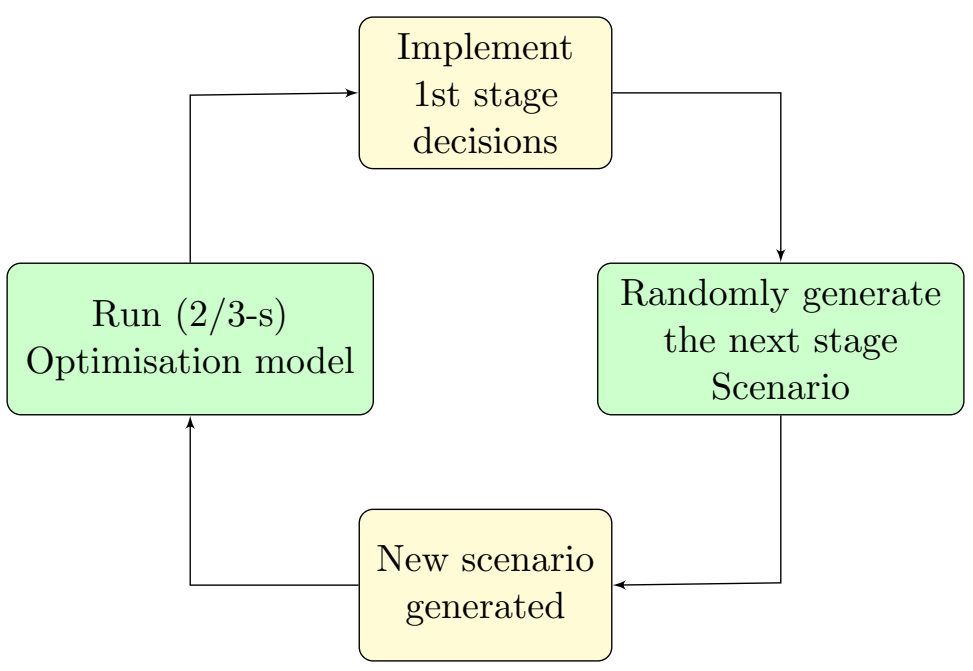

Figure 5.2: Optimisation and Simulation process

$s(k), k \in\{1, . ., p\})$ as the next stage scenario. Thereafter, we set the generated scenario as the current scenario and rerun the optimisation model one more time to gain the next stage decisions. This cycle, which is portrayed in Figure 5.2, is iterated until we reach the end of the planning horizon.

The two- and three-stage Simulation-Optimisation algorithms used in this study have been proposed in Sections 5.4.1 and 5.4.2, respectively, which is followed by a brief debate on the number of plausible meta-scenarios and required simulations in Section 5.4.3. Some illustrative examples and more discussion on theses results are also presented in Section 5.5.

\subsubsection{Simulating the two-stage moving horizon structure in a T-stage planning horizon}

Firstly, the two-stage model is used to obtain the first stage decisions $\left(\mathbf{X}_{0}\right)$. These decisions represent the initial decisions which will be implemented immediately after the decision-making process and before scenario realisation. Then the next stage's scenario is randomly generated (one of the identified plausible scenarios for the next stage is randomly chosen using the Monte Carlo method). Thereafter, without utilising the contingent/recourse decisions produced by the

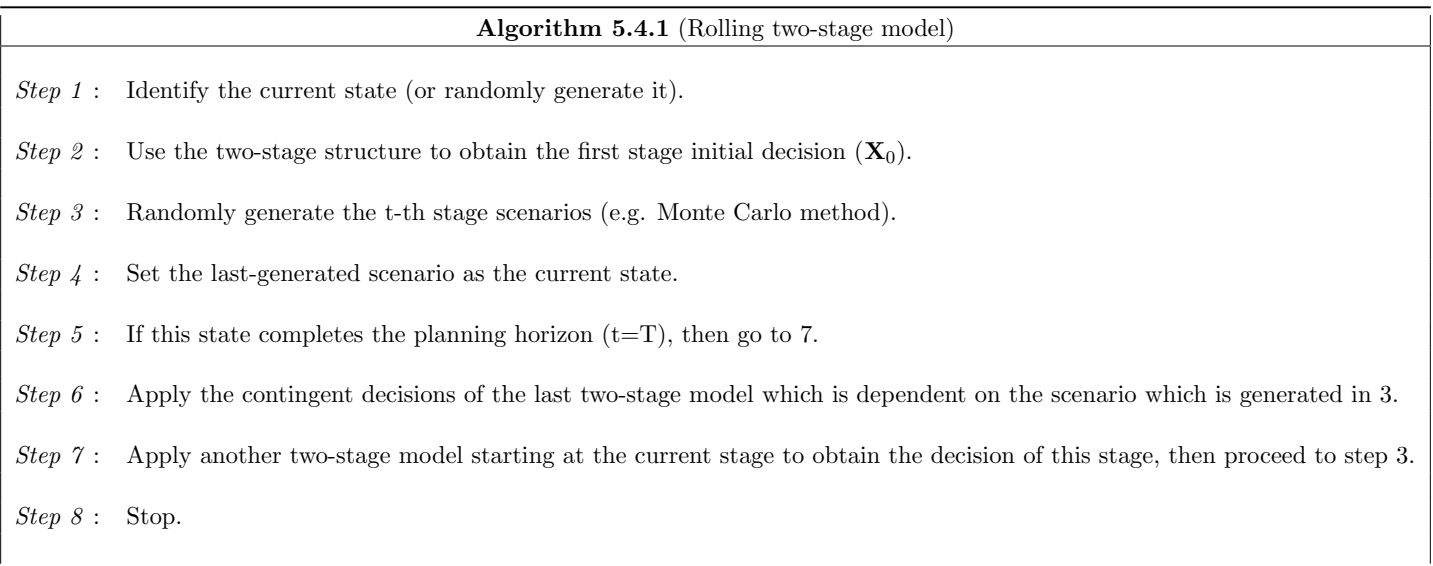


first two-stage model, this randomly-generated scenario will be set as the current scenario and another two-stage model will be run which is started from the second stage and cover the third stage. The initial decisions which are obtained from the second model contain both recourse decisions to the previous decisions as well as the initial decisions for the next stages. Then, the second stage scenario is randomly generated, and the algorithm is iterated until the entire horizon is covered. Finally, for the last two-stage model, the contingent decisions may also be considered. Figure 5.1 describes how to utilise these $T-1$ consecutive two-stage models in a T-stage problem. Moreover, Algorithm 5.4.1 describes more details and provides a step-by-step manuals of this moving horizon approach.

\subsubsection{Simulating three-stage moving horizon structure in a T-stage planning horizon}

In this structure, similar to the rolling two-stage models, the three-stage model is run to obtain the first stage decisions $\left(\mathbf{X}_{0}\right)$ then the scenario for the next stage is randomly generated or just used if it has already been generated, and another three-stage model starting at second stage is applied, second stage scenario is randomly generated, and so on. The first and second contingent decisions of the last three-stage model may also be considered. Figure 5.1 and Algorithm 5.4.2 explain how to use these $T-2$ successive three-stage models in a $T$-stage problem.

These algorithms help us to simulate the plausible meta-scenarios, suggested solutions and the outcomes. However, because the results need to be compared and analysed for both approaches, the number of required simulations is important. Accordingly, this issue has been investigated in the next section.

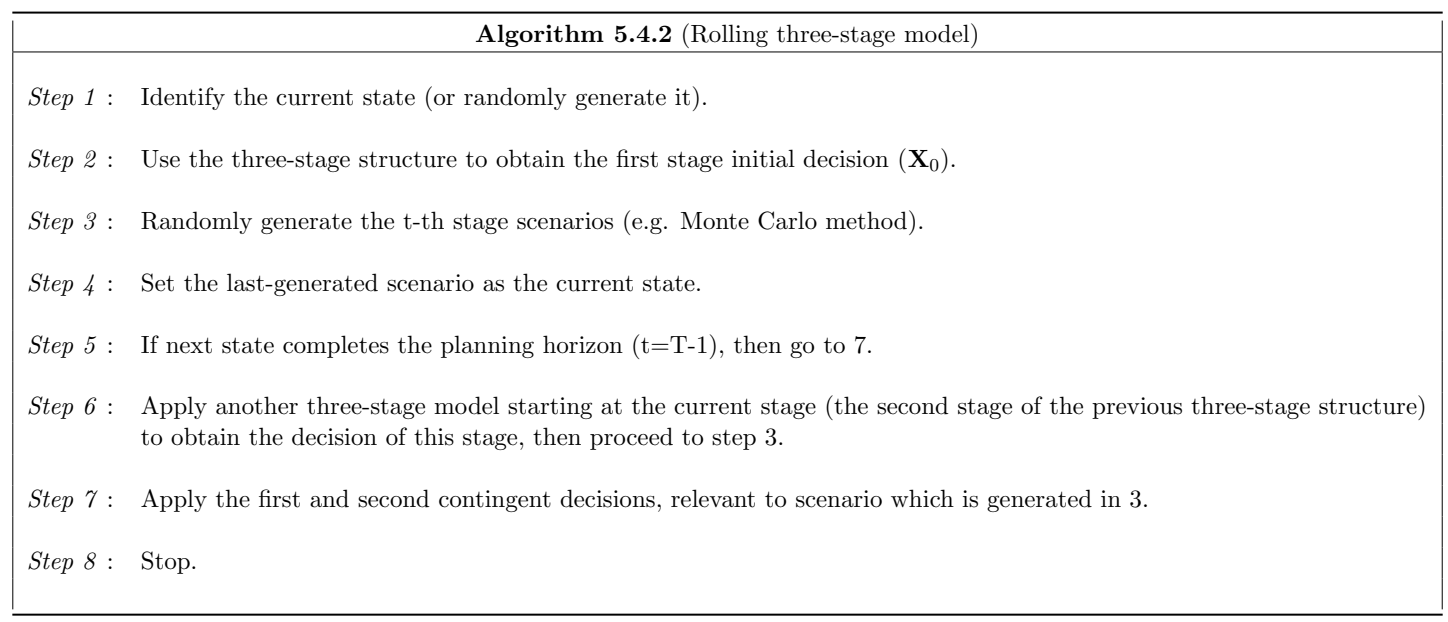

\subsubsection{How many simulations need to be run?}

The total number of different meta-scenarios is a subset of the combinations of all plausible scenarios in each stage and can be calculated as follows:

$$
p \leq p_{1} \times p_{2} \times \ldots \times p_{T-1} \leq p_{M a x}^{T-1}
$$

where $p$ is the total number of meta-scenarios, $p_{t}(t=1,2, \ldots, T-1)$, is the maximum number of plausible scenarios in the next stage $(t+1)$ for the scenarios in stage $t, p_{\max }=\operatorname{Maximum}\left\{p_{1}, p_{2}, \ldots, p_{T-1}\right\}$.

For example, if three, at the most, different plausible scenarios exist for any plausible scenario at the last stage, then, the total number of meta-scenarios would be less than or equal to $3^{5}=243$, 
$3^{6}=729$ and $3^{10}=59049$ in a horizon of 5,6 , and 10 stages.

Therefore, even if we suppose that all generated meta-scenarios would be monopolised, to produce all meta-scenarios we need to perform around 60000 simulations in a ten-stage horizon with only three particular plausible scenarios in each stage. Based on our experiences in some examples, this number would triple to 180 000. A problem like this would require several hours/days of computer computation, depending on the problem. Accordingly, applying a ten-stage horizon, especially in real problems, would be computationally expensive and may need several supercomputers to run for several hours/days. Alternatively, we have to reduce the number of simulations and iterate them for only a few times or more. Then, we may be able to simulate most of the plausible meta-scenarios and hope that they sufficiently cover a wide range of plausible meta-scenarios. However, this reduction may provide us with some surprises later.

Nonetheless, with the proposed frameworks we can split the horizon and run the simulations for a shorter period- perhaps five stages of a ten-stage planning horizon, for instance. Then, after the realisation of scenarios in these five stages, we would run the model for the next five stages to cover the planning window (i.e. all the ten stages). Since we are rolling the two or threestage model during the horizon, the length of the planning horizon does not affect the decisions. Therefore, instead of the analysing all plausible meta-scenarios for a planning horizon that is too long, we can divide the whole period into some shorter windows in which we can simply simulate and analyse all the meta-scenarios. Nevertheless, we are always able to simulate some meta-scenarios of the entire planning horizon and compare the consequences of the decisions over these meta-scenarios.

Finally, we may, nonetheless, need to note that the aim of simulation for the long-term horizons in this study is not to cover and analyse all plausible meta-scenarios in that window. Instead, we are attempting to illustrate how the proposed methods work and compare the results of that part of the simulated meta-scenarios (i.e. only some meta-scenarios have been simulated not all of them). However, by rolling the two or three-stage structure during the planning window, the number of comparisons are substantially reduced because only the effective meta-scenarios are compared not all of them (viz. at each stage only the next effective scenario is randomly generated and the calculations, comparisons, and analyses are limited to this scenario in this stage, not all meta-scenarios in the entire planning horizon).

\subsection{Illustrative example}

The primary aim of this section is to examine the efficiency of the proposed moving horizon approaches, with different length of future vision (two and three stages). Also, this section will explore the robustness of these approaches in a longer planning window. It will be demonstrated how the proposed models, algorithms, and structures are applied and how to compare the results. Then, a discussion on the trade-offs and Pareto front will be discussed to show that there is a need to find a combined picture of the results to be able to truly compare the performances. However, drawing the Pareto front for such a high dimensional problem is almost impossible. Therefore, we will start by looking at performance under conditions of some different metascenarios and, thereafter, try to simulate a $2 \mathrm{D}$ image of the Pareto frontier for both proposed frameworks that can provide us with a logical comparison. This will be followed by a description of the advantages of applying the proposed differential weighting, in Sections 5.3.1 and 5.3.2, as well as the robustness of the results in relation to the choice of weights. We may need to mention that, because the differences of the time processing between the two approaches for this example in a 5-stage horizon is less than 100 seconds, we are not going to present and discuss that in this section. However, as will be seen later, the information of the total processing time of the algorithms for this example in longer horizons have been compared in the next section.

Example 5.5.1. In this section, example 4.5.1 is revisited while the planning horizon includes five stages. We will roll the two-stage (Algorithm 1) and the three-stage (Algorithm 2) moving 
horizon models in this five-stage planning windows. According to the calculation in Section 5.4 .3 , there are 243 plausible meta-scenarios in a five-stage planning horizon that needs triple simulations to cover a wide range of these meta-scenarios. Therefore, seven hundred simulations have been run for both proposed algorithms, and the results have been described in Table 5.1 in which the means and standard deviations for the objective functions (available fund and withdrawal) of these two different approaches are compared and evaluated in the given planning horizon $(\mathrm{T}=6)$. Also, a more vivid picture of these results is portrayed in Figure 5.3. The mean of cash flow and withdrawal of the two proposed structures at the end of each period (just after scenario realisation) have been respectively compared from left to the right in this figure. It can be clearly seen that rolling the two-stage model provides us with more cash flow, on average, at the end of the stages in comparison with rolling the three-stage model, while the latter tends to provide more amount of withdrawal in each stage.

By comparing the results of the two proposed approaches in the last row of Table 5.1, it can be seen that the three-stage model results in 3 million less for available funds relative to the two-stage model, but only a 2.1 advantage in terms of withdrawals. To explain this, it must be noted that there is not any constraint in the model to optimise the average of objectives in all meta-scenarios. Also, no tendency has been applied to the model for optimising the aggregations of objectives over all plausible meta-scenarios. Therefore, as will be shown later in this chapter, the solutions to the two-stage framework may provide better achievements in a few meta-scenarios than the three-stage structure. However, the two-stage moving horizon approach generates fewer achievements for the others that contain most of the plausible meta-scenarios. Furthermore, the two-stage approach may fail to find a feasible solution for some meta-scenarios. Therefore, it is possible that large negative differences between the solutions $(3 \mathrm{~s}-2 \mathrm{~s})$ in those few meta-scenarios (that two-stage had better achievements than the three-stage) cannot undo the small positive differences in the rest of the meta-scenarios. Therefore, the total differences will be demonstrated by a negative number. In other words, the two-stage structure may provide some extreme achievements for only a few meta-scenarios while the three-stage structure, with a further future view, generates more balanced solutions that will have better achievements in most of the meta-scenarios, but not necessarily all, compared to the two-stage. Furthermore, the three-stage provides more robust solutions related to the two-stage, so, it guarantees better achievements in the worst cases. However, it may sacrifice higher achievements in a few meta-scenarios to provide better attainments in a vast majority of meta-scenarios. Therefore, the average of achievements over the plausible meta-scenarios may not provide a valid base for comparison between these models.

\subsubsection{Discussion on trade-offs and Pareto Front}

The trade-offs between the objectives can be clearly highlighted in Figure 5.3. As seen in Figure 5.3 and Table 5.1, the available fund at the beginning is five million Rands (one million in each

\begin{tabular}{|c|c|c|c|c|c|c|c|c|}
\hline \multirow{2}{*}{ Stages } & \multicolumn{4}{|c|}{ Available Fund(million Rands) } & \multicolumn{3}{c|}{ Withdrawal(million Rands) } \\
\cline { 2 - 9 } & Rolling 3s & \multicolumn{2}{c|}{ Rolling 2s } & \multicolumn{2}{c|}{ Rolling 3s } & \multicolumn{2}{c|}{ Rolling 2s } \\
\cline { 2 - 9 } & Mean & Std & Mean & Std & Mean & Std & Mean & Std \\
\hline 0 & 5.00 & 0.00 & 5.00 & 0.00 & 0.25 & 0.00 & 0.25 & 0.00 \\
\hline 1 & 5.35 & 0.27 & 5.34 & 0.27 & 1.09 & 0.59 & 0.33 & 0.12 \\
\hline 2 & 4.87 & 0.94 & 5.72 & 0.78 & 0.79 & 0.60 & 0.31 & 0.10 \\
\hline 3 & 4.69 & 1.50 & 6.25 & 1.47 & 0.85 & 0.60 & 0.42 & 0.28 \\
\hline 4 & 4.46 & 1.87 & 6.85 & 2.14 & 0.62 & 0.48 & 0.54 & 0.53 \\
\hline 5 & 4.45 & 2.43 & 7.45 & 2.80 & 0.99 & 0.39 & 0.64 & 0.46 \\
\hline Total & 4.45 & 2.43 & 7.45 & 2.80 & 4.59 & 0.95 & 2.49 & 0.80 \\
\hline
\end{tabular}

Table 5.1: E3 Comparison results of 700 simulation runs in a five-stage horizon 

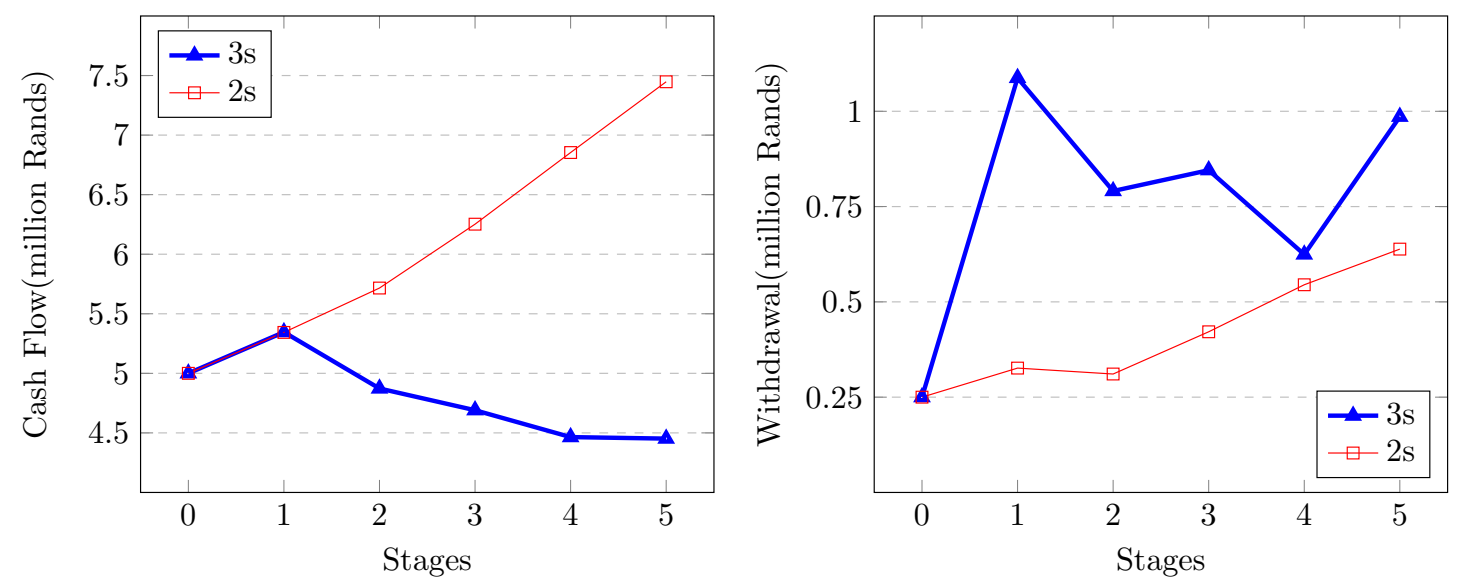

Figure 5.3: Mean of cash flow and withdrawal at the end of the stages in 700 simulation runs $(T=6)$, comparison of rolling two-stage and the three-stage structures.

investment option). The first initial decisions distribute this amount of capital into different investment options and withdrawal for consumption. Both approaches allocated exactly the minimum amount i.e. 250 thousand Rands (mean 250 with STD zero) of withdrawal. At the first stage, the available fund in both structures is almost the same while the consumption in the three-stage model is tripled. For the next steps, the total available fund in the three-stage model has decreased continuously while the opposite pattern has been formed in the two-stage model. Except for the fourth stage where the difference between the consumption's dedicated funds between the two methods is not too much, this difference is largely indicated better achievements which were obtained by applying the solutions of the three-stage model in the other stages. Comparing the total amount of withdrawal at the end of the given period shows that the threestage solutions provide $84 \%$ more achievement (on average) in this objective, while comparing the mean of total achievement of the first goal (available capital) described $67 \%$ more attainment in that goal for the two-stage solutions. Therefore, both approaches provide more achievement for one objective function and the less for the other but in an opposite manner. Although one may say that the three-stage solutions are preferred if the preferences of both criteria are assumed to be the same, we need a combined picture to be able to compare these solutions.

The best way to compare the results of these two frameworks in their multi-criteria structure is to draw the Pareto frontier of the solutions to these methods. However, identifying the hyperplane equations of the Pareto optimal solutions, or even the approximation one, needs a separate study because their structure may not be simply doable, and will lie in the future directions. Remember that we used scenarios as an additional dimension of preferences in the proposed structures which expanded the dimensions of the multifaceted surface of Pareto frontier. Therefore, the trade-offs must be evaluated and compared between all meta-criteria which would be too complex. For example, in a problem with only two criteria and three scenarios in each stage, there will be six meta-criteria for a one-stage problem, viz, the solutions need to be evaluated and compared for all of these six criteria. Even worse, for a planning horizon of five stages, 364 meta-scenario multiplied by two objectives (i.e. 728 meta-criteria) must be considered. Obviously, plotting all information in a 2D or 3D graph is impossible. Nevertheless, we may be able to sketch the results in a two-dimensional diagram for each meta-scenario, although it may not be widely useful. Still, it can help to illustrate the proposed structures and simulations. This diagram can also provide us with a vivid image of the Pareto frontier under conditions of any meta-scenario. Therefore, in the next section, we will introduce a procedure to approximate the Pareto front in each meta-scenario and this will be extended later to simulate a 2D image of the Pareto frontier representing a comparable part of performances. 


\subsubsection{Single-Scenario Pareto Frontier Approximation}

As seen in Chapter 3, we can generate various solutions on the Pareto frontier and observe the existing trade-offs by changing the values of the importance weights. Therefore, here, this property has been used to generate different Pareto solutions and approximate the Pareto front for a single meta-scenario in multi-stage multi-objective problems. In fact, various weights have been used to generate different solutions and simulate some parts of the Pareto front in the conditions of a single meta-scenario. Actually, we utilise a simulated meta-scenario and apply another simulation to approximate various solutions by changing the importance weights. Moreover, the differential weighting and discounted weights are examined. Their effects on generating solutions of the proposed models have been illustrated and analysed in some cases as well.

To reach this purpose, the intended meta-scenario is chosen and the two- and three-stage moving horizon models are run to obtain the solutions and related performances in each criterion. Then, new weights are set/generated and the proposed structures are rerun to create new solutions. After obtaining a suitable number of solutions, the values of the objectives for all generated solutions in a 2D or 3D plot can show the image of some points in the Pareto Front under conditions of the selected meta-scenario. This procedure has been summarised by Algorithm 5.5.1.1.

In the three-stage moving horizon model, the first stage's weights are randomly multiplied (using the Monte Carlo method) by a number between 1 and 30 in such a way that the simulated scenarios cover a broad range of solutions to demonstrate a wide range of trade-offs between different optimal solutions (1000 simulations have been done). Furthermore, discounted weights, in order of $5,4,3,2,1$ on the existing five stages, have been applied over the planning horizon to exert time preferences on the models. The importance weights related to the next stages are also reduced in a discounting manner. Note that, although we use the Monte Carlo method to randomly generate different weights for the first stage, this weighting still follows the rules which were already defined for discounted weights in Section 5.3.2, i.e. more weights on the first and less on the last stage. Multiplying the first stage weights by a number greater than one raises the weights for the first stage which, together with applying discounted weights for the stages, exerts some sort of reduction to the future events in comparison with the first stage.

The opposite framework is applied to the proposed two-stage moving horizon model to apply time preferences that would be helpful to develop the comparability of the solutions between the two and three-stage structures. In fact, both frameworks, defined in Section 5.3, are simultane-

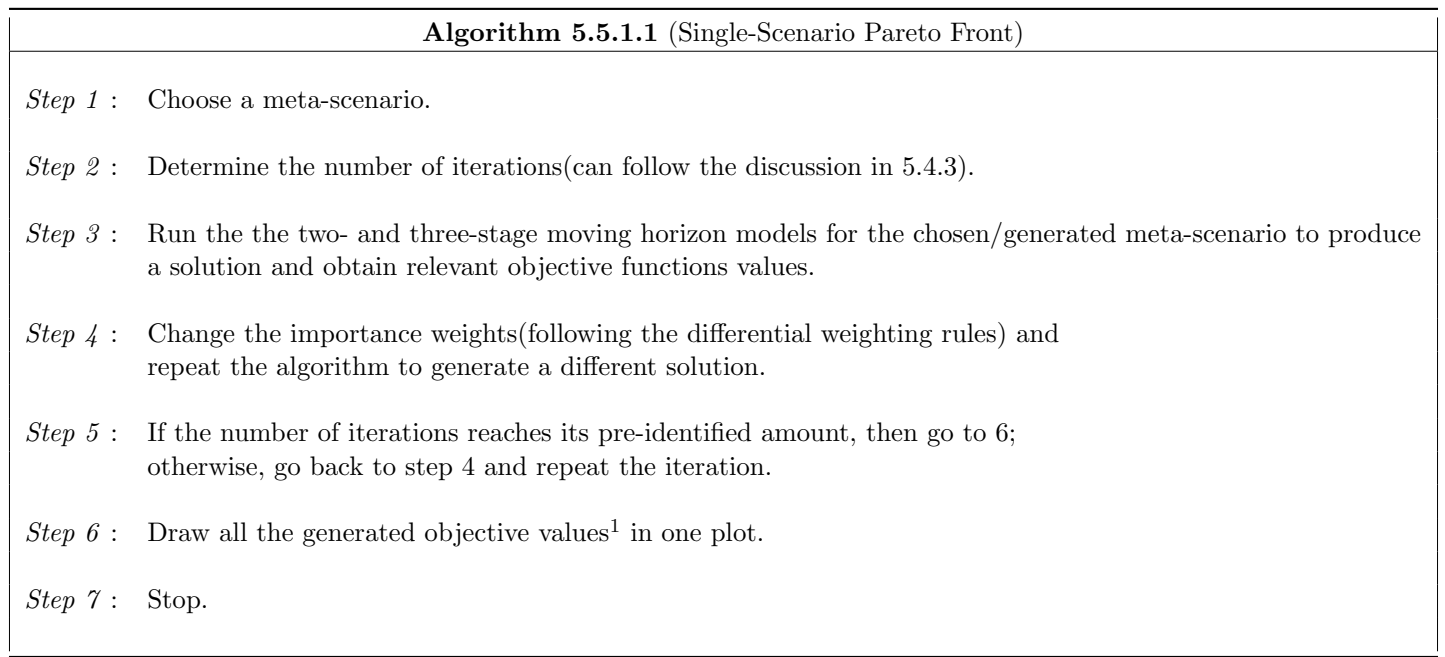

\footnotetext{
${ }^{1}$ Note that, in the case of problems with more than three objectives, we need to choose two or three criteria to be
} compared. Otherwise, we cannot sketch the graph. 
ously applied in this study.

Therefore, to examine this method, let us start with the worst plausible meta-scenario in a five-stage planning horizon of the example. i.e. a situation in which the current economics falls in each stage until they reach the worst scenario $\left(s_{1}\right)$; then keep the recession and stay on this worst scenario $\left(s_{1}\right)$ for the rest stages. Meta-scenario $s_{3}-s_{2}-s_{1}-s_{1}-s_{1}-s_{1}$ represents this situation. This meta-scenario could be an interesting case, and perhaps the most important one for the DM in some instances because some policy-makers are considerably interested in observing the results and analysis in this case and even make their decision depending on the outcomes of the worst case scenario. Then, comparing the Pareto front to the proposed models, in this particular case, would be interesting and may even be crucial for some DMs. Therefore, the solutions of the two proposed methods are examined and the differences between them have been compared in the worst case. Figure 5.4 compared the estimated Pareto frontiers of the two proposed methods in example 5.5.1 under conditions of the worst meta-scenario of 364 plausible meta-scenarios. As seen in this graph, the Pareto Front drawn for the three-stage model dominates the twostage frontier as expected. Moreover, according to the large differences in this meta-scenario, the efficiency of the three-stage model seems to be satisfactory. So, the efficiency of the threestage moving horizon model compared to the two-stage structure, in one of the most important scenario, is clearly seen in this picture.

However, to convince the rest of management who are looking for the overall performances over all plausible meta-scenarios, and to be able to generalise this conclusion, the efficiency of the three-stage moving horizon approach must also be proved for all the other meta-scenarios. Thus, we will examine the test for a few more meta-scenarios and the results will be shown in Figures

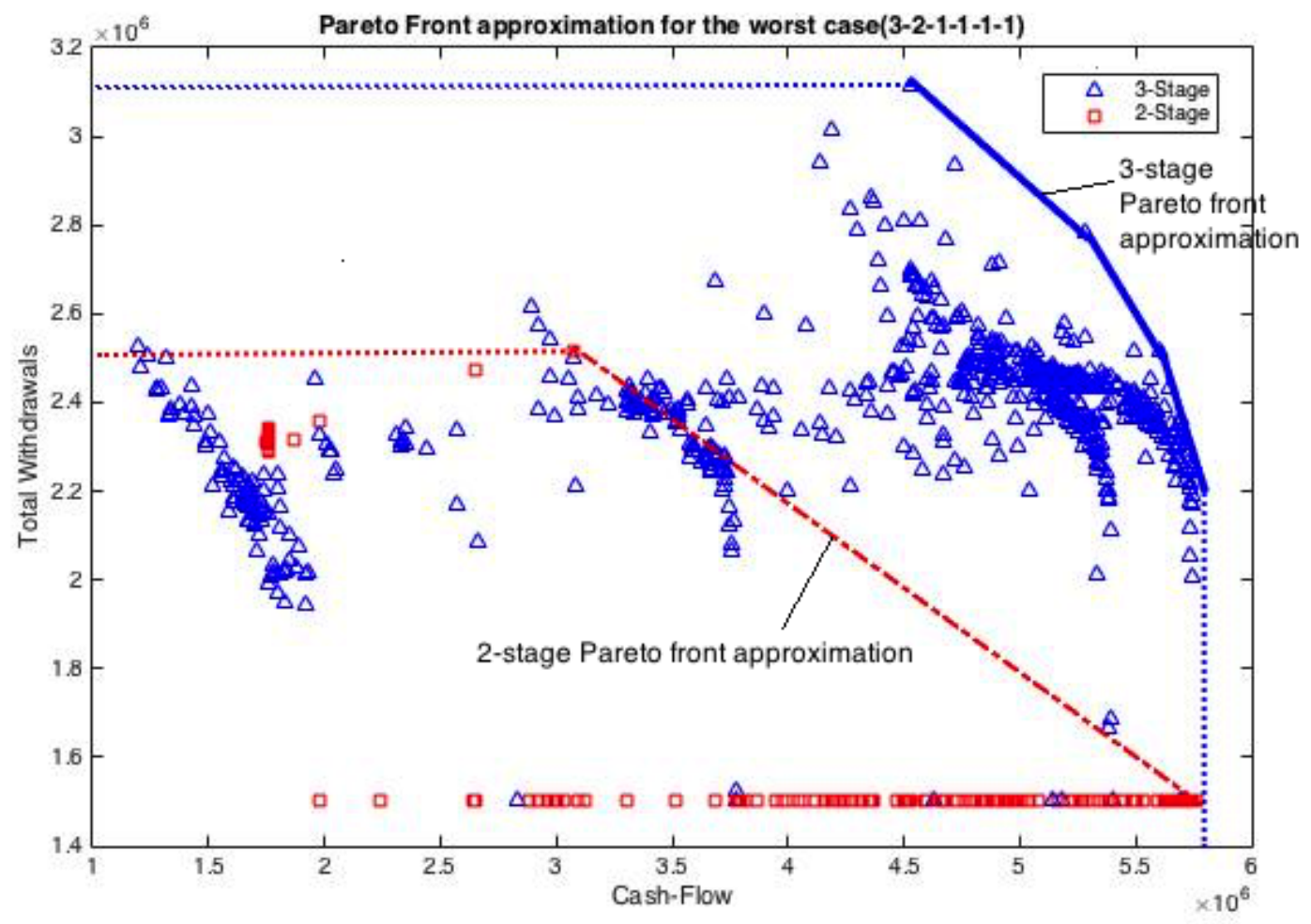

Figure 5.4: Simulated Pareto frontiers of rolling two-stage and the three-stage structures in the worst case meta-scenario (3-2-1-1-1-1) (1000 simulation runs with random weights between 1 and 30 in a discounting manner for 5 -stage planning horizon $(T=6))$. 
5.5 and 5.6 for four other meta-scenarios. The first picture, in Figure 5.5, also demonstrates another interesting situation in which the current economics falls until they reach the worst case; then, after a short stay, the economics rise to their first condition at the beginning of the period. Both recession and expansion with equal magnitude can be observed over the horizon of this situation which is represented by meta-scenario $s_{3}-s_{2}-s_{1}-s_{1}-s_{2}-s_{3}$. As seen in this graph, the Pareto frontiers to the two-stage model are, again, dominated by the Pareto Front of the solutions to the three-stage structure.

The opposite situation has been indicated in the second picture $\left(s_{3}-s_{4}-s_{5}-s_{5}-s_{4}-s_{3}\right)$ in which the current economics grows until reaching the best case; then, after a short stay, the economics fell down to the initial situation. In this meta-scenario, the three-stage Pareto frontier is dominated by the Pareto front of the two-stage structure. It must be noted that, as discussed earlier, the three-stage model should be somewhat dominant over most meta-scenarios, but not necessarily all, as we observed in some meta-scenarios here. However, the solutions to the three-stage structure dominate the corresponding solutions to the two-stage framework in the worst case meta-scenarios, as with as all the robust solutions, although it is dominated in a few non-extreme meta-scenarios.

The plot at the top of Figure 5.6 indicates the Pareto front for the most stable meta-scenario. In this meta-scenario, current economics has no change and is stable during the given planning window. Meta-scenario $s_{3}-s_{3}-s_{3}-s_{3}-s_{3}-s_{3}$ represents this situation. This meta-scenario could be an interesting case for comparison purposes as it is the most stable meta-scenario with no exchange penalties and the same conditions in each stage. As seen in this graph, no dominance relation could be found between the two proposed methods in this meta-scenario.

Finally, the best plausible meta-scenario has been portrayed in the second picture in Figure $5.6\left(s_{3}-s_{4}-s_{5}-s_{5}-s_{5}-s_{5}\right)$. In this situation, the current economics situation grows and keeps expanding for the next stages until they reach the best case scenario $\left(s_{5}\right)$, then they remain stable for the remaining stages. Comparing Pareto frontiers in this chart explains no dominance relations between the objectives, although the two-stage solutions are so close to the solutions of the three-stage pattern, and even tend to be better in some senses. It is worth remembering, once again, that the solutions to the two-stage model are feasible to the three-stage model. One reason to choose different solutions by the three-stage structure, beyond getting better achievements for objectives, is to provide the more robust solutions for all other meta-scenarios that may end with the fewer achievements in some other meta-scenarios. The cause of choosing these sub-optimal solutions is that the feasibility of the solutions in all meta-scenarios over the three stages has higher priority than better accomplishments because of some sever constraints in the model. These reasons will justify the above-mentioned results of the Pareto frontiers in the last two plots.

A comparison between the performances of the two proposed approaches indicates that the efficiency of the three-stage structure in some meta-scenarios is represented by a large difference between the Pareto front of the three-stage and the two-stage frontier. However, this difference is not big enough to conclude the efficiency of the three-stage moving horizon model in some other meta-scenarios. Moreover, no dominance relations could be found between the two methods in some meta-scenarios. In other words, it seems that the three-stage model has advantages, but these are not considerable relative to the two-stage model. This needs to be contrasted with the greater computational and data collection demands. However, we need to perform this kind of Pareto frontier simulation for all plausible meta-scenarios and compare them all which will be complex work even for a simple example. Hence, simulating a single-scenario Pareto front, in practice, would not be widely useful and the comparison based on that would not be practical. Therefore, even if we can generalise the results in this experimental setup to wider classes of problems, we still need a combined picture of objectives under conditions of all meta-scenarios to compare these two methods. In the next section, we will suggest a combined picture that helps us compare the overall performances between the two proposed structures. Further discussions are also presented after introducing this picture. 

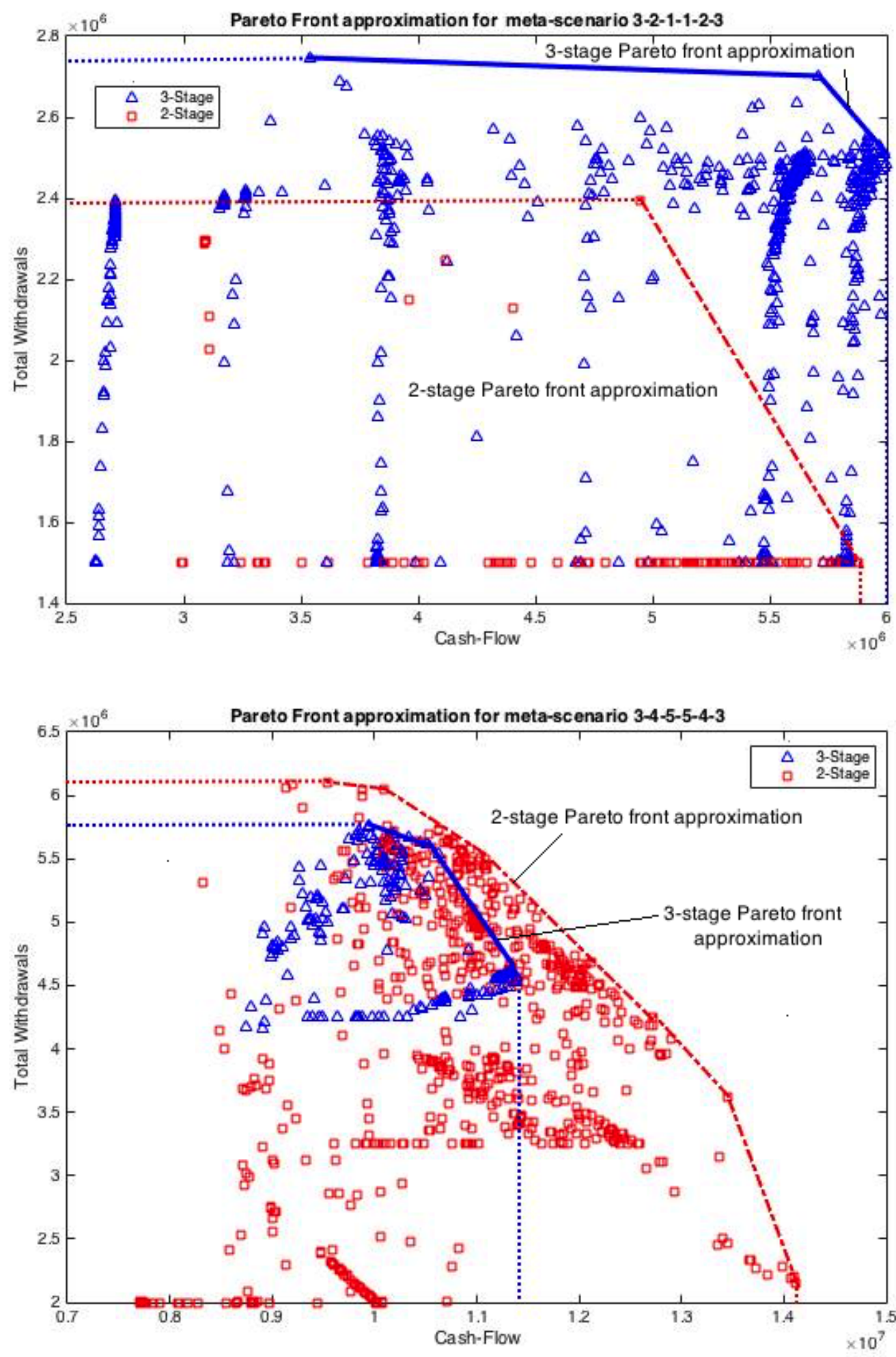

Figure 5.5: Simulated Pareto frontiers of rolling two-stage and the three-stage structures in some specific meta-scenarios (1000 simulation runs with random weights between 1 and 30 in a discounting manner for 5 -stage planning horizon $(T=6))$. 

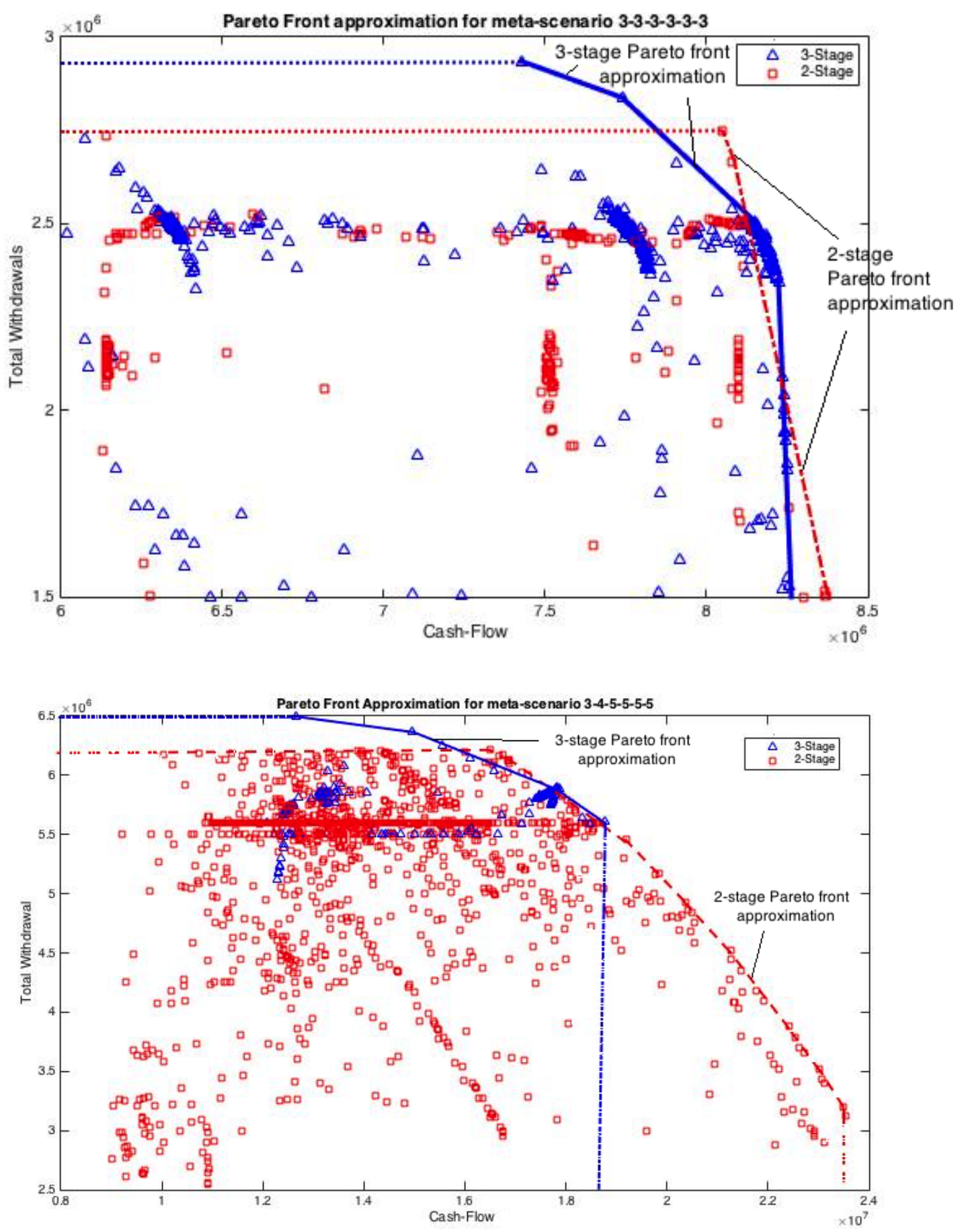

Figure 5.6: Simulated Pareto frontiers of rolling two-stage and the three-stage structures in some specific meta-scenarios (1000 simulation runs with random weights between 1 and 30 in a discounting manner for 5-stage planning horizon $(T=6))$. 


\subsubsection{A Combined Picture of Performances}

As discussed above, single-scenario Pareto fronts are suitable to compare some specific metascenarios; for example, in the case that some particular meta-scenarios are considerably important to the DM (e.g. the worst case meta-scenario). In these cases, comparing the outcomes and performances in few meta-scenarios might be enough to convince the DM about the choice of methodology. It also would be enough to observe the effects of various weights in different metascenarios and find a set of suitable weights. However, comparing the overall performance of the two proposed methods with the single-scenario Pareto fronts is almost impossible and we need to show all the performances in a combined picture for a trustworthy comparison.

According to the high dimensional aspect of the problem, plotting a two or three-dimensional picture of the results is impossible and, as mentioned before, we are able to sketch the Pareto front for only a single scenario. This cannot provide us with an overall performance comparison. Therefore, instead of drawing the Pareto front, we suggest sketching one ordered pair (or triple) of performances/objective functions for a simulated meta-scenario; then simulate another metascenarios and add the new point (solution) to the plot while the set of weights is the same for all of them. In fact, we are plotting and comparing the ordered multi-sets of performances, provided by the proposed methods, in terms of these two(or three) criteria under conditions pertaining to the simulated meta-scenario. Algorithm 5.5.1.2 describes the suggested procedure in more detail. This algorithm is also applicable for all plausible meta-scenarios, if they are enumerable, to cover all the meta-criteria. The result will be a plot representing all the performances of different criteria under conditions of all meta-scenarios. It is also possible to use the mean of the performances from the simulated single-scenario Pareto front in the previous section.

Therefore, this algorithm is utilised to exhibit the performances of example 5.5 and the results will be compared while different sets of weights have been chosen to highlight the effects of applying time preferences and choice of weights on solutions.

Accordingly, Figure 5.7 portrays two separate combined pictures of the final solutions of the two structures(rolling the two- and three-stage) in which each point represents a pair of the goal achievements of the two objectives in simulated meta-scenarios. i.e. each point represents the goal achievements of the two criteria regarding one of the 1000 simulated meta-scenarios. Note that all 1000 simulated meta-scenarios utilised in both approaches are the same. To highlight the effects of applying time preferences on the solutions, the first graph shows the results without

Algorithm 5.5.1.2 (Performances in different meta-scenarios)

Step 1: Determine the set of weights.

Step 2: Identify the number of simulations(can follow the discussion in 5.4.3).

Step 3: Run the Simulation-Optimisation algorithms(algorithms 5.4.1 and 5.4.2) to generate the solutions and obtain the relevant objective functions values.

Step 4: Sketch the generated objective values ${ }^{1}$ at the plot.

Step 5: If the number of simulations reaches its pre-identified amount, then go to 6, Otherwise, go back to step 3 .

Step 6: Stop.

${ }^{1}$ Note that presenting the results is limited to two or three criteria. Then, for problems with more than three criteria, it is impossible to display all performances in one picture. However, in this case, we can choose two or three criteria and sketch the relevant graph. 
applying the time preferences while the time preferences are applied to the second plot, in which the importance weights follow the rule of discounted weights in both approaches, as mentioned in the previous section.

As seen in the first graph, the solutions to the three-stage model (indicated by blue triangles) strictly tend to provide more achievements in the second objective (withdrawals) and fewer attainments for the first one (cash flow/available fund). However, the opposite pattern has been obtained for the two-stage structure (shown by red squares), viz. more in cash flow and less in withdrawals. Then, neither of these models represents the balance between the metacriteria. Also, the dominated areas by the solutions of both methods have been shown in these
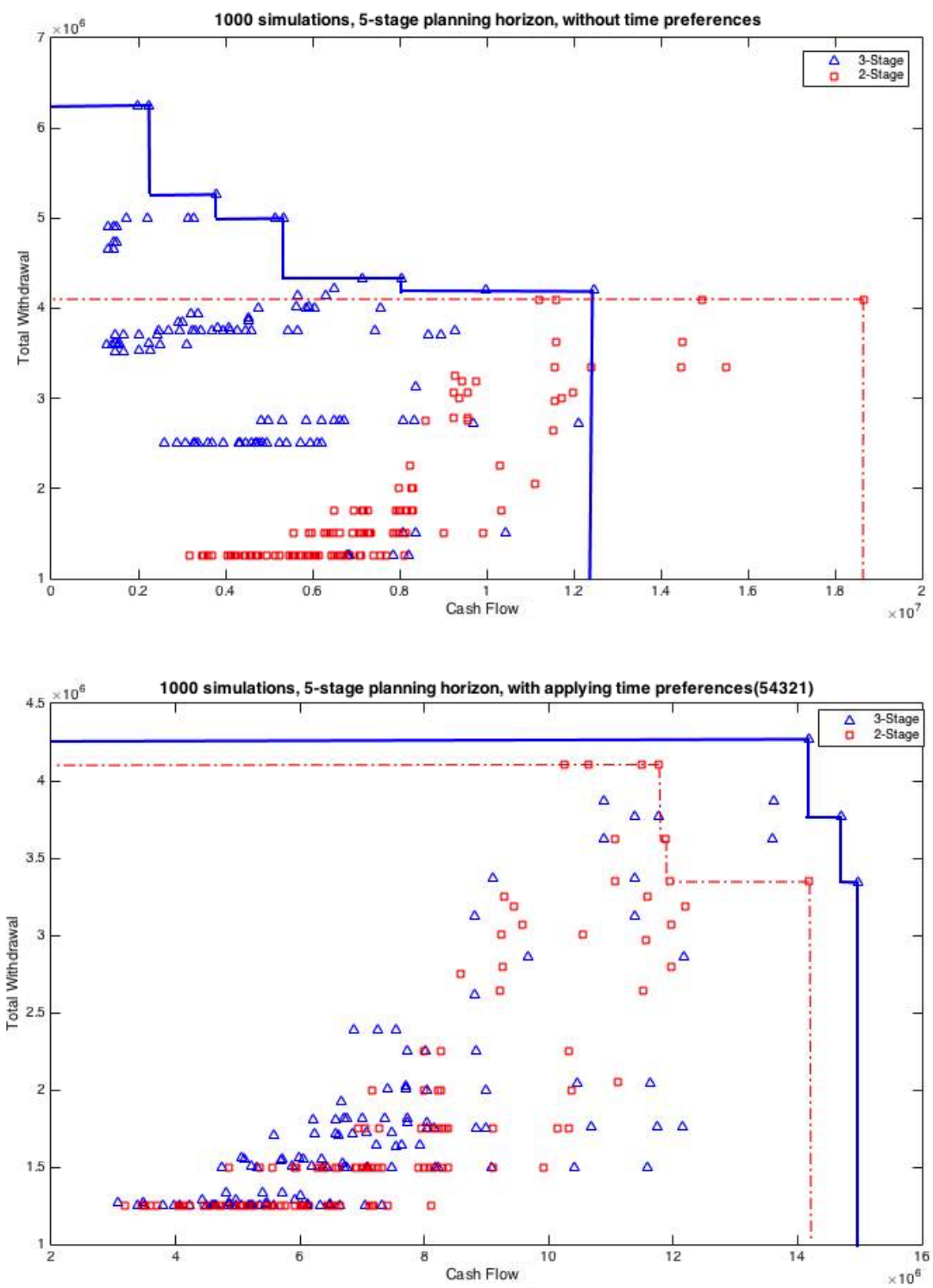

Figure 5.7: Combined picture of cash flow and total withdrawal at the end of the fifth stage in 1000 simulation runs $(T=6)$, showing a comparison between the rolling two- and the three-stage structures with and without applying time preferences. 
plots (Figure 5.7). The solid lines describe the area dominated by the solutions of the threestage model, while the dominated area for the two-stage approach is indicated by the dash-dot. Obviously, no dominance relation between the solutions of the two structures, in the first graph, can be imagined for this case. Therefore, without applying time preferences, the models lead us to some unbalanced solutions with no dominance relations.

Now, let us describe the second graph and compare the effects of applying time preferences on results. As mentioned earlier, time preferences have been applied in a discounting manner, viz. $5,4,3,2,1$ on the existing five stages, by using the differential weighting in various stages of the planning horizon in example 5.5.1. A relevant combined picture of the results has been presented in the second graph of Figure 5.7.

As clearly seen in this plot, by applying the time preferences to the models, the solutions of the simulated meta-scenarios are more balanced and the overall shape seems to come closer to the midpoint line(bisector of the first quarter) in both approaches. Furthermore, the dominance area of the solutions to the two-stage model has been dominated by the dominance area created by the solutions to the three-stage model. Thus, as expected, the solutions to the three-stage model dominate the solutions to the two-stage model. Although the differences in most metascenarios may not be practical important, these variations for some other meta-scenarios may not be negligible and might persuade the DM to utilise the three-stage model, despite its complexity and requirement for greater computation.

Then, the application of the time preferences in the problem helps to make the solutions to the two proposed models directly comparable, and also leads it to generate more preferred solutions. Furthermore, the application of time preferences can also provide us with balanced solutions in both structures. It also makes more sense to focus on the first stage circumstances while still considering the future and consequences of the current decisions on every plausible meta-scenario.

Moreover, the differences between the solutions to these models are not the issues of risk aversion but of future flows. These differences are affected by using the different length of future vision (two or three) in these structures. In fact, there are some trade-offs between the shortand long-term objectives because of the difference of time modelling that affects the solutions to the models with various time windows. In other words, when a problem includes the element of time, then the term risk-averse decisions does not make sense. In this case, the most effective factor is time, not the risk measures. In fact, the risk-aversion decision is better matched with the once off decision-making problems in which time is not involved as a vital factor. This result has been pointed out in some other studies (see Stewart [1988], and Mardle and Pascoe [2002] for more details). Therefore, it seems that this result can be generalised to wider classes of decision-making problems with long-term planning horizons. Accordingly, we recommend that future research should consider the consequences of this effective factor (time) in modelling and analysing the results.

The next section is allocated to a sensitivity analysis which investigates the robustness of the results generated under various weights.

\subsubsection{Analysis of various weights}

In this section, the robustness of the proposed approaches to the variations in weights for the previous example will be investigated. This will be achieved via a sensitivity analysis on importance weights ${ }^{2}$, and the results of the two proposed structures will be compared. It will be shown, as expected, that the solutions to the three-stage structure are more robust to variations in weights than the two-stage models.

\footnotetext{
${ }^{2}$ As mentioned earlier, in this study, we do not focus on the system of goal and weight choice. As we believe, because of existing too many goals/weights, elicitation of the DM preferences is hardly possible. Therefore, here, we only use some different weights(and goals) to show some specific situations or cover a wide range of possible consequences.
} 
Consider the five-stage planning horizon of the example 5.5.1. One thousand simulations have been run for the two- and three-stage moving horizon algorithms while the time preferences, in the form of discounted weights, are also applied at different stages $(5,4,3,2,1)$. This simulation is repeated using various weights to study the robustness of the results when the importance weights of any particular criterion, scenario, or meta-criterion are increased. Note that time preferences have been applied in the form of discounted weights $(5,4,3,2,1)$ at all stages, viz. the first stage has the weight of ' 5 ' and the weight decreases to ' 4 ', ' 3 ', '2', and ' 1 ' at the second, third, fourth and fifth stage, respectively. These weights will be multiplied by $w_{i k}$ to construct the final importance weights. Therefore, even if equal numbers are considered for $w_{i k}$ (e.g. ' $w_{i k}=1$ '), final weights follow the identified discount rate over the stages.

Table 5.2 compares the mean of cash-flow, total withdrawals, and net profit for both approaches in various weights of any particular criterion, scenario, or meta-criterion of the second stage. The differences between the achievements of the two models are described in the fourth, seventh, and the last column of the table as well. To start the sensitivity analysis of variations in importance weights, the first case is allocated to presenting the neutral preferences on meta-criteria, viz. the equal preferences of the DM on all meta-criteria in the next stage. ${ }^{3}$. The second and third cases evaluate the effects of increasing the importance weights regarding each criterion $(i, i=1,2)$ in all scenarios $(k, k=1,2,3)$. In each case, the importance weights relating to one criterion for all three scenarios is raised to fifteen and the other weights are kept at their initial values. Cash-flow and total withdrawals are, respectively, indicated by the first $(i=1)$ and $\operatorname{second}(i=2)$ criterion in this example. The next three rows of Table 5.2 are allocated to different scenarios in which the importance weights to the objective functions, related to the determined scenario $(k, k=1,2,3)$, are raised $(\times 15)$ while these weights for the other scenarios have no changes. Finally, the comparison has been continued for each meta-criterion $(i k, i=1,2 ; k=1,2,3)$, which is described in the last six rows. In each case, only the importance weight relating to one determined meta-criteria was enhanced while no variations have been experienced by the others. The relevant situation, which is explained by meta-criteria $i k$, have been defined in Table 5.3.

Comparing the results in Table 5.2 indicates that no substantial changes between different parts could be found and the conclusions seem robust despite the weight variations, especially in the three-stage moving horizon models. If we evaluate the different amounts of cash-flow in the second and third columns of the Table 5.2, it can be seen that this value changes between ' 7.18 '

\footnotetext{
${ }^{3}$ Remember that the first-order preferences of the DM have been applied to the model by choosing the goals. The importance weights (as the second order) only exert the time preferences in this case.
}

\begin{tabular}{|c|c|c|c|c|c|c|c|c|c|}
\hline \multirow{2}{*}{$\begin{array}{c}\text { Weights }\left(w_{i k}\right) \\
\left(w_{11}, w_{12}, w_{13}, w_{21}, w_{22}, w_{23}\right)\end{array}$} & \multicolumn{3}{|c|}{ Cash-Flow(million Rands) } & \multicolumn{3}{|c|}{ Withdrawal(million Rands) } & \multicolumn{3}{|c|}{ Net Profit(million Rands) } \\
\hline & $3 s^{1}$ & $2 \mathrm{~s}^{2}$ & Diff $^{3}$ & $3 \mathrm{~s}$ & $2 \mathrm{~s}$ & Diff & $3 \mathrm{~s}$ & $2 \mathrm{~s}$ & Diff \\
\hline$(1,1,1,1,1,1)$ & 7.46 & 7.83 & -0.37 & 2.76 & 2.49 & 0.27 & 5.22 & 5.32 & -0.10 \\
\hline$(15,15,15,1,1,1)$ & 7.65 & 7.62 & 0.03 & 2.91 & 2.56 & 0.35 & 5.56 & 5.18 & 0.38 \\
\hline$(1,1,1,15,15,15)$ & 7.29 & 8.36 & -1.07 & 3.16 & 2.21 & 0.95 & 5.45 & 5.57 & -0.12 \\
\hline$(15,1,1,15,1,1)$ & 7.22 & 6.74 & 0.48 & 2.86 & 2.16 & 0.70 & 5.08 & 3.90 & 1.18 \\
\hline$(1,15,1,1,15,1)$ & 7.80 & 8.09 & -0.29 & 3.11 & 2.51 & 0.60 & 5.91 & 5.60 & 0.31 \\
\hline$(1,1,15,1,1,15)$ & 7.31 & 7.47 & -0.16 & 2.80 & 2.38 & 0.42 & 5.11 & 4.85 & 0.26 \\
\hline$(15,1,1,1,1,1)$ & 7.18 & 6.41 & 0.77 & 2.81 & 2.15 & 0.66 & 4.99 & 3.56 & 1.43 \\
\hline$(1,15,1,1,1,1)$ & 7.93 & 8.31 & -0.38 & 3.11 & 2.39 & 0.72 & 6.04 & 5.70 & 0.34 \\
\hline$(1,1,15,1,1,1)$ & 7.65 & 7.81 & -0.16 & 2.96 & 2.50 & 0.46 & 5.61 & 5.31 & 0.30 \\
\hline$(1,1,1,15,1,1)$ & 7.47 & 7.78 & -0.31 & 2.81 & 2.30 & 0.51 & 5.28 & 5.08 & 0.20 \\
\hline$(1,1,1,1,15,1)$ & 7.20 & 7.60 & -0.40 & 3.05 & 2.41 & 0.64 & 5.25 & 5.01 & 0.24 \\
\hline$(1,1,1,1,1,15)$ & 7.25 & 8.20 & -0.95 & 3.16 & 2.32 & 0.84 & 5.41 & 5.52 & -0.09 \\
\hline
\end{tabular}

Table 5.2: Analysis of various weights 
and ' 7.93 ' in the three-stage moving horizon models with a maximum difference of ' 0.75 ' million Rands, while the corresponding difference in rolling the two-stage structure is ' 1.95 ' million Rands. This represents a very large difference. The same analysis for the second criteria, i.e. total withdrawals, reveals only a small difference of 0.4 , after five stages, for both approaches. Moreover, the variation between the mean of net profit for these two frameworks is double in the two-stage moving horizon model.Therefore, rolling the three-stage models provided us with more robust solutions to choice of weights than the two-stage moving horizon model in this example.

This result can be generalised to all problems because of the natural robust orientation of the three-stage structure and the broader range of meta-scenarios it considers compared to the two-stage framework. It is important to see that the conclusions are robust to choice of weights, because this robustness can satisfy a wide range of preferences of the $\mathrm{DM}(\mathrm{s})$ or various parties with specific preferences. Therefore, the three-stage moving horizon structure works better than the other models in terms of generating the solutions which are robust to choice of weights.

Existing Meta-criteria in the second stage in example 5.5.1

$M C_{1}$ : Available amount of funds at the end of the second stage after subtracting the withdrawals, if current economics faces a recession scenario.

$M C_{2}$ : Available amount of funds at the end of the second stage after subtracting the withdrawals, if current economics stay the same.

$M C_{3}$ : Available amount of funds at the end of the second stage after subtracting the withdrawals, if current economics face an expansion scenario.

$M C_{4}$ : Total withdrawals in the first two stages, if current economics face a recession scenario.

$M C_{5}$ : Total withdrawals in the first two stages, if current economics stay the same.

$M C_{6}$ : Total withdrawals in the first two stages, if current economics face an expansion scenario.

Table 5.3: Meta-criteria of the second stage in a two or three-stage model.

\subsection{Effects of the Length of Planning Horizon}

As seen in the previous chapter, although the superiority of the three-stage structure in comparison with the two-stage moving horizon models is observed in some meta-scenarios, the superiority/efficiency of utilising the three-stage model needs qualification, as it is not clearly apparent in some other meta-scenario. i.e., the differences between the solutions to the two- and threestage structures are not always substantial which cannot justify the additional complexity and calculations which appear when the three-stage framework is used. As described in previous sections, this issue is frequently observed when comparing these methods in problems with a short-term(less than five stages) planning window. Nevertheless, the efficiency of the three-stage framework is a little more visible in comparisons between the rolling two- and three-stage structures which have been used in problems with a planning window of five stages. It seems that utilising the three-stage model is more efficient for longer periods, because the differences between the solutions of the two proposed structures increase with every iteration of the algorithm in moving horizon models. Then, if the planning window is long enough (say more than ten stages), it is possible that the efficiency of the three-stage manifests itself more clearly.

Therefore, in this section, the length of the planning horizon, and its effects on the solutions generated by the proposed frameworks, will be examined and the above arguments will be illus- 
trated by an example. An attempt will be made to answer the following two questions: Which structure works better in longer horizons? How large should the length of the horizon be?

We examined the two proposed structures and changed the duration of the horizon to find the answers to these questions. Accordingly, 300 simulations were carried out for various planning horizons of 10,15,20,25, and 30 stages, and discounted weights are used to apply time preferences in the models. Note that, because the number of scenarios increases remarkably by appending the stages, running 1000 simulations in a long-term period (which includes many stages) greatly increases the time and memory required to these simulations. So, to do the study with limited available memory in a reasonable time, we can decrease the number of simulations to 300 in this example. To reach this purpose, the relevant program has been written in Matlab2015a and run on a MacBook Pro which has a $2.5 \mathrm{GHz}$ Intel Core i5 processor and 4GB RAM. The results have been presented in Table 5.4 as well as in Figures 5.8. The mean of cash-flow, total withdrawals, and net profit for both approaches with varying planning horizons $(5,10,15,20,25$, and 30$)$ have been compared in Table 5.4. The differences between the achievements of the two models (3S-2S) are also described in the fourth, seventh, and the tenth column of the table. Processing times are also described in the last column.

As seen in Table 5.4 and Figure 5.8, the differences between the solutions to the two proposed models in a moving horizon structure, in all categories, increase as the length of the planning horizon is raised. For instance, the differences between the mean of cash-flow, provided by the solutions to the three-stage and the two-stage frameworks, grow from -370000 Rands at the end of the five stages to the +150.26 million Rands after thirty stages. Also, comparing the dominance area of the two proposed methods in all graphs of Figure 5.8 confirms the superiority of the three-stage structure.

On the other hand, during the long-term calculations, we noticed that the two-stage algorithm failed to find the optimal solutions for some iterations while the three-stage algorithm finds the optimal value in all stages. We may also need to note that the constraints in the proposed example do not severely restrict the problems over different meta-scenarios and will be fixed by some financial penalties. Therefore, this example is hardly infeasible because of the consequences of the previous decisions (i.e. choosing improper decisions in previous stages). However, in many problems, especially in real-life problems, we may face severe limitations caused by prior decisions which can considerably affect the feasibility of the solutions in the next stages. This infeasibility may not be able to recover from this with a reasonable financial penalty. Therefore, in such a problem we recommend utilising the three-stage structure if the DM accepts the cost of additional complexity and computations for the quite modest improvements. Utilising the three-stage structure seems to be more efficient in problems with a long-term planning horizon (particularly for more than ten stages) if the complexity of the problem is not too computationally expensive. Nonetheless, expensive computations and extreme complexity that could be faced in real problems may well prevent us from considering more than three stages. Our experiences in using the proposed methodology in real-world applications, such as one that will be explained in the next chapter, indicates that, even in a three-stage structure, this issue may occur in some complex problems, especially with more than two or three uncertain parameters.

\begin{tabular}{|c|c|c|c|c|c|c|c|c|c|c|}
\hline \multirow{2}{*}{$\begin{array}{c}\text { Length of } \\
\text { horizon }\end{array}$} & \multicolumn{3}{|c|}{ Cash-Flow(million Rands) } & \multicolumn{3}{|c|}{ Withdrawal(million Rands) } & \multicolumn{3}{|c|}{ Net Profit(million Rands) } & \multirow{2}{*}{$\begin{array}{c}\text { Processing } \\
\text { time }(\mathrm{sec})\end{array}$} \\
\hline & $3 \mathrm{~s}$ & $2 \mathrm{~s}$ & Diff & $3 \mathrm{~s}$ & $2 \mathrm{~s}$ & Diff & $3 \mathrm{~s}$ & $2 \mathrm{~s}$ & Diff & \\
\hline 5 & 7.46 & 7.83 & -0.37 & 2.76 & 2.49 & 0.27 & 5.22 & 5.32 & -0.10 & 160 \\
\hline 10 & 12.10 & 11.39 & 0.71 & 6.86 & 6.00 & 0.87 & 12.86 & 11.62 & 1.24 & 500 \\
\hline 15 & 20.03 & 16.18 & 3.85 & 11.24 & 10.31 & 0.93 & 25.21 & 20.76 & 4.45 & 734 \\
\hline 20 & 46.98 & 32.75 & 14.23 & 15.61 & 14.45 & 1.15 & 56.48 & 41.55 & 14.93 & 1040 \\
\hline 25 & 151.05 & 94.55 & 56.50 & 21.16 & 20.10 & 1.06 & 166.18 & 109.05 & 57.13 & 1307 \\
\hline 30 & 247.09 & 150.26 & 96.83 & 26.23 & 25.28 & 0.95 & 267.34 & 169.96 & 97.38 & 1661 \\
\hline
\end{tabular}

Table 5.4: Comparison results of 300 simulations in long-term horizons(5, 10, 15, 20, 25, and 30 stages) 

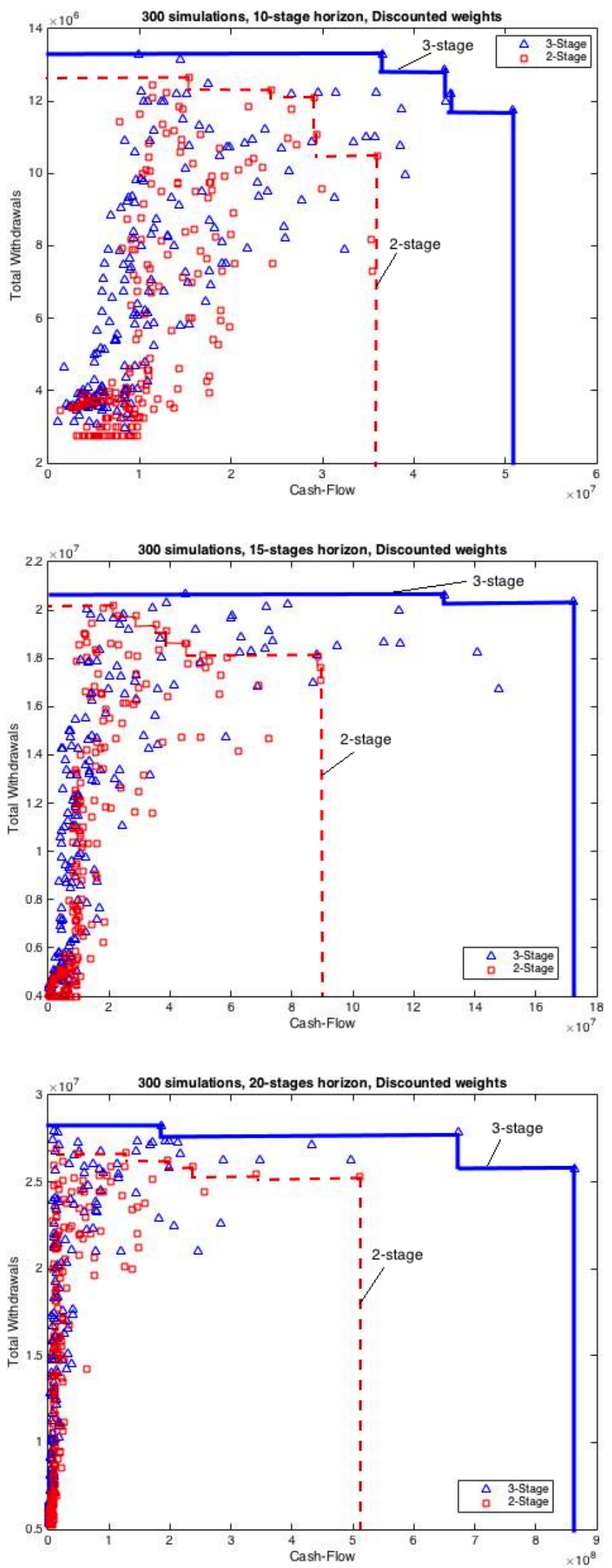

Figure 5.8: Combined picture of cash flow and total withdrawal at the end of the different stages with discounted weights in 300 simulation runs $(T=11,16,21)$, to compare the rolling two-stage and the three-stage structures. 
Overall, as seen in this chapter and the previous one, in problems with a shorter planning window (e.g. five stages or less), the differences between the solutions to the two- and three-stage models is small, and the improvement that is gained by utilising the three-stage approach may not be efficient. It must be noted that by the "efficiency" of the three-stage framework in our context, we want to know if utilising this approach and its solutions is worth the additional complexity and computation. Remember that the solutions to the three-stage pattern must be better in goal achievements, but not much better than the other solutions in short-term horizons. The solutions of the three-stage model also provided more robustness than the solution generated by the two-stage model. This difference between the solutions to these models is not an issue of risk aversion but of future flows of objective function performance that is mostly affected by the element of time which is typically involved in these kinds of problems. These differences represent trade-offs between the short and long-term objectives. On the other hand, a substantial problem was comparability of these methods. This can be solved by modelling time preferences in multistage problems. Therefore, importance weights have been utilised to apply time preferences in the proposed methodology. These weights, then, primarily use to make the two- and three-stage models more directly comparable, and only secondarily as a measure of risk preference. Thus, a comparison of the results of the proposed methods highlights certain features. In particular, at the beginning of the project (or in a short term window) the solutions to the two-stage model are very similar to the solutions of the three-stage method, or are better in some cases. However, over a longer period, utilising the solutions which were generated by a model with an extended future vision (such as the three-stage model) can justify the additional complexity and more computations. Then, the differences between the solutions of the two proposed methods, in long-term windows, increase remarkably, which confirms the efficiency of using the three-stage moving horizon structure in these problems. Therefore, it seems that if the planning horizon is long enough (especially for more than ten stages), the efficiency of the three-stage model is worth the additional complexity and computation. Nevertheless, if the DM prefers to not use the three-stage structure because of the complexity and/or expensive calculations, the two-stage moving horizon model can still provide some reasonable solutions, although they might not as good as the solutions generated by a three-stage framework.

\subsection{Conclusion}

As mentioned in the previous chapters, the main aim for this thesis was to find a way of dealing with deep uncertainty in multi-criteria decision analytic models/multi-objective optimisation in long-term strategic planning problems. To reach our purposes, we first utilised scenarios as a dimension of preferences (a component of the meta-criteria) to avoid problems of evaluating probabilities under deep uncertainty. Then, we introduced the two-stage structure that looks one step ahead, postpones part of the decision and leaves room for possible adaptation later after scenario realisation. This interesting framework not only matches well enough with scenarios and the concept of meta-criteria in MCDM problems but also, by nature, provides us with some dynamic-robust decisions for every plausible scenario. After constructing this powerful structure to treat deep uncertainty in MCDM problems, the proposed structure has been successfully extended to the three-stage (multi-stage in general) structure, in the previous chapter, to act in problems with a three-stage planning horizon.

However, expanding the proposed structure to problems with more than three stages which usually have too many meta-scenarios may lead us to a computationally expensive model that cannot be easily solved, if it all. Moreover, drawing a horizon that is too long could not be an exact plan, as nothing in nature is predictable, and we are always surprised by new events. Therefore, beyond the expensive computation in a multi-stage structure for more than three stages, defining plausible scenarios for far stages is not logical or even possible. However, guessing plausible scenarios for the next two stages is easier and more acceptable. Therefore, to solve the last part of the puzzle, we needed to extend this structure to longer periods. In the proposed 
frameworks, stages are artificial to structure thinking of an indefinite future. They distinguish the planning horizon (which includes T-stages) into different periods that are separated by the time of various scenario realisations in which we will get new knowledge by unfolding an unknown scenario. This structuring, together with the dynamic nature of the decisions, inherently matches with the moving horizon models (rolling two- or three-stage models in a T-stage planning window). This motivated us to extend our proposed philosophy and context to a moving horizon structure. Nevertheless, the important question is: How many stages we should consider in advance (two or three) in an infinite horizon?

Therefore, in this chapter, we introduced the moving horizon models in a T-stage planning window and two rolling frameworks (two- and three-stage model) have been proposed. A comparison of these methods has shown that the solutions of the two-stage model are feasible in the other pattern (three-stage). Also, the optimal solution of the three-stage model is not dominated by any solutions of the two-stage model. So, with no doubt, the three-stage model must find better, or at least the same, goal achievement compared to the two-stage model. On the other hand, in the previous chapter, we indicated that the three-stage structure provides more robust solutions than the two-stage pattern, because the three-stage structure considers the consequences of the decisions of the initial stages and the conditions of different meta-scenarios with a longer future vision. Accordingly, the three-stage model evaluates and compares the optimal solution of the corresponding two-stage model to the other feasible solutions. Then, if it selects anything else, it must either be better in goal achievement or be robust in some future scenarios, or provide a combination of both. Thus, the superiority of rolling the three-stage model in a T-stage planning horizon would be expected. However, the cost of these supremacies must be considered (as it may lead us to a computationally expensive problem), and the efficiency of applying this structure still needs qualification. The experiments in this chapter showed that the three-stage model has advantages under most circumstances (meta-scenarios), but that the gains are quite modest.

Modelling time preferences in multi-stage problems has been introduced to solve the fundamental problem of comparability of the two proposed methodologies by applying a differential weighting strategy. Importance weights, then, are used, primarily, to make the two- and threestage models more directly comparable, and only secondarily as a measure of risk preference. Differential weighting can help us apply more preferences on the model and lead it to generate more preferred solutions. It also makes more sense to focus on the first stage circumstances while still being mindful of the future and considering the consequences of the current decisions on every plausible meta-scenario. Furthermore, by utilising the differential weighting, we could expect the solutions for both approaches (two- and three-stage) to be balanced between the meta-criteria. As an interesting extension to the differential weighting, it would be more meaningful to apply the differential weighting in a discounting manner, i.e. put the most weight on the first stage, then decrease the weights for the second stage and so on. Descending weighting in long-term problems seems more meaningful as some surprising outcomes may arise when considering a far unknown future. We always need to update and adopt the decisions as we are moving ahead. Therefore, most concentration should be placed on the near future whilst still looking further ahead to avoid, or at least reduce, some difficulties that may be predictable. This concept also matches with the dynamic-robust orientation of the proposed structures.

To analyse all plausible meta-scenarios and compare the solutions to these two moving horizon models in a T-stage planning window, too many models need to be run. Even for five or six stages, hundreds and thousands of multi-objective comparisons are required and this is not easily conceivable, if ever possible. This issue motivated us to perform a simulation study so that we could evaluate, compare and analyse these methods for problems with the T-stage planning horizon. Accordingly, a simulation-optimisation approach was introduced in Section 5.4 to compare the proposed structures in a T-stage planning horizon. After that, the investment example 4.5.1 was revisited to examine the proposed structures and algorithms. This was followed by a detailed discussion on trade-offs and Pareto frontiers in Section 5.5.1. The best way of 
comparing the results of these two frameworks in their multi-criteria structure is to draw the Pareto frontier of the solutions to these methods. However, in this problem, the trade-offs must be evaluated and compared between all meta-criteria which would be too complex. Obviously, plotting all information in a $2 \mathrm{D}$ or $3 \mathrm{D}$ graph is impossible. Nevertheless, we can sketch the results in a two-dimensional diagram for each meta-scenario. Although this may not widely useful, this picture can still help to illustrate the proposed structures and simulations. It can also provide us with a vivid image of the Pareto frontier under conditions of any meta-scenario. This procedure, introduced as the single-scenario Pareto front approximation algorithm, can approximate the Pareto front in each meta-scenario. These plots are suitable to compare some important meta-scenarios, such as the worst case, in more detail.It also helps to observe the effects of various weights in different meta-scenarios and find a set of suitable weights. However, comparing overall performance of the two proposed methods with the single-scenario Pareto fronts is almost impossible and we need to show all the performances in a combined picture for a trustworthy comparison.

However, according to the high dimensional aspect of the problem, plotting a two- or threedimensional picture of the results is impossible. To draw a combined picture of performances, an algorithm has been suggested in Section 5.5.1.2 in which, instead of drawing the Pareto front, we suggest sketching and comparing the ordered multi-sets of performances, provided by the proposed methods, in terms of two (or three) criteria under conditions of the simulated meta-scenarios. This algorithm can also be applied for all plausible meta-scenarios, if they are enumerable, to cover all the meta-criteria. The result will be a plot representing all the performances of different criteria under conditions of all meta-scenarios. Comparing the results of our example in a relevant combined picture highlighted the fact that applying time preferences in the problem helps to make the solutions to the two proposed models directly comparable, and allows for the inclusion of more preferences in the model to generate more preferred solutions. In addition, applying time preferences can also provide us with balanced solutions in both structures.

The differences between the solutions to these models are not the issues of risk aversion but of future flows and are affected by using the different lengths of future vision (two or three) in these structures. In fact, there are some trade-offs between the short- and long-term objectives, because the difference of time modelling affects the solutions to the models with various time windows. Therefore, when the element of the time is involved in a problem, the term "risk-averse decisions" does not make sense. In this case, the most effective factor is time. The risk-aversion decision matches with the once off decision-making problems in which time is not involved as a vital factor.

Furthermore, from an analysis of various weights we concluded that the solutions to the threestage structure are more robust to variations in weights compared to the two-stage models. Then, the use of the three-stage moving horizon structure better satisfies a wide range of preferences of the $\mathrm{DM}(\mathrm{s})$ or various parties with specific preferences.

Nevertheless, analysis of the length of the planning horizon and its effects on the solutions to the proposed frameworks, in the final section of this chapter, indicates that utilising the three-stage models is more efficient for longer periods (say more than ten stages) because every time the the algorithms in moving horizon models are run, the difference between the solutions increases. Moreover, during the long-term calculations, we noticed that the two-stage algorithm failed to find the optimal solutions for some iterations while the three-stage algorithm found the optimal value in all cases.

Thus, it seems that for a planning horizon with more than ten stages, the efficiency of the three-stage model may be worth the additional complexity and computation. Nevertheless, if the DM prefers to not use the three-stage structure because of the complexity and/or calculations, the two-stage moving horizon model can provide us with some reasonable solutions, although they might not be as good as the solutions generated by a three-stage framework. Furthermore, expensive computations and the extreme complexity that will be faced in real problems may well prevent us from applying more than three stages. Our experiences in using the proposed 
methodology in real-world applications, such as one that will be explained in the next chapter, indicate that, even in a three-stage structure, this issue may occur in some complex problems, especially with more than two or three uncertain parameters. Therefore, to apply the proposed methodology in a complex strategic design of sugar-bioethanol supply chains under deep uncertainty in the next chapter, we utilised the two-stage structure to avoid extreme complexity and expensive computation. 


\section{Chapter 6}

\section{Multi-Objective Optimisation of the Sugar and Bioethanol Supply Chain under Deep Uncertainty}

\subsection{Introduction}

Nowadays, environmental problems such as pollution, global warming, ozone layer depletion, climate change, and public health issues represent some of the most controversial matters facing modern society. One of the main driving factors behind these problems is the large volume of Green House Gas (GHG) emissions that is produced from burning the fossil-related sources of energy. Various international agreements such as the Kyoto Protocol ${ }^{1}$ show the widespread commitment to reducing GHG and a number of internationally mandatory GHG reduction goals have been set.

One of the successful goals has been to drive a shift away from the use of fossil-fuels towards biofuels and, especially, the use of bioethanol which is suitable for spark-ignition engines [Awudu and Zhang, 2012]. Utilising ethanol or blending ethanol with regular fuel, such as petrol, can significantly reduce carbon dioxide $\left(\mathrm{CO}_{2}\right)$ emissions and and this has led to an increased demand for ethanol in recent years [Kostin et al., 2012].

Currently, more than $72 \%$ of all bioethanol is produced from the fermentation of sugarcane, mainly in Brasil, or corn, mainly in the US. However, sugarcane-based ethanol is known as one of the most energy-efficient and sustainable products [Goldemberg et al., 2008].

However, the advent of biofuel and other renewable sources of energy need adapted design of the old systems within the supply chain. This modification needs decision-support tools to ensure the satisfaction of different stakeholders in terms of the possibility and profitability of new designs. For biofuels and bioethanol to be considered feasible alternatives to current fuels, there is a need to show that utilising these kinds of fuels can reduce the GHG and other environmental effects that may arise along the supply chain. Therefore, the optimisation of the bioethanol supply chain is an important and interesting problem and this has been studied by several researchers. Therefore, mathematical programming, particularly linear(LP) and mixedinteger linear programming(MILP), has been recognised as the most suitable strategy to handle and find solutions for such a complex problem. Different models have been suggested to optimise various parts such as transportations([Ioannou, 2005, Kawamura et al., 2006, Milan et al., 2006, Dunnett et al., 2008], distributions([Yoshizaki et al., 1996]), scheduling([Grunow et al., 2007, Colin, 2009]), and production planning([Paiva and Morabito, 2009]) in the bioethanol/biofuel

\footnotetext{
${ }^{1}$ An international agreement (named the Kyoto Protocol) that adopted in Kyoto, Japan, on 11 December 1997 and linked to the United Nations Framework Convention on Climate Change (UNFCC).
} 
supply chain.

As mentioned above, the most important and interesting aspect of utilising biofuel and bioethanol is their potential to reduce the environmental issues and mainly the GHG emissions associated with the fossil-fuel industry. Nonetheless, the profitability of the projects is the only(or the most) important part for almost all investors. These two objectives/criteria (i.e. environmental and economic benefits) are conflicting goals (at least with the current technologies). Therefore, the use of a multi-objective optimisation programming approach could be useful (or even be necessary) to solve these kinds of problems. Accordingly, several multi-objective optimisation models have been proposed and they mainly focus on these two major criteria of economics and environmental benefits (see, for example, [Zamboni et al., 2009, de Vries et al., 2010, Mele et al., 2011, Giarola et al., 2011]). Most other strategies have considered only the certain parameters which are known in advance. Nevertheless, as discussed in this thesis, such strategies cannot be applied to real-world problems which face a lot of uncertainty. Optimisation models considering uncertain parameters have also been introduced by some authors (see an informative review in [Sahinidis, 2004]). However, as discussed in Chapter 2, none of them are able to handle deep uncertainty faced in real problems. In these previous studies, uncertainty is treated by utilising the two-stage stochastic models ([Liu and Sahinidis, 1996]), robust stochastic formulations ([Li et al., 2008]), or Fuzzy programming ([Zimmermann, 1991]).

To the best of our knowledge, very little published research has investigated the biofuel Supply Chains (SCs) and infrastructure optimisation under uncertainty. Furthermore, only two studies have used multi-objective optimisation ([Dal-Mas et al., 2011], and [Osmani and Zhang, 2014]). Some recent research which has proposed various models for optimising biofuel SCs under uncertainty have been cited in Table 6.1 which details the objective(s), the uncertain parameter(s), application, and methodology used in each study. As seen in the last column of Table 6.1, almost all of these studies utilised the two-stage stochastic models. These models are able to cope with only mild uncertainty and not deep (or even moderate) uncertainty. Therefore, it would interesting to apply the two-stage structure (proposed in the current study) to treat the deep uncertainty in this biofuel supply-chain problem.

Therefore, the main purpose of this chapter was to concentrate on applying the introduced definitions and concepts in a two-stage structure (as the simplest form of our methodology), as well as to examine the reliability and validity of the proposed methodology in a real-world problem. We limited this example to the two-stage structure to avoid extreme complexity and expensive computation. For purposes of demonstrating our approach, there is no immediate necessity to apply the three-stage or moving horizon models in this study, although consideration

\begin{tabular}{|c|c|c|c|c|}
\hline Author(s) & Objective(s) & Uncertainty & case study & Method \\
\hline Dal-Mas et al. [2011] & $\begin{array}{l}\text { Max Expected Profit } \\
\text { Min Financial Risk }\end{array}$ & $\begin{array}{l}\text { Biomass production cost } \\
\text { Product selling price }\end{array}$ & $\begin{array}{l}\text { Corn to ethanol } \\
\text { production SC } \\
\text { in Northern Italy }\end{array}$ & $\begin{array}{c}\text { Dynamic, spatially explicit } \\
\text { and multi-echelon } \\
\text { MILP modelling }\end{array}$ \\
\hline Kim et al. [2011] & Max Expected Profit & $\begin{array}{l}14 \text { Parameters such as } \\
\text { demand, sale price, etc. }\end{array}$ & $\begin{array}{c}\text { Biomass supply chain } \\
\text { network for biofuel }\end{array}$ & $\begin{array}{c}\text { Two-stage } \\
\text { stochastic model }\end{array}$ \\
\hline Kostin et al. [2012] & $\begin{array}{l}\text { Max Expected Net } \\
\text { present value }\end{array}$ & Demand & $\begin{array}{l}\text { Bioethanol and sugar } \\
\text { production supply chain }\end{array}$ & $\begin{array}{c}\text { Two-stage } \\
\text { stochastic model }\end{array}$ \\
\hline Osmani and Zhang [2013] & Max Profit & $\begin{array}{c}\text { Switch-grass Yield } \\
\text { Crop residue purchase price } \\
\text { Bioethanol demand } \\
\text { Sales price }\end{array}$ & $\begin{array}{c}\text { Bioethanol SC } \\
\text { North Dakota } \\
\text { state } \\
\text { in the US }\end{array}$ & $\begin{array}{c}\text { Two-stage } \\
\text { stochastic model }\end{array}$ \\
\hline Osmani and Zhang [2014] & $\begin{array}{c}\text { Max Profit } \\
\text { Min Carbon Emissions }\end{array}$ & $\begin{array}{c}\text { Supply biomass } \\
\text { Purchase price } \\
\text { Bioethanol demand } \\
\text { Sale price }\end{array}$ & Bioethanol SC & $\begin{array}{c}\text { Two-stage } \\
\text { stochastic } \\
\text { MILP model }\end{array}$ \\
\hline Awudu and Zhang [2013] & Max Profit & $\begin{array}{c}\text { Price } \\
\text { demand }\end{array}$ & $\begin{array}{c}\text { Biofuel SC } \\
\text { North Dakota, USA }\end{array}$ & $\begin{array}{l}\text { stochastic } \\
\text { model }\end{array}$ \\
\hline
\end{tabular}

Table 6.1: Some recent related works proposing models for optimising biofuel SCs under uncertainty 
of these models would an interesting direction for future research. Finally, this chapter also aims to investigate the possibility of utilising the proposed structure as a decision support tool in MCDA problems.

In a biofuel supply system, the design and planning decisions, such as refinery and terminal locations and sizes, need to be made before the uncertainty is unfolded (scenario-free decisions). These decisions cannot be simply adapted at a later stage. However, operational decisions such as raw material procurement, ethanol production, and transportation can be adjusted with some penalties (scenario dependent or recourse decisions). Therefore, in the proposed two-stage structure, the former decisions (scenario free) are classified as the initial decisions at the first stage (stage ' 0 '). While the latter decisions will be counted as the contingent (recourse) decisions that can be implemented after scenario realisation in the second stage (stage ' 1 ').

In this chapter, the strategic planning of sugar-bioethanol supply chains (SCs) under deep uncertainty has been addressed by applying the two-stage methodology that was proposed in Chapter 3. In this study, we analysed the whole infrastructure of the sugar-bioethanol supply chain in such a way that all economic, environmental and social aspects could be optimised. We were also interested to find the best place(s) to build the ethanol plant(s) amongst available areas. The problem is formulated as a scenario-based mixed-integer two-stage multi-objective optimisation problem and is solved by utilising the Generalised goal programming approach. Three objectives (Max profit, Min $\mathrm{CO}_{2}$-emissions, and Max job-creation) are considered in this problem under six uncertain parameters (sugarcane yield, ethanol and refined sugar demands and prices, and the exchange rate). The South African sugarcane industry is utilised to study and examine the proposed methodology. Unfortunately, some real data were highly classified by the South African Sugar Association (SASA) so we decided to make this study slightly hypothetical and used some approximation for some data. The problem is still is real and valid.

The next section, Section 6.2, details a general sugar-ethanol Supply Chain (SC) infrastructure and provides some key assumptions, such as existing uncertainties, which exist for this problem. In Section 6.3, the proposed two-stage scenario-based model for this general problem is structured. Stages, scenarios, and variables which are matched with this two-stage structure are also fully described, and the objective functions and constraints are also formulated. The solution method is also explained in this section. A real case-study, based on the South African sugarcane industry, is used to represent the capability of the proposed structure in real problems and this is elaborated on in Section 6.4. In Section 7.2, the case-study results are described, interpreted, and discussed, in terms of the valuable insights obtained, by a sensitivity analysis of various goals. Finally, in the last section (Section 6.6), the conclusions of the work and future directions are presented.

\subsection{Problem description}

The sugar and bioethanol supply chain (SC) structure used in this study is motivated by previous literature studies. This structure is portrayed in Figure 6.1. Generating ethanol from sugar cane and its productions is closely linked with the sugar industry and some parts of their respective infrastructure are common to both industries. This study includes an analysis of the possibility of providing suitable infrastructures for generating ethanol and integrating these with the existing sugar production system. Ethanol, raw and refined(white) sugar, as well as some by-products, are the final products of the system. They may be sent either directly to the central depot/terminal for delivery to the market or may be converted to other products (depend on the product, possibility and requirements) after being delivered to the existing refineries or ethanol plant(s) that will be set up in the future.

Suppose that $l \in L$ suitable areas of land are available that could be used to cultivate sugar cane. Harvested sugarcane is transport to the nearest mill, in the same area, where it could be either converted to raw sugar or sent to the ethanol plant $r \in L$ which has the relevant technology to produce ethanol. $L$ is the set of all sites (areas of land), $l$ and $r$ indices representing specific 


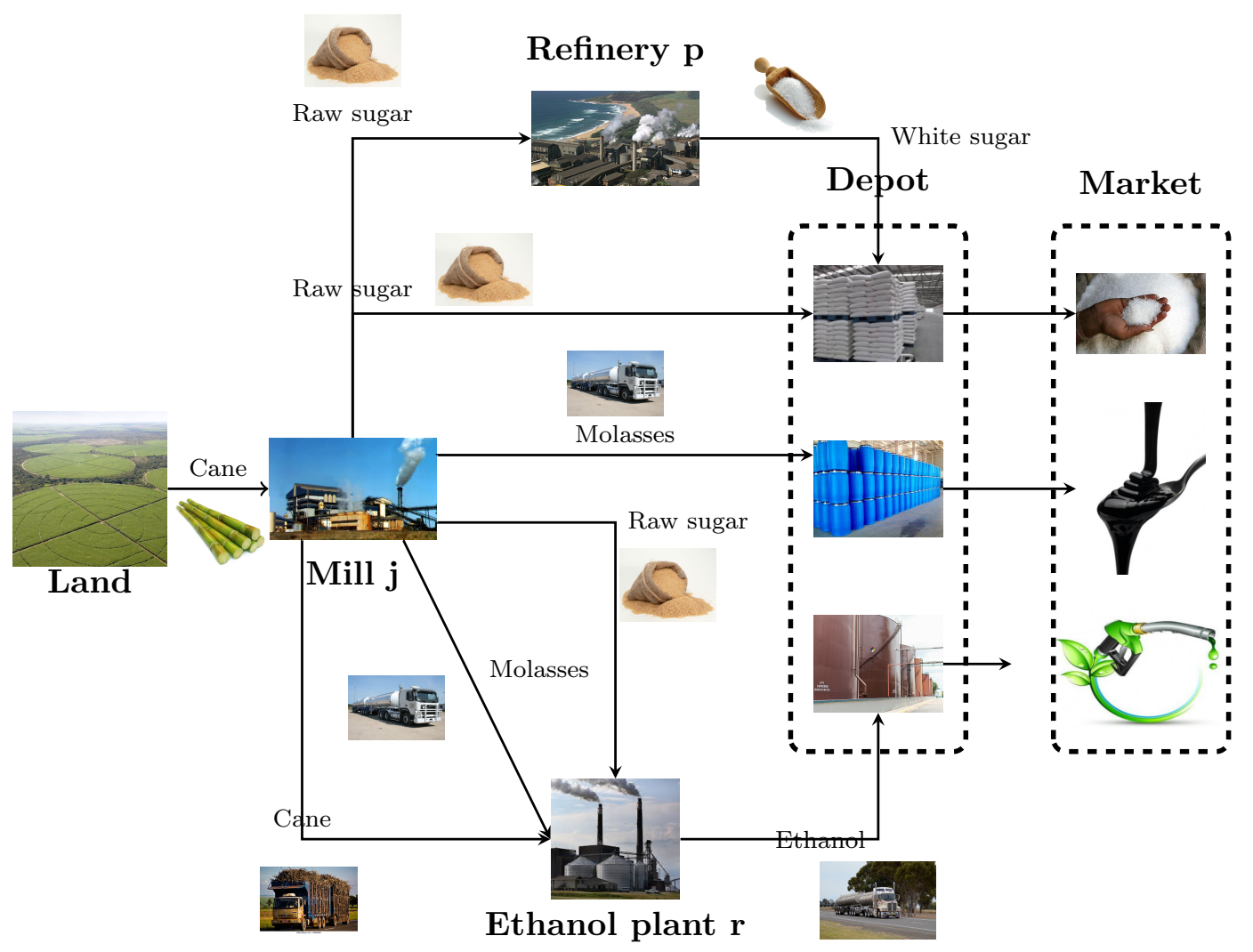

Figure 6.1: Sugar and Bioethanol SC

sites. It is important to note that harvested sugarcane stalks cannot be stored for more than a few days, so transporting sugarcane to far areas is almost impossible and the stalks decay fast.

In this study, only one production technology has been considered in each mill. This technology uses sugar cane stalks as feedstock to generate raw sugar and molasses as by-products. This is a basic technology which is used in some countries such as South Africa ([SASAwebsite, 2017]). Figure 6.2 shows this technology, as well as the technology of the refineries, including relevant coefficients in detail. Different technologies can also be simply appended to the model. Furthermore, sugarcane and its products (such as sugar and fermented products) have been utilising in making and preserving various kind of food and medicines like sugarcane juice, syrup, molasses, jaggery, falernum, rum, rock candy, ethyl and butyl alcohol, and capsules. However, in this study, we are not going to consider all of them. There are also some fibrous residues (known as bagasse) which are produced during the processing of sugar cane to sugar. Bagasse is mostly used for packaging and heating ${ }^{2}$. Recently, with new technologies, it is possible to generate biofuel from bagasse which makes the process more profitable. However, this study does not consider this technology which may be too expensive, and which needs further research to confirm its feasibility. Nevertheless, it would be an interesting direction for our future work to extend our proposed methodology.

Three destinations are imagined for the produced raw sugar. They could be sent either directly to the depot, forwarded to the ethanol plant $r$, or transported to refinery $p \in P$ to generate white(refined) sugar. Molasses is sent either to the ethanol plant $r$ to generate bioethanol

\footnotetext{
${ }^{2}$ Note that bagasse is known as a sustainable and renewable by-product that can use for packaging, tree-free paper, composts. Most importantly, it can be utilised as a biofuel and in the manufacture of pulp and building materials.
} 

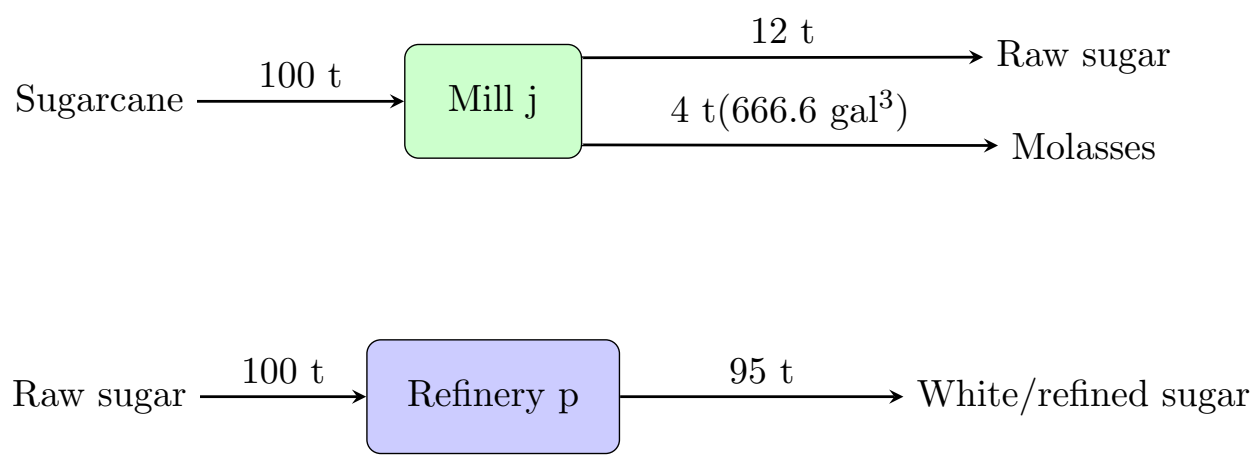

Figure 6.2: Sugar and ethanol production technologies used in mills and refineries

or transported to the depot. Refinery-produced white(refined) sugar would be transport to the depot. No more molasses production is considered in this step.

There are four technologies that would be deemed suitable to produce bioethanol from sugarcane, molasses, raw sugar and refined sugar, respectively. Here, due to the similar processes of bioethanol conversion from raw/refined sugar and molasses, we consider two different technologies (see Figure 6.3. One technology, refers to $q=1$, and this produces ethanol from sugarcane stalks. The second technology $(\mathrm{q}=2)$ generates ethanol and a by-product during the ethanol production process from sugar (raw/white) and molasses. Converting refined sugar to ethanol is the most expensive way of producing ethanol; therefore, we will not consider this technology unless we have to perform a recourse action. Finally, the produced bioethanol is transported from an ethanol plant $r$ to the terminal/depot in which bioethanol could be sold locally, by blending it with petrol, used directly as a biofuel, or could be exported.

In this study, we analysed the whole infrastructure of the sugar and bioethanol supply chain in such a way that all economics, environmental and social aspects could be optimised. We also searched for the best place(s) to build the ethanol plant(s) amongst existing areas which leads us to a Multi-Objective Mixed Integer Linear Programming (MOMILP) model.

Furthermore, the literature review demonstrated that most of the key parameters of the study would be uncertain at the time of decision-making and, specifically, most of them are beyond all expectations and have a deep uncertain nature. To the best of our knowledge, there appear to be no published studies that deal with deep uncertainty, not only in the biofuel supply chain literature but also in the literature concerning MCDM. Most of the previous work has been limited to probabilistic and stochastic programming which is classified as mild uncertainty. The next section will describe the deeply uncertain parameters that were considered in this study.

\subsubsection{Existing Uncertainties}

As mentioned earlier, different kinds of uncertainty can be found in the biofuel SC literature. These uncertainties include, but are not limited to, uncertainty in sugar and ethanol production costs, demand and prices that largely depend on oil prices, uncertain sugarcane yields that would be weather dependent, currency fluctuations that have been highly volatile recently mainly due to global economic crises, and uncertainty in new technologies in our fast growing world. In this study, six key uncertain parameters have been considered: 1)sugarcane yield; 2)ethanol demand; 3)sugar demand; 4)ethanol sale price; 5)sugar sale price, and 6)currency fluctuations.

\footnotetext{
${ }^{3}$ As mentioned earlier, the US and Brazil are the world leaders in ethanol production from corn and sugar, respectively. Therefore, most of the international deals in stock markets perform in US gallons and existing prices deal in USD. Thus, this thesis uses US gallons for ethanol and USD for prices which are converted to South African Rand in models by multiplication with the relevant conversion factor.
} 

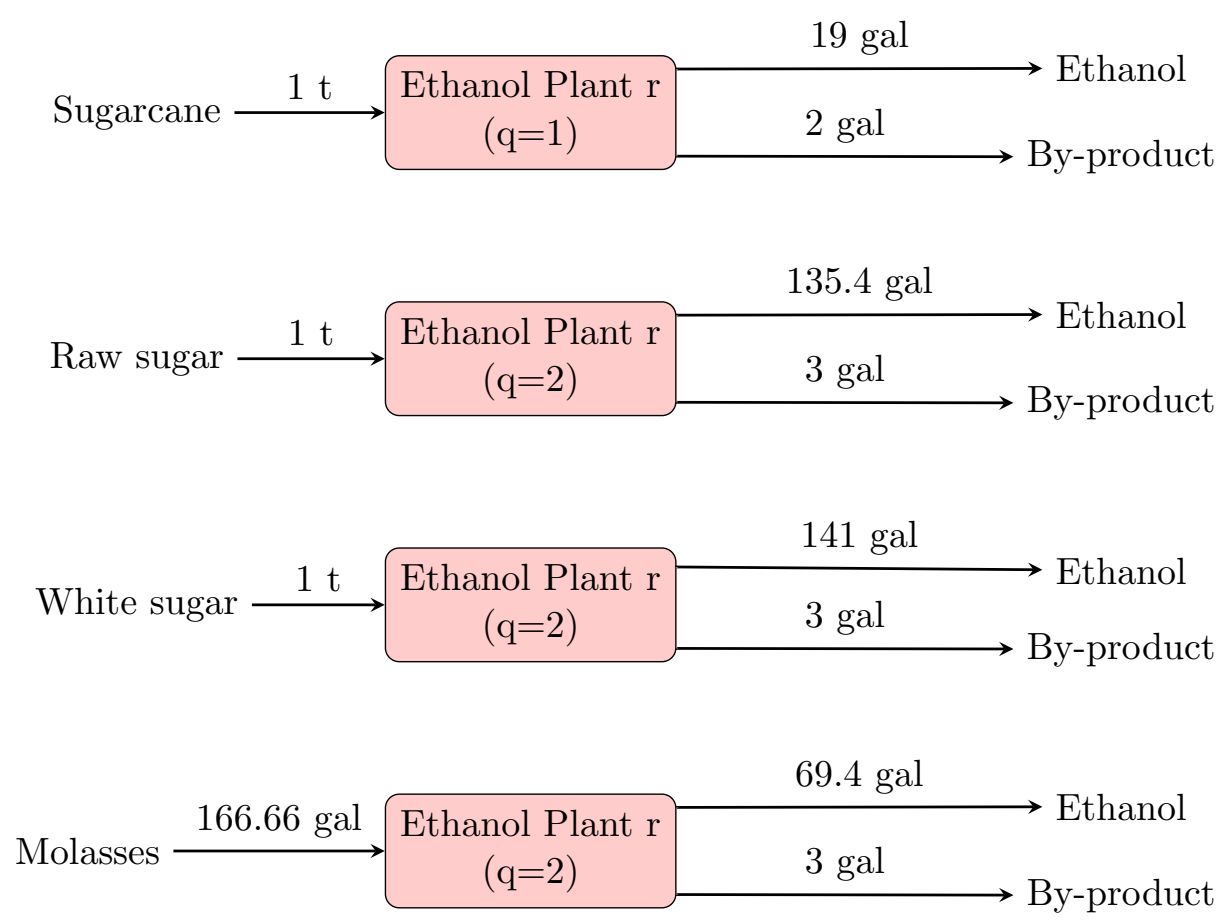

Figure 6.3: Set of production technologies in an ethanol plant

\subsubsection{Sugarcane Yields}

Crop annual yields are always affected by weather conditions such as rainfall level, global warming and climate changes. This uncertainty can affect the decision of how many hectares of arable land should be utilised to grow sugarcane in each region. This decision is an initial decision that needs to be made before scenario realisation, and hence it is common among all plausible yield scenarios. Sugar and ethanol productions are also dependent on the sugarcane yields. We define $s\left(k_{1}\right)$ to present plausible scenarios of sugarcane yields.

\subsubsection{Ethanol and Sugar Demand}

The uncertainty in product demand has been known as one of the principal sources of uncertainty in any SC [Kostin et al., 2012]. A manager always aims to avoid both excess production and failure to satisfy customer demand. Sugar and ethanol consumptions as a result of human behaviour are not deterministic and vary in different years. Although governments usually approve a certain percentage of ethanol blending to petrol in advance, petrol consumption is also uncertain and, as a result, makes ethanol demand uncertain too. $s\left(k_{2}\right)$ represent the different scenarios of ethanol demand.

In this study, to reduce the independent uncertain parameters that can increase the size of our model, we assume that the demand for raw and refined(white) sugar have a relative correlation (raw demand $=\vartheta \times$ white demand). Therefore, various scenarios of refined sugar (and hence raw sugar) are explained by $s\left(k_{3}\right)$. It is always possible to separate these two by defining a new uncertain variable for raw sugar demand. However, it will raise the model's size exponentially. 


\subsubsection{Ethanol and Sugar Price}

Another important source of uncertainty is the uncertainty in the product selling price that has a major impact on the financial part of the system and, specifically, on the final revenue. Oil price largely determines biofuel, and hence ethanol, demand and price. The oil price fluctuated extensively over the last decade (US\$ 30 - 140 for a barrel) which led to fluctuations in the ethanol price. So, the fourth independent uncertain parameter which is considered in this study is the world ethanol price, and its randomness is represented by different plausible scenarios $s\left(k_{4}\right)$.

In the sugar industry, in addition to local demand and weather dependent yields affecting the local prices, world sugar prices significantly depend on Brasil sugar production because this country contributes to around 40 percent of global sugar production. Thus, another independent uncertain parameter is the price of white sugar which is dictated by the selling price of sugar. Here, we suppose that the world price of raw sugar can be calculated as a correlated function of the world white sugar price. Moreover, the local prices for raw and white sugar and molasses are computed by multiplying the relevant conversion factor to the world price of the white(refined) sugar in each and every plausible scenario $s\left(k_{5}\right)$.

\subsubsection{Exchange rate}

Recently, the global economics crisis has caused extensive fluctuations in most currencies. For example, in South Africa, the US dollar to ZAR exchange rate increased sharply from 6.6 in 2012 to 16.8 in 2016 and this has had a massive impact on all commodity prices in SA. Therefore, the sixth independent uncertain element considered in this study is currency fluctuations. Different plausible scenarios for currency have been determined by $s\left(k_{6}\right)$.

\subsection{Model structure and formulation}

This section is allocated to matching the problem in the proposed two-stage structure, defining stages, scenarios, and variables matching this two-stage structure, as well as formulating the objective functions and constraints.

\subsubsection{Two-stage structure}

In a two-stage decision-making process, decisions are divided into two different groups. The first group is known as a "here-and-now" decision which is made under conditions of uncertainty. The second group is called "wait-and-see" decision which is undertaken by the DM after the unfolding of scenarios. This delayed decision-making allows the DM to modify his/her previous decisions by paying some penalties and this corresponds to the recourse variables in Chapter 3. These two groups and the relevant variables that lie on each division, in our problem, are described below:

\subsubsection{Initial decisions (Stage ' 0 '):}

As discussed earlier, these decisions must be made before scenario realisation. In this problem, the following decisions must be made and implemented at the beginning as the initial decisions:

- Hectares of land that should be utilised to grow sugarcane in each region.

- Number, technology, and location of ethanol plants that should be set up.

- The volume of the sugarcane converted to raw sugar and the optimal amount of sugarcane that should be sent to the ethanol plants for ethanol production. 
- The portion of generated raw sugar that should be transported from each mill to every refinery, ethanol plant and the depot.

- The amount of generated molasses in each mill that must be sent to each ethanol plant and the depot.

Although it may possible to adapt the last two decisions later, these decisions still need to be made before scenario realisation and at the beginning of the period.

\subsubsection{Contingent/recourse decisions (Stage ' 1 ):}

After scenario realisation and obtaining new information in stage ' 1 ', a group of contingent/recourse decisions relevant to the scenario which has unfolded must be implemented in the second stage (stage ' 1 '). These decisions have been identified previously but not implemented because of the uncertain scenarios. In this problem, the following recourse decisions must be implemented in the second stage:

- The volume of raw sugar, white sugar, molasses, by-products, and ethanol that needs to be sold locally, exported, or imported in each scenario

- The amount of raw sugar, white sugar, and molasses that must be transported from depot to ethanol plants for extra ethanol production

- The portion of extra sugarcane yield in each region that should be sent to the mill for more raw sugar generation

After obtaining information about the uncertain parameters, the above actions could be implemented as contingent/recourse steps to recover the unsatisfied initial goals as much as possible. Of course, there may be some penalties such as more transportation costs and emissions, higher importation costs and emissions, wasted crops, loss of market share, and additional production processes. For instance, the excess amount of sugarcane which is harvested and transported later (as a recourse decision) only can be utilised to produce raw sugar. In this production, molasses is also generated as a by-product, but both raw sugar and molasses are generated by 50 percent less than the regular conversion factor. In other words, the volume production of the raw sugar and molasses are half of the regular production. This happens mainly due to the late harvesting time and the resultant loss of sugarcane quality. This is the penalty that must be paid for this recourse decision. No further ethanol generation is possible from this poor quality sugarcane.

In fact, at the first stage, we make and implement the above-mentioned initial decisions before having any idea about what may happen in the future. Then, we wait and observe which scenario will unfold for each of the uncertain parameters. After obtaining information about the unfolding meta-scenario $s(k), k \in S^{1}$, the relevant decisions from the second list(second stage) can be implemented.

\subsubsection{Objective functions}

It is important to remember that, in this section, we attempted to formulate the general model of the problems, which are presented in section 6.2, based on our proposed methodology. A similar pattern can be used in modelling similar problems. Therefore, no specific numerical values have been considered at this point. Later, in Section 6.4, this general model will be utilised to model a case study based on the South African sugar-bioethanol supply chains. Then, relevant values will be substituted with the general variables in this model. Table 6.8 in Section 6.7 is allocated to the definition and notation of these general variables.

As discussed in previous chapters, different performances would be reached if various scenarios are revealed and different objective functions need to be evaluated in the conditions of each and every single scenario. In other words, in a scenario-based multi-criteria decision-making problem, performances should be assessed under conditions of all meta-criteria. 
As mentioned earlier, the performances in this study will be evaluated with three criteria/objectives classified as economic, environmental, and social benefits.

We also consider six uncertain parameters that are completely independent from each other. So, if different scenarios $\left(k_{t}, t=1, . ., 6\right)$ are determined for each parameter, there will be $k=$ $k_{1} \times k_{2} \times k_{3} \times k_{4} \times k_{5} \times k_{6}$ meta-scenarios that can construct $i \times k$ meta-criteria. Suppose that the scenario space is $S^{1}$ including all meta-scenarios showing by $s(k), k \in S^{1}$. Therefore, depending on model 3.2 in 3 , there will be $i \times k$ objective functions described by $Z_{i k}$ which represent preferences in terms of criterion $i$ under conditions pertaining to scenario $k \in S^{1}$. These objective functions for our biofuel SC problem have been formulated in the next sections.

\subsubsection{Economic objectives}

In terms of the economics objective, we are looking to maximise the profit which equates to maximising the difference between revenue and cost of the entire SC. These objectives are shown by $Z_{1 k}, k \in S^{1}$, and is formulated as follows:

(Total Profit) $(Z A R)$

$\operatorname{Max} Z_{1 k}=\operatorname{Rev}(\text { local }- \text { sale })_{k}+\operatorname{Rev}(\text { int }- \text { sale })_{k}+\operatorname{Rev}($ Tax - exemption $)-\operatorname{Cost}(\text { Agri })_{k}$ $-\operatorname{Cost}(\text { production })_{k}-\operatorname{Cost}(\text { EPlant })_{k}-\operatorname{Cost}(\text { transport })_{k}-\operatorname{Cost}(\text { import })_{k} ; \quad k \in S^{1}$

The total income includes the total sales of raw sugar, white(refined) sugar, molasses, ethanol and its by-product in the local and international markets as well as the tax exemption from selling ethanol locally. All products would be sold in the local market while only raw sugar, refined sugar, and ethanol would be exported after satisfying local demands. The international prices of refined sugar $\left(P S_{k}^{1}\right)$ and ethanol $\left(P E_{k}^{1}\right)$, as well as the conversion rate Dollar to $\operatorname{Rand}\left(R_{k}^{1}\right)^{4}$, are uncertain in scenario $k$. The local prices of products are calculated by multiplying the relevant coefficients in international price. Therefore, the local and international revenue in scenario $k$ can be formulated as follows:

$$
\operatorname{Rev}(\text { local }- \text { sale })_{k}=\xi \cdot P S_{k}^{1} \cdot R_{k}^{1}\left(X_{11 k}^{1}+\mu X_{12 k}^{1}+\iota X_{21 k}^{1}\right)+\rho P E_{k}^{1} \cdot R_{k}^{1}\left(X_{13 k}^{1}+\nu X_{17 k}^{1}\right) ; \quad k \in S^{1}
$$

$$
\operatorname{Rev}(\text { int }- \text { sale })_{k}=P S_{k}^{1} \cdot R_{k}^{1}\left(X_{14 k}^{1}+\mu X_{15 k}^{1}\right)+P E_{k}^{1} \cdot R_{k}^{1} X_{16 k}^{1} ; \quad k \in S^{1}
$$

where $\xi$ and $\rho$ represent the conversion factors for local price of refined sugar and ethanol, respectively. $\mu, \iota$, and $\nu$ are the conversion factor for prices of raw sugar, molasses and ethanol's by-product, respectively.

Tax exemption is allocated to the amount of ethanol that will be sold in the local market as a blend with fuel. $\tau$ shows the tax of ethanol that would be applied to the volume of ethanol selling in the local market $\left(X_{13 k}^{1}\right)$ in each meta-scenario $k \in S^{1}$. This revenue is calculated by equation 6.4.

\footnotetext{
${ }^{4}$ In this study, we are using the conversion rate of US Dollar to South African Rand that could be replaced by any other rates.
} 


$$
\operatorname{Rev}(\text { Tax - exemption })=\tau . \rho \cdot P E_{k}^{1} \cdot R_{k}^{1} X_{13 k}^{1} \quad k \in S^{1}
$$

The following equations compute the different type of costs that must be considered in this study. The agricultural costs include the cost of cultivating, harvesting and transporting to the relevant mill. These costs can be calculated, as described in equation 6.5 , by multiplying the overall cost by the total yield in every scenario.

$$
\operatorname{Cost}(\text { Agri })_{k}=\sum_{l} c_{1 l}^{0} Y_{k}^{0} X_{1 l}^{0} \quad k \in S^{1}
$$

Note that, alternatively, the agricultural costs may be replaced by the cost of buying the sugarcane stalks from farmers.

Equations in 6.6 describe the production costs in each scenario $k \in S^{1}$ that contain raw and white sugar production costs plus the expenses/costs of generating ethanol and its by-product. Ethanol and its by-product can be produced directly from sugarcane or can be generated from the produced raw and white sugar, or molasses in an ethanol plant. The potential costs of the recourse actions are also included.

$$
\begin{array}{ll}
\operatorname{Cost}(\text { production })_{k}=\sum_{j} c_{2}^{0} \alpha\left(X_{2 j}^{0}+X_{23 j k}^{1}\right)+\sum_{j} \sum_{r} c_{3}^{0} \beta X_{3 j r}^{0}+\sum_{r} \sum_{j} c_{4}^{0} \sigma\left(X_{7 j r}^{0}+X_{22 r k}^{1}\right) & \\
+\sum_{r} \sum_{j} c_{5}^{0} \theta\left(X_{6 j r}^{0}+X_{19 r k}^{1}\right)+\sum_{j} \sum_{p} c_{6}^{0} \gamma X_{4 j p}^{0}+\sum_{r} c_{20 k}^{1} \lambda X_{18 r k}^{1} & \\
\left.+\sum_{r} \sum_{j} c_{7}^{0}\left(\kappa_{1} X_{3 j r}^{0}\right)+\sum_{r} \sum_{j} \kappa_{2}\left[\left(X_{7 j r}^{0}+X_{22 r k}^{1}\right)+\left(X_{6 j r}^{0}+X_{19 r k}^{1}+X_{18 r k}^{1}\right)\right]\right) & k \in S^{1}
\end{array}
$$

The cost of transporting products/feedstocks between mills, refineries, ethanol plant(s) (already installed), and the depot (for both initial and recourse decisions) in each plausible metascenario can be calculated by 6.7. This cost includes transportation of sugarcane stalks, raw sugar, and molasses from a mill to an ethanol plant (with relevant technology for each), raw sugar from a mill to a refinery or the depot, molasses from a mill to the depot, white/refined sugar from a refinery to the depot, ethanol and its by-product from an ethanol plant (to be set up in future) to the terminal/depot. The cost of sending molasses, raw and white sugar back to an ethanol plant as a recourse decision is also computed. Note that two different types of transport for solid products (sugarcane stalks, raw and white sugar) and the liquids (ethanol and molasses) are considered.

$$
\begin{aligned}
& \text { Cost(transport })_{k}=\sum_{j} \sum_{r} c_{9 j r}^{0} X_{3 j r}^{0}+\sum_{j} \sum_{r} c_{10 j r}^{0} X_{6 j r}^{0}+\sum_{j} \sum_{p} c_{11 j p}^{0} X_{4 j p}^{0}+\sum_{j} c_{12 j}^{0}\left(X_{5 j}^{0}+\alpha X_{23 j k}^{1}\right) \\
& +\sum_{j} \sum_{r} c_{13 r}^{0}\left[\left(\beta+\kappa_{1}\right) X_{3 j r}^{0}+\left(\sigma+\kappa_{2}\right) \sum_{j}\left(X_{7 j r}^{0}+X_{22 r k}^{1}\right)+\left(\theta+\kappa_{2}\right)\left(X_{6 j r}^{0}+X_{19 r k}^{1}\right)+\left(\lambda+\kappa_{2}\right) X_{18 r k}^{1}\right] \\
& +\sum_{j} \sum_{p} c_{14 p}^{0} \gamma X_{4 j p}^{0}+\sum_{j} \sum_{r} c_{15 j r}^{0} X_{7 j r}^{0}+\sum_{r} c_{16 r}^{1}\left(X_{18 r k}^{1}+X_{19 r k}^{1}\right) \\
& +\sum_{j} c_{21 j}^{0} X_{20 j}^{0}+\sum_{r} c_{13 r}^{0} X_{22 r k}^{1}
\end{aligned}
$$$$
k \in S^{1}
$$ 
Finally, equations 6.8 and 6.9 represent, respectively, the expenses of constructing an ethanol plant with technology $q$ in each area, and the charge of imported ethanol, raw and white sugar in meta-scenario $k \in S^{1}$.

$$
\begin{aligned}
& \operatorname{Cost}(\text { EPlant })=\sum_{r} \sum_{q} c_{8 r q}^{0} \Gamma_{r q}^{0} \quad r \in L ; q=1,2,3 ; \\
& \operatorname{Cost}(\text { import })_{k}=c_{17}^{1} \xi P S_{k}^{1} R_{k}^{1} X_{9 k}^{1}+c_{18}^{1} P S_{k}^{1} R_{k}^{1} X_{8 k}^{1}+c_{19}^{1} P E_{k}^{1} R_{k}^{1} X_{10 k}^{1} \quad k \in S^{1}
\end{aligned}
$$

\subsubsection{Environmental objectives}

As the environmental objective, we are looking to minimise the total $\mathrm{CO}_{2}$ emissions from the entire SC. These emissions include total $\mathrm{CO}_{2}$ emissions of agricultural, productions, transportations, construction of ethanol plant(s), and importations in each scenario. We also seek to maximise total saving in $\mathrm{CO}_{2}$ emissions which would be reached by consuming bioethanol as a fuel (i.e. further reducing $\mathrm{CO}_{2}$ emissions if bioethanol is used instead of fossil fuels). These objectives are shown by $Z_{2 k}, k \in S^{1}$ and formulated as follows:

(Total $\mathrm{CO}_{2}$ emissions))

Min $Z_{2 k}=\mathrm{CO}_{2}$ Emissions $(\text { Agri })_{k}+\mathrm{CO}_{2}$ Emissions $(\text { production })_{k}+\mathrm{CO}_{2}$ Emissions $(\text { EPlant })_{k}$ $+\mathrm{CO}_{2}$ Emissions(transport $)_{k}+\mathrm{CO}_{2}$ Emissions(import $)_{k}-\mathrm{CO}_{2}$ saving(biofuel $)_{k} \quad k \in S^{1}$

Equations 6.11-6.15 describe the different type of emissions considered in our model for each meat-scenario.

$$
\mathrm{CO}_{2} \text { Emissions }(\text { Agri })_{k}=\sum_{l} d_{1 l}^{0} Y_{k}^{0} X_{1 l}^{0} \quad k \in S^{1}
$$

$$
\begin{array}{ll}
\left.C O_{2} \text { Emissions(production }\right)_{k}=\sum_{j} d_{2}^{0} \alpha\left(X_{2 j}^{0}+X_{23 j k}^{1}\right)+\sum_{j} \sum_{r} d_{3}^{0} \beta X_{3 j r}^{0}+\sum_{j} \sum_{r} d_{4}^{0} \sigma\left(X_{7 j r}^{0}+X_{22 r k}^{1}\right) & \\
+\sum_{j} \sum_{r} d_{5}^{0} \theta\left(X_{6 j r}^{0}+X_{19 r k}^{1}\right)+\sum_{j} \sum_{p} d_{6}^{0} \gamma X_{4 j p}^{0}+\sum_{r} d_{20}^{1} \lambda X_{18 r k}^{1} & \\
+\sum_{j} \sum_{r} d_{7}^{0}\left(\kappa_{1} X_{3 j r}^{0}+\kappa_{2}\left[\left(X_{7 j r}^{0}+X_{22 r k}^{1}\right)+\left(X_{6 j r}^{0}+X_{19 r k}^{1}+X_{18 r k}^{1}\right)\right)\right] & k \in S^{1}
\end{array}
$$

$$
\left.C \mathrm{O}_{2} \text { Emissions(EPlant }\right)=\sum_{r} \sum_{q} d_{8 r q}^{0} \Gamma_{r q}^{0} \quad r \in L ; q=1,2,3
$$




$$
\begin{aligned}
& \left.C_{2} \text { Emissions(transport }\right)_{k}=\sum_{j} \sum_{r} d_{9 j r}^{0} X_{3 j r}^{0}+\sum_{j} \sum_{r} d_{10 j r}^{0} X_{6 j r}^{0}+\sum_{j} \sum_{p} d_{11 j p}^{0} X_{4 j p}^{0}+\sum_{j} d_{12 j}^{0}\left(X_{5 j}^{0}+\alpha X_{23 j k}^{1}\right) \\
& +\sum_{j} \sum_{r} d_{13 r}^{0}\left[\left(\beta+\kappa_{1}\right) X_{3 j r}^{0}+\left(\sigma+\kappa_{2}\right)\left(X_{7 j r}^{0}+X_{22 r k}^{1}\right)+\left(\theta+\kappa_{2}\right)\left(X_{6 j r}^{0}+X_{19 r k}^{1}\right)+\left(\lambda+\kappa_{2}\right) X_{18 r k}^{1}\right] \\
& +\sum_{j} \sum_{p} d_{14 p}^{0} \gamma X_{4 j p}^{0}+\sum_{j} \sum_{r} d_{15 j r}^{0} X_{7 j r}^{0}+\sum_{r} d_{16 r}^{1}\left(X_{18 r k}^{1}+X_{19 r k}^{1}\right) \\
& +\sum_{j} d_{21 j}^{0} X_{20 j}^{0}+\sum_{r} d_{13 r}^{0} X_{22 r k}^{1}
\end{aligned}
$$

$$
C \mathrm{O}_{2} \text { Emissions }(\text { import })_{k}=d_{17}^{1} X_{9 k}^{1}+d_{18}^{1} X_{8 k}^{1}+d_{19}^{1} X_{10 k}^{1} \quad k \in S^{1}
$$

Moreover, 6.16 displays the total saving in $\mathrm{CO}_{2}$ emissions that would be reached by blending fuel with ethanol in meta-scenario $k \in S^{1}$ and this is calculated from the product of factor $e^{0}$ and the amount of ethanol sold locally for this purpose.

$$
\left.C O_{2} \text { saving (biofuel }\right)_{k}=e^{0} X_{13 k}^{1} \quad k \in S^{1}
$$

\subsubsection{Social benefit objectives}

Maximising employment is considered as the social benefit objective which includes permanent and seasonal employees at the agricultural level as well as construction and operational workers of ethanol plant(s) and production lines in each meta-scenario. These objectives are shown by $Z_{3 k}, k \in S^{1}$ and are formulated as follows:

(Total number of employees))

Max $Z_{3 k}=\operatorname{Jobs}(\text { Agri })_{k}+$ Jobs $(\text { production })_{k}+$ Jobs $(\text { EPlant })_{k}+$ Jobs $(\text { transport })_{k} \quad k \in S^{1}$

$$
\operatorname{Jobs}(\text { Agri })_{k}=\sum_{l}\left(h_{1}^{0}+h_{2}^{0}\right) Y_{k}^{0} X_{1 l}^{0} \quad k \in S^{1}
$$

$$
\begin{aligned}
& \text { Jobs(production })_{k}=\sum_{j} h_{3}^{0} \alpha\left(X_{2 j}^{0}+X_{23 j k}^{1}\right)+\sum_{j} \sum_{p} h_{4}^{0} \gamma X_{4 j p}^{0} \\
& +\sum_{j} \sum_{r} h_{51}^{0} \beta X_{3 j r}^{0}+\sum_{j} \sum_{r} h_{52}^{0} \sigma\left(X_{7 j r}^{0}+X_{22 r k}^{1}\right)+\sum_{j} \sum_{r} h_{52}^{0} \theta\left(X_{6 j r}^{0}+X_{19 r k}^{1}\right)+\sum_{r} h_{52}^{1} \lambda X_{18 r k}^{1} \quad k \in S^{1} \\
& +\sum_{j} \sum_{r} h_{61}^{0} \kappa_{1} X_{3 j r}^{0}+h_{62}^{0}\left[\sum_{r} \sum_{j} \kappa_{2}\left(X_{7 j r}^{0}+X_{22 r k}^{1}\right)+\sum_{r} \sum_{j} \kappa_{2}\left(X_{6 j r}^{0}+X_{19 r k}^{1}\right)+\sum_{r} \kappa_{2} X 18 r k^{1}\right] ;
\end{aligned}
$$




$$
\operatorname{Jobs}(\text { EPlant })=\sum_{r} \sum_{q}\left(h_{7 q}^{0}+h_{8 q}^{0}\right) \Gamma_{r q}^{0} \quad r \in L ; q=1,2,3
$$

$$
\begin{aligned}
& \text { Jobs(transport })_{k}=\sum_{j} \sum_{r} h_{9 j r}^{0} X_{3 j r}^{0}+\sum_{j} \sum_{r} h_{10 j r}^{0} X_{6 j r}^{0}+\sum_{j} \sum_{p} h_{11 j p}^{0} X_{4 j p}^{0}+\sum_{j} h_{12 j}^{0}\left(X_{5 j}^{0}+\alpha X_{23 j k}^{1}\right) \\
& +\sum_{j} \sum_{r} h_{13 r}^{0}\left[\left(\beta+\kappa_{1}\right) X_{3 j r}^{0}+\left(\sigma+\kappa_{2}\right)\left(X_{7 j r}^{0}+X_{22 r k}^{1}\right)+\left(\theta+\kappa_{2}\right)\left(X_{6 j r}^{0}+X_{19 r k}^{1}\right)+\left(\lambda+\kappa_{2}\right) X_{18 r k}^{1}\right] \\
& +\sum_{j} \sum_{p} h_{14 p}^{0} \gamma X_{4 j p}^{0}+\sum_{j} \sum_{r} h_{15 j r}^{0} X_{7 j r}^{0}+\sum_{r} h_{16 r}^{1}\left(X_{18 r k}^{1}+X_{19 r k}^{1}\right) \\
& +\sum_{j} h_{21 j}^{0} X_{20 j}^{0}+\sum_{r} h_{13 r}^{0} X_{22 r k}^{1}
\end{aligned}
$$$$
k \in S^{1}
$$

\subsubsection{Constraints}

All constraints considered in this study, including land availability, sugar and ethanol demands, sale/production constraints, and capacity limitations, can be divided into two broader groups of scenario-free and scenario-dependant constraints, as well as binary and non-negativity constraints. These have been described as follows:

\subsubsection{Scenario-free constraints:}

Land availability

$$
X_{1 l}^{0} \leq b_{1 l}^{0}, \quad l \in L
$$

Equations 6.22 assure that the hectares of land allocated to sugarcane cultivation are less than or equal to the available arable land in each area.

Sale/production balance

$$
\begin{aligned}
& X_{5 j}^{0}+\sum_{p} X_{4 j p}^{0}+\sum_{r} X_{6 j r}^{0}=\alpha X_{2 j}^{0}, \quad j \in L \\
& X_{20 j}^{0}+\sum_{r} X_{7 j r}^{0}=\sum_{j} \zeta X_{2 j}^{0}, \quad j \in L
\end{aligned}
$$

In every single mill $j \in L$, the volume of raw sugar that is sent to the depot plus that which is transported to all refineries and ethanol plants must be equal to total raw sugar output(6.23). Equations 6.24 ensures that the entire amount of molasses sent out from each mill $j$ to all refineries and the depot is the same as its production.

\section{Capacity}

Minimum and maximum levels of the white/refined sugar production in refinery $r \in P$, and ethanol output from sugarcane in ethanol plant with technology $q=1$, have been guaranteed in equations 6.26 and 6.25 , respectively. 


$$
\begin{aligned}
& \varsigma_{1}^{\text {min }}\left(\Gamma_{r 1}+\Gamma_{r 3}\right) \leq \sum_{j} \beta X_{3 j r}^{0} \leq \varsigma_{1}^{\max }\left(\Gamma_{r 1}+\Gamma_{r 3}\right) \quad r \in L ; \\
& \varrho_{p}^{\text {min }} \leq \sum_{j} \gamma X_{4 j p}^{0} \leq \varrho_{p}^{\text {max }} \quad p \in P
\end{aligned}
$$

The group of equations in 6.27 certify the fact that only one ethanol plant can be set up in each area.

$$
\sum_{q} \Gamma_{r q}^{0} \leq 1 ; \quad r \in L ; q=1,2,3 ;
$$

\subsubsection{Scenario-dependant constraints:}

Sugar and ethanol demands are satisfied by equations $6.28-6.30$ as follows:

White sugar demand

$$
X_{11 k}^{1}=D S_{k}^{1}, \quad k \in S^{1} ;
$$

Raw sugar demand

$$
X_{12 k}^{1}=\vartheta D S_{k}^{1}, \quad k \in S^{1} ;
$$

\section{Ethanol demand}

$$
X_{13 k}^{1}=D E_{k}^{1}, \quad k \in S^{1} ;
$$

Furthermore, the mass balance of white and raw sugar, molasses, ethanol, and by-product in the depot are checked by equations 6.31-6.35, respectively.

Sale/production balance

$$
\begin{aligned}
& X_{11 k}^{1}+X_{14 k}^{1}+\sum_{r} X_{18 r k}^{1}=\sum_{j} \sum_{p} \gamma X_{4 j p}^{0}+X_{8 k}^{1}, \quad k \in S^{1} ; \\
& X_{12 k}^{1}+X_{15 k}^{1}+\sum_{r} X_{19 r k}^{1}=\sum_{j} X_{5 j}^{0}+X_{9 k}^{1}+\sum_{j} \alpha X_{23 j k}^{1}, \quad k \in S^{1} ; \\
& X_{21 k}^{1}+\sum_{r} X_{22 r k}^{1}=\sum_{j}\left(X_{20 j}^{0}+\zeta X_{23 j k}^{1}\right), \quad k \in S^{1} ;
\end{aligned}
$$

$X_{13 k}^{1}+X_{16 k}^{1}=\sum_{j} \sum_{r} \beta X_{3 j r}^{0}+\sum_{j} \sum_{r} \sigma\left(X_{7 j r}^{0}+X_{22 r k}^{1}\right)+\sum_{j} \sum_{r} \theta\left(X_{6 j r}^{0}+X_{19 r k}^{1}\right)+\sum_{r} \lambda X_{18 r k}^{1}+X_{10 k}^{1}, \quad k \in S^{1} ;$ 


$$
X_{17 k}^{1}=\sum_{j} \sum_{r} \kappa_{1} X_{3 j r}^{0}+\sum_{j} \sum_{r} \kappa_{2}\left(X_{7 j r}^{0}+X_{22 r k}^{1}\right)+\sum_{j} \sum_{r} \kappa_{2}\left(X_{6 j r}^{0}+X_{19 r k}^{1}+X_{18 r k}^{1}\right), \quad k \in S^{1} ;
$$

\section{Capacity}

Equations in 6.36 ensure that all sugarcane yields in all meta-scenarios are utilised, by initial and recourse decisions.

$$
X_{2 j}^{0}+\sum_{r} X_{3 j r}^{0}+X_{23 j k}^{1}=Y_{k}^{0} X_{1 l}^{0}, \quad l=j \in L ; k \in S^{1}
$$

Ethanol and production limitations via technology number two $(q=2)$, and mill restrictions of generating raw sugar are guaranteed by equations in 6.37 and 6.38 , respectively.

$$
\varsigma_{2}^{\min }\left(\Gamma_{r 2}+\Gamma_{r 3}\right) \leq \sum_{j} \sigma\left(X_{7 j r}^{0}+X_{22 r k}^{1}\right)+\sum_{j} \theta\left(X_{6 j r}^{0}+X_{19 r k}^{1}\right)+\lambda X_{18 r k}^{1} \leq \varsigma_{2}^{\max }\left(\Gamma_{r 2}+\Gamma_{r 3}\right) \quad r \in L ; k \in S^{1} ;
$$

$$
\varpi_{j}^{\min } \leq \alpha\left(X_{2 j}^{0}+X_{23 j k}^{1}\right) \leq \varpi_{j}^{\max } \quad j \in L ; k \in S^{1}
$$

\subsubsection{Binary and non-negativity constraints:}

Binary

$$
\gamma_{r q} \in 0,1 ; \quad r \in L ; q=1,2,3
$$

$$
\begin{aligned}
& \text { Non-negativity } \\
& \qquad \begin{aligned}
x_{1 l}^{0}, x_{2 j}^{0}, x_{3 j r}^{0}, x_{4 j p}^{0}, x_{5 j}^{0}, x_{6 j r}^{0}, x_{7 p r}^{0}, x_{20 p}^{0} \geq 0, & \forall l, j, r, p ; \\
\gamma_{r q}, x_{n k}^{1}, x_{18 r k}^{1}, x_{19 r k}^{1}, x_{22 r k}^{1}, X_{23 j k}^{1} \geq 0 . & n \in\{8,9, \ldots, 17,21\} ; \\
& r \in L ; k \in S^{1} ; \\
& q=1,2,3 .
\end{aligned}
\end{aligned}
$$

\subsubsection{Solving by Generalised Goal Programming (GGP)}

If the goals of each objective in each scenario are determined by $g_{i k}^{1}$ and all weights are set by 1 , the goal programming model of the above model can be formulated as follows: 


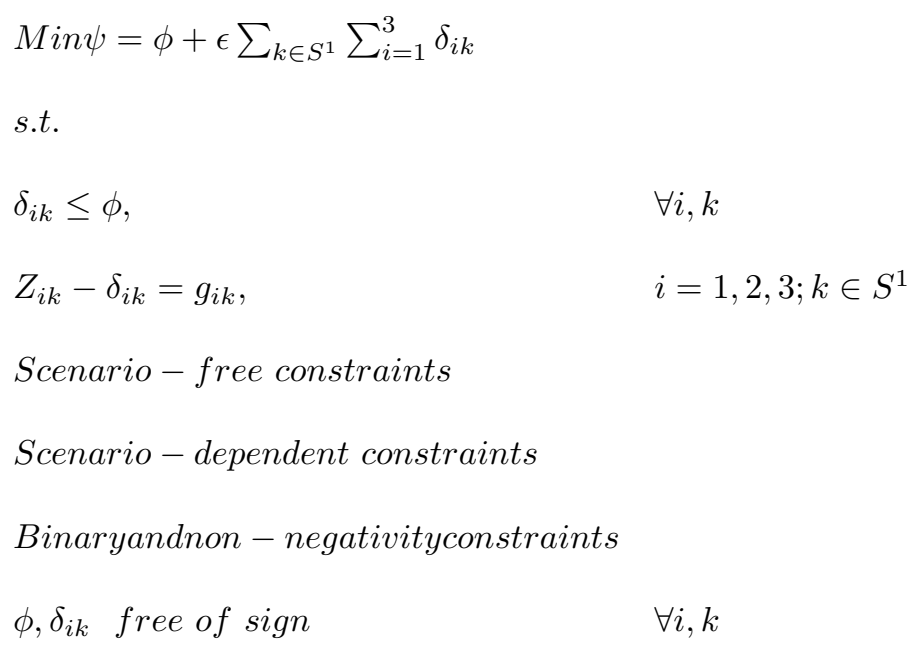

\subsection{Case study (SA sugar-ethanol supply chain)}

In this section, the proposed scenario-based mixed-integer two-stage multi-objective optimisation model for strategic planning of sugar-bioethanol supply chains (SC) under deep uncertainty has been examined in the South African sugarcane industry to represent the capability of the proposed structure in real problems. Unfortunately, some real data were highly classified by the South African Sugar Association (SASA), so we decided to make this study slightly hypothetical and approximated some data. Nevertheless, the problem remains a real problem that can be addressed by the proposed framework.

The sugar industry in South Africa has been producing sugar for more than 100 years. There are about 50000 cane growers in SA and statistics show that, out of 29130 registered sugarcane growers, 22500 of these operate in KwaZulu-Natal. Unofficial statistics show that South Africa produces 20 million tonnes of sugarcane annually [SASAwebsite, 2017]. Currently, about 371662 hectares of arable land is dedicated to grow sugarcane in SA, while the suitable land for additional farms is estimated to be more than 4 million hectares. However, the ability to successfully grow sugarcane on all of this land is limited due to water scarcity.

Six companies with 14 sugar mills manufacture 2.2 million tonnes of sugar seasonally. Southern African Customs Union (SACU) has about $60 \%$ of the market share, while the rest has been exported to markets outside the country. The sugar industry in SA contributes to R 8 billion to the country's annual income and about 79000 direct and 350000 indirect employment makes a significant contribution to the national economy [SASAwebsite, 2017].

The South African sugar industry is highly dependent on the world sugar price mainly because sugar is its only significant commercial product, although it has some other by-products [Letete, 2009]. Germinshuis [2006] pointed out in its first national biofuels report that ethanol production from sugarcane "has the potential to enhance the sustainability of the sugar industry in South Africa and to stimulate growth both industrially and agriculturally in the areas where the industry operates". SA's Department of Minerals and Energy approved 2\% level of biofuels in national liquid fuel supply in 2007 [Website, 2017]. However, it still not implemented mainly due to the fact that sugar companies, which are an important stakeholder, doubt the profitability of the project. In terms of government strategies, sugarcane and sugar beet are the two targeted crops to drive the bioethanol industry in South Africa, and a 100\% fuel tax exemption is proposed for bioethanol as it can also be used in markets other than petrol (For example, ethanol gels [Website, 2017]). Possible issues that ought to be considered in the government's plan are food security, animal feed, land issue, and water resources. 
In this section, the capabilities of the proposed two-stage framework are examined through a case study based on the South African Sugar Industry. We are considering the integrated South African infrastructures for joint productions of sugar and ethanol in a way that all economics (mainly producer company goal), environmental (the world unions agreements), and social benefits (government target) are optimised while key uncertainties have also been considered. Overall, the potential profitability of the project, as the most important result of this study, could encourage the SA's sugar companies to operate this project in South Africa.

\subsubsection{Modelling assumptions}

The different assumptions applied to proposed Mixed Integer two-stage model are reviewed below. The uncertain parameters are explained in the next section, while the other parameters are either described in chapter or thesis Appendix. Note that, because we did not have access to all real data, some of them are real (most of the real data extract from SASA website in [SASAwebsite, 2017]), some are estimated, and some are guessed.

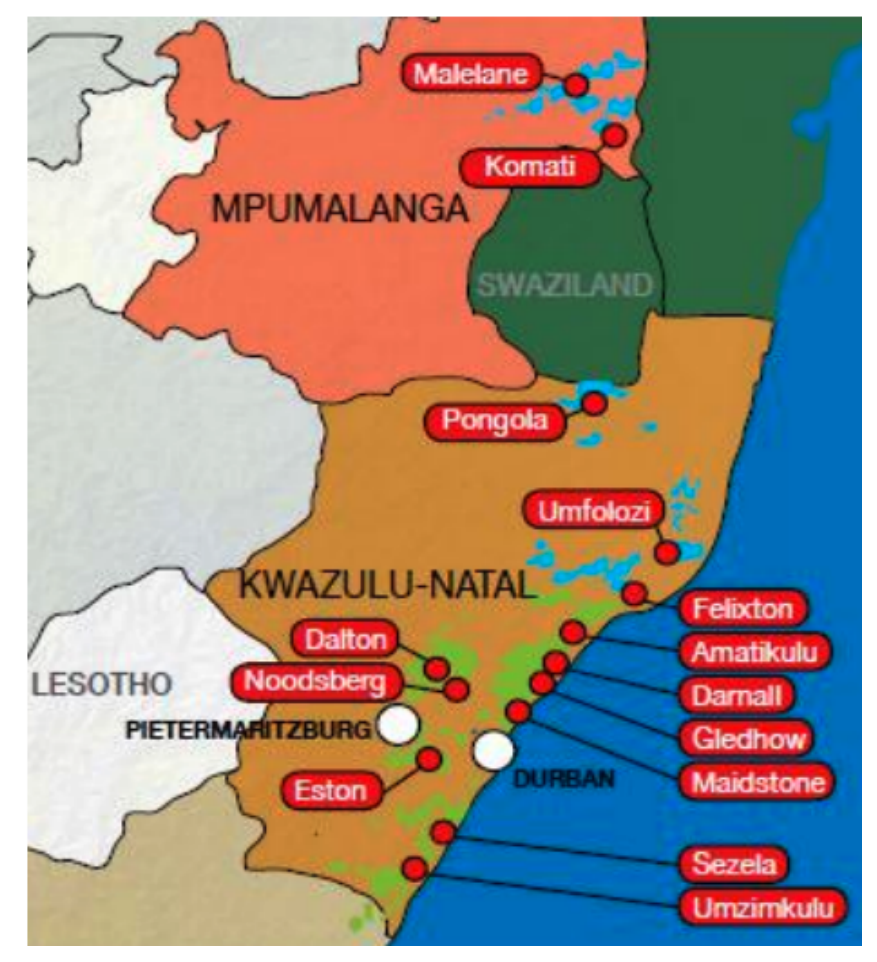

Figure 6.4: SA sugar Mills and the central refinery locations

1. As stated earlier, 14 sugar mills are operating in SA and these are situated in an agricultural belt extending from the Eastern Cape to Mpumalanga. There is also a central sugar refinery located in Durban, KwaZulu-Natal, which produces white/refined sugar. There are also three other white end refineries placing in Umzimkulu, Sezela (both in KwaZuluNatal) and Komati (in Mpumalanga) [SASAwebsite, 2017]. Figure 6.4 shows the location of these 15 points and their surroundings. Each sugar mill is located in a region that is specifically utilised for cultivating sugarcane in that area. Therefore, we consider $L=15$ different regions that, in the exception of Durban, all available arable lands for growing sugarcane are located in these regions. Table 6.9 (in chapter Appendix) describes the estimated arable lands (in the areas mentioned above) that can be considered to growing 
sugarcane ${ }^{5}$.

2. Assume that all sugarcane growing in region $j$ is directly transported to the sugar mill that is located in the same area. Then, after the preparation step in the mill, the product can either be consumed as feedstock for the production of raw sugar or be sent to the ethanol plant $r \in L$ in surrounding area (less than $100 \mathrm{~km}$ drive), if its setup is complete and has a relevant technology, to produce ethanol. Table 6.10, in chapter Appendix, portrays the distances between these 15 regions. These distances were calculated as the road distance between two points in Google Maps ([googlemap, 2017]).

3. All 14 sugar mills are raw sugar ended (i.e. only produce raw sugar from sugarcane). The production capacities of these mills, as well as the capacities of the four operating refineries and potential ethanol plants in the different regions, are shown in Table 6.11, in chapter Appendix. These capacities are guessed by comparing them with similar options worldwide.

4. No limitations have been considered for the depot/terminal capacity.

5. All demands must be completely met in every scenario and only the excess volume of productions, if any, can be exported. Nevertheless, the amount of exported ethanol does not qualify for tax exemption.

6. There are some (artificial) taxes on sugar and ethanol importations which have been approved to support the local sugar companies ( $150 \%$ on ethanol and $400 \%$ on sugar importations, respectively).

7. No further direct job creation has been supposed for exportations/importations.

8. No specific demand for molasses and ethanol by-product has been considered and we assume that all product (i.e. molasses and by-product) would be sold on the market.

\subsubsection{Uncertainties and scenarios}

As discussed earlier in section 6.2.1.3, the prices of ethanol and white sugar have been set as independent uncertain parameters and the rest can be determined as a correlated function of them. These functions have been described as follows:

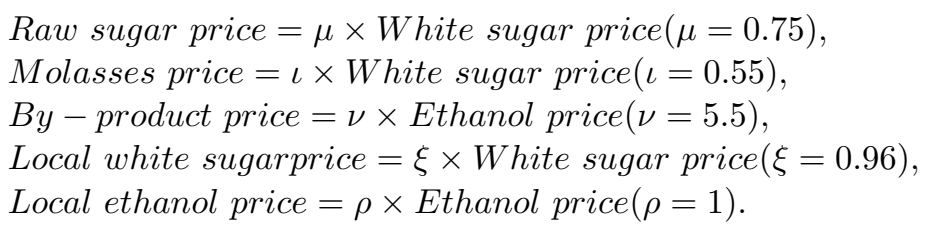

Except for sugar demands and prices for which two plausible scenarios are considered, three plausible scenarios (Low, Normal, and High) are determined for the other uncertain parameters. Tables 6.2 and 6.3 show the demand and selling prices for all products in every plausible scenario, respectively. Sugarcane yields in three plausible scenarios (poor, normal, and good) are respectively equal to 50,60, 70 tonnes per hectare. Also, three levels of 10, 14, and 17 are set for the exchange rate of US dollar to ZAR. Table 6.4 summarises these amounts.

Accordingly, $k_{1}=k_{2}=k_{4}=k_{6}=3$ and $k_{3}=k_{5}=2$. Therefore, the total number of meta-scenarios(k) is equals to $k=3 \times 3 \times 2 \times 3 \times 2 \times 3=324$.

\footnotetext{
${ }^{5}$ As noted earlier the available arable lands are more than what is showed in Table 6.9 in chapter Appendix. However, it is impossible to expand sugarcane growing to more than $50 \%$ of the currently underutilised lands
} 


\begin{tabular}{lccc}
\hline \multirow{2}{*}{ Product } & \multicolumn{3}{c}{ Demand } \\
\cline { 2 - 4 } & Low & Normal & High \\
\hline White sugar & 150 & - & 250 \\
Raw sugar & 225 & - & 375 \\
Ethanol & 350 & 400 & 450 \\
\hline
\end{tabular}

Table 6.2: Demand (estimated)

\begin{tabular}{lccc}
\hline \multirow{2}{*}{ Product } & \multicolumn{3}{c}{ Price } \\
\cline { 2 - 4 } & Low & Normal & High \\
\hline White sugar $(\$ / \mathrm{t})$ & 300 & - & 600 \\
Raw sugar $\$ / \mathrm{t})$ & 225 & - & 375 \\
Molasses $(\$ /$ gal $)$ & 165 & - & 330 \\
Ethanol $(\$ /$ gal $)$ & 1.3 & 1.7 & 3.5 \\
By-product $(\$ /$ gal $)$ & 7.15 & 9.35 & 19.25 \\
\hline
\end{tabular}

Table 6.3: Prices (estimated from last 30 years prices)

\begin{tabular}{|ccc|}
\hline \multicolumn{3}{|c|}{ Yield(t/he)(k1) } \\
\hline Poor & Normal & Good \\
\hline 50 & 60 & 70 \\
\hline
\end{tabular}

\begin{tabular}{|ccc|}
\hline \multicolumn{3}{|c|}{ Exchange rate(US\$-ZAR)(k6) } \\
\hline Low & Normal & High \\
\hline 10 & 14 & 17 \\
\hline
\end{tabular}

Table 6.4: Sugarcane yields and exchange rate in different scenarios (estimated from last 30 years statistics) 


\subsubsection{Solution procedure and goals}

To be able to solve our Multi-Objective Mixed Integer problem which includes $972(3 \times 324)$ metacriteria/objectives, the Generalised Goal Programming $(G G P)$ is applied(model 6.41). Therefore, the DM needs to set 972 goals (one for each meta-criterion/objective) which would be significantly confusing, especially with this size of the model. Generally, to specify the goal levels, the following options might be used by the $\operatorname{DM}(\mathrm{s})$ :

- The DM determines the goals from his/her experience, expectations and preferences.

- Solve the single objective models (one for each meta-scenario/objective) and utilise the optimal solutions as the goal of that meta-scenario.

- Set one goal for each criterion (not each meta-criterion) and apply it to all scenarios.

- A combination of the above options.

The first option above might best describe the real preferences of the DM. However, in the problems with too many plausible meta-scenarios, identifying all the goal levels for every meta-scenario will be an exhausting and non-effective venture. The second option could be a suggestible choice, although this may not reflect all the $\mathrm{DM}(\mathrm{s})$ preferences, especially in some meta-scenarios where the DM preferred some specific goals (not the optimum). In this case, the last option could be helpful. The third option could be utilised in the case where the DM only looks for particular goals for each criterion in all meta-scenarios. This option may largely simplify the process of identifying the goal levels, although it may limit or completely ignore the chance of applying all preferences in the model. Nevertheless, as we will discuss later in Section 6.5.4, further preferences can be appended later by a sensitive analysis of the goals level.

With this option (the third one), the goals of this problem can be divided into three groups (economics, environmental, and social benefit) that are shown in Table 6.5. More discussion on various goals and their effects on the final results will be presented later through a sensitivity analysis in Section 6.5.4.

These goals, together with the other parameters(summarised in Table 1 in thesis Appendix A), have been utilised in model 6.41. The 342 meta-scenarios used in this MILP model possess 24396 continues variables, 45 binary variables, and 27945 constrains (19 817 inequity +8128 equity).

The model 6.41 is coded in Matlab2015b and solved by MILP solver intlinprog(Optimality tolerance gap ) on a MacBook Pro with 2,5 GHz Intel Core i5 and $4 \mathrm{~GB}$ of RAM. The final results have been described in the next section.

\begin{tabular}{|l|l|}
\hline Objectives & Goals \\
\hline Economic & $g_{1 k}=0\left(\right.$ break-even point $\left.^{a}\right)$ \\
\hline Environmental & $g_{1 k}=750($ million kg) \\
\hline Jobs & $g_{1 k}=400($ million man $/ \mathrm{hrs})$ \\
\hline \hline
\end{tabular}

Table 6.5: Sugarcane yields and exchange rate in different scenarios

${ }^{a}$ Break-Even Point (BEF), in economics and finance, is the point at which revenue is equal to the cost and there is neither no profit nor net loss [Boldrin and Levine, 2008]. Here, the DM would be satisfied if the capital invested has returned in one year after operating the installed ethanol plant(s).

\subsection{Case study results and discussion}

This section is allocated to representing the results that were obtained by applying the proposed two-stage structure in the South African sugar-ethanol SC. The decisions have been divided into 
two major groups related to each stage of the two-stage model.

\subsubsection{First-stage/initial decisions}

The first-stage (initial) decisions, made before scenario realisations, include design and planning decisions. Design decisions in our problem are dedicated to finding the optimal number and the best place(s) for setting up the ethanol plant(s). There is no limitation on the number, or places have been assumed in this study. Figure 6.5 shows the locations of the four ethanol plants that could be constructed in the future. Two factories will be operated with the first technology, and will use sugarcane as feedstock $(q=1)$. These would be installed in Noodsberg and Darnall (both in KwaZulu-Natal). Another two factories will be operated with the second technology $(q=2)$, and would be setup in Komati (in Mpumalanga) and Durban (in KwaZulu-Natal).

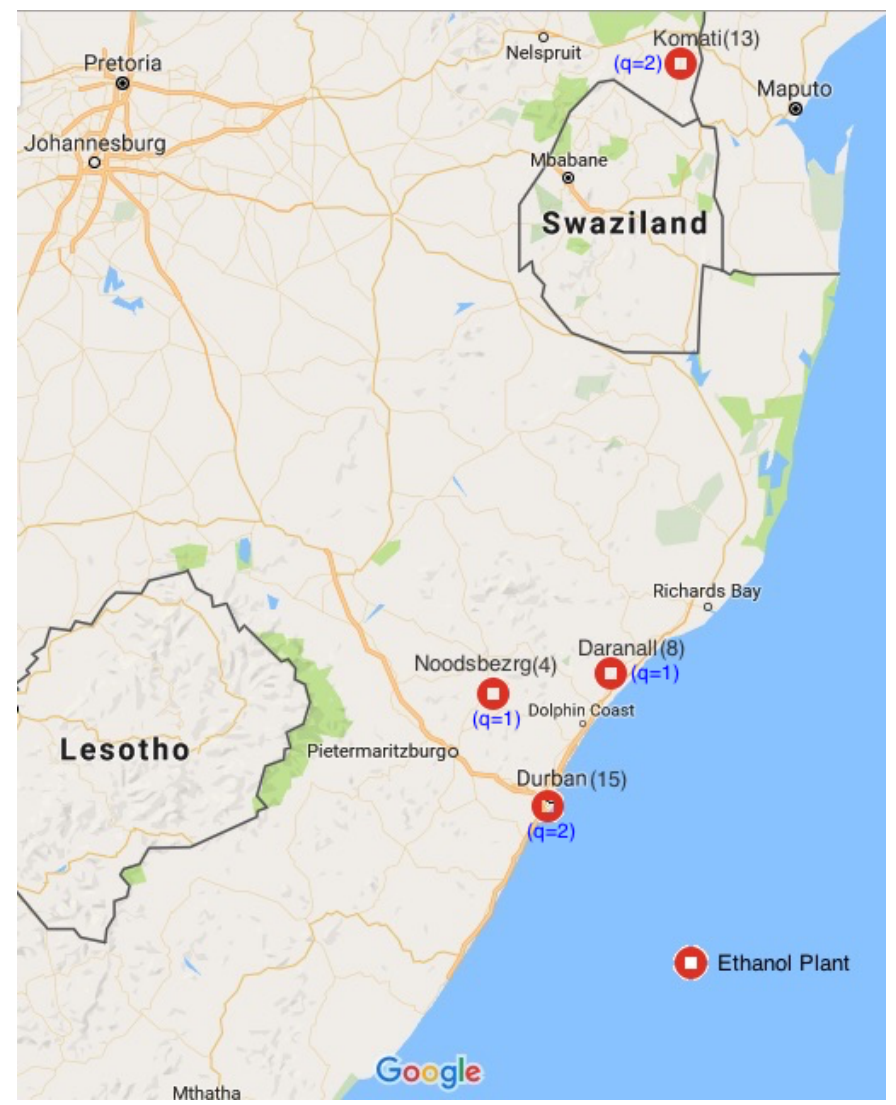

Figure 6.5: Ethanol plants locations

A summary of the land-use planning decisions, as one of the planning decisions in this study, has been described in Table 6.6. Results showed that by applying the solutions which were obtained by solving the proposed two-stage model for this problem (i.e. model 6.41), 377803 hectares of arable lands would be cultivated by sugarcane. Therefore, depending on the weather (and the particular scenario that will eventually unfold) we could harvest 18890143,22668172 , and 26446200 tonnes of sugarcane in various scenarios.

Another planning decision is allocated to the distribution of raw materials. In total, 13 million tonnes of sugarcane yields are assigned to producing raw sugar in all fourteen mills which could generate 1.57 million tonnes of raw sugar together with 87.22 million gallons of molasses (See Table 2 in 7.2). While the total amount of sugarcane transported to ethanol plants is only 5.89 


\begin{tabular}{lc}
\hline Region & Land used in each region \\
\hline Umzimkulu & 18772 \\
Sezela & 55240 \\
Eston & 43749 \\
Noodsberg & 18772 \\
Union-Coop & 13333 \\
Maidstone & 58749 \\
Gledhow & 43749 \\
Darnall & 18772 \\
Amatikulu & 16666 \\
Felixton & 25000 \\
Umfolozi & 16666 \\
Pongola & 15 \\
Komati & 16666 \\
Malelane & 16666 \\
Durban & 0 \\
\hline total & 377802 \\
\hline
\end{tabular}

Table 6.6: Land use

million tonnes, this produces 110.329 million gallons of ethanol in two brand-new cane-to-ethanol plants during the first operating year).

At this stage, 930550 tonnes of produced raw sugar, as well as 63.593 million gallons of molasses, could be sent to the depot. Ethanol production from raw sugar and molasses, at this time, equates to 29.571 and 9.763 million gallons, respectively (see Table 2 in 7.2 ).

Each of four refineries generates their minimum capacity of 100000 tonnes of white/refined sugar (400 000 tonnes in total) which is transported to the depot.

\subsubsection{Second-stage/recourse decisions}

There is no need to import white sugar in any scenario, and there is also no need to import raw sugar except in scenario 152 and $169^{6}$ in which, respectively, 17711 tonnes and 14685 tonnes of raw sugar would need to be imported.

Ethanol importation in various scenarios is recorded between 0 to 300.340 million gallons. More details can be found in Figure 1, in thesis Appendix B, as well as in Table 3 in thesis Appendix C.

All demands have been satisfied completely in each and every scenario that is equal to the local sale. The amounts of by-product and molasses that are sold are portrayed in Figures 2 and 3. Figures 7 and 8 , in thesis Appendix B, are explained the different volume of exportations of white and raw sugar in various scenarios, respectively. There would be no more ethanol left to export under any circumstances.

The volume of molasses, white and raw sugar that is transported back to ethanol plants (with q=2) to generate more ethanol can be found in Table 4 in thesis Appendix $\mathrm{C}$ or Figures 4-5 in thesis Appendix B. More raw sugar and molasses could be produced as a recourse option if there is an extra amount of sugarcane in some scenarios that have been shown in Table 4 in thesis Appendix C or Figures 9 and 10 in thesis Appendix B.

\footnotetext{
${ }^{6}$ Scenario $152: k_{1}=2, k_{2}=2, k_{3}=1, k_{4}=2, k_{5}=1, k_{6}=2$. Scenario $169: k_{1}=2, k_{2}=2, k_{3}=2, k_{4}=2, k_{5}=1, k_{6}=1$.
} 


\subsubsection{Objective functions and achievements}

Figure 6.6 represents a combined picture of the three objective values in different scenarios from two various angles. As seen in these pictures, these objective values lay on three groups with major differences of job creation. These differences are related to various plausible levels of yield. To get more details, let us study these values separately in each criterion. The trade-offs between these three criteria will be investigated later in the next section.

As described in Figure 6.7, economic objective functions are partly satisfied, and this satis-
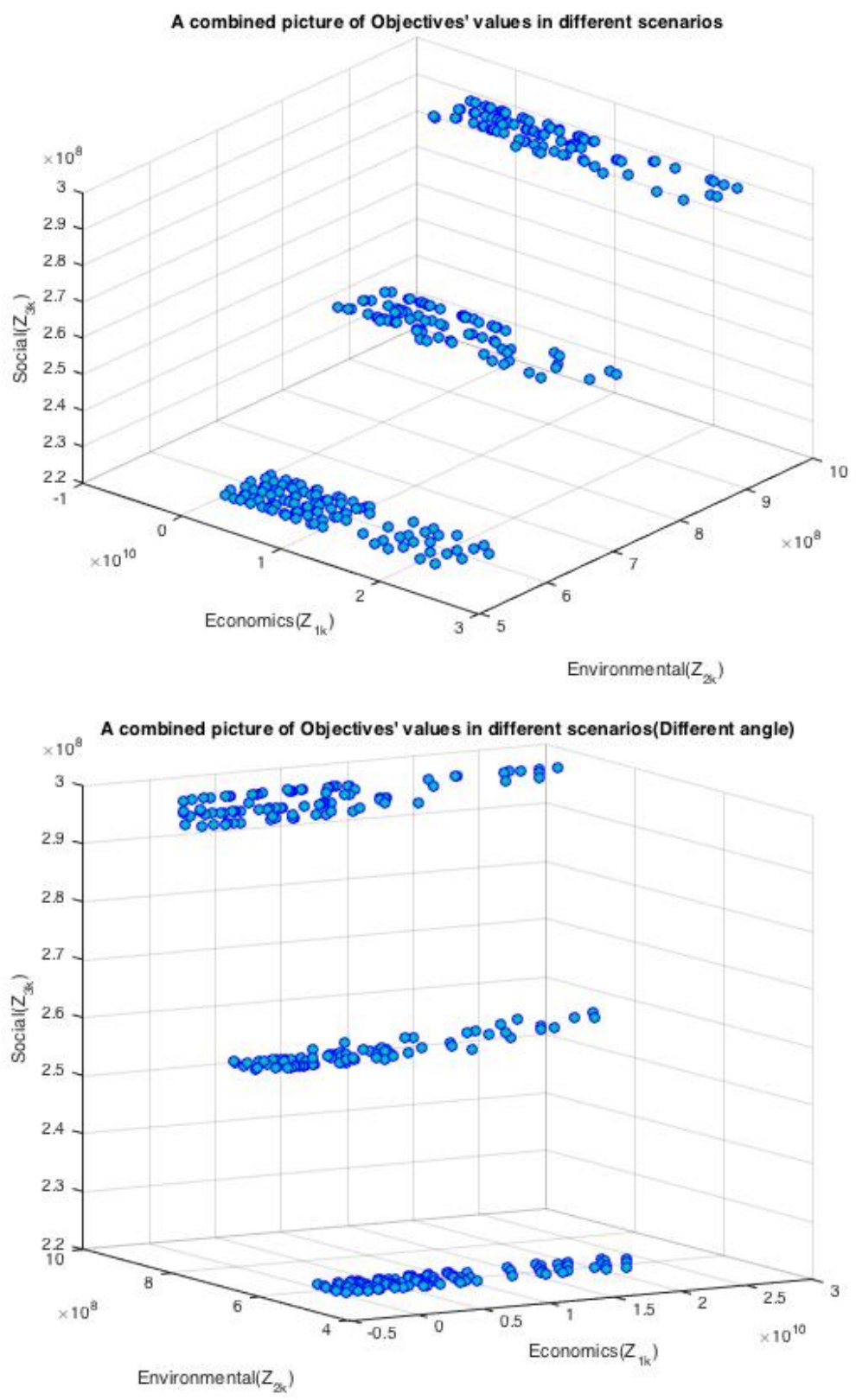

Figure 6.6: A combined picture of objectives in different scenarios(from two various angles) 


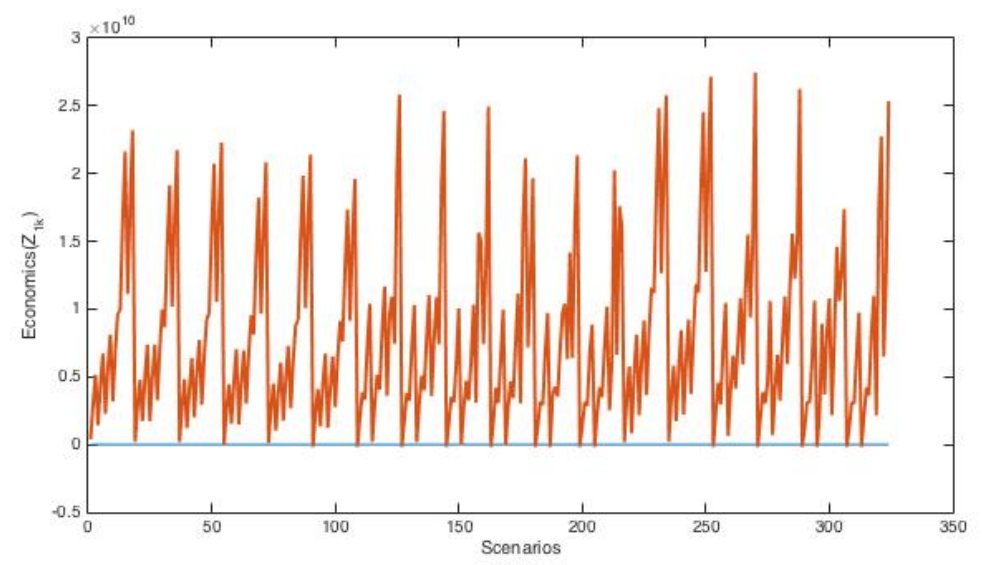

Figure 6.7: Economics objective in different scenarios $\left(Z_{1 k}\right)$

faction assures the DMs that, in more than 300 plausible scenarios $(95 \%)$, they can get back their initial investment within a year after operating the ethanol plants. Furthermore, in the worst case, they may lose 179 million Rands in the first year but this definitely will be compensated in the second year of operating the project. They also can make money up to 2.75 billion SA Rands for the best scenario. The profit would likely increase over the following years because further construction capital would not be needed for the next periods' operations. This economic achievement proves the economic profitability and sustainability of the project (see more discussions on the economic justification of the project in Section 6.5.4). Moreover, the last three factors of uncertainty, i.e. ethanol $\left(k_{4}\right)$ and $\operatorname{sugar}\left(k_{5}\right)$ prices as well as the exchange $\operatorname{rate}\left(k_{6}\right)$, have more effects on the economic objectives. This effects can be followed up by tracking the fluctuations of the graph in Figure 6.7.

By taking a look at the other objectives in Figures 6.8 and 6.9 , it can be clearly seen that the uncertainty relating to sugarcane yields has the most significant influence on both of these objectives. Ethanol and sugar demand uncertainties $\left(k_{2}\right.$ and $\left.k_{3}\right)$ impose two other major impacts on $\mathrm{CO}_{2}$ emissions. The variations in the lowest level of sugarcane yields scenario $\left(k_{1}=1\right)$ belong to the interval $[560,630]$ thousand tonnes of $\mathrm{CO}_{2}$. This interval for $k_{1}=2$ and $k_{1}=3$ has been recorded as $[720,820]$ and $[870,930]$ million $\mathrm{kg}$ of $\mathrm{CO}_{2}$, respectively.

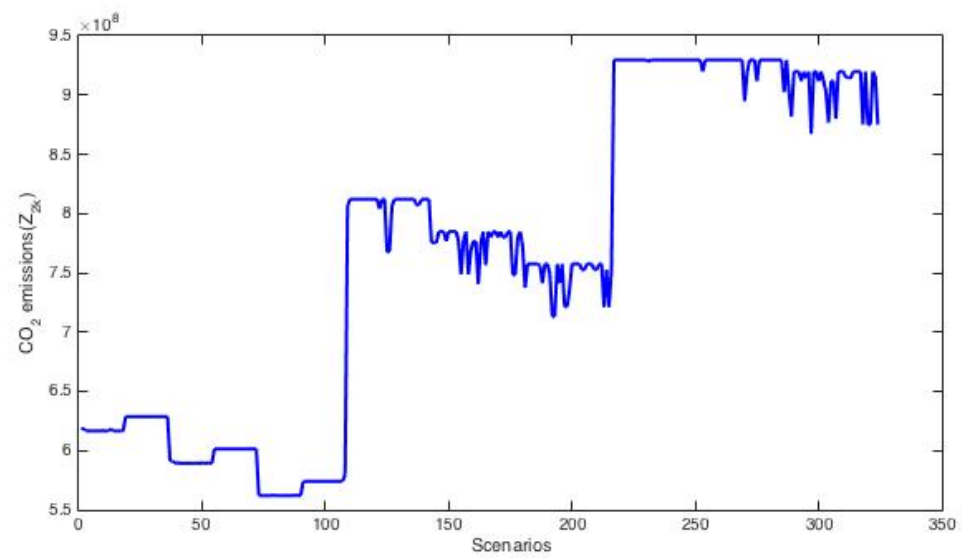

Figure 6.8: Environmental objective in different $\operatorname{scenarios}\left(Z_{2 k}\right)$ 
The total number of jobs created will have no change if scenario $s\left(k_{1}\right)=1$ unfolds and keeps its value of 110000 direct job (220 million hours a year ${ }^{7}$ ) opportunities over all other parameter variations. In a normal yields $\operatorname{scenario}\left(s\left(k_{1}\right)=2\right)$, the amount of the third objective function fluctuated slightly between 127000 and 130000 employment, while this interval for the high yield scenario $\left(s\left(k_{1}\right)=3\right)$ fluctuates between 146000 and 149000 jobs. Therefore, even in the worst case scenario, about 31000 new jobs will be created(38\% grows) and this would still satisfy the DM's preferences in this criterion.

More discussion and comparison between different criteria and their trade-offs will be presented in the next section.

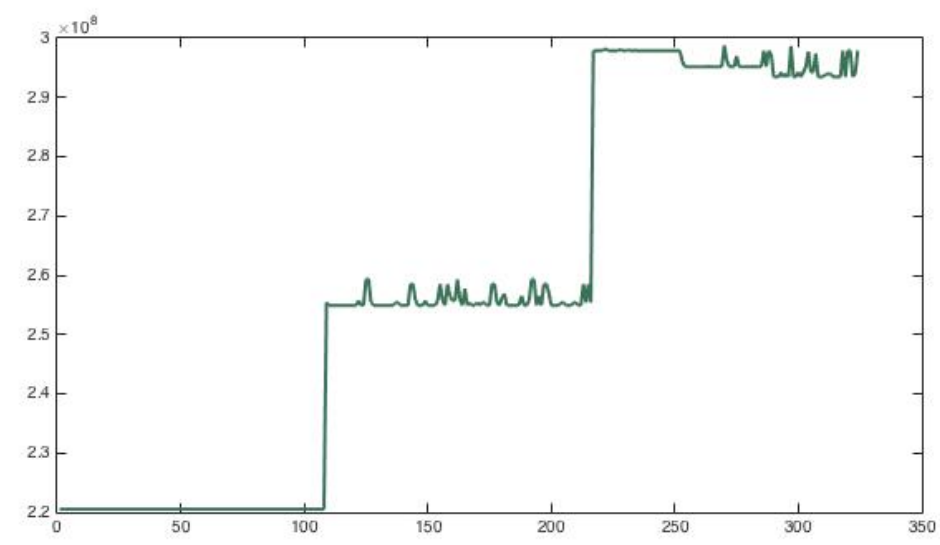

Figure 6.9: Social benefits objective in different scenarios $\left(Z_{3 k}\right)$

\subsubsection{Sensitivity analysis on various goals}

As mentioned in earlier chapters, identifying the goals in our proposed GP-based methodology is reflected by the first-order preferences of the $\mathrm{DM}(\mathrm{s})$ in models. However, a large number of scenarios in the proposed structure may prevent the DMs from choosing a suitable set of goals for the model. Analysis of variations in goals could be useful as we attempt to reach this purpose. Moreover, this kind of analysis helps us to observe the trade-offs between different meta-criteria and to evaluate the costs of improvements in one objective, compared to the others, that would be significantly advantageous in the process of decision-making in such a high dimensional problem. Accordingly, in this section, we analysed the variations of goals in our real-world application that was utilised to understand the value of the proposed two-stage model. Table 6.7 portrays the results of nine different cases from a sensitivity analysis executing on various aspirations levels.

As seen in the previous section, sugarcane yield is the most effective factor in the second and third criteria ( $\mathrm{CO}_{2}$ emissions and job creation); therefore, it would be logical to set different weights on various scenarios of sugarcane yield. Therefore, we considered three different groups of low, normal, and high yield in each case and tried various amount of goals regarding these groups. More diversity is also applied in the third and fifth case in which ' $\mathrm{EP}$ ' and 'SP' described ethanol and sugar prices while ' $R$ ' shows the exchange rate of ZAR in comparison with USD. As expected, comparing the first five cases in Table 6.7 indicates that the economic profits will grow by increasing the initial goals on this criteria, while the other goals remain fixed at the same values. This increase also affected the other two criteria and boosted their values, although this improvement in the second criterion is on the contrary to optimality of this objective function (minimising $\mathrm{CO}_{2}$ emissions) and would not satisfy the DMs. In other words, if the initial goals

\footnotetext{
${ }^{7}$ Each 20000 hours considered as one full-year job.
} 
for the first criteria (economics) increase enough, then the optimistic values could be reached for the first and the third criteria while, at the same time, an evaluation of the solutions shows the pessimistic values for the second objective function/criterion under conditions of most plausible scenarios. This represents a direct relationship between the first and the third criteria, as well as an inverse correlation between these two objectives/criteria and the second criterion.

However, the ideal values for $\mathrm{CO}_{2}$ emissions can be obtained under conditions of a few particular scenarios of the 324 plausible scenarios. Negative values in the amount of the second objective function, in case ' 5 ', represent that saving $\mathrm{CO}_{2}$ emissions in blending ethanol and petrol is greater than the rate of this emission in all other parts of the project. Although this situation, which occurs in some scenarios, would completely satisfy the environmental stakeholders, the large amount of emissions in most of the other scenarios could not satisfy the DMs. In the worst case scenario 1.4 billion $\mathrm{kg}$ of $\mathrm{CO}_{2}$, on average, would be recorded which is double compared to the initial goals for this objective.

Therefore, this analysis can help to investigate the effects and consequences of applying different goals and identify the correlation between different criteria in all plausible scenarios. Furthermore, it is also possible to trace the trade-offs between the optimistic and pessimistic values for different criteria in various scenarios. Overall, all these features would help the DM(s) in making better decisions.

By taking a second look at this example and analysing the information in Table 6.7 further discussion is warranted. If the environmental criterion was not important at all or all the stakeholders accepted an unsatisfactory amount of $\mathrm{CO}_{2}$ emissions in some plausible meta-scenarios, then case ' 5 ' could be the ideal case in which a considerable financial profit (of at least 2.8 billion Rands) and acceptable job creation (at least 168 thousand jobs (336 million hours)) would be guaranteed in the worst case. Ideally, in the best scenario, these objectives would provide some remarkable values (in this example, the profit would be about 49 billion Rands and also 232000 jobs would be created). Unfortunately, the high level of pollution generated in this case (with a mean of 1.4 billion kilogrammes $\mathrm{CO}_{2}$ emission) may cause certain policy-makers to stop the project. Nevertheless, the ability to make this large profit, within one year after the opening of new factories, can fully justify the project from an economical standpoint and might encourage sugar companies to execute this project, if the green party (environmentally friendly organisations) accepts the risk of that amount of emissions in the worst case scenario. If this example were a real project, the conclusion could assure the sugar companies about the profitability of the project and could economically support the decision of producing ethanol from sugarcane in South Africa. In the past, uncertainty about the economic implications of such a venture has thwarted the implementation of this project for more than 10 years. Finally, to convince the environmental stakeholders, similar analyses can be helpful and this will be described in the the next paragraphs. These analyses show how the sensitivity analysis can help in the decision support structure.

As seen in this hypothetical example, the proposed two-stage structure has a strong potential to be used as a Decision Support System (DSS) in decision-making, especially in MCDM problems under deep uncertainty which could be an interesting direction for future research. Moreover, the sensitivity analysis can help to analyse different aspects of the decisions in various (and perhaps conflicting) criteria of stakeholders under conditions of all plausible scenarios. This analysis is well fitted in the decision support structure.

Moreover, analysis of the goal levels can also help to investigate the impacts of different parameters in each criterion/meta-criterion. For example, in this bioethanol supply chain problem, the most effective factor in job creation is land use because planting cane in additional hectares of land, together with harvesting the sugarcane yield, needs more workers than constructing the ethanol plants and productions. Jobs created by building ethanol plants is the second effective factor. It can be clearly seen that, as expected, by increasing the third criteria (job creation) all available land would be used and ethanol plants with both technologies would be constructed in all areas. This boosts environmental pollution. Also, the profit will be decrease substantially 


\begin{tabular}{|c|c|c|c|c|c|c|c|c|c|c|c|c|c|c|c|}
\hline \multirow{2}{*}{ Cases } & \multicolumn{4}{|c|}{ Setting Goals } & \multirow{2}{*}{\multicolumn{2}{|c|}{ Objective Functions }} & \multicolumn{2}{|c|}{ Ethanol Plant(s) } & \multirow{2}{*}{\begin{tabular}{|l} 
Land Used \\
(hectares) \\
\end{tabular}} & \multicolumn{3}{|c|}{ Sugar Productions } & \multicolumn{3}{|c|}{ Ethanol Production } \\
\hline & Yield & $g_{1 k}$ & $g_{2 k}$ & $g_{3 k}$ & & & Number & Place(s)[Tech] & & White & Raw & Molasses & Cane & Raw & Molasses \\
\hline Case 1 & $\begin{array}{l}\text { Poor } \\
\text { Normal } \\
\text { Good }\end{array}$ & $\begin{array}{l}0 \\
0 \\
0\end{array}$ & $\begin{array}{l}7.5 \times 10^{8} \\
7.5 \times 10^{8} \\
7.5 \times 10^{8}\end{array}$ & $\begin{array}{l}4 \times 10^{8} \\
4 \times 10^{8} \\
4 \times 10^{8}\end{array}$ & $\begin{array}{l}Z_{1}=[-179,27445] \times 10^{6} \\
Z_{2}=[562,929] \times 10^{6} \\
Z_{2}=[220,299] \times 10^{6}\end{array}$ & $\begin{array}{r}7.9 \times 10^{9} \\
764.4 \times 10^{6} \\
25.4 \times 10^{6}\end{array}$ & 4 & $\begin{array}{c}(4[1]) \\
(8[1], 13[2])\end{array}$ & 377803 & 400000 & 1570000 & 87220 & 110587 & 33072 & 6657 \\
\hline Case 2 & \begin{tabular}{|l|} 
Poor \\
Normal \\
Good
\end{tabular} & $\begin{array}{l}0 \\
0 \\
0\end{array}$ & $\begin{array}{l}5 \times 10^{8} \\
6.5 \times 10^{8} \\
8 \times 10^{8}\end{array}$ & $\begin{array}{l}2.5 \times 10^{8} \\
3 \times 10^{8} \\
3.5 \times 10^{8}\end{array}$ & $\begin{array}{l}Z_{1}=[-84,24153] \times 10^{6} \\
Z_{2}=[416,867] \times 10^{6} \\
Z_{3}=[193,267] \times 10^{6}\end{array}$ & $\begin{array}{r}7.5 \times 10^{9} \\
701.8 \times 10^{6} \\
229.8 \times 10^{6}\end{array}$ & 5 & $\begin{array}{c}(2[1], 8[1]) \\
(10[3]) \\
(13[2], 15[2])\end{array}$ & 345800 & 400000 & 1570000 & 87220 & 79926 & 104792 & 18824 \\
\hline Case 3 & \begin{tabular}{|l|} 
Poor \\
If EP high, \\
If SP high, \\
Normal \\
If EP high, \\
If SP high, \\
Good \\
If EP high, \\
If SP high, \\
\end{tabular} & $\begin{array}{l}0 \\
g_{1 k}=1 \times 10^{9} ; \\
g_{1 k}=2 \times 10^{9} ; \\
0 \\
g_{1 k}=1 \times 10^{9} ; \\
g_{1 k}=2 \times 10^{9} ; \\
0 \\
g_{1 k}=1 \times 10^{9} ; \\
g_{1 k}=2 \times 10^{9} ;\end{array}$ & $\begin{array}{l}5 \times 10^{8} \\
\text { If R Low, } \\
6.5 \times 10^{8} \\
\text { If R Low, } \\
8 \times 10^{8} \\
\text { If R Low, }\end{array}$ & $\begin{array}{l}2.5 \times 10^{8} \\
g_{1 k}=3 \times 10^{9} \\
3 \times 10^{8} \\
g_{1 k}=3 \times 10^{9} \\
3.5 \times 10^{8} \\
g_{1 k}=3 \times 10^{9}\end{array}$ & $\begin{array}{l}Z_{1}=[-145,28342] \times 10^{6} \\
Z_{2}=[362,945] \times 10^{6} \\
Z_{3}=[223,305] \times 10^{6}\end{array}$ & $\begin{array}{l}8.8 \times 10^{9} \\
767 \times 10^{6} \\
264 \times 10^{6}\end{array}$ & 7 & $\begin{array}{l}(2[1]) \\
(3[1]) \\
(4[1]) \\
(8[1]) \\
(10[2]) \\
(14[2]) \\
(15[3])\end{array}$ & 385884 & 400000 & 1570000 & 87220 & 118000 & 109597 & 19938 \\
\hline Case 4 & \begin{tabular}{|l|} 
Poor \\
Normal \\
Good
\end{tabular} & $\begin{array}{l}0 \\
1 \times 10^{9} \\
2 \times 10^{9}\end{array}$ & $\begin{array}{l}5 \times 10^{8} \\
6.5 \times 10^{8} \\
8 \times 10^{8}\end{array}$ & $\begin{array}{l}2.5 \times 10^{8} \\
3 \times 10^{8} \\
3.5 \times 10^{8}\end{array}$ & $\begin{array}{l}Z_{1}=[194,35070] \times 10^{6} \\
Z_{2}=[270,1106] \times 10^{6} \\
Z_{3}=[245,340] \times 10^{6}\end{array}$ & $\begin{array}{r}12.44 \times 10^{9} \\
898 \times 10^{6} \\
292 \times 10^{6}\end{array}$ & 1 & $(7[3])$ & 456125 & 400000 & 1570000 & 87220 & 184735 & 129605 & 2915 \\
\hline Case 5 & \begin{tabular}{|l|} 
Poor \\
If EP high, \\
If SP high, \\
Normal \\
If EP high, \\
If SP high, \\
Good \\
If EP high, \\
If SP high, \\
\end{tabular} & $\begin{array}{l}1 \times 10^{9} \\
g_{1 k}=2 \times 10^{9} ; \\
g_{1 k}=4 \times 10^{9} ; \\
3 \times 10^{9} \\
g_{1 k}=5 \times 10^{9} ; \\
g_{1 k}=7 \times 10^{9} ; \\
6 \times 10^{9} \\
g_{1 k}=8 \times 10^{9} ; \\
g_{1 k}=10 \times 10^{9} ;\end{array}$ & $\begin{array}{l}5 \times 10^{8} \\
\text { If R Low, } \\
6.5 \times 10^{8} \\
\text { If R Low, } \\
8 \times 10^{8} \\
\text { If R Low, }\end{array}$ & $\begin{array}{l}2.5 \times 10^{8} \\
g_{1 k}=8 \times 10^{9} \\
3 \times 10^{8} \\
g_{1 k}=10 \times 10^{9} \\
3.5 \times 10^{8} \\
g_{1 k}=15 \times 10^{9}\end{array}$ & $\begin{array}{l}Z_{1}=[2.8,49] \times 10^{9} \\
Z_{2}=[-176,1712] \times 10^{6} \\
Z_{3}=[336,464] \times 10^{6}\end{array}$ & $\begin{array}{r}19.3 \times 10^{9} \\
1406 \times 10^{6} \\
400 \times 10^{6}\end{array}$ & 4 & $\begin{array}{c}(2[1]) \\
(6[3], 10[1]) \\
(14[1])\end{array}$ & 619162 & 1000000 & 1580000 & 87777 & 338037 & 0 & 3627 \\
\hline Case 6 & \begin{tabular}{|l|} 
Poor \\
Normal \\
Good
\end{tabular} & $\begin{array}{l}0 \\
0 \\
0\end{array}$ & $\begin{array}{l}5 \times 10^{8} \\
6.5 \times 10^{8} \\
8 \times 10^{8}\end{array}$ & $\begin{array}{l}12.5 \times 10^{8} \\
30 \times 10^{8} \\
50 \times 10^{8}\end{array}$ & $\begin{array}{l}Z_{1}=[-4479,43655] \times 10^{6} \\
Z_{2}=[731,1971] \times 10^{6} \\
Z_{3}=[382,520] \times 10^{6}\end{array}$ & $\begin{array}{r}12.9 \times 10^{9} \\
1508 \times 10^{6} \\
451 \times 10^{6}\end{array}$ & 15 & $\begin{array}{c}(1,2,3,4,4,5)[3] \\
(6,7,8,9,10)[3] \\
(11,12,13,14,15)[3]\end{array}$ & 627495 & 1000000 & 1817006 & 100940 & 308427 & 0 & 9070 \\
\hline Case 7 & \begin{tabular}{|l|} 
Poor \\
Normal \\
Good \\
\end{tabular} & $\begin{array}{l}0 \\
0 \\
0 \\
\end{array}$ & $\begin{array}{l}5 \times 10^{8} \\
6.5 \times 10^{8} \\
8 \times 10^{8} \\
\end{array}$ & $\begin{array}{l}2500 \times 10^{8} \\
6000 \times 10^{8} \\
150000 \times 10^{8}\end{array}$ & 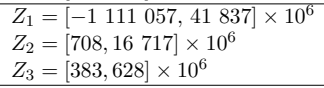 & $\begin{array}{r}-185 \times 10^{9} \\
6333 \times 10^{6} \\
487 \times 10^{6} \\
\end{array}$ & 12 & $\begin{array}{c}(2,4,5,6)[3] \\
(7,8,10,11)[3] \\
(12,13,14,15)[3]\end{array}$ & 627495 & 1000000 & 1570000 & 87220 & 347537 & 7276 & 1712 \\
\hline Case 8 & \begin{tabular}{|l|} 
Poor \\
Normal \\
Good
\end{tabular} & $\begin{array}{l}0 \\
0 \\
0\end{array}$ & $\begin{array}{l}3 \times 10^{8} \\
5 \times 10^{8} \\
6 \times 10^{8}\end{array}$ & $\begin{array}{l}2.5 \times 10^{8} \\
3 \times 10^{8} \\
3.5 \times 10^{8}\end{array}$ & $\begin{array}{l}Z_{1}=[-220,16373] \times 10^{6} \\
Z_{2}=[79.5,780] \times 10^{6} \\
Z_{3}=[156,217] \times 10^{6}\end{array}$ & $\begin{array}{l}5.4 \times 10^{9} \\
620 \times 10^{6} \\
186 \times 10^{6}\end{array}$ & 1 & $(6[3])$ & 290724 & 400000 & 1570000 & 87220 & 27604 & 104792 & 36042 \\
\hline Case 9 & \begin{tabular}{|l|} 
Poor \\
Normal \\
Good
\end{tabular} & $\begin{array}{l}0 \\
0 \\
0\end{array}$ & $\begin{array}{l}0 \\
0 \\
0\end{array}$ & $\begin{array}{l}2.5 \times 10^{8} \\
3 \times 10^{8} \\
3.5 \times 10^{8}\end{array}$ & $\begin{array}{l}Z_{1}=[-700,14307] \times 10^{6} \\
Z_{2}=[-490,700] \times 10^{6} \\
Z_{3}=[141,197] \times 10^{6}\end{array}$ & $\begin{array}{r}4 \times 10^{9} \\
573 \times 10^{6} \\
168 \times 10^{6}\end{array}$ & 2 & $\begin{array}{l}(6[2]) \\
(13[2])\end{array}$ & 261666 & 400000 & 1570000 & 87220 & 0 & 109576 & 36042 \\
\hline
\end{tabular}

Table 6.7: Sensitivity analysis on various goals 
(see cases ' 6 ' and ' 7 ' in which the aspiration levels related to the third criteria have been raised while the others keep their previous values). Therefore, by changing the goal levels and tracking the trade-offs between the criteria/objectives, the most suitable decisions can be made by the $\mathrm{DM}(\mathrm{s})$ to satisfy all the stakeholders as much as possible.

In contrast, the last two cases, especially the ninth case, are the most environmentallyfriendly cases (the maximum emissions, in the worst-case scenario, would be less than '780 000' and '700 000' tonnes of $\mathrm{CO}_{2}$, respectively). However, in the worst-case scenario of the last case, we may also face a financial loss of '700' million Rands, while job creation would be between ' 70 ' and ' 100 ' thousand jobs. This amount of jobs (70 000) will be even less than the current number of jobs available within the sugar industry. Thus, if the aspiration levels of the second criteria are decreased too much, then some non-reasonable solutions, including severe economic and social effects, will emerge.

Overall, it seems that setting the goals in the fourth case could be the most reasonable choice since this provides us with some balanced solutions which not only guarantee the profitability of the project, but also create a considerable number of jobs and produce limited $\mathrm{CO}_{2}$ emissions (that will be less than ' 900000 ' tonnes in average).

Therefore, sensitivity analysis on various goals represents different trade-offs between the meta-criteria and helps DMs to study and interpret the results that can lead them to choose a more suitable strategy. Such analysis will also provide the DMs with a complete analysis which highlights the consequences of changes in their initial goals and the effects of these variations in result. This can help us discuss the management insights that are generated, and on how the sensitivity analysis needs to fit into the decision support structure.

Another important factor that we need to consider is the water consumption for planting too many hectares of the land, which is a real issue for the future of this project. This issue can be classified as an environmental criterion or could be applied as a completely separate criterion/objective. Nonetheless, we are not going to consider this issue in this study, but it would be an interesting direction for future work.

\subsection{Conclusions}

The first aim of this chapter was to examine the simplest form of the proposed methodology (i.e. two-stage structure) and check its reliability and validity in a real-life problem. The second aim was to investigate the possibility of utilising the proposed structure as a decision support tool in MCDA problems. Thirdly, we looked to provide a general model to that considers deep uncertainty in multi-objective optimisation problems in biofuel supply chains management.

In this chapter, the capability of the proposed two-stage framework to provide solutions for real world problems was investigated by applying the proposed methodology to a strategic planning problem involving sugar-bioethanol supply chains (SCs) under deep uncertainty. The infrastructure of the entire sugar-bioethanol supply chains was analysed in such a way that all economics ( $\max$ profit), environmental $\left(\min \mathrm{CO}_{2}\right.$ ), and social benefits (max job creation) were optimised under six key uncertainties, sugarcane yield, ethanol and refined sugar demands and prices, and the exchange rate. Moreover, one of the critical design questions, designing the optimal number and technologies as well as the best place(s) for setting up the ethanol plant(s), is also addressed in this study. Furthermore, a case study based on the South African Sugar Industry is also utilised to study and examine the proposed methodology. We have considered the integrated South African infrastructures for joint productions of sugar and ethanol under deep uncertainty. This problem was formulated as a scenario-based mixed-integer two-stage multi-objective optimisation problem and solved by utilising the Generalised Goal Programming approach.

The first-stage (initial) decisions, made before scenario realisation, included design and planning decisions such as finding the optimal number, technologies, and the best place(s) for setting up the ethanol plant(s), hectares of cultivated lands, and the volume of feedstock to be used for 
every production. Moreover, after scenario realisation, the unfilled ethanol demand could now be recovered by the importation of ethanol or by sending back some stored sugar/molasses from the depot to ethanol plants for more ethanol generation. These decisions, together with some other recourse decisions (such as the amount of each product that should be sold, or extra raw sugar production from excess sugarcane yields), made the second-stage/recourse decisions of the proposed two-stage structure. In fact, these recourse decisions can be described as "contingency plans" 8 . In other words, the initial decision(s) are made and implemented immediately at the beginning of the project, and one group of recourse decisions is identified for each plausible scenario as a contingency plan. Then, after scenario realisation, the relevant plan will be implemented. These contingency plans and relevant scenarios can be stored in a tabular form for better explanation and comparisons.

Fourteen sugar mills and four refineries are operating in SA. Except for sugar demands and prices for which two plausible scenarios (low and high) were considered, three plausible scenarios (low, normal, and high) were determined for the other uncertain parameters that construct 324 meta-scenarios. Therefore, by setting some goals, the Multi-Objective Mixed Integer problem including 972 meta-criteria/objectives was solved by applying the Generalised Goal Programming.

The results included the suggestion to build four new ethanol plants. Two factories with the first technology that utilises sugarcane as feedstock $(\mathrm{q}=1)$ that would be installed in Noodsberg and Darnall (in KwaZulu-Natal). The other two plants would operate with the second technol$\operatorname{ogy}(\mathrm{q}=2)$, and would be set up in Komati (in Mpumalanga) and Durban (in KwaZulu-Natal).

Cultivating sugar cane in 377803 hectares of arable lands would give rise to harvesting 18890143,22668172 , and 26446200 tonnes of sugarcane depending on the weather in various scenarios. An interesting observation was that currently almost the same hectares of land are underutilised of sugar cane. This means that this project can be simply executed without any significant extension of land-use and/or further sugarcane production (and hence water consumption). That is, by only constructing the four suggested ethanol plants and reallocation in sources, this project can be simply operated.

Furthermore, almost no sugar importation is needed, and all demands are fully satisfied with local sugar production which maintains the economic self-sufficiency of the sugar industry. There is still enough sugars for exportation. However, we may need some ethanol importation in some scenarios.

Tracking the fluctuations in achievements in different criteria shows that the uncertainty in ethanol and sugar demands plus currency volatility have significant influences on the economic objectives. However, the most effective uncertain parameter in both environmental and social objectives is sugarcane yields. Environmental target is also more or less affected by existing uncertainty in ethanol and sugar demands and these variations are more visible in certain scenarios including the low sugarcane yield scenario.

Analysis of variations in goals can help to find a suitable set of aspiration levels that gives rise to balanced solutions; furthermore, this analysis helps us to observe the trade-offs between different meta-criteria and evaluate the costs of improvements in one objective, compared to the others, that would be beneficial in the process of decision-making.

It is also shown that by setting some suitable goals some balanced solutions would be reached. These balance solutions provide satisfactory performances for almost all meta-criteria and can be recognised from the deviations between the goals and achievements. By utilising these balanced solutions, the profitability of the project will be guaranteed, a remarkable number of jobs will be created, and a more reasonable amount of $\mathrm{CO}_{2}$ emissions will be produced. Therefore, sensitivity analysis on various goals represents different trade-offs between the meta-criteria and helps the DMs to study and interpret the results. This can lead them to choose a more suitable strategy.

The first-order preferences of the $\mathrm{DM}(\mathrm{s})$ have been applied to the model by choosing the

\footnotetext{
${ }^{8} \mathrm{~A}$ plan (Plan B) that is usually utilised for risk management and would be implemented in the case of surprises when the original plan (Plan A) is no longer working well.
} 
initial goals for the relevant GP model which were used to solve the proposed two-stage model. After obtaining the solutions, the second-order preferences can be exerted by a sensitivity analysis on variations in goals (such as one we have been performing in this section). In fact, at this stage, the $\mathrm{DM}(\mathrm{s})$ can observe the solutions which were generated in terms of their first-order preferences. Furthermore, the sensitivity analysis can provide the opportunity for the $\mathrm{DM}(\mathrm{s})$ to compare the trade-offs between different criteria under conditions of all plausible scenarios. Therefore, they have a chance to modify their initial goals and improve the decisions regarding their preferences (i.e. they are interactively involved during all steps of the decision-making process and generating the solutions). This is the golden opportunity that the proposed two-stage model, together with the sensitivity analysis, can provide for the $\mathrm{DM}(\mathrm{s})$.

The most important concepts and insights that could be extracted from using the proposed two-stage structure to model and solve this real-world problem can be summarised as follows:

- The possibility of analysing the effects and consequences of applying different goals which could help the $\mathrm{DM}(\mathrm{s})$ to improve the decisions.

- The possibility of analysing the correlation between different criteria and observing the variations in all plausible scenarios.

- The possibility of improvements in results by observing the trade-offs between different criteria in various scenarios.

- The possibility of observing and analysing the trade-offs between the optimistic and pessimistic values for different criteria in various scenarios.

- Identifying the balanced solutions for all meta-criteria.

- The possibility of analysing the solutions in the case of eliminating one (or more) criterion from the problem.

- Identifying the impacts of different parameters in each criterion/meta-criterion.

- The probabilistic and stochastic methods may only be able to demonstrate the tradeoffs between the criteria in a possible scenario. However, with the proposed two-stage structure we can observe and analyse the trade-offs between all meta-criteria (i.e. the trade-offs between different criteria in all plausible scenarios). This highlights one of the most important superiorities of the proposed two-stage structure compared to probabilistic and stochastic methods.

- The possibility of using as a Decision Support Tool (DST) (or Decision Support System (DSS)) by providing an analysis of effects of various decisions in a broad range of plausible scenarios.

Finally, during the modelling and coding of the two-stage structure, we faced some computation difficulties (such as an "out of memory" error) because of the complexity of dealing with deep uncertainty and handling many meta-criteria. Thus, this chapter is limited to the two-stage structure to avoid extreme complexity and expensive computation. However, by applying the three-stage or moving horizon models we can investigate the results of the problem in longer planning periods if the computations are efficient. In a longer planning horizon we may expect to achieve more clear justification for supporting the decision of producing bioethanol and blend with oil fuels. Such expansion would be an interesting direction for future study. Considering other uncertain factors such as water consumption would also be another interesting direction for future work. The methodology could also be applied to other supply chain problems under deep uncertainty. Furthermore, only the first generation of biofuel SCs is investigated in this study. The second generation, or even the third generation that will be able to operate in a near future, could be an interesting and useful area to extend the proposed methodology in the future. 


\subsection{Notation}

Table 6.8: Variables notation

\begin{tabular}{|c|c|}
\hline \multicolumn{2}{|l|}{ Notations } \\
\hline Sets: & \\
\hline$L:$ & The set of different areas. \\
\hline$P:$ & The set of working refineries locating in different areas $P \subseteq L$. \\
\hline$N_{j}:$ & The set of lands locating in the neighbourhood of the Land $j \in L$. \\
\hline Indexes: & \\
\hline$i,(i \in\{1,2,3\}):$ & represent the objective function $i$. \\
\hline$j,(j \in L):$ & represent the Mill $j$. \\
\hline$k \in S^{1}:$ & represent the scenario that revealed. \\
\hline$l,(l \in L):$ & represent the land area $l$. \\
\hline$p,(p \in P):$ & represent the refinery $p$. \\
\hline$q,(q \in\{1,2,3\}):$ & represent the division/technology $q$ utilizing to produce ethanol. \\
\hline$r,(r \in L):$ & represent the ethanol plant/biorefinery $r$. \\
\hline Decision Variables & \\
\hline Stage 0: & \\
\hline$X_{1 l}^{0} \in \Re:$ & Hectares of land used for cane in area $l \in L$. \\
\hline$X_{2 j}^{0} \in \Re,:$ & Tonnage of sugar cane turned into raw sugar in Mill $j \in L$. \\
\hline$X_{3 j r}^{0} \in \Re,:$ & Tonnage of sugar cane transport from Mill $j \in L$ to ethanol plant $r \in N_{j}$ (with technology $q, q=1,3$ ) to produce ethanol. \\
\hline$X_{4 j p}^{0} \in \Re,:$ & Tonnage of raw sugar transport from Mill $j \in L$ to refinery $p \in P$ to produce white sugar. \\
\hline$X_{5 j}^{0} \in \Re,:$ & Tonnage of raw sugar transport from Mill $j \in L$ to depot to sale. \\
\hline$X_{6 j r}^{0} \in \Re,:$ & Tonnage of raw sugar transport from Mill $j \in L$ to ethanol plant $r \in L$ (with technology $q, q=2,3$ ) to produce ethanol. \\
\hline$X_{7 j r}^{0} \in \Re,:$ & $\begin{array}{l}\text { A thousand gallons of molasses transport from Mill } j \in L \text { to ethanol plant } r \in L \text { (with technology } q, q=2,3 \text { ) } \\
\text { to produce ethanol. }\end{array}$ \\
\hline$X_{20 j}^{0} \in \Re,:$ & A thousand gallons of molasses transport from Mill $j \in L$ to Depot to sale locally as animal feeds. \\
\hline$\Gamma_{r q}^{0} \in\{0,1\}($ binary $)$ & $\begin{array}{l}\{1 \text { : if ethanol plant with conversion technology } q, q=1,2,3 ; \text { installed in area } r \\
0: \text { otherwise. }\}\end{array}$ \\
\hline
\end{tabular}


Table 6.8 - Continued from previous page

\begin{tabular}{|c|c|}
\hline Stage 1: & \\
\hline$X_{8 k}^{1} \in \Re,:$ & Tonnage of refined sugar to import if scenario $k, k \in S^{1}$ revealed. \\
\hline$X_{9 k}^{1} \in \Re,:$ & Tonnage of raw sugar to import if scenario $k, k \in S^{1}$ revealed. \\
\hline$X_{10 k}^{1} \in \Re,:$ & A thousand gallons of ethanol to import if scenario $k, k \in S^{1}$ revealed. \\
\hline$X_{11 k}^{1} \in \Re,:$ & Tonnage of refined sugar to sale locally if scenario $k, k \in S^{1}$ revealed. \\
\hline$X_{12 k}^{1} \in \Re,:$ & Tonnage of raw sugar to sale locally if scenario $k, k \in S^{1}$ revealed. \\
\hline$X_{13 k}^{1} \in \Re,:$ & A thousand gallons of ethanol to sale locally if scenario $k, k \in S^{1}$ revealed. \\
\hline$X_{14 k}^{1} \in \Re,:$ & Tonnage of refined sugar to export if scenario $k, k \in S^{1}$ revealed. \\
\hline$X_{15 k}^{1} \in \Re,:$ & Tonnage of raw sugar to export if scenario $k, k \in S^{1}$ revealed. \\
\hline$X_{16 k}^{1} \in \Re,:$ & A thousand gallons of ethanol to export if scenario $k, k \in S^{1}$ revealed. \\
\hline$X_{17 k}^{1} \in \Re,:$ & $\begin{array}{l}\text { A thousand gallons of by-product produced by technology } q, q=1,2,3 \text {; to sale (locally/internationally), } \\
\text { if scenario } k, k \in S^{1} \text { revealed. }\end{array}$ \\
\hline$X_{21 k}^{1} \in \Re,:$ & A thousand gallons of molasses to sale (locally/internationally), if scenario $k, k \in S^{1}$ revealed. \\
\hline$X_{18 r k}^{1} \in \Re,:$ & $\begin{array}{l}\text { Tonnage of refined sugar to transport from depot to ethanol plant } r \in L \text { to produce more ethanol, } \\
\text { if scenario } k, k \in S^{1} \text { revealed. }\end{array}$ \\
\hline$X_{19 r k}^{1} \in \Re,:$ & $\begin{array}{l}\text { Tonnage of raw sugar to transport from depot to ethanol plant } r \in L \text { to produce more ethanol, } \\
\text { if scenario } k, k \in S^{1} \text { revealed. }\end{array}$ \\
\hline$X_{22 r k}^{1} \in \Re,:$ & $\begin{array}{l}\text { A thousand gallons of molasses to transport from depot to ethanol plant } r \in L \text { to produce more ethanol, } \\
\text { if scenario } k, k \in S^{1} \text { revealed. }\end{array}$ \\
\hline$X_{23 j k}^{1} \in \Re,:$ & Tonnage of sugarcane to transport from Mill $j \in L$ to depot, if scenario $k, k \in S^{1}$ revealed. \\
\hline Uncertain Parameters & \\
\hline$Y_{k}^{0} \in \Re,:$ & Tonnage of sugar cane yield from a hectare if scenario $k, k i n S^{1}$ revealed. \\
\hline$\tilde{D E} E_{k}^{1} \in \Re,:$ & Ethanol demand if scenario $k, k \in S^{1}$ revealed. \\
\hline$D S_{k}^{1} \in \Re,:$ & Refined sugar demand if scenario $k, k \in S^{1}$ revealed. \\
\hline$P E_{k}^{1} \in \Re,:$ & Ethanol Price if scenario $k, k \in S^{1}$ revealed. \\
\hline$P S_{k}^{1} \in \Re,:$ & Refined sugar Price if scenario $k, k i n S^{1}$ revealed. \\
\hline$R_{k}^{1} \in \Re,:$ & Exchange rate of Dollar to Rand. \\
\hline Certain Parameters & \\
\hline$\alpha \in \Re,:$ & Conversion factor for cane to raw sugar. \\
\hline$\beta \in \Re,:$ & Conversion factor for cane to ethanol. \\
\hline$\gamma \in \Re,:$ & Conversion factor for raw sugar to refined sugar. \\
\hline$\zeta \in \Re,:$ & Conversion factor for raw sugar to molasses. \\
\hline$\theta \in \Re,:$ & Conversion factor for raw sugar to ethanol. \\
\hline
\end{tabular}


Table 6.8 - Continued from previous page

\begin{tabular}{|c|c|}
\hline$\lambda \in \Re,:$ & Conversion factor for refined sugar to ethanol. \\
\hline$\sigma \in \Re,:$ & Conversion factor for molasses to ethanol. \\
\hline$\mu \in \Re,:$ & Conversion factor for price of raw sugar(compare to refined sugar price). \\
\hline$\nu \in \Re,:$ & Conversion factor for price of by-product of ethanol(compare to ethanol price). \\
\hline$\iota \in \Re,:$ & Conversion factor for price of molasses(compare to refined sugar price). \\
\hline$\xi \in \Re,:$ & Conversion factor for local price of refined sugar. \\
\hline$\rho \in \Re,:$ & Conversion factor for local price of ethanol. \\
\hline$\kappa_{q} \in \Re,:$ & Conversion factor for produce by-product with technology $q, q \in\{1,2,3\}$. \\
\hline$\tau \in \Re,:$ & Tax exemption factor for local sale of ethanol. \\
\hline$\varpi_{j}^{\min } \in \Re,:$ & Minimum capacity of raw sugar production in Mill $j \in L$. \\
\hline$\varpi_{j}^{\max } \in \Re,:$ & Maximum capacity of raw sugar production in Mill $j \in L$. \\
\hline$\varrho_{p}^{\text {min }} \in \Re,:$ & Minimum capacity of refined sugar production in refinery $p \in P$. \\
\hline$\varrho_{p}^{\max } \in \Re,:$ & Maximum capacity of refined sugar production in refinery $p \in P$. \\
\hline$\varsigma_{q}^{\min } \in \Re,:$ & Minimum capacity of ethanol production in ethanol plant with technology $q,(q=1,2,3)$ if installed. \\
\hline$\varsigma_{q}^{\max } \in \Re,:$ & Maximum capacity of ethanol production in ethanol plant with technology $q,(q=1,2,3)$ if installed. \\
\hline$b_{1 l}^{0} \in \Re,:$ & Available hectare of land in area $l \in L$. \\
\hline$c_{1}^{0} \in \Re,:$ & Cost of renting, growing, harvesting and transporting a tonne of sugar cane to the Mill in area $l$. \\
\hline$c_{2}^{0} \in \Re,:$ & Cost of producing a tonne of raw sugar. \\
\hline$c_{3}^{0} \in \Re,:$ & Cost of producing a thousand gallons of ethanol from cane. \\
\hline$c_{4}^{0} \in \Re,:$ & Cost of producing a thousand gallons of ethanol from molasses. \\
\hline$c_{5}^{0} \in \Re,:$ & Cost of producing a thousand gallons of ethanol from raw sugar. \\
\hline$c_{6}^{0} \in \Re,:$ & Cost of producing a tonne of refined sugar from raw sugar. \\
\hline$c_{7}^{0} \in \Re,:$ & Cost of producing a thousand gallons of by-product. \\
\hline$c_{8 r q}^{0} \in \Re,:$ & Cost of installation and operation of an ethanol plant with division/technology $q,(q=1,2,3)$ in area $r \in L$. \\
\hline$c_{9 j r}^{0} \in \Re,:$ & Cost of transporting a tonne of cane from Mill $j \in L$ to plant $r \in N_{j}$ (with division/technology $q=1,3$ ). \\
\hline$c_{10 j r}^{0} \in \Re,:$ & Cost of transporting a tonne of raw sugar from Mill $j \in L$ to plant $r \in L$ (with division/technology $q=2,3$ ). \\
\hline$c_{11 j p}^{0} \in \Re,:$ & Cost of transporting a tonne of raw sugar from Mill $j \in L$ to refinery $p \in P$. \\
\hline$c_{12 j}^{0} \in \Re,:$ & Cost of transporting a tonne of raw sugar from Mill $j \in L$ to depot. \\
\hline$c_{13 r}^{0} \in \Re,:$ & Cost of transporting a thousand gallons of ethanol/molasses/by-product between plant $r \in L$ and depot. \\
\hline
\end{tabular}

Continued on next page 
Table 6.8 - Continued from previous page

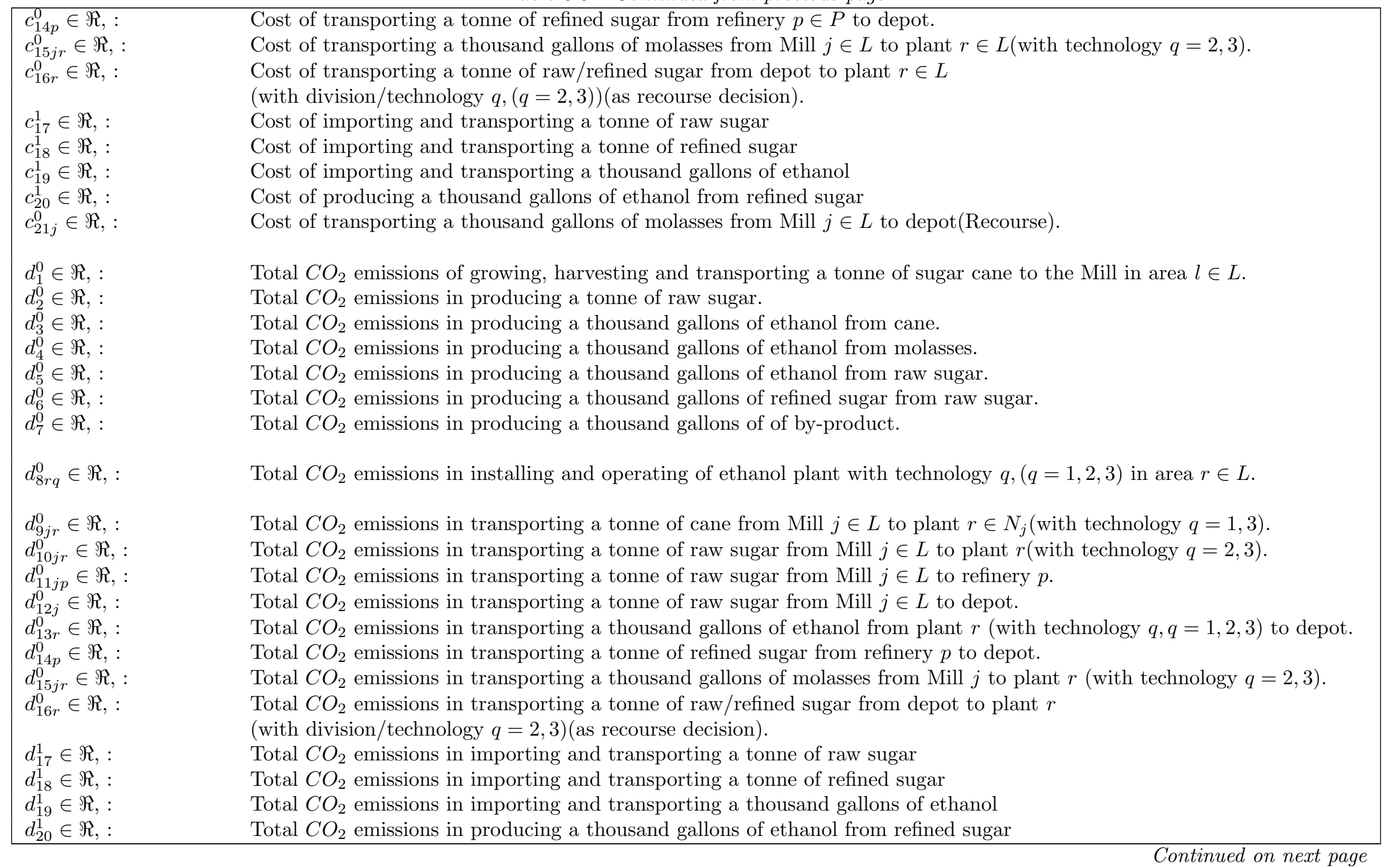


Table 6.8 - Continued from previous page

\begin{tabular}{|c|c|}
\hline $\begin{array}{l}d_{21 j}^{0} \in \Re,: \\
e^{0} \in \Re,:\end{array}$ & $\begin{array}{l}\text { Total } \mathrm{CO}_{2} \text { emissions in transporting a thousand gallons of molasses from Mill } j \in L \text { to depot. } \\
\text { Total } \mathrm{CO}_{2} \text { saving in blending a thousand gallons of ethanol in petrol. }\end{array}$ \\
\hline$h_{1}^{0} \in W,:$ & Total number of permanent jobs that secured/created per hectare of cultivated land. \\
\hline$h_{2}^{0} \in W,:$ & Total number of seasonal jobs that secured/created per hectare of cultivated land. \\
\hline$h_{3}^{0} \in W,:$ & Total number of permanent jobs that secured/created to producing a tonne of raw sugar at a Mill. \\
\hline$h_{4}^{0} \in W,:$ & Total number of permanent jobs that secured/created to producing a tonne of refined sugar at a refinery. \\
\hline$h_{5 q}^{0} \in W,:$ & $\begin{array}{l}\text { Total number of permanent jobs that created to producing a thousand gallons of ethanol in plant } \\
\text { with division/technology } q \text {. }\end{array}$ \\
\hline$h_{6 q}^{0} \in W,:$ & $\begin{array}{l}\text { Total number of permanent jobs that created to producing a thousand gallons of by-product in plant } \\
\text { with division/technology } q \text {. }\end{array}$ \\
\hline$h_{7 q}^{0} \in W,:$ & Total number of temporary jobs that created to constructing an ethanol plant with division/technology $q$. \\
\hline$h_{8 q}^{0} \in W,:$ & $\begin{array}{l}\text { Total number of permanent jobs that created to operating an ethanol plant with } \\
\text { division/technology } q \text { (exclude production jobs). }\end{array}$ \\
\hline$h_{9 j r}^{0} \in \Re,:$ & $\begin{array}{l}\text { Total number of permanent jobs that secured/created to transporting a tonne of } \\
\left.\text { cane from Mill } j \in L \text { to plant } r \in N_{j} \text { (with division/technology } q=1,3\right) \text {. }\end{array}$ \\
\hline$h_{10 j r}^{0} \in \Re,:$ & $\begin{array}{l}\text { Total number of permanent jobs that secured/created to transporting a tonne of } \\
\text { raw sugar from Mill } j \in L \text { to plant } r \text { (with division/technology } q=2,3 \text { ). }\end{array}$ \\
\hline$h_{11 j p}^{0} \in \Re,:$ & Total number of permanent jobs that created to transporting a tonne of raw sugar from Mill $j \in L$ to refinery $p \in P$. \\
\hline$h_{12 j}^{0} \in \Re,:$ & Total number of permanent jobs that secured/created to transporting a tonne of raw sugar from Mill $j \in L$ to depot. \\
\hline$h_{13 r}^{0} \in \Re,:$ & $\begin{array}{l}\text { Total number of permanent jobs that created to transporting a thousand gallons of ethanol } \\
\text { from plant } r \in L \text { (with division/technology } q, q=1,2,3 \text { ) to depot. }\end{array}$ \\
\hline$h_{14 p}^{0} \in \Re,:$ & Total number of permanent jobs that created to transporting a tonne of refined sugar from refinery $p \in P$ to depot. \\
\hline$h_{15 j r}^{0} \in \Re,:$ & $\begin{array}{l}\text { Total number of permanent jobs that created to transporting a thousand gallons of molasses from Mill } j \in L \\
\text { to plant } r \in L \text { (with division/technology } q=2,3 \text { ). }\end{array}$ \\
\hline$h_{16 r}^{0} \in \Re,:$ & $\begin{array}{l}\text { Total number of permanent jobs that created to transporting a tonne of raw/refined sugar from depot } \\
\text { to plant } r \in L \text { (with division/technology } q=2,3) \text { (as recourse decision). }\end{array}$ \\
\hline$h_{21 j}^{0} \in \Re,:$ & $\begin{array}{l}\text { Total number of permanent jobs that created to transporting a thousand gallons of molasses } \\
\text { from Mill } j \in L \text { to depot. }\end{array}$ \\
\hline$g_{i k} \in \Re,:$ & Goal $i$ in scenario $k$. \\
\hline$\delta_{i k} \in \Re,:$ & Deviations from the goal $i$ in scenario $k$. \\
\hline
\end{tabular}




\subsection{Appendix}

\begin{tabular}{lcc}
\hline \multirow{2}{*}{ regions } & \multicolumn{2}{c}{ Available arable land(Thousand hectares) } \\
\cline { 2 - 3 } & In use & Max practical \\
\hline Umzimkulu & 26.667 & 40.0005 \\
Sezela & 48.333 & 72.4995 \\
Eston & 29.166 & 43.749 \\
Noodsberg & 33.333 & 49.9995 \\
Union Coop & 16.667 & 25.0005 \\
Maidstone & 39.166 & 58.749 \\
Gledhow & 29.166 & 43.749 \\
Darnall & 29.166 & 43.749 \\
Amatikulu & 33.333 & 49.9995 \\
Felixton & 41.667 & 62.5005 \\
Umfolozi & 23.333 & 34.9995 \\
Pongola & 20 & 30 \\
Komati & 25 & 37.5 \\
Malelane & 33.333 & 49.9995 \\
Durban & 0 & 0 \\
Total & 428.33 & 642.495 \\
\hline
\end{tabular}

Table 6.9: Estimated available land(Thousand hectares) 


\begin{tabular}{|c|c|c|c|c|c|c|c|c|c|c|c|c|c|c|c|}
\hline Areas & Umzimkulu & Sezela & Eston & Noodsberg & Union Coop & Maidstone & Gledhow & Darnall & Amatikulu & Felixton & Umfolozi & Pongola & Komati & Malelane & Durban \\
\hline Umzimkulu & 0 & 52.5 & 141 & 212 & 220 & 163 & 195 & 204 & 245 & 280 & 329 & 489 & 907 & 847 & 122 \\
\hline Sezela & 52.5 & 0 & 97 & 190 & 198 & 119 & 150 & 160 & 201 & 235 & 285 & 522 & 863 & 810 & 77 \\
\hline Eston & 141 & 97 & 0 & 85 & 93 & 111 & 142 & 152 & 193 & 227 & 277 & 436 & 755 & 708 & 76 \\
\hline Noodsberg & 212 & 190 & 85 & 0 & 8 & 72 & 92 & 111 & 152 & 186 & 236 & 396 & 694 & 634 & 122 \\
\hline Union Coop & 220 & 198 & 93 & 8 & 0 & 78 & 99 & 118 & 158 & 193 & 243 & 402 & 680 & 626 & 124 \\
\hline Maidstone & 163 & 119 & 111 & 72 & 78 & 0 & 40 & 48 & 89 & 123 & 173 & 332 & 742 & 689 & 49 \\
\hline Gledhow & 195 & 150 & 142 & 92 & 99 & 40 & 0 & 21 & 62 & 96 & 146 & 305 & 715 & 662 & 73 \\
\hline Darnall & 204 & 160 & 152 & 111 & 118 & 48 & 21 & 0 & 42 & 83 & 133 & 292 & 702 & 648 & 90 \\
\hline Amatikulu & 245 & 201 & 193 & 152 & 158 & 89 & 62 & 42 & 0 & 51 & 111 & 271 & 668 & 615 & 124 \\
\hline Felixton & 280 & 235 & 227 & 186 & 193 & 123 & 96 & 83 & 51 & 0 & 59 & 218 & 681 & 628 & 158 \\
\hline Umfolozi & 329 & 285 & 277 & 236 & 243 & 173 & 146 & 133 & 111 & 59 & 0 & 208 & 625 & 573 & 208 \\
\hline Pongola & 489 & 522 & 436 & 396 & 402 & 332 & 305 & 292 & 271 & 218 & 208 & 0 & 466 & 413 & 374 \\
\hline Komati & 907 & 863 & 755 & 694 & 680 & 742 & 715 & 702 & 668 & 681 & 625 & 466 & 0 & 57 & 785 \\
\hline Malelane & 847 & 810 & 708 & 634 & 626 & 689 & 662 & 648 & 615 & 628 & 573 & 413 & 57 & 0 & 741 \\
\hline Durban & 122 & 77 & 76 & 122 & 124 & 49 & 73 & 90 & 124 & 158 & 208 & 374 & 785 & 741 & 0 \\
\hline
\end{tabular}

Table 6.10: Distances between land areas $(\mathrm{km})$ (calculated from roads in google map)

\begin{tabular}{|c|c|c|c|c|c|c|c|c|c|c|}
\hline \multirow{3}{*}{ Regions } & \multicolumn{4}{|c|}{ Sugar production capacity(Thousand Tonnes) } & \multicolumn{6}{|c|}{ Ethanol production capacity(Thousand Tonnes) } \\
\hline & \multicolumn{2}{|c|}{ Raw sugar } & \multicolumn{2}{|c|}{ White sugar } & \multicolumn{2}{|c|}{ From Cane $(q=1)$} & \multicolumn{2}{|c|}{ From sugar(Raw/white) $(q=2)$} & \multicolumn{2}{|c|}{ Both Technologies $(q=3)$} \\
\hline & Minimum & Maximum & Minimum & Maximum & Minimum & Maximum & Minimum & Maximum & Minimum & Maximum \\
\hline Umzimkulu & 100 & 160 & 100 & 250 & 2 & 300 & 2 & 300 & 3 & 600 \\
\hline Sezela & 200 & 290 & 100 & 250 & 2 & 300 & 2 & 300 & 3 & 600 \\
\hline Eston & 100 & 175 & 0 & 0 & 2 & 300 & 2 & 300 & 3 & 600 \\
\hline Noodsberg & 100 & 200 & 0 & 0 & 2 & 300 & 2 & 300 & 3 & 600 \\
\hline Union-Coop & 80 & 100 & 0 & 0 & 2 & 300 & 2 & 300 & 3 & 600 \\
\hline Maidstone & 150 & 235 & 0 & 0 & 2 & 300 & 2 & 300 & 3 & 600 \\
\hline Gledhow & 100 & 175 & 0 & 0 & 2 & 300 & 2 & 300 & 3 & 600 \\
\hline Darnall & 100 & 175 & 0 & 0 & 2 & 300 & 2 & 300 & 3 & 600 \\
\hline Amatikulu & 100 & 200 & 0 & 0 & 2 & 300 & 2 & 300 & 3 & 600 \\
\hline Felixton & 150 & 250 & 0 & 0 & 2 & 300 & 2 & 300 & 3 & 600 \\
\hline Umfolozi & 100 & 140 & 0 & 0 & 2 & 300 & 2 & 300 & 3 & 600 \\
\hline Pongola & 90 & 120 & 0 & 0 & 2 & 300 & 2 & 300 & 3 & 600 \\
\hline Komati & 100 & 150 & 0 & 0 & 2 & 300 & 2 & 300 & 3 & 600 \\
\hline Malelane & 100 & 200 & 100 & 250 & 2 & 300 & 2 & 300 & 3 & 600 \\
\hline Durban & 0 & 0 & 100 & 250 & 2 & 300 & 2 & 300 & 3 & 600 \\
\hline Total & 1570 & 2570 & 400 & 1000 & 30 & 4500 & 30 & 4500 & 45 & 9000 \\
\hline
\end{tabular}

Table 6.11: Production Capacity (Thousand Tonnes) (guessed) 



\section{Chapter 7}

\section{Conclusions and Future Directions}

\subsection{Summary and Conclusions}

The first and the main motivating factor for embarking on this research project was that we were concerned about dealing with higher degrees of uncertainty in multi-criteria decision-making (MCDM) problems. This issue has been frequently observed in many real-world processes such as strategic planning. In many real-life problems, policy-makers must be able to satisfy several conflicting criteria/objectives. Moreover, in most of these problems, the decisions must be made before one can begin to explore the consequences of the decision or gain sufficient awareness of all other options. In our fast-growing and highly competitive world, ignoring the consequences of our decisions over the uncertain future could lead us to some devastating penalties and, in some cases, the losses may not be recoverable at all. Reducing or predicting this so-called deep uncertainty is almost impossible. Deep uncertainty is also not statistical in nature. Therefore, all statistical and predictive modelling will fail to deal with deep uncertainty. However, most of the previous works in this field of study ignore deep uncertainty because of its complexity, and are limited to a low level of uncertainty with known probability distributions (namely mild uncertainty). To the best of our knowledge, there is limited or no literature or scientific work concerning deep uncertainty in MCDM/Multi-Objective Optimisation (MOO) problems and how these problems can be addressed. This is a crucial gap in the literature and the DMs must face it one way or another. Therefore, this thesis tried to provide a way forward as well as further understanding of the complex problems associated with deep uncertainty in MCDM/MOO.

The literature review (Chapter 2) on uncertainty indicated that different dimensions of uncertainty (such as nature, location and degrees/levels of uncertainty) can be studied. However, it seems that the degrees of uncertainty have a crucial role in modelling the multi-criteria decisionmaking and optimisation problems. We defined three intermediate uncertainties between complete certainty and total ignorance as mild, moderate, and deep uncertainty. In this study, the term deep uncertainty refers to a situation in which "outcomes cannot be completely enumerated, so that, probabilities are not definable". We believe that this classification can help us to choose the most suitable strategy and improve the decision-making process in real-world problems.

To deal with this kind of uncertainty, especially in problems with a long-term planning window, we need planning strategies that are both robust (can perform satisfactorily under a wide variety of plausible futures) and dynamic (can be adapted to changing variable future conditions). The review of optimisation problems in the literature review revealed only a few publications which utilise this so-called dynamics robustness approach to handle the uncertainty in multi-objective optimisation, but none of them can deal with deep uncertainty. This was 
identified as an important gap in the literature and was an interesting direction for our research. Nevertheless, a high level of complexity has prevented other authors from working on this kind of problem. Therefore, our study needed to be well structured and carefully developed.

To achieve this purpose, this thesis (in Chapter 3) introduced a novel robust-dynamic optimisation approach, named the scenario-based two-stage framework, to deal with multi-objective optimisation problems under deep uncertainty. The main idea was extended by the two-stage stochastic programming with recourse to address the capability of dealing with deep uncertainty through the use of scenario planning rather than statistical expectation. Scenarios are utilised as a dimension of preference (a component of the meta-criteria) to avoid problems of evaluating probabilities under deep uncertainty. Moreover, to some extent, the meta-criteria concept is extended to find a robust decision. Such scenario-based thinking involves a multi-objective representation of performance under different future conditions as an alternative to expectation, which fits naturally into the broader multi-objective problem context. This dynamic-robust multiobjective optimisation structure can help us handle moderate and deep uncertainty in complex MCDA problems. The proposed methodology introduces the concept of dynamic-robust decisions by extending the robust decision philosophy. These kinds of decisions reduce the shortcoming of the regular robust strategies such as generating a risk-averse solution because of a bad scenario. Also, the advantages of the robust solutions are developed. A dynamic-robust decision in our philosophy is a split decision and contains two subgroups of decisions, namely the initial decision(s) followed by recourse decision(s), in which the full decision is robust across scenarios.

It is shown that the proposed two-stage structure not only helps us cope with higher degrees of uncertainty but also can contribute to solving the scenario-based problems without a feasible nor robust solution. This approach does not look for a solution that could be feasible in every single scenario. Such a solution, if it exists, may cost too much as it needs to satisfy all constraints of all plausible scenarios, while only one scenario will happen.

In the proposed two-stage structure, rather than formulating a predictive model, we try to identify a reasonable initial decision (which is compensable, whatever happens in the future) together with an appropriate scenario-dependent recourse decision for every single plausible scenario, implemented after scenario realisation. In fact, we break the decision into two parts and defer one part of the decision long enough to realise the uncertain parameter(s). In other words, in the two-stage structure, we look one step ahead, postpone part of the decision and leave room for possible adaptation later (after scenario realisation), while we identified suitable adaptive plans for every plausible scenario in advance. The aggregation of the decisions in both stages provides us with the overall optimality to the two-stage problem. Therefore, by using the proposed method, any specific cost would be postponed to the second stage and after scenario realisation. Thus, there is no need to pay costs for some scenarios that would never be revealed.

To be able to deal with the deep uncertainty in strategic planning problems, which regularly have more than two stages, the three-stage structure and moving horizon algorithms were also introduced as extensions of the two-stage framework (Chapter 4). "Stages", in this study, are artificial constructs to structure thinking of an indefinite future in which scenarios are not independent events by stages and continuously unfold, generally with some depending.

Philosophically, the proposed two-stage structure always plans and looks one step ahead while the three-stage structure considers the conditions and consequences of two upcoming steps in advance. Ignoring long-term consequences of decisions, as well as likely conditions, could not be a robust strategic approach. Therefore, generally, by utilising the three-stage structure, we may expect a more robust decision than with a two-stage representation.

In comparing the two-stage versus the three-stage structure, the fundamental problem was comparability of the two methods because of the different time horizon, as the two-stage model is ignorant of the third stage. We needed to compare these methods in the same period, for which a two-stage moving horizon approach is introduced. In the two-stage moving horizon model, we iterate a two-stage structure twice. i.e. at the first iteration, one two-stage model is applied which covers the first two stages (stages 1 and 2). Then, another (second) two-stage 
model starting at the end of the first stage, is re-iterated to cover the entire period of three stages (stages 2 and 3 ).

Modelling time preferences in multi-stage problems was also introduced to solve the fundamental problem of comparability of the two proposed methodologies because of the different time horizon (the two-stage model is ignorant of the third stage). This concept has been applied by differential weighting in the models. Importance weights, then, are primarily used to make the two- and three-stage models more directly comparable, and only secondarily as a measure of risk preference. Differential weighting can help us apply further preferences in the model and lead it to generate more preferred solutions.

The problem with having more than three stages is that this will generate too many metascenarios which would lead us to a computationally expensive model that cannot easily be solved, if it all. Furthermore, identifying the plausible meta-scenarios would be challenging if the length of the planning window increases. Therefore, the two- and three-stage moving horizon models in a T-stage planning window was introduced (Chapter 5. Due to the large number of plausible metascenarios, it is hardly possible to analyse and evaluate the proposed moving horizon algorithms in problems with more than five stages. Therefore, a Simulation-Optimisation study was performed to simulate the reasonable number of meta-scenarios and enable evaluation, comparison and analysis of the proposed methods for the problems with a T-stage planning horizon. In this Simulation-Optimisation study, we started by setting the current scenario, the scenario that we were facing at the beginning of the period. Then, the optimisation model was run to get the first-stage decisions which can be implemented immediately. Thereafter, the next scenario was randomly generated by using Monte Carlo simulation methods. In deep uncertainty, we do not have enough knowledge about the likelihood of plausible scenarios nor the probability space; therefore, to simulate the deep uncertainty we did not use anything of scenario likelihoods in the decision models. The two- and three-stage Simulation-Optimisation algorithms were also proposed.

A comparison of these algorithms showed that the solutions to the two-stage moving horizon model are feasible when compared to the three-stage model. Also, the optimal solution to the three-stage moving horizon model is not dominated by any solutions of the other model. So, with no doubt, it must find better, or at least the same, goal achievement compared to the twostage moving horizon model. Accordingly, the three-stage moving horizon model evaluates and compares the optimal solution of the corresponding two-stage moving horizon model to the other feasible solutions. Then, if it selects anything else, it must either be better in goal achievement or be robust in some future scenarios or a combination of both. However, the cost of these supremacies must be considered (as it may lead us to a computationally expensive problem), and the efficiency of applying this structure needs to be approved.

Obviously, using the three-stage structure in comparison with the two-stage approach brings more complexity and calculations to the models. It was also shown that the solutions to the three-stage model would be preferred to the solutions provided by the two-stage model under most circumstances. However, by the "efficiency" of the three-stage framework in our context, we wanted to know whether utilising this approach and its solutions is worth the expense of the additional complexity and computation.

The experiments in this study showed that the three-stage model has advantages under most circumstances(meta-scenarios), but that the gains are quite modest. This issue is frequently observed when comparing these methods in problems with a short-term (say less than five stages) planning window. Nevertheless, analysis of the length of the planning horizon and its effects on the solutions to the proposed frameworks indicate that utilising the three-stage models is more efficient for longer periods because the differences between the solutions of the two proposed structures increase with every iteration of the algorithm in moving horizon models. Moreover, during the long-term calculations, we noticed that the two-stage algorithm failed to find the optimal solutions for some iterations while the three-stage algorithm found the optimal value in all cases. 
Thus, it seems that for the planning horizons with more than ten stages, the efficiency of the three-stage model may be worth the expenses associated with additional complexity and computation. Nevertheless, if the DM prefers to not use the three-stage structure because of the complexity and/or calculations, the two-stage moving horizon model can provide us with some reasonable solutions, although they might not be as good as the solutions generated by a three-stage framework.

Finally, in Chapter 6, the power of the proposed methodology when applied to real cases was examined. The proposed two-stage structure was applied in the sugarcane industry to analyse the whole infrastructure of the sugar and bioethanol Supply Chain (SC) in such a way that all economics ( $\max$ profit), environmental $\left(\min \mathrm{CO}_{2}\right.$ ), and social benefits (max job-creation) were optimised under six key uncertainties, namely sugarcane yield, ethanol and refined sugar demands and prices, and the exchange rate. Moreover, one of the critical design questions that is, to design the optimal number and technologies as well as the best place(s) for setting up the ethanol plant(s) - was also addressed in this study. The general model for the strategic planning of sugar-bioethanol supply chains (SC) under deep uncertainty was formulated and also examined in a case study based on the South African Sugar Industry. This problem is formulated as a Scenario-Based Mixed-Integer Two-Stage Multi-Objective Optimisation problem and solved by utilising the Generalised Goal Programming Approach.

In conclusion, the traditional MCDM approaches involving uncertainty about the future are usually divided into two groups: 1) probabilistic and stochastic approaches, 2) predictive models. Predictive models are usually failed by surprises, as nothing is completely predictable in real life. Furthermore, probabilistic methods struggle with setting probabilities and distributions which are not only difficult to compute, but also limited the real problems with some artificial assumptions that may not really exist in these problems. Therefore, the outcomes of the decisions may not fit well in reality.

The proposed scenario-based methodology brings novel insight for decision makers, provides a number of important advantages and opens a new viewpoint in decision-making problems. In this structure, we do not use the models to predict the future; instead, we are utilising the predictions to improve the robustness without getting involved in identifying the inaccurate probabilities which increase the vagueness and calculations. In fact, in the proposed methodology, the DMs can freely think about plausible scenarios without getting stuck in the probabilities trap.

Moreover, long-term decision-making has a dynamic environment and thus needs dynamic planning. In such a problem, decisions need to be adaptable in the case of surprises; therefore, dynamic decision-making is necessary. Furthermore, decisions need to be robust to provide a sustainable plan that works well in a broad range of variations/scenarios. However, in typical robust approaches, the robust decision usually plans to work reasonably in the worst case. This insight can be too costly and too risk-averse which would waste a lot of resources if any other scenario unfolded.

In contrast, the proposed scenario-based decision-making structure is not focused on the worst, best, or the most plausible scenario. Instead, it provides a dynamic environment for decision-making by dividing the entire decision into some contingent decisions depending on what may happen in the future. In other words, we provide some contingency plans (one for each plausible scenario) in each stage. After scenario realisation the relevant plan/decision will be implemented immediately. The aggregation of these contingency plans (which were implemented in different stages of the planning horizon of a problem relevant to the unfolded scenarios), together with the initial decision(s) (which were implemented at the beginning of the planning window), will make the optimal overall decision. In fact, we do not change our initial plan; instead, in each stage we choose the relevant contingency plan (depending on what happens during the project) from the plans which were already identified. However, at the end of the planning horizon, the aggregation of all the implemented decisions will be optimal. The proposed methodology in this thesis has changed the old insight of decision-making from finding the best decision that works well in an unknown future (perhaps with some variations), to the insight of 
dynamic decision-making which includes a series of some consecutive decisions.

Therefore, the proposed methodology is, to the best of our knowledge, the only approach that can successfully handle deep uncertainty in MCDM/MOO problems with both short- and long-term planning horizons. It is generic enough to be used in all MCDM problems under deep uncertainty. The two- or three-stage structures can be used properly in the MCDM/Decision Support problems with a planning window of two or three stages. Furthermore, moving horizon models are recommended to be utilised in problems with more than three stages such as strategic planning. Depending on the complexity of the problem and the efficiency of moving the two- or three-stage structure in a T-stage planning window, the $\mathrm{DM}(\mathrm{s})$ can choose one of the proposed moving horizon algorithms. Modelling time preferences can help the $\mathrm{DM}(\mathrm{s})$ to apply further preferences in the model and improves the solutions.

However, there remain many theoretical and applicational challenges which need to be addressed. The next section points out some of these challenges, and provides some other interesting directions for future research.

\section{2 $\quad$ Future Directions}

The concepts, structures, and methodology that were introduced in this thesis were generic and there will be various opportunities for further examination and improvements. Moreover, some challenges in theory and/or applications still need to be considered and addressed. Some of these challenges and the most interesting topics for future research can be listed as follows:

1. Handling a large number of scenarios: The most challenging issue on this methodology is the limited number of scenarios that can be handled. Further study on this issue seems to be necessary and would improve the structure. Some scenario reduction algorithm or utilising a novel solution method may help to achieve this purpose.

2. Surprises are out of our expectations: This thesis considers the surprises as plausible scenarios; however, it is possible that some surprises come from outside of the considered scenarios. Dealing with these kinds of uncertainty could be challenging and may need some changes in structuring.

3. Non-linear Problems: In this thesis, the proposed structure is applied in only Linear Problems (LP). Non-linear problems would be an important direction for future research. Different solution methods may also need to be examined to solve the non-linear problems.

4. Examine different solution methods: The generalised goal programming approach is used, in this study, to solve the MOLP and MILP problems. There are some other interesting methods, such as NAUTILUS and NIMBUS, that could contribute to the proposed methodology, and also some multi-objective evolutionary algorithms, such as NSGA.

5. Finding the Pareto hyperplanes in moving horizon methods: Identifying (or even the approximating) the hyperplane equations of the Pareto optimal solutions would be useful and interesting. However, because of the complexity of the problem, the required computation may expensive and hardly possible. Nonetheless, it could be an interesting subject for future research.

6. Comparing with dynamic programming: Although this framework has a dynamic nature, it is different to dynamic programming. For example, the stages in dynamic programming must be the same (eg. annually) while there is no limitation for this in the proposed methodology (i.e. each stage can have its own distance). Therefore, a complete comparison (mathematically and conceptually) could be interesting and educational.

7. Improving the robustness of the proposed methodology: Finding a generalised robust two- and three-stage moving horizon model needs to pass many tests on different 
real-world problems, and maybe a classification of the involved problems is required. In this thesis, some simple (but useful) ideas have been raised that could be used as a starting point in future work.

8. Expansion on Sugar-bioethanol supply chains: This study considers the infrastructures of the sugar-ethanol SCs. Other biofuel SCs such as corn-ethanol can also be considered by applying the proposed methodology. Considering other uncertain factors, such as water consumption, would also be another interesting direction for future work. It is likely that this method can be applied to all other supply chain problems under deep uncertainty. Furthermore, only the first generation of biofuel SCs was investigated in this study. The second generation, or even the third generation that will be able to operate in a near future, could be an interesting and useful area to extend the proposed methodology in the future. More importantly, applying the three-stage and moving horizon models in $\mathrm{SC}$ problems can be considered as one of the most interesting directions for our future work.

9. Real-life applications: As the proposed methodology has a very generic structure, it can be easily utilised in various real-world problems that face deep uncertainty and which include several conflicting criteria/objectives, such as socio-economic risks, environmental risk, climate change, portfolio management, scheduling, energy saving, power systems, water management, supply chain network design, energy retrofits, and decision support systems. However, some new application areas could be challenging and could require some restructuring of the model.

10. Application for basic OR problems: Expand and examine the proposed methodology in some basic OR problems such as multi-objective knapsack, assignment, and minimum cost/maximum flow problems under conditions of deep uncertainty. Applying the proposed methodology in these basic problems could be an interesting direction that may be challenging in a different way.

11. Comparing with other approaches: Using other GP variants such as extended, meta or multi-choice GP as a comparison with GGP would be an interesting study. Moreover, comparing these models with stochastic (probability-based) models to examine effect/benefit of knowing the probability would also be interesting.

12. Setting too many goals and weights by the DM: As mentioned earlier, this study does not concentrate on the system of goal (and weight) choice. Considering all the metacriteria in the proposed framework may lead us to solve problems with too many objectives. Therefore, the DM needs to set too many goals (and importance weights). In practice, eliciting much information from the DM is hardly possible, if ever. Accordingly, further study on this issue seems to be necessary and very interesting.

13. New visualisation: It is clear that drawing the Pareto front for a high dimensional problem such as what we are facing in scenario-based approaches is almost impossible. Although a few ways for comparing the results and visualisation of them are suggested in this thesis, still a more clear visualisation seems to be necessary for better comparisons, analysis and decision-making in multi-objective scenario-based methods. 


\section{Appendix A: Case study data}

Table 1: SA sugar-ethanol SC data

\begin{tabular}{|c|c|}
\hline $\begin{array}{l}\text { Uncertain Parameters } \\
Y_{k}^{0}=[50,60,70] ;: \\
D E_{k}^{1}=10^{5} *[3.5,4,4.5] \\
D S_{k}^{1}=10^{5} *[1.5,2.6] ; \\
P E_{k}^{1}=10^{3} *[1.3,1.7,3.5] \\
P S_{k}^{1}[300,600] ; \\
R_{k}^{1}=[10,14,17] ;:\end{array}$ & $\begin{array}{l}\text { SugarCane Yield }(\mathrm{T} / \mathrm{ha}), \text { if } s\left(k_{1}\right) \text { unfolded, } k_{1}=[\text { Poor Normal Good }] \\
\text { Ethanol demand }(1000 \text { gal }) \text { if } s\left(k_{2}\right) \text { revealed, } k_{2}=[\text { Low Normal High }] \\
\text { Refined sugar demand (Tonnes) if } s\left(k_{3}\right) \text { revealed, } k_{3}=[\text { Low High }] \\
\text { Ethanol price }\left(\$ / 1000 \text { ga), if } s\left(k_{4}\right) \text { revealed, } k_{4}=[\text { Low Normal High }]\right. \\
\text { Refined sugar price }(\$ / \text { Ton }) \text {, if } s\left(k_{5}\right) \text { revealed, } k_{5}=[\text { Low High }] \\
\text { Exchange rate of US Dollar to Rand. }\end{array}$ \\
\hline $\begin{array}{l}\text { Certain Parameters } \\
\text { (Conversion factors) } \\
\alpha=0.12 \\
\beta=0.019 \\
\zeta=(0.04 * 166.66) / 1000 \\
\theta=0.1354 \\
\lambda=0.141 \\
\sigma=0.41324 \\
\nu=5.5 \\
\iota=0.55 \\
\xi=0.96 \\
\rho=1\end{array}$ & $\begin{array}{l}\text { Cane to Raw sugar } \\
\text { Raw sugar to Refined sugar } \\
\text { Cane to Molasses (1000 ga of molasses) } \\
\text { Raw sugar to Ethanol (1 Ton of raw sugar yields } 135.4 \text { gallons of ethanol) } \\
\text { Refined sugar to Ethanol (1 Ton of refined sugar yields } 141 \text { gallons of ethanol) } \\
\text { Raw sugar price compare to Refined sugar price } \\
\text { By-product of Ethanol price compare to Ethanol price } \\
\text { Molasses price compare to Refined sugar price } \\
\text { Local price of Refined sugar } \\
\text { Local price of Ethanol }\end{array}$ \\
\hline
\end{tabular}


Table 1 - Continued from previous page

\begin{tabular}{|c|c|}
\hline $\begin{array}{l}\vartheta=1.5 \\
\kappa_{q}=[0.002,0.003] \\
\tau=0.2\end{array}$ & $\begin{array}{l}\text { Raw sugar demand compare to Refined sugar demand } \\
\text { Produce by-product with technology q=1,2 } \\
\text { Tax exemption factor for local sell of Ethanol (20\%) }\end{array}$ \\
\hline $\begin{array}{l}\text { Costs } \\
c_{1}^{0}=225,: \\
c_{2}^{0}=50,: \\
c_{3}^{0}=300,: \\
c_{4}^{0}=410,: \\
c_{5}^{0}=350,: \\
c_{6}^{0}=80,: \\
c_{7}^{0}=400,: \\
c_{20}^{1}=330,: \\
c_{8 r q}^{0}=10^{6} *[150,160,240],:\end{array}$ & $\begin{array}{l}\text { Cost of renting, growing, harvesting and transporting a tonne of sugar cane to the Mill. } \\
\text { Cost of producing a tonne of raw sugar. } \\
\text { Cost of producing a thousand gallons of ethanol from cane. } \\
\text { Cost of producing a thousand gallons of ethanol from molasses. } \\
\text { Cost of producing a thousand gallons of ethanol from raw sugar. } \\
\text { Cost of producing a tonne of refined sugar from raw sugar. } \\
\text { Cost of producing a thousand gallons of by-product. } \\
\text { Cost of producing a thousand gallons of ethanol from refined sugar } \\
\text { Cost of installation and operation of an ethanol plant with technology } q,(q=1,2,3) \text {. }\end{array}$ \\
\hline $\begin{array}{l}c_{1} 7^{1}=4 \\
c_{1} 8^{1}=4 \\
c_{1} 9^{1}=1.5\end{array}$ & $\begin{array}{l}\text { Cost of importing a tonne of raw sugar }(200 \% \operatorname{tax}) \\
\text { Cost of importing a tonne of refined sugar }(200 \% \operatorname{tax}) \\
\text { Cost of importing a thousand gallons of ethanol( } 50 \% \operatorname{tax})\end{array}$ \\
\hline $\begin{array}{l}0.5 \\
0.5\end{array}$ & $\begin{array}{l}\text { The cost of transporting a tonne of any product for a } \mathrm{Km} \text {. } \\
\text { The cost of transporting } 1000 \text { gallons of any liquid product for a } \mathrm{Km} \text {. }\end{array}$ \\
\hline $\begin{array}{l}C O_{2} \text { emissions } \\
d_{1}^{0}=26.5,: \\
d_{2}^{0}=135,: \\
d_{3}^{0}=211,: \\
d_{4}^{0}=211,: \\
d_{5}^{0}=211,: \\
d_{6}^{0}=382,: \\
d_{7}^{0}=500,: \\
d_{20}^{1}=211,: \\
d_{8 r q}^{0}=10^{3} *[150380450],:\end{array}$ & $\begin{array}{l}\mathrm{kg} C O_{2} \text { emissions of renting, growing, harvesting and transporting a tonne of sugarcane to the Mill. } \\
\mathrm{kg} C \mathrm{O}_{2} \text { emissions of producing a tonne of raw sugar. } \\
\mathrm{kg} C \mathrm{O}_{2} \text { emissions of producing a thousand gallons of ethanol from cane. } \\
\mathrm{kg} C O_{2} \text { emissions of producing a thousand gallons of ethanol from molasses. } \\
\mathrm{kg} C O_{2} \text { emissions of producing a thousand gallons of ethanol from raw sugar. } \\
\mathrm{kg} C O_{2} \text { emissions of producing a tonne of refined sugar from raw sugar. } \\
\mathrm{kg} C O_{2} \text { emissions of producing a thousand gallons of by-product. } \\
\mathrm{kgCO} \text { emissions of producing a thousand gallons of ethanol from refined sugar } \\
\mathrm{kg} C O_{2} \text { emissions of installation and operation of an ethanol plant with technology } q .\end{array}$ \\
\hline
\end{tabular}

Continued on next page 
Table 1 - Continued from previous page

\begin{tabular}{|c|c|}
\hline$d 17=435$, & $\mathrm{kg} \mathrm{CO}_{2}$ emissions of importing a tonne of raw sugar \\
\hline$d 18=682$, & $\mathrm{kg} \mathrm{CO}_{2}$ emissions of importing a tonne of refined sugar \\
\hline$d 19=451$ & $\mathrm{~kg} \mathrm{CO}_{2}$ emissions of importing a thousand gallons of ethanol \\
\hline 0.01072, & The $\mathrm{kg} C \mathrm{C}_{2}$ emissions of transporting a tonne of any product for a $\mathrm{Km}$. \\
\hline 0.00846 & The $\mathrm{kg} \mathrm{CO}_{2}$ emissions of transporting 1000 gallons of any liquid product for a $\mathrm{Km}$. \\
\hline$e^{0}=996,:$ & Total $\mathrm{kg} \mathrm{CO}_{2}$ saving in blending a thousand gallons of ethanol in petrol. \\
\hline \multicolumn{2}{|l|}{ Jobs } \\
\hline$h_{1}^{0}=0.08,:$ & Total number of permanent jobs that created per hectare of cultivated land. \\
\hline$h_{2}^{0}=121 / 12,:$ & Total number of seasonal jobs that created per hectare of cultivated land. \\
\hline$h_{3}^{0}=0.1964,:$ & Total number of permanent jobs that created to producing a tonne of raw sugar at a Mill. \\
\hline$h_{4}^{0}=0.2143,:$ & Total number of permanent jobs that created to producing a tonne of white sugar at a refinery. \\
\hline$h_{5 q}^{0}=[22.82,22.82],:$ & $\begin{array}{l}\text { Total number of permanent jobs that created to producing a thousand gallons of ethanol in plant } \\
\text { with division/technology } q \text {. }\end{array}$ \\
\hline$h_{6 q}^{0}=[0,0],:$ & $\begin{array}{l}\text { Total number of permanent jobs that created to producing a thousand gallons of by-product in plant } \\
\text { with division/technology } q \text {. }\end{array}$ \\
\hline $\begin{array}{l}h_{7 q}^{0}=2000 *[1000,1000,1500],: \\
h_{8 q}^{0}=2000 *[100,100,175],:\end{array}$ & $\begin{array}{l}\text { Total number of temporary jobs that created to constructing an ethanol plant with technology } q \text {. } \\
\text { Total number of permanent jobs that created to operating an ethanol plant with } \\
\text { division/technology } q \text { (exclude production jobs). }\end{array}$ \\
\hline 0.002 , & Jobs (man-hrs/t) created to transporting a tonne of any product for a $\mathrm{Km}$. \\
\hline 0.002 , & Jobs (man-hrs/t) created to transporting 1000 gallons of any liquid product for a Km. \\
\hline
\end{tabular}




\section{Appendix B: Case study results (Figures)}

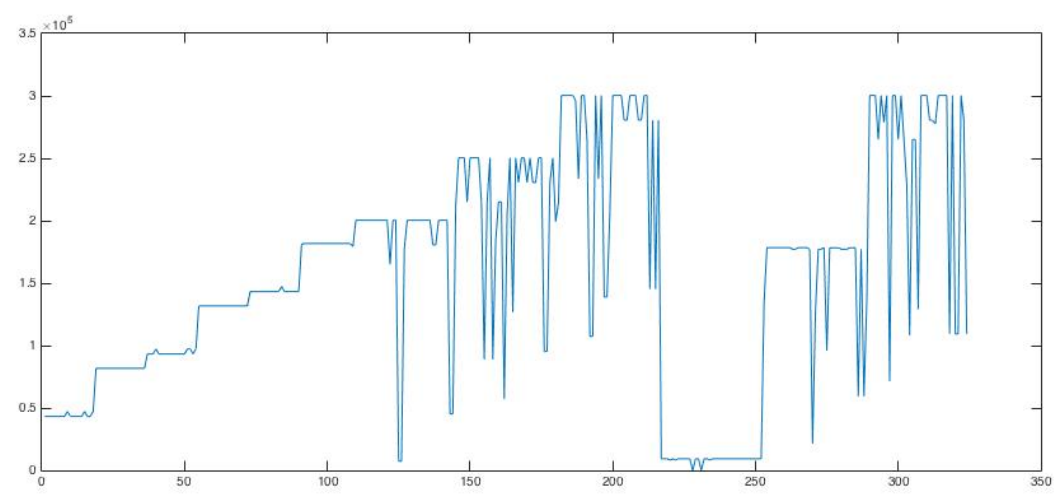

Figure 1: Ethanol importation in different meta-scenarios

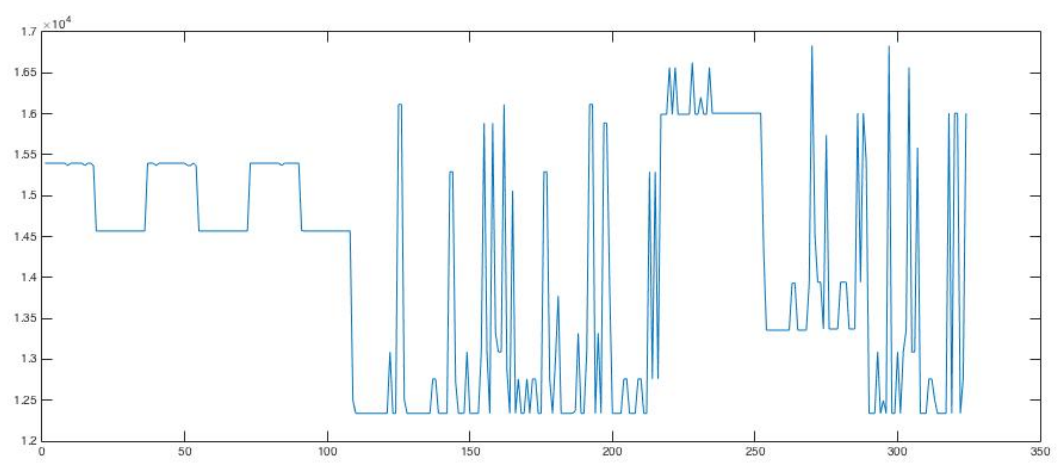

Figure 2: By-product Sale in different meta-scenarios 


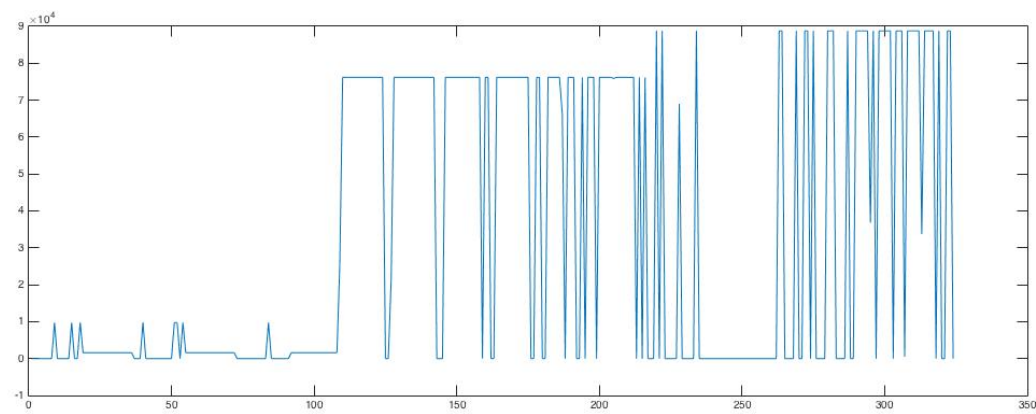

Figure 3: Molasses Sale in different meta-scenarios

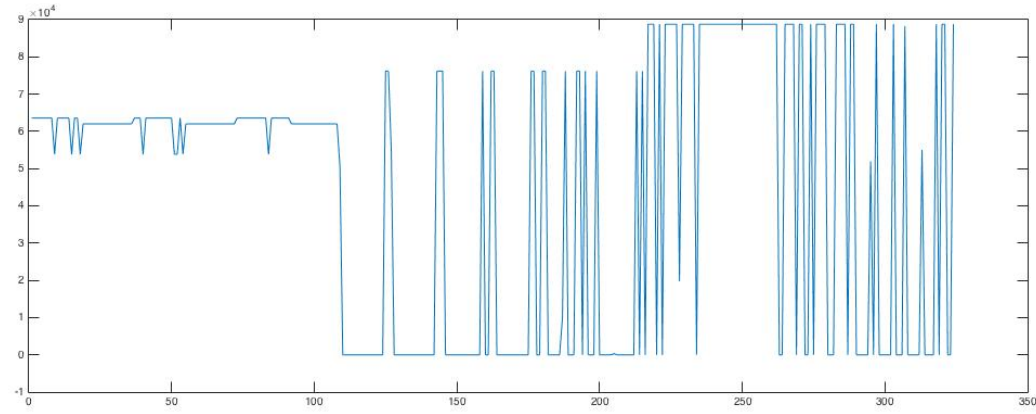

Figure 4: Molasses send back to ethanol plants (Recourse decision) in different metascenarios

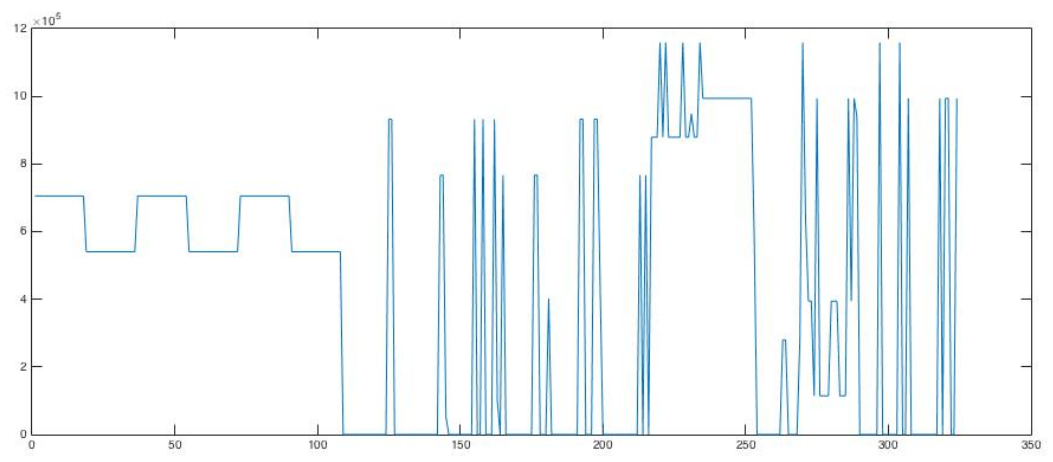

Figure 5: Raw sugar send back to ethanol plants (Recourse decision) in different metascenarios 


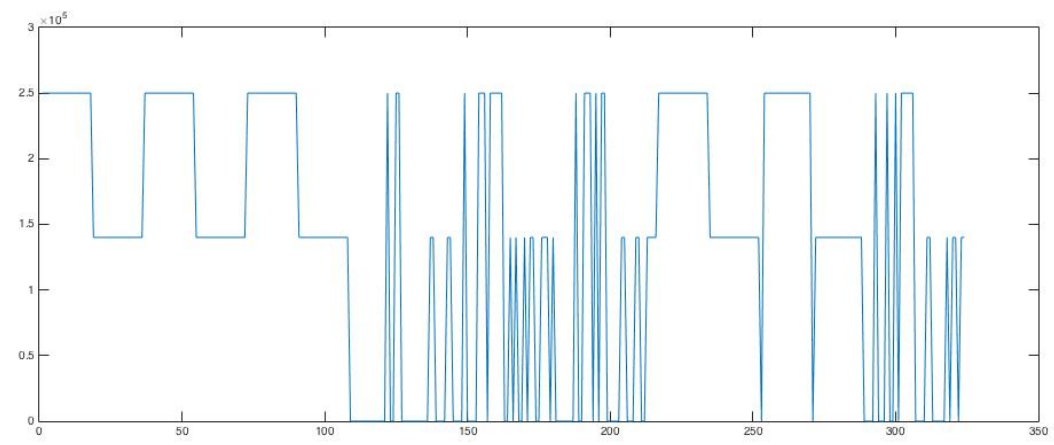

Figure 6: White sugar send back to ethanol plants (Recourse decision) in different metascenarios

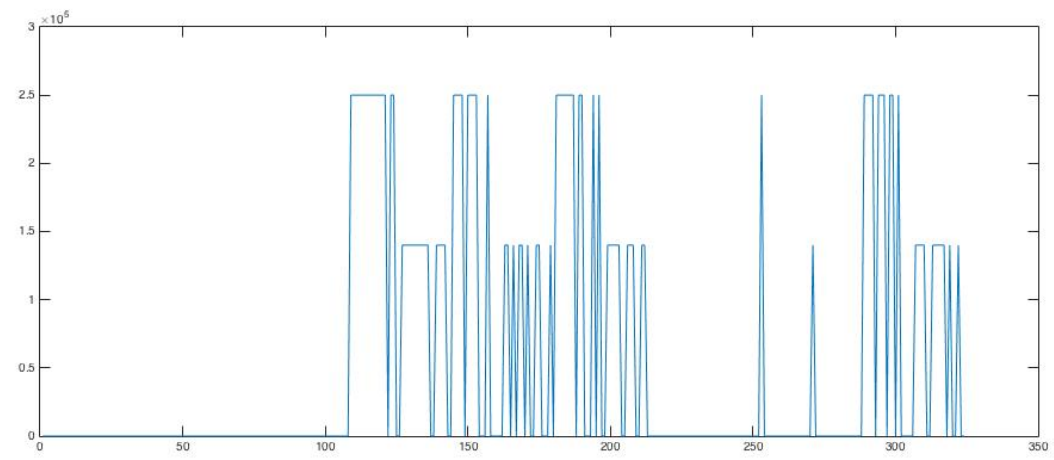

Figure 7: White sugar Exportation in different meta-scenarios

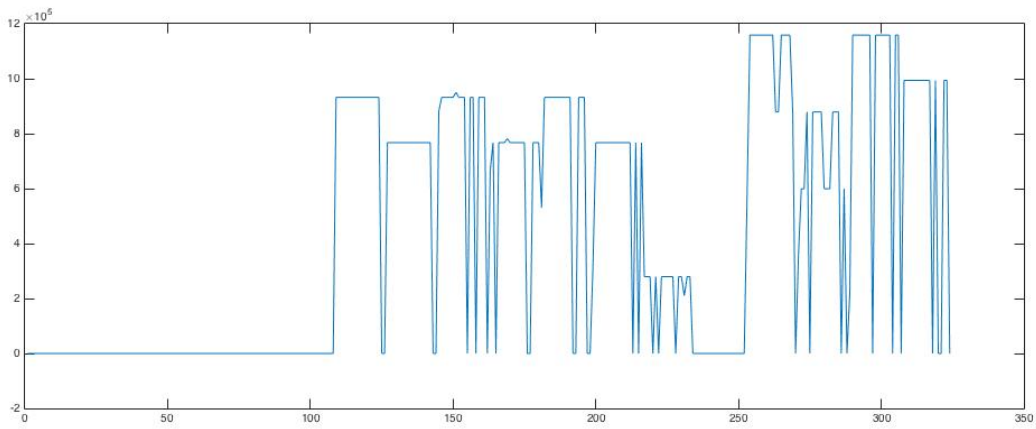

Figure 8: Raw sugar Exportation in different meta-scenarios 


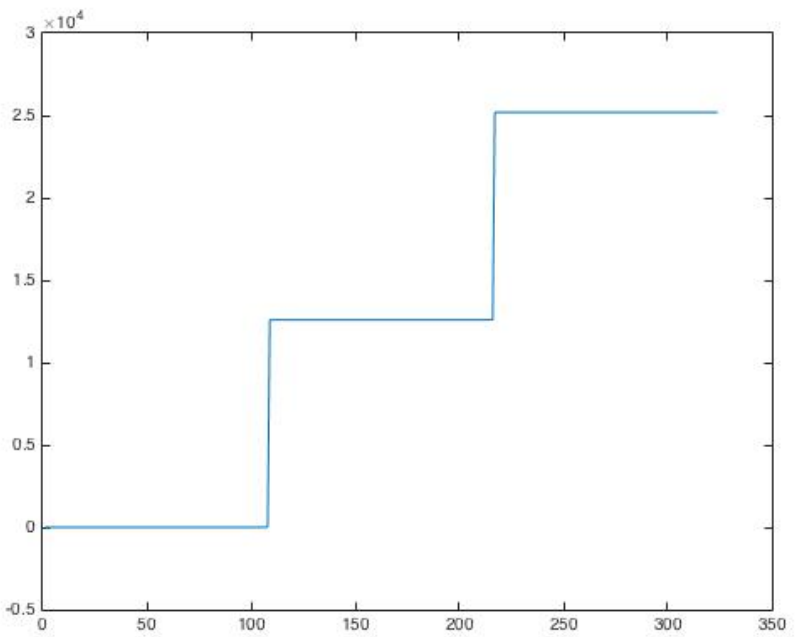

Figure 9: Total Molasses Production Recourse in different meta-scenarios

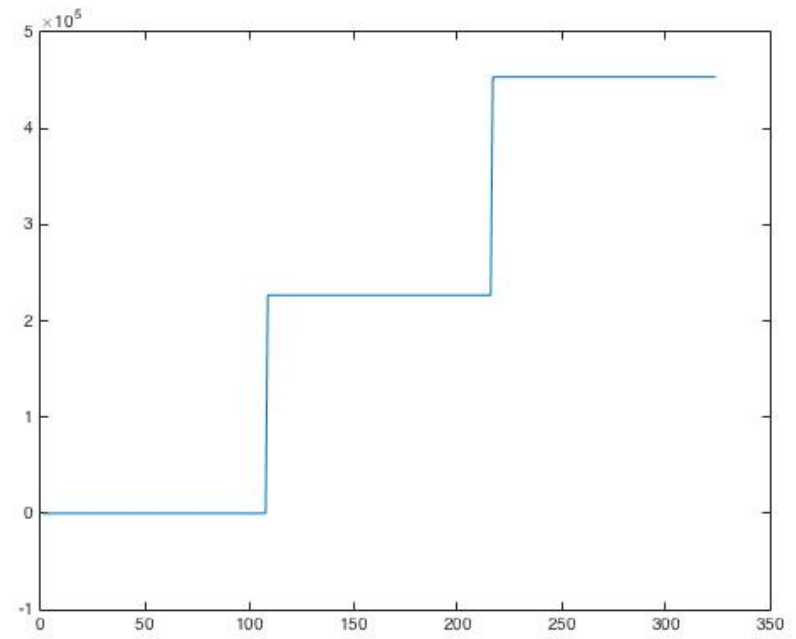

Figure 10: Total Raw sugar Production Recourse in different meta-scenarios 


\section{Appendix C: Case study results (Tables)}

Table 2: Mills Operations

\begin{tabular}{|c|c|c|c|c|c|c|}
\hline \multirow{2}{*}{ Number } & \multirow{2}{*}{ Mills } & \multirow{2}{*}{$\begin{array}{l}\text { Cane converted to } \\
\text { raw sugar in mill } \mathrm{j}\end{array}$} & \multicolumn{2}{|c|}{ Productions } & \multicolumn{2}{|c|}{ Transported from mill $\mathrm{j}$ to the Depot } \\
\hline & & & Raw sugar(t) & Molasses(m ga) & Raw sugar $(\mathrm{t})$ & Molasses (m ga) \\
\hline 1 & Umzimkulu & 833333 & 100000 & 5.55 & 0 & 5.555 \\
\hline 2 & Sezela & 1666667 & 200000 & 11.11 & 89470 & 11.111 \\
\hline 3 & Eston & 833333 & 100000 & 5.55 & 100000 & 5.555 \\
\hline 4 & Noodsberg & 833333 & 100000 & 5.55 & 0 & 5.555 \\
\hline 5 & Union-Coop & 666667 & 80000 & 4.44 & 80000 & 4.444 \\
\hline 6 & Maidstone & 1250000 & 150000 & 8.33 & 126340 & 8.333 \\
\hline 7 & Gledhow & 833333 & 100000 & 5.555 & 100000 & 5.555 \\
\hline 8 & Darnall & 833333 & 100000 & 5.55 & 100000 & 5.555 \\
\hline 9 & Amatikulu & 833333 & 100000 & 5.55 & 100000 & 5.555 \\
\hline 10 & Felixton & 1250000 & 150000 & 8.33 & 144740 & 1.374 \\
\hline 11 & Umfolozi & 833333 & 100000 & 5.55 & 0 & 0 \\
\hline 12 & Pongola & 750000 & 90000 & 4.99 & 90000 & 5 \\
\hline 13 & Komati & 833333 & 100000 & 5.55 & 0 & 0 \\
\hline 14 & Malelane & 833333 & 100000 & 5.55 & 0 & 0 \\
\hline Total & - & 13083333 & 1570000 & 87.15 & 930550 & 63.592 \\
\hline
\end{tabular}

Table 3: Recourse decisions part 1

\begin{tabular}{cccccc}
\hline \multirow{2}{*}{ Meta-scenario } & Importation & \multicolumn{2}{c}{ Sale } & \multicolumn{2}{c}{ Exportation } \\
\cline { 2 - 6 } & Ethanol & By-product $(\times 10)$ & Molasses $(\times 10)$ & White $\left(\times 10^{5}\right)$ & Raw $\left(\times 10^{6}\right)$ \\
\hline 1 & 0.4328 & 1.5397 & 0 & 0 & 0 \\
2 & 0.4328 & 1.5397 & 0 & 0 & 0 \\
3 & 0.4328 & 1.5397 & 0 & 0 & 0 \\
4 & 0.4328 & 1.5397 & 0 & 0 & 0 \\
5 & 0.4328 & 1.5397 & 0 & 0 & 0 \\
6 & 0.4328 & 1.5397 & 0 & 0 & 0 \\
7 & 0.4328 & 1.5397 & 0 & 0 & 0 \\
8 & 0.4328 & 1.5397 & 0 & 0 & 0 \\
9 & 0.4731 & 1.5368 & 0.9754 & 0 & 0 \\
10 & 0.4328 & 1.5397 & 0 & 0 & 0 \\
11 & 0.4328 & 1.5397 & 0 & 0 & 0 \\
12 & 0.4328 & 1.5397 & 0 & 0 & 0 \\
13 & 0.4328 & 1.5397 & 0 & 0 & 0 \\
14 & 0.4328 & 1.5397 & 0 & 0 & 0 \\
15 & 0.4731 & 1.5368 & 0.9754 & 0 & 0 \\
16 & 0.4328 & 1.5397 & 0 & 0 & 0 \\
\hline
\end{tabular}


Table 3 - Continued from previous page

\begin{tabular}{|c|c|c|c|c|c|}
\hline \multirow{2}{*}{ Meta-scenario } & \multirow{2}{*}{$\begin{array}{c}\text { Importation } \\
\text { Ethanol }\end{array}$} & \multicolumn{2}{|c|}{ Sale } & \multicolumn{2}{|c|}{ Exportation } \\
\hline & & By-product $(\times 10)$ & Molasses $(\times 10)$ & White $\left(\times 10^{5}\right)$ & $\operatorname{Raw}\left(\times 10^{6}\right)$ \\
\hline 17 & 0.4328 & 1.5397 & 0 & 0 & 0 \\
\hline 18 & 0.4731 & 1.5368 & 0.9754 & 0 & 0 \\
\hline 19 & 0.8178 & 1.4567 & 0.1584 & 0 & 0 \\
\hline 20 & 0.8178 & 1.4567 & 0.1584 & 0 & 0 \\
\hline 21 & 0.8178 & 1.4567 & 0.1584 & 0 & 0 \\
\hline 22 & 0.8178 & 1.4567 & 0.1584 & 0 & 0 \\
\hline 23 & 0.8178 & 1.4567 & 0.1584 & 0 & 0 \\
\hline 24 & 0.8178 & 1.4567 & 0.1584 & 0 & 0 \\
\hline 25 & 0.8178 & 1.4567 & 0.1584 & 0 & 0 \\
\hline 26 & 0.8178 & 1.4567 & 0.1584 & 0 & 0 \\
\hline 27 & 0.8178 & 1.4567 & 0.1584 & 0 & 0 \\
\hline 28 & 0.8178 & 1.4567 & 0.1584 & 0 & 0 \\
\hline 29 & 0.8178 & 1.4567 & 0.1584 & 0 & 0 \\
\hline 30 & 0.8178 & 1.4567 & 0.1584 & 0 & 0 \\
\hline 31 & 0.8178 & 1.4567 & 0.1584 & 0 & 0 \\
\hline 32 & 0.8178 & 1.4567 & 0.1584 & 0 & 0 \\
\hline 33 & 0.8178 & 1.4567 & 0.1584 & 0 & 0 \\
\hline 34 & 0.8178 & 1.4567 & 0.1584 & 0 & 0 \\
\hline 35 & 0.8178 & 1.4567 & 0.1584 & 0 & 0 \\
\hline 36 & 0.8178 & 1.4567 & 0.1584 & 0 & 0 \\
\hline 37 & 0.9328 & 1.5397 & 0 & 0 & 0 \\
\hline 38 & 0.9328 & 1.5397 & 0 & 0 & 0 \\
\hline 39 & 0.9328 & 1.5397 & 0 & 0 & 0 \\
\hline 40 & 0.9731 & 1.5368 & 0.9754 & 0 & 0 \\
\hline 41 & 0.9328 & 1.5397 & 0 & 0 & 0 \\
\hline 42 & 0.9328 & 1.5397 & 0 & 0 & 0 \\
\hline 43 & 0.9328 & 1.5397 & 0 & 0 & 0 \\
\hline 44 & 0.9328 & 1.5397 & 0 & 0 & 0 \\
\hline 45 & 0.9328 & 1.5397 & 0 & 0 & 0 \\
\hline 46 & 0.9328 & 1.5397 & 0 & 0 & 0 \\
\hline 47 & 0.9328 & 1.5397 & 0 & 0 & 0 \\
\hline 48 & 0.9328 & 1.5397 & 0 & 0 & 0 \\
\hline 49 & 0.9328 & 1.5397 & 0 & 0 & 0 \\
\hline 50 & 0.9328 & 1.5397 & 0 & 0 & 0 \\
\hline 51 & 0.9731 & 1.5368 & 0.9754 & 0 & 0 \\
\hline 52 & 0.9731 & 1.5368 & 0.9754 & 0 & 0 \\
\hline 53 & 0.9328 & 1.5397 & 0 & 0 & 0 \\
\hline 54 & 0.9731 & 1.5368 & 0.9754 & 0 & 0 \\
\hline 55 & 1.3178 & 1.4567 & 0.1584 & 0 & 0 \\
\hline 56 & 1.3178 & 1.4567 & 0.1584 & 0 & 0 \\
\hline 57 & 1.3178 & 1.4567 & 0.1584 & 0 & 0 \\
\hline 58 & 1.3178 & 1.4567 & 0.1584 & 0 & 0 \\
\hline 59 & 1.3178 & 1.4567 & 0.1584 & 0 & 0 \\
\hline 60 & 1.3178 & 1.4567 & 0.1584 & 0 & 0 \\
\hline 61 & 1.3178 & 1.4567 & 0.1584 & 0 & 0 \\
\hline 62 & 1.3178 & 1.4567 & 0.1584 & 0 & 0 \\
\hline 63 & 1.3178 & 1.4567 & 0.1584 & 0 & 0 \\
\hline 64 & 1.3178 & 1.4567 & 0.1584 & 0 & 0 \\
\hline 65 & 1.3178 & 1.4567 & 0.1584 & 0 & 0 \\
\hline 66 & 1.3178 & 1.4567 & 0.1584 & 0 & 0 \\
\hline 67 & 1.3178 & 1.4567 & 0.1584 & 0 & 0 \\
\hline 68 & 1.3178 & 1.4567 & 0.1584 & 0 & 0 \\
\hline 69 & 1.3178 & 1.4567 & 0.1584 & 0 & 0 \\
\hline 70 & 1.3178 & 1.4567 & 0.1584 & 0 & 0 \\
\hline
\end{tabular}


Table 3 - Continued from previous page

\begin{tabular}{|c|c|c|c|c|c|}
\hline \multirow{2}{*}{ Meta-scenario } & \multirow{2}{*}{$\begin{array}{c}\text { Importation } \\
\text { Ethanol }\end{array}$} & \multicolumn{2}{|c|}{ Sale } & \multicolumn{2}{|c|}{ Exportation } \\
\hline & & By-product $(\times 10)$ & Molasses $(\times 10)$ & White $\left(\times 10^{5}\right)$ & $\operatorname{Raw}\left(\times 10^{6}\right)$ \\
\hline 71 & 1.3178 & 1.4567 & 0.1584 & 0 & 0 \\
\hline 72 & 1.3178 & 1.4567 & 0.1584 & 0 & 0 \\
\hline 73 & 1.4328 & 1.5397 & 0 & 0 & 0 \\
\hline 74 & 1.4328 & 1.5397 & 0 & 0 & 0 \\
\hline 75 & 1.4328 & 1.5397 & 0 & 0 & 0 \\
\hline 76 & 1.4328 & 1.5397 & 0 & 0 & 0 \\
\hline 77 & 1.4328 & 1.5397 & 0 & 0 & 0 \\
\hline 78 & 1.4328 & 1.5397 & 0 & 0 & 0 \\
\hline 79 & 1.4328 & 1.5397 & 0 & 0 & 0 \\
\hline 80 & 1.4328 & 1.5397 & 0 & 0 & 0 \\
\hline 81 & 1.4328 & 1.5397 & 0 & 0 & 0 \\
\hline 82 & 1.4328 & 1.5397 & 0 & 0 & 0 \\
\hline 83 & 1.4328 & 1.5397 & 0 & 0 & 0 \\
\hline 84 & 1.4731 & 1.5368 & 0.9754 & 0 & 0 \\
\hline 85 & 1.4328 & 1.5397 & 0 & 0 & 0 \\
\hline 86 & 1.4328 & 1.5397 & 0 & 0 & 0 \\
\hline 87 & 1.4328 & 1.5397 & 0 & 0 & 0 \\
\hline 88 & 1.4328 & 1.5397 & 0 & 0 & 0 \\
\hline 89 & 1.4328 & 1.5397 & 0 & 0 & 0 \\
\hline 90 & 1.4328 & 1.5397 & 0 & 0 & 0 \\
\hline 91 & 1.8113 & 1.4572 & 0 & 0 & 0 \\
\hline 92 & 1.8178 & 1.4567 & 0.1584 & 0 & 0 \\
\hline 93 & 1.8178 & 1.4567 & 0.1584 & 0 & 0 \\
\hline 94 & 1.8178 & 1.4567 & 0.1584 & 0 & 0 \\
\hline 95 & 1.8178 & 1.4567 & 0.1584 & 0 & 0 \\
\hline 96 & 1.8178 & 1.4567 & 0.1584 & 0 & 0 \\
\hline 97 & 1.8178 & 1.4567 & 0.1584 & 0 & 0 \\
\hline 98 & 1.8178 & 1.4567 & 0.1584 & 0 & 0 \\
\hline 99 & 1.8178 & 1.4567 & 0.1584 & 0 & 0 \\
\hline 100 & 1.8178 & 1.4567 & 0.1584 & 0 & 0 \\
\hline 101 & 1.8178 & 1.4567 & 0.1584 & 0 & 0 \\
\hline 102 & 1.8178 & 1.4567 & 0.1584 & 0 & 0 \\
\hline 103 & 1.8178 & 1.4567 & 0.1584 & 0 & 0 \\
\hline 104 & 1.8178 & 1.4567 & 0.1584 & 0 & 0 \\
\hline 105 & 1.8178 & 1.4567 & 0.1584 & 0 & 0 \\
\hline 106 & 1.8178 & 1.4567 & 0.1584 & 0 & 0 \\
\hline 107 & 1.8178 & 1.4567 & 0.1584 & 0 & 0 \\
\hline 108 & 1.8178 & 1.4567 & 0.1584 & 0 & 0 \\
\hline 109 & 1.7940 & 1.2492 & 2.5516 & 2.5000 & 0.9322 \\
\hline 110 & 2.0034 & 1.2340 & 7.6186 & 2.5000 & 0.9322 \\
\hline 111 & 2.0034 & 1.2340 & 7.6186 & 2.5000 & 0.9322 \\
\hline 112 & 2.0034 & 1.2340 & 7.6186 & 2.5000 & 0.9322 \\
\hline 113 & 2.0034 & 1.2340 & 7.6186 & 2.5000 & 0.9322 \\
\hline 114 & 2.0034 & 1.2340 & 7.6186 & 2.5000 & 0.9322 \\
\hline 115 & 2.0034 & 1.2340 & 7.6186 & 2.5000 & 0.9322 \\
\hline 116 & 2.0034 & 1.2340 & 7.6186 & 2.5000 & 0.9322 \\
\hline 117 & 2.0034 & 1.2340 & 7.6186 & 2.5000 & 0.9322 \\
\hline 118 & 2.0034 & 1.2340 & 7.6186 & 2.5000 & 0.9322 \\
\hline 119 & 2.0034 & 1.2340 & 7.6186 & 2.5000 & 0.9322 \\
\hline 120 & 2.0034 & 1.2340 & 7.6186 & 2.5000 & 0.9322 \\
\hline 121 & 2.0034 & 1.2340 & 7.6186 & 2.5000 & 0.9322 \\
\hline 122 & 1.6509 & 1.3090 & 7.6186 & 0 & 0.9322 \\
\hline 123 & 2.0034 & 1.2340 & 7.6186 & 2.5000 & 0.9322 \\
\hline 124 & 2.0034 & 1.2340 & 7.6186 & 2.5000 & 0.9322 \\
\hline
\end{tabular}


Table 3 - Continued from previous page

\begin{tabular}{|c|c|c|c|c|c|}
\hline \multirow{2}{*}{ Meta-scenario } & \multirow{2}{*}{$\begin{array}{c}\text { Importation } \\
\text { Ethanol }\end{array}$} & \multicolumn{2}{|c|}{ Sale } & \multicolumn{2}{|c|}{ Exportation } \\
\hline & & By-product $(\times 10)$ & Molasses $(\times 10)$ & White $\left(\times 10^{5}\right)$ & $\operatorname{Raw}\left(\times 10^{6}\right)$ \\
\hline 125 & 0.0738 & 1.6115 & 0 & 0 & 0 \\
\hline 126 & 0.0738 & 1.6115 & 0 & 0 & 0 \\
\hline 127 & 1.7762 & 1.2505 & 2.1213 & 1.4000 & 0.7672 \\
\hline 128 & 2.0034 & 1.2340 & 7.6186 & 1.4000 & 0.7672 \\
\hline 129 & 2.0034 & 1.2340 & 7.6186 & 1.4000 & 0.7672 \\
\hline 130 & 2.0034 & 1.2340 & 7.6186 & 1.4000 & 0.7672 \\
\hline 131 & 2.0034 & 1.2340 & 7.6186 & 1.4000 & 0.7672 \\
\hline 132 & 2.0034 & 1.2340 & 7.6186 & 1.4000 & 0.7672 \\
\hline 133 & 2.0034 & 1.2340 & 7.6186 & 1.4000 & 0.7672 \\
\hline 134 & 2.0034 & 1.2340 & 7.6186 & 1.4000 & 0.7672 \\
\hline 135 & 2.0034 & 1.2340 & 7.6186 & 1.4000 & 0.7672 \\
\hline 136 & 2.0034 & 1.2340 & 7.6186 & 1.4000 & 0.7672 \\
\hline 137 & 1.8060 & 1.2760 & 7.6186 & 0 & 0.7672 \\
\hline 138 & 1.8060 & 1.2760 & 7.6186 & 0 & 0.7672 \\
\hline 139 & 2.0034 & 1.2340 & 7.6186 & 1.4000 & 0.7672 \\
\hline 140 & 2.0034 & 1.2340 & 7.6186 & 1.4000 & 0.7672 \\
\hline 141 & 2.0034 & 1.2340 & 7.6186 & 1.4000 & 0.7672 \\
\hline 142 & 2.0034 & 1.2340 & 7.6186 & 1.4000 & 0.7672 \\
\hline 143 & 0.4523 & 1.5290 & 0 & 0 & 0 \\
\hline 144 & 0.4523 & 1.5290 & 0 & 0 & 0 \\
\hline 145 & 2.1187 & 1.2723 & 0 & 2.5000 & 0.8807 \\
\hline 146 & 2.5034 & 1.2340 & 7.6186 & 2.5000 & 0.9322 \\
\hline 147 & 2.5034 & 1.2340 & 7.6186 & 2.5000 & 0.9322 \\
\hline 148 & 2.5034 & 1.2340 & 7.6186 & 2.5000 & 0.9322 \\
\hline 149 & 2.1509 & 1.3090 & 7.6186 & 0 & 0.9322 \\
\hline 150 & 2.5034 & 1.2340 & 7.6186 & 2.5000 & 0.9322 \\
\hline 151 & 2.5034 & 1.2340 & 7.6186 & 2.5000 & 0.9499 \\
\hline 152 & 2.5034 & 1.2340 & 7.6186 & 2.5000 & 0.9322 \\
\hline 153 & 2.5034 & 1.2340 & 7.6186 & 2.5000 & 0.9322 \\
\hline 154 & 2.1509 & 1.3090 & 7.6186 & 0 & 0.9322 \\
\hline 155 & 0.8886 & 1.5886 & 7.6186 & 0 & 0 \\
\hline 156 & 2.1509 & 1.3090 & 7.6186 & 0 & 0.9322 \\
\hline 157 & 2.5034 & 1.2340 & 7.6186 & 2.5000 & 0.9322 \\
\hline 158 & 0.8886 & 1.5886 & 7.6186 & 0 & 0 \\
\hline 159 & 1.8360 & 1.3318 & 0 & 0 & 0.9322 \\
\hline 160 & 2.1509 & 1.3090 & 7.6186 & 0 & 0.9322 \\
\hline 161 & 2.1509 & 1.3090 & 7.6186 & 0 & 0.9322 \\
\hline 162 & 0.5738 & 1.6115 & 0 & 0 & 0 \\
\hline 163 & 2.0504 & 1.2874 & 0 & 1.4000 & 0.6652 \\
\hline 164 & 2.5034 & 1.2340 & 7.6186 & 1.4000 & 0.7672 \\
\hline 165 & 1.2671 & 1.5061 & 7.6186 & 0 & 0 \\
\hline 166 & 2.5034 & 1.2340 & 7.6186 & 1.4000 & 0.7672 \\
\hline 167 & 2.3060 & 1.2760 & 7.6186 & 0 & 0.7672 \\
\hline 168 & 2.5034 & 1.2340 & 7.6186 & 1.4000 & 0.7672 \\
\hline 169 & 2.5034 & 1.2340 & 7.6186 & 1.4000 & 0.7819 \\
\hline 170 & 2.3060 & 1.2760 & 7.6186 & 0 & 0.7672 \\
\hline 171 & 2.5034 & 1.2340 & 7.6186 & 1.4000 & 0.7672 \\
\hline 172 & 2.3060 & 1.2760 & 7.6186 & 0 & 0.7672 \\
\hline 173 & 2.3060 & 1.2760 & 7.6186 & 0 & 0.7672 \\
\hline 174 & 2.5034 & 1.2340 & 7.6186 & 1.4000 & 0.7672 \\
\hline 175 & 2.5034 & 1.2340 & 7.6186 & 1.4000 & 0.7672 \\
\hline 176 & 0.9523 & 1.5290 & 0 & 0 & 0 \\
\hline 177 & 0.9523 & 1.5290 & 0 & 0 & 0 \\
\hline 178 & 2.3060 & 1.2760 & 7.6186 & 0 & 0.7672 \\
\hline
\end{tabular}


Table 3 - Continued from previous page

\begin{tabular}{|c|c|c|c|c|c|}
\hline \multirow{2}{*}{ Meta-scenario } & \multirow{2}{*}{$\begin{array}{c}\text { Importation } \\
\text { Ethanol }\end{array}$} & \multicolumn{2}{|c|}{ Sale } & \multicolumn{2}{|c|}{ Exportation } \\
\hline & & By-product $(\times 10)$ & Molasses $(\times 10)$ & White $\left(\times 10^{5}\right)$ & $\operatorname{Raw}\left(\times 10^{6}\right)$ \\
\hline 179 & 2.5034 & 1.2340 & 7.6186 & 1.4000 & 0.7672 \\
\hline 180 & 1.9911 & 1.2988 & 0 & 0 & 0.7672 \\
\hline 181 & 2.1440 & 1.3775 & 0 & 2.5000 & 0.5301 \\
\hline 182 & 3.0034 & 1.2340 & 7.6186 & 2.5000 & 0.9322 \\
\hline 183 & 3.0034 & 1.2340 & 7.6186 & 2.5000 & 0.9322 \\
\hline 184 & 3.0034 & 1.2340 & 7.6186 & 2.5000 & 0.9322 \\
\hline 185 & 3.0034 & 1.2340 & 7.6186 & 2.5000 & 0.9322 \\
\hline 186 & 3.0034 & 1.2340 & 7.6186 & 2.5000 & 0.9322 \\
\hline 187 & 2.9620 & 1.2370 & 6.6166 & 2.5000 & 0.9322 \\
\hline 188 & 2.3360 & 1.3318 & 0 & 0 & 0.9322 \\
\hline 189 & 3.0034 & 1.2340 & 7.6186 & 2.5000 & 0.9322 \\
\hline 190 & 3.0034 & 1.2340 & 7.6186 & 2.5000 & 0.9322 \\
\hline 191 & 2.6509 & 1.3090 & 7.6186 & 0 & 0.9322 \\
\hline 192 & 1.0738 & 1.6115 & 0 & 0 & 0 \\
\hline 193 & 1.0738 & 1.6115 & 0 & 0 & 0 \\
\hline 194 & 3.0034 & 1.2340 & 7.6186 & 2.5000 & 0.9322 \\
\hline 195 & 2.3360 & 1.3318 & 0 & 0 & 0.9322 \\
\hline 196 & 3.0034 & 1.2340 & 7.6186 & 2.5000 & 0.9322 \\
\hline 197 & 1.3886 & 1.5886 & 7.6186 & 0 & 0 \\
\hline 198 & 1.3886 & 1.5886 & 7.6186 & 0 & 0 \\
\hline 199 & 2.0757 & 1.3926 & 0 & 1.4000 & 0.3146 \\
\hline 200 & 3.0034 & 1.2340 & 7.6186 & 1.4000 & 0.7672 \\
\hline 201 & 3.0034 & 1.2340 & 7.6186 & 1.4000 & 0.7672 \\
\hline 202 & 3.0034 & 1.2340 & 7.6186 & 1.4000 & 0.7672 \\
\hline 203 & 3.0034 & 1.2340 & 7.6186 & 1.4000 & 0.7672 \\
\hline 204 & 2.8060 & 1.2760 & 7.6186 & 0 & 0.7672 \\
\hline 205 & 2.8046 & 1.2761 & 7.5865 & 0 & 0.7672 \\
\hline 206 & 3.0034 & 1.2340 & 7.6186 & 1.4000 & 0.7672 \\
\hline 207 & 3.0034 & 1.2340 & 7.6186 & 1.4000 & 0.7672 \\
\hline 208 & 3.0034 & 1.2340 & 7.6186 & 1.4000 & 0.7672 \\
\hline 209 & 2.8060 & 1.2760 & 7.6186 & 0 & 0.7672 \\
\hline 210 & 2.8060 & 1.2760 & 7.6186 & 0 & 0.7672 \\
\hline 211 & 3.0034 & 1.2340 & 7.6186 & 1.4000 & 0.7672 \\
\hline 212 & 3.0034 & 1.2340 & 7.6186 & 1.4000 & 0.7672 \\
\hline 213 & 1.4523 & 1.5290 & 0 & 0 & 0 \\
\hline 214 & 2.8060 & 1.2760 & 7.6186 & 0 & 0.7672 \\
\hline 215 & 1.4523 & 1.5290 & 0 & 0 & 0 \\
\hline 216 & 2.8060 & 1.2760 & 7.6186 & 0 & 0.7672 \\
\hline 217 & 0.0936 & 1.5993 & 0 & 0 & 0.2798 \\
\hline 218 & 0.0936 & 1.5993 & 0 & 0 & 0.2798 \\
\hline 219 & 0.0936 & 1.5993 & 0 & 0 & 0.2798 \\
\hline 220 & 0.0817 & 1.6566 & 8.8779 & 0 & 0 \\
\hline 221 & 0.0936 & 1.5993 & 0 & 0 & 0.2798 \\
\hline 222 & 0.0817 & 1.6566 & 8.8779 & 0 & 0 \\
\hline 223 & 0.0936 & 1.5993 & 0 & 0 & 0.2798 \\
\hline 224 & 0.0936 & 1.5993 & 0 & 0 & 0.2798 \\
\hline 225 & 0.0936 & 1.5993 & 0 & 0 & 0.2798 \\
\hline 226 & 0.0936 & 1.5993 & 0 & 0 & 0.2798 \\
\hline 227 & 0.0936 & 1.5993 & 0 & 0 & 0.2798 \\
\hline 228 & 0 & 1.6626 & 6.9009 & 0 & 0 \\
\hline 229 & 0.0936 & 1.5993 & 0 & 0 & 0.2798 \\
\hline 230 & 0.0936 & 1.5993 & 0 & 0 & 0.2798 \\
\hline 231 & 0 & 1.6201 & 0 & 0 & 0.2106 \\
\hline 232 & 0.0936 & 1.5993 & 0 & 0 & 0.2798 \\
\hline
\end{tabular}


Table 3 - Continued from previous page

\begin{tabular}{|c|c|c|c|c|c|}
\hline \multirow{2}{*}{ Meta-scenario } & \multirow{2}{*}{$\begin{array}{c}\text { Importation } \\
\text { Ethanol }\end{array}$} & \multicolumn{2}{|c|}{ Sale } & \multicolumn{2}{|c|}{ Exportation } \\
\hline & & By-product $(\times 10)$ & Molasses $(\times 10)$ & White $\left(\times 10^{5}\right)$ & $\operatorname{Raw}\left(\times 10^{6}\right)$ \\
\hline 233 & 0.0936 & 1.5993 & 0 & 0 & 0.2798 \\
\hline 234 & 0.0817 & 1.6566 & 8.8779 & 0 & 0 \\
\hline 235 & 0.0933 & 1.6008 & 0 & 0 & -0.0000 \\
\hline 236 & 0.0933 & 1.6008 & 0 & 0 & 0 \\
\hline 237 & 0.0933 & 1.6008 & 0 & 0 & -0.0000 \\
\hline 238 & 0.0933 & 1.6008 & 0 & 0 & 0 \\
\hline 239 & 0.0933 & 1.6008 & 0 & 0 & 0 \\
\hline 240 & 0.0933 & 1.6008 & 0 & 0 & 0 \\
\hline 241 & 0.0933 & 1.6008 & 0 & 0 & -0.0000 \\
\hline 242 & 0.0933 & 1.6008 & 0 & 0 & 0 \\
\hline 243 & 0.0933 & 1.6008 & 0 & 0 & 0 \\
\hline 244 & 0.0933 & 1.6008 & 0 & 0 & 0 \\
\hline 245 & 0.0933 & 1.6008 & 0 & 0 & 0 \\
\hline 246 & 0.0933 & 1.6008 & 0 & 0 & 0 \\
\hline 247 & 0.0933 & 1.6008 & 0 & 0 & -0.0000 \\
\hline 248 & 0.0933 & 1.6008 & 0 & 0 & 0 \\
\hline 249 & 0.0933 & 1.6008 & 0 & 0 & 0 \\
\hline 250 & 0.0933 & 1.6008 & 0 & 0 & 0 \\
\hline 251 & 0.0933 & 1.6008 & 0 & 0 & 0 \\
\hline 252 & 0.0933 & 1.6008 & 0 & 0 & 0 \\
\hline 253 & 1.3414 & 1.4368 & 0 & 2.5000 & 0.5717 \\
\hline 254 & 1.7840 & 1.3356 & 0 & 0 & 1.1589 \\
\hline 255 & 1.7840 & 1.3356 & 0 & 0 & 1.1589 \\
\hline 256 & 1.7840 & 1.3356 & 0 & 0 & 1.1589 \\
\hline 257 & 1.7840 & 1.3356 & 0 & 0 & 1.1589 \\
\hline 258 & 1.7840 & 1.3356 & 0 & 0 & 1.1589 \\
\hline 259 & 1.7840 & 1.3356 & 0 & 0 & 1.1589 \\
\hline 260 & 1.7840 & 1.3356 & 0 & 0 & 1.1589 \\
\hline 261 & 1.7840 & 1.3356 & 0 & 0 & 1.1589 \\
\hline 262 & 1.7840 & 1.3356 & 0.0000 & 0 & 1.1589 \\
\hline 263 & 1.7721 & 1.3929 & 8.8779 & 0 & 0.8791 \\
\hline 264 & 1.7721 & 1.3929 & 8.8779 & 0 & 0.8791 \\
\hline 265 & 1.7840 & 1.3356 & 0 & 0 & 1.1589 \\
\hline 266 & 1.7840 & 1.3356 & 0 & 0 & 1.1589 \\
\hline 267 & 1.7840 & 1.3356 & 0 & 0 & 1.1589 \\
\hline 268 & 1.7840 & 1.3356 & 0 & 0 & 1.1589 \\
\hline 269 & 1.7721 & 1.3929 & 8.8779 & 0 & 0.8791 \\
\hline 270 & 0.2148 & 1.6833 & 0 & 0 & 0 \\
\hline 271 & 1.2732 & 1.4519 & 0 & 1.4000 & 0.3563 \\
\hline 272 & 1.7718 & 1.3943 & 8.8779 & 0 & 0.5994 \\
\hline 273 & 1.7718 & 1.3943 & 8.8779 & 0 & 0.5994 \\
\hline 274 & 1.7837 & 1.3370 & 0 & 0 & 0.8791 \\
\hline 275 & 0.9602 & 1.5741 & 8.8779 & 0 & 0 \\
\hline 276 & 1.7837 & 1.3370 & 0 & 0 & 0.8791 \\
\hline 277 & 1.7837 & 1.3370 & 0 & 0 & 0.8791 \\
\hline 278 & 1.7837 & 1.3370 & 0 & 0 & 0.8791 \\
\hline 279 & 1.7837 & 1.3370 & 0 & 0 & 0.8791 \\
\hline 280 & 1.7718 & 1.3943 & 8.8779 & 0 & 0.5994 \\
\hline 281 & 1.7718 & 1.3943 & 8.8779 & 0 & 0.5994 \\
\hline 282 & 1.7718 & 1.3943 & 8.8779 & 0 & 0.5994 \\
\hline 283 & 1.7837 & 1.3370 & 0 & 0 & 0.8791 \\
\hline 284 & 1.7837 & 1.3370 & 0 & 0 & 0.8791 \\
\hline 285 & 1.7837 & 1.3370 & 0 & 0 & 0.8791 \\
\hline 286 & 0.5933 & 1.6008 & 0 & 0 & 0 \\
\hline
\end{tabular}


Table 3 - Continued from previous page

\begin{tabular}{|c|c|c|c|c|c|}
\hline \multirow{2}{*}{ Meta-scenario } & \multirow{2}{*}{$\begin{array}{c}\text { Importation } \\
\text { Ethanol }\end{array}$} & \multicolumn{2}{|c|}{ Sale } & \multicolumn{2}{|c|}{ Exportation } \\
\hline & & By-product $(\times 10)$ & Molasses $(\times 10)$ & White $\left(\times 10^{5}\right)$ & $\operatorname{Raw}\left(\times 10^{6}\right)$ \\
\hline 287 & 1.7718 & 1.3943 & 8.8779 & 0 & 0.5994 \\
\hline 288 & 0.5933 & 1.6008 & 0 & 0 & 0 \\
\hline 289 & 1.3667 & 1.5419 & 0 & 2.5000 & 0.2211 \\
\hline 290 & 3.0034 & 1.2340 & 8.8779 & 2.5000 & 1.1589 \\
\hline 291 & 3.0034 & 1.2340 & 8.8779 & 2.5000 & 1.1589 \\
\hline 292 & 3.0034 & 1.2340 & 8.8779 & 2.5000 & 1.1589 \\
\hline 293 & 2.6509 & 1.3090 & 8.8779 & 0 & 1.1589 \\
\hline 294 & 3.0034 & 1.2340 & 8.8779 & 2.5000 & 1.1589 \\
\hline 295 & 2.7887 & 1.2496 & 3.6832 & 2.5000 & 1.1589 \\
\hline 296 & 3.0034 & 1.2340 & 8.8779 & 2.5000 & 1.1589 \\
\hline 297 & 0.7148 & 1.6833 & 0 & 0 & 0 \\
\hline 298 & 3.0034 & 1.2340 & 8.8779 & 2.5000 & 1.1589 \\
\hline 299 & 3.0034 & 1.2340 & 8.8779 & 2.5000 & 1.1589 \\
\hline 300 & 2.6509 & 1.3090 & 8.8779 & 0 & 1.1589 \\
\hline 301 & 3.0034 & 1.2340 & 8.8779 & 2.5000 & 1.1589 \\
\hline 302 & 2.6509 & 1.3090 & 8.8779 & 0 & 1.1589 \\
\hline 303 & 2.2840 & 1.3356 & 0 & 0 & 1.1589 \\
\hline 304 & 1.0817 & 1.6566 & 8.8779 & 0 & 0 \\
\hline 305 & 2.6509 & 1.3090 & 8.8779 & 0 & 1.1589 \\
\hline 306 & 2.6509 & 1.3090 & 8.8779 & 0 & 1.1589 \\
\hline 307 & 1.2927 & 1.5586 & 0.0486 & 1.4000 & 0 \\
\hline 308 & 3.0034 & 1.2340 & 8.8779 & 1.4000 & 0.9939 \\
\hline 309 & 3.0034 & 1.2340 & 8.8779 & 1.4000 & 0.9939 \\
\hline 310 & 3.0034 & 1.2340 & 8.8779 & 1.4000 & 0.9939 \\
\hline 311 & 2.8060 & 1.2760 & 8.8779 & 0 & 0.9939 \\
\hline 312 & 2.8060 & 1.2760 & 8.8779 & 0 & 0.9939 \\
\hline 313 & 2.7759 & 1.2505 & 3.3737 & 1.4000 & 0.9939 \\
\hline 314 & 3.0034 & 1.2340 & 8.8779 & 1.4000 & 0.9939 \\
\hline 315 & 3.0034 & 1.2340 & 8.8779 & 1.4000 & 0.9939 \\
\hline 316 & 3.0034 & 1.2340 & 8.8779 & 1.4000 & 0.9939 \\
\hline 317 & 3.0034 & 1.2340 & 8.8779 & 1.4000 & 0.9939 \\
\hline 318 & 1.0933 & 1.6008 & 0 & 0 & 0 \\
\hline 319 & 3.0034 & 1.2340 & 8.8779 & 1.4000 & 0.9939 \\
\hline 320 & 1.0933 & 1.6008 & 0 & 0 & 0 \\
\hline 321 & 1.0933 & 1.6008 & 0 & 0 & 0 \\
\hline 322 & 3.0034 & 1.2340 & 8.8779 & 1.4000 & 0.9939 \\
\hline 323 & 2.8060 & 1.2760 & 8.8779 & 0 & 0.9939 \\
\hline 324 & 1.0933 & 1.6008 & 0 & 0 & 0 \\
\hline
\end{tabular}

Table 4: Recourse decisions part 2

\begin{tabular}{ccccccc}
\hline \multirow{2}{*}{ Meta-scenario } & \multicolumn{2}{c}{ Recourse (send back to more ethanol production) } & & \multicolumn{2}{c}{ Recourse $($ extra sugarcane $)$} \\
\cline { 2 - 3 } \cline { 6 - 7 } & White $\left(\times 10^{5}\right)$ & Raw $\left(\times 10^{6}\right)$ & Molasses $(\times 10)$ & & Raw $\left(\times 10^{6}\right)$ & Molasses $(\times 10)$ \\
\hline 1 & 2.5000 & 0.7056 & 6.3593 & & 0 & 0 \\
2 & 2.5000 & 0.7056 & 6.3593 & & 0 & 0 \\
3 & 2.5000 & 0.7056 & 6.3593 & & 0 & 0 \\
4 & 2.5000 & 0.7056 & 6.3593 & & 0 & 0 \\
5 & 2.5000 & 0.7056 & 6.3593 & & 0 & 0 \\
6 & 2.5000 & 0.7056 & 6.3593 & & 0 & 0 \\
7 & 2.5000 & 0.7056 & 6.3593 & & 0 & 0 \\
\hline
\end{tabular}


Table 4 - Continued from previous page

\begin{tabular}{|c|c|c|c|c|c|}
\hline \multirow{2}{*}{ Meta-scenario } & \multicolumn{3}{|c|}{ Recourse(send back to more ethanol production) } & \multicolumn{2}{|c|}{ Recourse(extra sugarcane) } \\
\hline & White $\left(\times 10^{5}\right)$ & $\operatorname{Raw}\left(\times 10^{6}\right)$ & Molasses $(\times 10)$ & $\operatorname{Raw}\left(\times 10^{6}\right)$ & Molasses $(\times 10)$ \\
\hline 8 & 2.5000 & 0.7056 & 6.3593 & 0 & 0 \\
\hline 9 & 2.5000 & 0.7056 & 5.3840 & 0 & 0 \\
\hline 10 & 2.5000 & 0.7056 & 6.3593 & 0 & 0 \\
\hline 11 & 2.5000 & 0.7056 & 6.3593 & 0 & 0 \\
\hline 12 & 2.5000 & 0.7056 & 6.3593 & 0 & 0 \\
\hline 13 & 2.5000 & 0.7056 & 6.3593 & 0 & 0 \\
\hline 14 & 2.5000 & 0.7056 & 6.3593 & 0 & 0 \\
\hline 15 & 2.5000 & 0.7056 & 5.3840 & 0 & 0 \\
\hline 16 & 2.5000 & 0.7056 & 6.3593 & 0 & 0 \\
\hline 17 & 2.5000 & 0.7056 & 6.3593 & 0 & 0 \\
\hline 18 & 2.5000 & 0.7056 & 5.3840 & 0 & 0 \\
\hline 19 & 1.4000 & 0.5406 & 6.2009 & 0 & 0 \\
\hline 20 & 1.4000 & 0.5406 & 6.2009 & 0 & 0 \\
\hline 21 & 1.4000 & 0.5406 & 6.2009 & 0 & 0 \\
\hline 22 & 1.4000 & 0.5406 & 6.2009 & 0 & 0 \\
\hline 23 & 1.4000 & 0.5406 & 6.2009 & 0 & 0 \\
\hline 24 & 1.4000 & 0.5406 & 6.2009 & 0 & 0 \\
\hline 25 & 1.4000 & 0.5406 & 6.2009 & 0 & 0 \\
\hline 26 & 1.4000 & 0.5406 & 6.2009 & 0 & 0 \\
\hline 27 & 1.4000 & 0.5406 & 6.2009 & 0 & 0 \\
\hline 28 & 1.4000 & 0.5406 & 6.2009 & 0 & 0 \\
\hline 29 & 1.4000 & 0.5406 & 6.2009 & 0 & 0 \\
\hline 30 & 1.4000 & 0.5406 & 6.2009 & 0 & 0 \\
\hline 31 & 1.4000 & 0.5406 & 6.2009 & 0 & 0 \\
\hline 32 & 1.4000 & 0.5406 & 6.2009 & 0 & 0 \\
\hline 33 & 1.4000 & 0.5406 & 6.2009 & 0 & 0 \\
\hline 34 & 1.4000 & 0.5406 & 6.2009 & 0 & 0 \\
\hline 35 & 1.4000 & 0.5406 & 6.2009 & 0 & 0 \\
\hline 36 & 1.4000 & 0.5406 & 6.2009 & 0 & 0 \\
\hline 37 & 2.5000 & 0.7056 & 6.3593 & 0 & 0 \\
\hline 38 & 2.5000 & 0.7056 & 6.3593 & 0 & 0 \\
\hline 39 & 2.5000 & 0.7056 & 6.3593 & 0 & 0 \\
\hline 40 & 2.5000 & 0.7056 & 5.3840 & 0 & 0 \\
\hline 41 & 2.5000 & 0.7056 & 6.3593 & 0 & 0 \\
\hline 42 & 2.5000 & 0.7056 & 6.3593 & 0 & 0 \\
\hline 43 & 2.5000 & 0.7056 & 6.3593 & 0 & 0 \\
\hline 44 & 2.5000 & 0.7056 & 6.3593 & 0 & 0 \\
\hline 45 & 2.5000 & 0.7056 & 6.3593 & 0 & 0 \\
\hline 46 & 2.5000 & 0.7056 & 6.3593 & 0 & 0 \\
\hline 47 & 2.5000 & 0.7056 & 6.3593 & 0 & 0 \\
\hline 48 & 2.5000 & 0.7056 & 6.3593 & 0 & 0 \\
\hline 49 & 2.5000 & 0.7056 & 6.3593 & 0 & 0 \\
\hline 50 & 2.5000 & 0.7056 & 6.3593 & 0 & 0 \\
\hline 51 & 2.5000 & 0.7056 & 5.3840 & 0 & 0 \\
\hline 52 & 2.5000 & 0.7056 & 5.3840 & 0 & 0 \\
\hline 53 & 2.5000 & 0.7056 & 6.3593 & 0 & 0 \\
\hline 54 & 2.5000 & 0.7056 & 5.3840 & 0 & 0 \\
\hline 55 & 1.4000 & 0.5406 & 6.2009 & 0 & 0 \\
\hline 56 & 1.4000 & 0.5406 & 6.2009 & 0 & 0 \\
\hline 57 & 1.4000 & 0.5406 & 6.2009 & 0 & 0 \\
\hline 58 & 1.4000 & 0.5406 & 6.2009 & 0 & 0 \\
\hline 59 & 1.4000 & 0.5406 & 6.2009 & 0 & 0 \\
\hline 60 & 1.4000 & 0.5406 & 6.2009 & 0 & 0 \\
\hline 61 & 1.4000 & 0.5406 & 6.2009 & 0 & 0 \\
\hline
\end{tabular}


Table 4 - Continued from previous page

\begin{tabular}{|c|c|c|c|c|c|}
\hline \multirow{2}{*}{ Meta-scenario } & \multicolumn{3}{|c|}{ Recourse(send back to more ethanol production) } & \multicolumn{2}{|c|}{ Recourse(extra sugarcane) } \\
\hline & White $\left(\times 10^{5}\right)$ & $\operatorname{Raw}\left(\times 10^{6}\right)$ & Molasses $(\times 10)$ & $\operatorname{Raw}\left(\times 10^{6}\right)$ & Molasses $(\times 10)$ \\
\hline 62 & 1.4000 & 0.5406 & 6.2009 & 0 & 0 \\
\hline 63 & 1.4000 & 0.5406 & 6.2009 & 0 & 0 \\
\hline 64 & 1.4000 & 0.5406 & 6.2009 & 0 & 0 \\
\hline 65 & 1.4000 & 0.5406 & 6.2009 & 0 & 0 \\
\hline 66 & 1.4000 & 0.5406 & 6.2009 & 0 & 0 \\
\hline 67 & 1.4000 & 0.5406 & 6.2009 & 0 & 0 \\
\hline 68 & 1.4000 & 0.5406 & 6.2009 & 0 & 0 \\
\hline 69 & 1.4000 & 0.5406 & 6.2009 & 0 & 0 \\
\hline 70 & 1.4000 & 0.5406 & 6.2009 & 0 & 0 \\
\hline 71 & 1.4000 & 0.5406 & 6.2009 & 0 & 0 \\
\hline 72 & 1.4000 & 0.5406 & 6.2009 & 0 & 0 \\
\hline 73 & 2.5000 & 0.7056 & 6.3593 & 0 & 0 \\
\hline 74 & 2.5000 & 0.7056 & 6.3593 & 0 & 0 \\
\hline 75 & 2.5000 & 0.7056 & 6.3593 & 0 & 0 \\
\hline 76 & 2.5000 & 0.7056 & 6.3593 & 0 & 0 \\
\hline 77 & 2.5000 & 0.7056 & 6.3593 & 0 & 0 \\
\hline 78 & 2.5000 & 0.7056 & 6.3593 & 0 & 0 \\
\hline 79 & 2.5000 & 0.7056 & 6.3593 & 0 & 0 \\
\hline 80 & 2.5000 & 0.7056 & 6.3593 & 0 & 0 \\
\hline 81 & 2.5000 & 0.7056 & 6.3593 & 0 & 0 \\
\hline 82 & 2.5000 & 0.7056 & 6.3593 & 0 & 0 \\
\hline 83 & 2.5000 & 0.7056 & 6.3593 & 0 & 0 \\
\hline 84 & 2.5000 & 0.7056 & 5.3840 & 0 & 0 \\
\hline 85 & 2.5000 & 0.7056 & 6.3593 & 0 & 0 \\
\hline 86 & 2.5000 & 0.7056 & 6.3593 & 0 & 0 \\
\hline 87 & 2.5000 & 0.7056 & 6.3593 & 0 & 0 \\
\hline 88 & 2.5000 & 0.7056 & 6.3593 & 0 & 0 \\
\hline 89 & 2.5000 & 0.7056 & 6.3593 & 0 & 0 \\
\hline 90 & 2.5000 & 0.7056 & 6.3593 & 0 & 0 \\
\hline 91 & 1.4000 & 0.5406 & 6.3593 & 0 & 0 \\
\hline 92 & 1.4000 & 0.5406 & 6.2009 & 0 & 0 \\
\hline 93 & 1.4000 & 0.5406 & 6.2009 & 0 & 0 \\
\hline 94 & 1.4000 & 0.5406 & 6.2009 & 0 & 0 \\
\hline 95 & 1.4000 & 0.5406 & 6.2009 & 0 & 0 \\
\hline 96 & 1.4000 & 0.5406 & 6.2009 & 0 & 0 \\
\hline 97 & 1.4000 & 0.5406 & 6.2009 & 0 & 0 \\
\hline 98 & 1.4000 & 0.5406 & 6.2009 & 0 & 0 \\
\hline 99 & 1.4000 & 0.5406 & 6.2009 & 0 & 0 \\
\hline 100 & 1.4000 & 0.5406 & 6.2009 & 0 & 0 \\
\hline 101 & 1.4000 & 0.5406 & 6.2009 & 0 & 0 \\
\hline 102 & 1.4000 & 0.5406 & 6.2009 & 0 & 0 \\
\hline 103 & 1.4000 & 0.5406 & 6.2009 & 0 & 0 \\
\hline 104 & 1.4000 & 0.5406 & 6.2009 & 0 & 0 \\
\hline 105 & 1.4000 & 0.5406 & 6.2009 & 0 & 0 \\
\hline 106 & 1.4000 & 0.5406 & 6.2009 & 0 & 0 \\
\hline 107 & 1.4000 & 0.5406 & 6.2009 & 0 & 0 \\
\hline 108 & 1.4000 & 0.5406 & 6.2009 & 0 & 0 \\
\hline 109 & 0 & 0 & 5.0670 & 2.2668 & 1.2593 \\
\hline 110 & 0 & 0 & 0 & 2.2668 & 1.2593 \\
\hline 111 & 0 & 0 & 0 & 2.2668 & 1.2593 \\
\hline 112 & 0 & 0 & 0 & 2.2668 & 1.2593 \\
\hline 113 & 0 & 0 & 0 & 2.2668 & 1.2593 \\
\hline 114 & 0 & 0 & 0 & 2.2668 & 1.2593 \\
\hline 115 & 0 & 0 & 0 & 2.2668 & 1.2593 \\
\hline
\end{tabular}


Table 4 - Continued from previous page

\begin{tabular}{|c|c|c|c|c|c|}
\hline \multirow{2}{*}{ Meta-scenario } & \multicolumn{3}{|c|}{ Recourse(send back to more ethanol production) } & \multicolumn{2}{|c|}{ Recourse(extra sugarcane) } \\
\hline & White $\left(\times 10^{5}\right)$ & $\operatorname{Raw}\left(\times 10^{6}\right)$ & Molasses $(\times 10)$ & $\operatorname{Raw}\left(\times 10^{6}\right)$ & Molasses $(\times 10)$ \\
\hline 116 & 0 & 0 & 0 & 2.2668 & 1.2593 \\
\hline 117 & 0 & 0 & 0 & 2.2668 & 1.2593 \\
\hline 118 & 0 & 0 & 0 & 2.2668 & 1.2593 \\
\hline 119 & 0 & 0 & 0 & 2.2668 & 1.2593 \\
\hline 120 & 0 & 0 & 0 & 2.2668 & 1.2593 \\
\hline 121 & 0 & 0 & 0 & 2.2668 & 1.2593 \\
\hline 122 & 2.5000 & 0 & 0 & 2.2668 & 1.2593 \\
\hline 123 & 0 & 0 & 0 & 2.2668 & 1.2593 \\
\hline 124 & 0 & 0 & 0 & 2.2668 & 1.2593 \\
\hline 125 & 2.5000 & 0.9322 & 7.6186 & 2.2668 & 1.2593 \\
\hline 126 & 2.5000 & 0.9322 & 7.6186 & 2.2668 & 1.2593 \\
\hline 127 & 0 & 0 & 5.4973 & 2.2668 & 1.2593 \\
\hline 128 & 0 & 0 & 0 & 2.2668 & 1.2593 \\
\hline 129 & 0 & 0 & 0 & 2.2668 & 1.2593 \\
\hline 130 & 0 & 0 & 0 & 2.2668 & 1.2593 \\
\hline 131 & 0 & 0 & 0 & 2.2668 & 1.2593 \\
\hline 132 & 0 & 0 & 0 & 2.2668 & 1.2593 \\
\hline 133 & 0 & 0 & 0 & 2.2668 & 1.2593 \\
\hline 134 & 0 & 0 & 0 & 2.2668 & 1.2593 \\
\hline 135 & 0 & 0 & 0 & 2.2668 & 1.2593 \\
\hline 136 & 0 & 0 & 0 & 2.2668 & 1.2593 \\
\hline 137 & 1.4000 & 0 & 0 & 2.2668 & 1.2593 \\
\hline 138 & 1.4000 & 0 & 0 & 2.2668 & 1.2593 \\
\hline 139 & 0 & 0 & 0 & 2.2668 & 1.2593 \\
\hline 140 & 0 & 0 & 0 & 2.2668 & 1.2593 \\
\hline 141 & 0 & 0 & 0 & 2.2668 & 1.2593 \\
\hline 142 & 0 & 0 & 0 & 2.2668 & 1.2593 \\
\hline 143 & 1.4000 & 0.7672 & 7.6186 & 2.2668 & 1.2593 \\
\hline 144 & 1.4000 & 0.7672 & 7.6186 & 2.2668 & 1.2593 \\
\hline 145 & 0 & 0.0516 & 7.6186 & 2.2668 & 1.2593 \\
\hline 146 & 0 & 0 & 0 & 2.2668 & 1.2593 \\
\hline 147 & 0 & 0 & 0 & 2.2668 & 1.2593 \\
\hline 148 & 0 & 0 & 0 & 2.2668 & 1.2593 \\
\hline 149 & 2.5000 & 0 & 0 & 2.2668 & 1.2593 \\
\hline 150 & 0 & 0 & 0 & 2.2668 & 1.2593 \\
\hline 151 & 0 & 0 & 0 & 2.2668 & 1.2593 \\
\hline 152 & 0 & 0 & 0 & 2.2668 & 1.2593 \\
\hline 153 & 0 & 0 & 0 & 2.2668 & 1.2593 \\
\hline 154 & 2.5000 & 0 & 0 & 2.2668 & 1.2593 \\
\hline 155 & 2.5000 & 0.9322 & 0 & 2.2668 & 1.2593 \\
\hline 156 & 2.5000 & 0 & 0 & 2.2668 & 1.2593 \\
\hline 157 & 0 & 0 & 0 & 2.2668 & 1.2593 \\
\hline 158 & 2.5000 & 0.9322 & 0 & 2.2668 & 1.2593 \\
\hline 159 & 2.5000 & 0 & 7.6186 & 2.2668 & 1.2593 \\
\hline 160 & 2.5000 & 0 & 0 & 2.2668 & 1.2593 \\
\hline 161 & 2.5000 & 0 & 0 & 2.2668 & 1.2593 \\
\hline 162 & 2.5000 & 0.9322 & 7.6186 & 2.2668 & 1.2593 \\
\hline 163 & 0 & 0.1020 & 7.6186 & 2.2668 & 1.2593 \\
\hline 164 & 0 & 0 & 0 & 2.2668 & 1.2593 \\
\hline 165 & 1.4000 & 0.7672 & 0 & 2.2668 & 1.2593 \\
\hline 166 & 0 & 0 & 0 & 2.2668 & 1.2593 \\
\hline 167 & 1.4000 & 0 & 0 & 2.2668 & 1.2593 \\
\hline 168 & 0 & 0 & 0 & 2.2668 & 1.2593 \\
\hline 169 & 0 & 0 & 0 & 2.2668 & 1.2593 \\
\hline
\end{tabular}


Table 4-Continued from previous page

\begin{tabular}{|c|c|c|c|c|c|}
\hline \multirow{2}{*}{ Meta-scenario } & \multicolumn{3}{|c|}{ Recourse(send back to more ethanol production) } & \multicolumn{2}{|c|}{ Recourse(extra sugarcane) } \\
\hline & White $\left(\times 10^{5}\right)$ & $\operatorname{Raw}\left(\times 10^{6}\right)$ & Molasses $(\times 10)$ & $\operatorname{Raw}\left(\times 10^{6}\right)$ & Molasses $(\times 10)$ \\
\hline 170 & 1.4000 & 0 & 0 & 2.2668 & 1.2593 \\
\hline 171 & 0 & 0 & 0 & 2.2668 & 1.2593 \\
\hline 172 & 1.4000 & 0 & 0 & 2.2668 & 1.2593 \\
\hline 173 & 1.4000 & 0 & 0 & 2.2668 & 1.2593 \\
\hline 174 & 0 & 0 & 0 & 2.2668 & 1.2593 \\
\hline 175 & 0 & 0 & 0 & 2.2668 & 1.2593 \\
\hline 176 & 1.4000 & 0.7672 & 7.6186 & 2.2668 & 1.2593 \\
\hline 177 & 1.4000 & 0.7672 & 7.6186 & 2.2668 & 1.2593 \\
\hline 178 & 1.4000 & 0 & 0 & 2.2668 & 1.2593 \\
\hline 179 & 0 & 0 & 0 & 2.2668 & 1.2593 \\
\hline 180 & 1.4000 & 0 & 7.6186 & 2.2668 & 1.2593 \\
\hline 181 & 0 & 0.4022 & 7.6186 & 2.2668 & 1.2593 \\
\hline 182 & 0 & 0 & 0 & 2.2668 & 1.2593 \\
\hline 183 & 0 & 0 & 0 & 2.2668 & 1.2593 \\
\hline 184 & 0 & 0 & 0 & 2.2668 & 1.2593 \\
\hline 185 & 0 & 0 & 0 & 2.2668 & 1.2593 \\
\hline 186 & 0 & 0 & 0 & 2.2668 & 1.2593 \\
\hline 187 & 0 & 0 & 1.0020 & 2.2668 & 1.2593 \\
\hline 188 & 2.5000 & 0 & 7.6186 & 2.2668 & 1.2593 \\
\hline 189 & 0 & 0 & 0 & 2.2668 & 1.2593 \\
\hline 190 & 0 & 0 & 0 & 2.2668 & 1.2593 \\
\hline 191 & 2.5000 & 0 & 0 & 2.2668 & 1.2593 \\
\hline 192 & 2.5000 & 0.9322 & 7.6186 & 2.2668 & 1.2593 \\
\hline 193 & 2.5000 & 0.9322 & 7.6186 & 2.2668 & 1.2593 \\
\hline 194 & 0 & 0 & 0 & 2.2668 & 1.2593 \\
\hline 195 & 2.5000 & 0 & 7.6186 & 2.2668 & 1.2593 \\
\hline 196 & 0 & 0 & 0 & 2.2668 & 1.2593 \\
\hline 197 & 2.5000 & 0.9322 & 0 & 2.2668 & 1.2593 \\
\hline 198 & 2.5000 & 0.9322 & 0 & 2.2668 & 1.2593 \\
\hline 199 & 0 & 0.4526 & 7.6186 & 2.2668 & 1.2593 \\
\hline 200 & 0 & 0 & 0 & 2.2668 & 1.2593 \\
\hline 201 & 0 & 0 & 0 & 2.2668 & 1.2593 \\
\hline 202 & 0 & 0 & 0 & 2.2668 & 1.2593 \\
\hline 203 & 0 & 0 & 0 & 2.2668 & 1.2593 \\
\hline 204 & 1.4000 & 0 & 0 & 2.2668 & 1.2593 \\
\hline 205 & 1.4000 & 0 & 0.0321 & 2.2668 & 1.2593 \\
\hline 206 & 0 & 0 & 0 & 2.2668 & 1.2593 \\
\hline 207 & 0 & 0 & 0 & 2.2668 & 1.2593 \\
\hline 208 & 0 & 0 & 0 & 2.2668 & 1.2593 \\
\hline 209 & 1.4000 & 0 & 0 & 2.2668 & 1.2593 \\
\hline 210 & 1.4000 & 0 & 0 & 2.2668 & 1.2593 \\
\hline 211 & 0 & 0 & 0 & 2.2668 & 1.2593 \\
\hline 212 & 0 & 0 & 0 & 2.2668 & 1.2593 \\
\hline 213 & 1.4000 & 0.7672 & 7.6186 & 2.2668 & 1.2593 \\
\hline 214 & 1.4000 & 0 & 0 & 2.2668 & 1.2593 \\
\hline 215 & 1.4000 & 0.7672 & 7.6186 & 2.2668 & 1.2593 \\
\hline 216 & 1.4000 & 0 & 0 & 2.2668 & 1.2593 \\
\hline 217 & 2.5000 & 0.8791 & 8.8779 & 4.5336 & 2.5186 \\
\hline 218 & 2.5000 & 0.8791 & 8.8779 & 4.5336 & 2.5186 \\
\hline 219 & 2.5000 & 0.8791 & 8.8779 & 4.5336 & 2.5186 \\
\hline 220 & 2.5000 & 1.1589 & 0.0000 & 4.5336 & 2.5186 \\
\hline 221 & 2.5000 & 0.8791 & 8.8779 & 4.5336 & 2.5186 \\
\hline 222 & 2.5000 & 1.1589 & -0.0000 & 4.5336 & 2.5186 \\
\hline 223 & 2.5000 & 0.8791 & 8.8779 & 4.5336 & 2.5186 \\
\hline
\end{tabular}


Table 4-Continued from previous page

\begin{tabular}{|c|c|c|c|c|c|}
\hline \multirow{2}{*}{ Meta-scenario } & \multicolumn{3}{|c|}{ Recourse(send back to more ethanol production) } & \multicolumn{2}{|c|}{ Recourse(extra sugarcane) } \\
\hline & White $\left(\times 10^{5}\right)$ & $\operatorname{Raw}\left(\times 10^{6}\right)$ & Molasses $(\times 10)$ & $\operatorname{Raw}\left(\times 10^{6}\right)$ & Molasses $(\times 10)$ \\
\hline 224 & 2.5000 & 0.8791 & 8.8779 & 4.5336 & 2.5186 \\
\hline 225 & 2.5000 & 0.8791 & 8.8779 & 4.5336 & 2.5186 \\
\hline 226 & 2.5000 & 0.8791 & 8.8779 & 4.5336 & 2.5186 \\
\hline 227 & 2.5000 & 0.8791 & 8.8779 & 4.5336 & 2.5186 \\
\hline 228 & 2.5000 & 1.1589 & 1.9770 & 4.5336 & 2.5186 \\
\hline 229 & 2.5000 & 0.8791 & 8.8779 & 4.5336 & 2.5186 \\
\hline 230 & 2.5000 & 0.8791 & 8.8779 & 4.5336 & 2.5186 \\
\hline 231 & 2.5000 & 0.9483 & 8.8779 & 4.5336 & 2.5186 \\
\hline 232 & 2.5000 & 0.8791 & 8.8779 & 4.5336 & 2.5186 \\
\hline 233 & 2.5000 & 0.8791 & 8.8779 & 4.5336 & 2.5186 \\
\hline 234 & 2.5000 & 1.1589 & 0 & 4.5336 & 2.5186 \\
\hline 235 & 1.4000 & 0.9939 & 8.8779 & 4.5336 & 2.5186 \\
\hline 236 & 1.4000 & 0.9939 & 8.8779 & 4.5336 & 2.5186 \\
\hline 237 & 1.4000 & 0.9939 & 8.8779 & 4.5336 & 2.5186 \\
\hline 238 & 1.4000 & 0.9939 & 8.8779 & 4.5336 & 2.5186 \\
\hline 239 & 1.4000 & 0.9939 & 8.8779 & 4.5336 & 2.5186 \\
\hline 240 & 1.4000 & 0.9939 & 8.8779 & 4.5336 & 2.5186 \\
\hline 241 & 1.4000 & 0.9939 & 8.8779 & 4.5336 & 2.5186 \\
\hline 242 & 1.4000 & 0.9939 & 8.8779 & 4.5336 & 2.5186 \\
\hline 243 & 1.4000 & 0.9939 & 8.8779 & 4.5336 & 2.5186 \\
\hline 244 & 1.4000 & 0.9939 & 8.8779 & 4.5336 & 2.5186 \\
\hline 245 & 1.4000 & 0.9939 & 8.8779 & 4.5336 & 2.5186 \\
\hline 246 & 1.4000 & 0.9939 & 8.8779 & 4.5336 & 2.5186 \\
\hline 247 & 1.4000 & 0.9939 & 8.8779 & 4.5336 & 2.5186 \\
\hline 248 & 1.4000 & 0.9939 & 8.8779 & 4.5336 & 2.5186 \\
\hline 249 & 1.4000 & 0.9939 & 8.8779 & 4.5336 & 2.5186 \\
\hline 250 & 1.4000 & 0.9939 & 8.8779 & 4.5336 & 2.5186 \\
\hline 251 & 1.4000 & 0.9939 & 8.8779 & 4.5336 & 2.5186 \\
\hline 252 & 1.4000 & 0.9939 & 8.8779 & 4.5336 & 2.5186 \\
\hline 253 & 0 & 0.5872 & 8.8779 & 4.5336 & 2.5186 \\
\hline 254 & 2.5000 & 0 & 8.8779 & 4.5336 & 2.5186 \\
\hline 255 & 2.5000 & 0 & 8.8779 & 4.5336 & 2.5186 \\
\hline 256 & 2.5000 & 0 & 8.8779 & 4.5336 & 2.5186 \\
\hline 257 & 2.5000 & 0 & 8.8779 & 4.5336 & 2.5186 \\
\hline 258 & 2.5000 & 0 & 8.8779 & 4.5336 & 2.5186 \\
\hline 259 & 2.5000 & 0 & 8.8779 & 4.5336 & 2.5186 \\
\hline 260 & 2.5000 & 0 & 8.8779 & 4.5336 & 2.5186 \\
\hline 261 & 2.5000 & 0 & 8.8779 & 4.5336 & 2.5186 \\
\hline 262 & 2.5000 & 0 & 8.8779 & 4.5336 & 2.5186 \\
\hline 263 & 2.5000 & 0.2798 & 0 & 4.5336 & 2.5186 \\
\hline 264 & 2.5000 & 0.2798 & 0 & 4.5336 & 2.5186 \\
\hline 265 & 2.5000 & 0 & 8.8779 & 4.5336 & 2.5186 \\
\hline 266 & 2.5000 & 0 & 8.8779 & 4.5336 & 2.5186 \\
\hline 267 & 2.5000 & 0 & 8.8779 & 4.5336 & 2.5186 \\
\hline 268 & 2.5000 & 0 & 8.8779 & 4.5336 & 2.5186 \\
\hline 269 & 2.5000 & 0.2798 & 0 & 4.5336 & 2.5186 \\
\hline 270 & 2.5000 & 1.1589 & 8.8779 & 4.5336 & 2.5186 \\
\hline 271 & 0 & 0.6376 & 8.8779 & 4.5336 & 2.5186 \\
\hline 272 & 1.4000 & 0.3945 & 0 & 4.5336 & 2.5186 \\
\hline 273 & 1.4000 & 0.3945 & 0 & 4.5336 & 2.5186 \\
\hline 274 & 1.4000 & 0.1148 & 8.8779 & 4.5336 & 2.5186 \\
\hline 275 & 1.4000 & 0.9939 & 0 & 4.5336 & 2.5186 \\
\hline 276 & 1.4000 & 0.1148 & 8.8779 & 4.5336 & 2.5186 \\
\hline 277 & 1.4000 & 0.1148 & 8.8779 & 4.5336 & 2.5186 \\
\hline
\end{tabular}


Table 4-Continued from previous page

\begin{tabular}{|c|c|c|c|c|c|}
\hline \multirow{2}{*}{ Meta-scenario } & \multicolumn{3}{|c|}{ Recourse(send back to more ethanol production) } & \multicolumn{2}{|c|}{ Recourse(extra sugarcane) } \\
\hline & White $\left(\times 10^{5}\right)$ & $\operatorname{Raw}\left(\times 10^{6}\right)$ & Molasses $(\times 10)$ & $\operatorname{Raw}\left(\times 10^{6}\right)$ & Molasses $(\times 10)$ \\
\hline 278 & 1.4000 & 0.1148 & 8.8779 & 4.5336 & 2.5186 \\
\hline 279 & 1.4000 & 0.1148 & 8.8779 & 4.5336 & 2.5186 \\
\hline 280 & 1.4000 & 0.3945 & 0 & 4.5336 & 2.5186 \\
\hline 281 & 1.4000 & 0.3945 & 0 & 4.5336 & 2.5186 \\
\hline 282 & 1.4000 & 0.3945 & 0 & 4.5336 & 2.5186 \\
\hline 283 & 1.4000 & 0.1148 & 8.8779 & 4.5336 & 2.5186 \\
\hline 284 & 1.4000 & 0.1148 & 8.8779 & 4.5336 & 2.5186 \\
\hline 285 & 1.4000 & 0.1148 & 8.8779 & 4.5336 & 2.5186 \\
\hline 286 & 1.4000 & 0.9939 & 8.8779 & 4.5336 & 2.5186 \\
\hline 287 & 1.4000 & 0.3945 & 0 & 4.5336 & 2.5186 \\
\hline 288 & 1.4000 & 0.9939 & 8.8779 & 4.5336 & 2.5186 \\
\hline 289 & 0 & 0.9378 & 8.8779 & 4.5336 & 2.5186 \\
\hline 290 & 0 & 0 & 0 & 4.5336 & 2.5186 \\
\hline 291 & 0 & 0 & 0 & 4.5336 & 2.5186 \\
\hline 292 & 0 & 0 & 0 & 4.5336 & 2.5186 \\
\hline 293 & 2.5000 & 0 & 0 & 4.5336 & 2.5186 \\
\hline 294 & 0 & 0 & 0 & 4.5336 & 2.5186 \\
\hline 295 & 0 & 0 & 5.1947 & 4.5336 & 2.5186 \\
\hline 296 & 0 & 0 & 0 & 4.5336 & 2.5186 \\
\hline 297 & 2.5000 & 1.1589 & 8.8779 & 4.5336 & 2.5186 \\
\hline 298 & 0 & 0 & 0 & 4.5336 & 2.5186 \\
\hline 299 & 0 & 0 & 0 & 4.5336 & 2.5186 \\
\hline 300 & 2.5000 & 0 & 0 & 4.5336 & 2.5186 \\
\hline 301 & 0 & 0 & 0 & 4.5336 & 2.5186 \\
\hline 302 & 2.5000 & 0 & 0 & 4.5336 & 2.5186 \\
\hline 303 & 2.5000 & 0 & 8.8779 & 4.5336 & 2.5186 \\
\hline 304 & 2.5000 & 1.1589 & 0 & 4.5336 & 2.5186 \\
\hline 305 & 2.5000 & 0 & 0 & 4.5336 & 2.5186 \\
\hline 306 & 2.5000 & 0 & 0 & 4.5336 & 2.5186 \\
\hline 307 & 0 & 0.9939 & 8.8293 & 4.5336 & 2.5186 \\
\hline 308 & 0 & 0 & 0 & 4.5336 & 2.5186 \\
\hline 309 & 0 & 0 & 0 & 4.5336 & 2.5186 \\
\hline 310 & 0 & 0 & 0 & 4.5336 & 2.5186 \\
\hline 311 & 1.4000 & 0 & 0 & 4.5336 & 2.5186 \\
\hline 312 & 1.4000 & 0 & 0 & 4.5336 & 2.5186 \\
\hline 313 & 0 & 0 & 5.5042 & 4.5336 & 2.5186 \\
\hline 314 & 0 & 0 & 0 & 4.5336 & 2.5186 \\
\hline 315 & 0 & 0 & 0 & 4.5336 & 2.5186 \\
\hline 316 & 0 & 0 & 0 & 4.5336 & 2.5186 \\
\hline 317 & 0 & 0 & 0 & 4.5336 & 2.5186 \\
\hline 318 & 1.4000 & 0.9939 & 8.8779 & 4.5336 & 2.5186 \\
\hline 319 & 0 & 0 & 0 & 4.5336 & 2.5186 \\
\hline 320 & 1.4000 & 0.9939 & 8.8779 & 4.5336 & 2.5186 \\
\hline 321 & 1.4000 & 0.9939 & 8.8779 & 4.5336 & 2.5186 \\
\hline 322 & 0 & 0 & 0 & 4.5336 & 2.5186 \\
\hline 323 & 1.4000 & 0 & 0 & 4.5336 & 2.5186 \\
\hline 324 & 1.4000 & 0.9939 & 8.8779 & 4.5336 & 2.5186 \\
\hline
\end{tabular}




\section{Bibliography}

F. B. Abdelaziz. Solution approaches for the multiobjective stochastic programming. European Journal of Operational Research, 216(1):1 - 16, 2012. ISSN 0377-2217. doi: http://dx.doi.org/ 10.1016/j.ejor.2011.03.033. URL http://www.sciencedirect.com/science/article/pii/ S0377221711002700.

F. B. Abdelaziz and H. Masri. Stochastic programming with fuzzy linear partial information on probability distribution. European Journal of Operational Research, 162(3):619 - 629, 2005. ISSN 0377-2217. doi: http://dx.doi.org/10.1016/j.ejor.2003.10.049. URL http://www . sciencedirect.com/science/article/pii/S0377221704000554. Decision-Aid to Improve Organisational Performance.

F. B. Abdelaziz and S. Mejri. Application of goal programming in a multi-objective reservoir operation model in tunisia. European Journal of Operational Research, 133(2):352 361, 2001. ISSN 0377-2217. doi: http://dx.doi.org/10.1016/S0377-2217(00)00303-9. URL http://www.sciencedirect.com/science/article/pii/S0377221700003039. Multiobjective Programming and Goal Programming.

F. B. Abdelaziz, B. Aouni, and R. E. Fayedh. Multi-objective stochastic programming for portfolio selection. European Journal of Operational Research, 177(3):1811 - 1823, 2007. ISSN 03772217. doi: http://dx.doi.org/10.1016/j.ejor.2005.10.021. URL http://www.sciencedirect. com/science/article/pii/S0377221705006648.

S. Ahmed, A. King, and G. Parija. A multi-stage stochastic integer programming approach for capacity expansion under uncertainty. Journal of Global Optimization, 26(1):3-24, 2003. ISSN 0925-5001. doi: 10.1023/A:1023062915106. URL http://dx.doi.org/10.1023/A\% 3A1023062915106.

O. Akz and D. Petrovic. A fuzzy goal programming method with imprecise goal hierarchy. European Journal of Operational Research, 181(3):1427 - 1433, 2007. ISSN 0377-2217. doi: http://dx.doi.org/10.1016/j.ejor.2005.11.049. URL http://www.sciencedirect.com/ science/article/pii/S0377221706001962.

M. Al-Zahrani and A. Ahmad. Stochastic goal programming model for optimal blending of desalinated water with groundwater. Water Resources Management, 18(4):339-352, 2004. ISSN 0920-4741. doi: 10.1023/B:WARM.0000048487.05662.88. URL http://dx.doi.org/ 10.1023/B\%3AWARM. 0000048487.05662 .88$.

A. Alonso-Ayuso, L. Escudero, A. Garn, M. Ortuo, and G. Prez. An approach for strategic supply chain planning under uncertainty based on stochastic 0-1 programming. Journal of Global Optimization, 26(1):97-124, 2003. ISSN 0925-5001. doi: 10.1023/A:1023071216923. URL http://dx.doi.org/10.1023/A\%3A1023071216923.

B. Aouni. Portfolio selection through the goal programming model: An overview. Journal of Financial Decision Making, 6(2):3-15, 2010a. 
B. Aouni. Portfolio selection through the goal programming model: An overview. Journal of Financial Decision Making, 6(2):3-15, 2010b.

B. Aouni, F. B. Abdelaziz, and J.-M. Martel. Decision-maker's preferences modeling in the stochastic goal programming. European Journal of Operational Research, 162(3):610 - 618, 2005. ISSN 0377-2217. doi: http://dx.doi.org/10.1016/j.ejor.2003.10.035. URL http://www . sciencedirect.com/science/article/pii/S0377221703008282. Decision-Aid to Improve Organisational Performance.

B. Aouni, J.-M. Martel, and A. Hassaine. Fuzzy goal programming model: an overview of the current state-of-the art. Journal of Multi-Criteria Decision Analysis, 16(5-6):149-161, 2009. ISSN 1099-1360. doi: 10.1002/mcda.448. URL http://dx.doi .org/10.1002/mcda. 448.

B. Aouni, C. Colapinto, and D. La Torre. Solving stochastic multi-objective programming in multi-attribute portfolio selection through the goal programming model. Journal of financial decision making, 6(2), 2010.

B. Aouni, F. Ben Abdelaziz, and D. La Torre. The stochastic goal programming model: Theory and applications. Journal of Multi-Criteria Decision Analysis, 19(5-6):185-200, 2012. ISSN 1099-1360. doi: 10.1002/mcda.1466. URL http://dx.doi.org/10.1002/mcda.1466.

B. Aouni, C. Colapinto, and D. La Torre. A cardinality constrained stochastic goal programming model with satisfaction functions for venture capital investment decision making. Annals of Operations Research, 205(1):77-88, 2013. ISSN 0254-5330. doi: 10.1007/s10479-012-1168-4. URL http://dx.doi.org/10.1007/s10479-012-1168-4.

B. Aouni, C. Colapinto, and D. L. Torre. Financial portfolio management through the goal programming model: Current state-of-the-art. European Journal of Operational Research, 234 (2):536 - 545, 2014. ISSN 0377-2217. doi: http://dx.doi.org/10.1016/j.ejor.2013.09.040. URL http://www.sciencedirect.com/science/article/pii/S0377221713007959. 60 years following Harry Markowitzs contribution to portfolio theory and operations research.

S. Arora and R. Gupta. Interactive fuzzy goal programming approach for bilevel programming problem. European Journal of Operational Research, 194(2):368 - 376, 2009. ISSN 0377-2217. URL http://www.sciencedirect.com/science/article/pii/S0377221707012179.

F. Arkan and Z. Gngr. An application of fuzzy goal programming to a multiobjective project network problem. Fuzzy Sets and Systems, 119(1):49 - 58, 2001. ISSN 0165-0114. doi: http://dx.doi.org/10.1016/S0165-0114(99)00119-0. URL http://www.sciencedirect.com/ science/article/pii/S0165011499001190.

J. Ascough II, H. Maier, J. Ravalico, and M. Strudley. Future research challenges for incorporation of uncertainty in environmental and ecological decision-making. Ecological Modelling, 219 (34):383-399, dec 2008. ISSN 0304-3800. URL http://www.sciencedirect.com/science/ article/pii/S0304380008003554.

I. Awudu and J. Zhang. Uncertainties and sustainability concepts in biofuel supply chain management: A review. Renewable and Sustainable Energy Reviews, 16(2):1359-1368, 2012.

I. Awudu and J. Zhang. Stochastic production planning for a biofuel supply chain under demand and price uncertainties. Applied Energy, 103:189-196, 2013.

A. Azaron, K. Brown, S. Tarim, and M. Modarres. A multi-objective stochastic programming approach for supply chain design considering risk. International Journal of Production Economics, 116(1):129 - 138, 2008. ISSN 0925-5273. doi: http://dx.doi.org/10.1016/j.ijpe.2008. 08.002. URL http://www. sciencedirect.com/science/article/pii/S0925527308002375. 
R. Azmi and M. Tamiz. A review of goal programming for portfolio selection. In D. Jones, M. Tamiz, and J. Ries, editors, New Developments in Multiple Objective and Goal Programming, volume 638 of Lecture Notes in Economics and Mathematical Systems, pages 15-33. Springer Berlin Heidelberg, 2010. ISBN 978-3-642-10353-7. doi: 10.1007/978-3-642-10354-4_2. URL http://dx.doi.org/10.1007/978-3-642-10354-4_2.

I. A. Baky. Fuzzy goal programming algorithm for solving decentralized bi-level multi-objective programming problems. Fuzzy Sets and Systems, 160(18):2701 - 2713, 2009. ISSN 01650114. doi: http://dx.doi.org/10.1016/j.fss.2009.02.022. URL http://www.sciencedirect. com/science/article/pii/S0165011409001171. Theme: Decision and Optimisation.

E. Ballestero. Stochastic goal programming: A meanvariance approach. European Journal of Operational Research, 131(3):476 - 481, 2001. ISSN 0377-2217. doi: http://dx.doi.org/ 10.1016/S0377-2217(00)00084-9. URL http://www.sciencedirect.com/science/article/ $\mathrm{pii/S0377221700000849.}$

E. Ballestero. Using stochastic goal programming: Some applications to management and a case of industrial production. Infor-Information Systems and Operational Research, 43(2):63-78, 2005.

E. Ballestero and A. Garcia-Bernabeu. Portfolio selection with multiple time horizons: A mean variance - stochastic goal programming approach. INFOR: Information Systems and Operational Research, 50(3):106-116, 2012.

E. Ballestero, B. Prez-Gladish, M. Arenas-Parra, and A. Bilbao-Terol. Selecting portfolios given multiple eurostoxx-based uncertainty scenarios: A stochastic goal programming approach from fuzzy betas. INFOR: Information Systems and Operational Research, 47(1):59-70, 2009.

S. C. Bankes. Tools and techniques for developing policies for complex and uncertain systems. In PNAS, volume 99, page 72637266. PNAS, 2002.

G. Barbarosoglu and Y. Arda. A two-stage stochastic programming framework for transportation planning in disaster response. J Oper Res Soc, 55(1):43-53, 2004. URL http://dx.doi.org/ 10.1057/palgrave.jors. 2601652 .

E. M. L. Beale. On minimizing a convex function subject to linear inequalities. Journal of the Royal Statistical Society. Series B (Methodological), 17(2):173-184, 1955. URL http: //www.jstor.org/stable/2983952.

R. E. Bellman and L. A. Zadeh. Decision-making in a fuzzy environment. Management Science, 17(4):141-164, 1970.

M. Belmokaddem, M. Mekidiche, and A. Sahed. Application of a fuzzy goal programming approach with different importance and priorities to aggregate production planning. Journal of applied quantitative methods, 4(3):317-331, 2009.

V. Belton and T. Stewart. Multiple criteria decision analysis: an integrated approach. Springer Science \& Business Media, 2002.

F. Ben Abdelaziz and M. Masmoudi. A multiobjective stochastic program for hospital bed planning. Journal of the Operational Research Society, 63(4):530-538, 2012. doi: doi:10. 1057/jors.2011.39. URL http://www.ingentaconnect.com/content/pal/01605682/2012/ 00000063/00000004/art00010.

F. Ben Abdelaziz, R. El Fayedh, and A. Rao. A discrete stochastic goal program for portfolio selection: The case of united arab emirates equity market. INFOR: Information Systems and Operational Research, 47(1):5-13, 2009. 
Y. Ben-Haim. Uncertainty, probability and information-gaps. Reliability Engineering $\&$ System Safety, 85(13):249 - 266, 2004. ISSN 0951-8320. doi: http://dx.doi.org/10.1016/j.ress.2004. 03.015. URL http://www.sciencedirect.com/science/article/pii/S095183200400064X. Alternative Representations of Epistemic Uncertainty.

Y. Ben-Haim. Info-Gap Decision Theory: Decisions Under Severe Uncertainty. Elsevier Science, 2006. ISBN 9780080465708. URL http://books.google.co.za/books?id=yR9H\_WbkIHkC.

A. Ben-Tal and A. Nemirovski. Robust truss topology design via semidefinite programming. SIAM Journal on Optimization, 7(4):991-1016, 1997. doi: 10.1137/S1052623495291951. URL http://dx.doi.org/10.1137/S1052623495291951.

A. Ben-Tal and A. Nemirovski. Robust convex optimization. Mathematics of operations research, 23(4):769-805, 1998.

A. Ben-Tal and A. Nemirovski. Robust solutions of uncertain linear programs. Operations Research Letters, 25(1):1 - 13, 1999. ISSN 0167-6377. doi: http://dx.doi.org/ 10.1016/S0167-6377(99)00016-4. URL http://www.sciencedirect.com/science/article/ pii/S0167637799000164.

A. Ben-Tal and A. Nemirovski. Robust solutions of linear programming problems contaminated with uncertain data. Mathematical Programming, 88(3):411-424, 2000a. ISSN 0025-5610.

A. Ben-Tal and A. Nemirovski. Robust solutions of linear programming problems contaminated with uncertain data. Mathematical programming, 88(3):411-424, 2000b.

D. Bertsimas and M. Sim. The price of robustness. Operations Research, 52(1):35-53, 2004.

D. Bertsimas, D. Brown, and C. Caramanis. Theory and applications of robust optimization. SIAM Review, 53(3):464-501, 2011.

A. Bilbao, M. Arenas, M. Rodrguez, and J. Antomil. On constructing expert betas for singleindex model. European Journal of Operational Research, 183(2):827 - 847, 2007. ISSN 03772217. doi: http://dx.doi.org/10.1016/j.ejor.2006.10.007. URL http://www.sciencedirect . com/science/article/pii/S037722170601040X.

A. Bilbao-Terol, M. Arenas-Parra, and V. Caal-Fernndez. Selection of socially responsible portfolios using goal programming and fuzzy technology. Information Sciences, 189(0): 110 - 125, 2012. ISSN 0020-0255. doi: http://dx.doi.org/10.1016/j.ins.2011.12.001. URL http://www.sciencedirect.com/science/article/pii/S0020025511006293.

A. Biswas and B. B. Pal. Application of fuzzy goal programming technique to land use planning in agricultural system. Omega, 33(5):391 - 398, 2005. ISSN 0305-0483. doi: http://dx.doi.org/10. 1016/j.omega.2004.07.003. URL http://www.sciencedirect.com/science/article/pii/ S0305048304000970. $\{\mathrm{OR}\}$ and its Applications.

M. Boldrin and D. Levine. Economic and game theory: Against intellectual monopoly. online book, Cambridge University Press, January, 2008.

M. Bravo and I. Gonzalez. Applying stochastic goal programming: A case study on water use planning. European Journal of Operational Research, 196(3):1123 - 1129, 2009. ISSN 03772217. doi: http://dx.doi.org/10.1016/j.ejor.2008.04.034. URL http://www.sciencedirect . com/science/article/pii/S0377221708003937.

P. L. Brockett, A. Charnes, W. W. Cooper, K.-H. Kwon, and T. W. Ruefli. Chance constrained programming approach to empirical analyses of mutual fund investment strategies*. Decision Sciences, 23(2):385-408, 1992. ISSN 1540-5915. doi: 10.1111/j.1540-5915.1992.tb00396.x. URL http://dx.doi.org/10.1111/j.1540-5915.1992.tb00396.x. 
T. Brown and R. Strauch. Dynamic programming in multiplicative lattices. Journal of Mathematical Analysis and Applications, 12(2):364 - 370, 1965. ISSN 0022-247X. doi: http://dx.doi.org/10.1016/0022-247X(65)90045-4. URL http://www.sciencedirect.com/ science/article/pii/0022247X65900454.

R. Caballero, E. Cerd, M. Muoz, L. Rey, and I. Stancu-Minasian. Efficient solution concepts and their relations in stochastic multiobjective programming. Journal of Optimization Theory and Applications, 110(1):53-74, 2001. ISSN 0022-3239. doi: 10.1023/A:1017591412366. URL http://dx.doi.org/10.1023/A\%3A1017591412366.

R. Caballero, T. Gmez, and F. Ruiz. Goal programming: realistic targets for the near future. Journal of Multi-Criteria Decision Analysis, 16(3-4):79-110, 2009. ISSN 1099-1360. doi: 10.1002/mcda.442. URL http://dx.doi.org/10.1002/mcda. 442.

G. A. Chalam. Fuzzy goal programming (fgp) approach to a stochastic transportation problem under budgetary constraint. Fuzzy Sets and Systems, 66(3):293 - 299, 1994. ISSN 0165-0114. doi: http://dx.doi.org/10.1016/0165-0114(94)90096-5. URL http://www.sciencedirect. com/science/article/pii/0165011494900965.

F. T. Chan and R. Swarnkar. Ant colony optimization approach to a fuzzy goal programming model for a machine tool selection and operation allocation problem in an \{FMS\}. Robotics and Computer-Integrated Manufacturing, 22(4):353 - 362, 2006. ISSN 0736-5845. doi: http: //dx.doi.org/10.1016/j.rcim.2005.08.001. URL http://www.sciencedirect.com/science/ article/pii/S073658450500061X.

F. T. S. Chan, R. Swarnkar, and M. K. Tiwari. Fuzzy goal-programming model with an artificial immune system (ais) approach for a machine tool selection and operation allocation problem in a flexible manufacturing system. International Journal of Production Research, 43 (19):4147-4163, 2005. doi: 10.1080/00207540500140823. URL http://dx.doi.org/10.1080/ 00207540500140823.

C.-T. Chang. Binary fuzzy goal programming. European Journal of Operational Research, 180 (1):29 - 37, 2007. ISSN 0377-2217. doi: http://dx.doi.org/10.1016/j.ejor.2006.03.030. URL http://www.sciencedirect.com/science/article/pii/S0377221706002116.

N.-B. Chang and S. Wang. A fuzzy goal programming approach for the optimal planning of metropolitan solid waste management systems. European Journal of Operational Research, 99 (2):303 - 321, 1997. ISSN 0377-2217. doi: http://dx.doi.org/10.1016/S0377-2217(96)00024-0. URL http://www.sciencedirect.com/science/article/pii/S0377221796000240.

C. Changchit and M. Terrell. A multiobjective reservoir operation model with stochatic inflows. Computers 83 Industrial Engineering, 24(2):303 - 313, 1993. ISSN 0360-8352. doi: http://dx.doi.org/10.1016/0360-8352(93)90016-Q. URL http://www. sciencedirect.com/ science/article/pii/036083529390016Q.

A. Charnes and W. Cooper. Chance constraints and normal deviates. J. Am. Stat. Assoc., 57: 134-148, 1952.

A. Charnes and W. Cooper. Chance-constrained programming. Management Science, 6:73-80, 1959.

A. Charnes and W. Cooper. Deterministic equivalents for optimising and satisfying under chance constraints. Operations Research, 11:1839, 1963.

A. Charnes, W. Cooper, and R. Ferguson. Optimal estimation of executive compensation by linear programming. Management Science, 1:138-351, 1955. 
A. Charnes, S. Duffuaa, and A. Al-Saffar. A dynamic goal programming model for planning food self-sufficiency in the middle east. Applied Mathematical Modelling, 13(2):86 - 93, 1989. ISSN 0307-904X. doi: http://dx.doi.org/10.1016/0307-904X(89)90019-X. URL http://www . sciencedirect.com/science/article/pii/0307904X8990019X.

A. C. Charnes et al. Management models and industrial applications of linear programming. Technical report, Wiley, New York, 1961.

H.-K. Chen. A note on a fuzzy goal programming algorithm by tiwari, dharmar, and rao. Fuzzy Sets and Systems, 62(3):287 - 290, 1994. ISSN 0165-0114. doi: http://dx.doi.org/10. 1016/0165-0114(94)90112-0. URL http://www.sciencedirect.com/science/article/pii/ 0165011494901120.

L.-H. Chen and F.-C. Tsai. Fuzzy goal programming with different importance and priorities. European Journal of Operational Research, 133(3):548 - 556, 2001. ISSN 0377-2217. doi: http://dx.doi.org/10.1016/S0377-2217(00)00201-0. URL http://www.sciencedirect.com/ science/article/pii/S0377221700002010.

L.-H. Chen and M.-C. Weng. An evaluation approach to engineering design in $\{$ QFD $\}$ processes using fuzzy goal programming models. European Journal of Operational Research, 172(1): 230 - 248, 2006. ISSN 0377-2217. doi: http://dx.doi.org/10.1016/j.ejor.2004.10.004. URL http://www.sciencedirect.com/science/article/pii/S0377221704007842.

C. Colapinto, R. Jayaraman, and S. Marsiglio. Multi-criteria decision analysis with goal programming in engineering, management and social sciences: a state-of-the art review. Annals of Operations Research, pages 1-34, 2015.

E. C. Colin. Mathematical programming accelerates implementation of agro-industrial sugarcane complex. European Journal of Operational Research, 199(1):232-235, 2009.

B. Contini. A stochastic approach to goal programming. Operations Research, 16(3):576-586, 1968.

H. Courtney. 20/20 foresight: Crafting strategy in an uncertain world. Harvard Business School Press, Boston., 2001.

M. Dal-Mas, S. Giarola, A. Zamboni, and F. Bezzo. Strategic design and investment capacity planning of the ethanol supply chain under price uncertainty. Biomass and Bioenergy, 35(5): 2059-2071, 2011.

G. Dantzig. Linear Programming and Extensions. Landmarks in Physics and Mathematics. Princeton University Press, 1998. ISBN 9780691059136. URL http://books.google.co.za/ books?id=2j46uCX5ZAYC.

G. B. Dantzig. Linear programming under uncertainty. Management Science, 1(3-4):197-206, 1955. doi: 10.1287/mnsc.1.3-4.197. URL http://dx.doi.org/10.1287/mnsc.1.3-4.197.

G. B. Dantzig. Linear programming and its extensions, 1963.

G. B. Dantzig and A. Medansky. On the solution of two-stage linear programs under uncertainty, 1961. URL http://projecteuclid.org/euclid.bsmsp/1200512165.

S. C. de Vries, G. W. van de Ven, M. K. van Ittersum, and K. E. Giller. Resource use efficiency and environmental performance of nine major biofuel crops, processed by first-generation conversion techniques. Biomass and Bioenergy, 34(5):588-601, 2010. 
K. Deb and K. Miettinen. Multiobjective optimization: interactive and evolutionary approaches, volume 5252. Springer Science \& Business Media, 2008.

M. H. DeGroot. Utility, pages 86-118. John Wiley \& Sons, Inc., 2005. ISBN 9780471729006. doi: 10.1002/0471729000.ch7. URL http://dx.doi.org/10.1002/0471729000.ch7.

A. K. Dhingra, S. S. Rao, and V. Kumar. Nonlinear membership functions in multiobjective fuzzy optimization of mechanical and structural systems. AIAA Journal, 30(1):251-260, 1992. URL http://dx.doi.org/10.2514/3.10906.

L. Diaz-Balteiro and C. Romero. Sustainability of forest management plans: a discrete goal programming approach. Journal of Environmental Management, 71(4):351 - 359, 2004. ISSN 0301-4797. doi: http://dx.doi.org/10.1016/j.jenvman.2004.04.001. URL http://www . sciencedirect.com/science/article/pii/S0301479704000659.

A. J. Dunnett, C. S. Adjiman, and N. Shah. A spatially explicit whole-system model of the lignocellulosic bioethanol supply chain: an assessment of decentralised processing potential. Biotechnology for biofuels, 1(1):13, 2008.

J. Dupačová. Multistage stochastic programs: The state-of-the-art and selected bibliography. Kybernetika, 31(2):151-174, 1995.

J. Dupacov. Stability in stochastic programming with recourse-estimated parameters. Mathematical Programming, 28(1):72-83, 1984. ISSN 0025-5610. doi: 10.1007/BF02612713. URL http://dx.doi.org/10.1007/BF02612713.

J. Dupacov. Stability in stochastic programming with recourse. contaminated distributions. In A. Prkopa and R.-B. Wets, editors, Stochastic Programming 84 Part I, volume 27 of Mathematical Programming Studies, pages 133-144. Springer Berlin Heidelberg, 1986. ISBN 978-3642-00924-2. doi: 10.1007/BFb0121117. URL http://dx.doi.org/10.1007/BFb0121117.

J. Dupacov, G. Consigli, and S. Wallace. Scenarios for multistage stochastic programs. Annals of Operations Research, 100(1-4):25-53, 2000. ISSN 0254-5330. doi: 10.1023/A:1019206915174. URL http://dx.doi .org/10.1023/A\%3A1019206915174.

I. Durbach and T. J. Stewart. Integrating scenario planning and goal programming. Journal of Multi-Criteria Decision Analysis, 12(4-5):261-271, 2003. ISSN 1099-1360.

I. N. Durbach and T. J. Stewart. Modeling uncertainty in multi-criteria decision analysis. European Journal of Operational Research, 223(1):1 - 14, 2012. ISSN 0377-2217. doi: http://dx.doi.org/10.1016/j.ejor.2012.04.038. URL http://www.sciencedirect.com/ science/article/pii/S0377221712003591.

F. F. Easton and D. F. Rossin. A stochastic goal program for employee scheduling*. Decision Sciences, 27(3):541-568, 1996. ISSN 1540-5915. doi: 10.1111/j.1540-5915.1996.tb00863.x. URL http://dx.doi.org/10.1111/j.1540-5915.1996.tb00863.x.

W. F. El-Wahed and M. A. Abo-Sinna. A hybrid fuzzy-goal programming approach to multiple objective decision making problems. Fuzzy Sets and Systems, 119(1):71 - 85, 2001. ISSN 0165-0114. doi: http://dx.doi.org/10.1016/S0165-0114(99)00050-0. URL http://www . sciencedirect.com/science/article/pii/S0165011499000500.

W. F. A. El-Wahed and S. M. Lee. Interactive fuzzy goal programming for multi-objective transportation problems. Omega, 34(2):158 - 166, 2006. ISSN 0305-0483. doi: http: //dx.doi.org/10.1016/j.omega.2004.08.006. URL http://www.sciencedirect.com/science/ article/pii/S0305048304001392. 
E. A. Eriksson and K. M. Weber. Adaptive foresight: Navigating the complex landscape of policy strategies. Technological Forecasting and Social Change, 75(4):462 - 482, 2008. ISSN 0040-1625. doi: http://dx.doi.org/10.1016/j.techfore.2008.02.006. URL http://www. sciencedirect.com/science/article/pii/s004016250800036X. Future-Oriented Technology Analysis (FTA): Impact on Policy and Decision Making The 2006 \{FTA $\}$ International Seville Seminar.

Y. Ermoliev. Numerical techniques for stochastic optimization. Springer-Verlag New York, Inc., 1988.

A. N. S. Freeling. Fuzzy sets and decision analysis. Systems, Man and Cybernetics, IEEE Transactions on, 10(7):341-354, July 1980. ISSN 0018-9472. doi: 10.1109/TSMC.1980.4308515.

S. French. Uncertainty and imprecision: Modelling and analysis. Journal of theOperational Research Society, 46:70-79, 1995.

S. Funtowicz and J. Ravetz. Uncertainty and Quality in Science for Policy. Law in Eastern Europe. Springer, 1990. ISBN 9780792307990. URL http://books.google.co.za/books? id=lINAsNfN7i $\mathrm{i}$ C.

V. Gabrel, C. Murat, and A. Thiele. Recent advances in robust optimization: An overview. European Journal of Operational Research, 235(3):471 - 483, 2014. ISSN 0377-2217.

M. Gen, K. Ida, Y. Tsujimura, and C. E. Kim. Large-scale 01 fuzzy goal programming and its application to reliability optimization problem. Computers $\mathcal{E}$ Industrial Engineering, 24(4): 539 - 549, 1993. ISSN 0360-8352. doi: http://dx.doi.org/10.1016/0360-8352(93)90196-5. URL http://www.sciencedirect.com/science/article/pii/0360835293901965.

H. Germinshuis. Republic of south africa, bio-fuels annual report 2006. Global Agriculture Information Network (GAIN), Pretoria, page 8, 2006.

A. Ghahtarani and A. A. Najafi. Robust goal programming for multi-objective portfolio selection problem. Economic Modelling, 33(1):588 - 592, 2013. ISSN 0264-9993.

I. Giannikos. Fuzzy goal programming models for analyzing demand coverage. TOP, 18(1): 185-202, 2010. ISSN 1134-5764. doi: 10.1007/s11750-009-0119-y. URL http://dx.doi.org/ $10.1007 / \mathrm{s} 11750-009-0119-\mathrm{y}$.

S. Giarola, A. Zamboni, and F. Bezzo. Spatially explicit multi-objective optimisation for design and planning of hybrid first and second generation biorefineries. Computers \& Chemical Engineering, 35(9):1782-1797, 2011.

P. K. Giri, M. K. Maiti, and M. Maiti. Fuzzy stochastic solid transportation problem using fuzzy goal programming approach. Computers 83 Industrial Engineering, 72(0):160 - 168, 2014. ISSN 0360-8352. doi: http://dx.doi.org/10.1016/j.cie.2014.03.001. URL http://www. sciencedirect.com/science/article/pii/S0360835214000746.

A. Goicoechea, D. Hansen, and L. Duckstein. Multiobjective Decision Analysis with Engineering and Business Applications. Wiley, New York, 1982.

J. Goldemberg, S. T. Coelho, and P. Guardabassi. The sustainability of ethanol production from sugarcane. Energy policy, 36(6):2086-2097, 2008.

googlemap. Googlemap, 2017. URL https://www.google.com/maps/.

M. Grunow, H.-O. Günther, and R. Westinner. Supply optimization for the production of raw sugar. International Journal of Production Economics, 110(1):224-239, 2007. 
M. Gupta and D. Bhattacharjee. Min sum weighted fuzzy goal programming model in investment management planning: a case study. International Research Journal of Finance and Economics, 56:76-81, 2010.

M. Gupta and D. Bhattacharya. Goal programming and fuzzy goal programming techniques in the bank investment plans under the scenario of maximizing profit and minimizing risk factor: A case study. Advances in Fuzzy Mathematics, 5(2), 2010.

M. Haasnoot, H. Middelkoop, E. van Beek, and W. P. A. van Deursen. A method to develop sustainable water management strategies for an uncertain future. Sustainable Development, 19(6):369-381, 2011. ISSN 1099-1719. doi: 10.1002/sd.438. URL http://dx.doi.org/10. $1002 / \mathrm{sd} .438$.

E. L. Hannan. On fuzzy goal programming*. Decision Sciences, 12(3):522-531, 1981a. ISSN 1540-5915. doi: 10.1111/j.1540-5915.1981.tb00102.x. URL http://dx.doi.org/10.1111/j . 1540-5915.1981.tb00102.x.

E. L. Hannan. Linear programming with multiple fuzzy goals. Fuzzy Sets and Systems, 6(3):235 - 248, 1981b. ISSN 0165-0114. doi: http://dx.doi.org/10.1016/0165-0114(81)90002-6. URL http://www.sciencedirect.com/science/article/pii/0165011481900026.

E. L. Hannan. Some further comments on fuzzy priorities. Decision Sciences, 12(3):539-541, 1981c. ISSN 1540-5915. doi: 10.1111/j.1540-5915.1981.tb00104.x. URL http://dx.doi.org/ $10.1111 / j .1540-5915.1981 . t b 00104 . x$.

E. L. Hannan. Contrasting fuzzy goal programming and fuzzy? multicriteria programming* . Decision Sciences, 13(2):337-339, 1982. ISSN 1540-5915. doi: 10.1111/j.1540-5915.1982. tb00153.x. URL http://dx.doi.org/10.1111/j.1540-5915.1982.tb00153.x.

E. Hawkins and R. Sutton. The potential to narrow uncertainty in regional climate predictions. Bulletin of the American Meteorological Society, 90(8):1095-1107, 2009/08/01 2009. doi: 10. 1175/2009BAMS2607.1. URL http://dx.doi.org/10.1175/2009BAMS2607.1. ISSN: 00030007 .

J. C. Helm, G. L. Curry, and G. L. Hogg. Capacity expansion of linked reservoir systems. Computers \& Industrial Engineering, 8(2):119 - 128, 1984. ISSN 0360-8352. doi: http:// dx.doi.org/10.1016/0360-8352(84)90004-4. URL http://www.sciencedirect.com/science/ article/pii/0360835284900044.

J. C. Helton and W. L. Oberkampf. Guest editorial: Alternative representations of epistemic uncertainty. Reliability Engineering and System Safety, 85(1-3):1-10, 2004.

J. C. Helton, J. D. Johnson, and W. L. Oberkampf. An exploration of alternative approaches to the representation of uncertainty in model predictions. Reliability Engineering and System Safety, 85(1-3):39-71, 2004.

M. Henrion and B. Fischhoff. Uncertainty assessment in the estimation of physical constants. American Journal of Physics, 54:791-798, 1986.

C.-F. Hu, C.-J. Teng, and S.-Y. Li. A fuzzy goal programming approach to multi-objective optimization problem with priorities. European Journal of Operational Research, 176(3):1319 - 1333, 2007. ISSN 0377-2217. doi: http://dx.doi.org/10.1016/j.ejor.2005.10.049. URL http: //www.sciencedirect.com/science/article/pii/S0377221705008817.

C.-L. Hwang and A. S. M. Masud. Multiple objective decision making?methods and applications: a state-of-the-art survey, volume 164. Springer Science \& Business Media, 2012. 
J. P. Ignizio. Goal programming and extensions, volume 26. Lexington Books Lexington, MA, 1976.

J. P. Ignizio. Linear programming in single-\&3 multiple-objective systems. Prentice-Hall Englewood Cliffs, NJ, 1982a.

J. P. Ignizio. On the (re)discovery of fuzzy goal programming. Decision Sciences, 13(2):331-336, 1982b. ISSN 1540-5915. doi: 10.1111/j.1540-5915.1982.tb00152.x. URL http://dx.doi.org/ 10.1111/j.1540-5915.1982.tb00152.x.

Y. Ijiri. Management goals and accounting for control, volume 3. North Holland Pub. Co., 1965.

G. Infanger. Planning under uncertainty: solving large-scale stochastic linear programs. Boyd \& Fraser Danvers, MA, 1994.

G. Ioannou. Streamlining the supply chain of the hellenic sugar industry. Journal of Food Engineering, 70(3):323-332, 2005.

A. Jamalnia and M. A. Soukhakian. A hybrid fuzzy goal programming approach with different goal priorities to aggregate production planning. Computers $\& 3$ Industrial Engineering, 56(4): 1474 - 1486, 2009. ISSN 0360-8352. doi: http://dx.doi.org/10.1016/j.cie.2008.09.010. URL http://www.sciencedirect.com/science/article/pii/S0360835208002155.

R. K. Jana and D. K. Sharma. Genetic algorithm-based fuzzy goal programming for class of chance-constrained programming problems. International Journal of Computer Mathematics, 87(4):733-742, 2010. doi: 10.1080/00207160801998934. URL http://dx.doi.org/10.1080/ 00207160801998934 .

B. Javadi, A. Rahimi-Vahed, M. Rabbani, and M. Dangchi. Solving a multi-objective mixed-model assembly line sequencing problem by a fuzzy goal programming approach. The International Journal of Advanced Manufacturing Technology, 39(9-10):975-982, 2008. ISSN 0268-3768. doi: 10.1007/s00170-007-1267-6. URL http://dx.doi.org/10.1007/ s00170-007-1267-6.

R. Jayaraman, C. Colapinto, D. Liuzzi, and D. La Torre. Planning sustainable development through a scenario-based stochastic goal programming model. Operational Research, pages $1-17,2016$.

X. Ji, S. Zhu, S. Wang, and S. Zhang. A stochastic linear goal programming approach to multistage portfolio management based on scenario generation via linear programming. IIE Transactions, 37(10):957-969, 2005. doi: 10.1080/07408170591008082. URL http://www . tandfonline.com/doi/abs/10.1080/07408170591008082.

A. Jinturkar and S. Deshmukh. A fuzzy mixed integer goal programming approach for cooking and heating energy planning in rural india. Expert Systems with Applications, 38(9):11377 - 11381, 2011. ISSN 0957-4174. doi: http://dx.doi.org/10.1016/j.eswa.2011.03.006. URL http://www.sciencedirect.com/science/article/pii/S0957417411004143.

D. Jones and M. Tamiz. Goal programming in the period 19902000. In M. Ehrgott and X. Gandibleux, editors, Multiple Criteria Optimization: State of the Art Annotated Bibliographic Surveys, volume 52 of International Series in Operations Research 85 Management Science, pages 129-170. Springer US, 2002. ISBN 978-1-4020-7128-7. doi: 10.1007/ 0-306-48107-3_3. URL http://dx.doi.org/10.1007/0-306-48107-3_3.

D. Jones and M. Tamiz. Practical Goal Programming. International Series in Operations Research \& Management Science. Springer, 2010. ISBN 9781441957719. URL http://books.google. co.za/books?id=-TgNzYtVZVgC. 
E. A. K. Darby-Dowman, S. Barker and D. Parsons. A two-stage stochastic programming with recourse model for determining robust planting plans in horticulture. The Journal of the Operational Research Society, 51(1):83-89, Jan. 2000. URL http://www.jstor.org/stable/ 253950.

P. Kall and D. Stoyan. Solving stochastic programming problems with recourse including error bounds. Mathematische Operationsforschung und Statistik. Series Optimization, 13(3):431447, 1982. doi: 10.1080/02331938208842805. URL http://www.tandfonline.com/doi/abs/ 10.1080/02331938208842805.

P. Kall and S. Wallace. Stochastic programming. Jonh Wiley and Sons, Chichester, 1994.

Y. Kara, T. Paksoy, and C.-T. Chang. Binary fuzzy goal programming approach to single model straight and u-shaped assembly line balancing. European Journal of Operational Research, 195(2):335 - 347, 2009. ISSN 0377-2217. doi: http://dx.doi.org/10.1016/j.ejor.2008.01.003. URL http://www.sciencedirect.com/science/article/pii/S0377221708001343.

M. Kawamura, D. Ronconi, and H. Yoshizaki. Optimizing transportation and storage of final products in the sugar and ethanol industry: a case study. International Transactions in Operational Research, 13(5):425-439, 2006.

A. J. Keown. A chance-constrained goal programming model for bank liquidity management. Decision Sciences, 9(1):93-106, 1978. ISSN 1540-5915. doi: 10.1111/j.1540-5915.1978.tb01369. x. URL http://dx.doi.org/10.1111/j.1540-5915.1978.tb01369.x.

A. J. Keown and B. W. T. III. A chance-constrained integer goal programming model for capital budgeting in the production area. The Journal of the Operational Research Society, 31(7): 579-589, Jul 1980. URL http://www.jstor.org/stable/2580845.

A. J. Keown and J. D. Martin. A chance constrained goal programming model for working capital management. The Engineering Economist, 22(3):153-174, 1977. doi: 10.1080/00137917708965174. URL http://www.tandfonline.com/doi/abs/10.1080/ 00137917708965174.

K. Khalili-Damghani, S. Sadi-Nezhad, and M. Tavana. Solving multi-period project selection problems with fuzzy goal programming based on \{TOPSIS \} and a fuzzy preference relation. Information Sciences, 252(0):42 - 61, 2013. ISSN 0020-0255. doi: http://dx.doi.org/ 10.1016/j.ins.2013.05.005. URL http://www.sciencedirect.com/science/article/pii/ S0020025513003794.

A. Kharrat, H. Chabchoub, B. Aouni, and S. Smaoui. Serial correlation estimation through the imprecise goal programming model. European Journal of Operational Research, 177(3): 1839-1851, 3/16/ 2007. doi: http://dx.doi.org/10.1016/j.ejor.2005.10.004. URL http://www. sciencedirect.com/science/article/pii/S0377221705006582.

C. S. Khor, A. Elkamel, K. Ponnambalam, and P. L. Douglas. Two-stage stochastic programming with fixed recourse via scenario planning with economic and operational risk management for petroleum refinery planning under uncertainty. Chemical Engineering and Processing: Process Intensification, 47(910):1744 - 1764, 2008. ISSN 0255-2701. doi: http://dx.doi.org/ 10.1016/j.cep.2007.09.016. URL http://www.sciencedirect.com/science/article/pii/ S0255270107003078.

J. Kim, M. J. Realff, and J. H. Lee. Optimal design and global sensitivity analysis of biomass supply chain networks for biofuels under uncertainty. Computers $\&$ Chemical Engineering, 35 (9):1738-1751, 2011. 
J. S. Kim and K.-S. Whang. A tolerance approach to the fuzzy goal programming problems with unbalanced triangular membership function. European Journal of Operational Research, 107 (3):614 - 624, 1998. ISSN 0377-2217. doi: http://dx.doi.org/10.1016/S0377-2217(96)00363-3. URL http://www.sciencedirect.com/science/article/pii/S0377221796003633.

P. O. Kim, K. J. Lee, and B. W. Lee. Selection of an optimal nuclear fuel cycle scenario by goal programming and the analytic hierarchy process. Annals of Nuclear Energy, 26(5):449 - 460, 1999. ISSN 0306-4549. doi: http://dx.doi.org/10.1016/S0306-4549(98)00081-4. URL http://www.sciencedirect.com/science/article/pii/S0306454998000814.

R. Klotzler. Multiobjective dynamic programming. Mathematische Operationsforschung und Statistik. Series Optimization, 9(3):423-426, 1978. doi: 10.1080/02331937808842507. URL http://dx.doi.org/10.1080/02331937808842507.

F. H. Knight. Risk, Uncertainty and Profit,. Mineola, New York, Dover Publication., 1921.

K. Kosmidou and C. Zopounidis. Combining goal programming model with simulation analysis for bank asset liability management. INFOR: Information Systems and Operational Research, 42(3):175-187, 2004.

A. Kostin, G. Guillén-Gosálbez, F. Mele, M. Bagajewicz, and L. Jiménez. Design and planning of infrastructures for bioethanol and sugar production under demand uncertainty. chemical engineering research and design, 90(3):359-376, 2012.

D. Kuchta. Robust goal programming. Control and Cybernetics, 33(3):501-510, 2004.

M. Kumar, P. Vrat, and R. Shankar. A fuzzy goal programming approach for vendor selection problem in a supply chain. Computers \& Industrial Engineering, 46(1):69 - 85, 2004. ISSN 0360-8352. doi: http://dx.doi.org/10.1016/j.cie.2003.09.010. URL http://www . sciencedirect.com/science/article/pii/S0360835203000986.

J. Kwakkel, W. Walker, and V. Marchau. From predictive modeling to exploratory modeling: How to use non- predictive models for decisionmaking under deep uncertainty. In 25th MiniEURO Conference on Uncertainty and Robustness in Planning and Decision Making (URPDM 2010), Coimbra - Portugal, April 2010.

J. H. Kwakkel and V. A. W. J. Walker, Warren E. Marchau. Classifying and communicating uncertainties in model-based policy analysis. International Journal of Technology, Policy and Management, 10(4):299-315, January 2010. doi: 10.1504/IJTPM.2010.036918. URL http://dx.doi.org/10.1504/IJTPM.2010.036918.

C.-S. Lee and C.-G. Wen. Fuzzy goal programming approach for water quality management in a river basin. Fuzzy Sets and Systems, 89(2):181 - 192, 1997. ISSN 0165-0114. doi: http://dx.doi.org/10.1016/S0165-0114(96)00089-9. URL http://www.sciencedirect.com/ science/article/pii/S0165011496000899.

S. Lee. Goal programming for decision analysis of multiple objectives. Sloan Manage. Rev., 14: 11-24, 1973.

S. Lee and D. Olson. Goal programming. In T. Gal, T. Stewart, and T. Hanne, editors, Multicriteria Decision Making, volume 21 of International Series in Operations Research E Management Science, pages 203-235. Springer US, 1999. ISBN 978-1-4613-7283-7. doi: 10.1007/978-1-4615-5025-9_8. URL http://dx.doi.org/10.1007/978-1-4615-5025-9_8.

S. M. Lee and E. R. Clayton. A goal programming model for academic resource allocation. Management Science, 18(8):B-395-B-408, 1972. doi: 10.1287/mnsc.18.8.B395. URL http: //dx.doi.org/10.1287/mnsc.18.8.B395. 
S. M. Lee and D. L. Olson. A gradient algorithm for chance constrained nonlinear goal programming. European Journal of Operational Research, 22(3):359 - 369, 1985. ISSN 0377-2217. doi: http://dx.doi.org/10.1016/0377-2217(85)90255-3. URL http://www. sciencedirect. com/science/article/pii/0377221785902553.

S. M. Lee, G. I. Green, and C. S. Kim. A multiple criteria model for the location-allocation problem. Computers $\& 3$ Operations Research, 8(1):1 - 8, 1981. ISSN 0305-0548. doi: http:// dx.doi.org/10.1016/0305-0548(81)90026-5. URL http://www. sciencedirect. com/science/ article/pii/0305054881900265.

R. Lempert, S. Popper, and S. Bankes. Shaping the Next One Hundred Years: New Methods for Quantitative, Long Term Policy Analysis. Santa Monica, CA, USA, Report MR-1626-RPC; RAND., 2003.

R. J. Lempert and M. T. Collins. Managing the risk of uncertain threshold responses: Comparison of robust, optimum, and precautionary approaches. Risk Analysis, 27(4):1009-1026, 2007. ISSN 1539-6924. doi: 10.1111/j.1539-6924.2007.00940.x. URL http://dx.doi.org/10.1111/ j.1539-6924.2007.00940.x.

R. J. Lempert, D. G. Groves, S. W. Popper, and S. C. Bankes. A general, analytic method for generating robust strategies and narrative scenarios. Management Science, 52(4):514-528, 2006. doi: 10.1287/mnsc.1050.0472. URL http://dx.doi.org/10.1287/mnsc. 1050.0472.

T. C. M. Letete. Multiobjective modelling of biofuel supply systems. PhD thesis, University of Cape Town, 2009.

R. R. Levary. Dynamic programming models with goal objectives. International Journal of Systems Science, 15(3):309-314, 1984. doi: 10.1080/00207728408926564. URL http://dx. doi.org/10.1080/00207728408926564.

G. Li. Fuzzy goal programming a parametric approach. Information Sciences, 195(0):287 295, 2012. ISSN 0020-0255. doi: http://dx.doi.org/10.1016/j.ins.2012.01.043. URL http: //www.sciencedirect.com/science/article/pii/S0020025512000710.

P. Li, H. Arellano-Garcia, and G. Wozny. Chance constrained programming approach to process optimization under uncertainty. Computers $\&$ chemical engineering, 32(1):25-45, 2008.

Y. Li, G. Huang, N. Zhang, and S. Nie. An inexact-stochastic with recourse model for developing regional economic-ecological sustainability under uncertainty. Ecological Modelling, 222(2):370 - 379, 2011. ISSN 0304-3800. doi: http://dx.doi.org/10.1016/j.ecolmodel.2009.12.010. URL http://www.sciencedirect.com/science/article/pii/S0304380009008400. Wetlands in China.

C.-C. Lin. A weighted max-min model for fuzzy goal programming. Fuzzy Sets and Systems, 142 (3):407 - 420, 2004. ISSN 0165-0114. doi: http://dx.doi.org/10.1016/S0165-0114(03)00092-7. URL http://www.sciencedirect.com/science/article/pii/S0165011403000927.

J. Linderoth. Stochastic programming. Lecture Notes), Lehigh University, IE495, 2003.

B. Liu and K. Iwamura. A note on chance constrained programming with fuzzy coefficients. Fuzzy Sets and Systems, 100(13):229 - 233, 1998. ISSN 0165-0114. doi: http://dx.doi.org/ 10.1016/S0165-0114(97)00291-1. URL http://www.sciencedirect.com/science/article/ $\mathrm{pii} / \mathrm{S} 0165011497002911$.

M. L. Liu and N. V. Sahinidis. Optimization in process planning under uncertainty. Industrial \&3 Engineering Chemistry Research, 35(11):4154-4165, 1996. 
M. Maggis and D. La Torre. A goal programming model with satisfaction function for risk management and optimal portfolio diversification. INFOR: Information Systems and Operational Research, 50(3):117-126, 2012.

N. Mansour, A. Rebai, and B. Aouni. Portfolio selection through imprecise goal programming model: Integration of the manager's preferences. Journal of Industrial Engineering International Islamic Azad University, 3(5):1-8, 2007.

V. Marchau, W. Walker, and G. van Wee. Dynamic adaptive transport policies for handling deep uncertainty. Technological Forecasting and Social Change, 77(6):940-950, jul 2010. ISSN 00401625. URL http://www.sciencedirect.com/science/article/pii/S0040162510000739.

S. Mardle and S. Pascoe. Modelling the effects of trade-offs between long and short-term objectives in fisheries management. Journal of Environmental Management, 65(1):49-62, 2002.

A. Martel and W. Price. Stochastic programming applied to human resource planning. The Journal of the Operational Research Society, 32(3):187-196, March 1981. URL http://www . jstor.org/stable/2581060.

J.-M. Martel and B. Aouni. Diverse imprecise goal programming model formulations. J. Global Opt, 12:127-138, 1998.

A. J. H. Martinez and A. G. Aguado. Stochastic goal programming wth recourse. Revista de la Real Academia de Ciencias Exactas, Fisicasy Naturales, 92(4):409-414, 1998.

M. Masmoudi and F. B. Abdelaziz. A recourse goal programming approach for the portfolio selection problem. INFOR: Information Systems and Operational Research, 50(3):134-139, 2012. doi: 10.3138/infor.50.3.134. URL http://dx.doi.org/10.3138/infor.50.3.134.

H. Masri, F. Ben Abdelaziz, and H. Alaya. A recourse stochastic goal programming approach for the multi-objective stochastic vehicle routing problem. Journal of Multi-Criteria Decision Analysis, pages n/a-n/a, 2016. ISSN 1099-1360. doi: 10.1002/mcda.1563. URL http://dx. doi.org/10.1002/mcda.1563. MCDA-15-0012.R3.

R. McDaniel and D. Driebe. Uncertainty and Suprise in Complex Systems: Questions on Working the Unexpected. Springer:Berlin, Germany, 2005.

D. McInerney, R. Lempert, and K. Keller. What are robust strategies in the face of uncertain climate threshold responses? Climatic Change, 112(3-4):547-568, 2012. ISSN 0165-0009. doi: 10.1007/s10584-011-0377-1. URL http://dx.doi.org/10.1007/s10584-011-0377-1.

F. D. Mele, A. M. Kostin, G. Guillén-Gosálbez, and L. Jiménez. Multiobjective model for more sustainable fuel supply chains. a case study of the sugar cane industry in argentina. Industrial E) Engineering Chemistry Research, 50(9):4939-4958, 2011.

K. Miettinen. Nonlinear multiobjective optimization, volume 12. Springer Science \& Business Media, 2012.

E. L. Milan, S. M. Fernandez, and L. M. P. Aragones. Sugar cane transportation in cuba, a case study. European Journal of Operational Research, 174(1):374-386, 2006.

S. Mishra, Prakash, M. K. Tiwari, and R. S. Lashkari. A fuzzy goal-programming model of machine-tool selection and operation allocation problem in fms: a quick converging simulated annealing-based approach. International Journal of Production Research, 44(1):43-76, 2006. doi: 10.1080/13528160500245772. URL http://dx.doi.org/10.1080/13528160500245772. 
R. H. Mohamed. The relationship between goal programming and fuzzy programming. Fuzzy Sets and Systems, 89(2):215 - 222, 1997. ISSN 0165-0114. doi: http://dx.doi.org/ 10.1016/S0165-0114(96)00100-5. URL http://www.sciencedirect.com/science/article/ $\mathrm{pii} / \mathrm{S} 0165011496001005$.

W. Mohammed. Chance constrained fuzzy goal programming with right-hand side uniform random variable coefficients. Fuzzy Sets and Systems, 109(1):107 - 110, 2000. ISSN 0165-0114. doi: http://dx.doi.org/10.1016/S0165-0114(98)00151-1. URL http://www. sciencedirect . com/science/article/pii/S0165011498001511.

M. Morgan, M. Henrion, and M. Small. Uncertainty: A Guide to Dealing with Uncertainty in Quantitative Risk and Policy Analysis. Cambridge University Press, 1992. ISBN 9780521427449. URL http://books.google.co.za/books?id=ajd1V305PgQC.

H. Mouslim, M. Belmokaddem, M. Benbouziane, and S. Melloul. A fuzzy goal programming formulation with multiple target levels. Journal of Multi-Criteria Decision Analysis, 21(3-4): 223-235, 2014. ISSN 1099-1360. doi: 10.1002/mcda.1511. URL http://dx.doi.org/10. $1002 /$ mcda. 1511.

A. P. Muhlemann, A. G. Lockett, and A. E. Gear. Portfolio modeling in multiple-criteria situations under uncertainty. Decision Sciences, 9(4):612-626, 1978. ISSN 1540-5915. doi: 10.1111/j.1540-5915.1978.tb00749.x. URL http://dx.doi.org/10.1111/j.1540-5915. 1978.tb00749.x.

M. Mkidiche, H. Mouslim, and A. Sahed. Application of tolerance approach to fuzzy goal programming to aggregate production planning. International Journal of Mathematics in Operational Research, 5(2):183-204, Jan. 2013. URL http://dx.doi.org/10.1504/IJMOR.2013. 052460.

R. Narasimhan. Goal programming in a fuzzy environment. Decision Sciences, 11(2):325-336, 1980. ISSN 1540-5915. doi: 10.1111/j.1540-5915.1980.tb01142.x. URL http://dx.doi.org/ $10.1111 / j .1540-5915.1980 . t b 01142 . x$.

R. Narasimhan. On fuzzy goal programmingsome comments. Decision Sciences, 12(3):532-538, 1981. ISSN 1540-5915. doi: 10.1111/j.1540-5915.1981.tb00103.x. URL http://dx.doi.org/ 10.1111/j.1540-5915.1981.tb00103.x.

C. Nembou and B. Murtagh. A chance-constrained programming approach to modelling hydrothermal electricity generation in papua new guinea. Asia-Pacific Journal of Operational Research, 13(2):105-114, 1996.

J. P. Oddoye, D. F. Jones, M. Tamiz, and P. Schmidt. Combining simulation and goal programming for healthcare planning in a medical assessment unit. European Journal of Operational Research, 193(1):250-261, 2009.

W. Ogryczak. A goal programming model of the reference point method. Annals of Operations Research, 51(1):33-44, 1994.

P. Olsen. Multistage stochastic programming with recourse: The equivalent deterministic problem. SIAM Journal on Control and Optimization, 14(3):495-517, 1976a. doi: 10.1137/0314033. URL http://dx.doi.org/10.1137/0314033.

P. Olsen. Multistage stochastic programming with recourse as mathematical programming in an $l_{p}$ space. SIAM Journal on Control and Optimization, 14(3):528-537, 1976b. doi: 10.1137/ 0314035. URL http://dx.doi.org/10.1137/0314035. 
A. Osmani and J. Zhang. Stochastic optimization of a multi-feedstock lignocellulosic-based bioethanol supply chain under multiple uncertainties. Energy, 59:157-172, 2013.

A. Osmani and J. Zhang. Economic and environmental optimization of a large scale sustainable dual feedstock lignocellulosic-based bioethanol supply chain in a stochastic environment. Applied Energy, 114:572-587, 2014.

U. Özcan and B. Toklu. Multiple-criteria decision-making in two-sided assembly line balancing: A goal programming and a fuzzy goal programming models. Computers 8 Operations Research, 36(6):1955-1965, 2009.

D. A. P. K. De and K. C. Sahu. A chance-constrained goal programming model for capital budgeting. The Journal of the Operational Research Society, 33(7):635-638, July 1982. URL http://www.jstor.org/stable/2581726.

R. P. Paiva and R. Morabito. An optimization model for the aggregate production planning of a brazilian sugar and ethanol milling company. Annals of Operations Research, 169(1):117-130, 2009 .

B. Pal, B. Moitra, and U. Maulik. A goal programming procedure for fuzzy multiobjective linear fractional programming problem. Fuzzy Sets and Systems, 139(2):395 - 405, 2003. ISSN 0165-0114. doi: http://dx.doi.org/10.1016/S0165-0114(02)00374-3. URL http://www . sciencedirect.com/science/article/pii/S0165011402003743.

B. B. Pal and B. N. Moitra. A fuzzy goal programming procedure for solving quadratic bilevel programming problems. International Journal of Intelligent Systems, 18(5):529-540, 2003. ISSN 1098-111X. doi: 10.1002/int.10102. URL http://dx.doi.org/10.1002/int.10102.

M. A. Parra, A. B. Terol, and M. R. Ur?a. A fuzzy goal programming approach to portfolio selection. European Journal of Operational Research, 133(2):287 - 297, 2001. ISSN 0377-2217. doi: http://dx.doi.org/10.1016/S0377-2217(00)00298-8. URL http://www.sciencedirect.com/ science/article/pii/S0377221700002988. Multiobjective Programming and Goal Programming.

J. B. Pickens and J. G. Hof. Fuzzy goal programming in forestry: An application with special solution problems. Fuzzy Sets and Systems, 39(3):239 - 246, 1991. ISSN 0165-0114. doi: http:// dx.doi.org/10.1016/0165-0114(91)90095-8. URL http://www.sciencedirect.com/science/ article/pii/0165011491900958.

S. Pramanik and T. K. Roy. Fuzzy goal programming approach to multilevel programming problems. European Journal of Operational Research, 176(2):1151 - 1166, 2007. ISSN 03772217. doi: http://dx.doi.org/10.1016/j.ejor.2005.08.024. URL http://www.sciencedirect . com/science/article/pii/S0377221705008398.

S. Pramanik and T. K. Roy. Multiobjective transportation model with fuzzy parameters: Priority based fuzzy goal programming approach. Journal of Transportation Systems Engineering and Information Technology, 8(3):40 - 48, 2008. ISSN 1570-6672. doi: http://dx.doi.org/ 10.1016/S1570-6672(08)60023-9. URL http://www.sciencedirect.com/science/article/ $\mathrm{pii/S1570667208600239.}$

A. Prkopa. Multi-stage stochastic programming problems. In Stochastic Programming, volume 324 of Mathematics and Its Applications, pages 425-446. Springer Netherlands, 1995. ISBN 978-90-481-4552-2. doi: 10.1007/978-94-017-3087-7_13. URL http://dx.doi.org/10.1007/ 978-94-017-3087-7_13.

E. S. Quade. Analysis for public decisions. Elsevier Science, New York, 1989. 
R. Rai, S. Kameshwaran, and M. K. Tiwari. Machine-tool selection and operation allocation in fms: Solving a fuzzy goal-programming model using a genetic algorithm. International Journal of Production Research, 40(3):641-665, 2002. doi: 10.1080/00207540110081515. URL http://dx.doi.org/10.1080/00207540110081515.

J. Ramik. Fuzzy goals and fuzzy alternatives in goal programming problems. Fuzzy Sets and Systems, 111(1):81-86, 2000. doi: http://dx.doi.org/10.1016/S0165-0114(98)00454-0. URL http://www.sciencedirect.com/science/article/pii/S0165011498004540.

S. Rao, K. Sundararaju, B. G. Prakash, and C. Balakrishna. Fuzzy goal programming approach for structural optimization. AIAA Journal, 30(5):1425-1432, 1992. URL http://dx.doi. org/10.2514/3.11079.

M. Rasmy, S. M. Lee, W. A. El-Wahed, A. M. Ragab, and M. El-Sherbiny. An expert system for multiobjective decision making: application of fuzzy linguistic preferences and goal programming. Fuzzy Sets and Systems, 127(2):209 - 220, 2002. ISSN 0165-0114. doi: http://dx.doi.org/10.1016/S0165-0114(01)00201-9. URL http://www.sciencedirect.com/ science/article/pii/S0165011401002019.

C. Romero. Handbook of Critical Issues in Goal Programming. Pergamon Press, 1991.

J. Rosenhead and J. Mingers. Rational Analysis of a Problematic World Revisted: Problem Structuring Methods for Complexity, Uncertainty, and Conflict,. Wiley and Sons, Chichester, UK, 2001.

P. A. Rubin and R. Narasimhan. Fuzzy goal programming with nested priorities. Fuzzy Sets and Systems, 14(2):115 - 129, 1984. ISSN 0165-0114. doi: http://dx.doi.org/10. 1016/0165-0114(84)90095-2. URL http://www.sciencedirect.com/science/article/pii/ 0165011484900952.

O. M. Saad. An iterative goal programming approach for solving fuzzy multiobjective integer linear programming problems. Applied Mathematics and Computation, 170(1):216 - 225, 2005. ISSN 0096-3003. doi: http://dx.doi.org/10.1016/j.amc.2004.11.026. URL http://www.sciencedirect.com/science/article/pii/S0096300304009191.

T. L. Saaty. The analytic hierarchy process, ny. McGraw-Hill, USA. Cook WD and Seiford LM.(1978). Priority ranking and consensus formation, Management Science, 24:1721-1732, 1980.

N. V. Sahinidis. Optimization under uncertainty: state-of-the-art and opportunities. Computers \& Chemical Engineering, 28(6):971-983, 2004.

N. P. Sahoo and M. P. Biswal. Computation of some stochastic linear programming problems with cauchy and extreme value distributions. International Journal of Computer Mathematics, 82(6):685-698, 2005.

SASAwebsite. South aafrica sugar association, 2017. URL http://www.sasa.org.za/ HomePage1. aspx.

Y. Sawaragi, H. Nakayama, and T. Tanino. Theory of Multiobjective Optimization. Academic Press, New York, 1985.

H. Selim and I. Ozkarahan. A supply chain distribution network design model: An interactive fuzzy goal programming-based solution approach. The International Journal of Advanced Manufacturing Technology, 36(3-4):401-418, 2008. ISSN 0268-3768. doi: 10.1007/ s00170-006-0842-6. URL http://dx.doi.org/10.1007/s00170-006-0842-6. 
H. Selim, C. Araz, and I. Ozkarahan. Collaborative productiondistribution planning in supply chain: A fuzzy goal programming approach. Transportation Research Part E: Logistics and Transportation Review, 44(3):396 - 419, 2008. ISSN 1366-5545. doi: http://dx.doi.org/ 10.1016/j.tre.2006.11.001. URL http://www.sciencedirect.com/science/article/pii/ S1366554506000998.

A. Shapiro and T. Homem-de Mello. A simulation-based approach to two-stage stochastic programming with recourse. Mathematical Programming, 81(3):301-325, 1998. ISSN 0025-5610. doi: 10.1007/BF01580086. URL http://dx.doi.org/10.1007/BF01580086.

A. Shapiro, D. Dentcheva, and A. Ruszczy?ski. Lectures on Stochastic Programming: Modeling and Theory. MOS-SIAM series on optimization. Society for Industrial and Applied Mathematics (SIAM, 3600 Market Street, Floor 6, Philadelphia, PA 19104), 2009. ISBN 9780898718751. URL http://books.google.co.za/books?id=7h7v97qUwPYC.

D. K. Sharma and R. Jana. Fuzzy goal programming based genetic algorithm approach to nutrient management for rice crop planning. International Journal of Production Economics, 121(1):224 - 232, 2009. ISSN 0925-5273. doi: http://dx.doi.org/10.1016/j.ijpe.2009.05.009. URL http://www.sciencedirect.com/science/article/pii/S0925527309001662. Modelling and Control of Productive Systems: Concepts and Applications.

H. Sharma, D. K. Sharma, and R. Jana. Credit union portfolio management-an additive fuzzy goal programming approach. International Research Journal of Finance and Economics, 30: $18-29,2009$.

K. Sigel, B. Klauer, and C. Pahl-Wostl. Conceptualising uncertainty in environmental decisionmaking: The example of the eu water framework directive. Ecological Economics, 69(3):502510, jan 2010. ISSN 0921-8009. URL http://www.sciencedirect.com/science/article/ pii/S0921800909004613.

S. Sinha, K. Rao, and B. Mangaraj. Fuzzy goal programming in multi-criteria decision systems: A case study in agricultural planning. Socio-Economic Planning Sciences, 22(2):93 - 101, 1988. ISSN 0038-0121. doi: http://dx.doi.org/10.1016/0038-0121(88)90021-3. URL http: //www.sciencedirect.com/science/article/pii/0038012188900213.

A. L. Soyster. Technical note: Convex programming with set-inclusive constraints and applications to inexact linear programming. Operations Research, 21(5):1154-1157, 1973.

I. Stancu-Minasian. Stochastic Programming with Multiple Objective Functions. D. Reidel Publishing Company, Dordrecht., 1984.

T. J. Stewart. Experience with prototype multicriteria decision support systems for pelagic fish quota determination. Naval Research Logistics (NRL), 35(6):719-731, 1988.

T. J. Stewart. Dealing with uncertainties in mcda. In Multiple Criteria Decision Analysis: State of the Art Surveys, volume 78 of International Series in Operations Research 83 Management Science, pages 445-466. Springer New York, 2005. ISBN 978-0-387-23067-2. doi: 10.1007/ 0-387-23081-5_11. URL http://dx.doi.org/10.1007/0-387-23081-5_11.

T. J. Stewart, S. French, and J. Rios. Integrating multicriteria decision analysis and scenario planningreview and extension. Omega, 41(4):679 - 688, 2013. ISSN 0305-0483. doi: http: //dx.doi.org/10.1016/j.omega.2012.09.003. URL http://www.sciencedirect.com/science/ article/pii/S030504831200179X. 
S. J. Stoyan and R. H. Kwon. A stochastic-goal mixed-integer programming approach for integrated stock and bond portfolio optimization. Computers \& Industrial Engineering, 61(4): 1285 - 1295, 2011. ISSN 0360-8352. doi: http://dx.doi.org/10.1016/j.cie.2011.07.022. URL http://www.sciencedirect.com/science/article/pii/S0360835211002099.

N. Taleb. The Black Swan. Random House, New York, NY, USA, 2007.

M. Tamiz and M. Yaghoobi. Nurse scheduling by fuzzy goal programming. In D. Jones, M. Tamiz, and J. Ries, editors, New Developments in Multiple Objective and Goal Programming, volume 638 of Lecture Notes in Economics and Mathematical Systems, pages 151-163. Springer Berlin Heidelberg, 2010. ISBN 978-3-642-10353-7.

M. Tamiz, D. Jones, and C. Romero. Goal programming for decision making: An overview of the current state-of-the-art. European Journal of Operational Research, 111(3):569 - 581, 1998. ISSN 0377-2217. doi: http://dx.doi.org/10.1016/S0377-2217(97)00317-2. URL http: //www.sciencedirect.com/science/article/pii/S0377221797003172.

R. Tiwari, S. Dharmar, and J. Rao. Priority structure in fuzzy goal programming. Fuzzy Sets and Systems, 19(3):251 - 259, 1986. ISSN 0165-0114. doi: http://dx.doi.org/10. 1016/0165-0114(86)90054-0. URL http://www.sciencedirect.com/science/article/pii/ 0165011486900540.

R. Tiwari, S. Dharmar, and J. Rao. Fuzzy goal programming an additive model. Fuzzy Sets and Systems, 24(1):27 - 34, 1987. ISSN 0165-0114. doi: http://dx.doi.org/10.1016/0165-0114(87) 90111-4. URL http://www.sciencedirect.com/science/article/pii/0165011487901114.

S. Torabi1 and E. Hassini. Multi-site production planning integrating procurement and distribution plans in multi-echelon supply chains: an interactive fuzzy goal programming approach. International Journal of Production Research, 47(19):5475-5499, 2009. doi: 10.1080/00207540801905460. URL http://www.tandfonline.com/doi/abs/10.1080/ 00207540801905460.

T. Trzaskalik. Dynamic goal programming models. In R. Caballero, F. Ruiz, and R. Steuer, editors, Advances in Multiple Objective and Goal Programming, volume 455 of Lecture Notes in Economics and Mathematical Systems, pages 111-119. Springer Berlin Heidelberg, 1997. ISBN 978-3-540-63599-4.

W.-H. Tsai and S.-J. Hung. A fuzzy goal programming approach for green supply chain optimisation under activity-based costing and performance evaluation with a value-chain structure. International Journal of Production Research, 47(18):4991-5017, 2009.

M. van Asselt and J. Rotmans. Uncertainty in integrated assessment modelling. Climatic Change, 54(1-2):75-105, 2002. ISSN 0165-0009. doi: 10.1023/A:1015783803445. URL http://dx.doi. org/10.1023/A\%3A1015783803445.

M. B. A. Van Asselt. Perspectives on Uncertainty and Risk, chapter 10, pages 407-417. Springer Netherlands, 2000. doi: 10.1007/978-94-017-2583-5_10. URL http://dx.doi.org/10.1007/ 978-94-017-2583-5_10.

K. Van der Heijden. Scenarios: the art of strategic conversation. John Wiley \& Sons, 1996. ISBN 9780471966395. URL https://books.google.co.za/books?id=oZYoAQAAMAAJ.

J. W. G. M. Van der Pas, W. E. Walker, V. A. W. J. Marchau, G. P. Van Wee, and D. B. Agusdinata. Exploratory mcda for handling deep uncertainties: the case of intelligent speed adaptation implementation. Journal of Multi-Criteria Decision Analysis, 17(1-2):1-23, 2010. ISSN 1099-1360. doi: 10.1002/mcda.450. URL http://dx.doi.org/10.1002/mcda. 450. 
J. P. van der Sluijs. Anchoring amid uncertainty: On the management of uncertainties in risk assessment of anthropogenic climate change. PhD thesis, University of Utrecht, The Netherlands., 1997.

R. Van Slyke and R. Wets. L-shaped linear programs with applications to optimal control and stochastic programming. SIAM Journal on Applied Mathematics, 17(4):638-663, 1969. doi: 10.1137/0117061. URL http://dx.doi.org/10.1137/0117061.

W. Walker, P. Harremoes, J. Rotmans, J. van der Sluijs, M. van Asselt, P. Janssen, and M. K. von Krauss. Defining uncertainty: A conceptual basis for uncertainty management in model-based decision support. Integrated Assessment Journal, 4(1):5-17, 2003.

W. Walker, R. Lempert, and J. Kwakkel. Deep uncertainty. In S. Gass and M. Fu, editors, Encyclopedia of Operations Research and Management Science, pages 395-402. Springer US, 2013a. ISBN 978-1-4419-1137-7. doi: 10.1007/978-1-4419-1153-7_1140. URL http://dx.doi. org/10.1007/978-1-4419-1153-7_1140.

W. E. Walker, S. Rahman, and J. Cave. Adaptive policies, policy analysis, and policy-making. European Journal of Operational Research, 128(2):282 - 289, 2001. ISSN 0377-2217. doi: http://dx.doi.org/10.1016/S0377-2217(00)00071-0. URL http://www.sciencedirect.com/ science/article/pii/S0377221700000710. Complex Societal Problems.

W. E. Walker, M. Haasnoot, and J. H. Kwakkel. Adapt or perish: A review of planning approaches for adaptation under deep uncertainty. Sustainability, 5(3):955-979, 2013b. ISSN 2071-1050. doi: 10.3390/su5030955. URL http://www.mdpi.com/2071-1050/5/3/955.

H.-F. Wang and C.-C. Fu. A generalization of fuzzy goal programming with preemptive structure. Computers \& Operations Research, 24(9):819 - 828, 1997. ISSN 0305-0548. doi: http://dx.doi.org/10.1016/S0305-0548(96)00096-2. URL http://www.sciencedirect.com/ science/article/pii/S0305054896000962.

S. Wang and S. Zhu. On fuzzy portfolio selection problems. Fuzzy Optimization and Decision Making, 1(4):361-377, 2002. ISSN 1568-4539. doi: 10.1023/A:1020907229361.

Y.-M. Wang, J.-B. Yang, and D.-L. Xu. A two-stage logarithmic goal programming method for generating weights from interval comparison matrices. Fuzzy Sets and Systems, 152(3): 475 - 498, 2005. ISSN 0165-0114. doi: http://dx.doi.org/10.1016/j.fss.2004.10.020. URL http://www.sciencedirect.com/science/article/pii/S0165011404004634.

J. Watada. Fuzzy portfolio selection and its applications to decision making. Tatra Mountains Mathematical Publication, 13:219-248, 1997.

D. Watkins, D. McKinney, L. Lasdon, S. Nielsen, and Q. Martin. A scenario-based stochastic programming model for water supplies from the highland lakes. International Transactions in Operational Research, 7(3):211-230, 2000. ISSN 1475-3995. doi: 10.1111/j.1475-3995.2000. tb00195.x. URL http://dx.doi.org/10.1111/j.1475-3995.2000.tb00195.x.

Website. Food, agriculture and natural resources policy analysis network, 2017. URL http: //www.fanrpan.org/documents/d00472/.

R. Wets. Stochastic programming: Solution techniques and approximation schemes. In A. Bachem, B. Korte, and M. Grtschel, editors, Mathematical Programming The State of the Art, pages 566-603. Springer Berlin Heidelberg, 1983. ISBN 978-3-642-68876-8. doi: 10.1007/978-3-642-68874-4_22. URL http: //dx.doi .org/10.1007/978-3-642-68874-4_22.

D. White. Optimality and Ef?ciency. Wiley, Chichester., 1982. 
A. P. Wierzbicki. A mathematical basis for satisficing decision making. Mathematical Modelling, 3(5):391 - 405, 1982. ISSN 0270-0255. doi: http://dx.doi.org/10.1016/0270-0255(82)90038-0. URL http://www.sciencedirect.com/science/article/pii/0270025582900380. Special \{IIASA $\}$ Issue.

A. P. Wierzbicki. Reference point methods in vector optimization and decision support. Technical Report 43 pp, International Institute for Applied Systems Analysis, Laxenburg, Austria., 1998.

Z. Xu. Goal programming models for obtaining the priority vector of incomplete fuzzy preference relation. International Journal of Approximate Reasoning, 36(3):261 - 270, 2004. ISSN 0888613X. doi: http://dx.doi.org/10.1016/j.ijar.2003.10.011. URL http://www.sciencedirect . com/science/article/pii/S0888613X0300149X.

M. Yaghoobi and M. Tamiz. A method for solving fuzzy goal programming problems based on \{MINMAX\} approach. European Journal of Operational Research, 177(3):1580 - 1590, 2007. ISSN 0377-2217. doi: http://dx.doi.org/10.1016/j.ejor.2005.10.022. URL http://www . sciencedirect.com/science/article/pii/S0377221705006624.

M. A. Yaghoobi, D. F. JONES, and M. TAMIZ. Weighted additive models for solving fuzzy goal programming problems. Asia-Pacific Journal of Operational Research, 25(05):715-733, 2008. doi: 10.1142/S0217595908001973. URL http://www.worldscientific.com/doi/abs/ 10.1142/S0217595908001973.

T. Yang, J. P. Ignizio, and H.-J. Kim. Fuzzy programming with nonlinear membership functions: Piecewise linear approximation. Fuzzy Sets and Systems, 41(1):39 - 53, 1991. ISSN 0165-0114. doi: http://dx.doi.org/10.1016/0165-0114(91)90156-K. URL http://www.sciencedirect . com/science/article/pii/016501149190156K.

H. T. Yoshizaki, A. R. Muscat, and J. L. Biazzi. Decentralizing ethanol distribution in southeastern brazil. Interfaces, 26(6):24-34, 1996.

L. Zadeh. Fuzzy sets. Information and Control, 8(3):338 - 353, 1965. ISSN 0019-9958. doi: http://dx.doi.org/10.1016/S0019-9958(65)90241-X. URL http://www.sciencedirect.com/ science/article/pii/S001999586590241X.

A. Zamboni, F. Bezzo, and N. Shah. Spatially explicit static model for the strategic design of future bioethanol production systems. 2. multi-objective environmental optimization. Energy ES Fuels, 23(10):5134-5143, 2009.

H. Zimmermann. Description and optimization of fuzzy systems. International Journal of General Systems, 2:209-215, 1976.

H. Zimmermann. Fuzzy programming and linear programming with several objective functions. Fuzzy Sets and Systems, 1(1):45 - 55, 1978. ISSN 0165-0114. doi: http://dx.doi.org/10. 1016/0165-0114(78)90031-3. URL http://www. sciencedirect.com/science/article/pii/ 0165011478900313.

H. Zimmermann. Fuzzy mathematical programming. Computers \& Operations Research, 10(4): 291 - 298, 1983. ISSN 0305-0548. doi: http://dx.doi.org/10.1016/0305-0548(83)90004-7. URL http://www.sciencedirect.com/science/article/pii/0305054883900047.

H. Zimmermann. Fuzzy Set Theory and Its Applications. Kluwer-Nijhoff, Dordrecht., 3rd edition edition, 1996.

H.-J. Zimmermann. Fuzzy set theory - and its applications, 1991. 